Palladium-Catalyzed Enantioselective Domino Heck/Intermolecular C-H Bond Functionalization: Development and Application to the Synthesis of (+)Esermethole

\author{
Wangqing Kong, Qian Wang and Jieping Zhu \\ Laboratory of Synthesis and Natural Products, Institute of Chemical Sciences and \\ Engineering, Ecole Polytechnique Fédérale de Lausanne, EPFL-SB-ISIC-LSPN, BCH 5304, 1015 \\ Lausanne, Switzerland \\ e-mail: jieping.zhu@epfl.ch
}

Content

1. General information

2. Table S1: Optimization of the reaction conditions with achiral ligands

3. Synthesis and characterization data of aryl triflates 1 S4

4. KIE experiments

5. General procedure

6. Characterization data

7. Crystallographic data for compounds $(R)-6$ and $(R)-9$ aa

8. References

9. Copies of the ${ }^{1} \mathrm{H}$ and ${ }^{13} \mathrm{C}$ NMR spectra 


\section{General Information}

NMR spectra were recorded on AV2 400 or AV2 $500 \mathrm{MHz}$ Bruker spectrometers. Chemical shifts are given in ppm. The spectra are calibrated to the residual ${ }^{1} \mathrm{H}$ and ${ }^{13} \mathrm{C}$ signals of the solvents. Multiplicities are abbreviated as follows: singlet $(s)$, doublet $(d)$, triplet $(t)$, quartet (q), doublet-doublet (dd), quintet (quint), septet (sept), multiplet (m), and broad (b). Infrared spectra were recorded on a JASCO FT/IR-4100 spectrometer. Mass spectra were determined with a Waters ACQUITY H-class UPLC/MS ACQ-SQD by electron ionisation (El positive and negative) or a Finnigan TSQ7000 by electrospray ionization (ESI+). The accurate masses were measured by the mass spectrometry service of the EPFL by ESI-TOF using a QTOF Ultima from Waters or APPI-FT-ICR using a linear ion trap Fourier transform ion cyclotron resonance mass spectrometer from Thermo Scientific. Optical rotations $\alpha_{D}$ were obtained with a Jasco P-2000 polarimeter $(589 \mathrm{~nm})$. Enantiomeric excesses were determined with a Thar SFC Investigator system using chiral stationary phase columns by comparing the samples with the appropriate racemic samples, column and elution details specified in each entry. Melting points were measured using a Stuart SMP30.

Materials and Methods: Unless otherwise stated, starting materials were purchased from commercial sources (Aldrich, Acros, Merck, Fluka and VWR international). More sensitive compounds were stored in a desiccator or in a glove-box if required. Solvents were purchased in HPLC quality, degassed by purging thoroughly with nitrogen and dried over activated molecular sieves of appropriate size. Alternatively, they were purged with argon and passed through alumina columns in a solvent purification system (Innovative Technology). Reactions were monitored by thin layer chromatography (TLC) using Merck TLC silica gel 60 F254. Compounds were visualized by UV-light at $254 \mathrm{~nm}$ and by dipping the plates in an ethanolic vanillin/sulfuric acid solution or an aqueous potassium permanganate solution followed by heating. Flash column chromatography was performed over silica gel (230-400 mesh). 
2. Table S1: Optimization of the Reaction Conditions with Achiral Ligand:

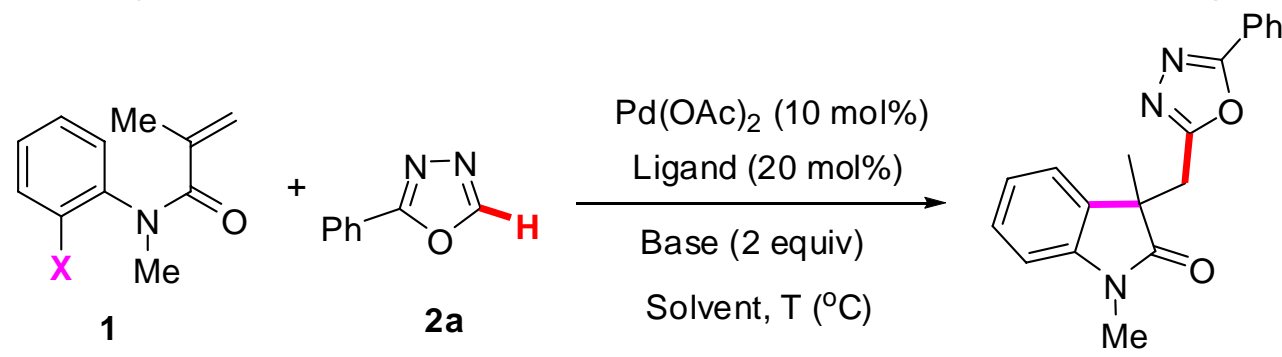

3 aa

\begin{tabular}{|c|c|c|c|c|c|c|}
\hline entry & $x$ & base & ligand & solvent & $\mathrm{T}\left({ }^{\circ} \mathrm{C}\right)$ & Yield (\%) of 3aa \\
\hline 1 & $\mathrm{Br}$ & $\mathrm{Cs}_{2} \mathrm{CO}_{3}$ & dppp & toluene & 100 & 76 \\
\hline 2 & $\mathrm{Br}$ & DBU & dppp & toluene & 100 & 40 \\
\hline 3 & $\mathrm{Br}$ & $\mathrm{KOBu}-t$ & dppp & toluene & 100 & no desired product \\
\hline 4 & $\mathrm{Br}$ & LiOBu-t & dppp & toluene & 100 & no desired product \\
\hline 5 & $\mathrm{Br}$ & $\mathrm{Cs}_{2} \mathrm{CO}_{3}$ & Xantphos & toluene & 100 & 70 \\
\hline 6 & $\mathrm{Br}$ & $\mathrm{Cs}_{2} \mathrm{CO}_{3}$ & dppp & $\mathrm{MeCN}$ & 100 & 85 \\
\hline 7 & $\mathrm{Br}$ & $\mathrm{Cs}_{2} \mathrm{CO}_{3}$ & dppp & THF & 100 & 96 \\
\hline 8 & $\mathrm{Br}$ & $\mathrm{Cs}_{2} \mathrm{CO}_{3}$ & dppp & DMF & 100 & 85 \\
\hline 9 & $\mathrm{Br}$ & $\mathrm{Cs}_{2} \mathrm{CO}_{3}$ & dppp & THF & 80 & 95 \\
\hline 10 & $\mathrm{Br}$ & $\mathrm{Cs}_{2} \mathrm{CO}_{3}$ & dppp & THF & 40 & no reaction \\
\hline 11 & $\mathbf{I}$ & $\mathrm{Cs}_{2} \mathrm{CO}_{3}$ & dppp & THF & 80 & 94 \\
\hline 12 & OTf & $\mathrm{Cs}_{2} \mathrm{CO}_{3}$ & dppp & THF & 80 & $80 \%$ \\
\hline 13 & OTf & $\mathrm{K}_{2} \mathrm{CO}_{3}$ & dppp & THF & 80 & $76 \%$ \\
\hline 14 & OTf & $\mathrm{K}_{3} \mathrm{PO}_{4}$ & $d p p p$ & THF & 80 & $81 \%$ \\
\hline 15 & OTf & TMG & dppp & $\mathrm{CH}_{3} \mathrm{CN}$ & 80 & $84 \%$ \\
\hline 16 & OTf & $\mathrm{K}_{2} \mathrm{HPO}_{4}$ & dppp & THF & 80 & no reaction \\
\hline
\end{tabular}




\section{Synthesis and Characterization Data of Aryl Triflates 1}

\subsection{General procedure for the synthesis of aryl triflates $1:^{1}$}

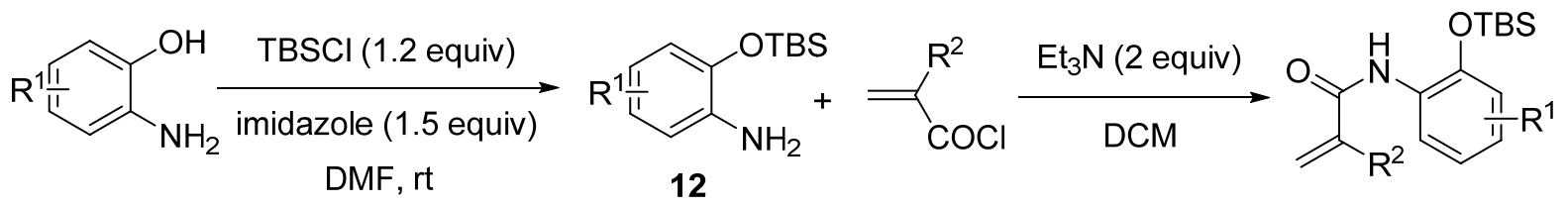

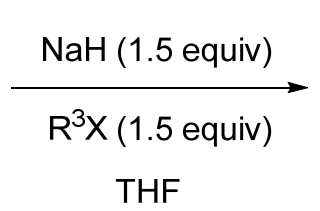

THF<smiles>[R]C(=C)C(=O)N([R])C1=CC=[R1]#[R1]=C1OC</smiles>

14

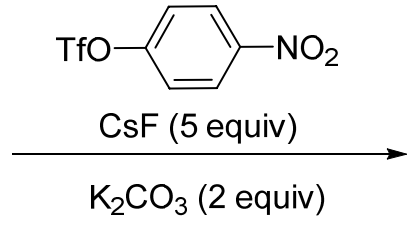

DMF<smiles></smiles>

1

To a solution of 2-aminophenol $(100 \mathrm{mmol})$ and imidazole (100 mmol, $10.2 \mathrm{~g}$ ) in DMF (100 $\mathrm{mL}$ ) was added a solution of TBSCl $(110 \mathrm{mmol}, 16.5 \mathrm{~g})$ in DMF (50 mL). The mixture was stirred at room temperature overnight. After the reaction was complete (monitored by TLC), the mixture was quenched with saturated $\mathrm{NH}_{4} \mathrm{Cl}$ solution $(100 \mathrm{~mL})$ and extracted with EtOAc $(100 \mathrm{~mL} \times 3)$. The combined organic layers were washed with brine $(150 \mathrm{~mL} \times 2)$, dried over anhydrous $\mathrm{Na}_{2} \mathrm{SO}_{4}$ and filtrated. Removal of the solvent under reduced pressure gave a yellow oily residue $\mathbf{1 2}$ which was used for the next step without further purification.

To a solution of TBS-protected aminophenol $12(30 \mathrm{mmol})$ and triethylamine $(60 \mathrm{mmol}, 8.7$ $\mathrm{mL}$ ) in DCM (40 mL) at $0{ }^{\circ} \mathrm{C}$ was added dropwise a solution of acryloyl chloride (36 mmol) in DCM (10 mL) over $15 \mathrm{~min}$. After stirring for another $30 \mathrm{~min}$, the mixture was warmed to room temperature and stirred until the amine was consumed completely (monitored by TLC). The resulting solution was concentrated, and the residue was dissolved in EtOAc $(50 \mathrm{~mL})$ and filtered. The organic layer was washed with $5 \% \mathrm{HCl}$ solution $(3 \times 20 \mathrm{~mL})$, saturated $\mathrm{NaHCO}_{3}$ solution $(50 \mathrm{~mL})$ and brine $(50 \mathrm{~mL})$, dried over anhydrous $\mathrm{Na}_{2} \mathrm{SO}_{4}$, filtered, and concentrated in vacuo. The residue (yellow oil) 13 was used for the next step without further purification.

To a stirred mixture of $\mathrm{NaH}$ (60\% dispersion in mineral oil) (18 mmol, $0.72 \mathrm{~g}, 1.5$ equiv) in dry THF (50 mL) was added amide $13(30 \mathrm{mmol})$ at $0{ }^{\circ} \mathrm{C}$ under $\mathrm{N}_{2}$. After being stirred at $\mathrm{rt}$ for 30 min, the reaction was cooled to $0{ }^{\circ} \mathrm{C}$ and $R^{3} X(1.2$ equiv) was added dropwise. The mixture was then stirred at room temperature overnight. After the reaction was complete 
(monitored by $\mathrm{TLC}$ ), the mixture was quenched with saturated aqueous $\mathrm{NH}_{4} \mathrm{Cl}$ at $0{ }^{\circ} \mathrm{C}$ and extracted with EtOAc $(40 \mathrm{~mL} \times 3)$. The combined extracts were washed with water and brine, dried over $\mathrm{Na}_{2} \mathrm{SO}_{4}$, filtered, and concentrated in vacuo. The residue was purified by column chromatography on silica gel to afford the $N$-protected amide 14.

To a solution of $14(5 \mathrm{mmol})$ in DMF $(30 \mathrm{~mL})$ was added CsF $(25 \mathrm{mmol})$. After stirring at $0{ }^{\circ} \mathrm{C}$ for $30 \mathrm{~min}$, 4-nitrophenyltriflate $(6 \mathrm{mmol})$ and $\mathrm{K}_{2} \mathrm{CO}_{3}(10 \mathrm{mmol})$ were introduced. The reaction mixture became yellow due to the generation of 4-nitrophenol. At the end of the reaction (followed by $T L C$ ), the yellow solution was diluted with $\mathrm{H}_{2} \mathrm{O}(40 \mathrm{~mL})$ and extracted with EtOAc. The combined organic phases were washed with $1 \mathrm{~N} \mathrm{NaOH}$ solution and saturated brine, dried over $\mathrm{Na}_{2} \mathrm{SO}_{4}$, and evaporated. The residue was purified by column chromatography on silica gel to afford the aryl triflate 1.

\subsection{Characterization data of aryl triflates 1:}

2-(N-Methylmethacrylamido)phenyl trifluoromethanesulfonate (1b)<smiles>C=C(C)C(=O)N(C)c1ccccc1[O-]</smiles>

Chemical Formula: $\mathrm{C}_{12} \mathrm{H}_{12} \mathrm{~F}_{3} \mathrm{NO}_{4} \mathrm{~S}$; Molecular Weight: 323.2882

According to general procedure, $\mathbf{1 b}$ was obtained in $61 \%$ yield over four steps as a white solid. b.p. $71^{\circ} \mathrm{C} ;{ }^{1} \mathrm{H}$ NMR (400 MHz, $\mathrm{CDCl}_{3}$ ): $\delta$ 7.48-7.27 (m, 4H), $5.06(\mathrm{~s}, 1 \mathrm{H}), 4.79(\mathrm{~s}, 1 \mathrm{H})$, $3.35(\mathrm{~s}, 3 \mathrm{H}), 1.88(\mathrm{~s}, 3 \mathrm{H}) ;{ }^{13} \mathrm{C}$ NMR $\left(100 \mathrm{MHz}, \mathrm{CDCl}_{3}\right): \delta 171.5,144.4,139.7,137.8,129.3$, $129.1,128.8,122.6,119.7,118.5(q, J=320.2 \mathrm{~Hz}), 37.5,19.8 ;{ }^{19} \mathrm{~F} \mathrm{NMR}\left(376 \mathrm{MHz}, \mathrm{CDCl}_{3}\right): \delta-$ 73.74 (s); IR: $v\left(\mathrm{~cm}^{-1}\right)$ 2984, 2930, 1660, 1629, 1497, 1422, 1363, 1249, 1211, 1167, 1158, 1138, 1088, 895, 864; HRMS: (ESI) calcd for $\mathrm{C}_{12} \mathrm{H}_{12} \mathrm{~F}_{3} \mathrm{NO}_{4} \mathrm{SH}^{+}[\mathrm{M}+\mathrm{H}]^{+}$324.0517; found 324.0517 .

2-(N-Benzylmethacrylamido)phenyl trifluoromethanesulfonate (1d) 
$\underbrace{\mathrm{N}}_{\substack{1 \\ \mathrm{Bn}}}$

Chemical Formula: $\mathrm{C}_{18} \mathrm{H}_{16} \mathrm{~F}_{3} \mathrm{NO}_{4} \mathrm{~S}$; Molecular Weight: 399.3841

According to general procedure, $\mathbf{1 d}$ was obtained in 51\% yield over four steps as a yellow oil. ${ }^{1} \mathrm{H}$ NMR $\left(400 \mathrm{MHz}, \mathrm{CDCl}_{3}\right): \delta$ 7.42-7.28 (m, 7H), $7.24(\mathrm{dt}, J=8.6,4.4 \mathrm{~Hz}, 1 \mathrm{H}), 7.00(\mathrm{~d}, J=7.8$ $\mathrm{Hz}, 1 \mathrm{H}), 5.80(\mathrm{~s}, 1 \mathrm{H}), 5.12(\mathrm{~s}, 1 \mathrm{H}), 4.91(\mathrm{~s}, 1 \mathrm{H}), 4.15(\mathrm{~s}, 1 \mathrm{H}), 1.93(\mathrm{~s}, 3 \mathrm{H}) ;{ }^{13} \mathrm{C} N M R(101 \mathrm{MHz}$, $\left.\mathrm{CDCl}_{3}\right): \delta 171.4,144.5,139.8,137.4,136.4,130.1,129.1,128.8,128.7,128.4,127.7,122.3$, 119.9, 118.5 (q, $\left.J=320.2 \mathrm{~Hz}), 52.6,19.9 ;{ }^{19} \mathrm{~F} \mathrm{NMR} \mathrm{(376} \mathrm{MHz,} \mathrm{CDCl}_{3}\right): \delta-73.75(\mathrm{~s}) ; \mathrm{IR}: v\left(\mathrm{~cm}^{-1}\right)$ 2987, 2972, 2921, 2901, 1661, 1631, 1494, 1455, 1421, 1409, 1394, 1384, 1326, 1249, 1210, 1137, 1102, 1079, 1067, 1047, 1030, 1029, 1018, 889, 852; HRMS: (ESI) calcd for $\mathrm{C}_{18} \mathrm{H}_{16} \mathrm{~F}_{3} \mathrm{NO}_{4} \mathrm{SH}^{+}[\mathrm{M}+\mathrm{H}]^{+}$400.0830; found 400.0830 .

2-(2-Benzyl-N-methylacrylamido)phenyl trifluoromethanesulfonate (1g)<smiles>C=C(Cc1ccccc1)C(=O)N(C)c1ccccc1[OH2+]</smiles>

Chemical Formula: $\mathrm{C}_{18} \mathrm{H}_{16} \mathrm{~F}_{3} \mathrm{NO}_{4} \mathrm{~S}$; Molecular Weight: 399.3841

According to general procedure, $\mathbf{1 g}$ was obtained in $56 \%$ yield over four steps as a yellow oil. ${ }^{1} \mathrm{H}$ NMR $\left(400 \mathrm{MHz}, \mathrm{CDCl}_{3}\right): \delta$ 7.51-6.60 (m, 9H), 5.64-4.55 (m, 2H), 3.90-2.91 (m, 5H); ${ }^{13} \mathrm{C}$ NMR (101 MHz, $\left.\mathrm{CDCl}_{3}\right): \delta$ 171.1, 144.4, 143.6, 137.7, 137.4, 129.6, 129.2, 129.0, 128.5, 126.6, 122.4, 119.5, 118.5 (q, $J=320.2 \mathrm{~Hz}$ ), 39.6, 37.5; ${ }^{19} \mathrm{~F} \mathrm{NMR} \mathrm{(376} \mathrm{MHz,} \mathrm{CDCl}_{3}$ ): $\delta-73.78$ (s); IR: $v$ $\left(\mathrm{cm}^{-1}\right)$ 3030, 1657, 1629, 1604, 1495, 1421, 1364, 1249, 1210, 1156, 1138, 1082, 895, 871, 780, 768, 749, 740, 702; HRMS: (ESI) calcd for $\mathrm{C}_{18} \mathrm{H}_{16} \mathrm{~F}_{3} \mathrm{NO}_{4} \mathrm{SH}^{+}[\mathrm{M}+\mathrm{H}]^{+}$400.0830; found 400.0830 .

2-(N-Methyl-2-methyleneoctanamido)phenyl trifluoromethanesulfonate (1h) 


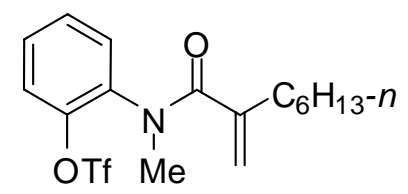

Chemical Formula: $\mathrm{C}_{17} \mathrm{H}_{22} \mathrm{~F}_{3} \mathrm{NO}_{4} \mathrm{~S}$; Molecular Weight: 393.4211

According to general procedure, $1 \mathrm{~h}$ was obtained in $63 \%$ yield over four steps as a yellow oil. ${ }^{1} \mathrm{H} \mathrm{NMR}\left(400 \mathrm{MHz}, \mathrm{CDCl}_{3}\right): \delta$ 7.65-7.10 (m, 4H), 5.74-4.50 (m, 2H), $3.35(\mathrm{~s}, 3 \mathrm{H}), 2.53-1.91(\mathrm{~m}$, $2 \mathrm{H}), 1.71-1.05(\mathrm{~m}, 8 \mathrm{H}), 0.86(\mathrm{t}, J=6.0 \mathrm{~Hz}, 3 \mathrm{H}) ;{ }^{13} \mathrm{C} \mathrm{NMR}\left(101 \mathrm{MHz}, \mathrm{CDCl}_{3}\right): \delta 171.8,144.4$, 137.7, 129.6, 129.2, 129.0, 122.5, 118.5 (q, $J=320.2 \mathrm{~Hz}$ ), 118.0, 37.5, 33.1, 31.6, 29.0, 27.5, 22.6, 14.1; ${ }^{19} \mathrm{~F} \mathrm{NMR}\left(376 \mathrm{MHz}, \mathrm{CDCl}_{3}\right.$ ): $\delta$-73.82 (s); IR: $v\left(\mathrm{~cm}^{-1}\right)$ 2987, 2970, 2927, 2910, 2902, $1659,1658,1497,1456,1421,1395,1375,1365,1249,1211,1166,1157,1140,1079,1067$, 1057, 1049, 1029, 894, 872; HRMS: (ESI) calcd for $\mathrm{C}_{17} \mathrm{H}_{22} \mathrm{~F}_{3} \mathrm{NO}_{4} \mathrm{SH}^{+}[\mathrm{M}+\mathrm{H}]^{+}$394.1300; found 394.1300.

2-(2-(Methoxymethyl)-N-methylacrylamido)phenyl trifluoromethanesulfonate (1i)<smiles>C=C(COC)C(=O)N(C)c1ccccc1[O-]</smiles>

Chemical Formula: $\mathrm{C}_{13} \mathrm{H}_{14} \mathrm{~F}_{3} \mathrm{NO}_{5} \mathrm{~S}$; Molecular Weight: 353.3142

According to general procedure, $1 \mathbf{i}$ was obtained in $48 \%$ yield over four steps as a yellow oil. ${ }^{1} \mathrm{H}$ NMR $\left(400 \mathrm{MHz}, \mathrm{CDCl}_{3}\right)$ : $\delta$ 7.61-7.14 (m, 4H), $5.29(\mathrm{~s}, 1 \mathrm{H}), 4.98(\mathrm{~s}, 1 \mathrm{H}), 4.17(\mathrm{~s}, 1 \mathrm{H}), 3.92(\mathrm{~s}$, 1H), 3.34 (s, 3H), 3.31 (s, 3H); ${ }^{13} \mathrm{C}$ NMR (101 MHz, $\left.\mathrm{CDCl}_{3}\right): \delta$ 169.5, 144.2, 140.6, 137.4, 129.9, 129.3, 129.0, 122.5, 119.3, 118.5 (q, $J=320.2 \mathrm{~Hz}), 72.3,58.6,37.4 ;{ }^{19} \mathrm{~F} \mathrm{NMR}\left(376 \mathrm{MHz}, \mathrm{CDCl}_{3}\right)$ : $\delta-73.85$ (s); IR: $v\left(\mathrm{~cm}^{-1}\right)$ 2987, 2972, 2921, 2920, 2901, 1662, 1630, 1605, 1497, 1454, 1419, $1395,1378,1249,1211,1158,1138,1106,1079,1068,1057,1029,936,899,872$; HRMS: (ESI) calcd for $\mathrm{C}_{13} \mathrm{H}_{14} \mathrm{~F}_{3} \mathrm{NO}_{5} \mathrm{SH}^{+}[\mathrm{M}+\mathrm{H}]^{+} 354.0623$; found 354.0623 .

2-(N,3-Dimethyl-2-methylenebutanamido)phenyl trifluoromethanesulfonate (1j) 
<smiles>C=C(C(=O)N(C)c1ccccc1OC)C(C)C</smiles>

Chemical Formula: $\mathrm{C}_{14} \mathrm{H}_{16} \mathrm{~F}_{3} \mathrm{NO}_{4} \mathrm{~S}$; Molecular Weight: 351.3413; Two rotamers

According to general procedure, $\mathbf{1} \mathbf{j}$ was obtained in $58 \%$ yield over four steps as a white solid. b.p. $56^{\circ} \mathrm{C} ;{ }^{1} \mathrm{H}$ NMR $\left(400 \mathrm{MHz}, \mathrm{CDCl}_{3}\right): \delta 7.51-7.27(\mathrm{~m}, 4 \mathrm{H}), 5.74-4.57(\mathrm{~m}, 2 \mathrm{H}), 3.35(\mathrm{~s}, 3 \mathrm{H})$, 2.96-2.27 (m, 1H), 1.04 (s, 3H); ${ }^{13} \mathrm{C}$ NMR (101 MHz, $\left.\mathrm{CDCl}_{3}\right): \delta 172.0,150.2,144.5,137.4$, 130.0, 129.1, 122.4, 118.5 (q, $J=320.1 \mathrm{~Hz}$ ), 116.4, 113.4, 39.6, 37.5, 31.0, 21.3, 21.2; ${ }^{19} \mathrm{~F}$ $\operatorname{NMR}\left(376 \mathrm{MHz}, \mathrm{CDCl}_{3}\right): \delta$ - 73.87 (s); IR: $v\left(\mathrm{~cm}^{-1}\right)$ 2987, 2971, 2920, 2901, 1656, 1496, 1419, $1395,1383,1371,1249,1210,1166,1139,1077,1068,1057,1029,900,879,780,768,738$; HRMS: (ESI) calcd for $\mathrm{C}_{14} \mathrm{H}_{16} \mathrm{~F}_{3} \mathrm{NO}_{4} \mathrm{SH}^{+}[\mathrm{M}+\mathrm{H}]^{+}$352.0830; found 352.0830 .

2-(N-Methyl-2-phenylacrylamido)phenyl trifluoromethanesulfonate (1k)<smiles>C=C(C(=O)N(C)c1ccccc1O)c1ccccc1</smiles>

Chemical Formula: $\mathrm{C}_{17} \mathrm{H}_{14} \mathrm{~F}_{3} \mathrm{NO}_{4} \mathrm{~S}$; Molecular Weight: 385.3576

According to general procedure, $\mathbf{1} \mathbf{k}$ was obtained in $65 \%$ yield over four steps as a yellow oil. Two rotamers were observed in 2.9:1 ratio. ${ }^{1} \mathrm{H}$ NMR $\left(400 \mathrm{MHz}, \mathrm{CDCl}_{3}\right): \delta 7.60-7.50(\mathrm{~m}$, 0.64H), 7.48-7.28 (m, 2.6H), 7.26-7.13 (m, 4,7H), 7.11-7.01 (m, 2.6H), $6.89(\mathrm{~d}, J=7.7 \mathrm{~Hz}, 1 \mathrm{H})$, $5.88(\mathrm{~s}, 0.3 \mathrm{H}), 5.64(\mathrm{~s}, 0.3 \mathrm{H}), 5.51(\mathrm{~s}, 1 \mathrm{H}), 5.44(\mathrm{~s}, 1 \mathrm{H}), 3.36(\mathrm{~s}, 3 \mathrm{H}), 3.25(\mathrm{~s}, 1 \mathrm{H}) ;{ }^{13} \mathrm{C}$ NMR $(101$ $\left.\mathrm{MHz}, \mathrm{CDCl}_{3}\right): \delta 170.4,145.4,144.6,136.5,136.2,130.5,129.3,129.0,128.8,128.6,128.3$, $128.1,125.8,122.3,121.9,118.8,118.4(d, J=319.7 \mathrm{~Hz}), 115.4,39.4,37.1 ;{ }^{19} \mathrm{~F}$ NMR (376 $\left.\mathrm{MHz}, \mathrm{CDCl}_{3}\right): \delta-73.65$ (s), -73.97 (s); IR: $v\left(\mathrm{~cm}^{-1}\right)$ 1659, 1496, 1420, 1367, 1249, 1210, 1157, 1138, 1080, 919, 902, 881, 768, 700; HRMS: (ESI) calcd for $\mathrm{C}_{17} \mathrm{H}_{14} \mathrm{~F}_{3} \mathrm{NO}_{4} \mathrm{SH}^{+}[\mathrm{M}+\mathrm{H}]^{+} 386.0674$; found 386.0674 .

2-(2-(4-Fluorophenyl)-N-methylacrylamido)phenyl trifluoromethanesulfonate (1I) 
<smiles>C=C(C(=O)N(C)c1ccccc1OCC)c1ccc(F)cc1</smiles>

Chemical Formula: $\mathrm{C}_{17} \mathrm{H}_{13} \mathrm{~F}_{4} \mathrm{NO}_{4} \mathrm{~S}$; Molecular Weight: 403.3480

According to general procedure, $\mathbf{1 l}$ was obtained in $55 \%$ yield over four steps as a yellow oil. Two rotamers were observed in 3.5:1 ratio. ${ }^{1} \mathrm{H}$ NMR $\left(400 \mathrm{MHz}, \mathrm{CDCl}_{3}\right): \delta 7.65-7.35(\mathrm{~m}, 1.7 \mathrm{H})$, $7.29(\mathrm{t}, J=7.7 \mathrm{~Hz}, 1 \mathrm{H}), 7.22(\mathrm{~d}, J=8.1 \mathrm{~Hz}, 1 \mathrm{H}), 7.16(\mathrm{t}, J=7.6 \mathrm{~Hz}, 1 \mathrm{H}), 7.13-7.04(\mathrm{~m}, 2.3 \mathrm{H})$, $6.96(\mathrm{~d}, J=7.9 \mathrm{~Hz}, 1 \mathrm{H}), 6.89(\mathrm{t}, J=8.3 \mathrm{~Hz}, 2 \mathrm{H}), 5.84(\mathrm{~s}, 0.3 \mathrm{H}), 5.64(\mathrm{~s}, 0.3 \mathrm{H}), 5.49(\mathrm{~s}, 1 \mathrm{H}), 5.42$ $(\mathrm{s}, 1 \mathrm{H}), 3.39(\mathrm{~s}, 3 \mathrm{H}), 3.29(\mathrm{~s}, 1 \mathrm{H}) ;{ }^{13} \mathrm{C} N M R\left(101 \mathrm{MHz}, \mathrm{CDCl}_{3}\right): \delta 170.2,163.8,161.4,144.5$, $144.1,136.3,132.7,130.4,129.4,128.7,127.7,127.7,122.3,122.0,120.0,118.8,116.7$, $115.9,115.4,115.2,39.5,37.2 ;{ }^{19} \mathrm{~F}$ NMR $\left(376 \mathrm{MHz} \mathrm{CDCl}_{3}\right): \delta-73.61$ (s), -73.95 (s), $-112.63(\mathrm{~s})$, -113.56 (s); IR: $v\left(\mathrm{~cm}^{-1}\right)$ 1741, 1656, 1603, 1509, 1497, 1421, 1370, 1248, 1212, 1160, 1138, 1100, 1079, 920, 903, 881, 843, 784, 783, 768, 735; HRMS: (ESI) calcd for $\mathrm{C}_{17} \mathrm{H}_{13} \mathrm{~F}_{4} \mathrm{NO}_{4} \mathrm{SH}^{+}$ $[\mathrm{M}+\mathrm{H}]^{+}$404.0580; found 404.0580.

2-(2-(4-Methoxyphenyl)-N-methylacrylamido)phenyl trifluoromethanesulfonate (1m)<smiles>C=C(C(=O)N(C)c1ccccc1OC)c1ccc(OC)cc1</smiles>

Chemical Formula: $\mathrm{C}_{18} \mathrm{H}_{16} \mathrm{~F}_{3} \mathrm{NO}_{5} \mathrm{~S}$; Molecular Weight: 415.3835

According to general procedure, $1 \mathrm{~m}$ was obtained in $51 \%$ yield over four steps as a yellow oil. Two rotamers were observed in 2.5:1 ratio. ${ }^{1} \mathrm{H} \mathrm{NMR}\left(400 \mathrm{MHz}, \mathrm{CDCl}_{3}\right): \delta 7.57-7.30(\mathrm{~m}, 2.8 \mathrm{H})$, $7.23(\mathrm{~d}, J=8.2 \mathrm{~Hz}, 1 \mathrm{H}), 7.19(\mathrm{~d}, J=7.3 \mathrm{~Hz}, 1 \mathrm{H}), 7.11(\mathrm{t}, J=7.5 \mathrm{~Hz}, 1 \mathrm{H}), 7.02(\mathrm{~d}, J=8.6 \mathrm{~Hz}, 2 \mathrm{H})$, 6.98-6.86 (m, 1.8H), $6.77(\mathrm{~d}, J=8.7 \mathrm{~Hz}, 0.3 \mathrm{H}), 6.71(\mathrm{~d}, J=8.6 \mathrm{~Hz}, 1.8 \mathrm{H}), 5.76(\mathrm{~s}, 0.3 \mathrm{H}), 5.52(\mathrm{~s}$, $0.3 \mathrm{H}), 5.37(\mathrm{~s}, 1 \mathrm{H}), 5.35(\mathrm{~s}, 1 \mathrm{H}), 3.83(\mathrm{~s}, 1.2 \mathrm{H}), 3.77(\mathrm{~s}, 3 \mathrm{H}), 3.36(\mathrm{~s}, 3 \mathrm{H}), 3.26(\mathrm{~s}, 1 \mathrm{H}) ;{ }^{13} \mathrm{C} N M R$ $\left(101 \mathrm{MHz}, \mathrm{CDCl}_{3}\right) \delta 171.3,170.8,160.1,159.6,144.6,136.3,130.8,130.5,130.1,130.0,129.5$, $129.4,129.3,129.0,128.6,127.2,122.8,122.2,121.8,120.0,116.8,114.4,113.8,113.7$, 113.2, 55.3, 37.1; $\left.{ }^{19} \mathrm{~F} \mathrm{NMR} \mathrm{(376} \mathrm{MHz,} \mathrm{CDCl}_{3}\right): \delta-73.61(\mathrm{~s}),-73.97(\mathrm{~s}) ; \mathrm{IR}: v\left(\mathrm{~cm}^{-1}\right)$ 1657, 1606, $1512,1496,1419,1369,1295,1248,1210,1181,1169,1156,1137,1107,1079,1033,902$, 
880, 879, 838, 781, 768, 733; HRMS: (ESI) calcd for $\mathrm{C}_{18} \mathrm{H}_{16} \mathrm{~F}_{3} \mathrm{NO}_{5} \mathrm{SH}^{+}[\mathrm{M}+\mathrm{H}]^{+}$416.0780; found 416.0779 .

2-(N-methyl-2-(naphthalen-2-yl)acrylamido)phenyl trifluoromethanesulfonate (1n)<smiles>C=C(C(=O)N(C)c1ccccc1[O-])c1ccc2ccccc2c1</smiles>

Chemical Formula: $\mathrm{C}_{21} \mathrm{H}_{16} \mathrm{~F}_{3} \mathrm{NO}_{4} \mathrm{~S}$; Molecular Weight: 435.4162

According to general procedure, 1n was obtained in $56 \%$ yield over four steps as a yellow oil. Two rotamers were observed in 3:1 ratio. ${ }^{1} \mathrm{H}$ NMR $\left(400 \mathrm{MHz}, \mathrm{CDCl}_{3}\right): \delta 8.03-7.93(\mathrm{~m}, 0.36 \mathrm{H})$, 7.92-7.83 (m, 1.35H), 7.81-7.69 (m, 2.4H), 7.66 (d, J = 8.6 Hz, 1H), $7.61(\mathrm{~s}, 1 \mathrm{H}), 7.56-7.34(\mathrm{~m}$, 4.7H), 7.22-7.10 (m, 2.7H), $6.95(\mathrm{t}, J=6.3 \mathrm{~Hz}, 1 \mathrm{H}), 6.88(\mathrm{~d}, J=7.7 \mathrm{~Hz}, 1 \mathrm{H}), 6.02(\mathrm{~s}, 0.35 \mathrm{H})$, $5.74(\mathrm{~s}, 0.35 \mathrm{H}), 5.58(\mathrm{~s}, 2 \mathrm{H}), 3.42(\mathrm{~s}, 3 \mathrm{H}), 3.29(\mathrm{~s}, 1 \mathrm{H}) ;{ }^{13} \mathrm{C} \mathrm{NMR}\left(101 \mathrm{MHz}, \mathrm{CDCl}_{3}\right) \delta$ 170.5, $145.1,144.6,136.3,133.7,133.1,132.9,130.5,129.4,129.4,128.9,128.9,128.6,128.5$, $128.2,128.1,127.7,127.5,126.7,126.6,126.3,125.4,125.3,123.5,123.1,122.3,121.9$, 120.1, 118.8, 115.8, 39.5, 37.2; $\left.{ }^{19} \mathrm{~F} \mathrm{NMR} \mathrm{(376} \mathrm{MHz,} \mathrm{CDCl}_{3}\right): \delta$-73.57 (s), $-73.90(\mathrm{~s}) ; \mathrm{IR}: v\left(\mathrm{~cm}^{-1}\right)$ $3057,2960,2934,2934,2933,1715,1613,1493,1486,1471,1373,1348,1259,1129,1090$, 1090, 1016, 1009, 910, 848, 769, 754, 735, 695; HRMS: (ESI) calcd for $\mathrm{C}_{21} \mathrm{H}_{16} \mathrm{~F}_{3} \mathrm{NO}_{4} \mathrm{SH}^{+}[\mathrm{M}+\mathrm{H}]^{+}$ 436.0830; found 436.0830 .

5-Methyl-2-(N-methylmethacrylamido)phenyl trifluoromethanesulfonate (10)<smiles>C=C(C)C(=O)N(C)c1ccc(C)cc1[OH2+]</smiles>

Chemical Formula: $\mathrm{C}_{13} \mathrm{H}_{14} \mathrm{~F}_{3} \mathrm{NO}_{4} \mathrm{~S}$; Molecular Weight: 337.3148

According to general procedure, 10 was obtained in $64 \%$ yield over four steps as a yellow oil. ${ }^{1} \mathrm{H}$ NMR $\left(400 \mathrm{MHz} \mathrm{CDCl}_{3}\right): \delta$ 7.25-7.14 (m, 2H), 7.13-7.03 (m, 1H), $5.02(\mathrm{~s}, 1 \mathrm{H}), 4.78(\mathrm{~s}, 1 \mathrm{H})$, $3.31(\mathrm{~s}, 3 \mathrm{H}), 2.38(\mathrm{~s}, 3 \mathrm{H}), 1.84(\mathrm{~s}, 3 \mathrm{H}) ;{ }^{13} \mathrm{C} \mathrm{NMR}\left(101 \mathrm{MHz}, \mathrm{CDCl}_{3}\right): \delta 171.5,144.0,139.7,134.9$, 
130.0, 128.7, 122.8, 119.4, 118.5 (q, J = 320.1 Hz), 37.3, 21.0, 19.8; ${ }^{19} \mathrm{~F} \mathrm{NMR}\left(376 \mathrm{MHz}^{\mathrm{C}} \mathrm{CDCl}_{3}\right)$ : $\delta-73.89$ (s); IR: $v\left(\mathrm{~cm}^{-1}\right)$ 2987, 2972, 2921, 2901, 1661, 1632, 1514, 1455, 1418, 1395, 1380, 1364, 1250, 1209, 1138, 1080, 1067, 1057, 1029, 954, 933, 893, 874, 828; HRMS: (ESI) calcd for $\mathrm{C}_{13} \mathrm{H}_{14} \mathrm{~F}_{3} \mathrm{NO}_{4} \mathrm{SH}^{+}[\mathrm{M}+\mathrm{H}]^{+} 338.0674$; found 338.0674.

4-Methyl-2-(N-methylmethacrylamido)phenyl trifluoromethanesulfonate (1p)<smiles>C=C(C)C(=O)N(C)c1cc(C)ccc1OCC</smiles>

Chemical Formula: $\mathrm{C}_{13} \mathrm{H}_{14} \mathrm{~F}_{3} \mathrm{NO}_{4} \mathrm{~S}$; Molecular Weight: 337.3148

According to general procedure, $1 \mathrm{p}$ was obtained in $67 \%$ yield over four steps as a yellow oil. ${ }^{1} \mathrm{H}$ NMR $\left(400 \mathrm{MHz}, \mathrm{CDCl}_{3}\right)$ : $\delta 7.21-6.98(\mathrm{~m}, 3 \mathrm{H}), 5.06(\mathrm{~s}, 1 \mathrm{H}), 4.79(\mathrm{~s}, 1 \mathrm{H}), 3.34(\mathrm{~s}, 3 \mathrm{H}), 2.38(\mathrm{~s}$, 3H), 1.89 (s, 3H); $\left.{ }^{13} \mathrm{C} \mathrm{NMR} \mathrm{(101} \mathrm{MHz,} \mathrm{CDCl}_{3}\right): \delta$ 171.5, 142.2, 139.8, 137.4, 129.4, 122.1, 119.5, $118.5\left(\mathrm{q}, J=320.2 \mathrm{~Hz}\right.$ ); 37.5, 20.9, 19.9; $\left.{ }^{19} \mathrm{~F} \mathrm{NMR} \mathrm{(376} \mathrm{MHz,} \mathrm{CDCl}_{3}\right): \delta-73.80(\mathrm{~s}) ; \mathrm{IR}: v\left(\mathrm{~cm}^{-1}\right)$ 2987, 2973, 2944, 2927, 2901, 1660, 1631, 1605, 1500, 1421, 1358, 1249, 1210, 1169, 1140, 1088, 1044, 912, 874, 820; HRMS: (ESI) calcd for $\mathrm{C}_{13} \mathrm{H}_{14} \mathrm{~F}_{3} \mathrm{NO}_{4} \mathrm{SH}^{+}[\mathrm{M}+\mathrm{H}]^{+}$338.0674; found 338.0674 .

4-Chloro-2-(N-methylmethacrylamido)phenyl trifluoromethanesulfonate (1q)<smiles>C=C(C)C(=O)N(C)c1cc(Cl)ccc1OCC</smiles>

Chemical Formula: $\mathrm{C}_{12} \mathrm{H}_{11} \mathrm{ClF}_{3} \mathrm{NO}_{4} \mathrm{~S}$; Molecular Weight: 357.7332

According to general procedure, 1q was obtained in $49 \%$ yield over four steps as a yellow oil. ${ }^{1} \mathrm{H}$ NMR $\left(400 \mathrm{MHz}, \mathrm{CDCl}_{3}\right): \delta 7.39(\mathrm{~d}, J=2.3 \mathrm{~Hz}, 1 \mathrm{H}), 7.35(\mathrm{dt}, J=8.8,1.9 \mathrm{~Hz}, 1 \mathrm{H}), 7.30-7.23$ $(\mathrm{m}, 1 \mathrm{H}), 5.19(\mathrm{~s}, 1 \mathrm{H}), 4.91(\mathrm{~s}, 1 \mathrm{H}), 3.37(\mathrm{~s}, 3 \mathrm{H}), 1.95(\mathrm{~s}, 3 \mathrm{H}) ;{ }^{13} \mathrm{C} \mathrm{NMR}\left(101 \mathrm{MHz}, \mathrm{CDCl}_{3}\right): \delta$ 
171.2, 143.1, 139.4, 138.7, 134.6, 129.0, 128.7, 123.6, 119.7, 118.5 (q, J = 320.4 Hz), 37.8, 19.7; ${ }^{19}$ F NMR (376 MHz, CDCl 3 ): $\delta$-73.57 (s); IR: $v\left(\mathrm{~cm}^{-1}\right)$ 2983, 2931, 1662, 1631, 1598, 1488, 1423, 1353, 1249, 1210, 1168, 1138, 1100, 864; HRMS: (ESI) calcd for $\mathrm{C}_{12} \mathrm{H}_{11} \mathrm{Cl}^{35} \mathrm{~F}_{3} \mathrm{NO}_{4} \mathrm{SH}^{+}$ $[\mathrm{M}+\mathrm{H}]^{+}$358.0128; found 358.0128; $\mathrm{C}_{12} \mathrm{H}_{11} \mathrm{Cl}^{37} \mathrm{~F}_{3} \mathrm{NO}_{4} \mathrm{SH}^{+}[\mathrm{M}+\mathrm{H}]^{+} 360.0098$; found 360.0100 .

3-Methyl-2-(N-methylmethacrylamido)phenyl trifluoromethanesulfonate (1r)<smiles>C=C(C)C(=O)N(C)c1c(O)cccc1O</smiles>

Chemical Formula: $\mathrm{C}_{13} \mathrm{H}_{14} \mathrm{~F}_{3} \mathrm{NO}_{4} \mathrm{~S}$; Molecular Weight: 337.3148

According to general procedure, $1 \mathrm{r}$ was obtained in $66 \%$ yield over four steps as a yellow oil. Two rotamers were observed in 3:1 ratio, NMR data of the major rotamer were reported. ${ }^{1} \mathrm{H}$ NMR (400 MHz, $\left.\mathrm{CDCl}_{3}\right): \delta$ 7.35-7.25 (m, 2H), 7.24-7.12 (m, 1H), $4.99(\mathrm{~s}, 1 \mathrm{H}), 4.73(\mathrm{~s}, 1 \mathrm{H})$, $3.24(\mathrm{~s}, 3 \mathrm{H}), 2.37$ (s, 3H), $1.84(\mathrm{~s}, 3 \mathrm{H}) ;{ }^{13} \mathrm{C}$ NMR (101 MHz, $\left.\mathrm{CDCl}_{3}\right): \delta 171.5,145.2,139.6,138.6$, 136.4, 131.1, 128.8, 119.7, 118.3, 118.1 (q, $J=320.0 \mathrm{~Hz}), 36.4,19.6,18.0 ;{ }^{19} \mathrm{~F} \mathrm{NMR}(376 \mathrm{MHz}$, $\left.\mathrm{CDCl}_{3}\right): \delta-73.93$ (s); IR: $v\left(\mathrm{~cm}^{-1}\right)$ 2980, 2960, 2930, 1660, 1631, 1484, 1465, 1420, 1357, 1252, 1209, 1168, 1139, 1120, 1096, 1088, 1007, 950, 938, 827, 790; HRMS: (ESI) calcd for $\mathrm{C}_{13} \mathrm{H}_{14} \mathrm{~F}_{3} \mathrm{NO}_{4} \mathrm{SH}^{+}[\mathrm{M}+\mathrm{H}]^{+} 338.0674$; found 338.0674.

3-(N-Methylmethacrylamido)naphthalen-2-yl trifluoromethanesulfonate (1s)<smiles>C=C(C)C(=O)N(C)c1cc2ccccc2cc1OC</smiles>

Chemical Formula: $\mathrm{C}_{16} \mathrm{H}_{14} \mathrm{~F}_{3} \mathrm{NO}_{4} \mathrm{~S}$; Exact Mass: 373.0596;

According to general procedure, $1 \mathrm{~s}$ was obtained in $64 \%$ yield over four steps as a yellow oil. ${ }^{1} \mathrm{H}$ NMR $\left(400 \mathrm{MHz}^{\mathrm{C} C D C l}\right)$ ): $\delta$ 8.00-7.70 (m, 4H), 7.73-7.49 (m, 2H), $5.03(\mathrm{~s}, 1 \mathrm{H}), 4.85(\mathrm{~s}, 1 \mathrm{H})$, $3.46(\mathrm{~s}, 3 \mathrm{H}), 1.92(\mathrm{~s}, 3 \mathrm{H}) ;{ }^{13} \mathrm{C} \mathrm{NMR}\left(101 \mathrm{MHz}, \mathrm{CDCl}_{3}\right) \delta 171.64,142.61,139.88,135.60,132.47$, 
131.97, 127.91, 127.87, 127.76, 127.69, 120.66, 119.99, 118.59 (d, J = 320.3 Hz), 38.10, 20.00; ${ }^{19} \mathrm{~F} \mathrm{NMR}\left(376 \mathrm{MHz}, \mathrm{CDCl}_{3}\right): \delta$-73.58(s); IR: $v\left(\mathrm{~cm}^{-1}\right)$ 2977, 2929, 1659, 1626, 1473, 1421, 1385, 1370, 1330, 1249, 1210, 1138, 1097, 1076, 1045; HRMS: (ESI) calcd for $\mathrm{C}_{16} \mathrm{H}_{14} \mathrm{~F}_{3} \mathrm{NO}_{4} \mathrm{SH}^{+}$ $[\mathrm{M}+\mathrm{H}]^{+} 374.0674$; found 374.0674 . 


\section{KIE Experiments}

\subsection{Synthesis of $\left[D_{1}\right]-2 a:^{2}$}

$\mathrm{CDCl}_{3}(99.5 \% \mathrm{D})+\mathrm{C}_{2} \mathrm{H}_{5} \mathrm{OD}(98 \% \mathrm{D})$

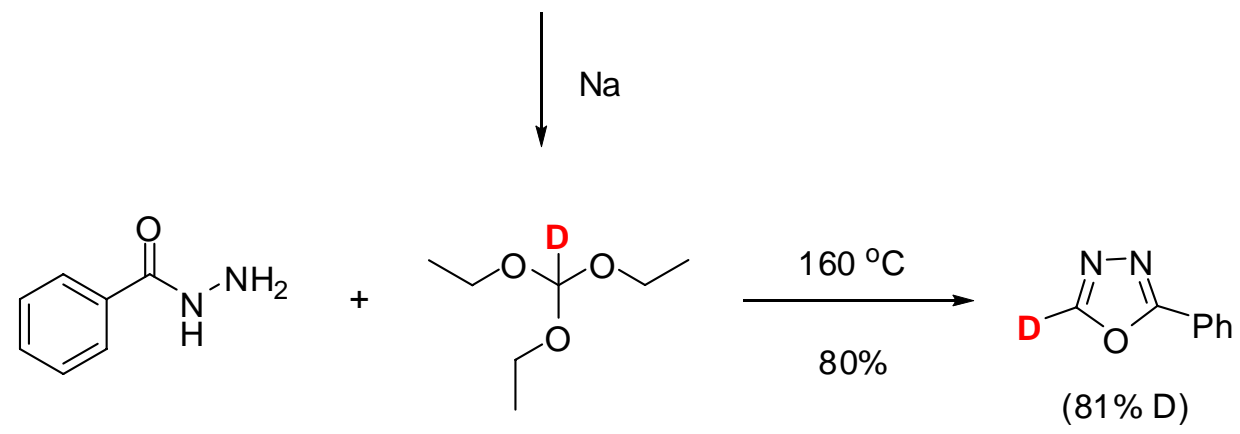

To a cooled solution of EtOD (50 mL, $0.86 \mathrm{~mol}, 98 \% \mathrm{D})$ and $\mathrm{CDCl}_{3}(16.9 \mathrm{~g}, 0.14 \mathrm{~mol}, 99.5 \%$ D ) in a dry $100 \mathrm{~mL}$ flask fitted with a magnetic stirrer, a condenser and a drying tube were added successively clean pieces of sodium (10.35 g, $0.45 \mathrm{~mol})$. After 12 hours the reaction mixture was poured into a solution of $\mathrm{NaHCO}_{3}(4 \mathrm{~g})$ in water $(100 \mathrm{~mL})$. The mixture was extracted twice with $n$-hexane $(60 \mathrm{~mL})$. The organic layers were dried over magnesium sulfate and the hexane was removed by gentle distillation. The residual oil was used directly without further purification.

The benzohydrazide $(1.3 \mathrm{~g})$ and triethyl orthoformate- $\mathrm{D}_{1}(5 \mathrm{~mL})$ from previous step were added to a round bottom flask with a magnetic stirrer bar. The mixture was stirred at $160^{\circ} \mathrm{C}$ for $7 \mathrm{~h}$. The residual triethyl orthoformate was removed under reduced pressure. Purification by column chromatography (petroleum ether/EtOAc $=5 / 1_{3}^{\sim} / 1$ ) yielded $\left[D_{1}\right]-2 a$ $(1.17 \mathrm{~g}, 80 \%, 81 \% \mathrm{D})$ as a white solid.

\subsection{Kinetic Isotope Effect Experiments:}<smiles>[2H]c1nnc(-c2ccccc2)o1</smiles>

$\left[D_{1}\right]-2 a(81 \% D)$

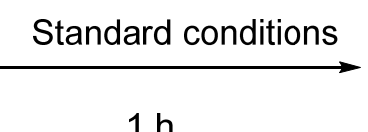

$1 \mathrm{~h}$<smiles>[2H]c1nnc(-c2ccccc2)o1</smiles>

$40 \%$ D

General procedure for control experiment: $\left[D_{1}\right]-2 a$ was submitted to the standard conditions in $\mathrm{CD}_{3} \mathrm{CN}$. After heating at $80{ }^{\circ} \mathrm{C}$ for $1 \mathrm{~h}$, only $40 \%$ deuterium residue was observed by ${ }^{1} \mathrm{H}$ NMR (No workup was conducted). 


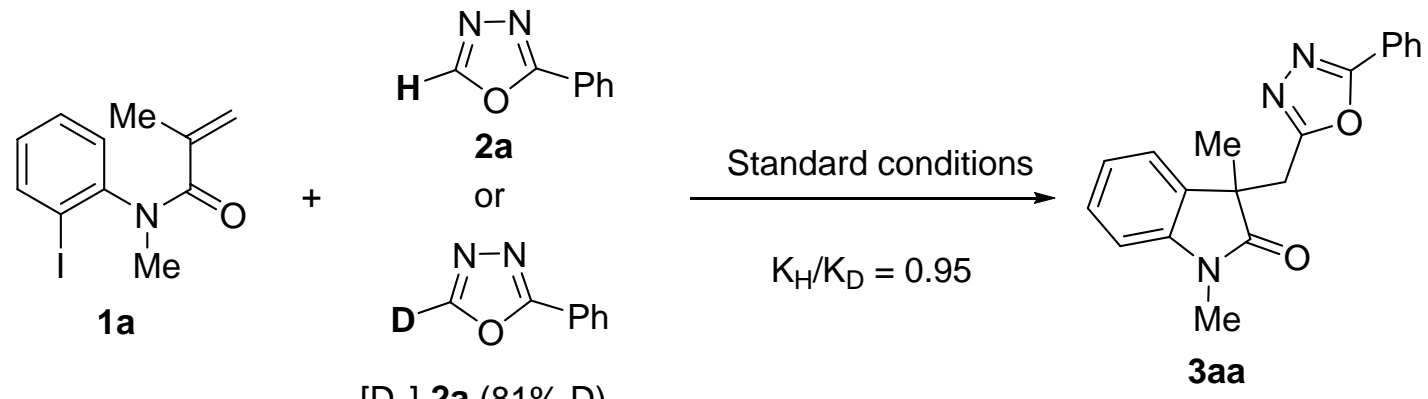

$\left[D_{1}\right]-2 \mathbf{a}(81 \% \mathrm{D})$

General procedure for KIE experiments: Two sets of reactions were carried out in parallel under the same conditions. In each case, amide $1 \mathrm{a}(0.2 \mathrm{mmol})$ was allowed to react with excess of oxadiazole $(2 \mathrm{a})$ and $\left[\mathrm{D}_{1}\right]-\mathbf{2 a}$, respectively, under standard conditions. The conversion was measured carefully at regular intervals by ${ }^{1} \mathrm{H}$ NMR using $1,3,5-$ trimethoxybenzene as internal standard to compare the initial reaction rates.

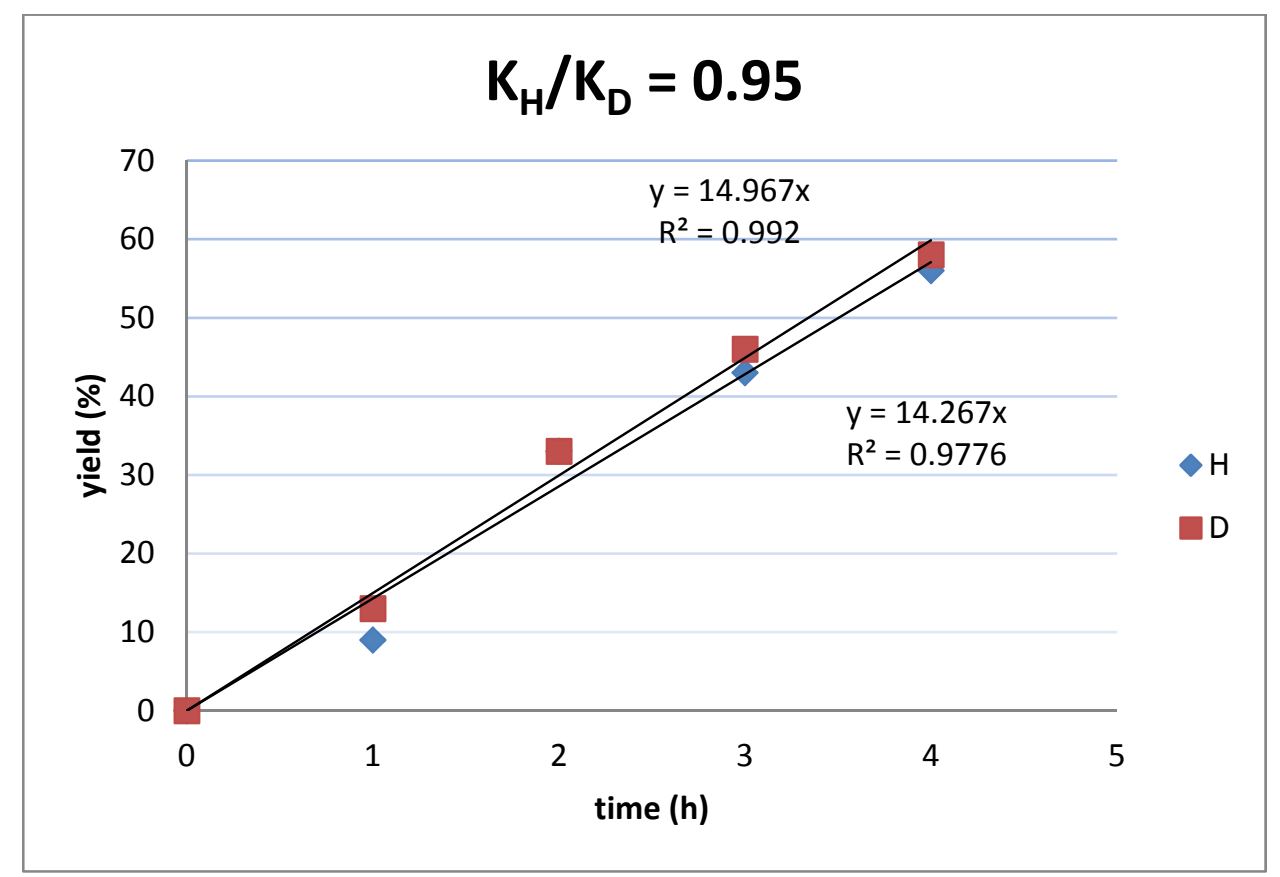

Figure S1. Kinetic Isotope Effect 


\section{General Procedure}

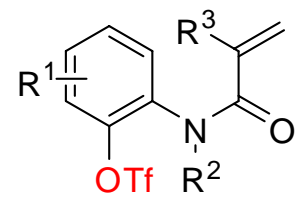

1

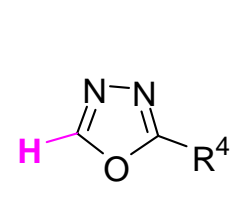

2
$\mathrm{PdCl}_{2}(\mathrm{MeCN})_{2}$ (10 mol\%)

(S)-t-BuPHOX (20 mol\%)

TMG (5 equiv), $\mathrm{CH}_{3} \mathrm{CN}$

$80{ }^{\circ} \mathrm{C}$

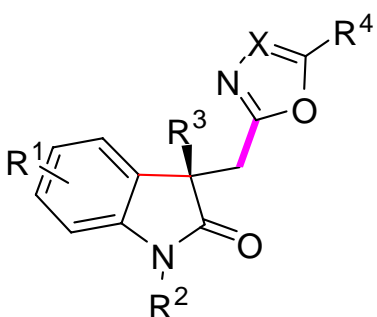

3

A mixture of $\mathrm{PdCl}_{2}(\mathrm{MeCN})_{2}$ (10 mol\%), (S)-t-BuPHOX (20 mol\%) and dry MeCN (0.05 M) was stirred in a sealed tube at room temperature for 1 hour under argon. To the resulting orange solution was added aryl triflate substrate $1(0.1 \mathrm{mmol})$, heterocycle 2 (1.2 equiv) and TMG (5 equiv), the reaction mixture was heated at $80{ }^{\circ} \mathrm{C}$ for 48 hours until the reaction was complete (monitored by TLC). The solvent was then removed under vacuum and the residue was purified by chromatography on silica gel, eluting with ethyl acetate/petroleum ether 1:3 1:1 (v/v) to afford the products 3 . 


\section{Characterization data}

1,3-Dimethyl-3-((5-phenyl-1,3,4-oxadiazol-2-yl)methyl)indolin-2-one (3aa)

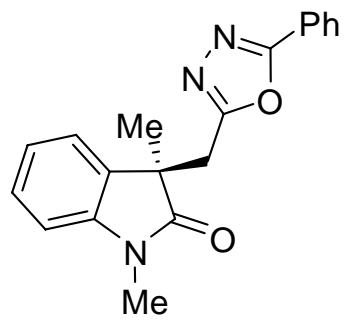

Chemical Formula: $\mathrm{C}_{19} \mathrm{H}_{17} \mathrm{~N}_{3} \mathrm{O}_{2}$; Molecular Weight: 319.3572

According to general procedure, 3aa was obtained in $71 \%$ yield $(22.5 \mathrm{mg})$ and $94 \%$ ee as a colorless oil. ${ }^{1} \mathrm{H}$ NMR $\left(400 \mathrm{MHz}, \mathrm{CDCl}_{3}\right): \delta 7.90(\mathrm{~d}, J=6.9 \mathrm{~Hz}, 2 \mathrm{H}), 7.58-7.38(\mathrm{~m}, 3 \mathrm{H}), 7.31-7.17$ $(\mathrm{m}, 2 \mathrm{H}), 7.05(\mathrm{t}, J=7.4 \mathrm{~Hz}, 1 \mathrm{H}), 6.83(\mathrm{~d}, J=7.7 \mathrm{~Hz}, 1 \mathrm{H}), 3.48(\mathrm{~d}, J=15.2 \mathrm{~Hz}, 1 \mathrm{H}), 3.43(\mathrm{~d}, J=$ $15.2 \mathrm{~Hz}, 1 \mathrm{H}), 3.27$ (s, 3H), 1.59 (s, 3H); $\left.{ }^{13} \mathrm{C} \mathrm{NMR} \mathrm{(100} \mathrm{MHz,} \mathrm{CDCl} 3\right): \delta$ 178.8, 164.7, 163.2, 143.0, 131.6, 129.0, 128.6, 126.7, 123.7, 123.2, 122.8, 108.3, 46.8, 33.3, 26.4, 23.6; IR: v (cm 1) 2972, 2929, 2900, 1711, 1613, 1569, 1552, 1493, 1470, 1450, 1422, 1378, 1350, 1254, 1125, 1066, 1012, 908; HRMS: (ESI) calcd for $\mathrm{C}_{19} \mathrm{H}_{17} \mathrm{~N}_{3} \mathrm{O}_{2} \mathrm{Na}^{+}[\mathrm{M}+\mathrm{Na}]^{+}$342.1218; found 342.1219.

SFC: $A D-H$ column, $10 \mathrm{ppm} \mathrm{MeOH}$ in supercritical $\mathrm{CO}_{2}$ as eluent, $4 \mathrm{~mL} / \mathrm{min}$. $\mathrm{tR}=6 \mathrm{~min}$ (major), 8 min (minor);

$[\alpha]_{D}^{29}=30(c=1.7, \mathrm{MeOH})$ for $94 \%$ ee.

\begin{tabular}{|l|l|l|l|l|l|l|l|l|l|}
\hline Instrument Method & Inj. Vol. & Solvent & Column & Sample & Well Location & Temp. & Flow & $\%$ Modifier & Pressure \\
\hline 10p methanol & 10 & $\mathrm{MeOH}$ & $\mathrm{AD}-\mathrm{H}$ & kwq-b-27 & $16 \mathrm{~A}$ & 31.3 & 4 & 10 & 150 \\
\hline
\end{tabular}

\section{Peak Information}

\begin{tabular}{|l|l|l|l|l|l|}
\hline Peak No & $\%$ Area & Area & Ret. Time & Height & Cap. Factor \\
\hline 1 & 50.0038 & 3293.6905 & $6.12 \mathrm{~min}$ & 99.846 & 6115.5833 \\
\hline 2 & 49.9962 & 3293.1897 & $8.02 \mathrm{~min}$ & 74.2849 & 8015.55 \\
\hline
\end{tabular}




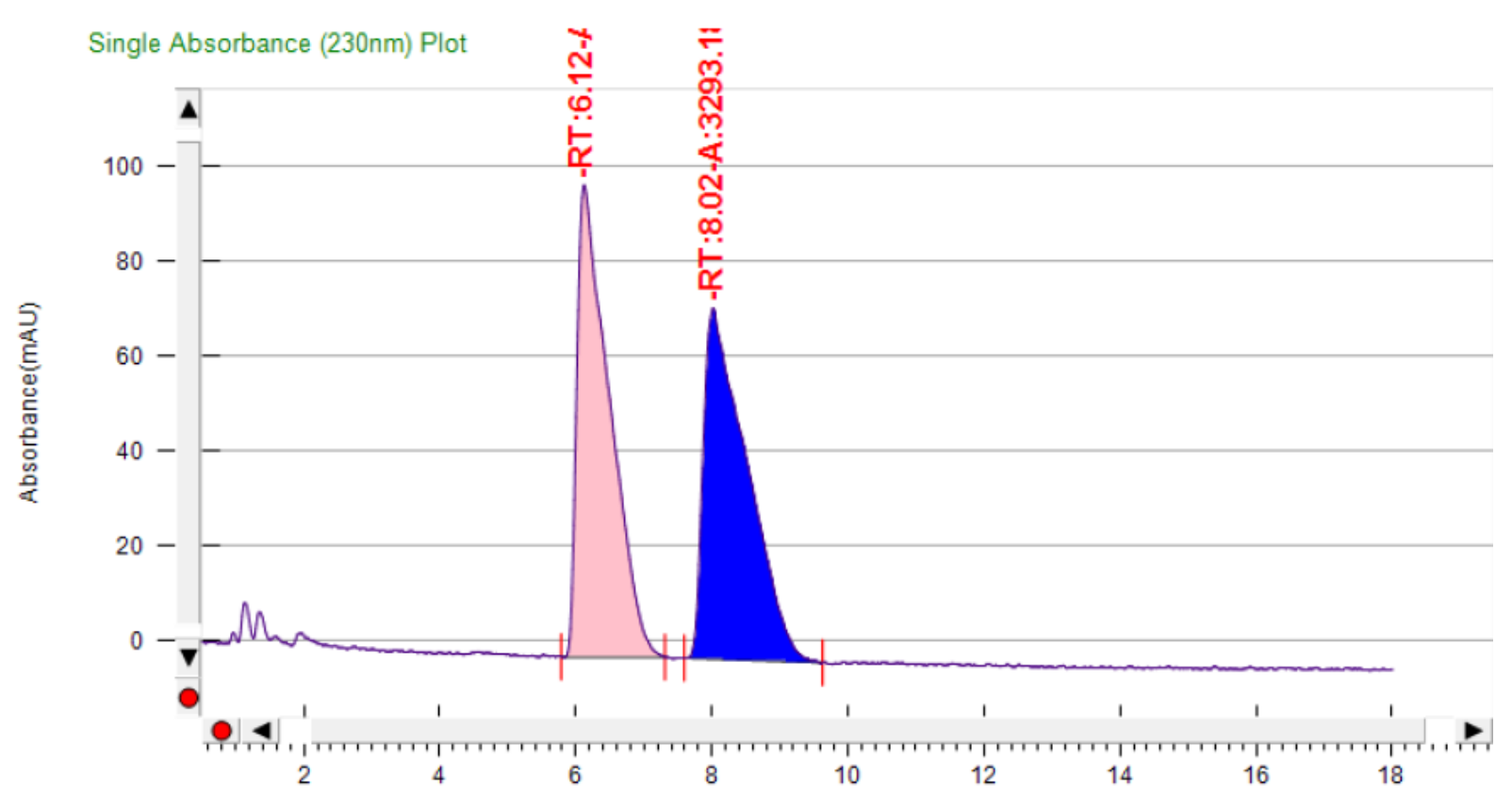

\begin{tabular}{|l|l|l|l|l|l|l|l|l|l|}
\hline Instrument Method & Inj. Vol. & Solvent & Column & Sample & Well Location & Temp. & Flow & $\%$ Modifier & Press \\
\hline 10p methanol & 10 & MeOH & AD-H & kwq-b-103-2 & $16 \mathrm{~B}$ & 30 & 4 & 10 & 150 \\
\hline
\end{tabular}
\begin{tabular}{|l|l|l|l|l|l|l|}
\hline Peak No & $\%$ Area & Area & Ret. Time & Height & Cap. Factor \\
\hline 1 & 96.7671 & $\begin{array}{l}18597.028 \\
6\end{array}$ & 6.23 min & 509.5117 & 6232.25 \\
\hline 2 & 3.2329 & 621.3072 & $8.21 \mathrm{~min}$ & 14.7297 & 8207.2167 \\
\hline
\end{tabular}

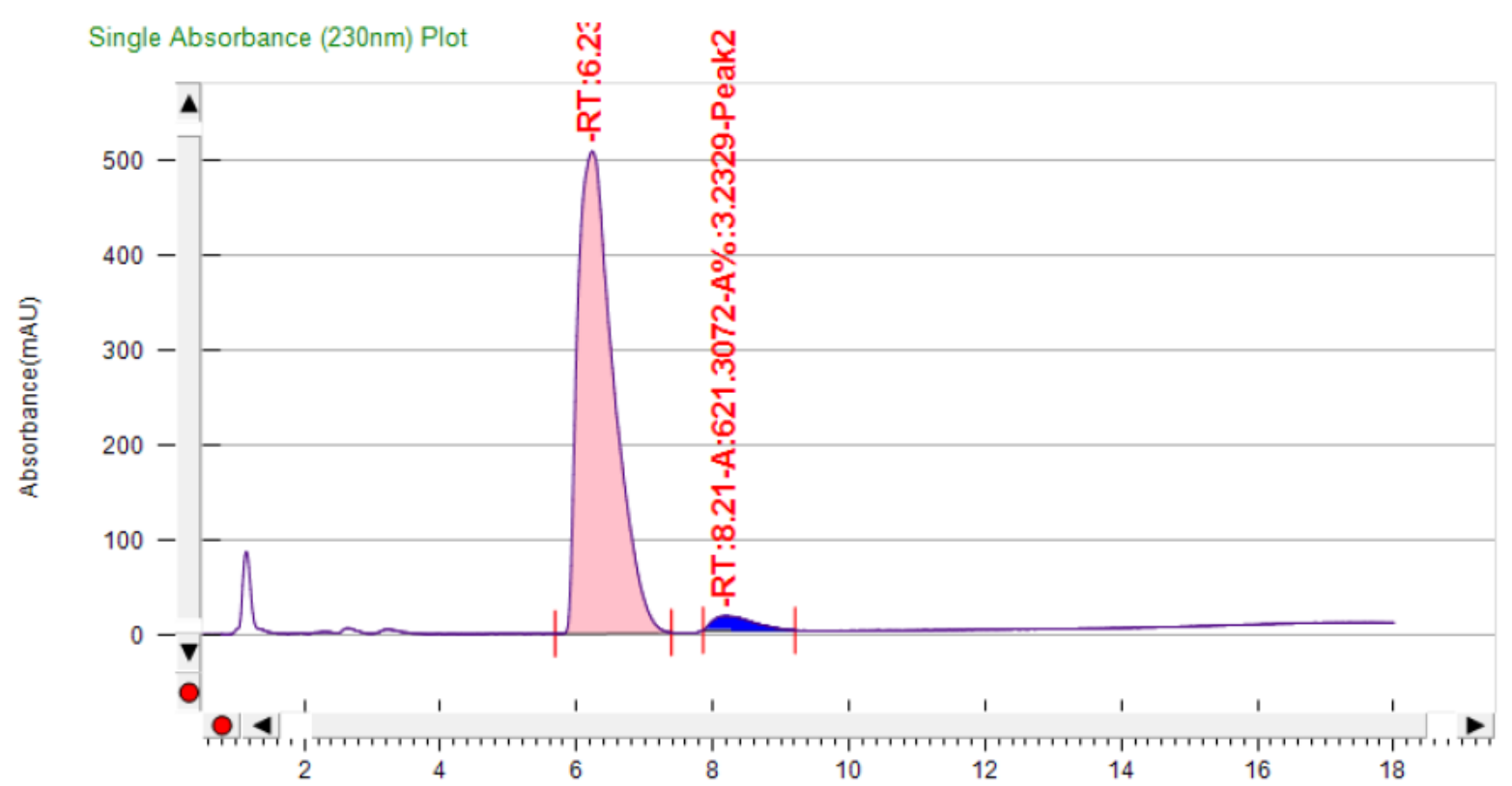




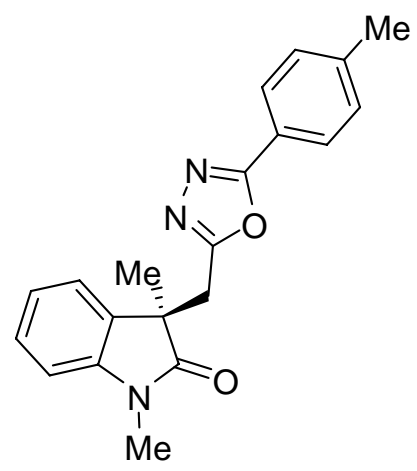

Molecular formula: $\mathrm{C}_{20} \mathrm{H}_{19} \mathrm{~N}_{3} \mathrm{O}_{2}$; Molecular Weight: 333.3838

According to general procedure, 3ab was obtained in 66\% yield (21.9 mg) and 98\% ee as a colorless oil. ${ }^{1} \mathrm{H}$ NMR $\left(400 \mathrm{MHz}, \mathrm{CDCl}_{3}\right): \delta 7.78(\mathrm{~d}, J=8.1 \mathrm{~Hz}, 2 \mathrm{H}), 7.33-7.16(\mathrm{~m}, 4 \mathrm{H}), 7.03(\mathrm{t}, J$ $=7.5 \mathrm{~Hz}, 1 \mathrm{H}), 6.81(\mathrm{~d}, J=7.8 \mathrm{~Hz}, 1 \mathrm{H}), 3.47(\mathrm{~d}, J=15.2 \mathrm{~Hz}, 1 \mathrm{H}), 3.41(\mathrm{~d}, J=15.2 \mathrm{~Hz}, 1 \mathrm{H}), 3.25(\mathrm{~s}$, 3H), 2.41 (s, 3H), 1.57 (s, 3H); ${ }^{13} \mathrm{C}$ NMR (101 MHz, $\left.\mathrm{CDCl}_{3}\right): \delta$ 178.9, 164.8, 162.9, 143.0, 142.2, 131.7, 129.7, 128.6, 126.6, 123.2, 122.8, 120.9, 108.3, 46.8, 33.3, 26.4, 23.6, 21.6; IR: $v\left(\mathrm{~cm}^{-1}\right)$ 2969, 2928, 1712, 1613, 1497, 1470, 1378, 1350, 1255, 1125, 1086, 1010, 910, 824, 727; HRMS: (ESI) calcd for $\mathrm{C}_{20} \mathrm{H}_{19} \mathrm{~N}_{3} \mathrm{O}_{2} \mathrm{H}^{+}[\mathrm{M}+\mathrm{H}]^{+}$334.1556; found 334.1555.

SFC: AD-H column, $15 \mathrm{ppm} \mathrm{MeOH}$ in supercritical $\mathrm{CO}_{2}$ as eluent, $4 \mathrm{~mL} / \mathrm{min}$. $\mathrm{tR}=4 \mathrm{~min}$ (major), 6 min (minor);

$[\alpha]_{D}^{27}=73\left(\mathrm{c}=1.0, \mathrm{CHCl}_{3}\right)$ for $98 \%$ ee.

\begin{tabular}{|l|l|l|l|l|l|l|l|l|l|}
\hline Instrument Method & Inj. Vol. & Solvent & Column & Sample & Well Location & Temp. & Flow & $\%$ Modifier & Pressure \\
\hline $15 \mathrm{p}$ methanol & 15 & $\mathrm{MeOH}$ & AD-H & kwq-b-125 & $16 \mathrm{D}$ & 29.8 & 4 & 15 & 150 \\
\hline Peak No & $\%$ Area & Area & Ret. Time & Height & Cap. Factor \\
\hline 1 & 49.8009 & $\begin{array}{l}22332.870 \\
7\end{array}$ & $4.82 \mathrm{~min}$ & 761.7079 & 0 & \\
\hline 2 & 50.1991 & $\begin{array}{l}22511.477 \\
8\end{array}$ & 6.37 min & 566.1874 & 0 & \\
\hline
\end{tabular}




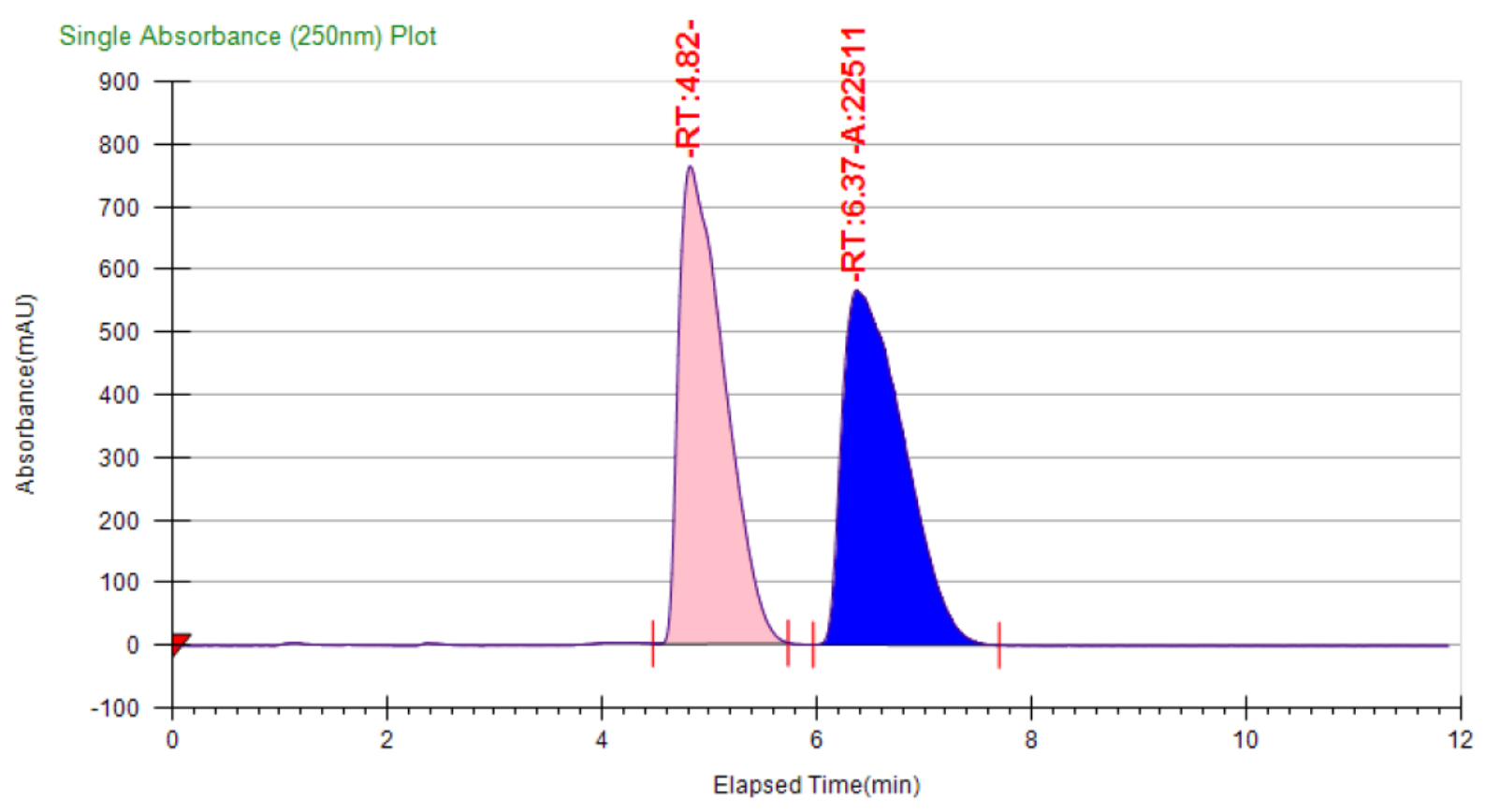

\begin{tabular}{|l|l|l|l|l|l|l|l|l|l|}
\hline Instrument Method & Inj. Vol. & Solvent & Column & Sample & Well Location & Temp. & Flow & $\%$ Modifier & Pressure \\
\hline $15 p$ methanol & 10 & MeOH & AD-H & kwq-b-128 & $16 \mathrm{C}$ & 30.2 & 4 & 15 & 150 \\
\hline Peak No & $\%$ Area & Area & Ret. Time & Height & Cap. Factor \\
\hline 1 & 99.2019 & 43.2148 & 4.75 min & 2.1098 & 0 & \\
\hline 2 & 0.7981 & 0.3477 & 6.03 min & 0.0243 & 0 \\
\hline
\end{tabular}

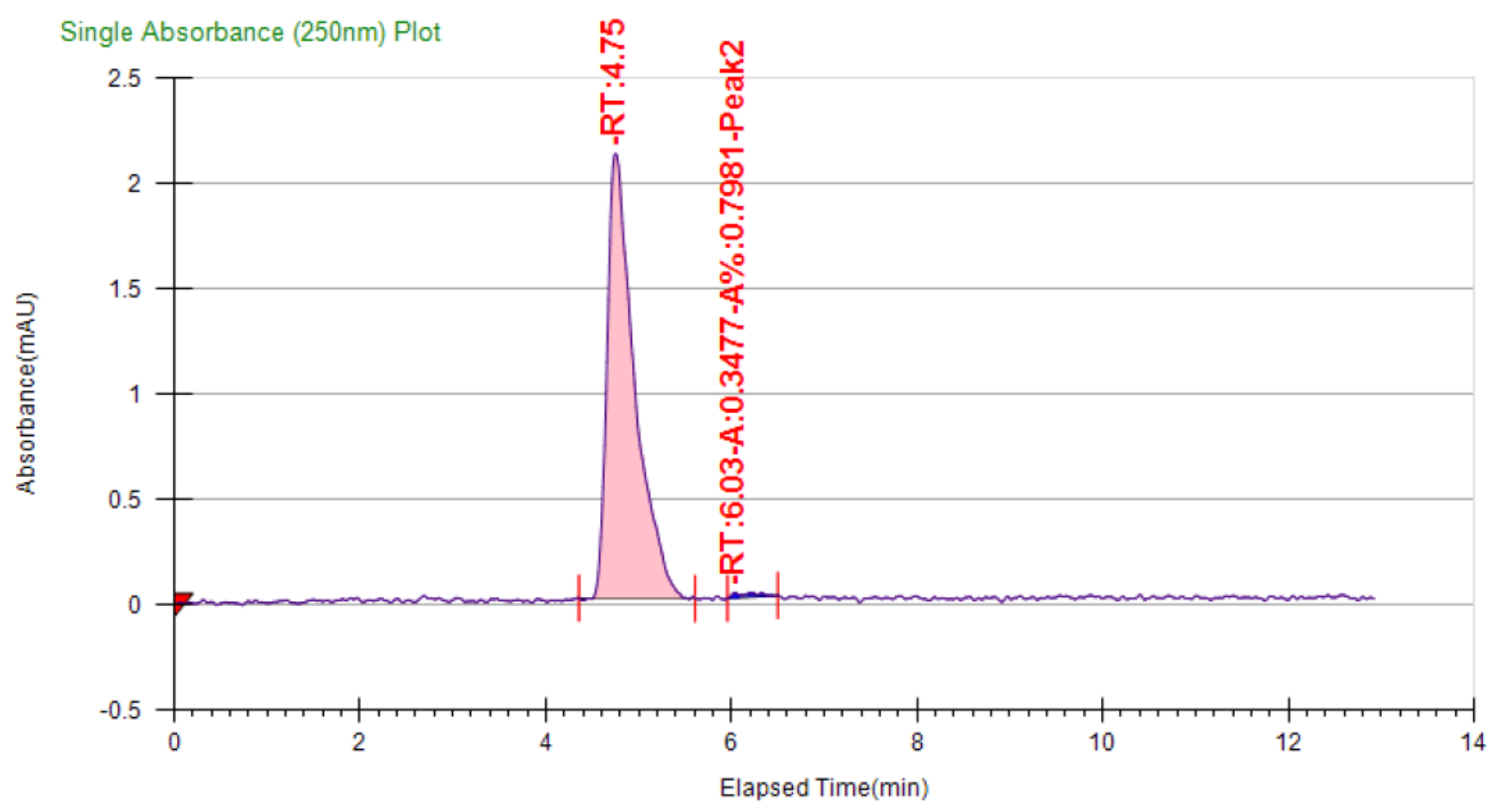


3-((5-(4-Tert-butylphenyl)-1,3,4-oxadiazol-2-yl)methyl)-1,3-dimethylindolin-2-one (3ac)

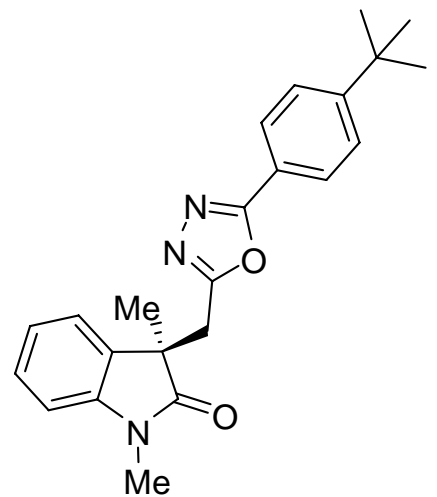

Molecular formula: $\mathrm{C}_{23} \mathrm{H}_{25} \mathrm{~N}_{3} \mathrm{O}_{2}$; Molecular Weight: 375.4635

According to general procedure, 3ac was obtained in $64 \%$ yield $(24.0 \mathrm{mg})$ and $94 \%$ ee as a colorless oil. ${ }^{1} \mathrm{H}$ NMR (400 MHz, $\left.\mathrm{CDCl}_{3}\right): \delta 7.83(\mathrm{~d}, J=8.3 \mathrm{~Hz}, 2 \mathrm{H}), 7.48(\mathrm{~d}, J=8.3 \mathrm{~Hz}, 2 \mathrm{H})$, 7.31-7.15 (m, 2H), $7.04(\mathrm{t}, J=7.5 \mathrm{~Hz}, 1 \mathrm{H}), 6.82(\mathrm{~d}, J=7.7 \mathrm{~Hz}, 1 \mathrm{H}), 3.48(\mathrm{~d}, J=15.2 \mathrm{~Hz}, 1 \mathrm{H})$, $3.41(\mathrm{~d}, J=15.2 \mathrm{~Hz}, 1 \mathrm{H}), 3.26(\mathrm{~s}, 3 \mathrm{H}), 1.57(\mathrm{~s}, 3 \mathrm{H}), 1.35(\mathrm{~s}, 9 \mathrm{H}) ;{ }^{13} \mathrm{C} \mathrm{NMR}\left(101 \mathrm{MHz}, \mathrm{CDCl}_{3}\right): \delta$ 178.9, 164.8, 162.9, 155.2, 143.0, 131.7, 128.6, 126.5, 126.0, 123.2, 122.8, 120.9, 108.3, 46.8, 35.1, 33.3, 31.1, 26.5, 23.6; IR: $v\left(\mathrm{~cm}^{-1}\right)$ 2965, 2905, 2871, 1712, 1614, 1494, 1470, 1378, 1350, 1267, 1114, 1009, 909, 843; HRMS: (ESI) calcd for $\mathrm{C}_{23} \mathrm{H}_{25} \mathrm{~N}_{3} \mathrm{O}_{2} \mathrm{H}^{+}[\mathrm{M}+\mathrm{H}]^{+} 376.2025$; found 376.2025 .

SFC: $\mathrm{AD}-\mathrm{H}$ column, $10 \mathrm{ppm} \mathrm{MeOH}$ in supercritical $\mathrm{CO}_{2}$ as eluent, $4 \mathrm{~mL} / \mathrm{min}$. $\mathrm{tR}=6 \mathrm{~min}$ (major), 14 min (minor);

$[\alpha]_{D}^{27}=102\left(\mathrm{c}=0.6, \mathrm{CHCl}_{3}\right)$ for $94 \%$ ee.

\begin{tabular}{|l|l|l|l|l|l|l|l|l|l|}
\hline Instrument Method & Inj. Vol. & Solvent & Column & Sample & Well Location & Temp. & Flow & $\%$ Modifier & Pressure \\
\hline $10 \mathrm{p}$ methanol & 10 & $\mathrm{MeOH}$ & AD-H & kwq-b-129 & $16 \mathrm{~B}$ & 30 & 4 & 10 & 150 \\
\hline \begin{tabular}{|l|l|l|l|l|l|}
\hline Peak No \\
\hline 1
\end{tabular} & $\%$ Area & Area & Ret. Time & Height & Cap. Factor \\
\hline 2 & 49.8616 & $\begin{array}{l}22223.943 \\
6\end{array}$ & 6.58 min & 652.1511 & 0 & \\
\hline
\end{tabular}




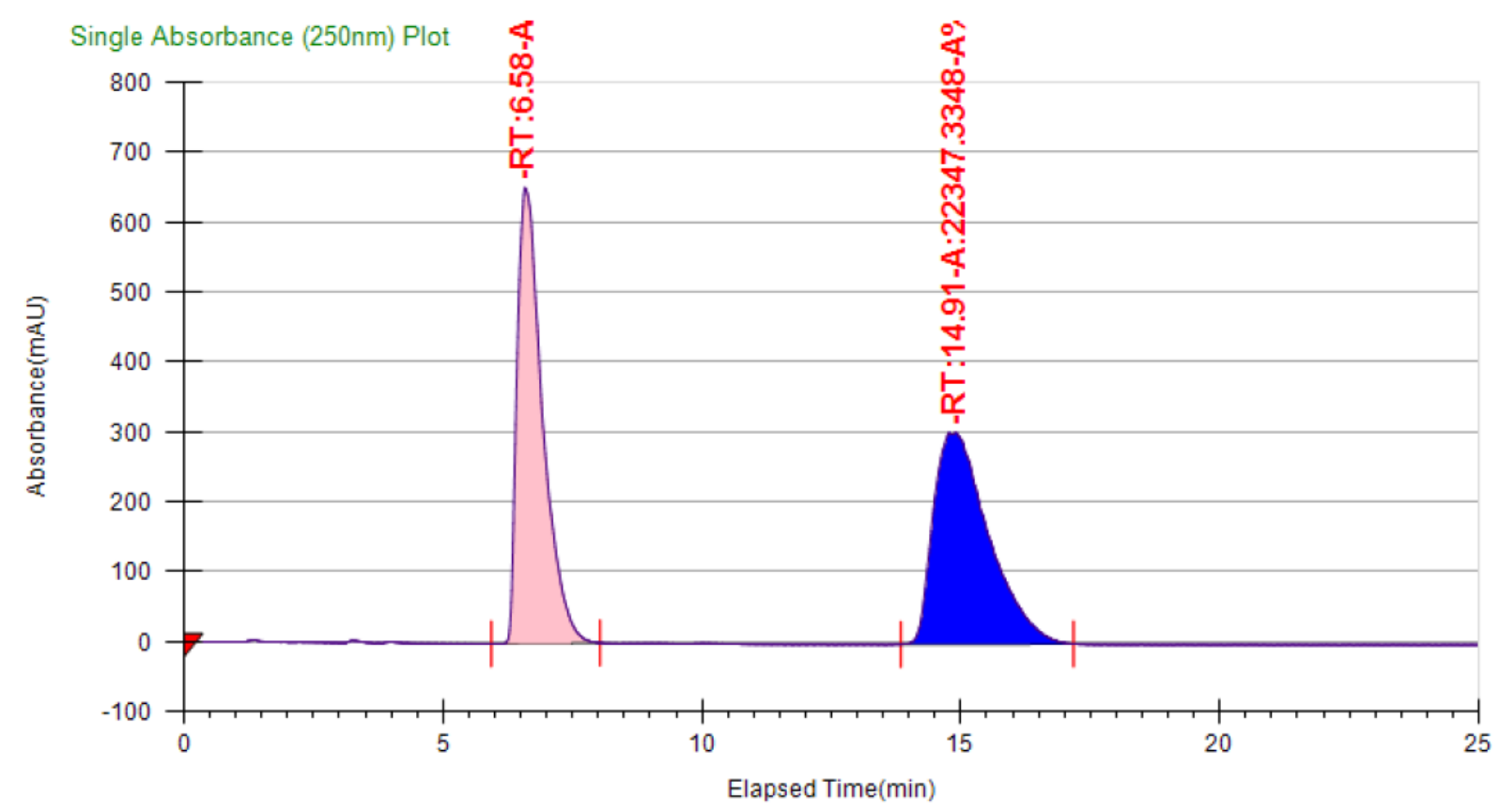

\begin{tabular}{|l|l|l|l|l|l|l|l|l|l|}
\hline Instrument Method & Inj. Vol. & Solvent & Column & Sample & Well Location & Temp. & Flow & $\%$ Modifier & Pressure \\
\hline $10 \mathrm{p}$ methanol & 10 & $\mathrm{MeOH}$ & $\mathrm{AD}-\mathrm{H}$ & kwq-b-130 & $16 \mathrm{~B}$ & 30.8 & 4 & 10 & 150 \\
\hline
\end{tabular}

\begin{tabular}{|l|l|l|l|l|l|}
\hline Peak No & \% Area & Area & Ret. Time & Height & Cap. Factor \\
\hline 1 & 97.1964 & $\begin{array}{l}30289.817 \\
3\end{array}$ & $6.32 \mathrm{~min}$ & 776.9075 & 6315.5833 \\
\hline 2 & 2.8036 & 873.6983 & $14.08 \mathrm{~min}$ & 11.827 & 14082.1333 \\
\hline
\end{tabular}

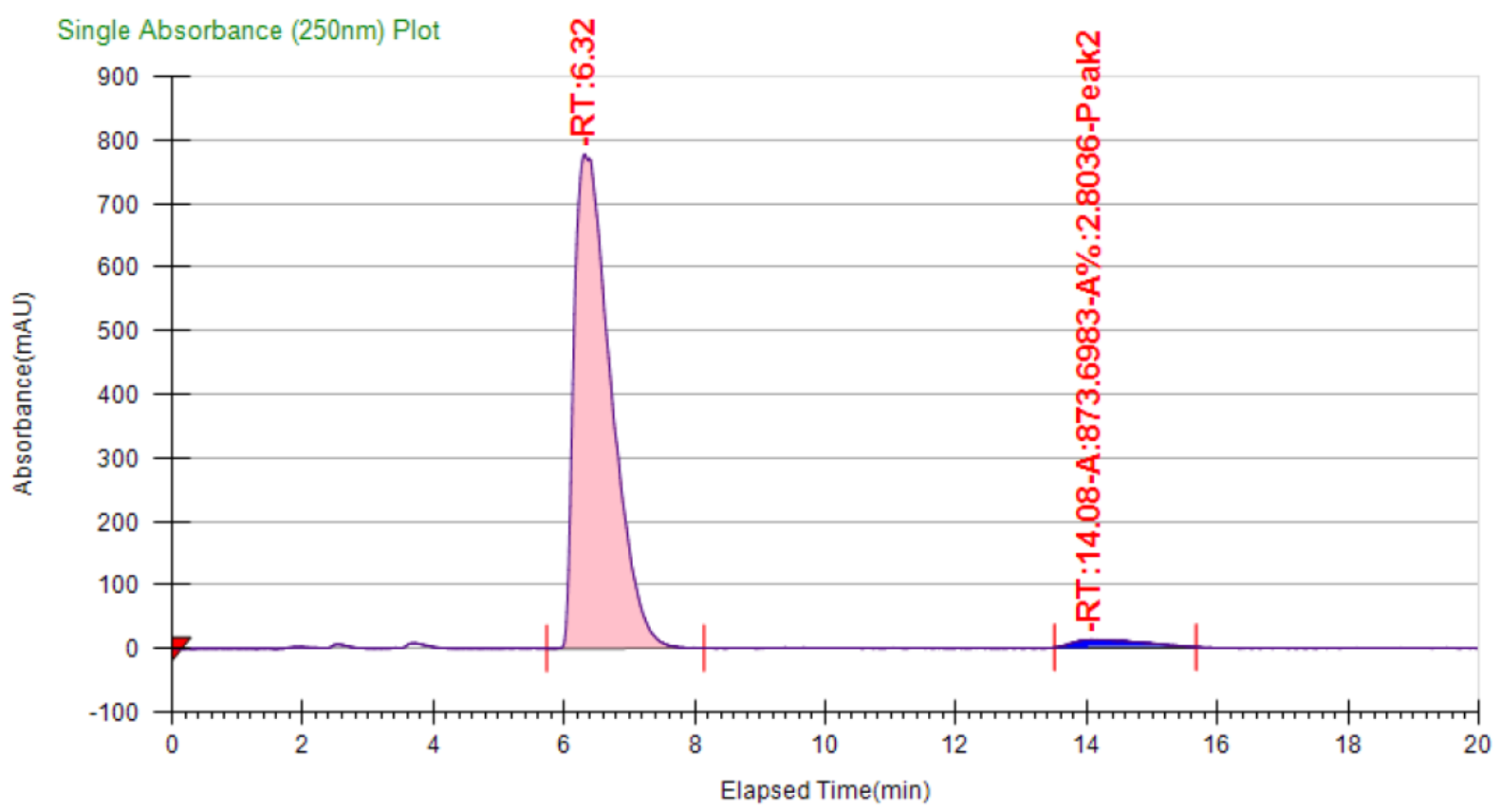




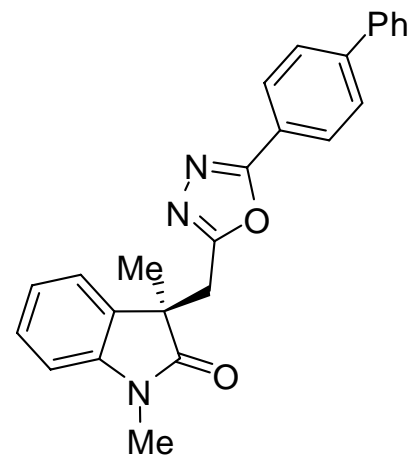

Molecular formula: $\mathrm{C}_{25} \mathrm{H}_{21} \mathrm{~N}_{3} \mathrm{O}_{2}$; Molecular Weight: 395.4531

According to general procedure, 3ad was obtained in 64\% yield (25.3 $\mathrm{mg}$ ) and $99 \%$ ee as a colorless oil. ${ }^{1} \mathrm{H}$ NMR $\left(400 \mathrm{MHz}, \mathrm{CDCl}_{3}\right): \delta 7.97(\mathrm{~d}, J=8.2 \mathrm{~Hz}, 2 \mathrm{H}), 7.70(\mathrm{~d}, J=8.2 \mathrm{~Hz}, 2 \mathrm{H}), 7.63$ (d, $J=7.5 \mathrm{~Hz}, 2 \mathrm{H}), 7.49$ (t, $J=7.5 \mathrm{~Hz}, 2 \mathrm{H}$ ), $7.41(\mathrm{t}, J=7.1 \mathrm{~Hz}, 1 \mathrm{H}), 7.28(\mathrm{~d}, J=7.2 \mathrm{~Hz}, 1 \mathrm{H}), 7.23$ (d, $J=7.5 \mathrm{~Hz}, 1 \mathrm{H}), 7.06(\mathrm{t}, J=7.5 \mathrm{~Hz}, 1 \mathrm{H}), 6.84(\mathrm{~d}, J=7.7 \mathrm{~Hz}, 1 \mathrm{H}), 3.50(\mathrm{~d}, J=15.2 \mathrm{~Hz}, 1 \mathrm{H})$, $3.45(\mathrm{~d}, J=15.2 \mathrm{~Hz}, 1 \mathrm{H}), 3.28(\mathrm{~s}, 3 \mathrm{H}), 1.60(\mathrm{~s}, 3 \mathrm{H}) ;{ }^{13} \mathrm{C} \mathrm{NMR}\left(101 \mathrm{MHz}, \mathrm{CDCl}_{3}\right): \delta 178.9,164.6$, 163.2 , 144.4, 143.0, 139.8, 131.7, 129.0, 128.6, 128.2, 127.7, 127.2, 127.1, 123.2, 122.8, 122.4, 108.4, 46.8, 33.3, 26.5, 23.6; IR: $v\left(\mathrm{~cm}^{-1}\right)$ 2970, 2930, 1712, 1613, 1484, 1378, 1350, 1255, 1125, 1008, 848; HRMS: (ESI) calcd for $\mathrm{C}_{25} \mathrm{H}_{21} \mathrm{~N}_{3} \mathrm{O}_{2} \mathrm{H}[\mathrm{M}+\mathrm{H}]^{+} 396.1712$; found 396.1712.

SFC: AD-H column, $20 \mathrm{ppm} \mathrm{MeOH}$ in supercritical $\mathrm{CO}_{2}$ as eluent, $4 \mathrm{~mL} / \mathrm{min}$. $\mathrm{tR}=8 \mathrm{~min}$ (major), $18 \mathrm{~min}$ (minor);

$[\alpha]_{D}^{28}=26(\mathrm{c}=1.1, \mathrm{MeOH})$ for $99 \%$ ee.

\begin{tabular}{|l|l|l|l|l|l|l|l|l|l|}
\hline Instrument Method & Inj. Vol. & Solvent & Column & Sample & Well Location & Temp. & Flow & $\%$ Modifier & Pressure \\
\hline 20p methanol & 10 & MeOH & AD-H & kwq-b-148 & $16 \mathrm{D}$ & 29.7 & 4 & 20 & 150 \\
\hline Peak No & $\%$ Area & Area & Ret. Time & Height & Cap. Factor \\
\hline 1 & 50.5554 & 5559.1999 & 8.83 min & 91.9706 & 0 & \\
\hline 2 & 49.4446 & 5437.0607 & 17.8 min & 24.1395 & 0 & \\
\hline
\end{tabular}




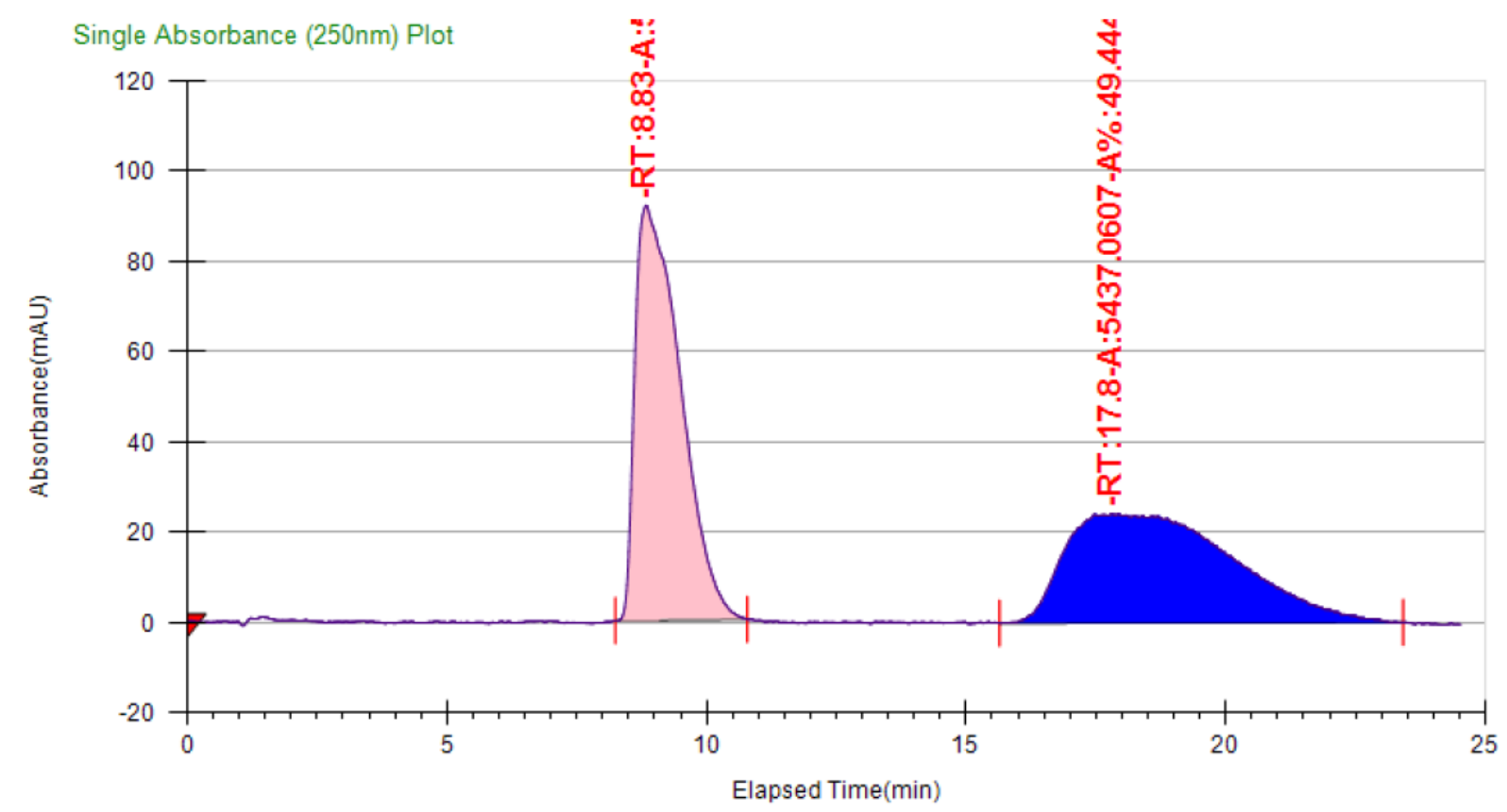

\begin{tabular}{|l|l|l|l|l|l|l|l|l|l|}
\hline Instrument Method & Inj. Vol. & Solvent & Column & Sample & Well Location & Temp. & Flow & $\%$ Modifier & Pressure \\
\hline $20 \mathrm{p}$ methanol & 10 & MeOH & AD-H & kwq-c-3 & $16 \mathrm{C}$ & 30 & 4 & 20 & 150 \\
\hline Peak No & $\%$ Area & Area & Ret. Time & Height & Cap. Factor \\
\hline 1 & 99.7347 & 2926.8556 & 8.9 min & 52.433 & 0 \\
\hline 2 & 0.2653 & 7.7867 & 20.37 min & 0.2669 & 0 & \\
\hline
\end{tabular}

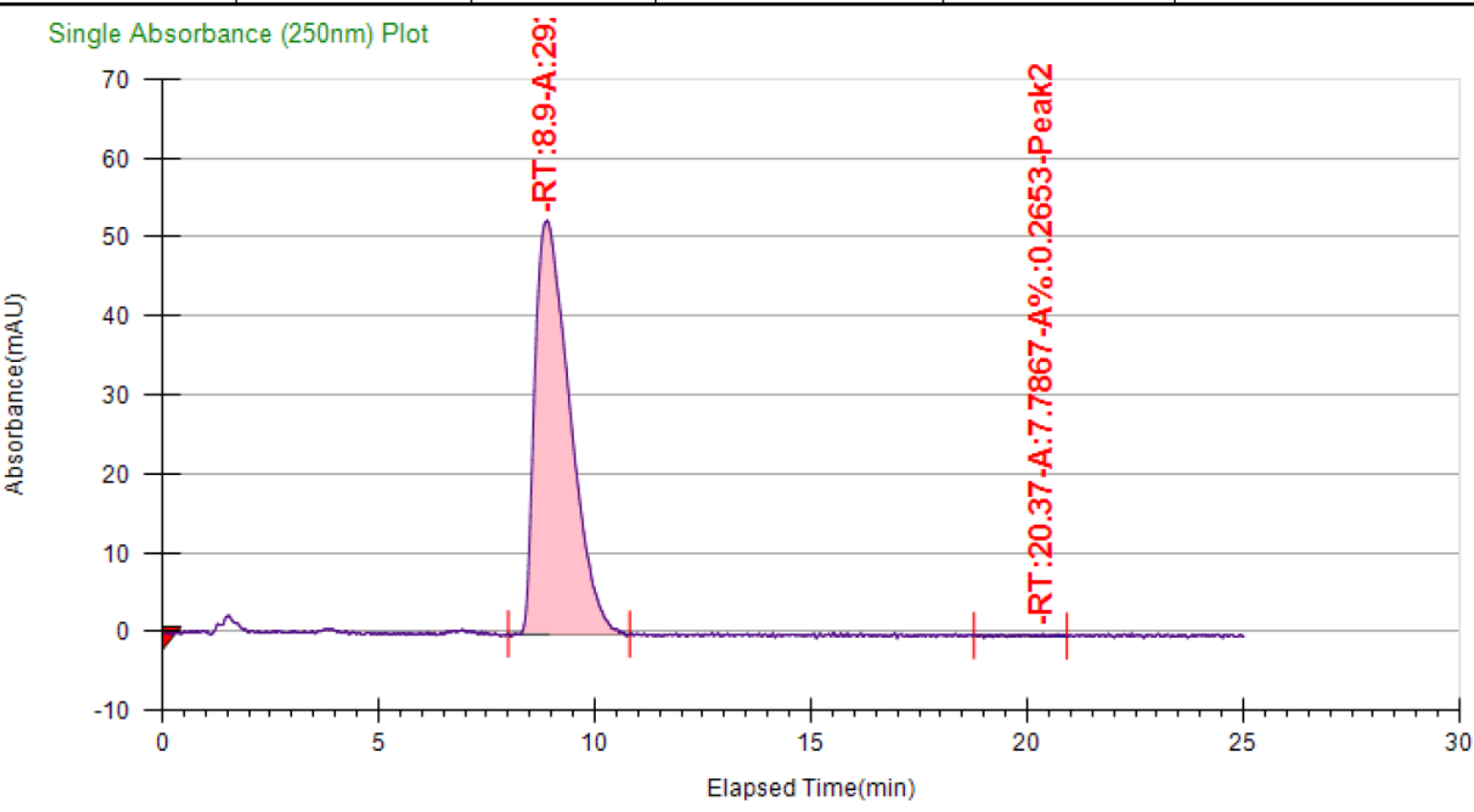


3-((5-(3-Methoxyphenyl)-1,3,4-oxadiazol-2-yl)methyl)-1,3-dimethylindolin-2-one (3ae)

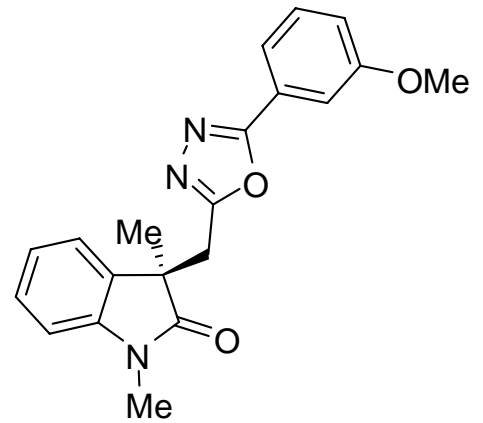

Molecular formula: $\mathrm{C}_{20} \mathrm{H}_{19} \mathrm{~N}_{3} \mathrm{O}_{3}$; Molecular Weight: 349.3832

According to general procedure, 3ae was obtained in $73 \%$ yield $(25.5 \mathrm{mg})$ and $93 \%$ ee as a colorless oil. ${ }^{1} \mathrm{H}$ NMR $\left(400 \mathrm{MHz}, \mathrm{CDCl}_{3}\right): \delta$ 7.49-7.40 (m, 2H), $7.37(\mathrm{t}, J=7.9 \mathrm{~Hz}, 1 \mathrm{H}), 7.29-7.16$ (m, 2H), 7.10-6.95 (m, 2H), $6.82(\mathrm{~d}, J=7.7 \mathrm{~Hz}, 1 \mathrm{H}), 3.85(\mathrm{~s}, 3 \mathrm{H}), 3.47(\mathrm{~d}, J=15.3 \mathrm{~Hz}, 1 \mathrm{H}), 3.42$ $(\mathrm{d}, J=15.3 \mathrm{~Hz}, 1 \mathrm{H}), 3.26(\mathrm{~s}, 3 \mathrm{H}), 1.58(\mathrm{~s}, 3 \mathrm{H}) ;{ }^{13} \mathrm{C} \mathrm{NMR}\left(101 \mathrm{MHz}, \mathrm{CDCl}_{3}\right): \delta 178.8,164.7$, 163.2, 159.9, 143.0, 131.6, 130.1, 128.6, 124.8, 123.2, 122.8, 119.0, 118.2, 111.3, 108.3, 55.5, 46.8, 33.3, 26.4, 23.6; IR: $v\left(\mathrm{~cm}^{-1}\right)$ 2969, 2931, 1711, 1613, 1551, 1493, 1469, 1378, 1350, 1245, 1125, 1035, 853, 728; HRMS: (ESI) calcd for $\mathrm{C}_{20} \mathrm{H}_{19} \mathrm{~N}_{3} \mathrm{O}_{3} \mathrm{Na}^{+}[\mathrm{M}+\mathrm{Na}]^{+}$372.1324; found 372.1324 .

SFC: AD-H column, $15 \mathrm{ppm} \mathrm{MeOH}$ in supercritical $\mathrm{CO}_{2}$ as eluent, $4 \mathrm{~mL} / \mathrm{min}$. $\mathrm{tR}=4 \mathrm{~min}$ (major), 5 min (minor);

$[\alpha]_{D}{ }^{27}=26(c=1.3, \mathrm{MeOH})$ for $93 \%$ ee.

\begin{tabular}{|l|l|l|l|l|l|l|l|l|l|}
\hline Instrument Method & Inj. Vol. & Solvent & Column & Sample & Well Location & Temp. & Flow & $\%$ Modifier & Pressure \\
\hline $15 p$ methanol & 10 & MeOH & AD-H & kwq-b-106 & 16 C & 29.9 & 4 & 15 & 150 \\
\hline Peak No & $\%$ Area & Area & Ret. Time & Height & Cap. Factor \\
\hline 1 & 49.7927 & $\begin{array}{l}10475.778 \\
8\end{array}$ & 3.87 min & 513.2513 & 0 & \\
\hline 2 & 50.2073 & $\begin{array}{l}10563.014 \\
6\end{array}$ & 5.42 min & 364.0277 & 0 \\
\hline
\end{tabular}




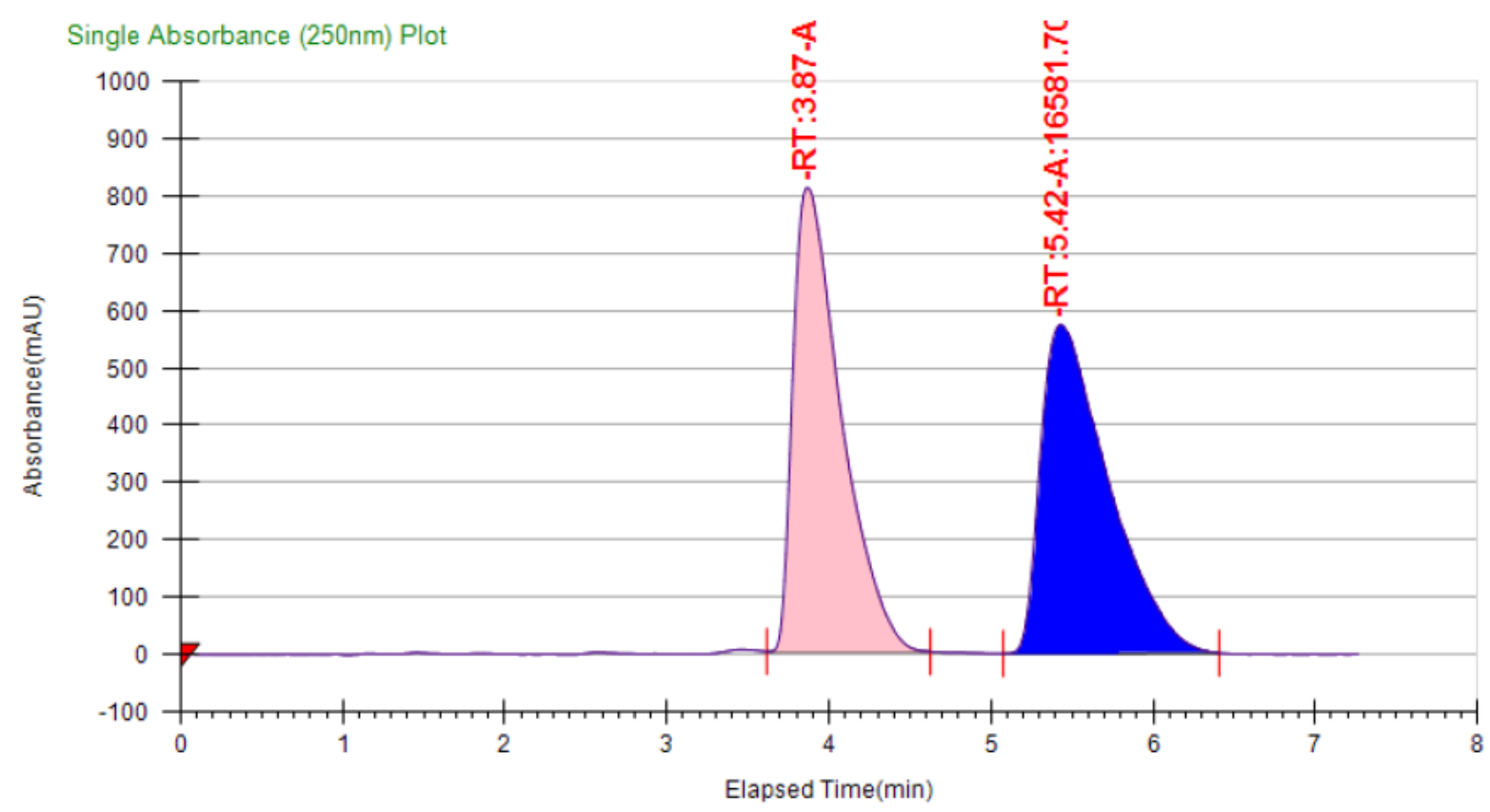

\begin{tabular}{|l|l|l|l|l|l|l|l|l|l|}
\hline Instrument Method & Inj. Vol. & Solvent & Column & Sample & Well Location & Temp. & Flow & $\%$ Modifier & Pressure \\
\hline $15 \mathrm{p}$ methanol & 10 & MeOH & AD-H & kwq-b-113 & $16 \mathrm{~B}$ & 31.4 & 4 & 15 & 150 \\
\hline \hline Peak No & $\%$ Area & Area & Ret. Time & Height & Cap. Factor \\
\hline 1 & 96.5544 & $\begin{array}{l}16678.018 \\
5\end{array}$ & $3.95 \mathrm{~min}$ & 731.1463 & 0 \\
\hline 2 & 3.4456 & 595.1655 & $5.27 \mathrm{~min}$ & 25.4767 & 0 & \\
\hline
\end{tabular}

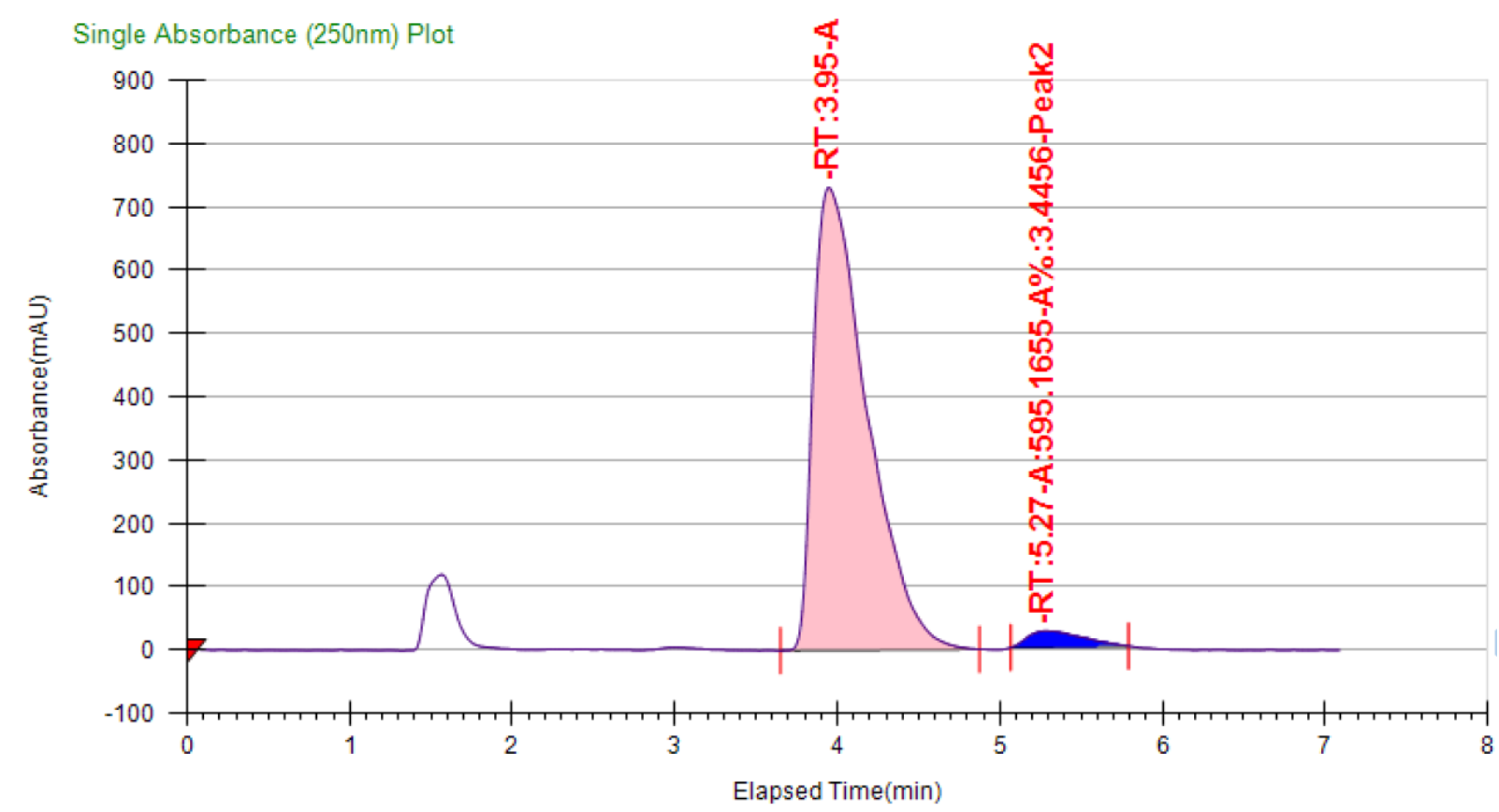




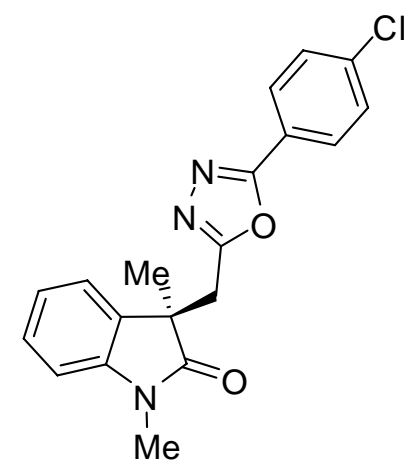

Molecular formula: $\mathrm{C}_{19} \mathrm{H}_{16} \mathrm{~N}_{3} \mathrm{O}_{2} \mathrm{Cl}$; Molecular Weight: 353.8022

According to general procedure, 3af was obtained in $64 \%$ yield $(22.7 \mathrm{mg})$ and $87 \%$ ee as a colorless oil. ${ }^{1} \mathrm{H}$ NMR $\left(400 \mathrm{MHz}, \mathrm{CDCl}_{3}\right): \delta 7.83(\mathrm{~d}, J=8.0 \mathrm{~Hz}, 2 \mathrm{H}), 7.45(\mathrm{~d}, J=8.0 \mathrm{~Hz}, 2 \mathrm{H})$, 7.32-7.15 (m, 2H), 7.05 (t, J = 7.2 Hz, 1H), $6.82(\mathrm{~d}, J=7.5 \mathrm{~Hz}, 1 \mathrm{H}), 3.45(\mathrm{~s}, 2 \mathrm{H}), 3.25(\mathrm{~s}, 3 \mathrm{H})$, $1.58(\mathrm{~s}, 3 \mathrm{H}) ;{ }^{13} \mathrm{C}$ NMR $\left(101 \mathrm{MHz}, \mathrm{CDCl}_{3}\right): \delta 178.7,163.9,163.3,143.0,137.9,131.6,129.4$, 128.7, 127.9, 123.1, 122.8, 122.2, 108.4, 46.8, 33.3, 26.4, 23.6; IR: $v\left(\mathrm{~cm}^{-1}\right)$ 2969, 2931, 2884, 1711, 1612, 1484, 1470, 1378, 1350, 1093, 1010, 909, 837; HRMS: (ESI) calcd for $\mathrm{C}_{19} \mathrm{H}_{16} \mathrm{~N}_{3} \mathrm{O}_{2} \mathrm{Cl}^{35} \mathrm{H}^{+}[\mathrm{M}+\mathrm{H}]^{+}$354.1009; found 354.1013; $\mathrm{C}_{19} \mathrm{H}_{16} \mathrm{~N}_{3} \mathrm{O}_{2} \mathrm{Cl}^{37} \mathrm{H}^{+}[\mathrm{M}+\mathrm{H}]^{+}$356.0980; found 356.0983 .

SFC: AD-H column, $15 \mathrm{ppm} \mathrm{MeOH}$ in supercritical $\mathrm{CO}_{2}$ as eluent, $4 \mathrm{~mL} / \mathrm{min}$. $\mathrm{tR}=5 \mathrm{~min}$ (major), 8 min (minor);

$[\alpha]_{D}^{28}=72\left(\mathrm{c}=0.7, \mathrm{CHCl}_{3}\right)$ for $87 \%$ ee.

\begin{tabular}{|l|l|l|l|l|l|l|l|l|l|}
\hline Instrument Method & Inj. Vol. & Solvent & Column & Sample & Well Location & Temp. & Flow & $\%$ Modifier & Pressure \\
\hline $15 p$ methanol & 10 & MeOH & AD-H & kwq-b-84 & $16 \mathrm{~B}$ & 30 & 4 & 15 & 150 \\
\hline Peak No & $\%$ Area & Area & Ret. Time & Height & Cap. Factor \\
\hline 1 & 49.8664 & $\begin{array}{l}11740.372 \\
5\end{array}$ & 5.47 min & 397.2888 & 0 & \\
\hline 2 & 50.1336 & $\begin{array}{l}11803.269 \\
5\end{array}$ & 7.81 min & 275.0286 & 0 & \\
\hline
\end{tabular}




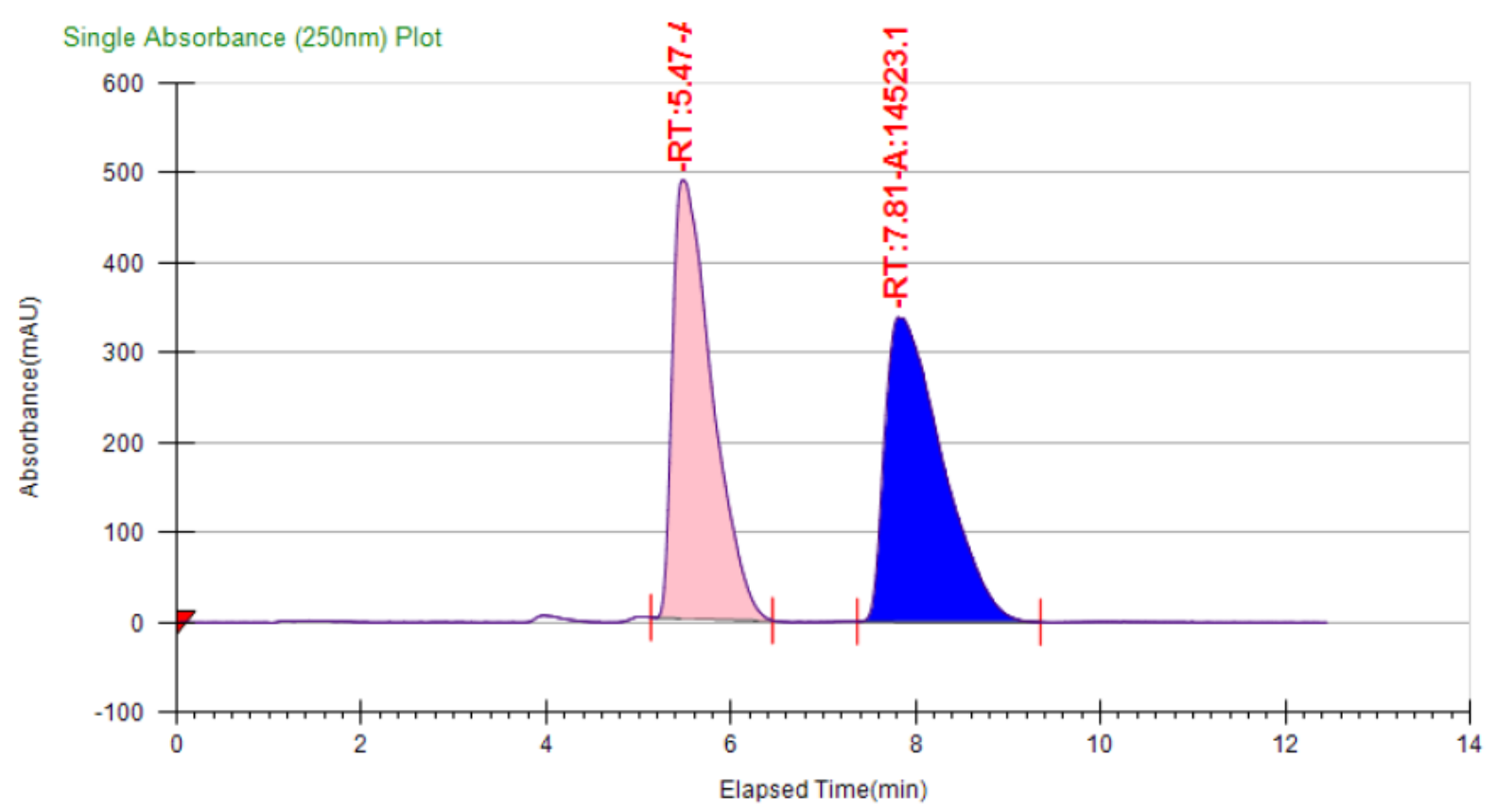

\begin{tabular}{|l|l|l|l|l|l|l|l|l|l|}
\hline Instrument Method & Inj. Vol. & Solvent & Column & Sample & Well Location & Temp. & Flow & $\%$ Modifier & Pressure \\
\hline $15 p$ methanol & 10 & MeOH & AD-H & kwq-b-118 & $16 \mathrm{C}$ & 29.9 & 4 & 15 & 150 \\
\hline Peak No & $\%$ Area & Area & Ret. Time & Height & Cap. Factor \\
\hline 1 & 93.5038 & $\begin{array}{l}32255.010 \\
8\end{array}$ & 5.28 min & 999.2633 & 0 & \\
\hline 2 & 6.4962 & 2240.9175 & 7.59 min & 61.1212 & 0 \\
\hline
\end{tabular}

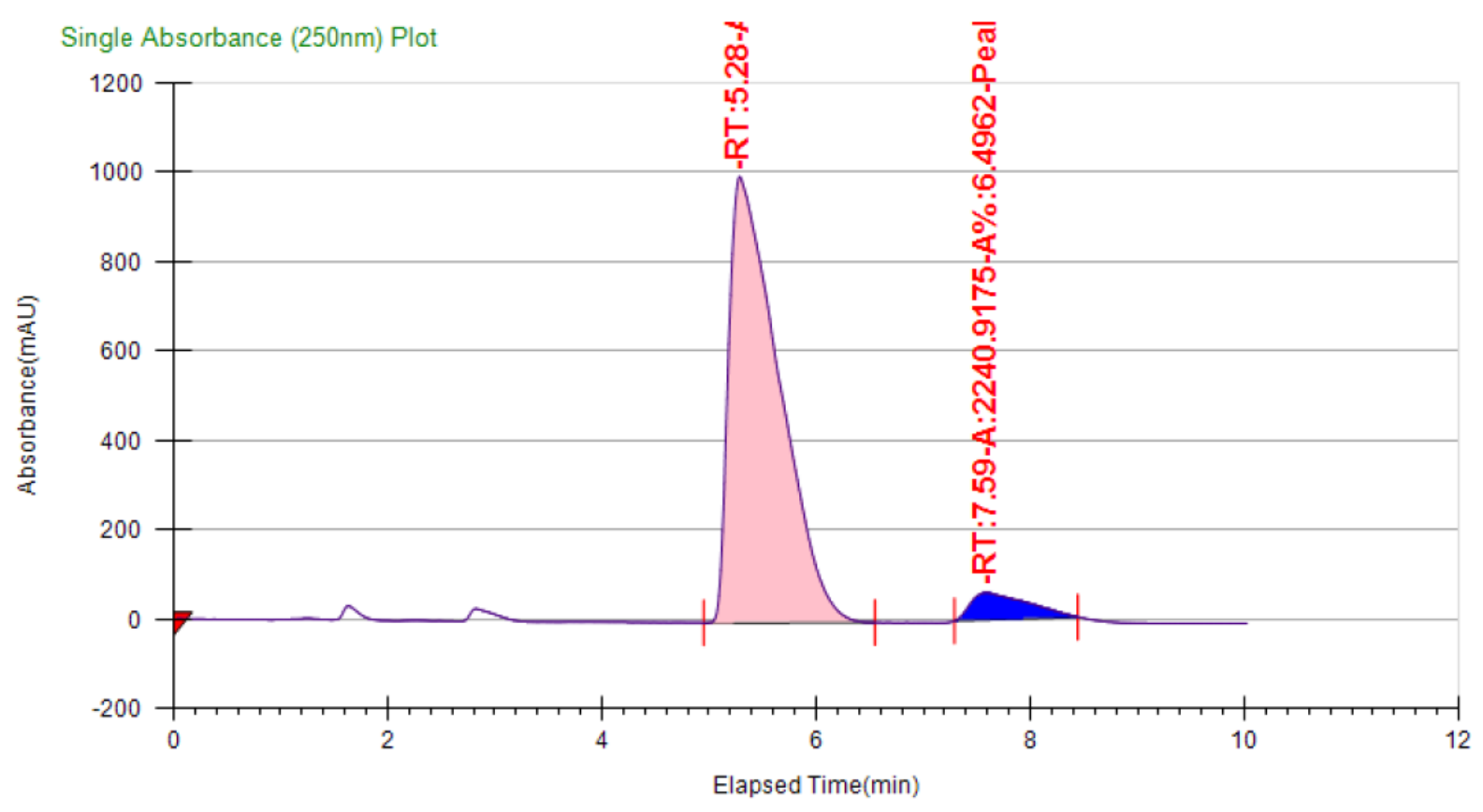


1,3-Dimethyl-3-((5-(4-(trifluoromethoxy)phenyl)-1,3,4-oxadiazol-2-yl)methyl)indolin-2-one (3ag)

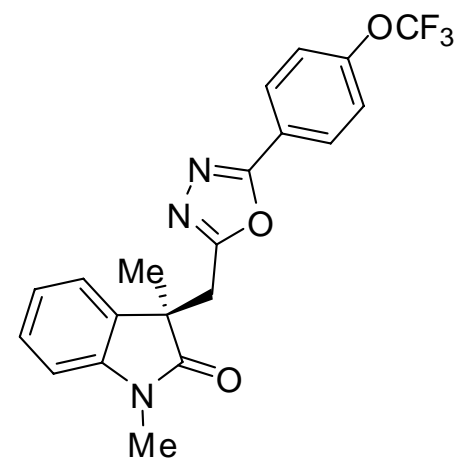

Molecular formula: $\mathrm{C}_{20} \mathrm{H}_{16} \mathrm{~F}_{3} \mathrm{~N}_{3} \mathrm{O}_{3}$; Molecular Weight: 403.3545

According to general procedure, 3ag was obtained in $75 \%$ yield $(30.2 \mathrm{mg}$ ) and $86 \%$ ee as a colorless oil. ${ }^{1} \mathrm{H}$ NMR (400 MHz, $\mathrm{CDCl}_{3}$ ): $\delta 7.94(\mathrm{~d}, J=8.8 \mathrm{~Hz}, 2 \mathrm{H}), 7.31(\mathrm{~d}, J=8.3 \mathrm{~Hz}, 2 \mathrm{H}), 7.26$ (t, $J=7.9 \mathrm{~Hz}, 1 \mathrm{H}), 7.21(\mathrm{~d}, J=7.3 \mathrm{~Hz}, 1 \mathrm{H}), 7.05(\mathrm{t}, J=7.5 \mathrm{~Hz}, 1 \mathrm{H}), 6.83(\mathrm{~d}, J=7.8 \mathrm{~Hz}, 1 \mathrm{H}), 3.45$ (s, 2H), 3.25 (s, 3H), $1.58(\mathrm{~s}, 3 \mathrm{H}) ;{ }^{13} \mathrm{C}$ NMR (101 MHz, $\left.\mathrm{CDCl}_{3}\right): \delta$ 178.7, 163.6, 163.4, 151.5, 143.0, 131.6, 128.7, 128.5, 123.1, 122.8, 122.3, 121.2, 120.3 (q, $J=258.8 \mathrm{~Hz}$ ), 108.4, 46.8, 33.3, 26.4, 23.6; $\left.{ }^{19} \mathrm{~F} \mathrm{NMR} \mathrm{(376} \mathrm{MHz,} \mathrm{CDCl}_{3}\right): \delta$-57.71 (s); IR: $v\left(\mathrm{~cm}^{-1}\right)$ 2972, 2931, 2899, 1712, 1614, 1496, 1471, 1379, 1351, 1254, 1211, 1166; HRMS: (ESI) calcd for $\mathrm{C}_{20} \mathrm{H}_{16} \mathrm{~F}_{3} \mathrm{~N}_{3} \mathrm{O}_{3} \mathrm{H}^{+}$ $[\mathrm{M}+\mathrm{H}]^{+}$404.1222; found 404.1238.

SFC: AD-H column, $8 \mathrm{ppm} \mathrm{MeOH}$ in supercritical $\mathrm{CO}_{2}$ as eluent, $4 \mathrm{~mL} / \mathrm{min} . \mathrm{tR}=3 \mathrm{~min}$ (major), 4 min (minor);

$[\alpha]_{D}^{29}=35(c=1.9, \mathrm{MeOH})$ for $86 \%$ ee.

\begin{tabular}{|l|l|l|l|l|l|l|l|l|l|}
\hline Instrument Method & Inj. Vol. & Solvent & Column & Sample & Well Location & Temp. & Flow & $\%$ Modifier & Pressure \\
\hline $8 \mathrm{p} \mathrm{methanol}$ & 10 & $\mathrm{MeOH}$ & $\mathrm{AD}-\mathrm{H}$ & kwq-b-84 & $16 \mathrm{C}$ & 29.6 & 4 & 8 & 150 \\
\hline Peak No & $\%$ Area & Area & Ret. Time & Height & Cap. Factor \\
\hline 1 & 49.8453 & $\begin{array}{l}17882.317 \\
2\end{array}$ & 3.62 min & 867.4178 & 3623.95 \\
\hline 2 & 50.1547 & $\begin{array}{l}17993.335 \\
2\end{array}$ & 4.69 min & 680.1837 & 4690.6 \\
\hline
\end{tabular}




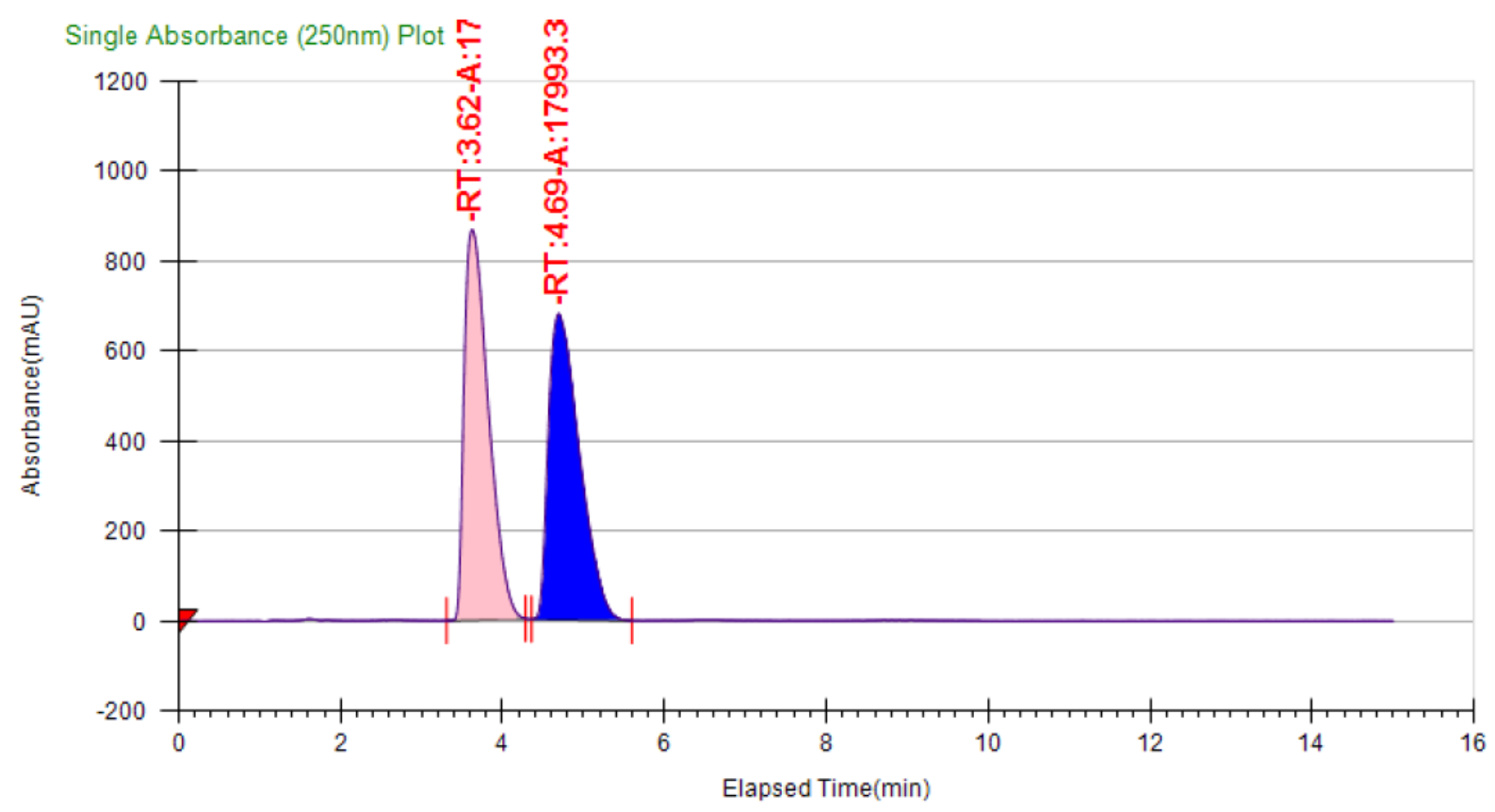

\begin{tabular}{|l|l|l|l|l|l|l|l|l|l|}
\hline Instrument Method & Inj. Vol. & Solvent & Column & Sample & Well Location & Temp. & Flow & $\%$ Modifier & Pressure \\
\hline $8 \mathrm{p}$ methanol & 10 & MeOH & AD-H & kwq-b-112 & $16 \mathrm{C}$ & 29.9 & 4 & 8 & 150 \\
\hline \hline Peak No & $\%$ Area & Area & Ret. Time & Height & Cap. Factor \\
\hline 1 & 92.9074 & $\begin{array}{l}29322.495 \\
8\end{array}$ & $3.43 \mathrm{~min}$ & 1328.2361 & 0 \\
\hline 2 & 7.0926 & 2238.4828 & $4.47 \mathrm{~min}$ & 91.2483 & 0 & \\
\hline
\end{tabular}

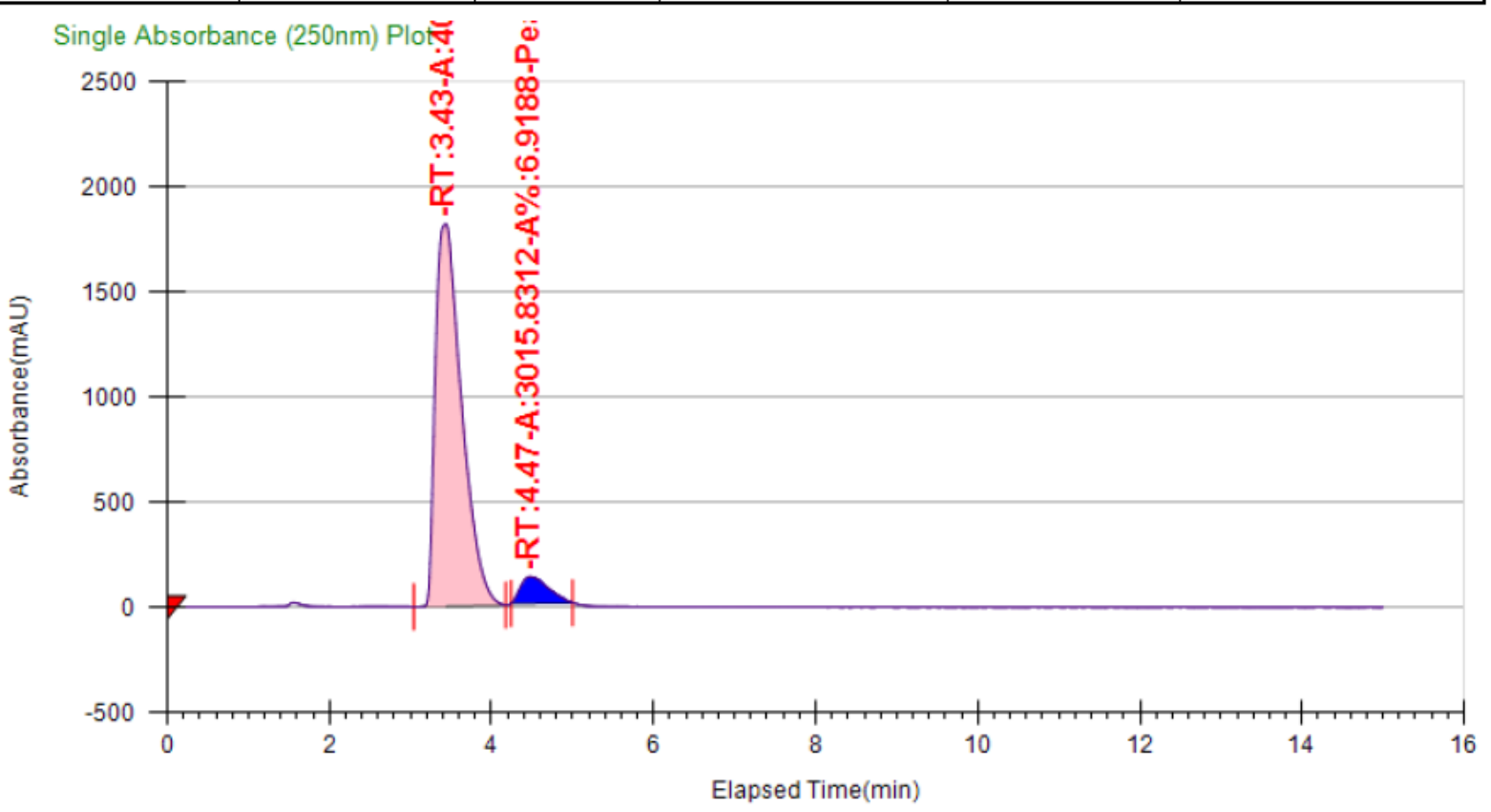


3-((5-Benzyl-1,3,4-oxadiazol-2-yl)methyl)-1,3-dimethylindolin-2-one (3ah)

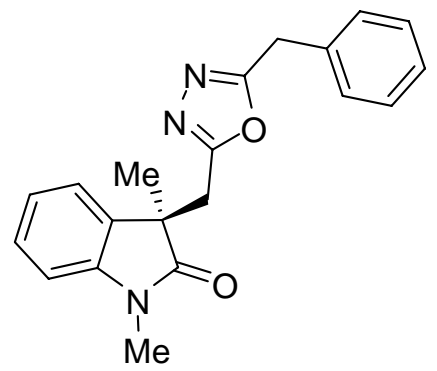

Molecular formula: $\mathrm{C}_{20} \mathrm{H}_{19} \mathrm{~N}_{3} \mathrm{O}_{2}$; Molecular Weight: 333.3838

According to general procedure, 3ah was obtained in $60 \%$ yield $(20.0 \mathrm{mg}$ ) and $94 \%$ ee as a colorless oil. ${ }^{1} \mathrm{H}$ NMR $\left(400 \mathrm{MHz}, \mathrm{CDCl}_{3}\right): \delta$ 7.40-7.25 (m, 3H), 7.21 (t, J= $\left.7.5 \mathrm{~Hz}, 1 \mathrm{H}\right), 7.12-$ $7.01(\mathrm{~m}, 3 \mathrm{H}), 6.95(\mathrm{t}, J=7.3 \mathrm{~Hz}, 1 \mathrm{H}), 6.65(\mathrm{~d}, J=7.7 \mathrm{~Hz}, 1 \mathrm{H}), 4.12(\mathrm{~d}, J=15.7 \mathrm{~Hz}, 1 \mathrm{H}), 3.98(\mathrm{~d}$, $J=15.7 \mathrm{~Hz}, 1 \mathrm{H}), 3.37(\mathrm{~d}, J=15.1 \mathrm{~Hz}, 1 \mathrm{H}), 3.31(\mathrm{~d}, J=15.1 \mathrm{~Hz}, 1 \mathrm{H}), 3.00(\mathrm{~s}, 3 \mathrm{H}), 1.48(\mathrm{~s}, 3 \mathrm{H})$; ${ }^{13} \mathrm{C}$ NMR $\left(101 \mathrm{MHz} \mathrm{CDCl}_{3}\right): \delta 178.7,165.2,163.8,142.8,133.8,131.3,128.9,128.6,128.4$, 127.4, 123.0, 122.7, 108.3, 46.7, 33.2, 31.6, 26.1, 23.6; IR: $v\left(\mathrm{~cm}^{-1}\right)$ 2971, 2929, 1711, 1613, $1494,1470,1454,1378,1350,1125,1030,911,727$; HRMS: (ESI) calcd for $\mathrm{C}_{20} \mathrm{H}_{19} \mathrm{~N}_{3} \mathrm{O}_{2} \mathrm{Na}^{+}$ $[\mathrm{M}+\mathrm{Na}]^{+}$356.1375; found 356.1375 .

SFC: IA column, $6 \mathrm{ppm} \mathrm{MeOH}$ in supercritical $\mathrm{CO}_{2}$ as eluent, $4 \mathrm{~mL} / \mathrm{min}$. $\mathrm{tR}=7 \mathrm{~min}$ (minor), 9 $\min ($ major);

$[\alpha]_{D}^{27}=59\left(\mathrm{c}=0.6, \mathrm{CHCl}_{3}\right)$ for $94 \%$ ee.

\begin{tabular}{|l|l|l|l|l|l|l|l|l|l|}
\hline Instrument Method & Inj. Vol. & Solvent & Column & Sample & Well Location & Temp. & Flow & $\%$ Modifier & Pressure \\
\hline 6p methanol & 15 & MeOH & IA & kwq-b-64 & $16 \mathrm{D}$ & 29.9 & 4 & 6 & 150 \\
\hline
\end{tabular}




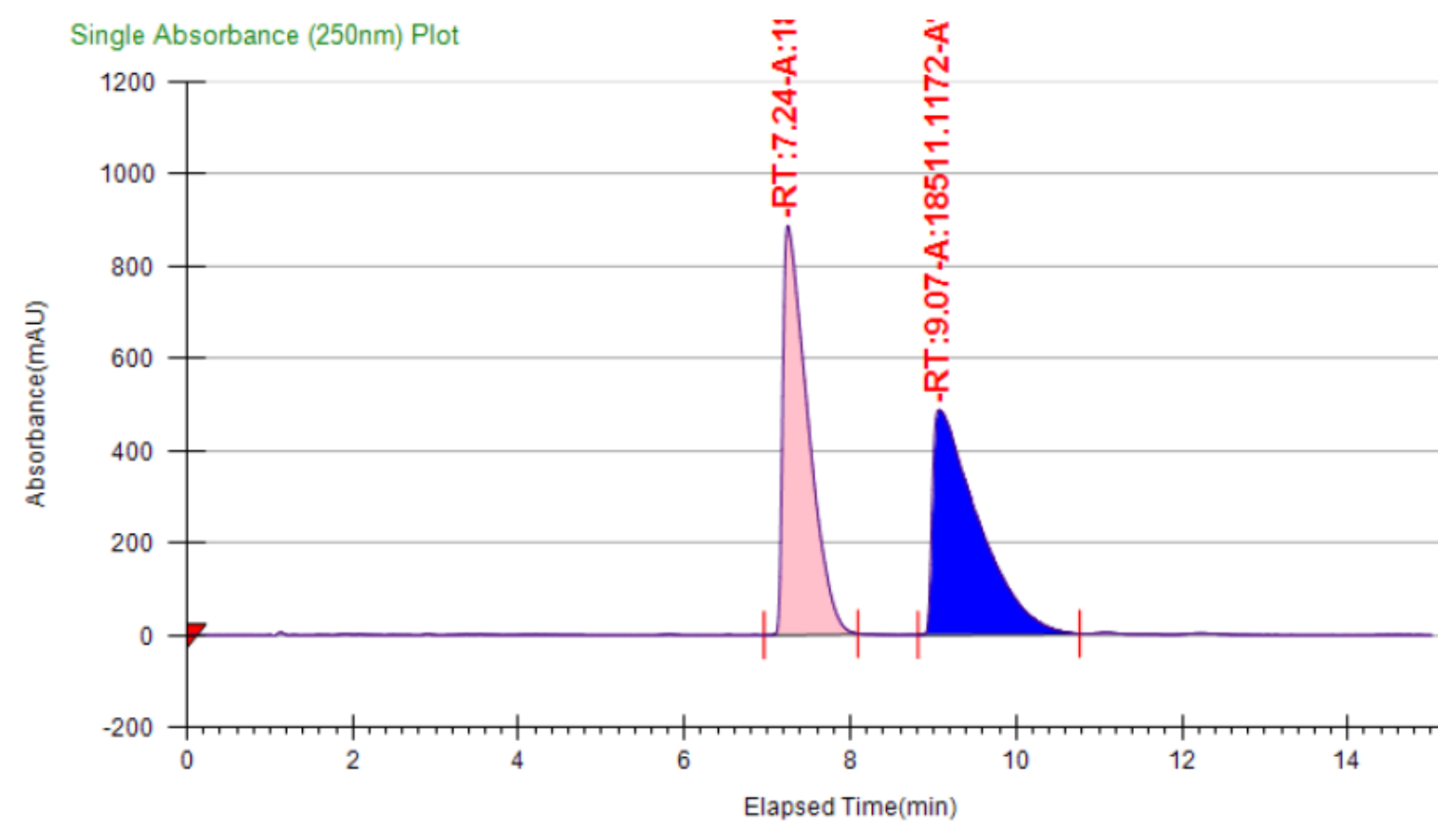

\begin{tabular}{|l|l|l|l|l|l|l|l|l|l|}
\hline Instrument Method & Inj. Vol. & Solvent & Column & Sample & Well Location & Temp. & Flow & $\%$ Modifier & Pressure \\
\hline 6p methanol & 15 & MeOH & IA & kwq-b-119 & $16 \mathrm{C}$ & 29.9 & 4 & 6 & 150 \\
\hline Peak No & $\%$ Area & Area & Ret. Time & Height & Cap. Factor \\
\hline 1 & 2.5649 & 81.092 & 7.63 min & 6.7926 & 0 & \\
\hline 2 & 97.4351 & 3080.4772 & 9.57 min & 113.8038 & 0 & \\
\hline
\end{tabular}

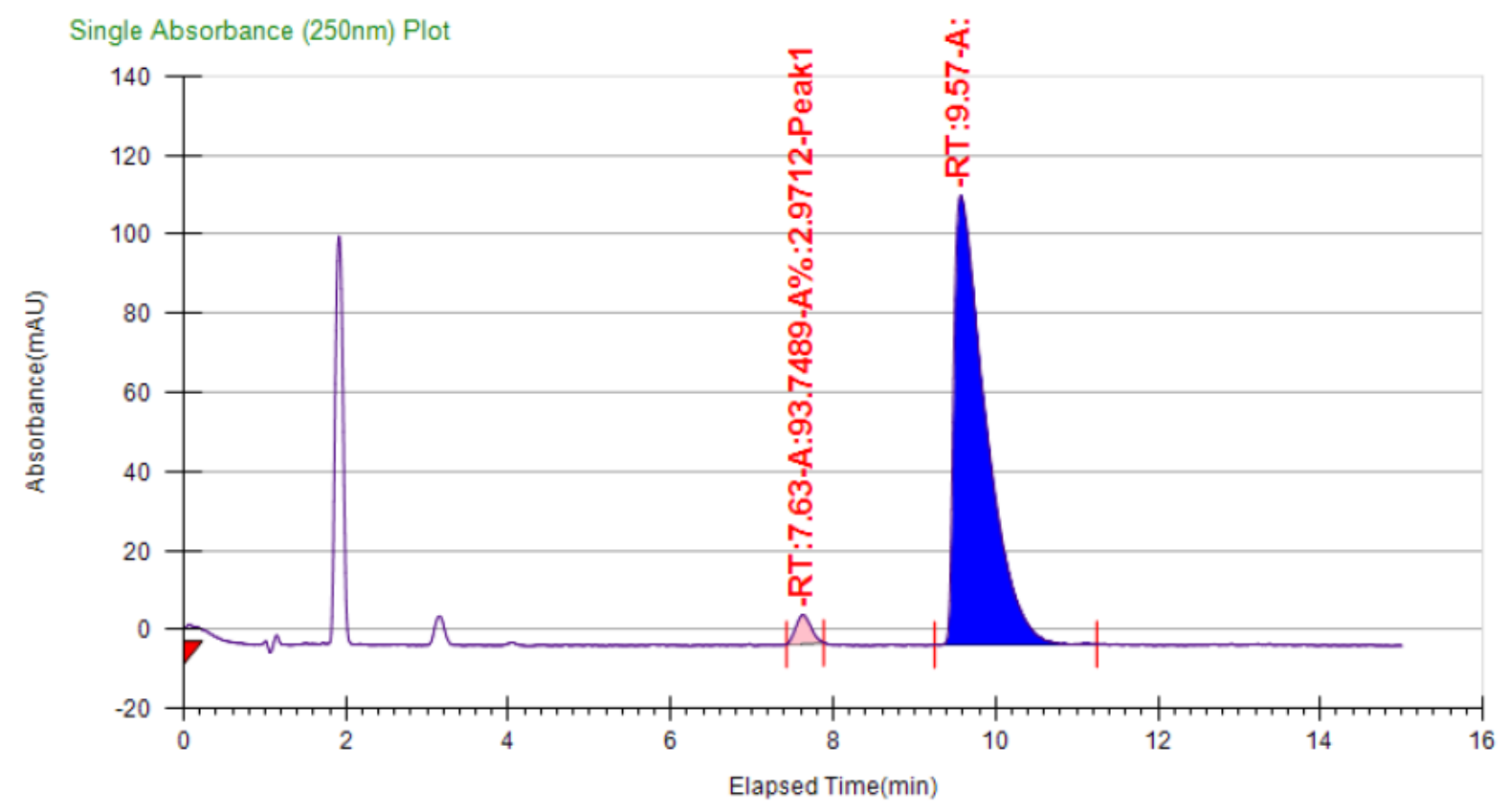




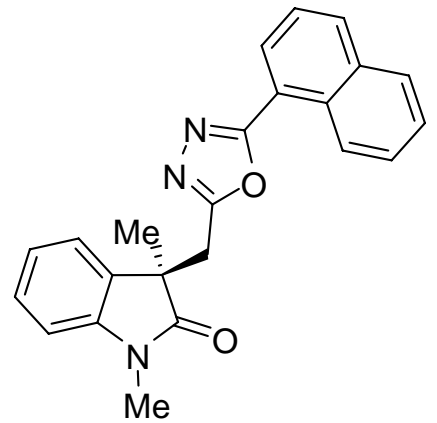

Molecular formula: $\mathrm{C}_{23} \mathrm{H}_{19} \mathrm{~N}_{3} \mathrm{O}_{2}$; Molecular Weight: 369.4159

According to general procedure, 3ai was obtained in $68 \%$ yield $(25.1 \mathrm{mg})$ and $94 \%$ ee as a colorless oil. ${ }^{1} \mathrm{H}$ NMR $\left(400 \mathrm{MHz}, \mathrm{CDCl}_{3}\right): \delta 9.04(\mathrm{~d}, J=8.5 \mathrm{~Hz}, 1 \mathrm{H}), 8.01(\mathrm{~d}, J=8.2 \mathrm{~Hz}, 1 \mathrm{H}), 7.96$ (d, $J=7.3 \mathrm{~Hz}, 1 \mathrm{H}), 7.91(\mathrm{~d}, J=8.0 \mathrm{~Hz}, 1 \mathrm{H}), 7.65(\mathrm{t}, J=7.2 \mathrm{~Hz}, 1 \mathrm{H}), 7.61-7.49(\mathrm{~m}, 2 \mathrm{H}), 7.34-$ $7.21(\mathrm{~m}, 2 \mathrm{H}), 7.07$ (t, J = $7.5 \mathrm{~Hz}, 1 \mathrm{H}), 6.84(\mathrm{~d}, J=7.9 \mathrm{~Hz}, 1 \mathrm{H}), 3.56$ (d, J = 15.3 Hz, 1H), 3.51 (d, $J=15.4 \mathrm{~Hz}, 1 \mathrm{H}), 3.26(\mathrm{~s}, 3 \mathrm{H}), 1.63(\mathrm{~s}, 3 \mathrm{H}) ;{ }^{13} \mathrm{C} \mathrm{NMR}\left(101 \mathrm{MHz}, \mathrm{CDCl}_{3}\right): \delta 178.9,164.8,162.8$, 143.1, 133.8, 132.5, 131.7, 129.9, 128.6, 128.6, 128.2, 128.1, 126.7, 126.1, 124.8, 123.2, 122.9, 120.3, 108.4, 46.9, 33.3, 26.5, 23.8; IR: $v\left(\mathrm{~cm}^{-1}\right)$ 2970, 2929, 2884, 1711, 1648, 1613, 1577, 1535, 1513, 1493, 1451, 1421, 1308, 1194, 1144, 1098, 1039; HRMS: (ESI) calcd for $\mathrm{C}_{23} \mathrm{H}_{19} \mathrm{~N}_{3} \mathrm{O}_{2} \mathrm{H}^{+}[\mathrm{M}+\mathrm{H}]^{+}$370.1556; found 370.1556.

SFC: $A D-H$ column, $15 \mathrm{ppm} \mathrm{MeOH}$ in supercritical $\mathrm{CO}_{2}$ as eluent, $4 \mathrm{~mL} / \mathrm{min}$. $\mathrm{tR}=9 \mathrm{~min}$ (major), 14 min (minor);

$[\alpha]_{D}^{29}=30(c=1.3, \mathrm{MeOH})$ for $94 \%$ ee.

\begin{tabular}{|c|c|c|c|c|c|c|c|c|c|c|}
\hline \multirow{2}{*}{\multicolumn{2}{|c|}{\begin{tabular}{|l|} 
Instrument Method \\
$15 p$ methanol
\end{tabular}}} & \multirow{2}{*}{\begin{tabular}{|l} 
Inj. Vol. \\
10
\end{tabular}} & \multirow{2}{*}{\begin{tabular}{|l} 
Solvent \\
$\mathrm{MeOH}$
\end{tabular}} & \multirow{2}{*}{\begin{tabular}{|l} 
Column \\
AD-H
\end{tabular}} & \multirow{2}{*}{\begin{tabular}{|l|} 
Sample \\
kwq-b-60
\end{tabular}} & Well Location & Temp. & \multirow{2}{*}{\begin{tabular}{|l} 
Flow \\
4
\end{tabular}} & \multirow{2}{*}{\begin{tabular}{|l}
$\%$ Modifier \\
15
\end{tabular}} & \multirow{2}{*}{$\begin{array}{l}\text { Pressure } \\
150\end{array}$} \\
\hline & & & & & & $16 \mathrm{C}$ & 30 & & & \\
\hline & \multicolumn{2}{|c|}{ Peak No } & $\%$ Area & Area & \multicolumn{3}{|c|}{ Ret. Time } & \multicolumn{2}{|c|}{ Cap. Factor } & \\
\hline & \multicolumn{2}{|c|}{1} & 49.9275 & $\begin{array}{l}14711.959 \\
4\end{array}$ & $9.03 \mathrm{~min}$ & \multicolumn{2}{|c|}{267.8575} & \multicolumn{2}{|l|}{9032.2} & \\
\hline & \multicolumn{2}{|c|}{2} & 50.0725 & $\begin{array}{l}14754.679 \\
6 \\
\end{array}$ & $15 \mathrm{~min}$ & \multicolumn{2}{|c|}{161.7114} & \multicolumn{2}{|c|}{14998.7833} & \\
\hline
\end{tabular}




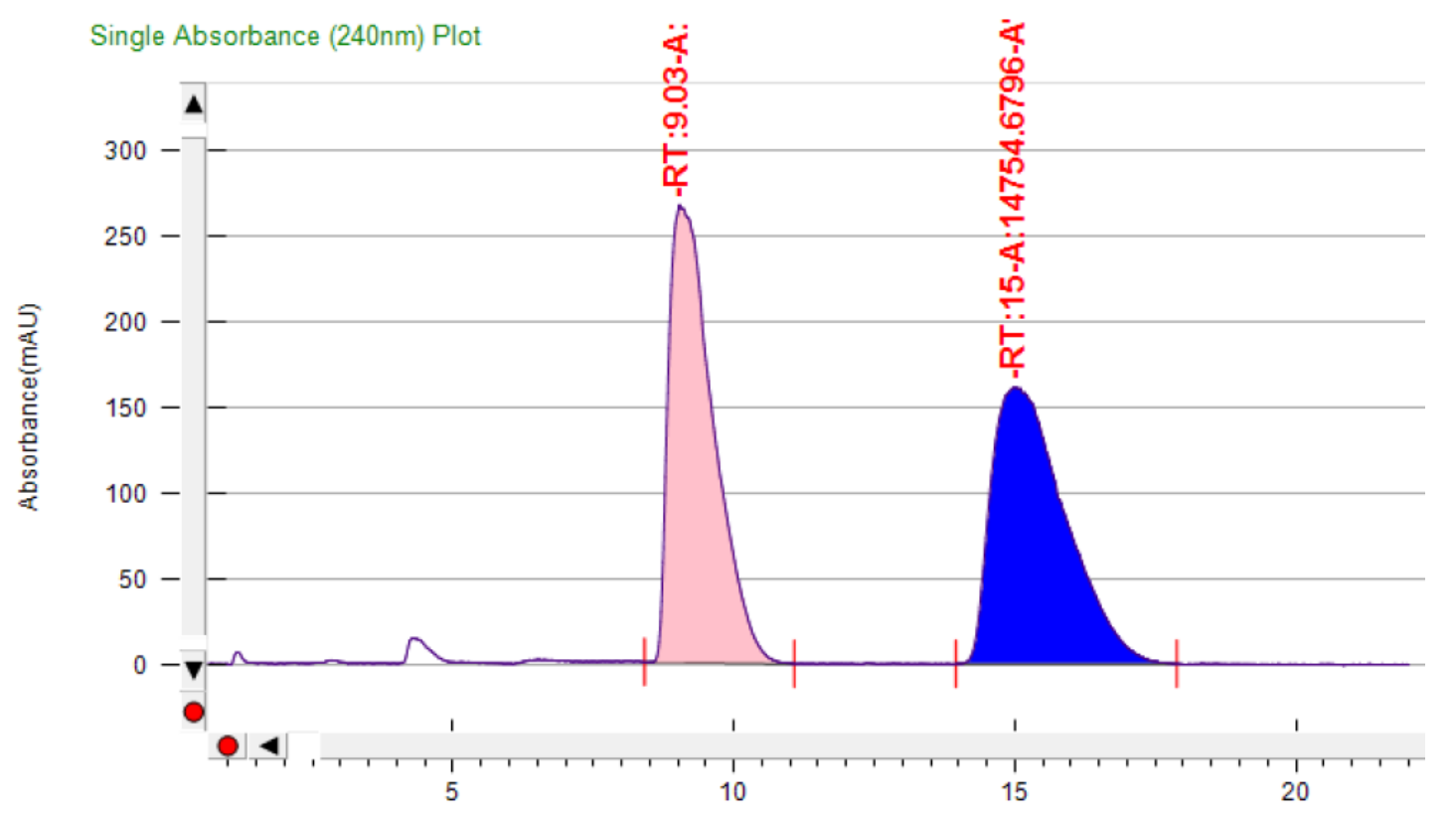

\begin{tabular}{|l|l|l|l|l|l|l|l|l|l|}
\hline Instrument Method & Inj. Vol. & Solvent & Column & Sample & Well Location & Temp. & Flow & $\%$ Modifier & Pressure \\
\hline 15p methanol & 10 & MeOH & AD-H & kwq-b-111 & $16 \mathrm{~B}$ & 31.2 & 4 & 15 & 150 \\
\hline Peak No & $\%$ Area & Area & Ret. Time & Height & Cap. Factor \\
\hline 1 & 96.948 & $\begin{array}{l}27554.768 \\
8\end{array}$ & 8.92 min & 571.0479 & 0 & \\
\hline
\end{tabular}

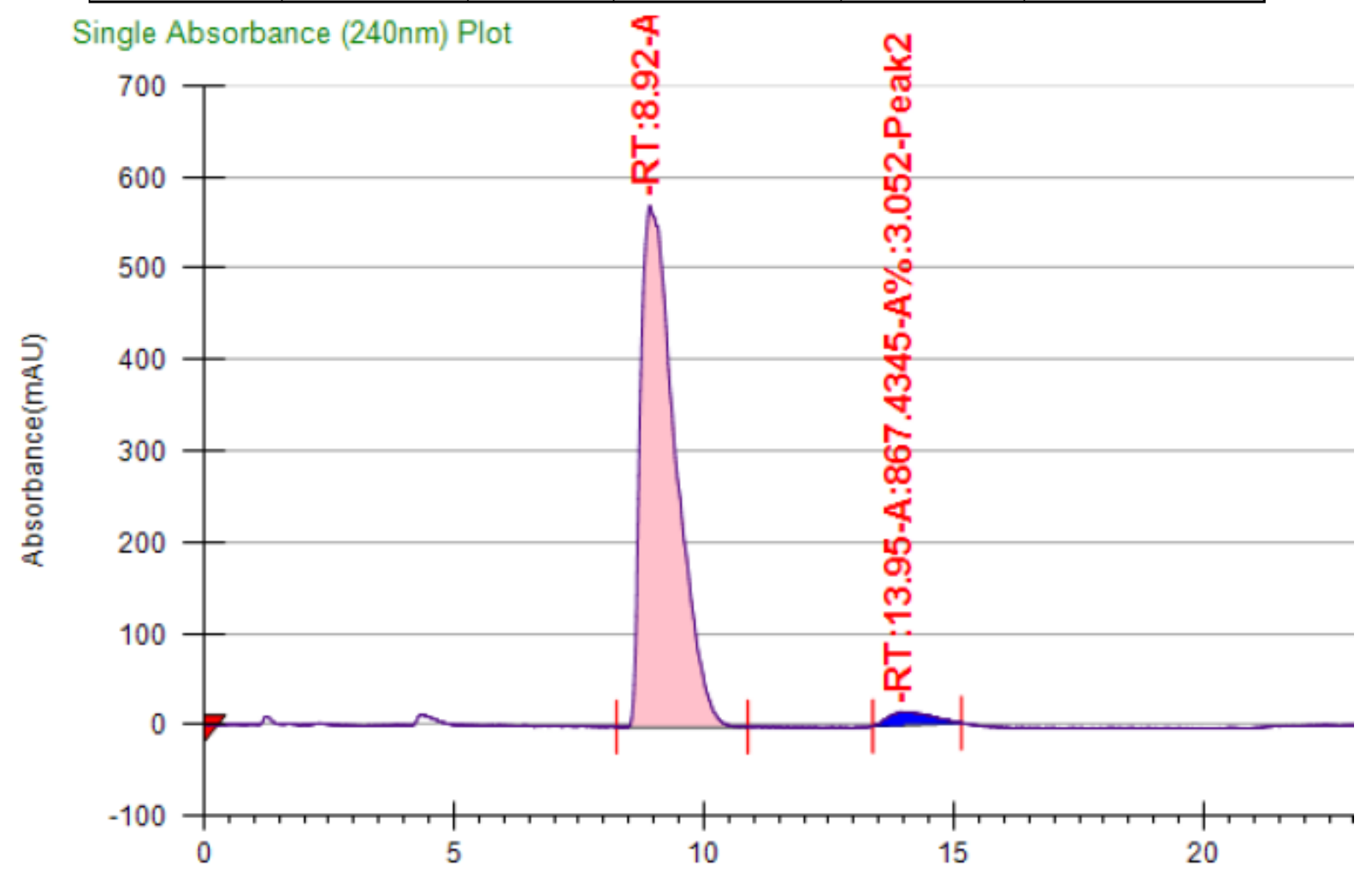


3-(Benzo[d]oxazol-2-ylmethyl)-1,3-dimethylindolin-2-one (3aj)

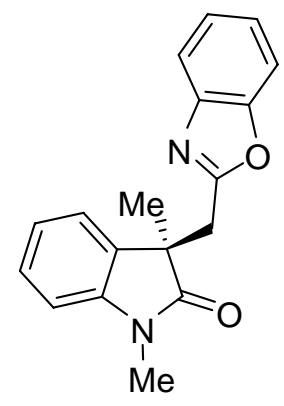

Molecular formula: $\mathrm{C}_{18} \mathrm{H}_{16} \mathrm{~N}_{2} \mathrm{O}_{2}$; Molecular Weight: 292.3318

According to general procedure, 3aj was obtained in 60\% yield $(17.5 \mathrm{mg})$ and $97 \%$ ee as a colorless oil. ${ }^{1} \mathrm{H}$ NMR $\left(400 \mathrm{MHz}, \mathrm{CDCl}_{3}\right)$ : $\delta$ 7.67-7.56 (m, $\left.1 \mathrm{H}\right), 7.44-7.36(\mathrm{~m}, 1 \mathrm{H})$, 7.33-7.18 (m, 3H), $7.14(\mathrm{~d}, J=7.3 \mathrm{~Hz}, 1 \mathrm{H}), 6.99(\mathrm{t}, J=7.5 \mathrm{~Hz}, 1 \mathrm{H}), 6.80(\mathrm{~d}, J=7.7 \mathrm{~Hz}, 1 \mathrm{H}), 3.47(\mathrm{~d}, J=15.0 \mathrm{~Hz}$, $1 \mathrm{H}), 3.42(\mathrm{~d}, J=14.9 \mathrm{~Hz}, 1 \mathrm{H}), 3.28(\mathrm{~s}, 3 \mathrm{H}), 1.59(\mathrm{~s}, 3 \mathrm{H}) ;{ }^{13} \mathrm{C} \mathrm{NMR}\left(101 \mathrm{MHz}, \mathrm{CDCl}_{3}\right): \delta$ 179.3, 162.9, 150.6, 143.1, 141.1, 132.2, 128.3, 124.7, 124.1, 123.2, 122.6, 119.9, 110.3, 108.2, 47.1, 36.4, 26.4, 23.5; IR: $v\left(\mathrm{~cm}^{-1}\right)$ : 2969, 2929, 1712, 1613, 1570, 1493, 1454, 1378, 1350, 1242, 1125, 855; HRMS: (ESI) calcd for $\mathrm{C}_{18} \mathrm{H}_{16} \mathrm{~N}_{2} \mathrm{O}_{2} \mathrm{H}^{+}[\mathrm{M}+\mathrm{H}]^{+}$293.1290; found 293.1285.

SFC: IA column, $4 \mathrm{ppm} \mathrm{MeOH}$ in supercritical $\mathrm{CO}_{2}$ as eluent, $4 \mathrm{~mL} / \mathrm{min} . \mathrm{tR}=8 \mathrm{~min}$ (major), 9 $\min ($ minor);

$[\alpha]_{D}^{27}=69\left(\mathrm{c}=0.5, \mathrm{CHCl}_{3}\right)$ for $97 \%$ ee.

\begin{tabular}{|l|l|l|l|l|l|l|l|l|l|}
\hline Instrument Method & Inj. Vol. & Solvent & Column & Sample & Well Location & Temp. & Flow & $\%$ Modifier & Pressure \\
\hline 4p methanol & 10 & MeOH & IA & $\begin{array}{l}\text { Unknown } \\
\text { Sample }\end{array}$ & $16 \mathrm{~B}$ & 29.9 & 4 & 4 & 150 \\
\hline \begin{tabular}{|l|l|l|l|l|l|}
\hline Peak No \\
\hline 1
\end{tabular} & $\%$ Area & Area & Ret. Time & Height & Cap. Factor \\
\hline 2 & 48.1658 & $\begin{array}{l}30971.297 \\
6\end{array}$ & 8.65 min & 2241.2546 & 0 & \\
\hline
\end{tabular}




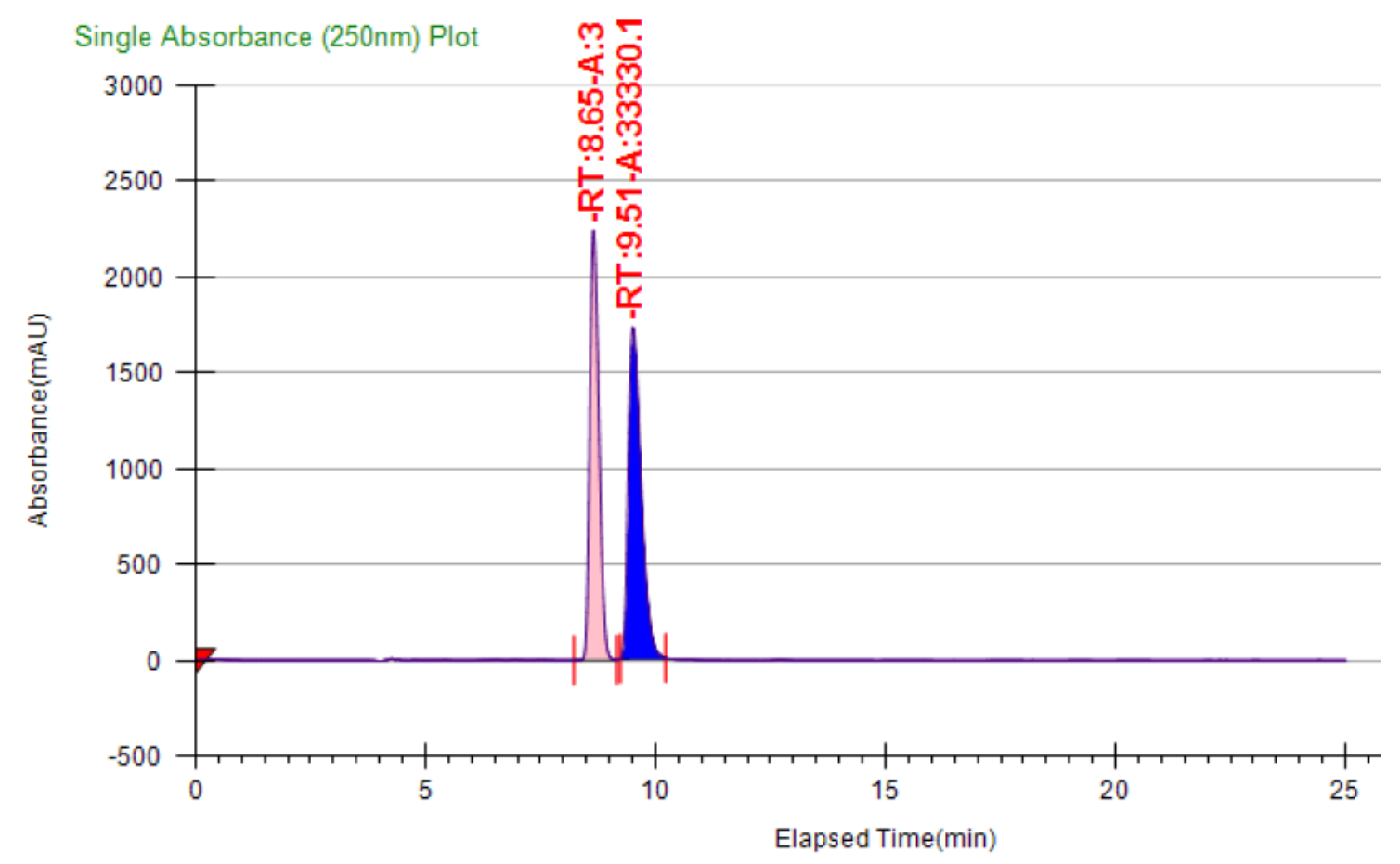

\begin{tabular}{|l|l|l|l|l|l|l|l|l|l|}
\hline Instrument Method & Inj. Vol. & Solvent & Column & Sample & Well Location & Temp. & Flow & $\%$ Modifier & Pressure \\
\hline $4 \mathrm{p}$ methanol & 10 & $\mathrm{MeOH}$ & $\mathrm{IA}$ & kwq-b-131 & $16 \mathrm{C}$ & 30.8 & 4 & 4 & 150 \\
\hline \hline Peak No & $\%$ Area & Area & Ret. Time & Height & Cap. Factor \\
\hline 1 & 98.2825 & 5653.6045 & $8.79 \mathrm{~min}$ & 411.4208 & 0 \\
\hline 2 & 1.7175 & 98.7992 & $9.8 \mathrm{~min}$ & 4.8247 & 0 & \\
\hline
\end{tabular}

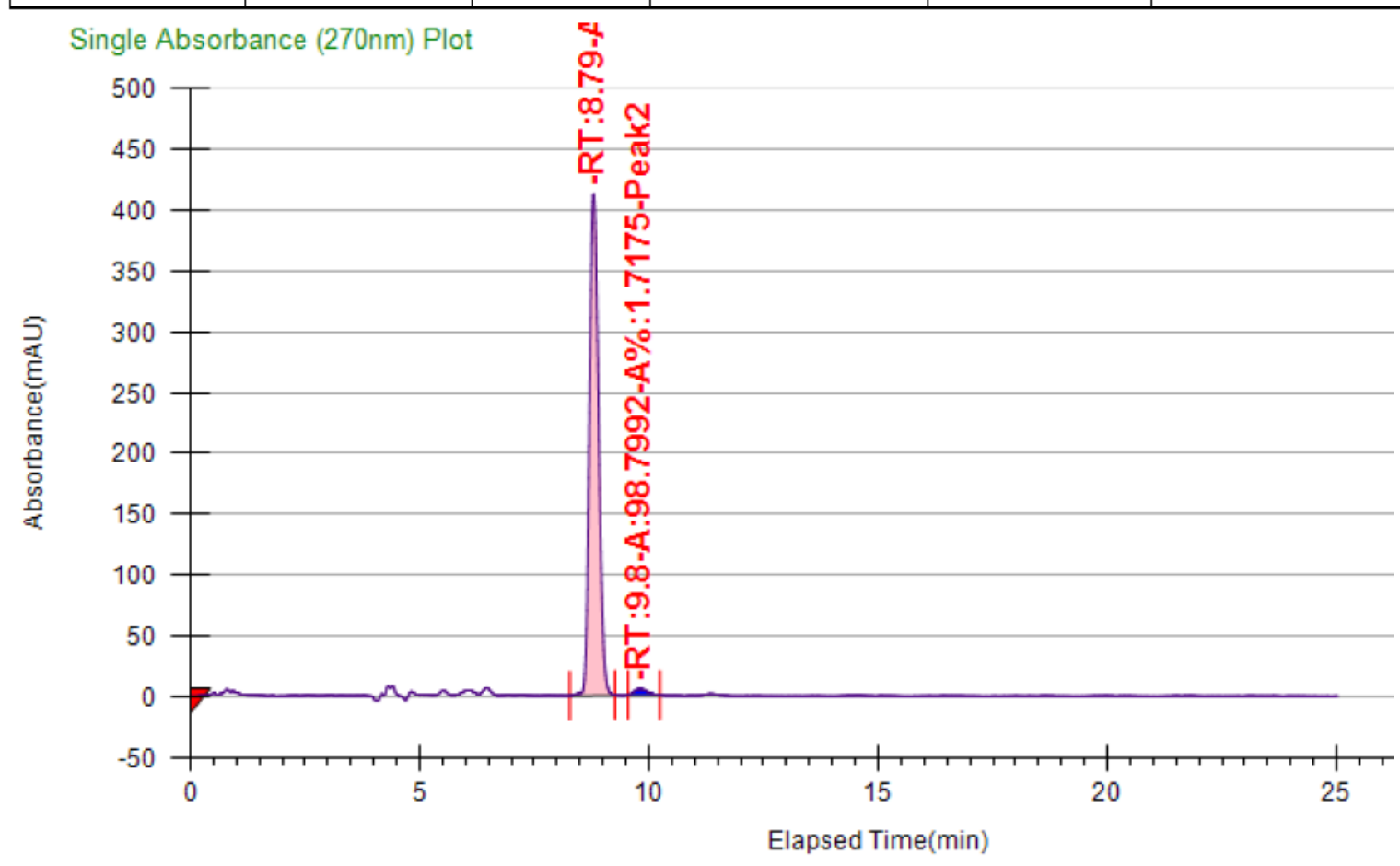




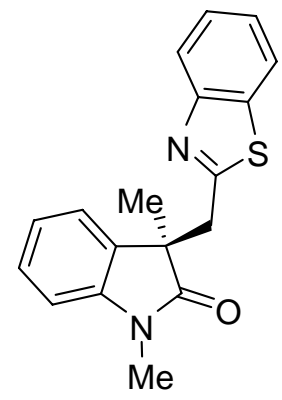

Molecular formula: $\mathrm{C}_{18} \mathrm{H}_{16} \mathrm{~N}_{2} \mathrm{OS}$; Molecular Weight: 308.3974

According to general procedure, 3ak was obtained in $61 \%$ yield $(18.8 \mathrm{mg}$ ) and $92 \%$ ee as a colorless oil. ${ }^{1} \mathrm{H}$ NMR $\left(400 \mathrm{MHz}, \mathrm{CDCl}_{3}\right): \delta 7.90(\mathrm{~d}, J=8.1 \mathrm{~Hz}, 1 \mathrm{H}), 7.74(\mathrm{~d}, J=7.9 \mathrm{~Hz}, 1 \mathrm{H}), 7.40$ (t, $J=7.6 \mathrm{~Hz}, 1 \mathrm{H}), 7.35-7.15(\mathrm{~m}, 3 \mathrm{H}), 7.05(\mathrm{t}, J=7.5 \mathrm{~Hz}, 1 \mathrm{H}), 6.77(\mathrm{~d}, J=7.7 \mathrm{~Hz}, 1 \mathrm{H}), 3.74(\mathrm{~d}, J$ $=14.5 \mathrm{~Hz}, 1 \mathrm{H}), 3.62(\mathrm{~d}, J=14.6 \mathrm{~Hz}, 1 \mathrm{H}), 3.22(\mathrm{~s}, 3 \mathrm{H}), 1.59(\mathrm{~s}, 3 \mathrm{H}) ;{ }^{13} \mathrm{C} \mathrm{NMR}\left(101 \mathrm{MHz}, \mathrm{CDCl}_{3}\right)$ : $\delta$ 179.4, 165.8, 143.3, 135.4, 132.1, 128.3, 125.7, 124.8, 123.4, 122.9, 122.6, 121.3, 108.1, 48.4, 41.8, 26.4, 24.2; IR: $v\left(\mathrm{~cm}^{-1}\right)$ 2967, 2966, 2927, 2926, 1711, 1614, 1493, 1471, 1455, 1437, 1436, 1379, 1351, 1125, 1060, 755, 754, 732; HRMS: (ESI) calcd for $\mathrm{C}_{18} \mathrm{H}_{16} \mathrm{~N}_{2} \mathrm{OSH}^{+}$ $[\mathrm{M}+\mathrm{H}]^{+}$309.1062; found 309.1062.

SFC: $A D-H$ column, $8 \mathrm{ppm} \mathrm{MeOH}$ in supercritical $\mathrm{CO}_{2}$ as eluent, $4 \mathrm{~mL} / \mathrm{min} . \mathrm{tR}=5 \mathrm{~min}$ (major), $8 \mathrm{~min}$ (minor);

$[\alpha]_{D}{ }^{24}=-83(c=0.2, \mathrm{MeOH})$ for $92 \%$ ee.

\begin{tabular}{|l|l|l|l|l|l|l|l|l|l|}
\hline Instrument Method & Inj. Vol. & Solvent & Column & Sample & Well Location & Temp. & Flow & $\%$ Modifier & Pressure \\
\hline $8 \mathrm{p}$ methanol & 10 & MeOH & AD-H & $\begin{array}{l}\text { Unknown } \\
\text { Sample }\end{array}$ & $16 \mathrm{~A}$ & 29.8 & 4 & 8 & 150 \\
\hline
\end{tabular}

\begin{tabular}{|l|l|l|l|l|l|}
\hline Peak No & \% Area & Area & Ret. Time & Height & Cap. Factor \\
\hline 1 & 50.7815 & 1646.5624 & $5.42 \mathrm{~min}$ & 69.0819 & 0 \\
\hline 2 & 49.2185 & 1595.8827 & $8.37 \mathrm{~min}$ & 46.3135 & 0 \\
\hline
\end{tabular}




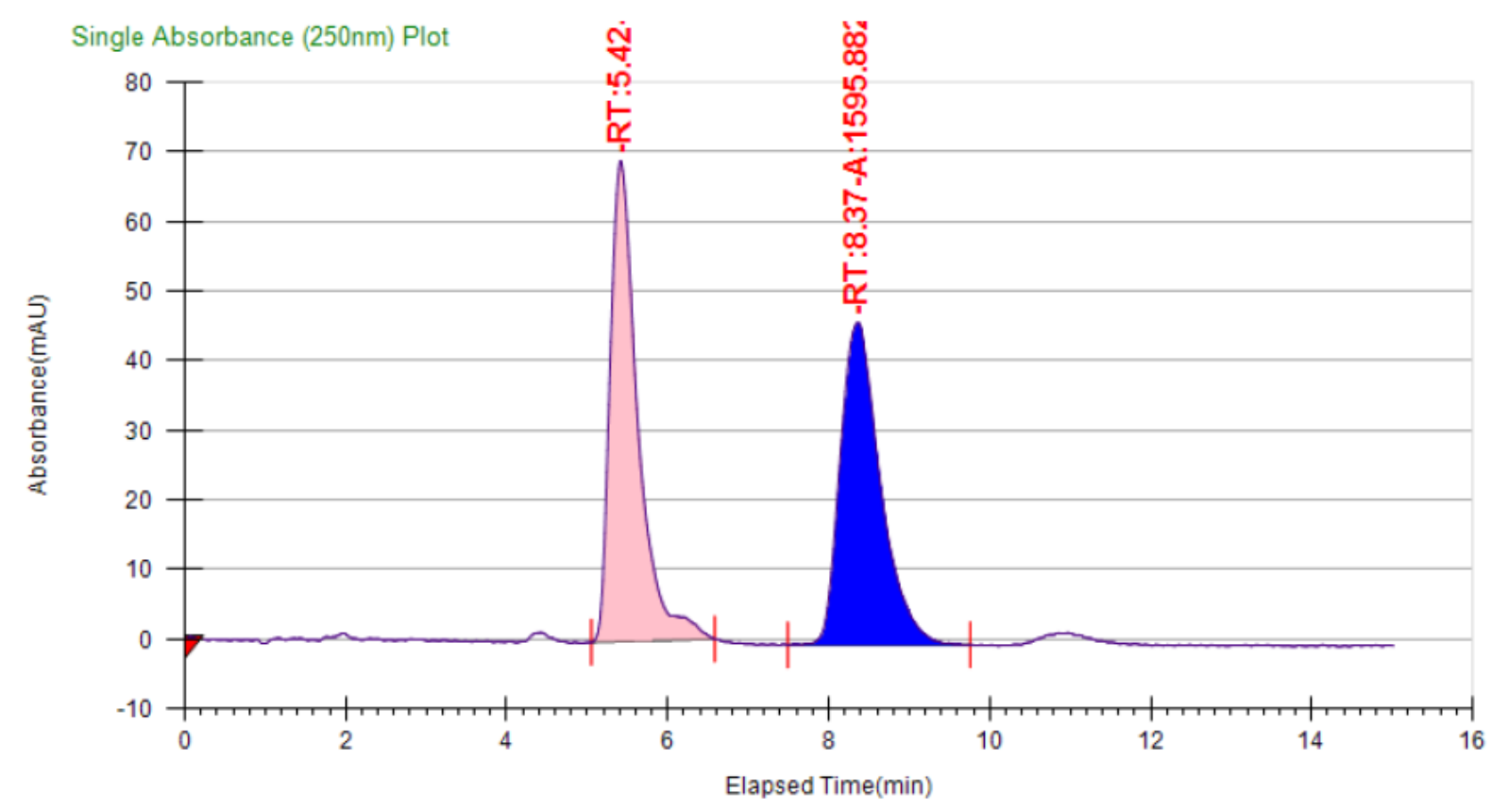

\begin{tabular}{|l|l|l|l|l|l|l|l|l|l|}
\hline Instrument Method & Inj. Vol. & Solvent & Column & Sample & Well Location & Temp. & Flow & $\%$ Modifier & Pressure \\
\hline $8 \mathrm{p} \mathrm{methanol}$ & 10 & $\mathrm{MeOH}$ & $\mathrm{AD}-\mathrm{H}$ & $\begin{array}{l}\text { Unknown } \\
\text { Sample }\end{array}$ & $16 \mathrm{~B}$ & 29.9 & 4 & 8 & 150 \\
\hline
\end{tabular}

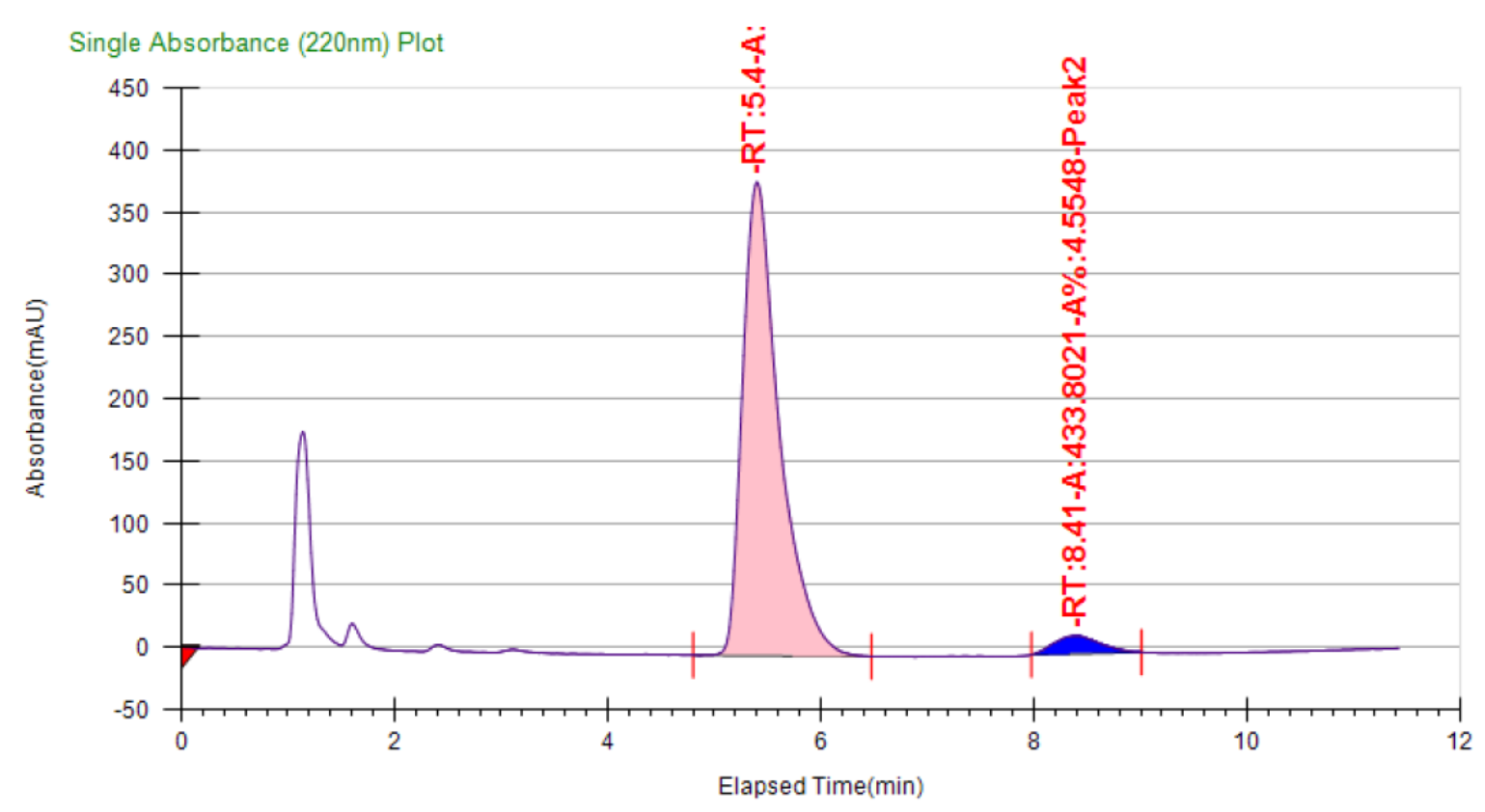


1-Benzyl-3-methyl-3-((5-phenyl-1,3,4-oxadiazol-2-yl)methyl)indolin-2-one (3da)

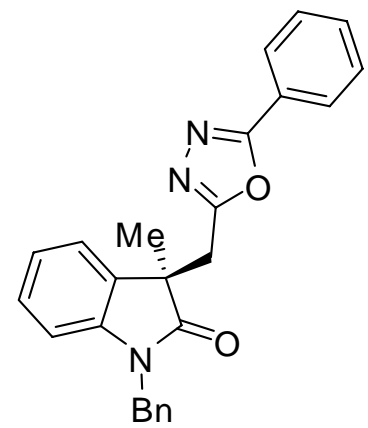

Molecular formula: $\mathrm{C}_{25} \mathrm{H}_{21} \mathrm{~N}_{3} \mathrm{O}_{2}$; Molecular Weight: 395.4531

According to general procedure, 3da was obtained in 67\% yield (26.4 mg) and 91\% ee as a colorless oil. ${ }^{1} \mathrm{H}$ NMR $\left(400 \mathrm{MHz}, \mathrm{CDCl}_{3}\right): \delta 7.87(\mathrm{~d}, J=7.3 \mathrm{~Hz}, 2 \mathrm{H}), 7.58-7.38(\mathrm{~m}, 3 \mathrm{H}), 7.33-$ $7.10(\mathrm{~m}, 7 \mathrm{H}), 7.04(\mathrm{t}, J=7.4 \mathrm{~Hz}, 1 \mathrm{H}), 6.71(\mathrm{~d}, J=7.7 \mathrm{~Hz}, 1 \mathrm{H}), 4.95(\mathrm{~s}, 2 \mathrm{H}), 3.53(\mathrm{~s}, 2 \mathrm{H}), 1.65(\mathrm{~s}$, $3 \mathrm{H}) ;{ }^{13} \mathrm{C}$ NMR $\left(101 \mathrm{MHz}, \mathrm{CDCl}_{3}\right): \delta 178.8,164.8,163.1,142.1,135.6,131.7,131.6,129.0$, $128.8,128.5,127.6,127.1,126.8,123.7,123.2,122.9,109.4,47.0,43.9,33.2,24.1 ;$ IR: $v\left(\mathrm{~cm}^{-}\right.$ $\left.{ }^{1}\right)$ 2971, 2928, 2901, 1710, 1611, 1552, 1488, 1450, 1380, 1361, 1176, 908, 728; HRMS: (ESI) calcd for $\mathrm{C}_{25} \mathrm{H}_{21} \mathrm{~N}_{3} \mathrm{O}_{2} \mathrm{H}^{+}[\mathrm{M}+\mathrm{H}]^{+}$396.1712; found 396.1704.

SFC: $A D-H$ column, $15 \mathrm{ppm} \mathrm{MeOH}$ in supercritical $\mathrm{CO}_{2}$ as eluent, $4 \mathrm{~mL} / \mathrm{min}$. $\mathrm{tR}=7 \mathrm{~min}$ (major), $12 \mathrm{~min}$ (minor);

$[\alpha]_{D}^{29}=22(c=1.6, \mathrm{MeOH})$ for $91 \%$ ee.

\begin{tabular}{|l|l|l|l|l|l|l|l|l|l|}
\hline Instrument Method & Inj. Vol. & Solvent & Column & Sample & Well Location & Temp. & Flow & $\%$ Modifier & Pressure \\
\hline 15p methanol & 10 & MeOH & AD-H & KWQ-B-75 & $16 \mathrm{~B}$ & 30.1 & 4 & 15 & 150 \\
\hline Peak No & $\%$ Area & Area & Ret. Time & Height & Cap. Factor \\
\hline 1 & 49.6641 & 6496.4349 & 7.23 min & 137.0563 & 0 & \\
\hline 2 & 50.3359 & 6584.3049 & $12.1 \mathrm{~min}$ & 85.4019 & 0 & \\
\hline
\end{tabular}




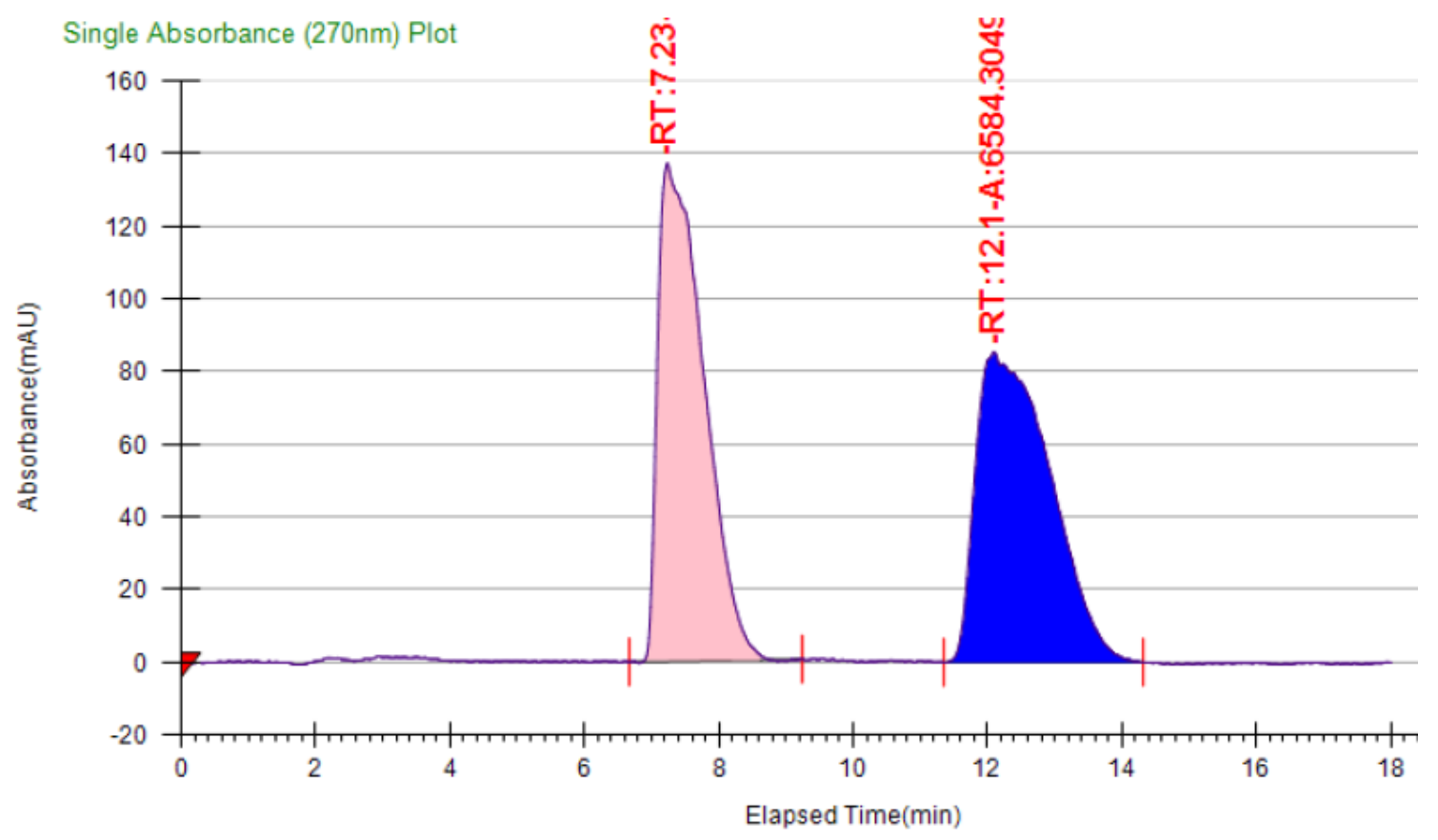

\begin{tabular}{|l|l|l|l|l|l|l|l|l|l|}
\hline Instrument Method & Inj. Vol. & Solvent & Column & Sample & Well Location & Temp. & Flow & $\%$ Modifier & Pressure \\
\hline 15p methanol & 10 & MeOH & AD-H & kwq-b-123 & $16 \mathrm{C}$ & 30.4 & 4 & 15 & 150 \\
\hline Peak No & $\%$ Area & Area & Ret. Time & Height & Cap. Factor \\
\hline 1 & 95.2647 & 78.2427 & 6.78 min & 1.8077 & 6782.2333 \\
\hline 2 & 4.7353 & 3.8892 & 11.46 min & 0.0738 & 11457.1667 \\
\hline
\end{tabular}

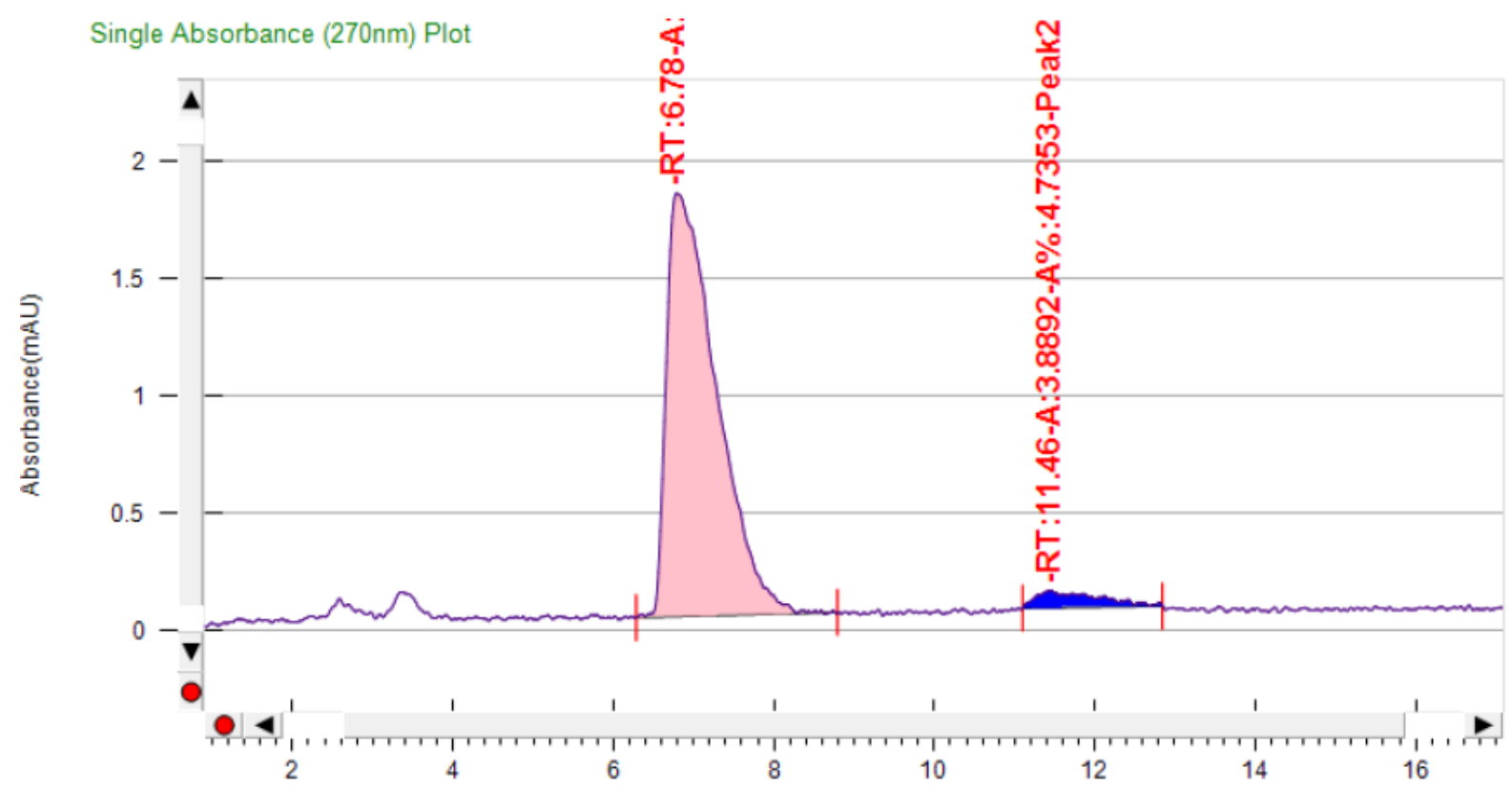




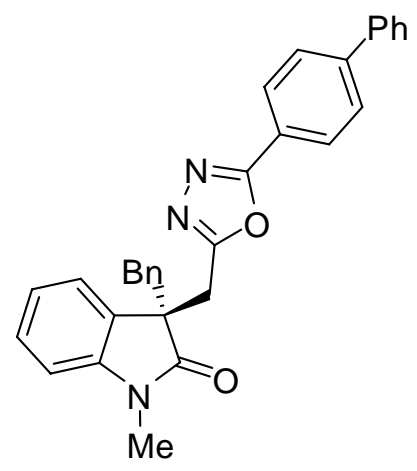

Molecular formula: $\mathrm{C}_{31} \mathrm{H}_{25} \mathrm{~N}_{3} \mathrm{O}_{2}$; Molecular Weight: 471.5491

According to general procedure, 3gd was obtained in $65 \%$ yield $(30.6 \mathrm{mg}$ ) and $85 \%$ ee as a colorless oil. ${ }^{1} \mathrm{H}$ NMR $\left(400 \mathrm{MHz}, \mathrm{CDCl}_{3}\right): \delta 7.92(\mathrm{~d}, J=8.4 \mathrm{~Hz}, 2 \mathrm{H}), 7.67(\mathrm{~d}, J=8.4 \mathrm{~Hz}, 2 \mathrm{H})$, 7.64-7.56 (m, 2H), $7.46(t, J=7.4 \mathrm{~Hz}, 2 \mathrm{H}), 7.39(\mathrm{t}, J=7.3 \mathrm{~Hz}, 1 \mathrm{H}), 7.21-7.12(\mathrm{~m}, 2 \mathrm{H}), 7.12-$ $7.04(\mathrm{~m}, 3 \mathrm{H}), 7.01$ (t, J= 7.5 Hz, 1H), 6.88 (dd, $J=7.6,1.5 \mathrm{~Hz}, 2 \mathrm{H}), 6.56(\mathrm{~d}, J=7.7 \mathrm{~Hz}, 1 \mathrm{H}), 3.62$ (s, 2H), $3.30(\mathrm{~d}, J=12.9 \mathrm{~Hz}, 1 \mathrm{H}), 3.25$ (d, $J=12.9 \mathrm{~Hz}, 1 \mathrm{H}), 3.01(\mathrm{~s}, 3 \mathrm{H}) ;{ }^{13} \mathrm{C} N M R(101 \mathrm{MHz}$, $\left.\mathrm{CDCl}_{3}\right): \delta 177.5,164.6,163.1,144.4,143.6,139.8,134.7,130.1,129.0,128.9,128.7,128.2$, 127.7, 127.6, 127.1, 126.9, 124.1, 122.4, 108.1, 52.5, 43.7, 32.1, 26.1; IR: $v\left(\mathrm{~cm}^{-1}\right)$ 2971, 2912, 1710, 1612, 1469, 1377, 1090, 908, 848; HRMS: (ESI) calcd for $\mathrm{C}_{31} \mathrm{H}_{25} \mathrm{~N}_{3} \mathrm{O}_{2} \mathrm{Na}^{+}[\mathrm{M}+\mathrm{Na}]^{+}$ 494.1844; found 494.1844.

SFC: IB column, $10 \mathrm{ppm} \mathrm{MeOH}$ in supercritical $\mathrm{CO}_{2}$ as eluent, $4 \mathrm{~mL} / \mathrm{min} . \mathrm{tR}=16 \mathrm{~min}$ (minor), $18 \min$ (major);

$[\alpha]_{D}^{28}=33(c=1.2, \mathrm{MeOH})$ for $85 \%$ ee.

\begin{tabular}{|l|l|l|l|l|l|l|l|l|l|}
\hline Instrument Method & Inj. Vol. & Solvent & Column & Sample & Well Location & Temp. & Flow & $\%$ Modifier & Pressure \\
\hline 10p methanol & 10 & MeOH & IB & $\begin{array}{l}\text { Unknown } \\
\text { Sample }\end{array}$ & $16 \mathrm{~A}$ & 30 & 4 & 10 & 150 \\
\hline
\end{tabular}




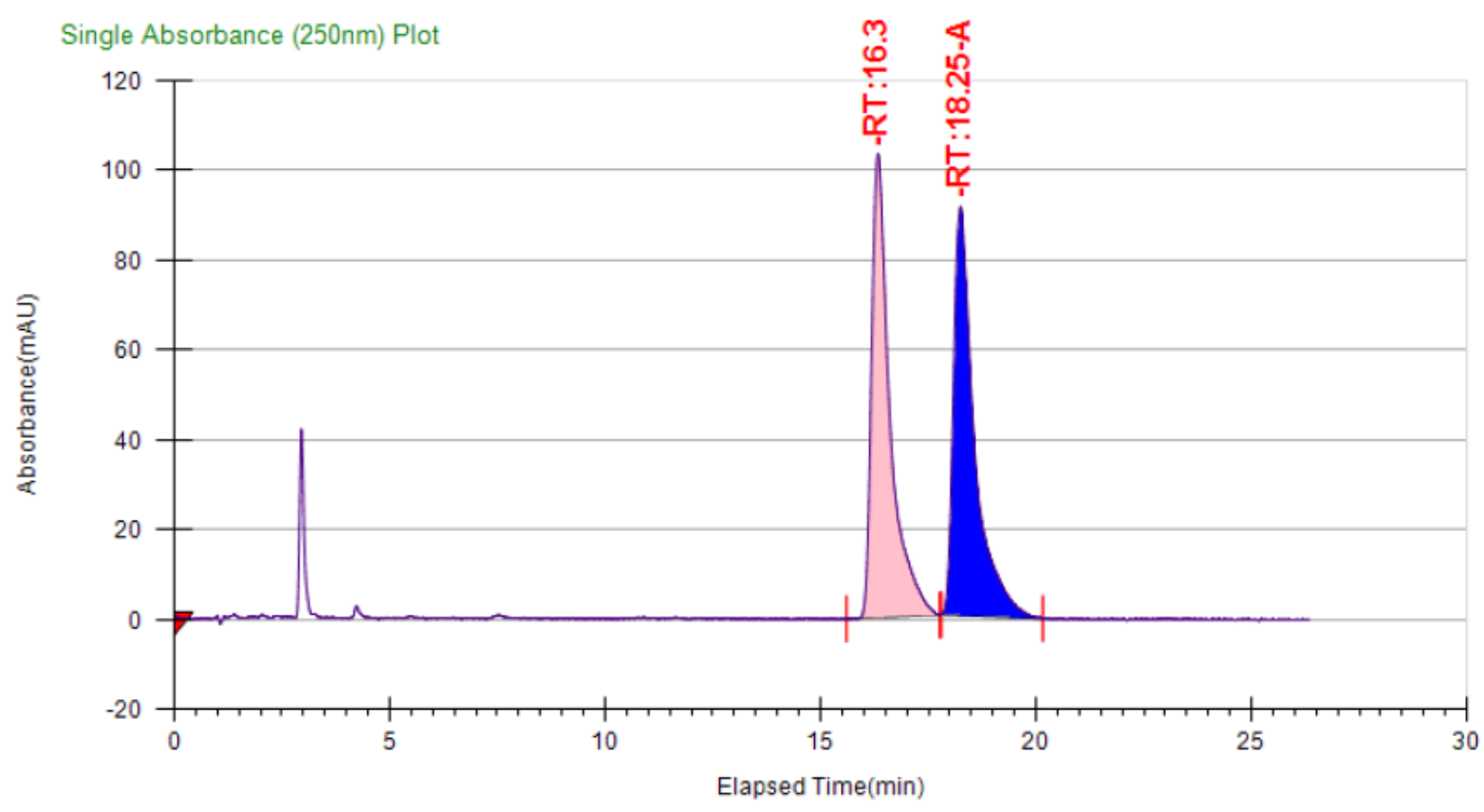

\begin{tabular}{|l|l|l|l|l|l|l|l|l|l|}
\hline Instrument Method & Inj. Vol. & Solvent & Column & Sample & Well Location & Temp. & Flow & $\%$ Modifier & Pressure \\
\hline 10p methanol & 10 & MeOH & IB & kwq-c-38 & $16 \mathrm{~B}$ & 30.5 & 4 & 10 & 150 \\
\hline \hline Peak No & $\%$ Area & Area & Ret. Time & Height & Cap. Factor \\
\hline 1 & 7.1393 & 223.3124 & 16.7 min & 8.7276 & 0 \\
\hline 2 & 92.8607 & 2904.63 & 18.45 min & 80.4956 & 0 \\
\hline
\end{tabular}

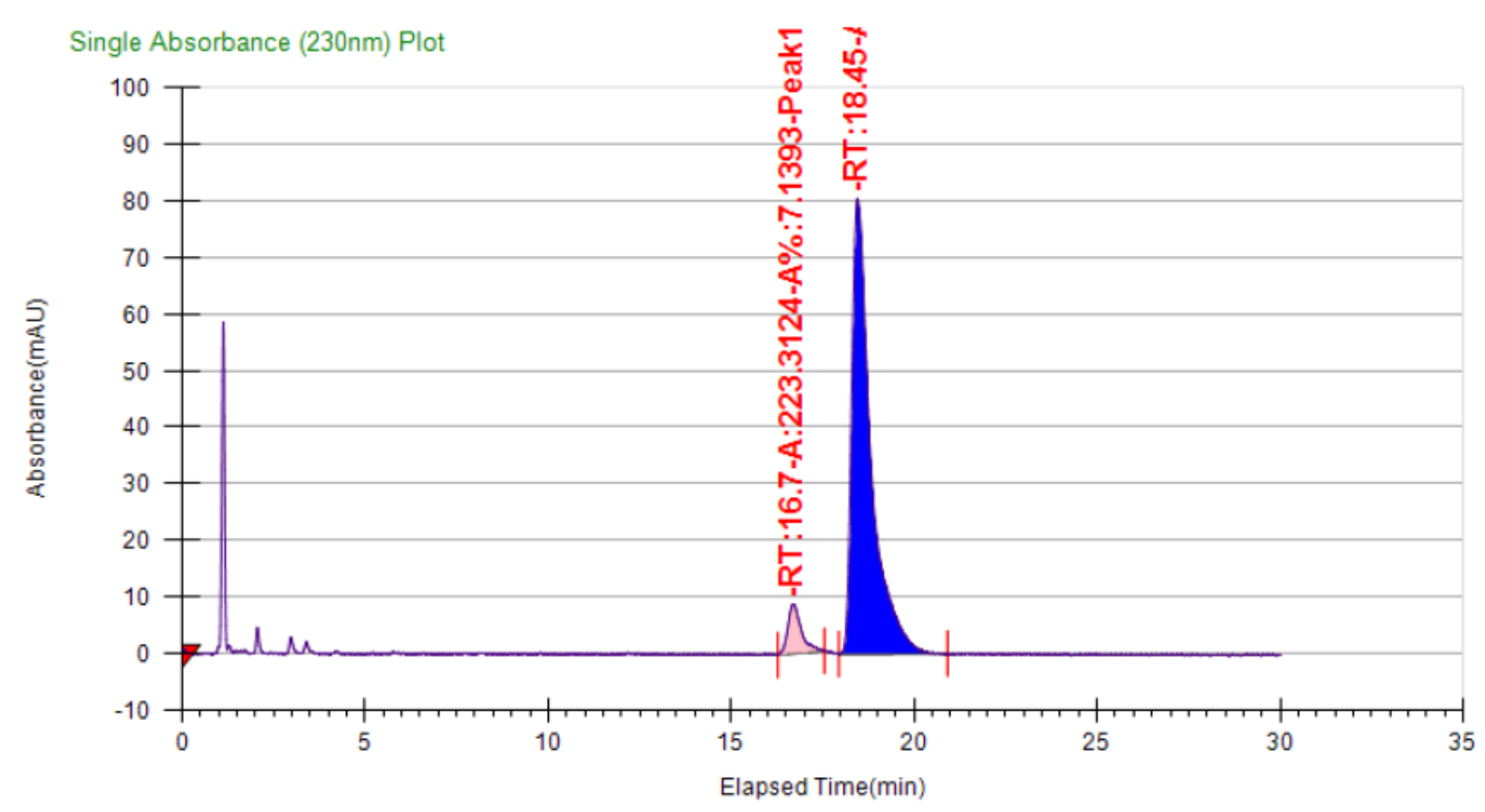


3-((5-(Biphenyl-4-yl)-1,3,4-oxadiazol-2-yl)methyl)-3-hexyl-1-methylindolin-2-one (3hd)

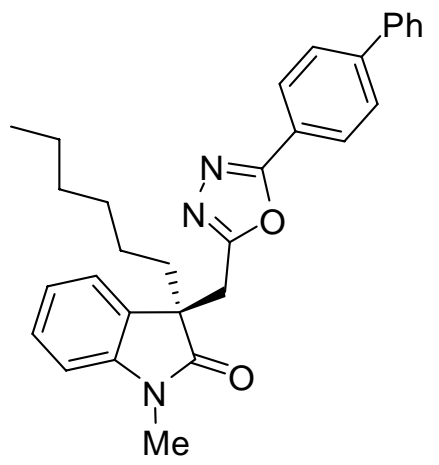

Molecular formula: $\mathrm{C}_{30} \mathrm{H}_{31} \mathrm{~N}_{3} \mathrm{O}_{2}$; Molecular Weight: 465.5860

According to general procedure, 3 hd was obtained in $75 \%$ yield $(34.8 \mathrm{mg}$ ) and $90 \%$ ee as a colorless oil. ${ }^{1} \mathrm{H}$ NMR $\left(400 \mathrm{MHz}, \mathrm{CDCl}_{3}\right): \delta 7.95(\mathrm{~d}, J=8.3 \mathrm{~Hz}, 2 \mathrm{H}), 7.70(\mathrm{~d}, J=8.3 \mathrm{~Hz}, 2 \mathrm{H}), 7.63$ (d, J = 7.3 Hz, 2H), $7.49(\mathrm{t}, J=7.4 \mathrm{~Hz}, 2 \mathrm{H}), 7.41(\mathrm{t}, J=7.3 \mathrm{~Hz}, 1 \mathrm{H}), 7.26(\mathrm{t}, J=8.1 \mathrm{~Hz}, 1 \mathrm{H}), 7.21$ (d, $J=7.2 \mathrm{~Hz}, 1 \mathrm{H}), 7.06(\mathrm{t}, J=7.4 \mathrm{~Hz}, 1 \mathrm{H}), 6.81(\mathrm{~d}, J=7.7 \mathrm{~Hz}, 1 \mathrm{H}), 3.51(\mathrm{~d}, J=15.2 \mathrm{~Hz}, 1 \mathrm{H})$, $3.45(\mathrm{~d}, J=15.2 \mathrm{~Hz}, 1 \mathrm{H}), 3.27(\mathrm{~s}, 3 \mathrm{H}), 2.11(\mathrm{td}, J=12.7,4.5 \mathrm{~Hz}, 1 \mathrm{H}), 1.96(\mathrm{td}, J=12.7,4.2 \mathrm{~Hz}$, $1 \mathrm{H}), 1.39-1.01(\mathrm{~m}, 8 \mathrm{H}), 0.84(\mathrm{t}, J=6.8 \mathrm{~Hz}, 3 \mathrm{H}) ;{ }^{13} \mathrm{C} \mathrm{NMR}\left(101 \mathrm{MHz}, \mathrm{CDCl}_{3}\right): \delta 178.3,164.5$, 163.2 , 144.4, 143.8, 139.8, 130.0, 129.0, 128.6, 128.2, 127.6, 127.1, 123.3, 122.8, 122.5, 108.1, 51.2, 37.6, 33.1, 31.5, 29.2, 26.4, 23.9, 22.5, 14.0; IR: $v\left(\mathrm{~cm}^{-1}\right)$ 2953, 2928, 2856, 1710, $1613,1084,1377,1350,1255,1087,908,848$; HRMS: (ESI) calcd for $\mathrm{C}_{30} \mathrm{H}_{31} \mathrm{~N}_{3} \mathrm{O}_{2} \mathrm{Na}^{+}[\mathrm{M}+\mathrm{Na}]^{+}$ 488.2314; found 488.2314 .

SFC: $\mathrm{AD}-\mathrm{H}$ column, $10 \mathrm{ppm} \mathrm{MeOH}$ in supercritical $\mathrm{CO}_{2}$ as eluent, $4 \mathrm{~mL} / \mathrm{min}$. $\mathrm{tR}=14 \mathrm{~min}$ (major), $23 \mathrm{~min}$ (minor);

$[\alpha]_{D}^{29}=70(c=1.4, \mathrm{MeOH})$ for $90 \%$ ee.

\begin{tabular}{|c|c|c|c|c|c|c|c|c|c|}
\hline Instrument Method & Inj. Vol & I. Solvent & \multirow{2}{*}{\begin{tabular}{|l} 
Column \\
AD-H
\end{tabular}} & \multirow{2}{*}{$\begin{array}{l}\text { Sample } \\
\text { KWQ-C-11 }\end{array}$} & Well Location & Temp. & \multirow{2}{*}{\begin{tabular}{|l} 
Flow \\
4
\end{tabular}} & \multirow{2}{*}{\begin{tabular}{|l} 
\% Modifier \\
10
\end{tabular}} & \multirow{2}{*}{$\begin{array}{l}\text { Pressure } \\
150\end{array}$} \\
\hline 10p methanol & 10 & $\mathrm{MeOH}$ & & & $16 \mathrm{~A}$ & 29.6 & & & \\
\hline & Peak No & $\%$ Area & Area & \multicolumn{3}{|l|}{ Ret. Time } & \multicolumn{2}{|c|}{ Cap. Factor } & \\
\hline & 1 & 49.5424 & $\begin{array}{l}20965.505 \\
5\end{array}$ & $14.36 \mathrm{~min}$ & \multicolumn{2}{|c|}{267.5597} & \multicolumn{2}{|c|}{14357.1167} & \\
\hline & 2 & 50.4576 & $\begin{array}{l}21352.834 \\
5\end{array}$ & $23.82 \mathrm{~min}$ & \multicolumn{2}{|c|}{161.8212} & \multicolumn{2}{|c|}{23823.65} & \\
\hline
\end{tabular}



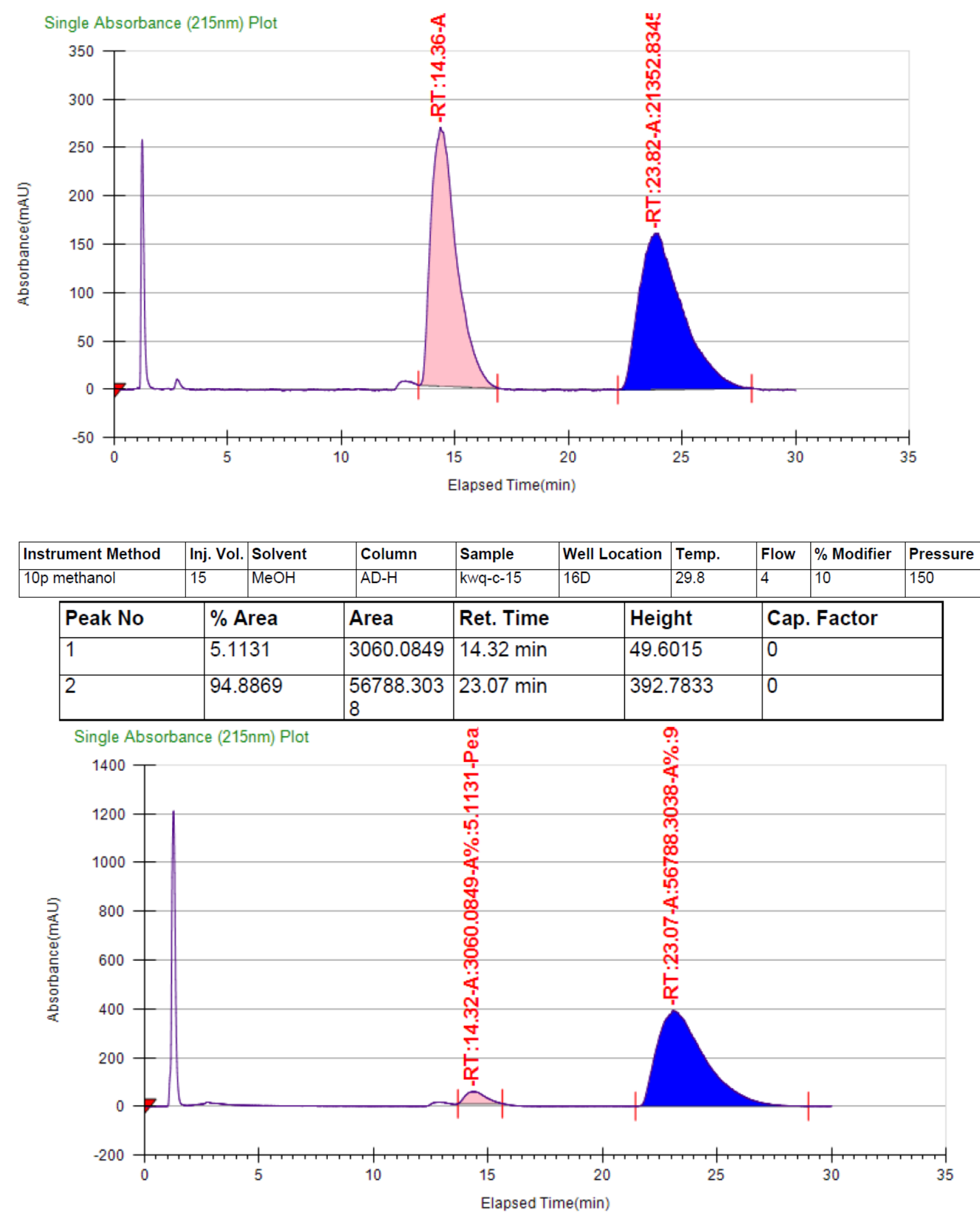
3-((5-(Biphenyl-4-yl)-1,3,4-oxadiazol-2-yl)methyl)-3-isopropyl-1-methylindolin-2-one (3jd)

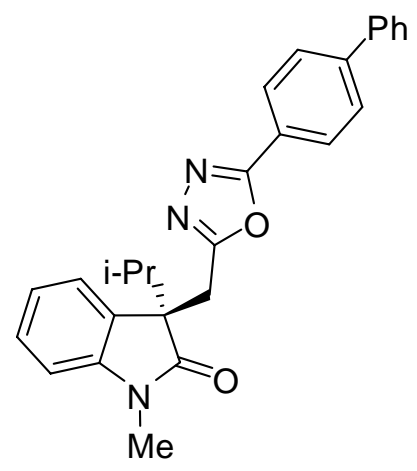

Molecular formula: $\mathrm{C}_{27} \mathrm{H}_{25} \mathrm{~N}_{3} \mathrm{O}_{2}$; Molecular Weight: 423.5063

According to general procedure, 3jd was obtained in $66 \%$ yield $(27.9 \mathrm{mg}$ ) and $90 \%$ ee as a colorless oil. ${ }^{1} \mathrm{H}$ NMR $\left(400 \mathrm{MHz}, \mathrm{CDCl}_{3}\right): \delta 7.90(\mathrm{~d}, J=8.3 \mathrm{~Hz}, 2 \mathrm{H}), 7.68(\mathrm{~d}, J=8.3 \mathrm{~Hz}, 2 \mathrm{H}), 7.63$ (d, J = 7.4 Hz, 2H), $7.49(\mathrm{t}, J=7.5 \mathrm{~Hz}, 2 \mathrm{H}), 7.41(\mathrm{t}, J=7.2 \mathrm{~Hz}, 1 \mathrm{H}), 7.28-7.23(\mathrm{~m}, 2 \mathrm{H}), 7.05(\mathrm{t}, J$ $=7.5 \mathrm{~Hz}, 1 \mathrm{H}), 6.78(\mathrm{~d}, J=7.7 \mathrm{~Hz}, 1 \mathrm{H}), 3.66(\mathrm{~d}, J=15.2 \mathrm{~Hz}, 1 \mathrm{H}), 3.54(\mathrm{~d}, J=15.2 \mathrm{~Hz}, 1 \mathrm{H}), 3.26$ (s, $3 \mathrm{H}), 2.48-2.25(\mathrm{~m}, 1 \mathrm{H}), 1.07(\mathrm{~d}, J=6.9 \mathrm{~Hz}, 3 \mathrm{H}), 0.90(\mathrm{~d}, J=6.7 \mathrm{~Hz}, 3 \mathrm{H}) ;{ }^{13} \mathrm{C} \mathrm{NMR}(101 \mathrm{MHz}$, $\left.\mathrm{CDCl}_{3}\right): \delta 178.2,164.4,163.4,144.3,144.1,139.8,129.0,128.6,128.2,127.6,127.1,127.1$, 124.0, 122.5, 108.0, 54.4, 35.3, 30.9, 26.2, 17.3, 17.1; IR: $v\left(\mathrm{~cm}^{-1}\right)$ 2967, 2883, 1709, 1611, 1493, 1469, 1376, 1258, 1079, 909, 848; HRMS: (ESI) calcd for $\mathrm{C}_{27} \mathrm{H}_{25} \mathrm{~N}_{3} \mathrm{O}_{2} \mathrm{H}^{+}[\mathrm{M}+\mathrm{H}]^{+}$ 424.2025; found 424.2025 .

SFC: $A D-H$ column, $10 \mathrm{ppm} \mathrm{MeOH}$ in supercritical $\mathrm{CO}_{2}$ as eluent, $4 \mathrm{~mL} / \mathrm{min}$. $\mathrm{tR}=18 \mathrm{~min}$ (major), $26 \mathrm{~min}$ (minor);

$[\alpha]_{D}^{28}=84(c=1.4, \mathrm{MeOH})$ for $90 \%$ ee.

\begin{tabular}{|l|l|l|l|l|l|l|l|l|l|}
\hline Instrument Method & Inj. Vol. & Solvent & Column & Sample & Well Location & Temp. & Flow & $\%$ Modifier & Pressure \\
\hline 10p methanol & 15 & MeOH & AD-H & kwq-c-56 & 16 B & 29.6 & 4 & 10 & 150 \\
\hline Peak No & $\%$ Area & Area & Ret. Time & Height & Cap. Factor \\
\hline 1 & 49.8886 & 8208.6828 & 18.23 min & 81.1558 & 0 \\
\hline 2 & 50.1114 & 8245.3387 & 26.39 min & 60.2036 & 0 & \\
\hline
\end{tabular}




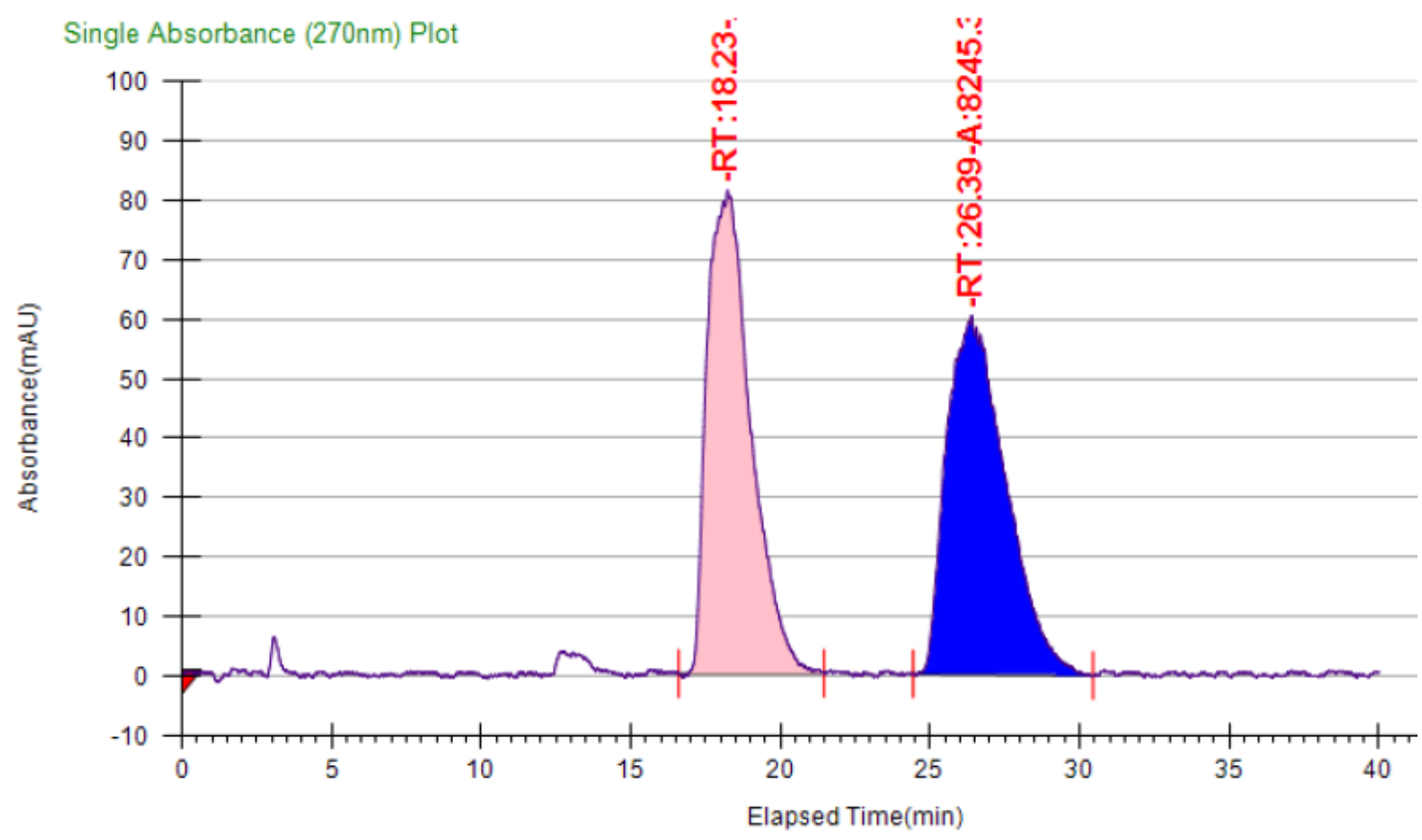

\begin{tabular}{|l|l|l|l|l|l|l|l|l|l|}
\hline Instrument Method & Inj. Vol. & Solvent & Column & Sample & Well Location & Temp. & Flow & \% Modifier & Pressure \\
\hline 10p methanol & 15 & $\mathrm{MeOH}$ & $\mathrm{AD}-\mathrm{H}$ & $\mathrm{kwq}-\mathrm{c}-55$ & $16 \mathrm{C}$ & 30.1 & 4 & 10 & 150 \\
\hline \hline Peak No & $\%$ Area & Area & Ret. Time & Height & Cap. Factor \\
\hline 1 & 94.8828 & $\begin{array}{l}17453.722 \\
7\end{array}$ & $17.82 \mathrm{~min}$ & 167.5488 & 0 \\
\hline 2 & 5.1172 & 941.303 & $26.55 \mathrm{~min}$ & 8.2283 & 0 & \\
\hline
\end{tabular}

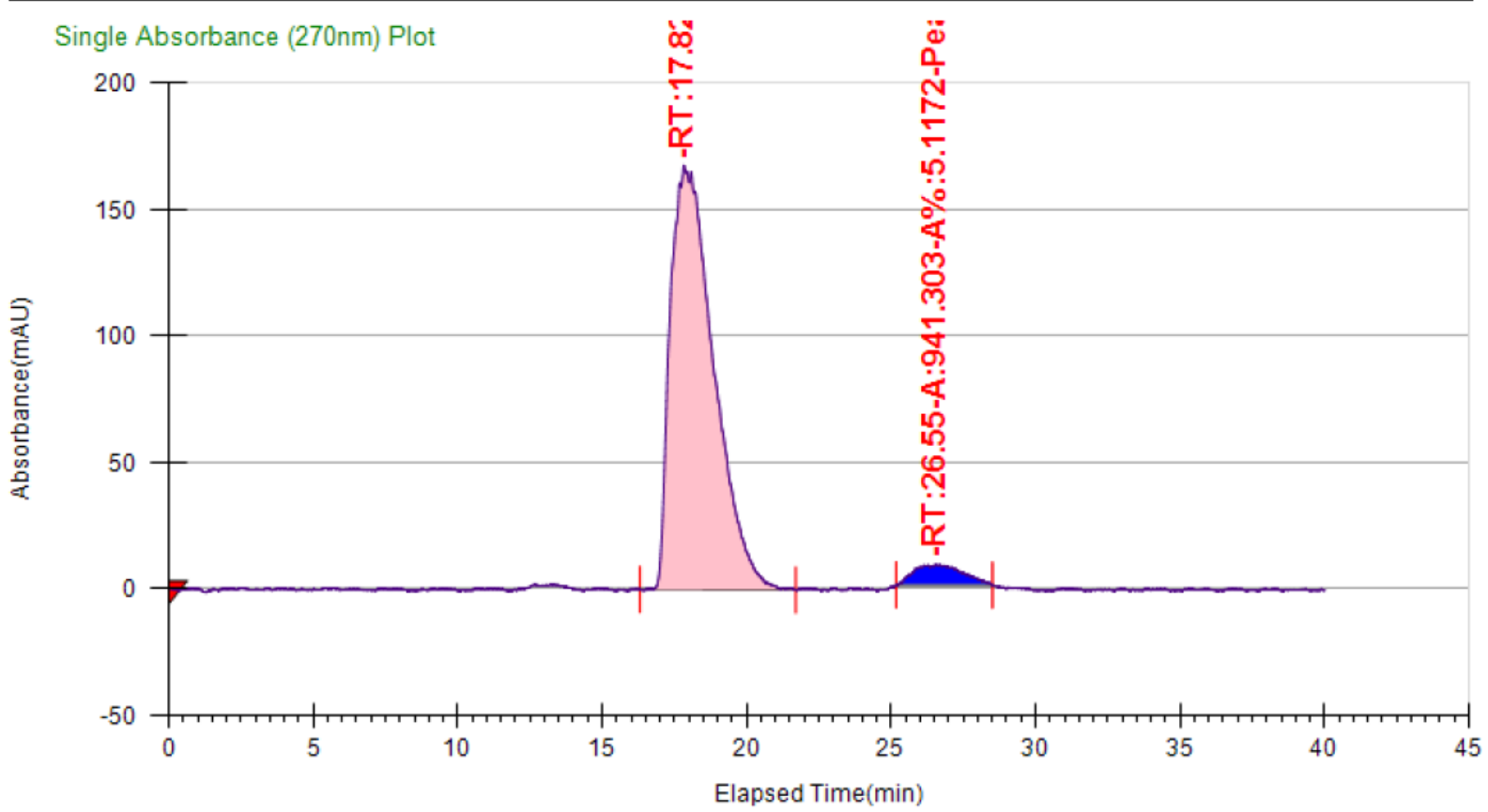


3-(Methoxymethyl)-1-methyl-3-((5-phenyl-1,3,4-oxadiazol-2-yl)methyl)indolin-2-one (3ia)

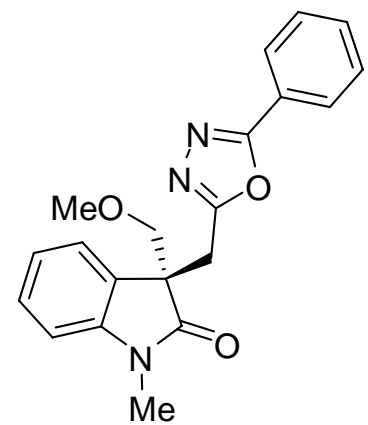

Molecular formula: $\mathrm{C}_{20} \mathrm{H}_{19} \mathrm{~N}_{3} \mathrm{O}_{3}$; Molecular Weight: 349.3832

According to general procedure, 3ia was obtained in $64 \%$ yield $(22.3 \mathrm{mg})$ and $87 \%$ ee as a colorless oil. ${ }^{1} \mathrm{H}$ NMR $\left(400 \mathrm{MHz}, \mathrm{CDCl}_{3}\right): \delta 7.88(\mathrm{~d}, J=6.8 \mathrm{~Hz}, 2 \mathrm{H}), 7.58-7.40(\mathrm{~m}, 3 \mathrm{H}), 7.35(\mathrm{~d}, J$ $=7.0 \mathrm{~Hz}, 1 \mathrm{H}), 7.26(\mathrm{t}, J=7.6 \mathrm{~Hz}, 1 \mathrm{H}), 7.04(\mathrm{t}, J=7.2 \mathrm{~Hz}, 1 \mathrm{H}), 6.81(\mathrm{~d}, J=7.5 \mathrm{~Hz}, 1 \mathrm{H}), 3.86(\mathrm{~d}, J$ $=8.7 \mathrm{~Hz}, 1 \mathrm{H}), 3.75-3.60(\mathrm{~m}, 2 \mathrm{H}), 3.46(\mathrm{~d}, J=15.4 \mathrm{~Hz}, 1 \mathrm{H}), 3.34(\mathrm{~s}, 3 \mathrm{H}), 3.26(\mathrm{~s}, 3 \mathrm{H}) ;{ }^{13} \mathrm{C} \mathrm{NMR}$ $\left(101 \mathrm{MHz}, \mathrm{CDCl}_{3}\right): \delta 176.3,164.7,163.0,143.8,131.6,129.0,128.9,126.7,124.3,123.7$, 122.7, 108.2, 75.8, 59.7, 51.8, 29.3, 26.5; IR: $v\left(\mathrm{~cm}^{-1}\right)$ 2930, 2891, 2830, 1711, 1613, 1552, 1493, 1470, 1376, 1351, 1255, 1120, 1093, 908; HRMS: (ESI) calcd for $\mathrm{C}_{20} \mathrm{H}_{19} \mathrm{~N}_{3} \mathrm{O}_{3} \mathrm{Na}^{+}$ $[\mathrm{M}+\mathrm{Na}]^{+}$372.1324; found 372.1324 .

SFC: AD-H column, $8 \mathrm{ppm} \mathrm{MeOH}$ in supercritical $\mathrm{CO}_{2}$ as eluent, $2 \mathrm{~mL} / \mathrm{min}$. $\mathrm{tR}=14 \mathrm{~min}$ (major), $18 \mathrm{~min}$ (minor);

$[\alpha]_{D}^{29}=24(c=1.7, \mathrm{MeOH})$ for $87 \%$ ee.

\begin{tabular}{|c|c|c|c|c|c|c|c|c|c|}
\hline \multirow{2}{*}{\begin{tabular}{|l|} 
Instrument Method \\
$8 \mathrm{p}$ methanol $2 \mathrm{ml}$ \\
\end{tabular}} & Inj. Vol. & \multirow{2}{*}{\begin{tabular}{|l|} 
Solvent \\
$\mathrm{MeOH}$
\end{tabular}} & \multirow{2}{*}{\begin{tabular}{|l|} 
Column \\
AD-H \\
\end{tabular}} & \multirow{2}{*}{\begin{tabular}{|l|} 
Sample \\
kwq-b-63
\end{tabular}} & Well Location & \multirow{2}{*}{\begin{tabular}{|l} 
Temp. \\
29.5
\end{tabular}} & \multirow{2}{*}{\begin{tabular}{|l|} 
Flow \\
2
\end{tabular}} & \multirow{2}{*}{\begin{tabular}{|l} 
\% Modifier \\
8
\end{tabular}} & \multirow{2}{*}{\begin{tabular}{|l} 
Pressure \\
150
\end{tabular}} \\
\hline & 10 & & & & $16 \mathrm{~B}$ & & & & \\
\hline Peak No & \multicolumn{2}{|c|}{$\%$ Area } & Area & \multicolumn{2}{|l|}{ Ret. Time } & Height & \multicolumn{3}{|c|}{ Cap. Factor } \\
\hline 1 & \multicolumn{2}{|c|}{50.5755} & $\begin{array}{l}35160.546 \\
1\end{array}$ & \multicolumn{2}{|l|}{$14.44 \mathrm{~min}$} & 376.0492 & \multicolumn{3}{|c|}{14440.45} \\
\hline 2 & \multicolumn{2}{|c|}{49.4245} & 34360.347 & \multicolumn{2}{|l|}{$18.01 \mathrm{~min}$} & 320.6708 & \multicolumn{3}{|c|}{18007.0667} \\
\hline
\end{tabular}




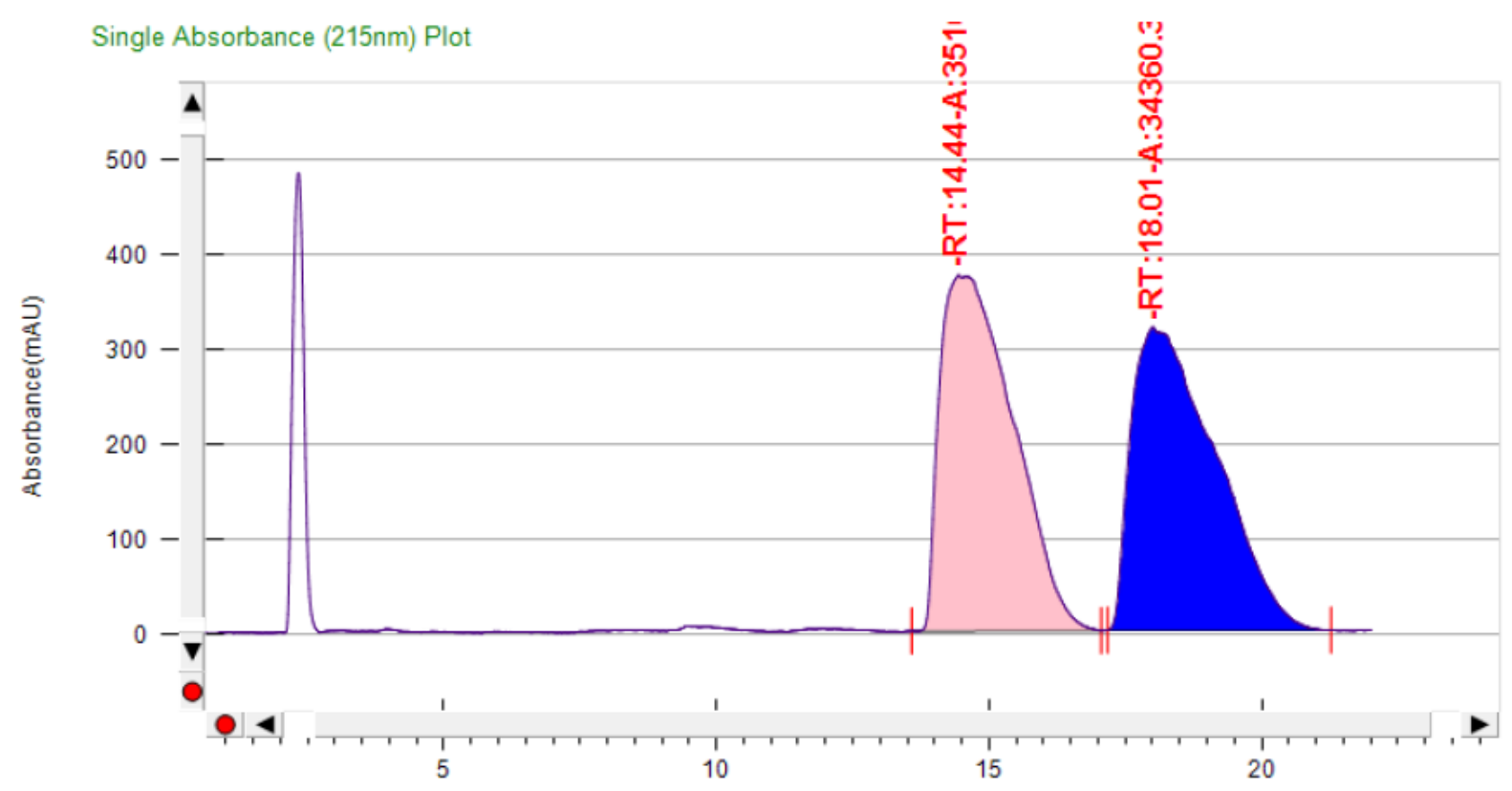

\begin{tabular}{|l|l|l|l|l|l|l|l|l|l|}
\hline Instrument Method & Inj. Vol. & Solvent & Column & Sample & Well Location & Temp. & Flow & $\%$ Modifier & Pressure \\
\hline $8 \mathrm{p}$ methanol 2 ML-MIN & 15 & $\mathrm{MeOH}$ & $\mathrm{AD}-\mathrm{H}$ & $\mathrm{kwq}-\mathrm{c}-17$ & $16 \mathrm{~B}$ & 30.2 & 2 & 8 & 150 \\
\hline \hline Peak No & $\%$ Area & Area & Ret. Time & Height & \multicolumn{1}{l|}{ Cap. Factor } \\
\hline 1 & 93.6178 & $\begin{array}{l}13900.126 \\
9\end{array}$ & $14.72 \mathrm{~min}$ & 213.1569 & 0 \\
\hline 2 & 6.3822 & 947.6168 & $18.62 \mathrm{~min}$ & 14.7562 & 0 \\
\hline
\end{tabular}

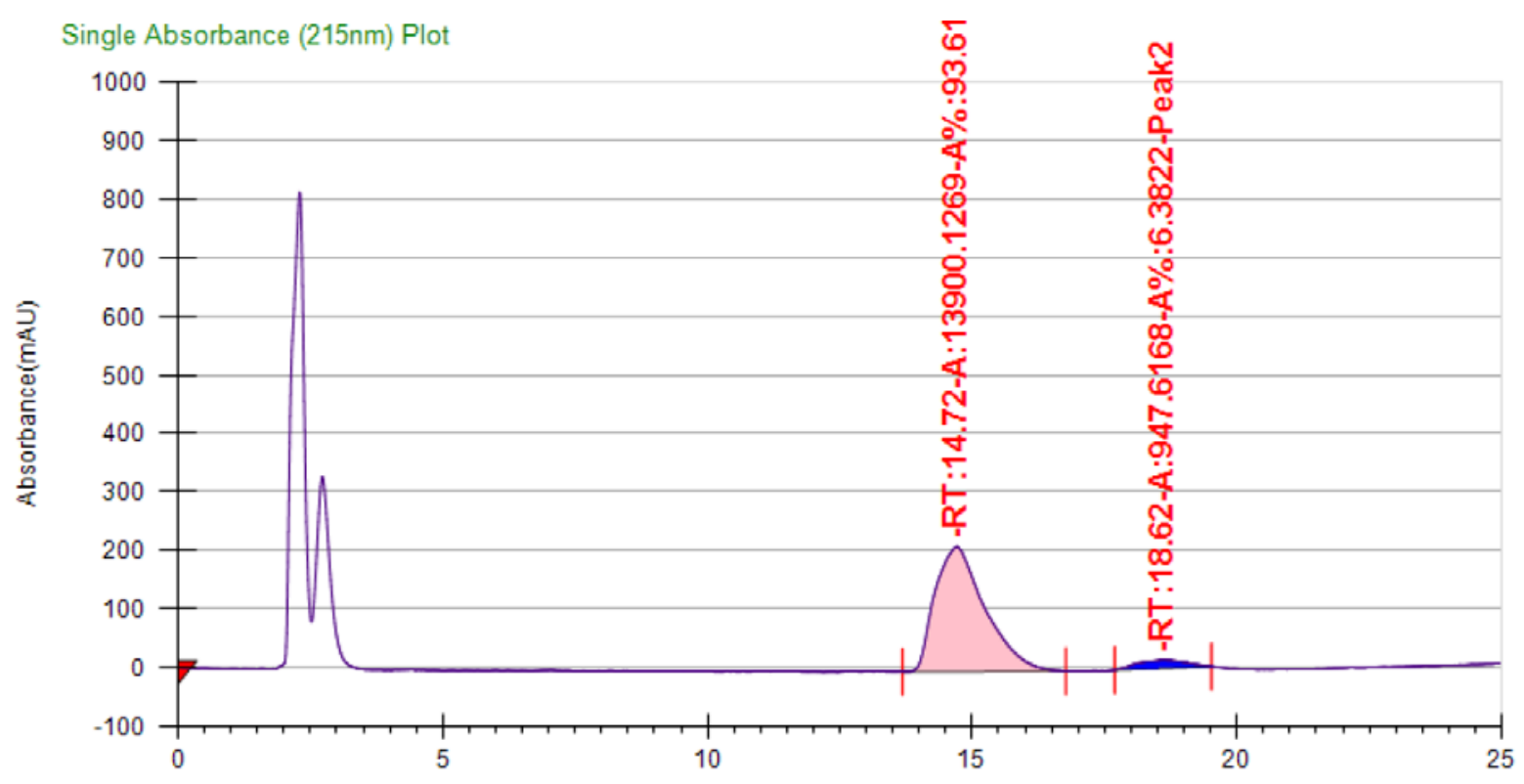


1-Methyl-3-phenyl-3-((5-phenyl-1,3,4-oxadiazol-2-yl)methyl)indolin-2-one (3ka)

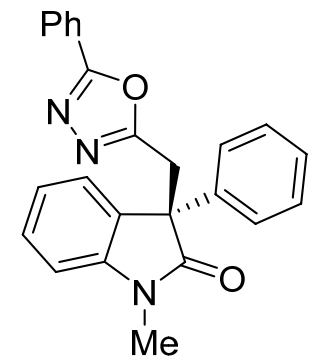

Molecular formula: $\mathrm{C}_{24} \mathrm{H}_{19} \mathrm{~N}_{3} \mathrm{O}_{2}$; Molecular Weight: 381.4266

According to general procedure, 3ka was obtained in $61 \%$ yield $(23.2 \mathrm{mg}$ ) and $94 \%$ ee as a colorless oil. ${ }^{1} \mathrm{H}$ NMR $\left(400 \mathrm{MHz}, \mathrm{CDCl}_{3}\right): \delta 7.82(\mathrm{~d}, J=7.0 \mathrm{~Hz}, 2 \mathrm{H}), 7.60-7.23(\mathrm{~m}, 10 \mathrm{H}), 7.12$ (t, $J=7.2 \mathrm{~Hz}, 1 \mathrm{H}), 6.86(\mathrm{~d}, J=7.7 \mathrm{~Hz}, 1 \mathrm{H}), 4.00(\mathrm{~d}, J=15.2 \mathrm{~Hz}, 1 \mathrm{H}), 3.95(\mathrm{~d}, J=15.2 \mathrm{~Hz}, 1 \mathrm{H}), 3.26$ (s, 3H); ${ }^{13} \mathrm{C}$ NMR $\left(101 \mathrm{MHz}, \mathrm{CDCl}_{3}\right): \delta 177.0,164.6,162.9,143.9,138.3,131.6,129.6,129.1$, 129.0, 128.8, 128.0, 126.9, 126.7, 125.5, 123.6, 122.9, 108.6, 54.5, 33.7, 26.7; IR: $v\left(\mathrm{~cm}^{-1}\right)$ 3057, 2970, 2926, 1713, 1612, 1552, 1485, 1449, 1373, 1349, 1257, 1090, 962, 908; HRMS: (ESI) calcd for $\mathrm{C}_{24} \mathrm{H}_{19} \mathrm{~N}_{3} \mathrm{O}_{2} \mathrm{Na}^{+}[\mathrm{M}+\mathrm{Na}]^{+} 404.1375$; found 404.1375 .

SFC: AD-H column, $15 \mathrm{ppm} \mathrm{MeOH}$ in supercritical $\mathrm{CO}_{2}$ as eluent, $4 \mathrm{~mL} / \mathrm{min}$. $\mathrm{tR}=8 \mathrm{~min}$ (major), 15 min (minor);

$[\alpha]_{D}^{27}=18\left(\mathrm{c}=0.2, \mathrm{CHCl}_{3}\right)$ for $94 \%$ ee.

\begin{tabular}{|l|l|l|l|l|l|l|l|l|l|}
\hline Instrument Method & Inj. Vol. & Solvent & Column & Sample & Well Location & Temp. & Flow & $\%$ Modifier & Pressure \\
\hline $15 p$ methanol & 10 & MeOH & AD-H & kwq-b-48 & $16 \mathrm{C}$ & 30 & 4 & 15 & 150 \\
\hline \hline Peak No & $\%$ Area & Area & Ret. Time & Height & Cap. Factor \\
\hline 1 & 50.0745 & 10628.657 & 8.2 min & 218.4649 & 0 & \\
\hline 2 & 79.9255 & 10597.014 & 15.32 min & 121.5476 & 0 \\
\hline
\end{tabular}




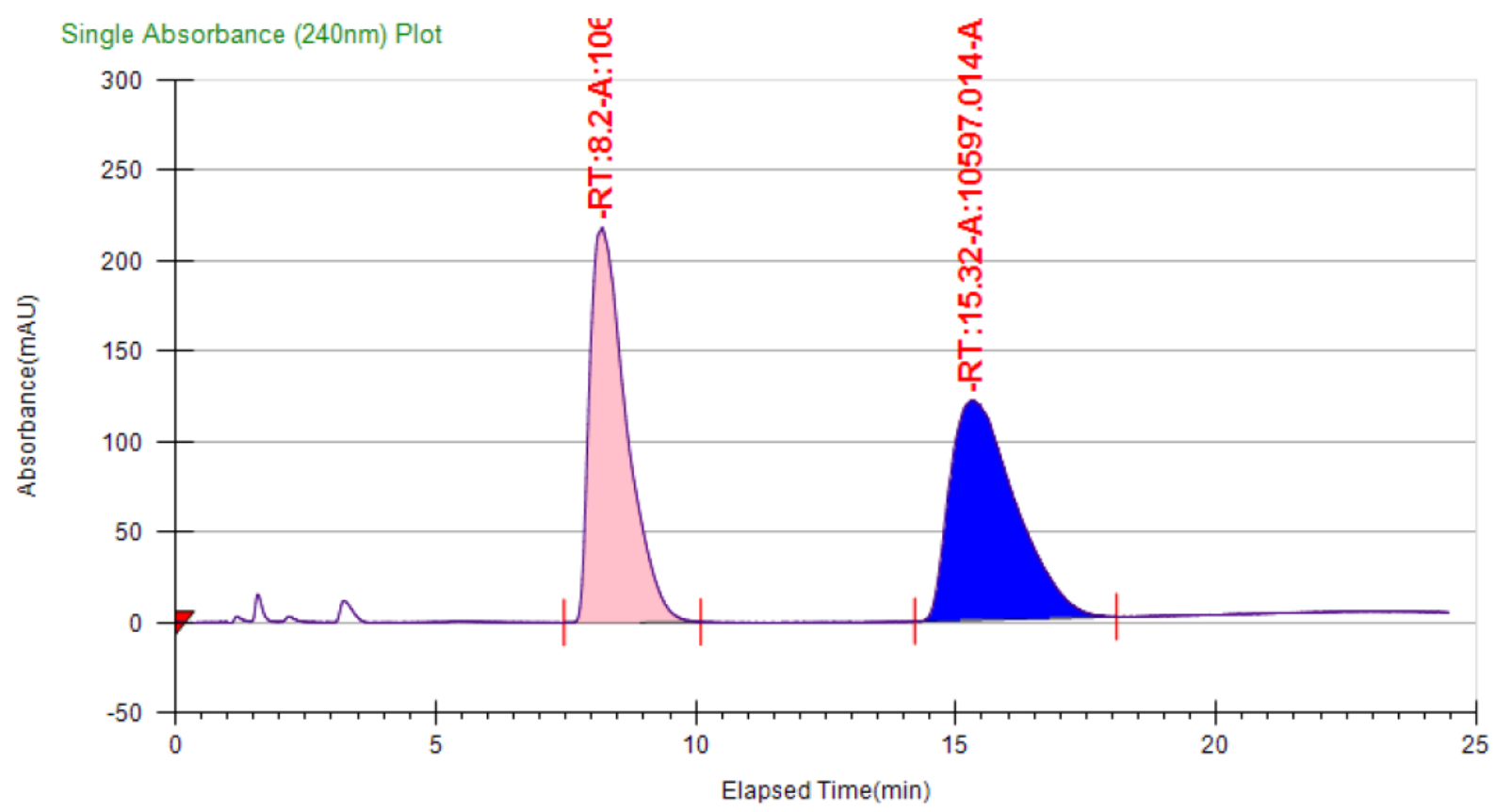

\begin{tabular}{|l|l|l|l|l|l|l|l|l|l|}
\hline Instrument Method & Inj. Vol. & Solvent & Column & Sample & Well Location & Temp. & Flow & $\%$ Modifier & Pressure \\
\hline $15 p$ methanol & 10 & MeOH & AD-H & kwq-b-124 & $16 \mathrm{D}$ & 29.8 & 4 & 15 & 150 \\
\hline \hline Peak No & $\%$ Area & Area & Ret. Time & Height & Cap. Factor \\
\hline 1 & 97.0171 & 6973.5308 & 7.81 min & 163.5388 & 0 \\
\hline 2 & 2.9829 & 214.4085 & 14.73 min & 4.3699 & 0 \\
\hline
\end{tabular}

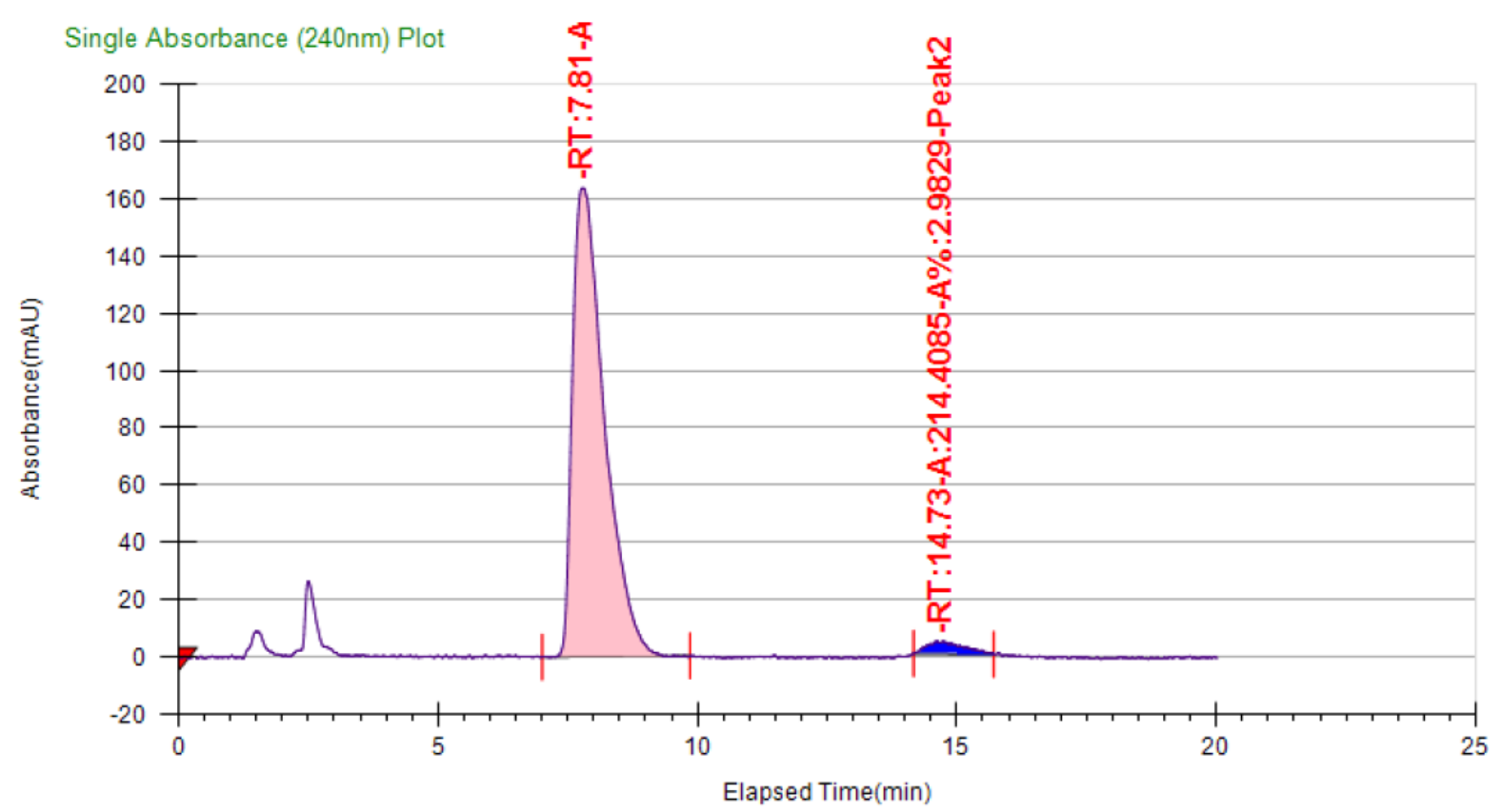


3-(4-Fluorophenyl)-1-methyl-3-((5-phenyl-1,3,4-oxadiazol-2-yl)methyl)indolin-2-one (3la)<smiles>CN1C(=O)[C@](Cc2nnc(-c3ccccc3)o2)(c2ccc(F)cc2)c2ccccc21</smiles>

Molecular formula: $\mathrm{C}_{24} \mathrm{H}_{18} \mathrm{FN}_{3} \mathrm{O}_{2}$; Molecular Weight: 399.4170

According to general procedure, 3la was obtained in $62 \%$ yield $(24.6 \mathrm{mg})$ and $91 \%$ ee as a colorless oil. ${ }^{1} \mathrm{H}$ NMR $\left(400 \mathrm{MHz}, \mathrm{CDCl}_{3}\right): \delta 7.82(\mathrm{~d}, J=7.1 \mathrm{~Hz}, 2 \mathrm{H}), 7.56-7.41(\mathrm{~m}, 5 \mathrm{H}), 7.38$ $7.26(\mathrm{~m}, 2 \mathrm{H}), 7.13(\mathrm{t}, J=7.5 \mathrm{~Hz}, 1 \mathrm{H}), 7.06(\mathrm{t}, J=8.6 \mathrm{~Hz}, 2 \mathrm{H}), 6.88(\mathrm{~d}, J=7.8 \mathrm{~Hz}, 1 \mathrm{H}), 3.92(\mathrm{~s}$, $2 \mathrm{H}), 3.26(\mathrm{~s}, 3 \mathrm{H}) ;{ }^{13} \mathrm{C}$ NMR $\left(101 \mathrm{MHz}, \mathrm{CDCl}_{3}\right): \delta$ 176.8, 164.7, 163.7, 162.7, 161.2, 143.8, 133.9, 131.7, 129.3, 129.3, 129.0, 128.8, 128.8, 126.6, 125.5, 123.5, 123.0, 115.8, 115.5, 108.8, 53.8, 33.9, 26.8; ${ }^{19} \mathrm{~F}$ NMR (376 MHz, $\left.\mathrm{CDCl}_{3}\right): \delta-114.31(\mathrm{~m})$; IR: $v\left(\mathrm{~cm}^{-1}\right)$ 2967, 2966, 2955, 2946, 2945, 2931, 2930, 2929, 2928, 2927, 2926, 2925, 2911, 1716, 1613, 1613, 1557, 1509, 1494, 1494, 1472, 1472, 1457, 1374, 1374, 1351, 1259, 1235, 1235, 1235, 1164, 1090, 1017, 755, 712, 690; HRMS: (ESI) calcd for $\mathrm{C}_{24} \mathrm{H}_{18} \mathrm{FN}_{3} \mathrm{O}_{2} \mathrm{H}^{+}[\mathrm{M}+\mathrm{H}]^{+} 400.1461$; found 400.1461.

SFC: AD-H column, $18 \mathrm{ppm} \mathrm{MeOH}$ in supercritical $\mathrm{CO}_{2}$ as eluent, $4 \mathrm{~mL} / \mathrm{min}$. $\mathrm{tR}=5 \mathrm{~min}$ (major), 7 min (minor);

$[\alpha]_{D}^{25}=146.3(c=2.0, \mathrm{MeOH})$ for $91 \%$ ee.

\begin{tabular}{|l|l|l|l|l|l|l|l|l|l|}
\hline Instrument Method & Inj. Vol. & Solvent & Column & Sample & Well Location & Temp. & Flow & $\%$ Modifier & Pressure \\
\hline $18 \mathrm{p}$ methanol & 15 & MeOH & AD-H & $\begin{array}{l}\text { Unknown } \\
\text { Sample }\end{array}$ & $16 \mathrm{~A}$ & 29.6 & 4 & 18 & 150 \\
\hline Peak No & $\%$ Area & Area & Ret. Time & Height & Cap. Factor \\
\hline 1 & 50.3548 & 3251.6898 & 5.17 min & 113.6581 & 0 & \\
\hline 2 & 49.6452 & 3205.8669 & 7.48 min & 80.9222 & 0 \\
\hline
\end{tabular}




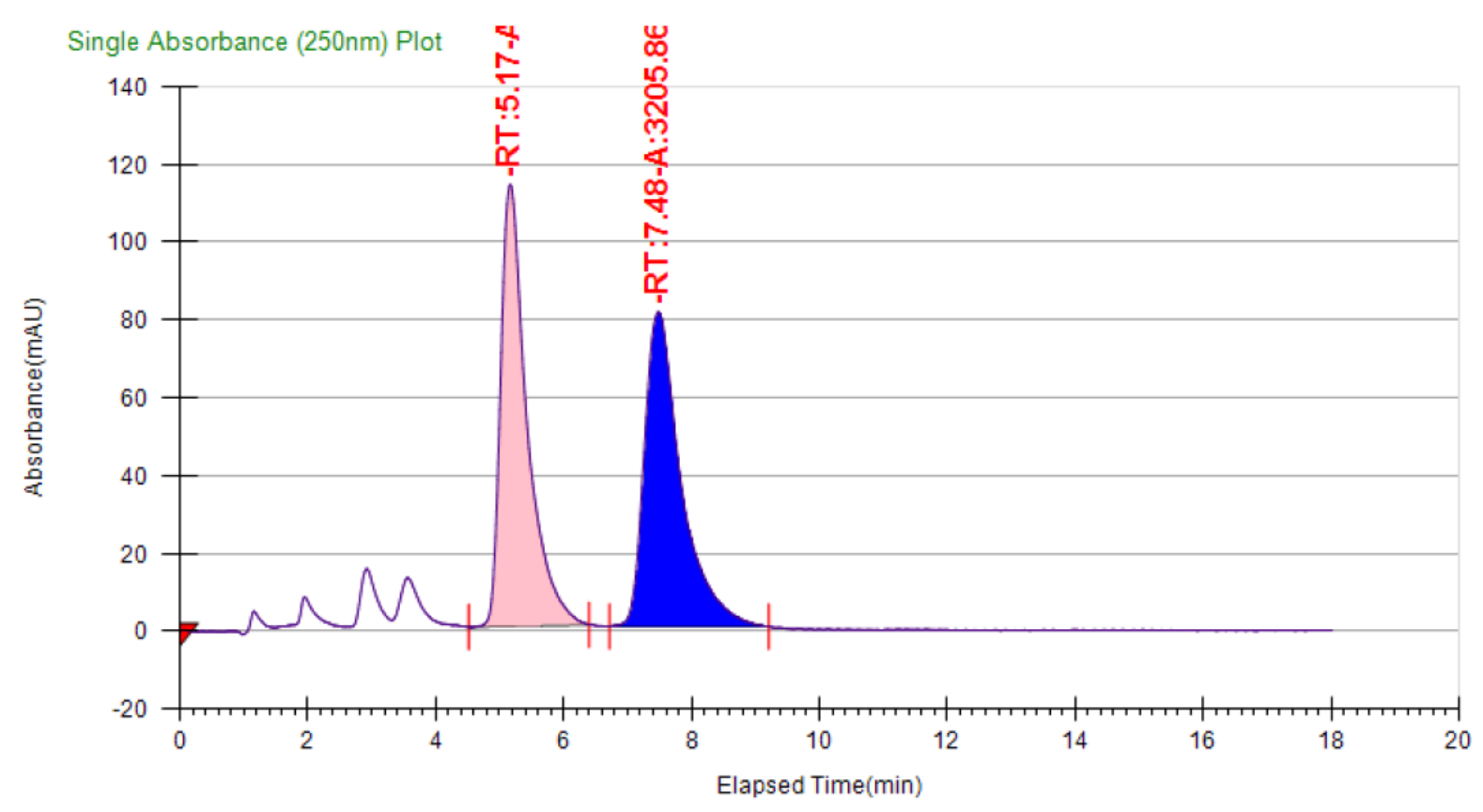

\begin{tabular}{|l|l|l|l|l|l|l|l|l|l|}
\hline Instrument Method & Inj. Vol. & Solvent & Column & Sample & Well Location & Temp. & Flow & $\%$ Modifier & Pressure \\
\hline $18 \mathrm{p} \mathrm{methanol}$ & 10 & $\mathrm{MeOH}$ & $\mathrm{AD}-\mathrm{H}$ & $\begin{array}{l}\text { Unknown } \\
\text { Sample }\end{array}$ & $16 \mathrm{~B}$ & 29.8 & 4 & 18 & 150 \\
\hline Peak No & $\%$ Area & Area & Ret. Time & Height & Cap. Factor \\
\hline 1 & 95.4045 & 1375.558 & $5.19 \mathrm{~min}$ & 44.2809 & 0 \\
\hline 2 & 4.5955 & 66.2584 & $7.5 \mathrm{~min}$ & 2.0601 & 0 \\
\hline
\end{tabular}

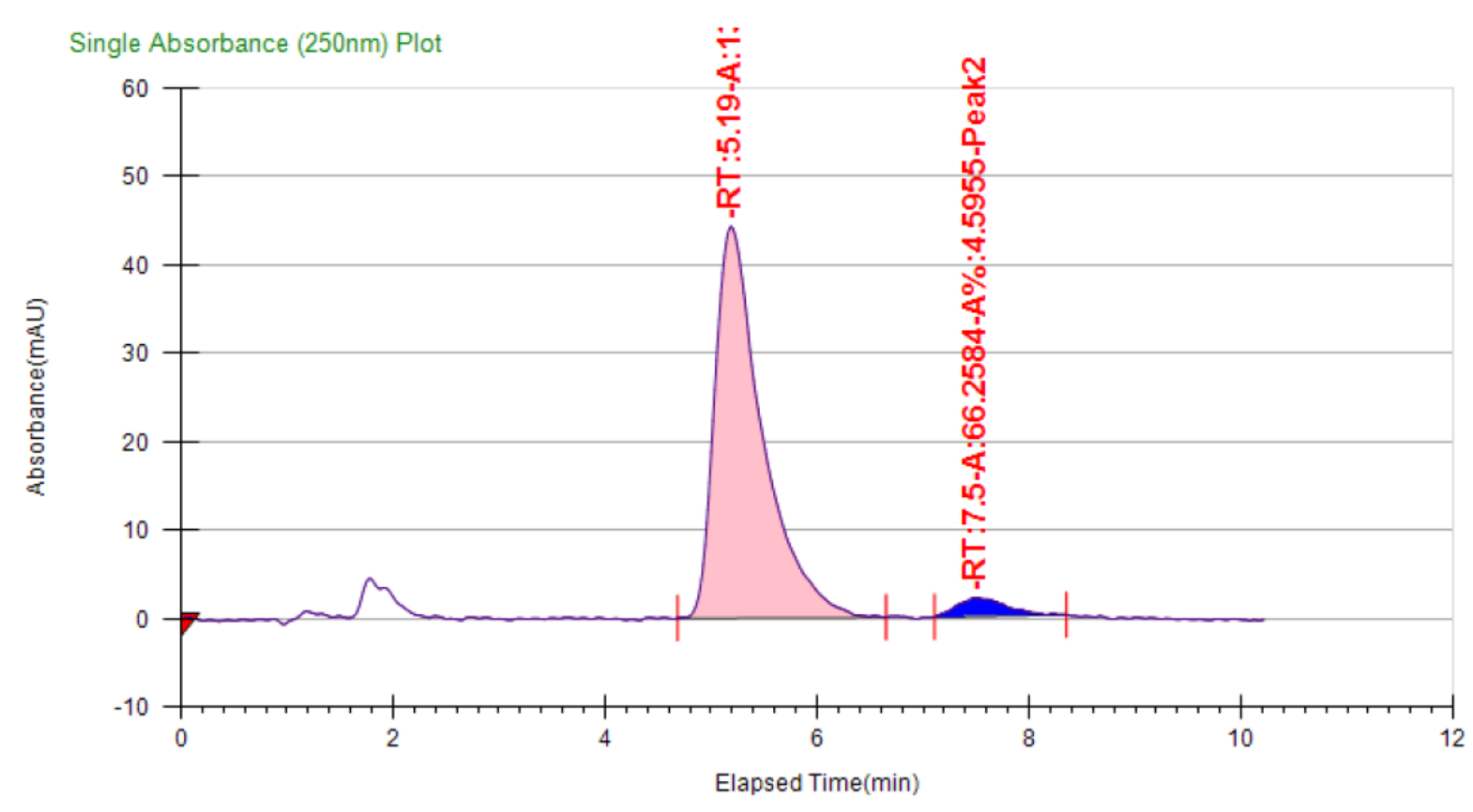


3-(4-Methoxyphenyl)-1-methyl-3-((5-phenyl-1,3,4-oxadiazol-2-yl)methyl)indolin-2-one (3ma)<smiles>COc1ccc(C2(Cc3nnc(-c4ccccc4)o3)C(=O)N(C)c3ccccc32)cc1</smiles>

Molecular formula: $\mathrm{C}_{25} \mathrm{H}_{21} \mathrm{~N}_{3} \mathrm{O}_{3}$; Molecular Weight: 411.4525

According to general procedure, 3ma was obtained in $66 \%$ yield $(27.1 \mathrm{mg}$ ) and $93 \%$ ee as a colorless oil. ${ }^{1} \mathrm{H}$ NMR $\left(400 \mathrm{MHz}, \mathrm{CDCl}_{3}\right): \delta 7.83(\mathrm{~d}, J=7.1 \mathrm{~Hz}, 2 \mathrm{H}), 7.55-7.35(\mathrm{~m}, 5 \mathrm{H}), 7.33-7.27$ (m, 2H), 7.12 (t, J= $7.5 \mathrm{~Hz}, 1 \mathrm{H}), 6.89$ (d, $J=8.8 \mathrm{~Hz}, 2 \mathrm{H}), 6.86(\mathrm{~d}, J=7.9 \mathrm{~Hz}, 1 \mathrm{H}), 3.96(\mathrm{~d}, J=$ $15.2 \mathrm{~Hz}, 1 \mathrm{H}), 3.90(\mathrm{~d}, J=15.1 \mathrm{~Hz}, 1 \mathrm{H}), 3.80(\mathrm{~s}, 3 \mathrm{H}), 3.25(\mathrm{~s}, 3 \mathrm{H}) ;{ }^{13} \mathrm{C} \mathrm{NMR}\left(101 \mathrm{MHz}, \mathrm{CDCl}_{3}\right): \delta$ 177.2, 164.6, 163.0, 159.3, 143.8, 131.6, 130.2, 129.7, 129.1, 129.0, 128.1, 126.7, 125.5, 123.6, 122.9, 114.1, 108.6, 55.3, 53.8, 33.8, 26.7; IR: $v\left(\mathrm{~cm}^{-1}\right)$ 2957, 2931, 2930, 2929, 1716, $1611,1553,1511,1492,1472,1458,1452,1374,1350,1253,1186,1091,1032,1032,1031$, 1031, 756, 713, 692; HRMS: (ESI) calcd for $\mathrm{C}_{25} \mathrm{H}_{21} \mathrm{~N}_{3} \mathrm{O}_{3} \mathrm{H}^{+}[\mathrm{M}+\mathrm{H}]^{+}$412.1661; found 412.1661.

SFC: $A D-H$ column, $15 \mathrm{ppm} \mathrm{MeOH}$ in supercritical $\mathrm{CO}_{2}$ as eluent, $4 \mathrm{~mL} / \mathrm{min}$. $\mathrm{tR}=11 \mathrm{~min}$ (major), $16 \mathrm{~min}$ (minor);

$[\alpha]_{D}^{25}=124.5(\mathrm{c}=2.0, \mathrm{MeOH})$ for $93 \%$ ee.

\begin{tabular}{|l|l|l|l|l|l|l|l|l|l|}
\hline Instrument Method & Inj. Vol. & Solvent & Column & Sample & Well Location & Temp. & Flow & $\%$ Modifier & Pressure \\
\hline $15 p$ methanol & 15 & MeOH & AD-H & $\begin{array}{l}\text { Unknown } \\
\text { Sample }\end{array}$ & $16 \mathrm{~A}$ & 29.6 & 4 & 15 & 150 \\
\hline
\end{tabular}

\begin{tabular}{|l|l|l|l|l|l|}
\hline Peak No & $\%$ Area & Area & Ret. Time & Height & Cap. Factor \\
\hline 1 & 49.5509 & 3089.3829 & $11.32 \mathrm{~min}$ & 50.0195 & 0 \\
\hline 2 & 50.4491 & 3145.3857 & $16.01 \mathrm{~min}$ & 34.8986 & 0 \\
\hline
\end{tabular}




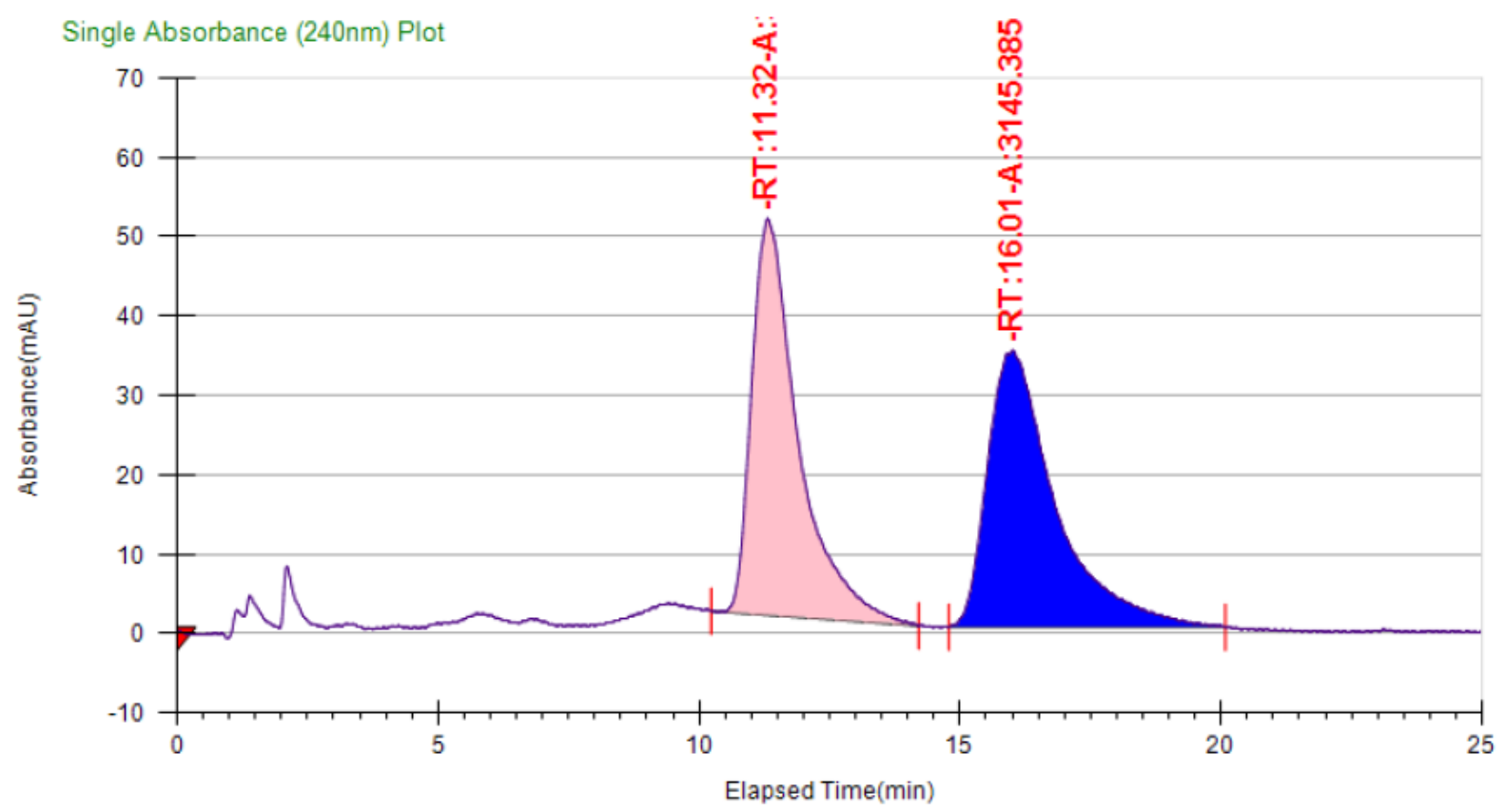

\begin{tabular}{|l|l|l|l|l|l|l|l|l|l|}
\hline Instrument Method & Inj. Vol. & Solvent & Column & Sample & Well Location & Temp. & Flow & $\%$ Modifier & Pressure \\
\hline $15 p$ methanol & 15 & MeOH & AD-H & $\begin{array}{l}\text { Unknown } \\
\text { Sample }\end{array}$ & $16 \mathrm{~B}$ & 30 & 4 & 15 & 150 \\
\hline Peak No & $\%$ Area & Area & Ret. Time & Height & Cap. Factor \\
\hline 1 & 96.5189 & 2699.4376 & 11.29 min & 42.4538 & 0 & \\
\hline 2 & 3.4811 & 97.3605 & 16.11 min & 1.4762 & 0 \\
\hline
\end{tabular}

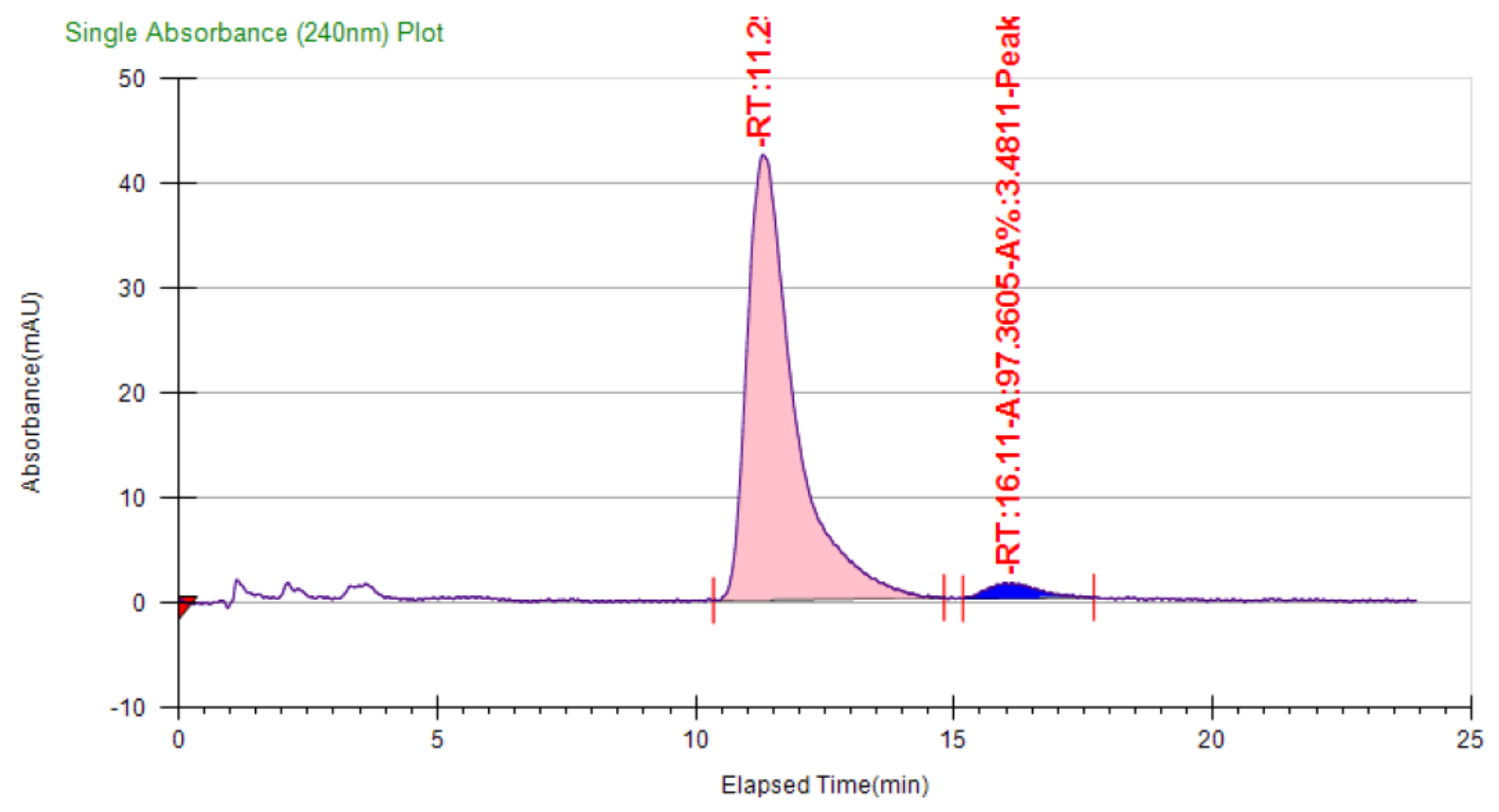


3-((5-(Biphenyl-4-yl)-1,3,4-oxadiazol-2-yl)methyl)-1-methyl-3-(naphthalen-1-yl)indolin-2-one (3nd)

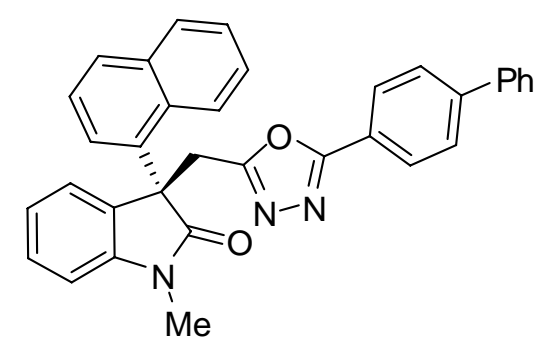

Molecular formula: $\mathrm{C}_{34} \mathrm{H}_{25} \mathrm{~N}_{3} \mathrm{O}_{2}$; Molecular Weight: 507.5812

According to general procedure, 3nd was obtained in $72 \%$ yield ( $36.5 \mathrm{mg}$ ) and $95 \%$ ee as a colorless oil. ${ }^{1} \mathrm{H}$ NMR $\left(400 \mathrm{MHz}, \mathrm{CDCl}_{3}\right): \delta$ 7.92-7.73 (m, 6H), 7.71-7.56 (m, 5H), 7.53-7.43 (m, $4 \mathrm{H}), 7.41(\mathrm{~d}, J=7.3 \mathrm{~Hz}, 1 \mathrm{H}), 7.38-7.30(\mathrm{~m}, 2 \mathrm{H}), 7.15(\mathrm{t}, J=7.5 \mathrm{~Hz}, 1 \mathrm{H}), 6.89(\mathrm{~d}, J=7.8 \mathrm{~Hz}, 1 \mathrm{H})$, $4.11(\mathrm{~d}, J=15.2 \mathrm{~Hz}, 1 \mathrm{H}), 4.05(\mathrm{~d}, J=15.1 \mathrm{~Hz}, 1 \mathrm{H}), 3.28(\mathrm{~s}, 3 \mathrm{H}) ;{ }^{13} \mathrm{C} \mathrm{NMR}\left(101 \mathrm{MHz}, \mathrm{CDCl}_{3}\right): \delta$ 176.9, 164.6, 162.9, 144.4, 143.9, 139.8, 135.6, 133.1, 132.8, 129.7, 129.3, 129.0, 128.7, $128.3,128.2$, 127.6, 127.5, 127.1, 127.1, 126.5, 126.4, 126.0, 125.6, 124.6, 123.0, 122.3, 108.7, 54.7, 33.6, 26.8; IR: $v\left(\mathrm{~cm}^{-1}\right)$ 3056, 1715, 1613, 1508, 1493, 1486, 1471, 1373, 1348, 1090, 1090, 1016, 1009, 910, 910, 848, 848, 769, 754, 735, 696; HRMS: (ESI) calcd for $\mathrm{C}_{34} \mathrm{H}_{25} \mathrm{~N}_{3} \mathrm{O}_{2} \mathrm{H}^{+}[\mathrm{M}+\mathrm{H}]^{+}$508.2025; found 508.2025.

SFC: IB column, $15 \mathrm{ppm} \mathrm{MeOH}$ in supercritical $\mathrm{CO}_{2}$ as eluent, $4 \mathrm{~mL} / \mathrm{min}$. $\mathrm{tR}=31 \mathrm{~min}$ (major), 36 min (minor);

$[\alpha]_{D}^{25}=142.0(\mathrm{c}=2.0, \mathrm{MeOH})$ for $95 \%$ ee.

\begin{tabular}{|c|c|c|c|c|c|c|c|c|c|}
\hline Instrument Method & Inj. Vol. & Solvent & Column & Sample & Well Location & Temp. & Flow & $\%$ Modifier & Pressure \\
\hline \begin{tabular}{|l|}
$15 p$ methanol \\
\end{tabular} & 5 & $\mathrm{MeOH}$ & IB & $\begin{array}{l}\text { Unknown } \\
\text { Sample }\end{array}$ & $16 \mathrm{~A}$ & 29.5 & 4 & 15 & 150 \\
\hline Peak No & \multicolumn{2}{|c|}{$\%$ Area } & Area & \multicolumn{2}{|c|}{ Ret. Time } & \multicolumn{2}{|c|}{ Height } & \multicolumn{2}{|c|}{ Cap. Factor } \\
\hline 1 & \multicolumn{2}{|c|}{50.4896} & 4250.0195 & \multicolumn{2}{|c|}{$31.74 \mathrm{~min}$} & \multicolumn{2}{|c|}{73.1444} & \multicolumn{2}{|c|}{0} \\
\hline 2 & \multicolumn{2}{|c|}{49.5104} & \begin{tabular}{|l|l|}
4167.5987 \\
\end{tabular} & \multicolumn{2}{|c|}{$35.43 \mathrm{~min}$} & \multicolumn{2}{|c|}{51.7279} & \multicolumn{2}{|c|}{0} \\
\hline
\end{tabular}




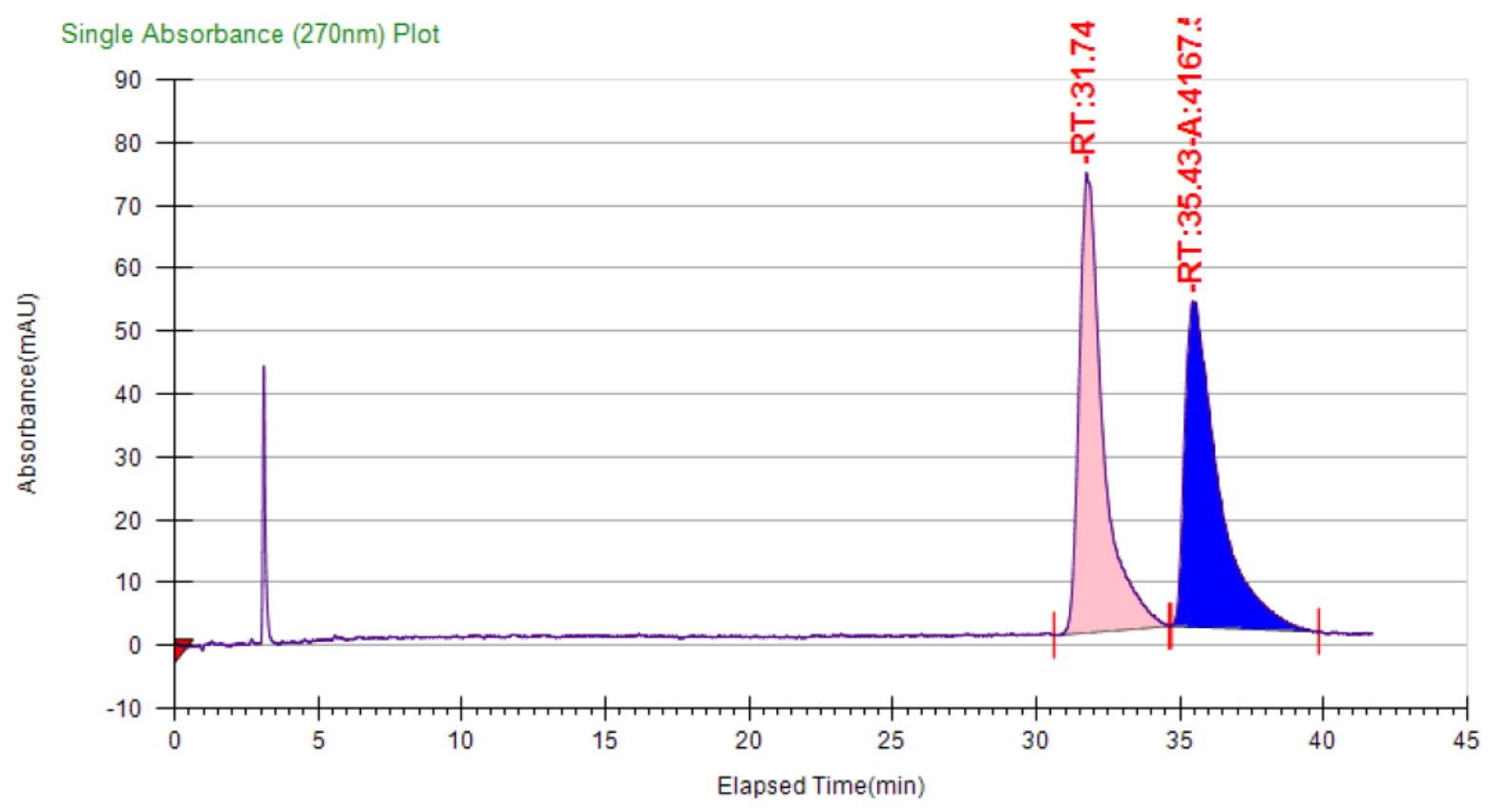

\begin{tabular}{|l|l|l|l|l|l|l|l|l|l|}
\hline Instrument Method & Inj. Vol. & Solvent & Column & Sample & Well Location & Temp. & Flow & $\%$ Modifier & Pressure \\
\hline $15 \mathrm{p}$ methanol & 5 & $\mathrm{MeOH}$ & $\mathrm{B}$ & $\begin{array}{l}\text { Unknown } \\
\text { Sample }\end{array}$ & \multicolumn{1}{|l|}{$16 \mathrm{~B}$} & 29.6 & 4 & 15 & 150 \\
\hline \hline Peak No & $\%$ Area & Area & Ret. Time & Height & Cap. Factor \\
\hline 1 & 97.6529 & 2336.6598 & $31.8 \mathrm{~min}$ & 39.4527 & 0 & \\
\hline 2 & 2.3471 & 56.1615 & $36.23 \mathrm{~min}$ & 1.0709 & 0 & \\
\hline
\end{tabular}

Single Absorbance (270nm) Plot

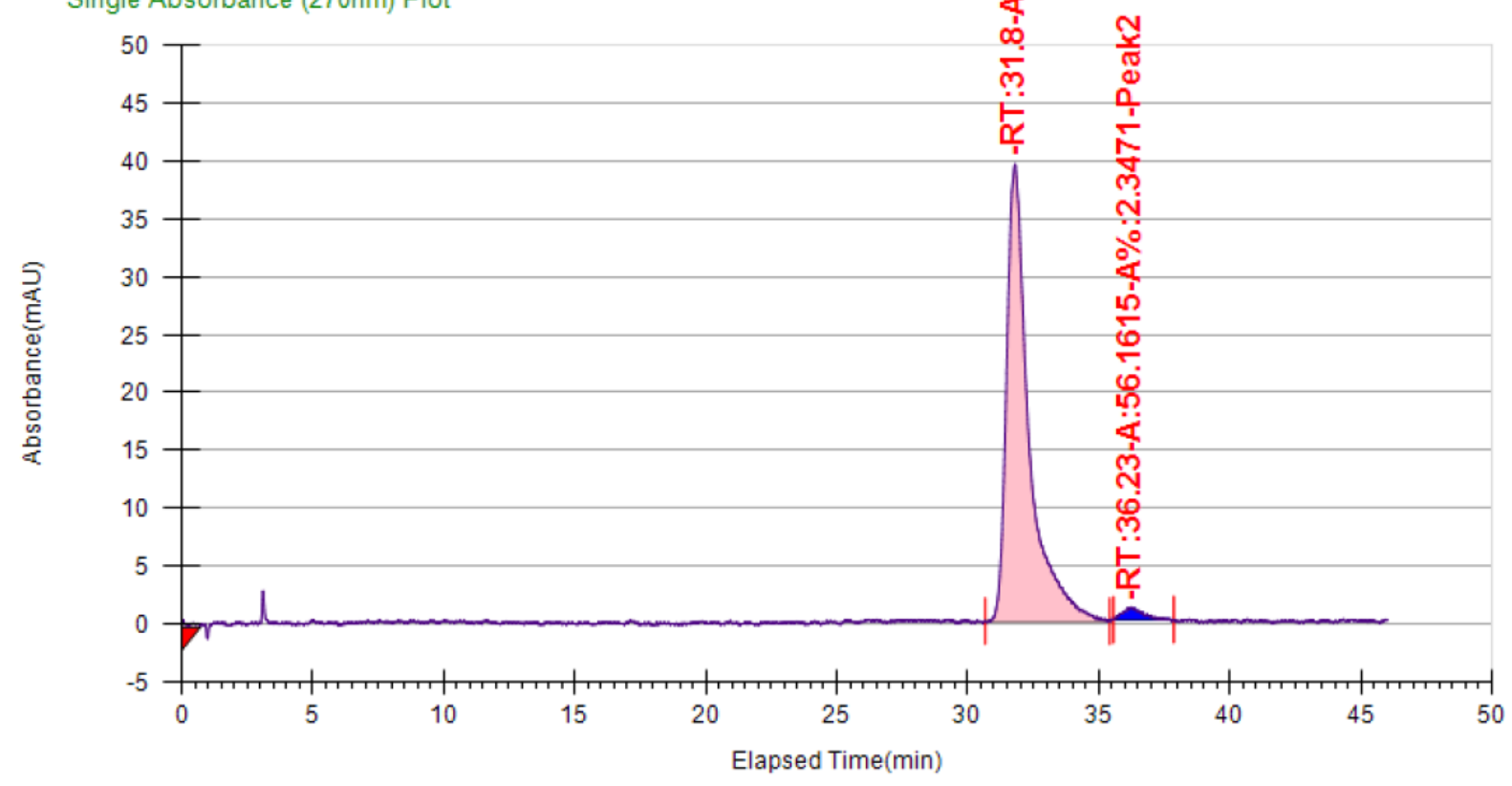


3-((5-(Biphenyl-4-yl)-1,3,4-oxadiazol-2-yl)methyl)-1,3,5-trimethylindolin-2-one (3od)

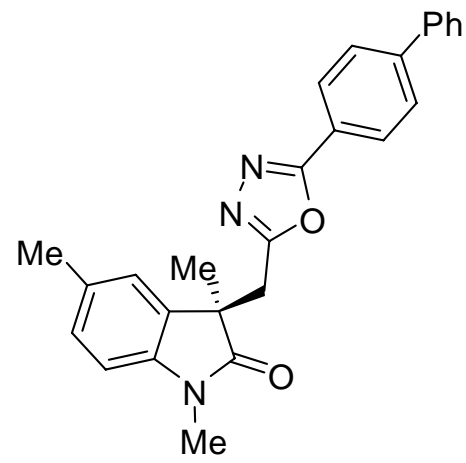

Molecular formula: $\mathrm{C}_{26} \mathrm{H}_{23} \mathrm{~N}_{3} \mathrm{O}_{2}$; Molecular Weight: 409.4797

According to general procedure, 3od was obtained in $72 \%$ yield $(29.4 \mathrm{mg}$ ) and $87 \%$ ee as a colorless oil. ${ }^{1} \mathrm{H}$ NMR $\left(400 \mathrm{MHz}, \mathrm{CDCl}_{3}\right): \delta 7.95(\mathrm{~d}, J=8.0 \mathrm{~Hz}, 2 \mathrm{H}), 7.68(\mathrm{~d}, J=8.0 \mathrm{~Hz}, 2 \mathrm{H}), 7.61$ $(\mathrm{d}, J=7.3 \mathrm{~Hz}, 2 \mathrm{H}), 7.46(\mathrm{t}, J=7.2 \mathrm{~Hz}, 2 \mathrm{H}), 7.39(\mathrm{~d}, J=7.0 \mathrm{~Hz}, 1 \mathrm{H}), 7.03(\mathrm{~s}, 2 \mathrm{H}), 6.69(\mathrm{~d}, J=8.1$ Hz, 1H), $3.46(\mathrm{~d}, J=15.2 \mathrm{~Hz}, 1 \mathrm{H}), 3.41(\mathrm{~d}, J=15.2 \mathrm{~Hz}, 1 \mathrm{H}), 3.23(\mathrm{~s}, 3 \mathrm{H}), 2.29(\mathrm{~s}, 3 \mathrm{H}), 1.55(\mathrm{~s}$, 3H); ${ }^{13} \mathrm{C}$ NMR $\left(101 \mathrm{MHz} \mathrm{CDCl}_{3}\right): \delta 178.8,164.6,163.2,144.4,140.6,139.7,132.3,131.7$, 129.0, 128.9, 128.2, 127.6, 127.1, 127.1, 124.1, 122.5, 108.1, 46.9, 33.4, 26.5, 23.7, 21.1; IR: $v$ $\left(\mathrm{cm}^{-1}\right)$ 2969, 2929, 1709, 1614, 1499, 1484, 1353, 1008, 909, 848, 808; HRMS: (ESI) calcd for $\mathrm{C}_{26} \mathrm{H}_{23} \mathrm{~N}_{3} \mathrm{O}_{2} \mathrm{Na}^{+}[\mathrm{M}+\mathrm{Na}]^{+}$432.1688; found 432.1688.

SFC: $\mathrm{AD}-\mathrm{H}$ column, $20 \mathrm{ppm} \mathrm{MeOH}$ in supercritical $\mathrm{CO}_{2}$ as eluent, $4 \mathrm{~mL} / \mathrm{min}$. $\mathrm{tR}=7 \mathrm{~min}$ (major), $10 \mathrm{~min}$ (minor);

$[\alpha]_{D}^{28}=116(c=1.2, \mathrm{MeOH})$ for $87 \%$ ee.

\begin{tabular}{|l|l|l|l|l|l|l|l|l|l|}
\hline Instrument Method & Inj. Vol. & Solvent & Column & Sample & Well Location & Temp. & Flow & $\%$ Modifier & Pressure \\
\hline 20p methanol & 10 & MeOH & AD-H & kwq-c-28 & $16 \mathrm{~A}$ & 30.9 & 4 & 20 & 150 \\
\hline Peak No & $\%$ Area & Area & Ret. Time & Height & \multicolumn{2}{l|}{ Cap. Factor } \\
\hline 1 & 49.8628 & $\begin{array}{l}13220.378 \\
1\end{array}$ & 6.77 min & 456.1791 & 0 \\
\hline 2 & 50.1372 & $\begin{array}{l}13293.129 \\
7\end{array}$ & 14.86 min & 217.787 & 0 & \\
\hline
\end{tabular}




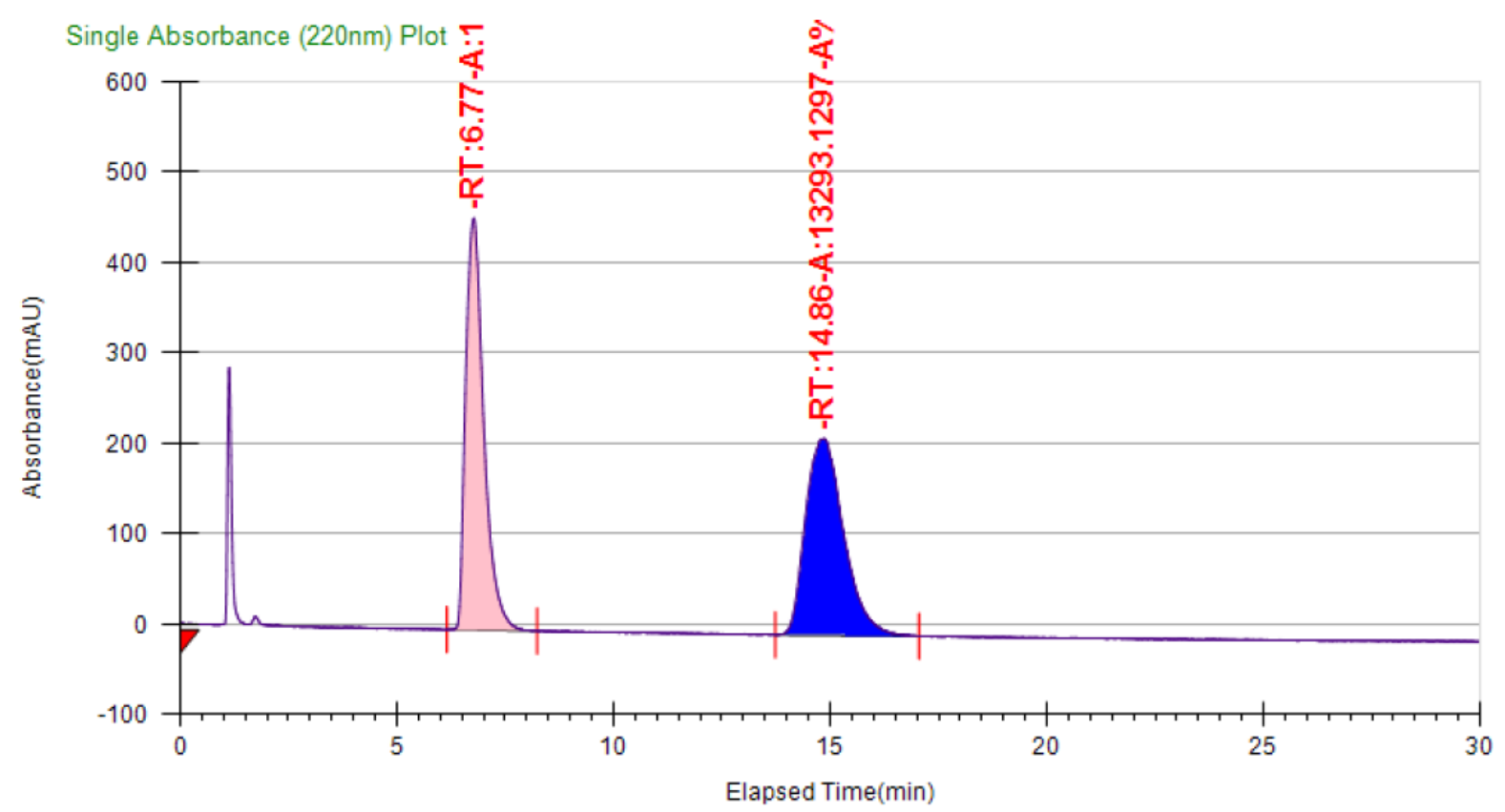

\begin{tabular}{|l|l|l|l|l|l|l|l|l|l|}
\hline Instrument Method & Inj. Vol. & Solvent & Column & Sample & Well Location & Temp. & Flow & $\%$ Modifier & Pressure \\
\hline $20 \mathrm{p}$ methanol & 10 & $\mathrm{MeOH}$ & $\mathrm{AD}-\mathrm{H}$ & kwq-c-30 & $16 \mathrm{~B}$ & 29.7 & 4 & 20 & 150 \\
\hline
\end{tabular}

\begin{tabular}{|l|l|l|l|l|l|}
\hline Peak No & \% Area & Area & Ret. Time & Height & Cap. Factor \\
\hline 1 & 92.8094 & 4410.1441 & $6.78 \mathrm{~min}$ & 151.2586 & 0 \\
\hline 2 & 7.1906 & 341.6836 & $14.75 \mathrm{~min}$ & 6.8762 & 0 \\
\hline
\end{tabular}

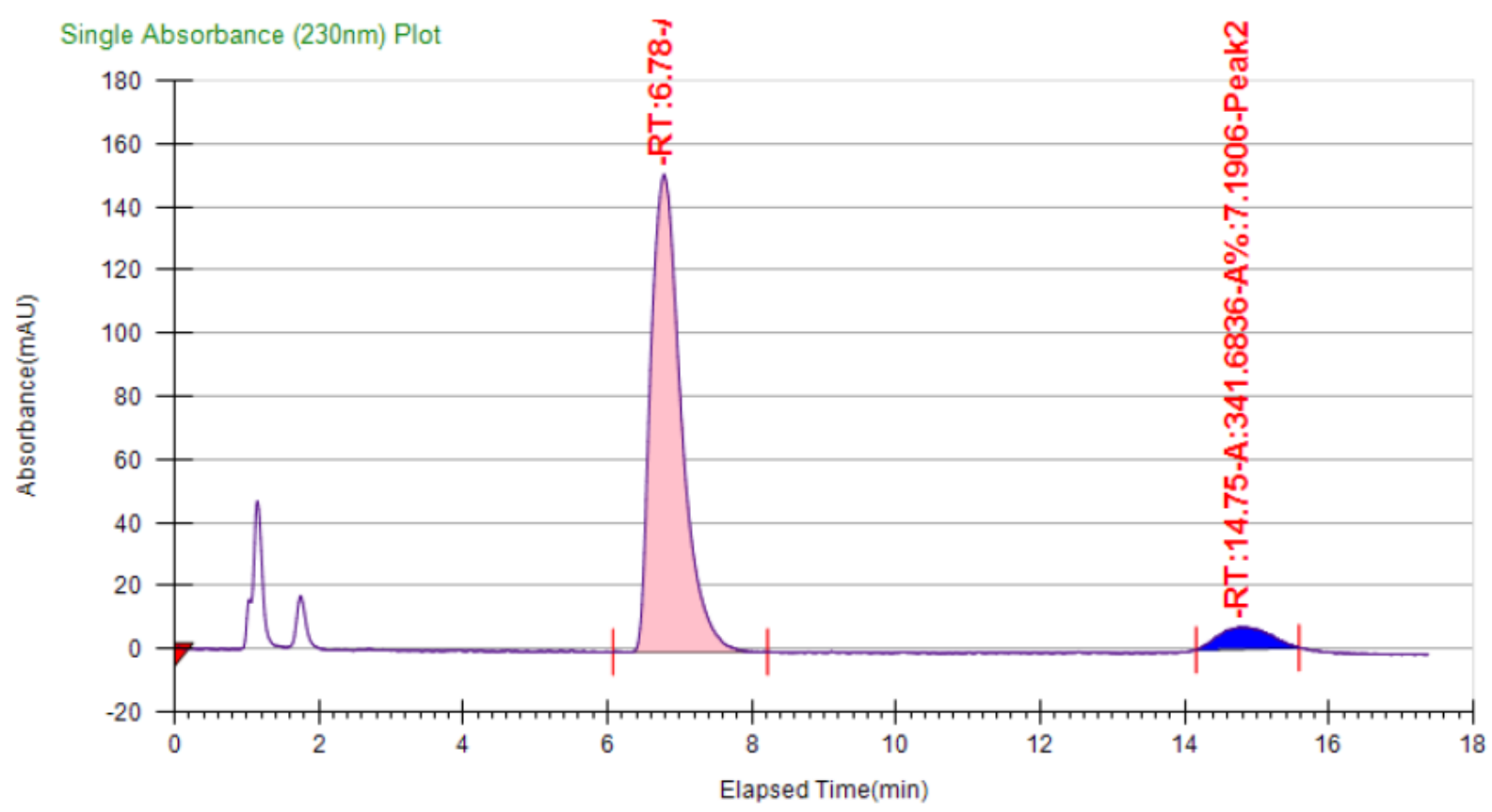


3-((5-(Biphenyl-4-yl)-1,3,4-oxadiazol-2-yl)methyl)-1,3,6-trimethylindolin-2-one (3pd)

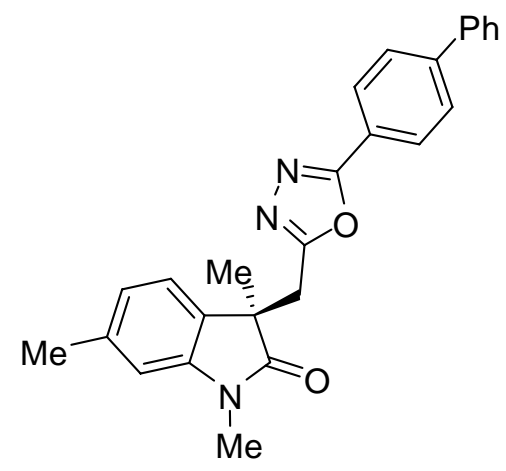

Molecular formula: $\mathrm{C}_{26} \mathrm{H}_{23} \mathrm{~N}_{3} \mathrm{O}_{2}$; Molecular Weight: 409.4797

According to general procedure, 3pd was obtained in $69 \%$ yield $(28.2 \mathrm{mg}$ ) and $85 \%$ ee as a colorless oil. ${ }^{1} \mathrm{H}$ NMR $\left(400 \mathrm{MHz}, \mathrm{CDCl}_{3}\right): \delta 7.96(\mathrm{~d}, J=8.3 \mathrm{~Hz}, 2 \mathrm{H}), 7.68(\mathrm{~d}, J=8.3 \mathrm{~Hz}, 2 \mathrm{H}), 7.62$ (d, J = 7.2 Hz, 2H), $7.47(\mathrm{t}, J=7.4 \mathrm{~Hz}, 2 \mathrm{H}), 7.39(\mathrm{t}, J=7.3 \mathrm{~Hz}, 1 \mathrm{H}), 7.08(\mathrm{~d}, J=7.5 \mathrm{~Hz}, 1 \mathrm{H}), 6.84$ (d, $J=7.5 \mathrm{~Hz}, 1 \mathrm{H}), 6.64$ (s, 1H), 3.46 (d, J=15.2 Hz, 1H), 3.40 (d, J = 15.2 Hz, 1H), $3.24(\mathrm{~s}, 3 \mathrm{H})$, 2.32 (s, 3H), $1.56(\mathrm{~s}, 3 \mathrm{H}) ;{ }^{13} \mathrm{C}$ NMR (101 MHz, CDCl 3 ): $\delta$ 179.2, 164.6, 163.3, 144.4, 143.1, $139.8,138.8,129.0,128.7,128.2,127.7,127.2$, 127.1, 123.4, 122.9, 122.5, 109.3, 46.7, 33.4, 26.4, 23.7, 21.8; IR: $v\left(\mathrm{~cm}^{-1}\right)$ 3032, 2968, 2927, 1712, 1618, 1484, 1449, 1381, 1304, 1117, 1008, 910; HRMS: (ESI) calcd for $\mathrm{C}_{26} \mathrm{H}_{23} \mathrm{~N}_{3} \mathrm{O}_{2} \mathrm{Na}^{+}[\mathrm{M}+\mathrm{Na}]^{+} 432.1688$; found 432.1688 .

SFC: AD-H column, $30 \mathrm{ppm} \mathrm{MeOH}$ in supercritical $\mathrm{CO}_{2}$ as eluent, $4 \mathrm{~mL} / \mathrm{min}$. $\mathrm{tR}=5 \mathrm{~min}$ (major), $12 \mathrm{~min}$ (minor);

$[\alpha]_{D}^{29}=48(\mathrm{c}=1.6, \mathrm{MeOH})$ for $85 \%$ ee.

\begin{tabular}{|l|l|l|l|l|l|l|l|l|l|}
\hline Instrument Method & Inj. Vol. & Solvent & Column & Sample & Well Location & Temp. & Flow & $\%$ Modifier & Pressure \\
\hline 30 methanol & 10 & MeOH & AD-H & kwq-c-16 & $16 \mathrm{~B}$ & 29.8 & 4 & 30 & 150 \\
\hline \hline Peak No & $\%$ Area & Area & Ret. Time & Height & Cap. Factor \\
\hline 1 & 49.9462 & 6452.937 & 5.26 min & 241.2444 & 0 \\
\hline 2 & 50.0538 & 6466.8279 & 12.24 min & 100.1796 & 0 \\
\hline
\end{tabular}




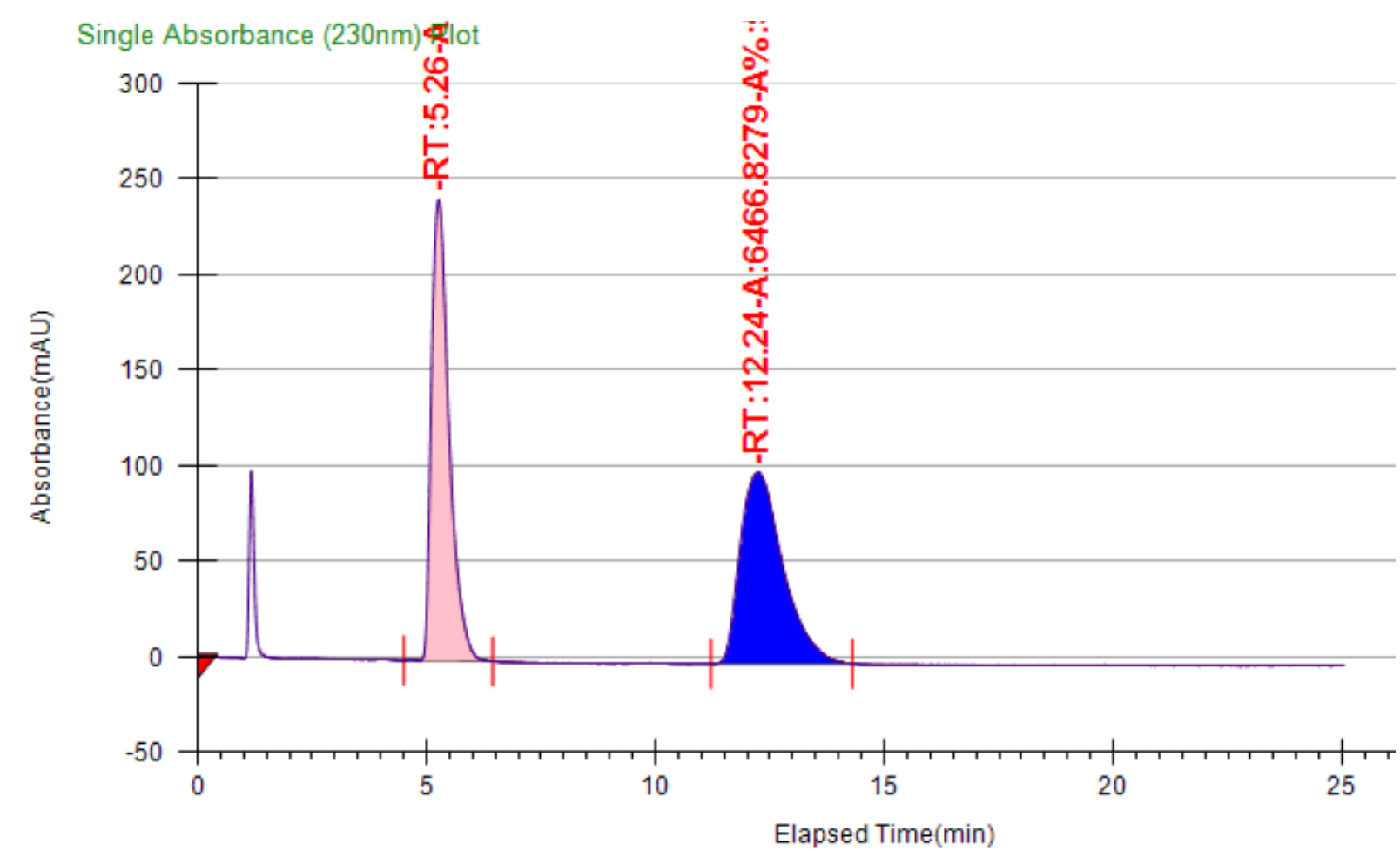

\begin{tabular}{|l|l|l|l|l|l|l|l|l|l|}
\hline Instrument Method & Inj. Vol. & Solvent & Column & Sample & Well Location & Temp. & Flow & $\%$ Modifier & Pressure \\
\hline 30p methanol & 10 & MeOH & AD-H & $\begin{array}{l}\text { kwq-C-16- } \\
\text { chiral }\end{array}$ & $16 \mathrm{C}$ & 29.6 & 4 & 30 & 150 \\
\hline \hline Peak No & $\%$ Area & Area & Ret. Time & Height & Cap. Factor \\
\hline 1 & 92.6784 & 4347.5224 & $5.28 \mathrm{~min}$ & 155.655 & 5282.2667 \\
\hline 2 & 7.3216 & 343.4568 & $12.43 \mathrm{~min}$ & 6.6227 & 12432.15 \\
\hline
\end{tabular}

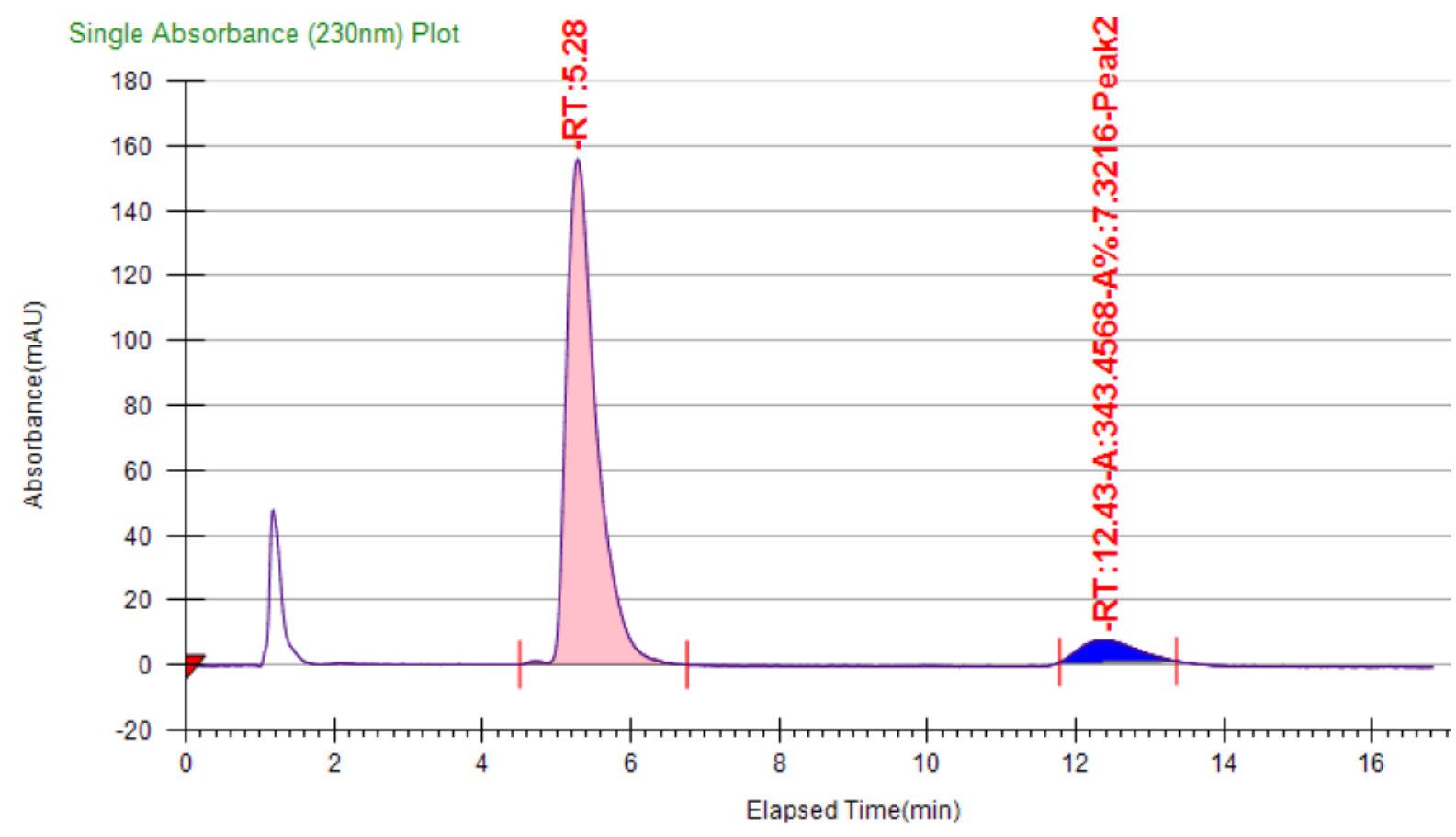


6-Chloro-1,3-dimethyl-3-((5-phenyl-1,3,4-oxadiazol-2-yl)methyl)indolin-2-one (3qd)

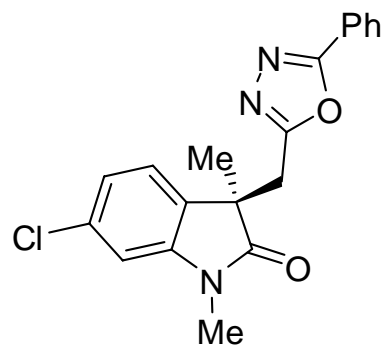

Molecular formula: $\mathrm{C}_{19} \mathrm{H}_{16} \mathrm{ClN}_{3} \mathrm{O}_{2}$; Molecular Weight: 353.8022

According to general procedure, 3qd was obtained in 55\% yield (19.5 mg) and $82 \%$ ee as a colorless oil. ${ }^{1} \mathrm{H}$ NMR $\left(400 \mathrm{MHz}, \mathrm{CDCl}_{3}\right): \delta 7.90(\mathrm{~d}, J=7.0 \mathrm{~Hz}, 2 \mathrm{H}), 7.64-7.38(\mathrm{~m}, 3 \mathrm{H}), 7.12(\mathrm{~d}, J$ $=7.9 \mathrm{~Hz}, 1 \mathrm{H}), 7.01(\mathrm{~d}, J=7.9 \mathrm{~Hz}, 1 \mathrm{H}), 6.81(\mathrm{~s}, 1 \mathrm{H}), 3.44(\mathrm{~d}, J=15.5 \mathrm{~Hz}, 1 \mathrm{H}), 3.39(\mathrm{~d}, J=15.4 \mathrm{~Hz}$, 1H), 3.23 (s, 3H), 1.55 (s, 3H); ${ }^{13} \mathrm{C}$ NMR (101 MHz, $\left.\mathrm{CDCl}_{3}\right): \delta$ 178.8, 164.8, 162.9, 144.2, 134.5, 131.8, 130.0, 129.1, 126.7, 124.1, 123.7, 122.6, 109.2, 46.5, 33.2, 26.6, 23.6; IR: $v\left(\mathrm{~cm}^{-1}\right)$ 2970, 2929, 1716, 1609, 1495, 1371, 1292, 1240, 1028, 812, 731; HRMS: (ESI) calcd for $\mathrm{C}_{19} \mathrm{H}_{16} \mathrm{~N}_{3} \mathrm{OCl}{ }^{35} \mathrm{Na}^{+}[\mathrm{M}+\mathrm{Na}]^{+}$376.0829; found 376.0829; $\mathrm{C}_{19} \mathrm{H}_{16} \mathrm{~N}_{3} \mathrm{OCl}^{37} \mathrm{Na}^{+}[\mathrm{M}+\mathrm{Na}]^{+}$378.0799; found 378.0806 .

SFC: $A D-H$ column, $10 \mathrm{ppm} \mathrm{MeOH}$ in supercritical $\mathrm{CO}_{2}$ as eluent, $4 \mathrm{~mL} / \mathrm{min}$. $\mathrm{tR}=7 \mathrm{~min}$ (major), $10 \mathrm{~min}$ (minor);

$[\alpha]_{D}^{28}=107\left(\mathrm{c}=0.4, \mathrm{CHCl}_{3}\right)$ for $83 \%$ ee.

\begin{tabular}{|l|l|l|l|l|l|l|l|l|l|}
\hline Instrument Method & Inj. Vol. & Solvent & Column & Sample & Well Location & Temp. & Flow & $\%$ Modifier & Pressure \\
\hline 10p methanol & 15 & MeOH & AD-H & kwq-b-149 & 16 E & 29.9 & 4 & 10 & 150 \\
\hline \hline Peak No & $\%$ Area & Area & Ret. Time & Height & Cap. Factor \\
\hline 1 & 50.0051 & 3495.4998 & 7.52 min & 67.9064 & 0 & \\
\hline 2 & 49.9949 & 3494.7827 & 10.54 min & 45.5892 & 0 & \\
\hline
\end{tabular}




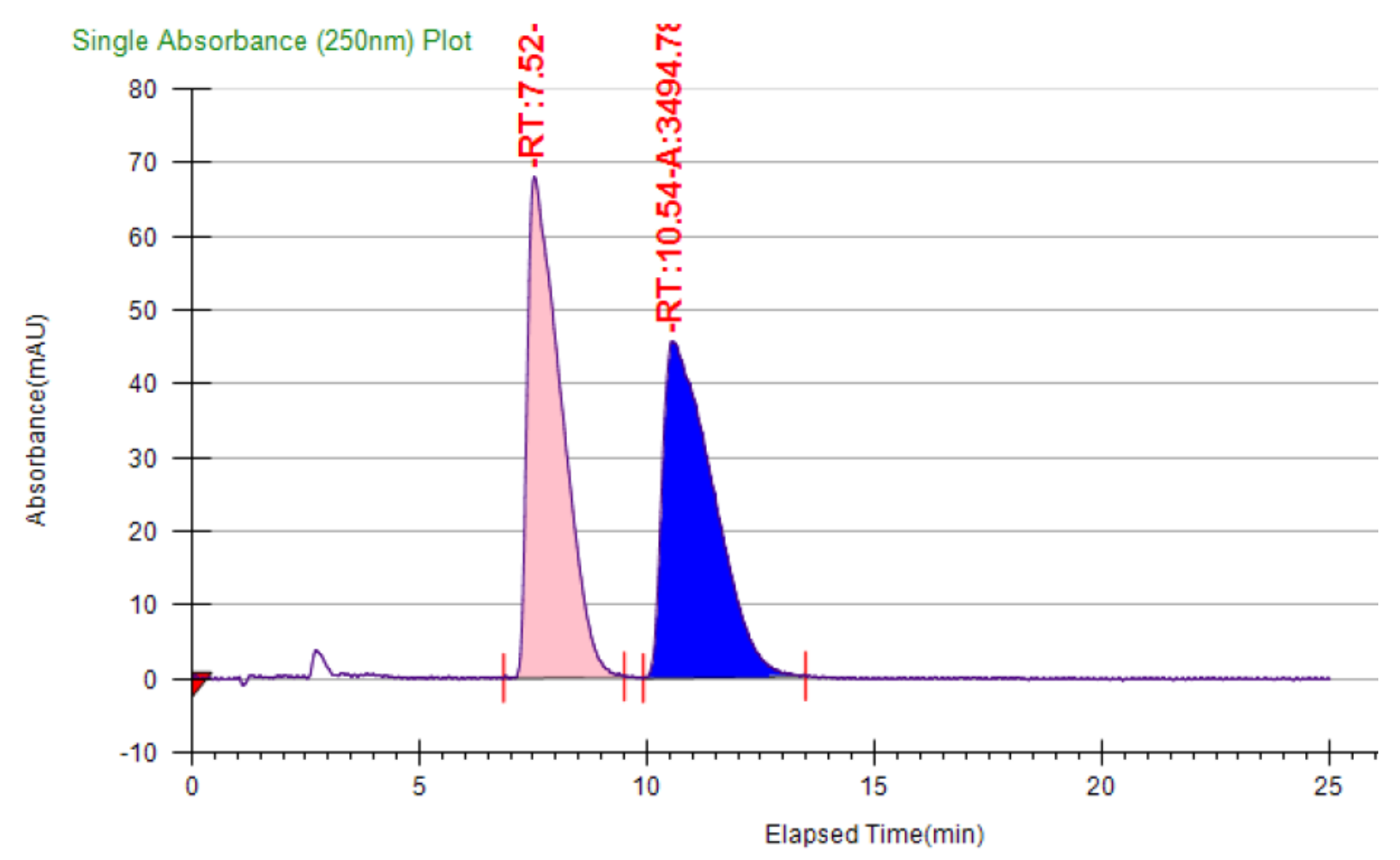

\begin{tabular}{|l|l|l|l|l|l|l|l|l|l|}
\hline Instrument Method & Inj. Vol. & Solvent & Column & Sample & Well Location & Temp. & Flow & $\%$ Modifier & Pressure \\
\hline 10p methanol & 15 & MeOH & AD-H & kwq-c-6 & $16 \mathrm{~A}$ & 31.2 & 4 & 10 & 150 \\
\hline
\end{tabular}

\begin{tabular}{|l|l|l|l|l|l|}
\hline Peak No & \% Area & Area & Ret. Time & Height & Cap. Factor \\
\hline 1 & 91.4225 & 3261.1611 & $7.82 \mathrm{~min}$ & 90.9718 & 7815.55 \\
\hline 2 & 8.5775 & 305.9711 & $11.05 \mathrm{~min}$ & 6.6243 & 11048.8333 \\
\hline
\end{tabular}

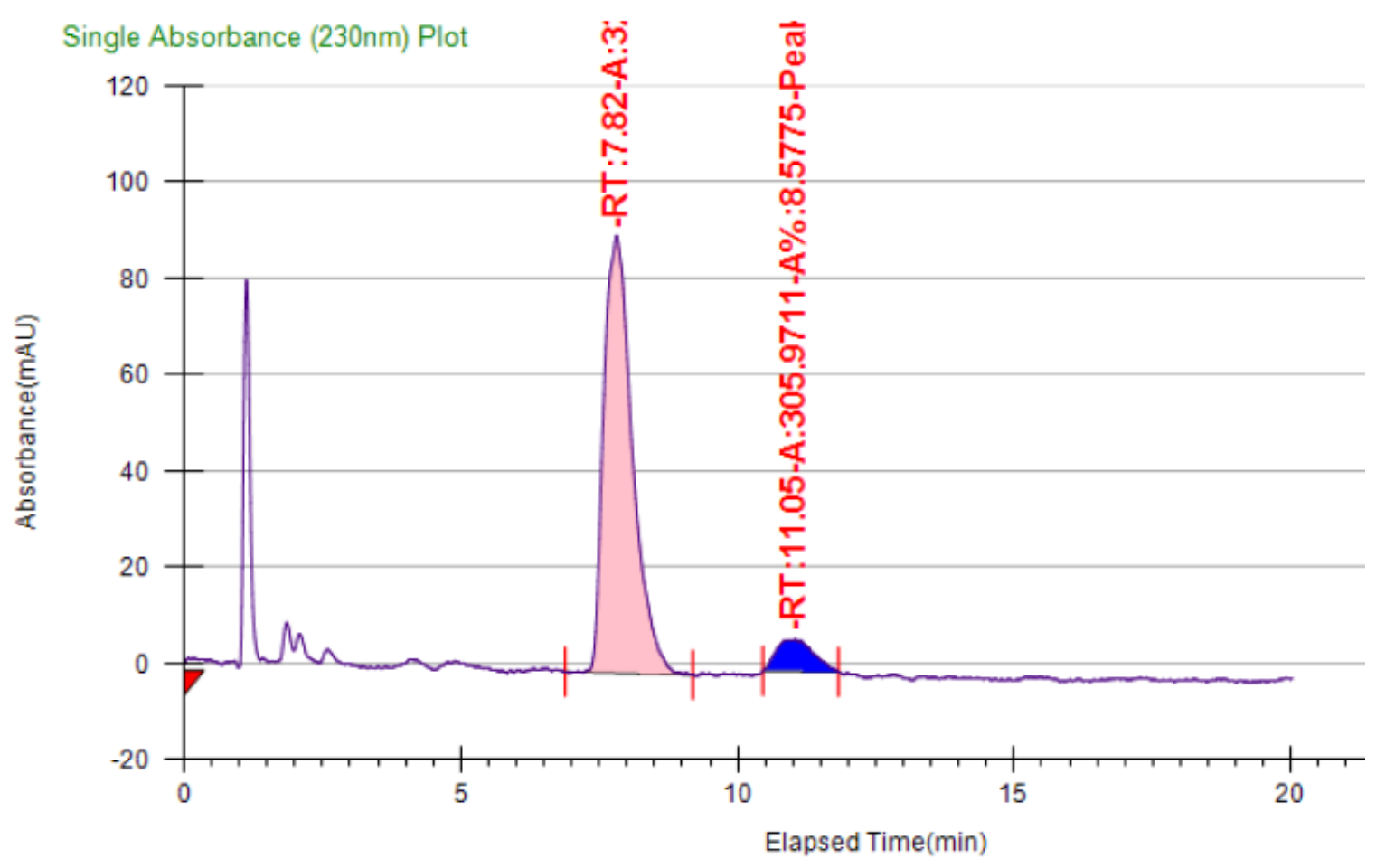


3-((5-(Biphenyl-4-yl)-1,3,4-oxadiazol-2-yl)methyl)-1,3,7-trimethylindolin-2-one (3rd)

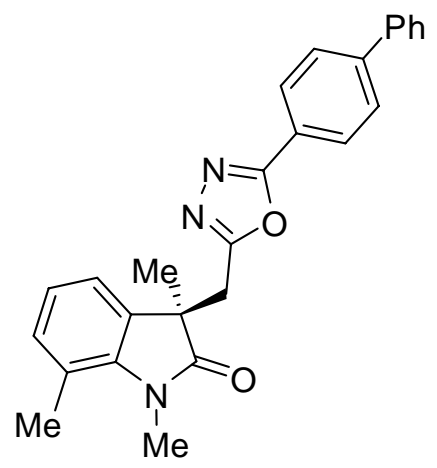

Molecular formula: $\mathrm{C}_{26} \mathrm{H}_{23} \mathrm{~N}_{3} \mathrm{O}_{2}$; Molecular Weight: 409.4797

According to general procedure, 3rd was obtained in $71 \%$ yield $(29.0 \mathrm{mg})$ and $92 \%$ ee as a colorless oil. ${ }^{1} \mathrm{H}$ NMR $\left(400 \mathrm{MHz}, \mathrm{CDCl}_{3}\right): \delta 7.96(\mathrm{~d}, J=8.2 \mathrm{~Hz}, 2 \mathrm{H}), 7.68(\mathrm{~d}, J=8.2 \mathrm{~Hz}, 2 \mathrm{H}), 7.61$ (d, J = 7.4 Hz, 2H), $7.46(\mathrm{t}, J=7.4 \mathrm{~Hz}, 2 \mathrm{H}), 7.39(\mathrm{t}, J=7.1 \mathrm{~Hz}, 1 \mathrm{H}), 7.02(\mathrm{~d}, J=6.9 \mathrm{~Hz}, 1 \mathrm{H}), 6.96$ (d, J = 7.2 Hz, 1H), $6.91(\mathrm{t}, J=7.4 \mathrm{~Hz}, 1 \mathrm{H}), 3.53(\mathrm{~s}, 3 \mathrm{H}), 3.43(\mathrm{~s}, 2 \mathrm{H}), 2.52(\mathrm{~s}, 3 \mathrm{H}), 1.55(\mathrm{~s}, 3 \mathrm{H})$; ${ }^{13} \mathrm{C}$ NMR $\left(101 \mathrm{MHz}, \mathrm{CDCl}_{3}\right): \delta 179.6,164.6,163.2,144.4,140.8,139.8,132.3,129.0,128.2$, 127.6, 127.2, 127.1, 122.7, 122.5, 121.0, 120.0, 46.2, 33.6, 29.8, 24.1, 19.0; IR: $v\left(\mathrm{~cm}^{-1}\right)$ 2969, 2928, 1706, 1602, 1484, 1459, 1364, 1127, 1072, 908, 848; HRMS: (ESI) calcd for $\mathrm{C}_{26} \mathrm{H}_{23} \mathrm{~N}_{3} \mathrm{O}_{2} \mathrm{Na}^{+}[\mathrm{M}+\mathrm{Na}]^{+}$432.1688; found 432.1688.

SFC: $A D-H$ column, $20 \mathrm{ppm} \mathrm{MeOH}$ in supercritical $\mathrm{CO}_{2}$ as eluent, $4 \mathrm{~mL} / \mathrm{min}$. $\mathrm{tR}=8 \mathrm{~min}$ (major), $22 \mathrm{~min}$ (minor);

$[\alpha]_{D}^{27}=-36(c=0.8, \mathrm{MeOH})$ for $92 \%$ ee.

\begin{tabular}{|l|l|l|l|l|l|l|l|l|l|}
\hline Instrument Method & Inj. Vol. & Solvent & Column & Sample & Well Location & Temp. & Flow & $\%$ Modifier & Pressure \\
\hline $20 \mathrm{p}$ methanol & 10 & $\mathrm{MeOH}$ & $\mathrm{AD}-\mathrm{H}$ & kwq-c-41 & $16 \mathrm{~B}$ & 30.1 & 4 & 20 & 150 \\
\hline \hline Peak No & $\%$ Area & Area & Ret. Time & Height & Cap. Factor \\
\hline 1 & 50.4664 & 8649.8614 & $8.51 \mathrm{~min}$ & 180.5869 & 8507.2167 \\
\hline 2 & 49.5336 & 8489.985 & $22.42 \mathrm{~min}$ & 71.0066 & 22423.6667 \\
\hline
\end{tabular}




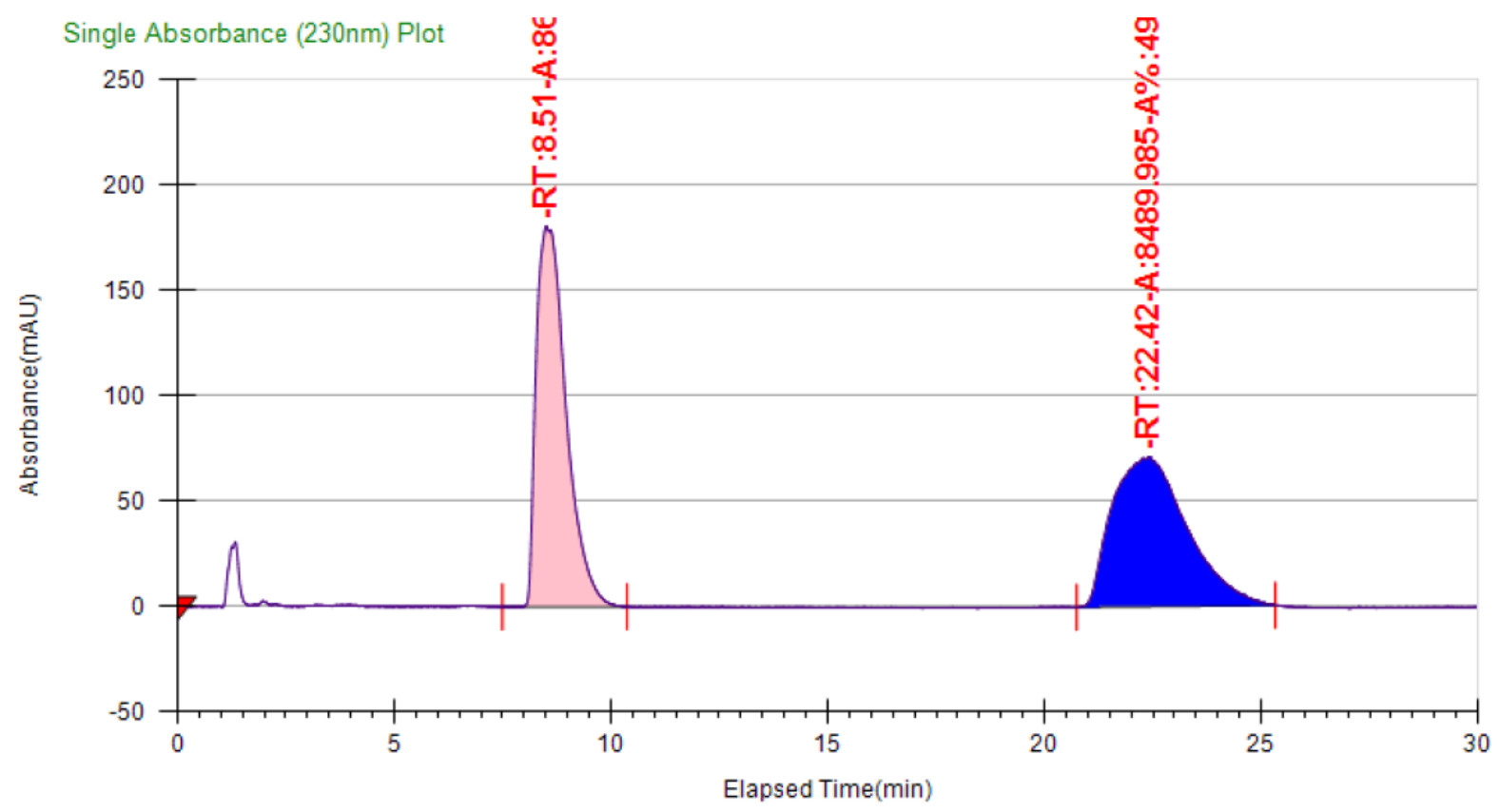

\begin{tabular}{|l|l|l|l|l|l|l|l|l|l|}
\hline Instrument Method & Inj. Vol. & Solvent & Column & Sample & Well Location & Temp. & Flow & $\%$ Modifier & Pressure \\
\hline $20 \mathrm{p}$ methanol & 10 & $\mathrm{MeOH}$ & $\mathrm{AD}-\mathrm{H}$ & kwq-c-39 & $16 \mathrm{C}$ & 30.2 & 4 & 20 & 150 \\
\hline \hline Peak No & $\%$ Area & Area & Ret. Time & Height & Cap. Factor \\
\hline 1 & 95.7966 & 2995.9524 & $8.45 \mathrm{~min}$ & 64.7603 & 0 & \\
\hline 2 & 4.2034 & 131.459 & $22.36 \mathrm{~min}$ & 1.6598 & 0 \\
\hline
\end{tabular}

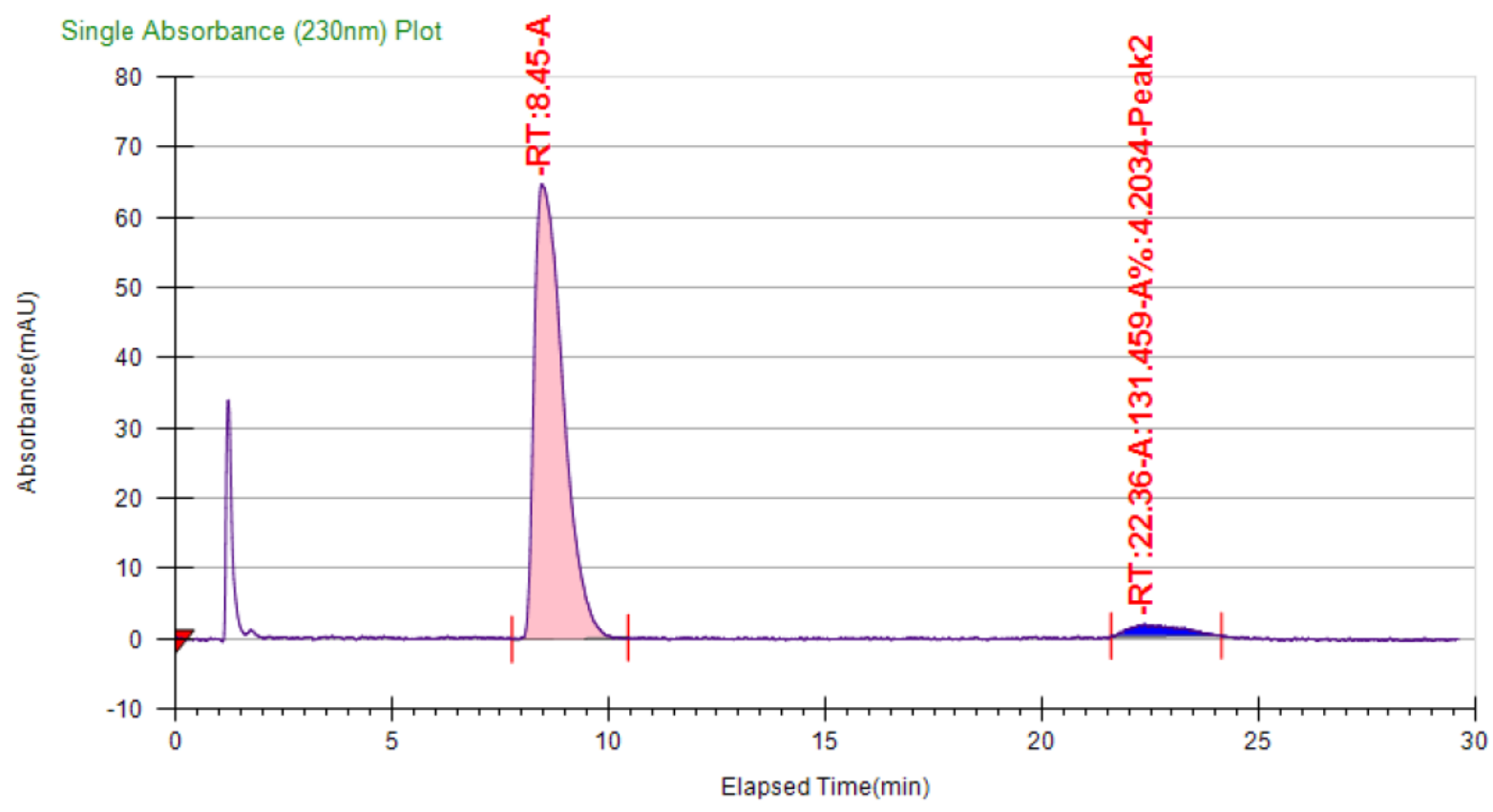


1,3-Dimethyl-3-((5-phenyl-1,3,4-oxadiazol-2-yl)methyl)-1,3-dihydro-2H-benzo[f]indol-2-one (3sa)

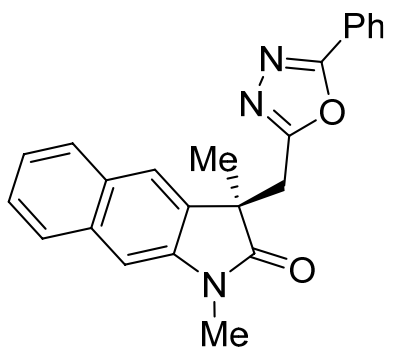

Chemical Formula: $\mathrm{C}_{23} \mathrm{H}_{19} \mathrm{~N}_{3} \mathrm{O}_{2}$; Exact Mass: 369.1477

According to general procedure, 3sa was obtained in $71 \%$ yield $(26.1 \mathrm{mg})$ and $82 \%$ ee as a colorless oil. ${ }^{1} \mathrm{H}$ NMR $\left(400 \mathrm{MHz}, \mathrm{CDCl}_{3}\right): \delta 7.83(\mathrm{~d}, J=7.3 \mathrm{~Hz}, 2 \mathrm{H}), 7.78-7.71(\mathrm{~m}, 2 \mathrm{H}), 7.65(\mathrm{~s}$, 1H), 7.51-7.31 (m, 5H), 7.09 (s, 1H), 3.56 (d, J = $15.4 \mathrm{~Hz}, 1 \mathrm{H}), 3.51(\mathrm{~d}, J=15.4 \mathrm{~Hz}, 1 \mathrm{H}), 3.35(\mathrm{~s}$, 3H), 1.65 (s, 3H); ${ }^{13} \mathrm{C}$ NMR (101 MHz, $\left.\mathrm{CDCl}_{3}\right): \delta 178.38,164.73,163.06,141.31,133.88$, $132.48,131.63,130.33,128.97,128.19,127.06,126.71,126.67,124.38,123.61,122.75$, 104.09, 46.27, 33.62, 26.71, 24.25; IR: $v\left(\mathrm{~cm}^{-1}\right)$ 2971, 2928, 2900, 1715, 1646, 1552, 1482, 1380, 1266, 1089, 1049, 907, 727; HRMS: (ESI) calcd for $\mathrm{C}_{23} \mathrm{H}_{19} \mathrm{~N}_{3} \mathrm{O}_{2} \mathrm{Na}{ }^{+}[\mathrm{M}+\mathrm{Na}]^{+} 392.1375$; found 392.1375 .

SFC: IB column, $6 \mathrm{ppm} \mathrm{MeOH}$ in supercritical $\mathrm{CO}_{2}$ as eluent, $4 \mathrm{~mL} / \mathrm{min}$. $\mathrm{tR}=15 \mathrm{~min}$ (major), 17 min (minor);

$[\alpha]_{D}^{28}=49(c=1.3, \mathrm{MeOH})$ for $82 \%$ ee.

\begin{tabular}{|l|l|l|l|l|l|l|l|l|l|}
\hline Instrument Method & Inj. Vol. & Solvent & Column & Sample & Well Location & Temp. & Flow & $\%$ Modifier & Pressure \\
\hline 6p methanol & 10 & MeOH & IB & $\begin{array}{l}\text { Unknown } \\
\text { Sample }\end{array}$ & $16 \mathrm{C}$ & 29.8 & 4 & 6 & 150 \\
\hline Peak No & $\%$ Area & Area & Ret. Time & Height & Cap. Factor \\
\hline 1 & 50.1993 & $\begin{array}{l}31323.777 \\
6\end{array}$ & 15.55 min & 1038.0594 & 0 \\
\hline 2 & 49.8007 & $\begin{array}{l}31075.058 \\
4\end{array}$ & 17.47 min & 921.1193 & 0 \\
\hline
\end{tabular}




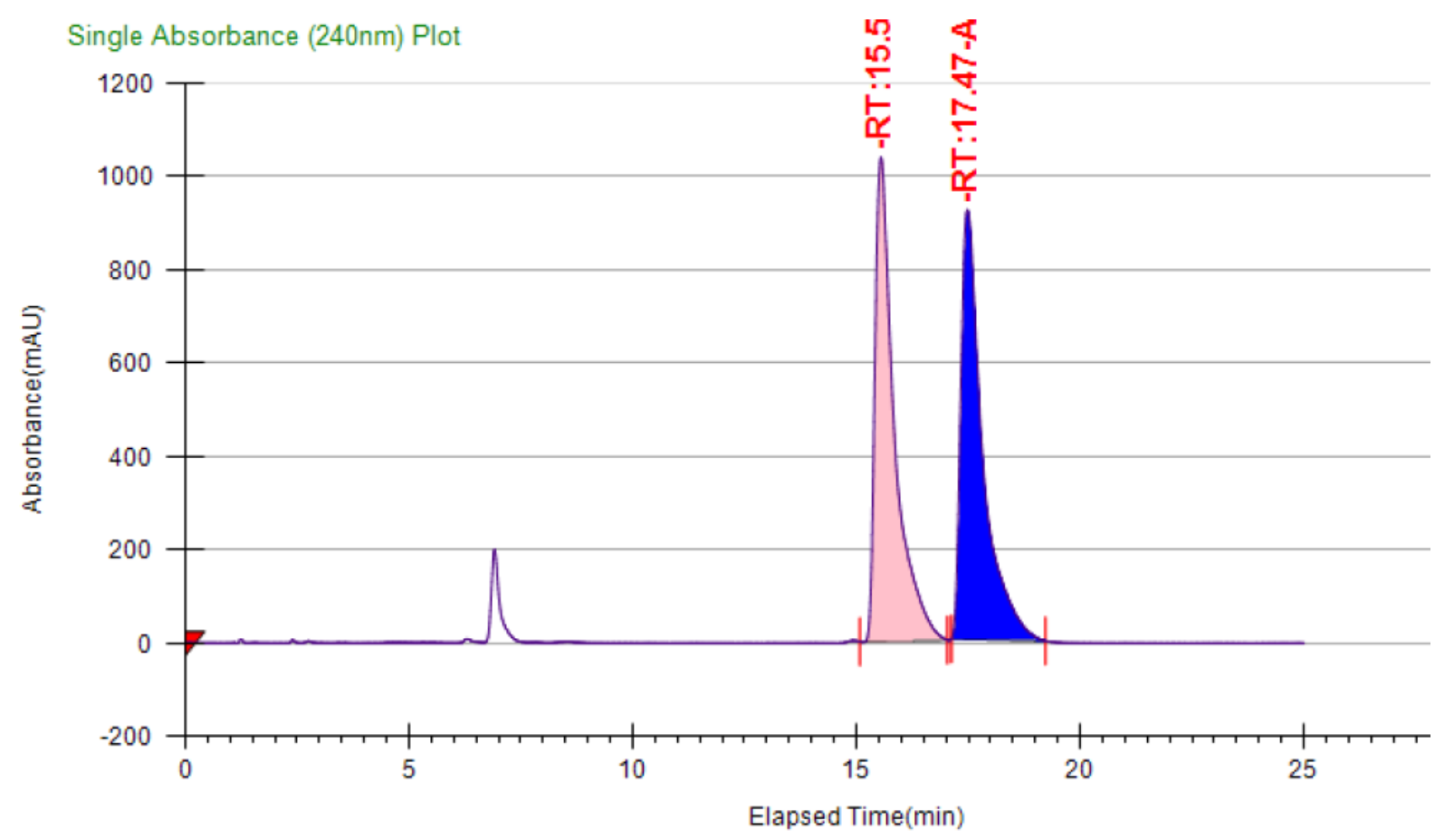

\begin{tabular}{|l|l|l|l|l|l|l|l|l|l|}
\hline Instrument Method & Inj. Vol. & Solvent & Column & Sample & Well Location & Temp. & Flow & $\%$ Modifier & Pressure \\
\hline $6 \mathrm{pp}$ methanol & 10 & $\mathrm{MeOH}$ & $\mathrm{IB}$ & $\mathrm{kwq}-\mathrm{C}-1-600 \mathrm{C}$ & $16 \mathrm{C}$ & 29.8 & 4 & 6 & 150 \\
\hline \hline Peak No & $\%$ Area & Area & Ret. Time & Height & Cap. Factor \\
\hline 1 & 91.1897 & $\begin{array}{l}21603.674 \\
5\end{array}$ & $15.52 \mathrm{~min}$ & 730.1703 & 0 \\
\hline 2 & 8.8103 & 2087.235 & $17.65 \mathrm{~min}$ & 81.1118 & 0 & \\
\hline
\end{tabular}

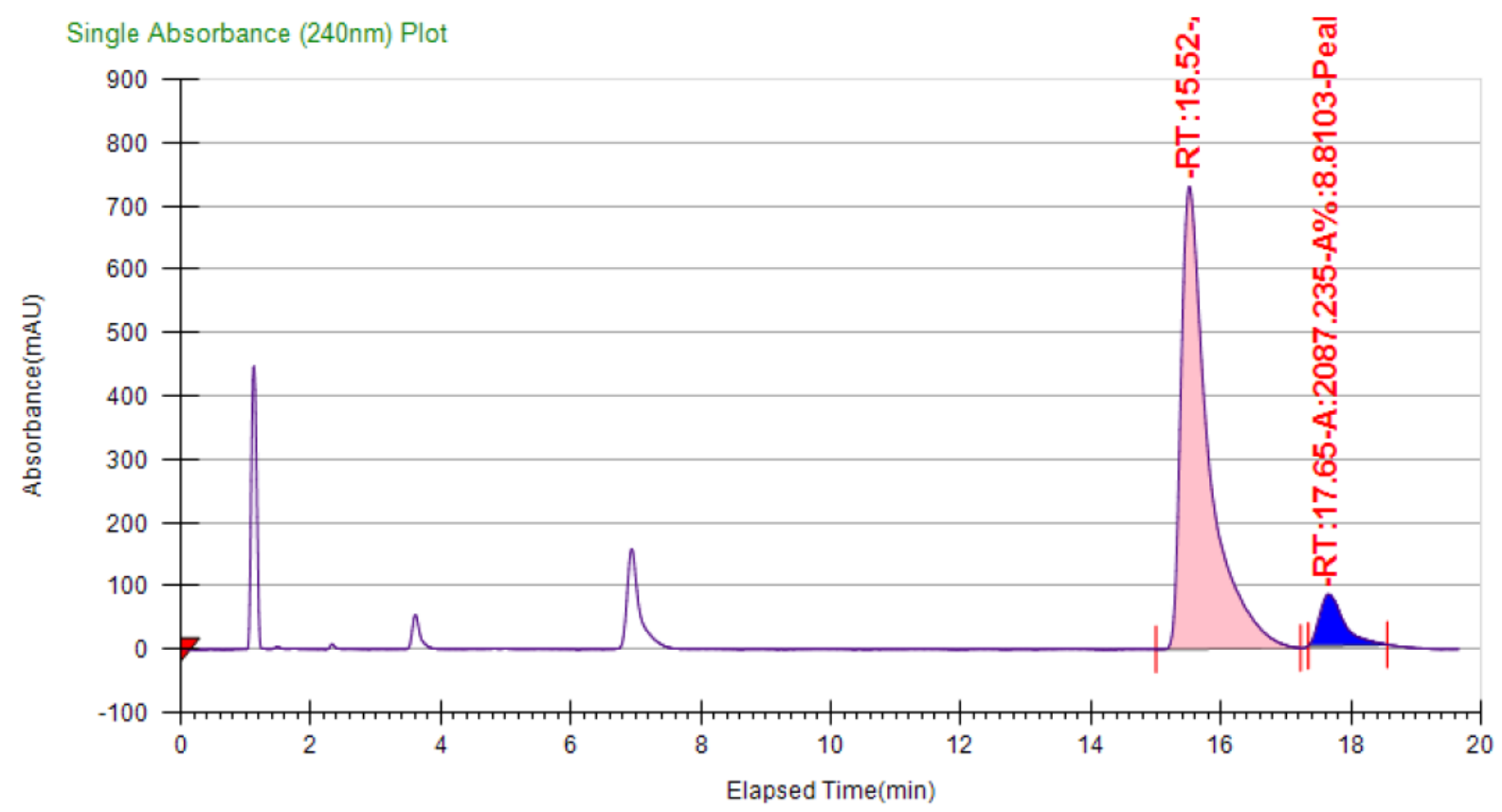


3-((5-(Biphenyl-4-yl)-1,3,4-oxadiazol-2-yl)methyl)-5-bromo-1,3-dimethylindolin-2-one [(R)-6]

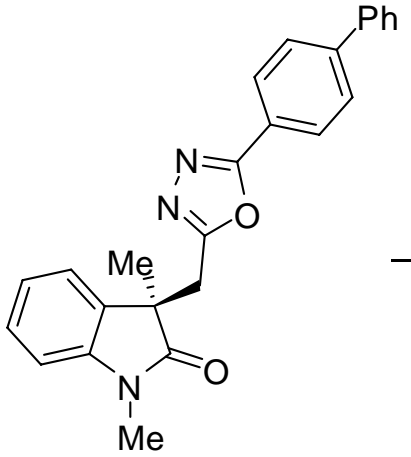

3ad $(99 \%$ ee)

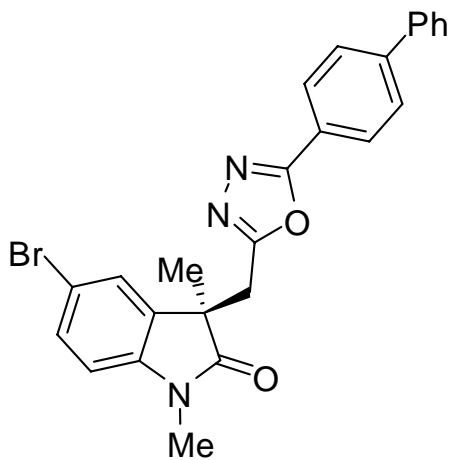

(R)-6 (94\% yield, 98\% ee)

To a suspension of $3 \mathrm{ad}(39.0 \mathrm{mg}, 0.1 \mathrm{mmol}, 94 \%$ ee) in MeCN $(5 \mathrm{~mL})$ was added NBS (27.8 $\mathrm{mg}, 0.15 \mathrm{mmol}$ ) at room temperature, and the reaction mixture was stirred at the same temperature overnight. The solvent was removed under vacuum, the residue was purified by flash chromatography on silica gel, eluting with ethyl acetate/petroleum ether 1:3 1:1 (v/v), to afford $(R)-6$ ( $44.6 \mathrm{mg}, 94 \%$ yield, $98 \%$ ee) as a white solid.

Molecular formula: $\mathrm{C}_{25} \mathrm{H}_{20} \mathrm{BrN}_{3} \mathrm{O}_{2}$; Molecular Weight: 474.3492

b.p. $143{ }^{\circ} \mathrm{C} ;{ }^{1} \mathrm{H}$ NMR (400 MHz, $\mathrm{CDCl}_{3}$ ): $\delta 7.97$ (d, J=8.3 Hz, 2H), $7.69(\mathrm{~d}, J=8.3 \mathrm{~Hz}, 2 \mathrm{H}), 7.62$ (d, $J=7.3 \mathrm{~Hz}, 2 \mathrm{H}$ ), 7.47 (t, $J=7.5 \mathrm{~Hz}, 2 \mathrm{H}), 7.40-7.38(\mathrm{~m}, 3 \mathrm{H}), 6.70$ (d, J=8.8 Hz, 1H), 3.44 (s, 2H), $3.23(\mathrm{~s}, 3 \mathrm{H}), 1.55$ (s, 3H); ${ }^{13} \mathrm{C}$ NMR (101 MHz, $\left.\mathrm{CDCl}_{3}\right): \delta$ 178.2, 164.7, 162.7, 144.5, 142.3, 139.8, 133.8, 131.5, 129.0, 128.2, 127.7, 127.2, 127.1, 126.5, 122.4, 115.4, 109.8, 46.9, 33.2, 26.6, 23.6; IR: $v\left(\mathrm{~cm}^{-1}\right)$ 2970, 2931, 1716, 1608, 1485, 1345, 1241, 1087, 909, 848; HRMS: (ESI) calcd for $\mathrm{C}_{25} \mathrm{H}_{20} \mathrm{~N}_{3} \mathrm{O}_{2} \mathrm{Br}{ }^{79} \mathrm{Na}[\mathrm{M}+\mathrm{Na}]^{+}$496.0637; found 496.0637; $\mathrm{C}_{25} \mathrm{H}_{20} \mathrm{~N}_{3} \mathrm{O}_{2} \mathrm{Br}^{81} \mathrm{Na}[\mathrm{M}+\mathrm{Na}]^{+}$ 498.0616; found 498.0620 .

SFC: $A D-H$ column, $30 \mathrm{ppm} \mathrm{MeOH}$ in supercritical $\mathrm{CO}_{2}$ as eluent, $4 \mathrm{~mL} / \mathrm{min}$. $\mathrm{tR}=7 \mathrm{~min}$ (major), $21 \mathrm{~min}$ (minor);

$[\alpha]_{D}^{27}=193\left(c=0.9, \mathrm{CHCl}_{3}\right)$ for $98 \%$ ee.

\begin{tabular}{|l|l|l|l|l|l|l|l|l|l|}
\hline Instrument Method & Inj. Vol. & Solvent & Column & Sample & Well Location & Temp. & Flow & $\%$ Modifier & Pressure \\
\hline 30p methanol & 5 & MeOH & AD-H & $\begin{array}{l}\text { Unknown } \\
\text { Sample }\end{array}$ & $16 \mathrm{~A}$ & 30 & 4 & 30 & 150 \\
\hline
\end{tabular}




\begin{tabular}{|l|l|l|l|l|l|}
\hline Peak No & $\%$ Area & Area & Ret. Time & Height & Cap. Factor \\
\hline 1 & 50.0832 & 2358.734 & $7.97 \mathrm{~min}$ & 54.9489 & 0 \\
\hline 2 & 49.9168 & 2350.8972 & $21.55 \mathrm{~min}$ & 21.4586 & 0 \\
\hline
\end{tabular}
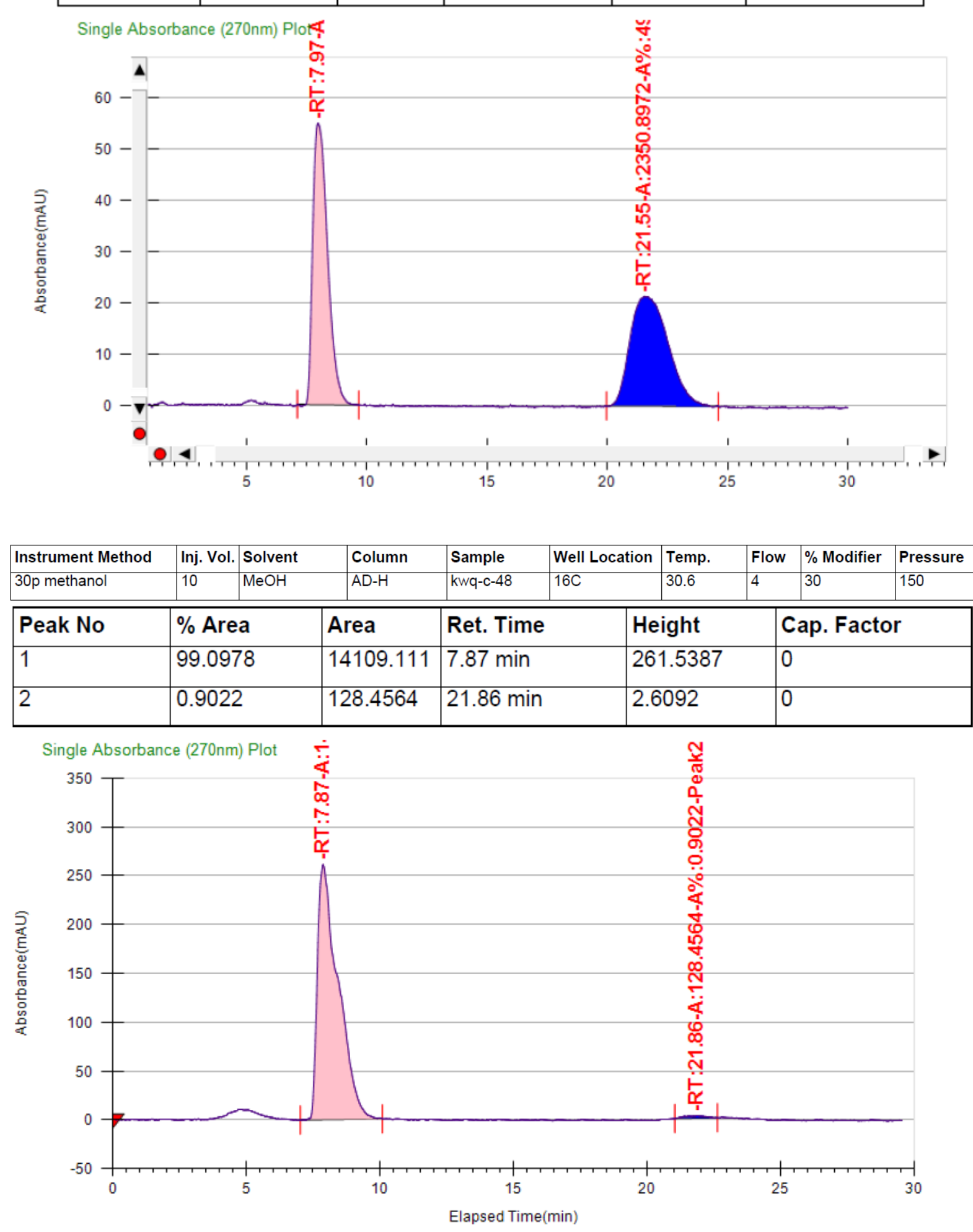
General procedure for the reductive cyclization by LAH:

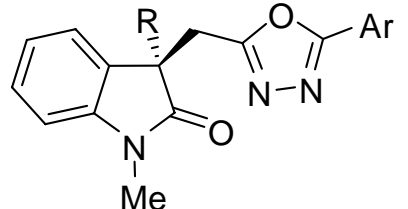

3

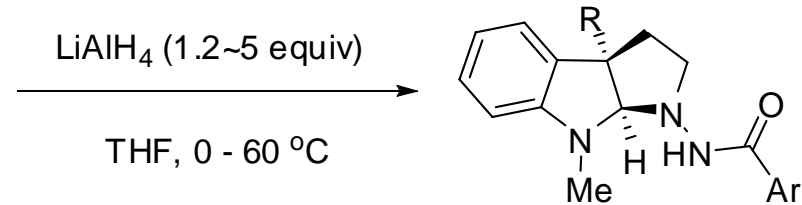

9

To a solution of $3(0.1 \mathrm{mmol})$ in THF $(2 \mathrm{~mL})$ was added a solution of $\mathrm{LiAlH}_{4}$ in THF $(2.4 \mathrm{M}, 1.2$ $\sim 5$ equiv) at $0{ }^{\circ} \mathrm{C}$. After stirring at $60{ }^{\circ} \mathrm{C}$ for $1 \sim 3 \mathrm{~h}$, the reaction mixture was quenched by pouring into ice water $(40 \mathrm{~mL})$, extracted with diethyl ether $(15 \mathrm{~mL})$ three times. The combined organic phases were dried with anhydrous $\mathrm{Na}_{2} \mathrm{SO}_{4}$. After evaporation of the solvent, the residue was purified by flash column chromatography on silica gel, eluting with ethyl acetate/petroleum ether 1:3 1:1 (v/v), to afford pyrrolidinoindoline 9. 
$N$-((3aS,8aR)-3a,8-dimethyl-3,3a,8,8a-tetrahydropyrrolo[2,3-b]indol-1(2H)-yl)benzamide [(R)9aa]

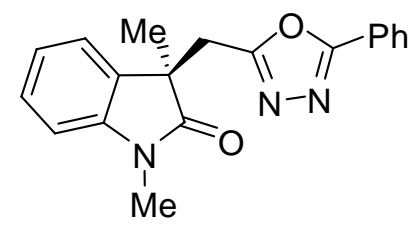

(R)-3aa (94\% ee)

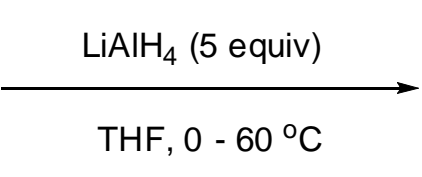

(R)-9aa (81\% yield, 92\% ee)

Molecular formula: $\mathrm{C}_{19} \mathrm{H}_{21} \mathrm{~N}_{3} \mathrm{O}$; Molecular Weight: 307.3895

According to general procedure, $(R)-9$ aa was obtained in $81 \%$ yield $(24.9 \mathrm{mg})$ and $92 \%$ ee as a white solid. b.p. $85{ }^{\circ} \mathrm{C} ;{ }^{1} \mathrm{H}$ NMR $\left(400 \mathrm{MHz}, \mathrm{CDCl}_{3}\right): \delta 7.81(\mathrm{~d}, J=7.3 \mathrm{~Hz}, 2 \mathrm{H}), 7.57-7.30(\mathrm{~m}$, $4 \mathrm{H}), 7.15(\mathrm{t}, J=7.6 \mathrm{~Hz}, 1 \mathrm{H}), 7.06(\mathrm{~d}, J=7.2 \mathrm{~Hz}, 1 \mathrm{H}), 6.82(\mathrm{t}, J=7.3 \mathrm{~Hz}, 1 \mathrm{H}), 6.59(\mathrm{~d}, J=7.8 \mathrm{~Hz}$, $1 \mathrm{H}), 4.65(\mathrm{~s}, 1 \mathrm{H}), 3.36(\mathrm{t}, J=6.8 \mathrm{~Hz}, 1 \mathrm{H}), 3.00(\mathrm{~s}, 3 \mathrm{H}), 2.72-2.60(\mathrm{~m}, 1 \mathrm{H}), 2.31-2.14(\mathrm{~m}, 1 \mathrm{H})$, $2.01(\mathrm{dd}, J=11.8,4.7 \mathrm{~Hz}, 1 \mathrm{H}), 1.54(\mathrm{~s}, 3 \mathrm{H}) ;{ }^{13} \mathrm{C} \mathrm{NMR}\left(101 \mathrm{MHz}, \mathrm{CDCl}_{3}\right): \delta$ 166.0, 151.6, 137.1, 133.6, 131.8, 128.7, 128.2, 127.1, 122.6, 119.9, 109.0, 95.0, 53.0, 51.9, 38.6, 38.2, 27.3; IR: $v$ $\left(\mathrm{cm}^{-1}\right)$ 3240, 3056, 2958, 2866, 1650, 1606, 1491, 1297, 1022, 910; HRMS: (ESI) calcd for $\mathrm{C}_{19} \mathrm{H}_{21} \mathrm{~N}_{3} \mathrm{ONa}[\mathrm{M}+\mathrm{Na}]^{+}$330.1582; found 330.1582;

SFC: IA column, $10 \mathrm{ppm} \mathrm{MeOH}$ in supercritical $\mathrm{CO}_{2}$ as eluent, $4 \mathrm{~mL} / \mathrm{min}$. $\mathrm{tR}=6 \mathrm{~min}$ (major), 10 min (minor);

$[\alpha]_{D}^{28}=62(\mathrm{c}=1.1, \mathrm{MeOH})$ for $92 \%$ ee.

\begin{tabular}{|l|l|l|l|l|l|l|l|l|l|}
\hline Instrument Method & Inj. Vol. & Solvent & Column & Sample & Well Location & Temp. & Flow & $\%$ Modifier & Pressure \\
\hline 10p methanol & 10 & MeOH & IA & kwq-c-50 & $16 \mathrm{D}$ & 30.8 & 4 & 10 & 150 \\
\hline Peak No & $\%$ Area & Area & Ret. Time & Height & Cap. Factor \\
\hline 1 & 49.4889 & 3838.983 & $6.21 \mathrm{~min}$ & 240.4924 & 0 & \\
\hline
\end{tabular}




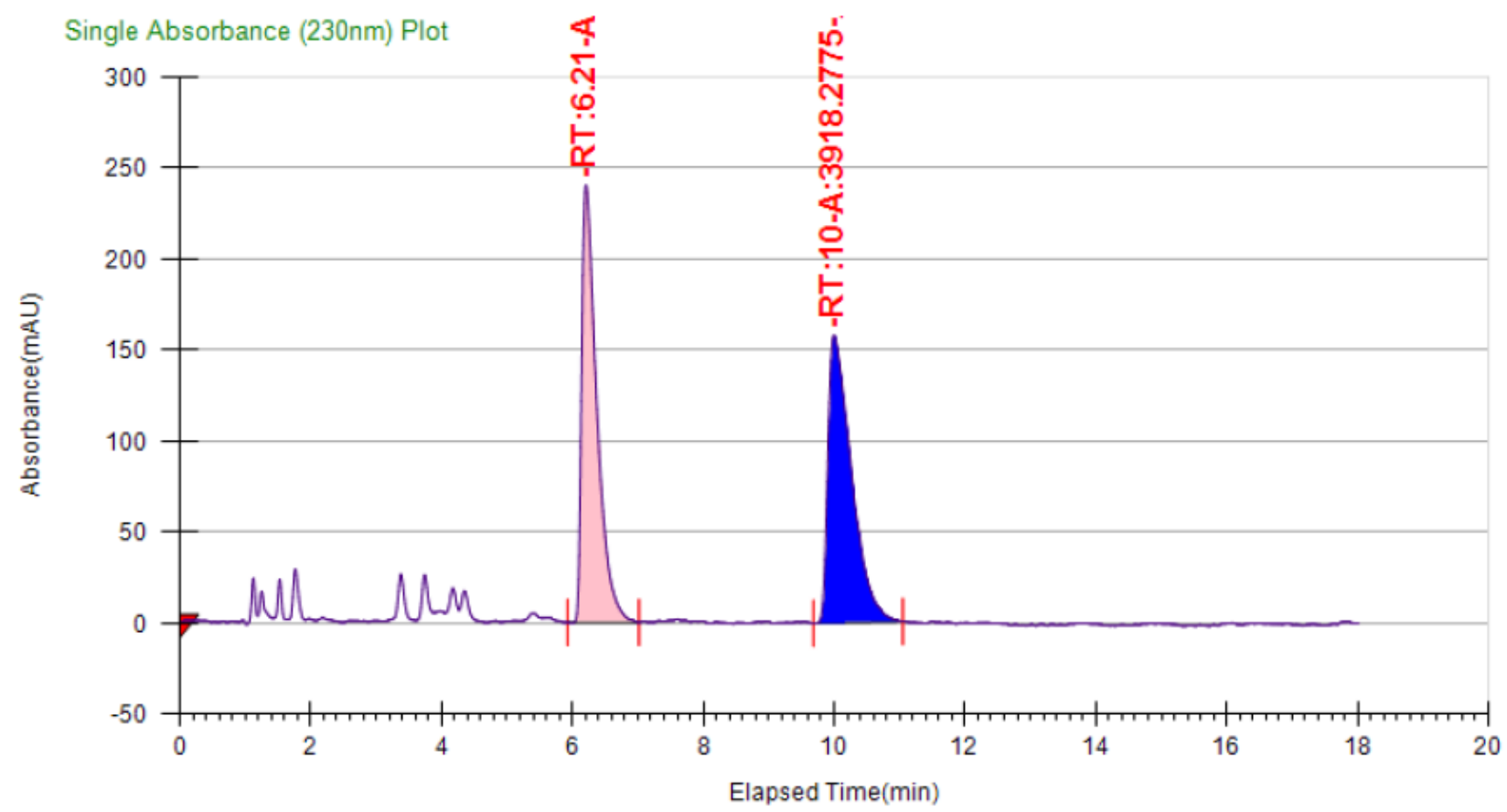

\begin{tabular}{|l|l|l|l|l|l|l|l|l|l|}
\hline Instrument Method & Inj. Vol. & Solvent & Column & Sample & Well Location & Temp. & Flow & $\%$ Modifier & Pressure \\
\hline $10 \mathrm{p}$ methanol & 10 & $\mathrm{MeOH}$ & $\mathrm{IA}$ & kwq-c-63 & $16 \mathrm{E}$ & 29.9 & 4 & 10 & 150 \\
\hline Peak No & $\%$ Area & Area & Ret. Time & Height & Cap. Factor \\
\hline 1 & 96.0196 & $\begin{array}{l}10217.781 \\
1\end{array}$ & $6.11 \mathrm{~min}$ & 572.3864 & 0 & \\
\hline 2 & 3.9804 & 423.5725 & 10.22 min & 22.6867 & 0 \\
\hline
\end{tabular}

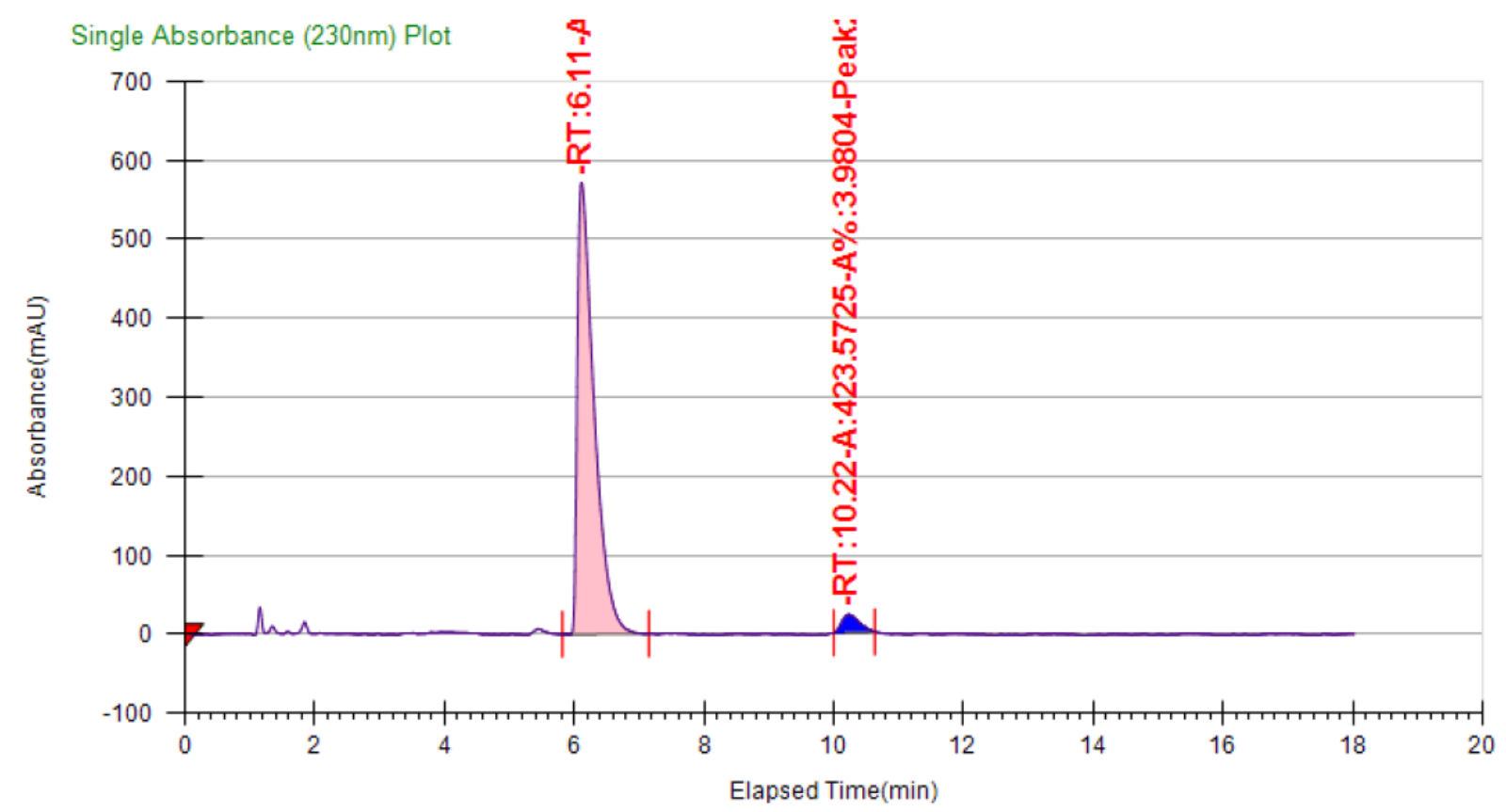


$N$-((3aR,8aR)-3a,8-dimethyl-3,3a,8,8a-tetrahydropyrrolo[2,3-b]indol-1(2H)-yl-2,2,8ad3)benzamide (9aa-3D)<smiles>CN1C(=O)C(C)(Cc2nnc(-c3ccccc3)o2)c2ccccc21</smiles>

3aa

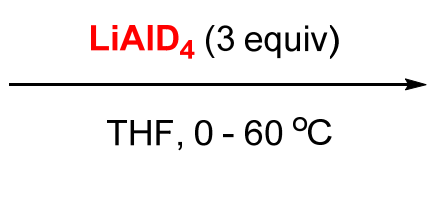

$\mathrm{THF}, 0-60^{\circ} \mathrm{C}$

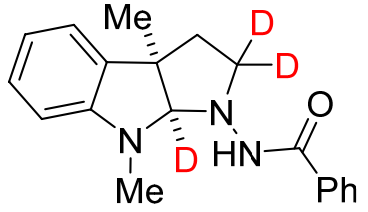

9aa $(78 \%$ yield $)$

Chemical Formula: $\mathrm{C}_{19} \mathrm{H}_{18} \mathrm{D}_{3} \mathrm{~N}_{3} \mathrm{O}$; Exact Mass: 310.1873

According to general procedure, 9aa-3D was obtained in 78\% yield $(23.9 \mathrm{mg})$ as a white solid. ${ }^{1} \mathrm{H}$ NMR $\left(400 \mathrm{MHz}, \mathrm{CDCl}_{3}\right): \delta 7.81(\mathrm{~d}, J=7.3 \mathrm{~Hz}, 2 \mathrm{H}), 7.57-7.30(\mathrm{~m}, 4 \mathrm{H}), 7.15(\mathrm{t}, J=7.6 \mathrm{~Hz}, 1 \mathrm{H})$, $7.06(\mathrm{~d}, J=7.2 \mathrm{~Hz}, 1 \mathrm{H}), 6.82(\mathrm{t}, J=7.3 \mathrm{~Hz}, 1 \mathrm{H}), 6.59$ (d, $J=7.8 \mathrm{~Hz}, 1 \mathrm{H}), 3.00(\mathrm{~s}, 3 \mathrm{H}), 2.22$ (d, $J=$ $12.0 \mathrm{~Hz}, 1 \mathrm{H}), 2.02(\mathrm{~d}, J=12.1 \mathrm{~Hz}, 1 \mathrm{H}), 1.54(\mathrm{~s}, 3 \mathrm{H}) ;{ }^{13} \mathrm{C} \mathrm{NMR}\left(101 \mathrm{MHz}, \mathrm{CDCl}_{3}\right): \delta 166.0,151.6$, 137.1, 133.6, 131.8, 128.7, 128.2, 127.1, 122.6, 119.9, 109.0, $95.0(\mathrm{t}, J=24.0 \mathrm{~Hz}), 52.5$ (quintet, $J=20.0 \mathrm{~Hz}$ ), 51.9, 38.6, 38.2, 27.3; 
N-((3aR,8aR)-3a-hexyl-8-methyl-3,3a,8,8a-tetrahydropyrrolo[2,3-b]indol-1(2H)-yl)biphenyl-4carboxamide $[(R)-9 h d]$<smiles>CN1C(=O)[C@@](Cc2nnc(-c3ccc(-c4ccccc4)cc3)o2)(c2ccccc2)c2ccccc21</smiles>

(R)-3hd (90\% ee)

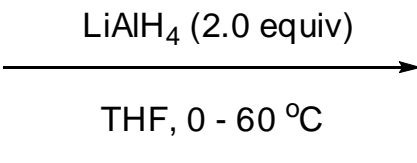

$\mathrm{THF}, 0-60^{\circ} \mathrm{C}$

Molecular formula: $\mathrm{C}_{30} \mathrm{H}_{35} \mathrm{~N}_{3} \mathrm{O}$; Molecular Weight: 453.6184

According to general procedure, $(R)-9 \mathrm{hd}$ was obtained in $71 \%$ yield $(32.1 \mathrm{mg})$ and $92 \%$ ee as a colorless oil. ${ }^{1} \mathrm{H}$ NMR $\left(400 \mathrm{MHz}, \mathrm{CDCl}_{3}\right): \delta 7.90(\mathrm{~d}, J=7.9 \mathrm{~Hz}, 2 \mathrm{H}), 7.76-7.55(\mathrm{~m}, 5 \mathrm{H}), 7.51-$ $7.35(\mathrm{~m}, 3 \mathrm{H}), 7.16(\mathrm{t}, J=7.6 \mathrm{~Hz}, 1 \mathrm{H}), 7.03(\mathrm{~d}, J=7.2 \mathrm{~Hz}, 1 \mathrm{H}), 6.84(\mathrm{t}, J=7.1 \mathrm{~Hz}, 1 \mathrm{H}), 6.61(\mathrm{~d}, J$ $=7.6 \mathrm{~Hz}, 1 \mathrm{H}), 4.72(\mathrm{~s}, 1 \mathrm{H}), 3.45-3.27(\mathrm{~m}, 1 \mathrm{H}), 2.99(\mathrm{~s}, 3 \mathrm{H}), 2.76-2.60(\mathrm{~m}, 1 \mathrm{H}), 2.35-2.17(\mathrm{~m}$, $1 \mathrm{H}), 2.06-1.92(\mathrm{~m}, 1 \mathrm{H}), 1.90-1.70(\mathrm{~m}, 2 \mathrm{H}), 1.32-1.06(\mathrm{~m}, 8 \mathrm{H}), 0.84(\mathrm{t}, J=6.5 \mathrm{~Hz}, 3 \mathrm{H}) ;{ }^{13} \mathrm{C} \mathrm{NMR}$ $\left(101 \mathrm{MHz} \mathrm{CDCl}_{3}\right): \delta 165.7,152.0,144.6,140.0,135.6,132.1,129.0,128.8,128.2,128.1$, 127.7, 127.5, 127.4, 127.3, 127.2, 127.1, 123.0, 120.4, 109.5, 92.8, 65.1, 56.3, 52.5, 39.8, 37.3, 31.7, 29.6, 25.8, 22.6, 14.1; IR: $v\left(\mathrm{~cm}^{-1}\right)$ 3264, 3256, 3255, 3240, 3230, 3049, 3030, 3029, 2955, 2927, 2869, 2868, 2855, 1649, 1607, 1606, 1543, 1487, 1467, 1449, 1378, 1326, 1297, 1122, 1022, 1008, 910, 856, 743, 697; HRMS: (ESI) calcd for $\mathrm{C}_{30} \mathrm{H}_{36} \mathrm{~N}_{3} \mathrm{O}[\mathrm{M}+\mathrm{H}]^{+} 454.2858$; found 454.2867;

SFC: IB column, $14 \mathrm{ppm} \mathrm{MeOH}$ in supercritical $\mathrm{CO}_{2}$ as eluent, $4 \mathrm{~mL} / \mathrm{min}$. $\mathrm{tR}=8 \mathrm{~min}$ (major), 10 min (minor);

$[\alpha]_{D}^{23}=-180(\mathrm{c}=1.5, \mathrm{MeOH})$ for $92 \%$ ee.

\begin{tabular}{|l|l|l|l|l|l|l|l|l|l|}
\hline Instrument Method & Inj. Vol. & Solvent & Column & Sample & Well Location & Temp. & Flow & $\%$ Modifier & Pressure \\
\hline $14 \mathrm{p}$ methanol & 15 & $\mathrm{MeOH}$ & $\mathrm{IB}$ & $\begin{array}{l}\text { Unknown } \\
\text { Sample }\end{array}$ & $16 \mathrm{~B}$ & 29.6 & 4 & 14 & 150 \\
\hline
\end{tabular}

\begin{tabular}{|l|l|l|l|l|l|}
\hline Peak No & $\%$ Area & Area & Ret. Time & Height & Cap. Factor \\
\hline 1 & 49.8204 & 3104.0161 & $8.96 \mathrm{~min}$ & 167.9157 & 0 \\
\hline 2 & 50.1796 & 3126.3969 & $10.62 \mathrm{~min}$ & 140.1772 & 0 \\
\hline
\end{tabular}




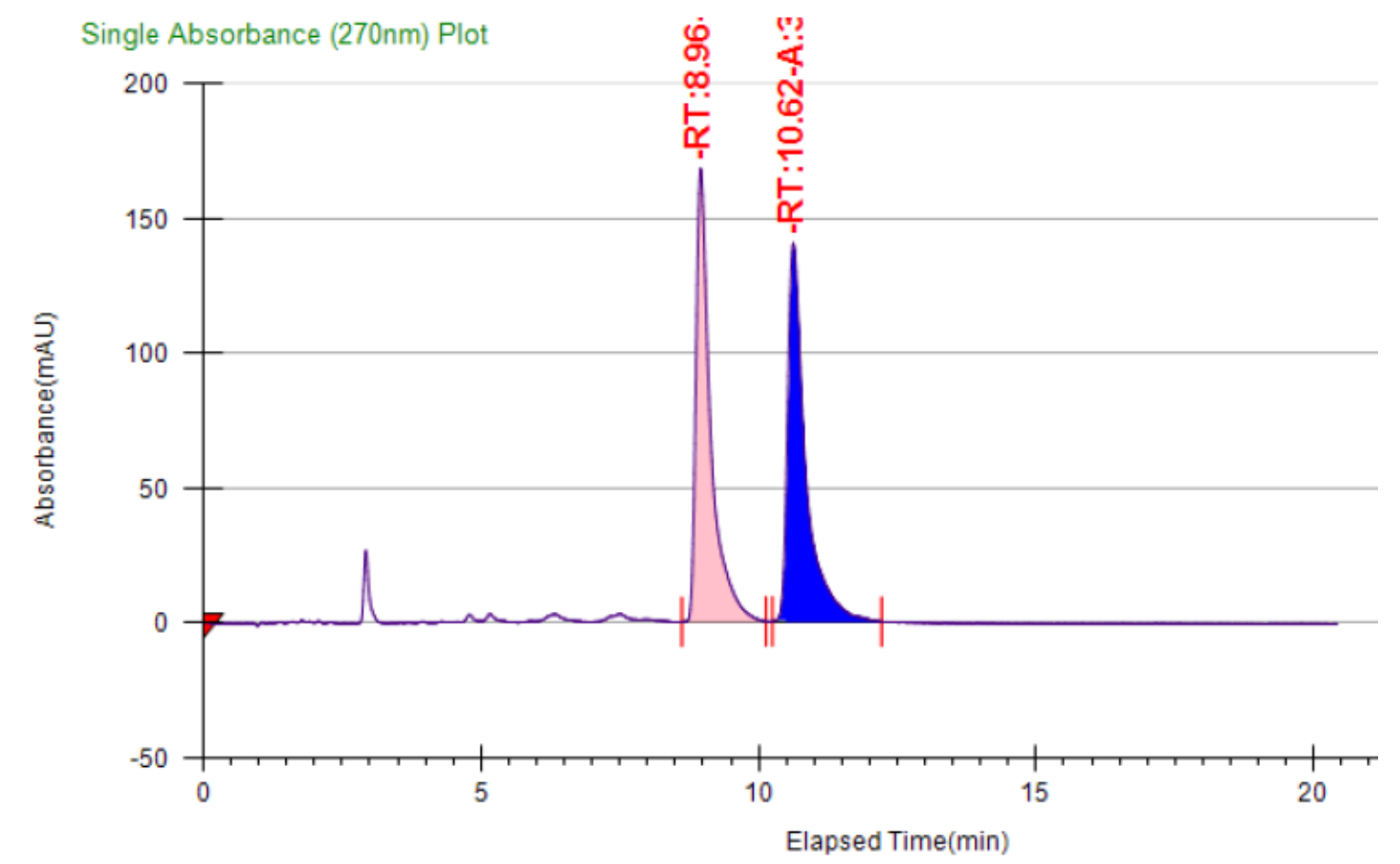

\begin{tabular}{|l|l|l|l|l|l|l|l|l|l|}
\hline Instrument Method & Inj. Vol. & Solvent & Column & Sample & Well Location & Temp. & Flow & $\%$ Modifier & Pressure \\
\hline $14 \mathrm{p}$ methanol & 10 & $\mathrm{MeOH}$ & IB & $\begin{array}{l}\text { Unknown } \\
\text { Sample }\end{array}$ & $16 \mathrm{C}$ & 29.8 & 4 & 14 & 150 \\
\hline \hline Peak No & $\%$ Area & Area & Ret. Time & Height & Cap. Factor \\
\hline 1 & 96.1551 & $\begin{array}{l}\text { 33732.199 } \\
9\end{array}$ & 8.97 min & 1465.902 & 0 \\
\hline 2 & 3.8449 & 1348.8411 & 10.76 min & 74.9669 & 0 \\
\hline
\end{tabular}

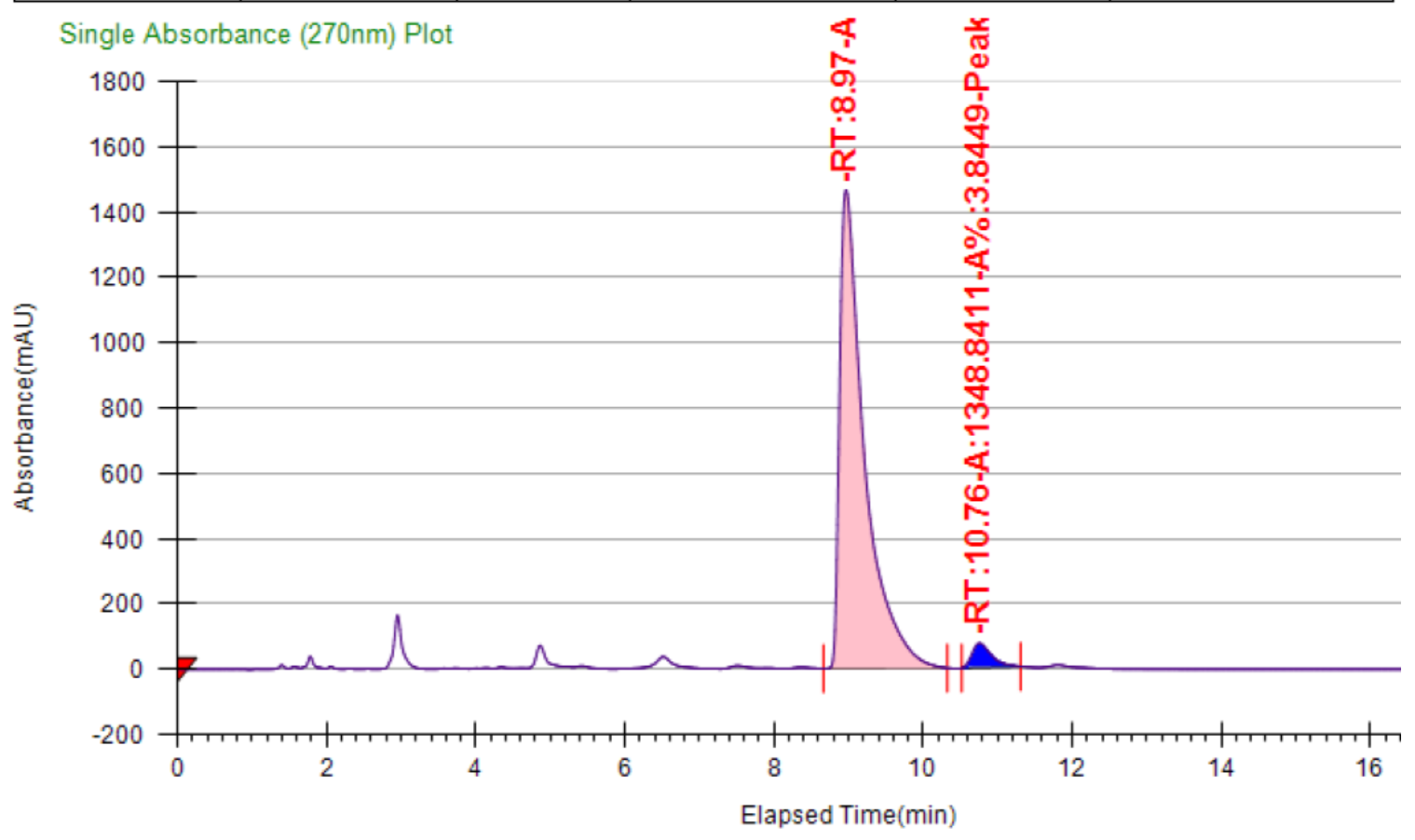


$N$-((3aS,8aR)-8-methyl-3a-phenyl-3,3a,8,8a-tetrahydropyrrolo[2,3-b]indol-1(2H)-

yl)benzamide $[(R)-9 k a]$

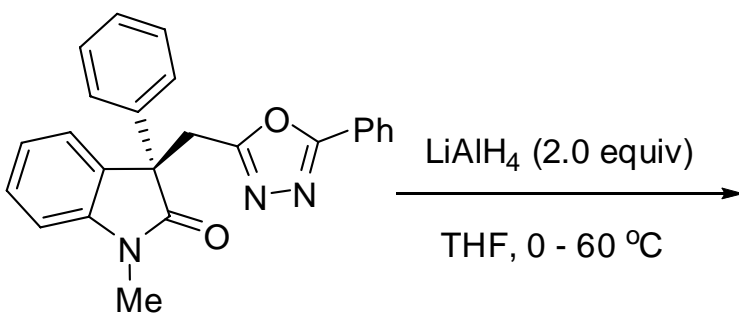

(R)-3ka (94\% ee)

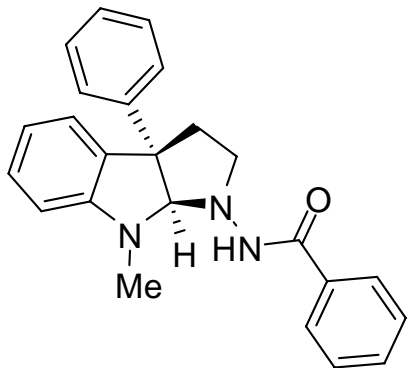

(R)-9ka (67\% yield, $98 \%$ ee)

Molecular formula: $\mathrm{C}_{24} \mathrm{H}_{23} \mathrm{~N}_{3} \mathrm{O}$; Molecular Weight: 369.4589

According to general procedure, $(R)-9$ ka was obtained in $67 \%$ yield $(24.7 \mathrm{mg}$ ) and $98 \%$ ee as a colorless oil. ${ }^{1} \mathrm{H}$ NMR $\left(400 \mathrm{MHz}, \mathrm{CDCl}_{3}\right): \delta 7.80(\mathrm{~d}, J=7.4 \mathrm{~Hz}, 2 \mathrm{H}), 7.57-7.49(\mathrm{~m}, 2 \mathrm{H}), 7.46(\mathrm{t}$, $J=7.4 \mathrm{~Hz}, 2 \mathrm{H}), 7.35-7.25(\mathrm{~m}, 4 \mathrm{H}), 7.25-7.12(\mathrm{~m}, 2 \mathrm{H}), 6.98(\mathrm{~d}, J=7.3 \mathrm{~Hz}, 1 \mathrm{H}), 6.79(\mathrm{t}, J=7.3 \mathrm{~Hz}$, $1 \mathrm{H}), 6.65(\mathrm{~d}, J=7.7 \mathrm{~Hz}, 1 \mathrm{H}), 5.02(\mathrm{~s}, 1 \mathrm{H}), 3.48(\mathrm{t}, J=7.1 \mathrm{~Hz}, 1 \mathrm{H}), 3.06(\mathrm{~s}, 3 \mathrm{H}), 3.00-2.87(\mathrm{~m}$, 1H), 2.86-2.72 (m, 1H), $2.37(\mathrm{dd}, J=11.7,4.3 \mathrm{~Hz}, 1 \mathrm{H}) ;{ }^{13} \mathrm{C} \mathrm{NMR}\left(101 \mathrm{MHz}, \mathrm{CDCl}_{3}\right): \delta$ 166.0, 152.2 , 145.3, 135.3, 133.7, 131.8, 128.7, 128.5, 128.4, 127.1, 126.6, 126.5, 124.6, 119.6, 108.9, 95.6, 60.2, 53.2, 37.7, 37.3; IR: $v\left(\mathrm{~cm}^{-1}\right)$; 3057, 2942, 1650, 1604, 1489, 1446, 1297 , 1025, 908, 728, 696; HRMS: (ESI) calcd for $\mathrm{C}_{24} \mathrm{H}_{24} \mathrm{~N}_{3} \mathrm{O}[\mathrm{M}+\mathrm{H}]^{+} 370.1919$; found 370.1919;

SFC: $\mathrm{AD}-\mathrm{H}$ column, $20 \mathrm{ppm} \mathrm{MeOH}$ in supercritical $\mathrm{CO}_{2}$ as eluent, $4 \mathrm{~mL} / \mathrm{min}$. $\mathrm{tR}=5 \mathrm{~min}$ (major), 8 min (minor);

$[\alpha]_{D}^{24}=103.0(c=7.5, \mathrm{MeOH})$ for $98 \%$ ee.

\begin{tabular}{|l|l|l|l|l|l|l|l|l|l|}
\hline Instrument Method & Inj. Vol. & Solvent & Column & Sample & Well Location & Temp. & Flow & $\%$ Modifier & Pressure \\
\hline 20p methanol & 15 & MeOH & AD-H & $\begin{array}{l}\text { Unknown } \\
\text { Sample }\end{array}$ & $16 \mathrm{~B}$ & 30 & 4 & 20 & 150 \\
\hline Peak No & $\%$ Area & Area & Ret. Time & Height & Cap. Factor \\
\hline 1 & 51.731 & 2573.267 & 5.75 min & 81.7195 & 0 & \\
\hline 2 & 48.269 & 2401.0592 & 8.32 min & 57.3272 & 0 & \\
\hline
\end{tabular}




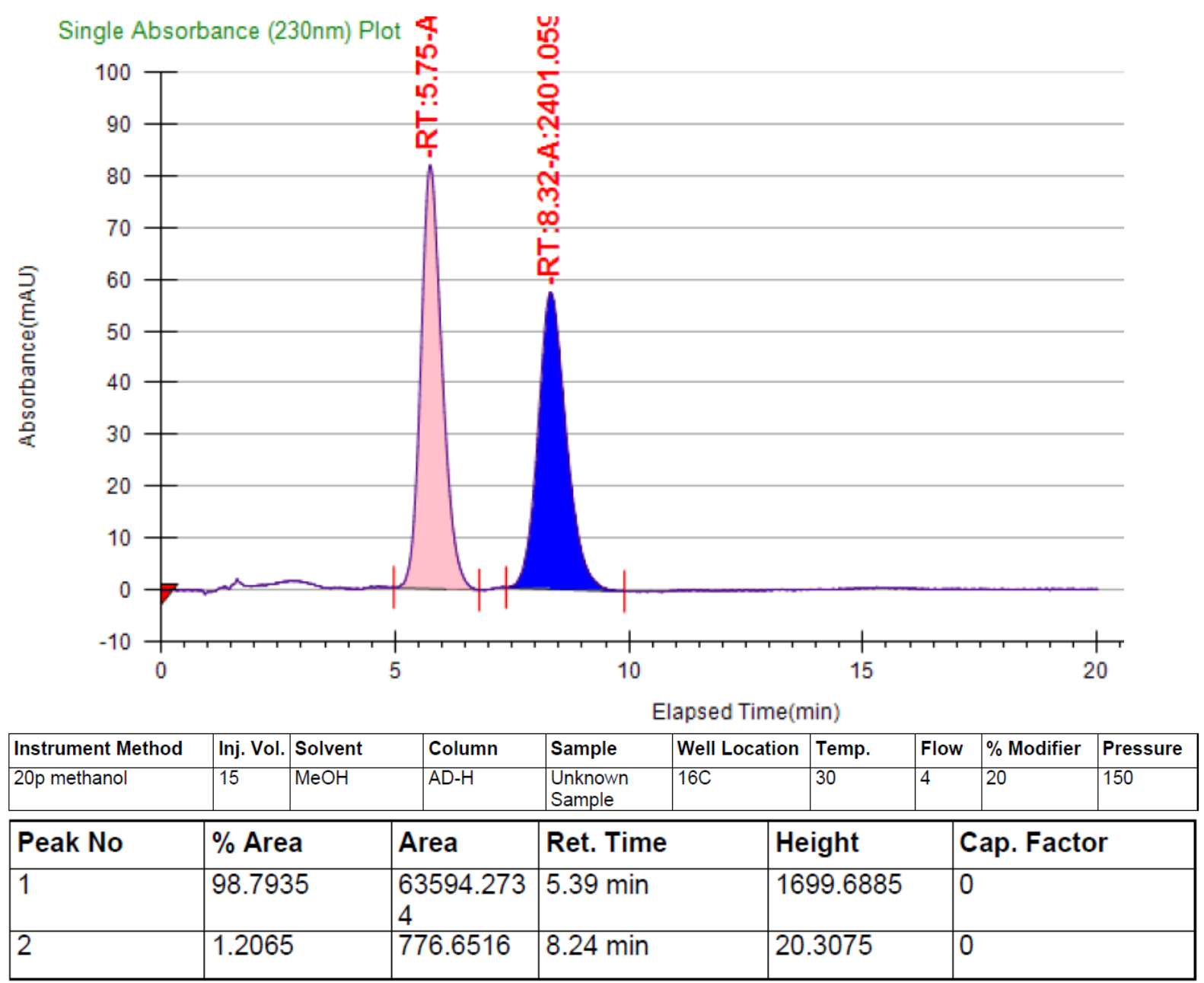

\begin{tabular}{|l|l|l|l|l|l|l|l|l|l|}
\hline Instrument Method & Inj. Vol. & Solvent & Column & Sample & Well Location & Temp. & Flow & $\%$ Modifier & Pressure \\
\hline 20p methanol & 15 & MeOH & AD-H & $\begin{array}{l}\text { Unknown } \\
\text { Sample }\end{array}$ & $16 \mathrm{C}$ & 30 & 4 & 20 & 150 \\
\hline \hline Peak No & $\%$ Area & Area & Ret. Time & Height & Cap. Factor \\
\hline 1 & 98.7935 & $\begin{array}{l}63594.273 \\
4\end{array}$ & $5.39 \mathrm{~min}$ & 1699.6885 & 0 \\
\hline 2 & 1.2065 & 776.6516 & $8.24 \mathrm{~min}$ & 20.3075 & 0 \\
\hline
\end{tabular}

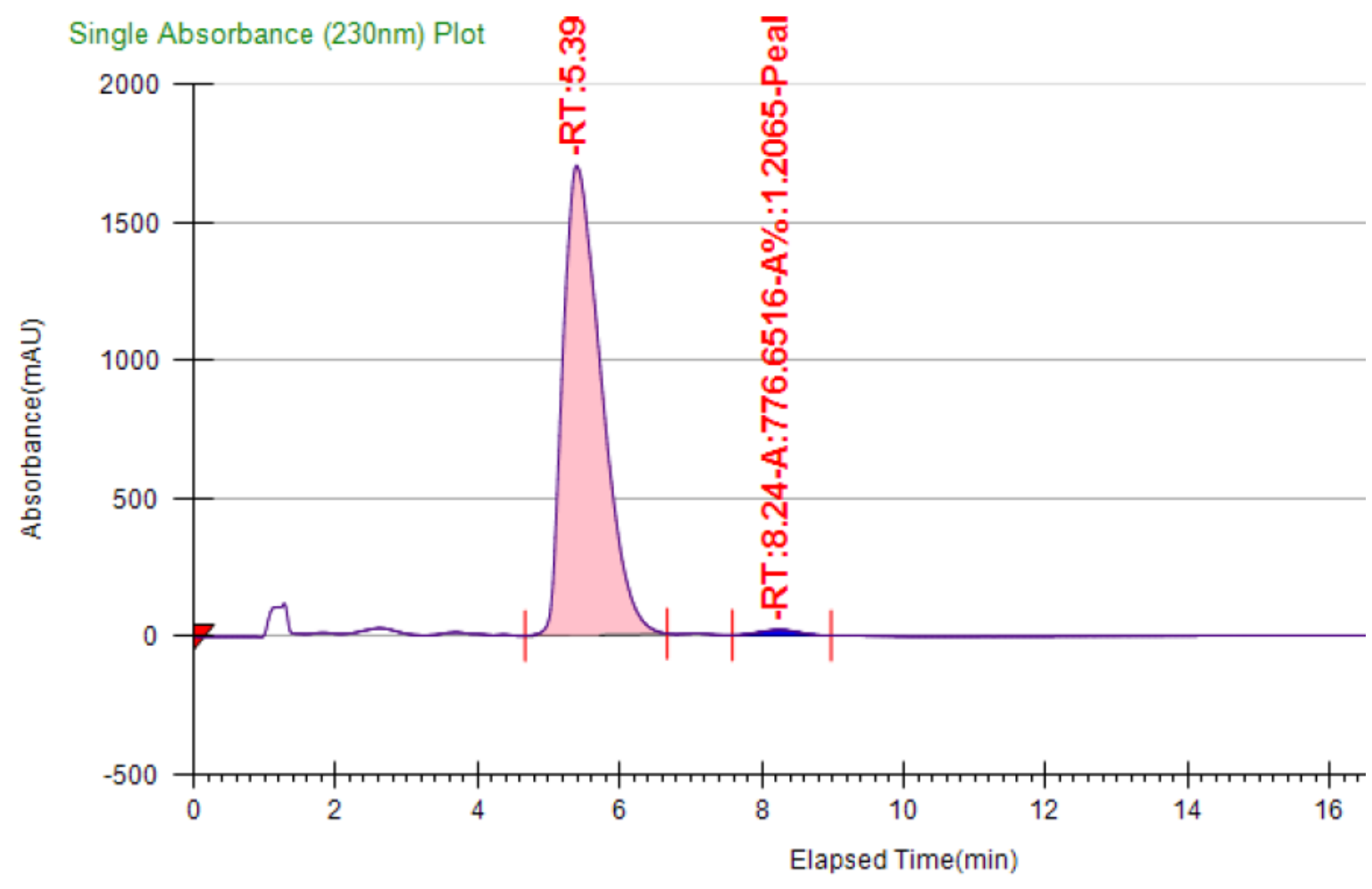


(3aR,8aS)-1,3a,8-Trimethyl-1,2,3,3a,8,8a-hexahydropyrrolo[2,3-b]indole (10)

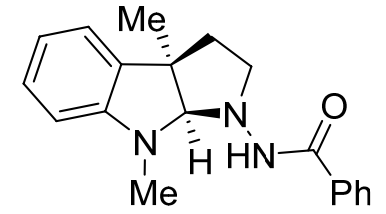

9aa

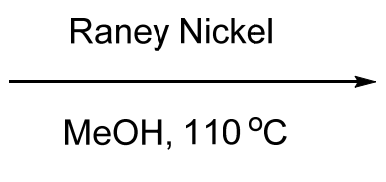

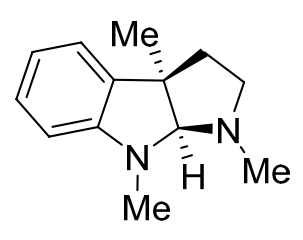

10

In a Schlenk tube fitted with a stirring bar was added a suspension of Raney-Ni in $\mathrm{H}_{2} \mathrm{O}$. After sedimentation of the nickel, supernatant water was carefully removed and the residue Nikel was washed with dry methanol twice. To the Raney-Ni ( $1.2 \mathrm{~g})$, 9aa (60 mg, $0.2 \mathrm{mmol})$ and methanol $(2 \mathrm{~mL})$ were added. After stirring at $110{ }^{\circ} \mathrm{C}$ for $21 \mathrm{~h}$, the reaction mixture was filtered and the filtrate was evaporated. Chromatography on silica gel, eluting with a $\mathrm{DCM} / \mathrm{MeOH}$ gradient, gave $10(25 \mathrm{mg}, 62 \%)$ as a colorless oil. The analytical data were in accordance with those reported in the literature. ${ }^{3}$

Molecular formula: $\mathrm{C}_{13} \mathrm{H}_{18} \mathrm{~N}_{2}$; Molecular Weight: 202.2954

${ }^{1} \mathrm{H} N M R\left(400 \mathrm{MHz}, \mathrm{CDCl}_{3}\right): \delta 7.10(\mathrm{t}, J=7.7 \mathrm{~Hz}, 1 \mathrm{H}), 7.01(\mathrm{~d}, J=7.3 \mathrm{~Hz}, 1 \mathrm{H}), 6.70(\mathrm{t}, J=7.4 \mathrm{~Hz}$, $1 \mathrm{H}), 6.45(\mathrm{~d}, J=7.8 \mathrm{~Hz}, 1 \mathrm{H}), 4.25(\mathrm{~s}, 1 \mathrm{H}), 2.97(\mathrm{~s}, 3 \mathrm{H}), 2.88-2.79(\mathrm{~m}, 1 \mathrm{H}), 2.68-2.58(\mathrm{~m}, 1 \mathrm{H})$, $2.58(\mathrm{~s}, 3 \mathrm{H}), 2.12-1.95(\mathrm{~m}, 2 \mathrm{H}), 1.46(\mathrm{~s}, 3 \mathrm{H}) ;{ }^{13} \mathrm{C} \mathrm{NMR}\left(101 \mathrm{MHz}, \mathrm{CDCl}_{3}\right)$ : $\delta$ 151.7, 136.3, 127.9, 122.3, 118.1, 106.9, 97.2, 53.2, 52.9, 40.4, 37.8, 36.8, 27.2; IR: $v\left(\mathrm{~cm}^{-1}\right) 3607,2957,2927$, $2865,2864,1606,1491,1464,1452,1346,1300,1124,1035,1022,958$, 957, 740; HRMS: (ESI) calcd for $\mathrm{C}_{13} \mathrm{H}_{18} \mathrm{~N}_{2}[\mathrm{M}+\mathrm{H}]^{+} 203.1548$; found 203.1548;

$[\alpha]_{D}^{24}=60\left(c=0.5, \mathrm{CDCl}_{3}\right)$. 


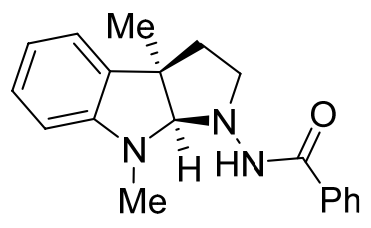

9aa

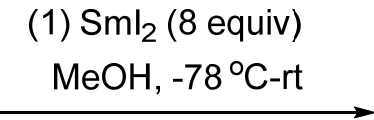

(2) $\mathrm{HCHO}, \mathrm{MeOH}$

$\mathrm{NaBH}(\mathrm{OAc})_{3}, 0^{\circ} \mathrm{C}-\mathrm{rt}$

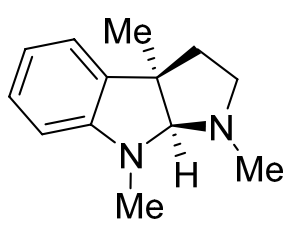

10

Compound 9aa $(0.2 \mathrm{mmol}, 61 \mathrm{mg})$ was added to a flame-dried round-bottomed flask and placed under an Ar atmosphere. Degassed anhydrous $\mathrm{MeOH}(2 \mathrm{~mL})$ was added and the solution was cooled to $-78^{\circ} \mathrm{C}$. $\mathrm{Sml}_{2}(0.1 \mathrm{M}$ in THF, 8 equiv, $8 \mathrm{~mL})$ was added dropwise until a persistent deep-green solution was obtained. The reaction mixture was allowed to warm to rt for half an hour, and quenched with saturated $\mathrm{NaHCO}_{3}$ solution $(10 \mathrm{~mL})$. The mixture was extracted with EtOAc $(15 \mathrm{~mL} \times 2)$. The combined organic layers were washed with brine (30 $\mathrm{mL} \times 2$ ), dried over $\mathrm{MgSO}_{4}$, filtered and concentrated under reduced pressure to give the crude product, which was purified by flash silica gel column chromatography, eluting with a $\mathrm{DCM} / \mathrm{MeOH}$ gradient, to give 15 (the deprotected product) $(34.0 \mathrm{mg}, 90 \%)$ as a white solid. The analytical data were in accordance with those reported in the literature. ${ }^{3}$

(3aR,8aS)-3a,8-Dimethyl-1,2,3,3a,8,8a-hexahydropyrrolo[2,3-b]indole (15)

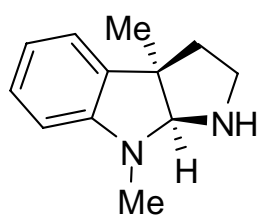

Chemical Formula: $\mathrm{C}_{12} \mathrm{H}_{16} \mathrm{~N}_{2}$; Exact Mass: 188.1313

${ }^{1} \mathrm{H}$ NMR $\left(400 \mathrm{MHz}, \mathrm{CDCl}_{3}\right): \delta 7.10(\mathrm{t}, J=7.5 \mathrm{~Hz}, 1 \mathrm{H}), 7.03(\mathrm{~d}, J=7.1 \mathrm{~Hz}, 1 \mathrm{H}), 6.69(\mathrm{t}, J=7.2 \mathrm{~Hz}$, 1H), $6.39(\mathrm{~d}, J=7.7 \mathrm{~Hz}, 1 \mathrm{H}), 4.72(\mathrm{~s}, 1 \mathrm{H}), 3.85$ (bs, 1H), 3.31-3.16 (m, 1H), $2.96(\mathrm{~s}, 3 \mathrm{H}), 2.84$ $(\mathrm{dt}, J=16.8,8.6 \mathrm{~Hz}, 1 \mathrm{H}), 2.13(\mathrm{dd}, J=12.4,5.9 \mathrm{~Hz}, 1 \mathrm{H}), 2.06-1.88(\mathrm{~m}, 1 \mathrm{H}), 1.48(\mathrm{~s}, 3 \mathrm{H}) ;{ }^{13} \mathrm{C}$ NMR $\left(101 \mathrm{MHz} \mathrm{CDCl}_{3}\right): \delta$ 150.5, 134.8, 128.2, 122.5, 117.8, 105.8, 91.6, 52.4, 45.4, 41.5, 32.7, 26.0;

To a solution of the deprotected product $15(0.2 \mathrm{mmol}, 34.0 \mathrm{mg})$ in $\mathrm{MeOH}(10 \mathrm{~mL})$ was added aqueous $\mathrm{HCHO}$ solution $(37 \%, 0.1 \mathrm{~mL}, 1 \mathrm{mmol})$ at $0{ }^{\circ} \mathrm{C}$ under Ar. After stirring for 5 minutes, $\mathrm{NaBH}(\mathrm{OAc})_{3}(212 \mathrm{mg}, 1 \mathrm{mmol})$ was added to the reaction mixture. The resulting 
solution was stirred at room temperature for 3 hours, and then treated with a saturated aqueous $\mathrm{NaHCO}_{3}$ solution at $0{ }^{\circ} \mathrm{C}$ and extracted with $\mathrm{DCM}$. The combined organic phases were washed with brine, dried over anhydrous $\mathrm{Na}_{2} \mathrm{SO}_{4}$ and evaporated under reduced pressure. The residue was purified by flash chromatography over silica gel to give desired product $10(37.0 \mathrm{mg}, 92 \%)$ as a colorless oil.

(3aS,8aS)-5-Bromo-1,3a,8-trimethyl-1,2,3,3a,8,8a-hexahydropyrrolo[2,3-b]indole (11)

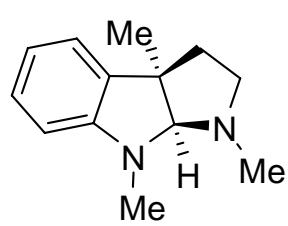

10

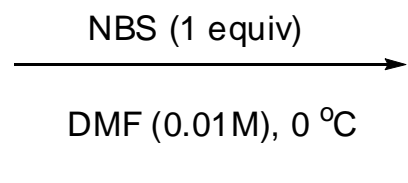

$\operatorname{DMF}(0.01 \mathrm{M}), 0{ }^{\circ} \mathrm{C}$

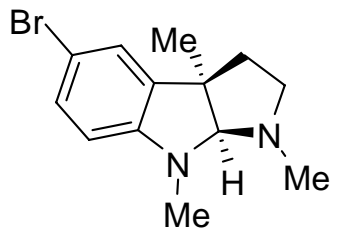

11

NBS $(0.15 \mathrm{mmol}, 26.8 \mathrm{mg})$ in DMF $(5 \mathrm{~mL})$ was added to a solution of 10 (0.15 mmol, $30.1 \mathrm{mg})$ in DMF $(10 \mathrm{~mL})$ at $0{ }^{\circ} \mathrm{C}$. The mixture was stirred at the same temperature for 2 hours. After addition of water, the mixture was extracted with ether. The combined organic extracts were washed with brine, dried over anhydrous $\mathrm{Na}_{2} \mathrm{SO}_{4}$, filtered, and evaporated. Purification by silica gel column chromatography with $\mathrm{DCM} / \mathrm{MeOH}=8 / 1$ provided 11 (34.5 $\mathrm{mg}, 82 \%$ ) as a yellow solid. The analytical data were in accordance with those reported in the literature. ${ }^{3}$

Chemical Formula: $\mathrm{C}_{13} \mathrm{H}_{17} \mathrm{BrN}_{2}$; Molecular Weight: 281.1915

${ }^{1} \mathrm{H}$ NMR $\left(400 \mathrm{MHz}, \mathrm{CDCl}_{3}\right): \delta 7.14(\mathrm{dd}, J=8.3,2.0 \mathrm{~Hz}, 1 \mathrm{H}), 7.05(\mathrm{~d}, J=2.0 \mathrm{~Hz}, 1 \mathrm{H}), 6.26(\mathrm{~d}, J=$ $8.3 \mathrm{~Hz}, 1 \mathrm{H}), 4.12(\mathrm{~s}, 1 \mathrm{H}), 2.91(\mathrm{~s}, 3 \mathrm{H}), 2.78-2.69(\mathrm{~m}, 1 \mathrm{H}), 2.62(\mathrm{dd}, J=15.6,8.4 \mathrm{~Hz}, 1 \mathrm{H}), 2.54(\mathrm{~s}$, 3H), 2.01-1.88 (m, 2H), 1.41 (s, 3H); ${ }^{13} \mathrm{C}$ NMR (101 MHz, $\left.\mathrm{CDCl}_{3}\right) \delta$ 150.8, 138.9, 130.3, 125.4, $109.1,107.9,97.5,53.2,52.7,40.6,38.5,36.3,27.1$; 


\section{(+)-Esermethole}

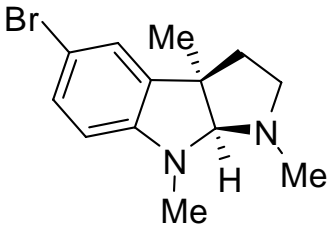

11

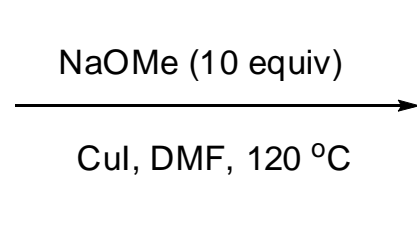

4b: (+)-Esermethole

To a suspension of $11(0.10 \mathrm{mmol}, 28.2 \mathrm{mg})$ and Cul $(0.10 \mathrm{mmol}, 20 \mathrm{mg})$ in DMF (1 mL) was added a sodium methoxide solution $(5.4 \mathrm{M}, 1.0 \mathrm{mmol}, 0.25 \mathrm{~mL})$. After stirring at $120{ }^{\circ} \mathrm{C}$ for 2 hours, the reaction mixture was cooled and the insoluble materials were filtered off. The filtrate was concentrated and $\mathrm{H}_{2} \mathrm{O}$ was added to the residue. The aqueous layer was extracted with ether and the extracts were washed with brine, dried over $\mathrm{MgSO}_{4}$, and concentrated under reduced pressure. The residue was purified by column chromatography on silica gel with $\mathrm{DCM} / \mathrm{MeOH}=5 / 1$ to give $(+)$-esermethole $(18.7 \mathrm{mg}, 81 \%)$ as white solid. The analytical data were in accordance with those reported in the literature. ${ }^{3}$

Chemical Formula: $\mathrm{C}_{14} \mathrm{H}_{20} \mathrm{~N}_{2} \mathrm{O}$; Molecular Weight: 232.3214

${ }^{1} \mathrm{H}$ NMR $\left(400 \mathrm{MHz}, \mathrm{CDCl}_{3}\right): \delta 6.75-6.57(\mathrm{~m}, 2 \mathrm{H}), 6.41(\mathrm{~d}, J=8.4 \mathrm{~Hz}, 1 \mathrm{H}), 4.24(\mathrm{~s}, 1 \mathrm{H}), 3.76(\mathrm{~s}$, $3 \mathrm{H}), 2.94(\mathrm{~s}, 3 \mathrm{H}), 2.88(\mathrm{ddd}, J=9.5,6.5,3.3 \mathrm{~Hz}, 1 \mathrm{H}), 2.69-2.60(\mathrm{~m}, 1 \mathrm{H}), 2.58(\mathrm{~s}, 3 \mathrm{H}), 2.12-$ $1.92(\mathrm{~m}, 2 \mathrm{H}), 1.48(\mathrm{~s}, 3 \mathrm{H}) ;{ }^{13} \mathrm{C}$ NMR (101 MHz, $\left.\mathrm{CDCl}_{3}\right) \delta$ 153.3, 146.1, 137.8, 112.5, 109.7, $107.9,97.8,56.0,53.1,53.0,40.4,38.2,37.3,27.2$.

$[\alpha]_{D}^{24}=134(c=0.6$, benzene). 
Crystallographic data for compounds $(R)-6$ and $(R)-9$ aa

(1) $(R)-6$

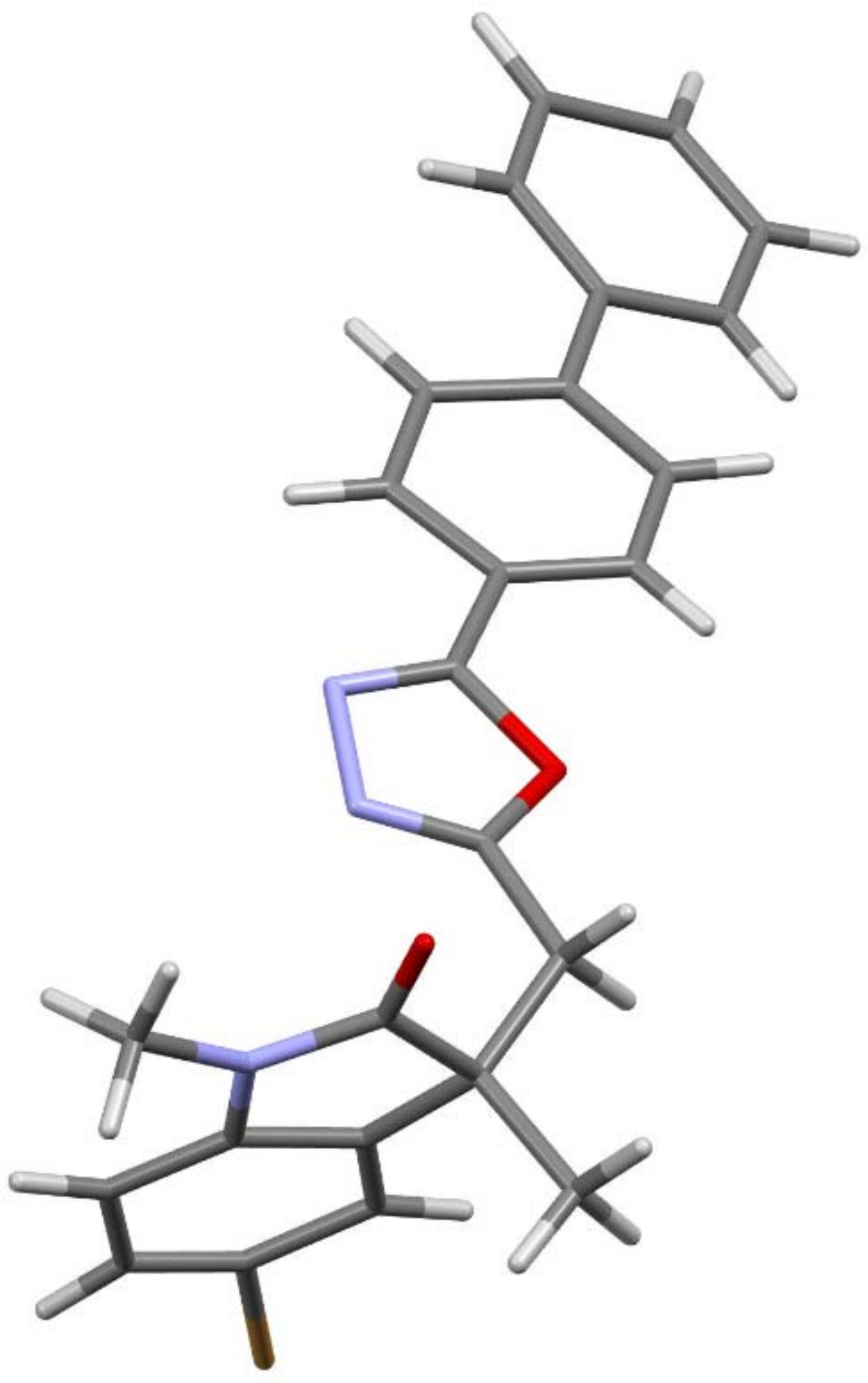


Table 1. Crystal data and structure refinement for kwq-c-48b.

Identification code

Empirical formula

Formula weight

Temperature

Wavelength

Crystal system

Space group

Unit cell dimensions

Volume

$\mathrm{Z}$

Density (calculated)

Absorption coefficient

$\mathrm{F}(000)$

Crystal size

Theta range for data collection

Index ranges

Reflections collected

Independent reflections

Completeness to theta $=27.51^{\circ}$

Absorption correction

Max. and min. transmission

Refinement method

Data / restraints / parameters

Goodness-of-fit on $\mathrm{F}^{2}$

Final R indices [I $>2 \operatorname{sigma}(\mathrm{I})]$

$\mathrm{R}$ indices (all data)

Absolute structure parameter

Extinction coefficient

Largest diff. peak and hole kwq-c-48b

$\mathrm{C}_{25} \mathrm{H}_{20} \mathrm{BrN}_{3} \mathrm{O}_{2}$

474.35

$100(2) \mathrm{K}$

$0.71073 \AA$

Monoclinic

$P 2_{1}$

$a=10.862(2) \AA \quad \alpha=90^{\circ}$.

$\mathrm{b}=8.9413(18) \AA \quad \beta=96.87(3)^{\circ}$.

$\mathrm{c}=44.757(9) \AA \quad \gamma=90^{\circ}$.

$4315.6(15) \AA^{3}$

8

$1.460 \mathrm{Mg} / \mathrm{m}^{3}$

$1.932 \mathrm{~mm}^{-1}$

1936

$0.47 \times 0.29 \times 0.16 \mathrm{~mm}^{3}$

1.37 to $27.51^{\circ}$.

$-14 \leq \mathrm{h} \leq 14,-11 \leq \mathrm{k} \leq 11,-58 \leq 1 \leq 57$

46444

$17585[\mathrm{R}(\mathrm{int})=0.0456]$

$97.1 \%$

Semi-empirical from equivalents

0.7456 and 0.5267

Full-matrix least-squares on $\mathrm{F}^{2}$

17585 / $721 / 1129$

1.157

$\mathrm{R} 1=0.0919, \mathrm{wR} 2=0.2181$

$\mathrm{R} 1=0.0993, \mathrm{wR} 2=0.2303$

0.00

$0.0039(9)$

6.281 and -0.934 e. $\AA^{-3}$ 
Table 2. Atomic coordinates ( $\times 10^{4}$ ) and equivalent isotropic displacement parameters $\left(\AA^{2} \times 10^{3}\right)$

for kwq-c-48b. $U(\mathrm{eq})$ is defined as one third of the trace of the orthogonalized $\mathrm{U}^{\mathrm{ij}}$ tensor.

\begin{tabular}{|c|c|c|c|c|}
\hline & $\mathrm{x}$ & $\mathrm{y}$ & $\mathrm{z}$ & $\mathrm{U}(\mathrm{eq})$ \\
\hline $\operatorname{Br}(1)$ & $5244(2)$ & $687(2)$ & 1391(1) & $47(1)$ \\
\hline $\operatorname{Br}(2)$ & $6745(1)$ & $-2506(1)$ & $-43(1)$ & $36(1)$ \\
\hline $\operatorname{Br}(3)$ & $-1189(1)$ & $-21809(1)$ & $-6402(1)$ & $35(1)$ \\
\hline $\operatorname{Br}(4)$ & $1827(1)$ & $-18729(1)$ & $-4959(1)$ & $28(1)$ \\
\hline $\mathrm{O}(1)$ & $560(8)$ & $-3514(12)$ & $267(2)$ & $39(2)$ \\
\hline $\mathrm{O}(2)$ & $1946(6)$ & $-5401(8)$ & $1266(2)$ & $15(1)$ \\
\hline $\mathrm{O}(3)$ & $338(8)$ & $-2128(10)$ & $-981(2)$ & $35(2)$ \\
\hline $\mathrm{O}(4)$ & $2755(6)$ & $-599(8)$ & $-1238(2)$ & $14(1)$ \\
\hline $\mathrm{O}(5)$ & $-4689(7)$ & $-17697(9)$ & $-5266(2)$ & $25(2)$ \\
\hline $\mathrm{O}(6)$ & $-4458(6)$ & $-15713(7)$ & $-6250(1)$ & $13(1)$ \\
\hline $\mathrm{O}(7)$ & $-3698(7)$ & $-19042(9)$ & $-4035(2)$ & $24(2)$ \\
\hline $\mathrm{O}(8)$ & $-967(6)$ & $-20553(8)$ & $-3770(2)$ & $15(1)$ \\
\hline $\mathrm{N}(1)$ & 992(9) & $-1205(11)$ & $472(2)$ & $28(2)$ \\
\hline $\mathrm{N}(2)$ & $795(8)$ & $-3524(11)$ & $1066(2)$ & $23(2)$ \\
\hline $\mathrm{N}(3)$ & $334(9)$ & $-3952(12)$ & $1348(2)$ & $29(2)$ \\
\hline $\mathrm{N}(4)$ & 1945(9) & $-3739(13)$ & $-846(2)$ & $28(2)$ \\
\hline $\mathrm{N}(5)$ & $4019(10)$ & $951(13)$ & $-991(2)$ & $34(2)$ \\
\hline $\mathrm{N}(6)$ & $4439(11)$ & $625(14)$ & $-1263(3)$ & $38(2)$ \\
\hline $\mathrm{N}(7)$ & $-4457(7)$ & $-20007(9)$ & $-5477(2)$ & $14(2)$ \\
\hline $\mathrm{N}(8)$ & $-5375(7)$ & $-17599(10)$ & $-6051(2)$ & $16(2)$ \\
\hline $\mathrm{N}(9)$ & $-6078(7)$ & $-17154(8)$ & $-6323(2)$ & $11(1)$ \\
\hline $\mathrm{N}(10)$ & $-2125(8)$ & $-17491(11)$ & $-4158(2)$ & $19(2)$ \\
\hline $\mathrm{N}(11)$ & $127(9)$ & $-22169(12)$ & $-4009(2)$ & $28(2)$ \\
\hline $\mathrm{N}(12)$ & $826(8)$ & $-21854(11)$ & $-3729(2)$ & $23(2)$ \\
\hline $\mathrm{C}(1)$ & $2527(9)$ & $-3096(11)$ & $605(2)$ & $17(2)$ \\
\hline$C(2)$ & 2794(9) & $-1685(11)$ & $775(2)$ & $16(2)$ \\
\hline$C(3)$ & $3818(9)$ & $-1322(12)$ & $997(2)$ & $20(2)$ \\
\hline$C(4)$ & $3801(10)$ & 191(12) & $1104(2)$ & $20(2)$ \\
\hline$C(5)$ & $3009(10)$ & $1199(15)$ & $1013(2)$ & $24(2)$ \\
\hline$C(6)$ & $1907(11)$ & $854(14)$ & 791(3) & $30(2)$ \\
\hline$C(7)$ & $1927(10)$ & $-585(12)$ & $685(2)$ & $20(2)$ \\
\hline$C(8)$ & $1254(10)$ & $-2697(13)$ & $432(2)$ & $24(2)$ \\
\hline
\end{tabular}




\begin{tabular}{|c|c|c|c|c|}
\hline$C(9)$ & $-84(13)$ & $-427(17)$ & $319(3)$ & $40(3)$ \\
\hline$C(10)$ & $3452(10)$ & $-3313(13)$ & $372(2)$ & $24(2)$ \\
\hline$C(11)$ & $2447(12)$ & $-4485(14)$ & 793(3) & $28(2)$ \\
\hline$C(12)$ & $1641(10)$ & $-4409(13)$ & $1029(2)$ & $21(2)$ \\
\hline$C(13)$ & $985(10)$ & $-5076(13)$ & $1438(2)$ & $21(2)$ \\
\hline$C(14)$ & $1082(11)$ & $-5974(14)$ & $1723(3)$ & $27(3)$ \\
\hline$C(15)$ & $1825(11)$ & $-7147(14)$ & $1782(3)$ & $27(2)$ \\
\hline$C(16)$ & $1805(12)$ & $-7911(14)$ & 2041(3) & $32(3)$ \\
\hline$C(17)$ & 1123(9) & $-7483(12)$ & $2279(2)$ & $16(2)$ \\
\hline$C(18)$ & $277(9)$ & $-6266(11)$ & $2200(2)$ & $14(2)$ \\
\hline$C(19)$ & 273(9) & $-5456(12)$ & $1931(2)$ & $18(2)$ \\
\hline$C(20)$ & $1173(10)$ & $-8268(12)$ & $2572(2)$ & $16(2)$ \\
\hline $\mathrm{C}(21)$ & $2170(10)$ & $-9175(13)$ & $2659(3)$ & $25(2)$ \\
\hline$C(22)$ & $2276(13)$ & $-9921(16)$ & 2944(3) & $34(3)$ \\
\hline$C(23)$ & $1454(9)$ & $-9713(11)$ & $3134(2)$ & $12(2)$ \\
\hline$C(24)$ & $314(12)$ & $-8792(16)$ & $3023(3)$ & $32(3)$ \\
\hline$C(25)$ & $262(14)$ & $-8104(18)$ & $2752(3)$ & $40(3)$ \\
\hline$C(26)$ & $2075(10)$ & $-1371(12)$ & $-602(2)$ & $21(2)$ \\
\hline$C(27)$ & $3249(10)$ & $-2215(12)$ & $-523(2)$ & $21(2)$ \\
\hline$C(28)$ & $4344(9)$ & $-1837(12)$ & $-340(2)$ & $20(2)$ \\
\hline$C(29)$ & $5243(10)$ & $-2936(13)$ & $-297(2)$ & $23(2)$ \\
\hline$C(30)$ & $5111(13)$ & $-4362(17)$ & $-430(3)$ & $38(3)$ \\
\hline $\mathrm{C}(31)$ & $3984(11)$ & $-4693(14)$ & $-616(3)$ & $28(2)$ \\
\hline$C(32)$ & $3092(9)$ & $-3653(13)$ & $-659(2)$ & $23(2)$ \\
\hline$C(33)$ & $1272(11)$ & $-2425(14)$ & $-833(3)$ & $28(2)$ \\
\hline$C(34)$ & $1440(14)$ & $-4920(17)$ & $-1051(3)$ & $40(3)$ \\
\hline$C(35)$ & $1338(10)$ & $-1208(13)$ & $-333(2)$ & $22(2)$ \\
\hline$C(36)$ & 2171(11) & 213(14) & $-738(3)$ & $27(2)$ \\
\hline$C(37)$ & $3025(10)$ & $250(12)$ & $-977(2)$ & $20(2)$ \\
\hline$C(38)$ & $3728(9)$ & $-296(12)$ & $-1393(2)$ & $17(2)$ \\
\hline$C(39)$ & $3708(9)$ & $-1056(11)$ & $-1687(2)$ & $16(2)$ \\
\hline$C(40)$ & $4664(11)$ & $-811(13)$ & $-1862(2)$ & $23(2)$ \\
\hline$C(41)$ & $4740(10)$ & $-1493(13)$ & $-2133(2)$ & $22(2)$ \\
\hline$C(42)$ & $3710(10)$ & $-2449(13)$ & $-2265(2)$ & $19(2)$ \\
\hline$C(43)$ & $2702(10)$ & $-2601(12)$ & $-2082(2)$ & $20(2)$ \\
\hline$C(44)$ & $2754(9)$ & $-1948(12)$ & $-1800(2)$ & $18(2)$ \\
\hline$C(45)$ & $3732(11)$ & $-3228(13)$ & $-2552(2)$ & $20(2)$ \\
\hline$C(46)$ & $4360(10)$ & $-2676(13)$ & $-2763(2)$ & $24(2)$ \\
\hline$C(47)$ & $4366(10)$ & $-3372(13)$ & $-3038(3)$ & $27(2)$ \\
\hline
\end{tabular}




\begin{tabular}{|c|c|c|c|c|}
\hline$C(48)$ & $3521(9)$ & $-4669(11)$ & $-3121(2)$ & $14(2)$ \\
\hline$C(49)$ & $2888(10)$ & $-5191(12)$ & $-2888(2)$ & $20(2)$ \\
\hline$C(50)$ & 2984(9) & $-4483(11)$ & $-2603(2)$ & $17(2)$ \\
\hline $\mathrm{C}(51)$ & $-3131(8)$ & $-18124(10)$ & $-5603(2)$ & $12(2)$ \\
\hline$C(52)$ & $-3011(8)$ & $-19532(10)$ & $-5786(2)$ & $9(2)$ \\
\hline $\mathrm{C}(53)$ & $-2271(8)$ & $-19859(10)$ & $-6007(2)$ & $10(2)$ \\
\hline$C(54)$ & $-2275(11)$ & $-21285(14)$ & $-6114(3)$ & $27(2)$ \\
\hline$C(55)$ & $-3072(9)$ & $-22390(13)$ & $-6018(2)$ & $19(2)$ \\
\hline$C(56)$ & $-3770(9)$ & $-22087(12)$ & $-5807(2)$ & $22(2)$ \\
\hline$C(57)$ & $-3777(9)$ & $-20612(11)$ & $-5689(2)$ & $15(2)$ \\
\hline $\mathrm{C}(58)$ & $-4216(8)$ & $-18498(11)$ & $-5433(2)$ & $15(2)$ \\
\hline $\mathrm{C}(59)$ & $-5368(11)$ & $-20862(15)$ & $-5327(3)$ & $31(2)$ \\
\hline$C(60)$ & $-1983(9)$ & $-17927(11)$ & $-5370(2)$ & $17(2)$ \\
\hline$C(61)$ & $-3319(8)$ & $-16632(9)$ & $-5782(2)$ & $8(2)$ \\
\hline$C(62)$ & $-4385(8)$ & $-16700(10)$ & $-6026(2)$ & $9(2)$ \\
\hline$C(63)$ & $-5517(8)$ & $-16039(10)$ & $-6434(2)$ & $10(2)$ \\
\hline$C(64)$ & $-5807(8)$ & $-15227(9)$ & $-6710(2)$ & $6(1)$ \\
\hline$C(65)$ & $-5158(9)$ & $-13891(12)$ & $-6752(2)$ & $23(2)$ \\
\hline$C(66)$ & $-5316(11)$ & $-13194(13)$ & $-7036(2)$ & $26(2)$ \\
\hline$C(67)$ & $-6185(8)$ & $-13702(12)$ & $-7251(2)$ & $12(2)$ \\
\hline$C(68)$ & $-6915(10)$ & $-14936(12)$ & $-7202(2)$ & $21(2)$ \\
\hline$C(69)$ & $-6741(9)$ & $-15661(12)$ & $-6931(2)$ & $18(2)$ \\
\hline$C(70)$ & $-6333(9)$ & $-12937(11)$ & $-7552(2)$ & $13(2)$ \\
\hline $\mathrm{C}(71)$ & $-5423(9)$ & $-12116(12)$ & $-7660(2)$ & $21(2)$ \\
\hline $\mathrm{C}(72)$ & $-5574(9)$ & $-11419(11)$ & $-7937(2)$ & $17(2)$ \\
\hline$C(73)$ & $-6776(9)$ & $-11505(13)$ & $-8104(2)$ & $18(2)$ \\
\hline$C(74)$ & $-7654(8)$ & $-12318(11)$ & $-8029(2)$ & $13(2)$ \\
\hline$C(75)$ & $-7484(9)$ & $-13015(10)$ & $-7744(2)$ & $13(2)$ \\
\hline$C(76)$ & $-2292(8)$ & $-19841(10)$ & $-4397(2)$ & $12(2)$ \\
\hline$C(77)$ & $-1181(8)$ & $-18998(10)$ & $-4478(2)$ & $13(2)$ \\
\hline $\mathrm{C}(78)$ & $-321(8)$ & $-19344(12)$ & $-4670(2)$ & $17(2)$ \\
\hline$C(79)$ & $583(10)$ & $-18285(13)$ & $-4714(2)$ & $23(2)$ \\
\hline$C(80)$ & $588(9)$ & $-16869(12)$ & $-4579(2)$ & $18(2)$ \\
\hline $\mathrm{C}(81)$ & $-295(10)$ & $-16492(13)$ & $-4393(2)$ & $27(2)$ \\
\hline$C(82)$ & $-1154(9)$ & $-17561(12)$ & $-4340(2)$ & $17(2)$ \\
\hline $\mathrm{C}(83)$ & $-2790(8)$ & $-18779(11)$ & $-4169(2)$ & $13(2)$ \\
\hline $\mathrm{C}(84)$ & $-2317(11)$ & $-16176(14)$ & $-3979(3)$ & $28(2)$ \\
\hline$C(85)$ & $-3285(9)$ & $-19983(12)$ & $-4673(2)$ & $20(2)$ \\
\hline$C(86)$ & $-1957(8)$ & $-21395(10)$ & $-4253(2)$ & $10(2)$ \\
\hline
\end{tabular}




\begin{tabular}{lcccc}
$C(87)$ & $-922(9)$ & $-21451(11)$ & $-4013(2)$ & $14(2)$ \\
$C(88)$ & $153(9)$ & $-20871(12)$ & $-3594(2)$ & $15(2)$ \\
$C(89)$ & $505(8)$ & $-20190(11)$ & $-3301(2)$ & $13(2)$ \\
$C(90)$ & $1637(10)$ & $-20337(13)$ & $-3137(2)$ & $22(2)$ \\
$C(91)$ & $1847(10)$ & $-19588(13)$ & $-2868(2)$ & $23(2)$ \\
$C(92)$ & $1002(8)$ & $-18699(11)$ & $-2757(2)$ & $9(2)$ \\
$C(93)$ & $-163(9)$ & $-18499(11)$ & $-2917(2)$ & $15(2)$ \\
$C(94)$ & $-478(9)$ & $-19262(11)$ & $-3194(2)$ & $16(2)$ \\
$C(95)$ & $1237(8)$ & $-17937(10)$ & $-2457(2)$ & $8(2)$ \\
$C(96)$ & $2330(9)$ & $-18264(11)$ & $-2250(2)$ & $14(2)$ \\
$C(97)$ & $2533(9)$ & $-17541(12)$ & $-1967(2)$ & $18(2)$ \\
$C(98)$ & $1783(9)$ & $-16495(12)$ & $-1908(2)$ & $18(2)$ \\
$C(99)$ & $693(11)$ & $-16075(14)$ & $-2094(3)$ & $28(3)$ \\
$C(100)$ & $489(11)$ & $-16806(14)$ & $-2366(3)$ & $26(2)$ \\
& & & & \\
\hline
\end{tabular}


Table 3. Bond lengths $[\AA]$ and angles $\left[{ }^{\circ}\right]$ for kwq-c- $48 b$.

\begin{tabular}{|c|c|}
\hline $\mathrm{Br}(1)-\mathrm{C}(4)$ & $1.955(11)$ \\
\hline $\operatorname{Br}(2)-C(29)$ & $1.912(11)$ \\
\hline $\operatorname{Br}(3)-C(54)$ & $1.907(12)$ \\
\hline $\operatorname{Br}(4)-C(79)$ & $1.879(11)$ \\
\hline $\mathrm{O}(1)-\mathrm{C}(8)$ & $1.228(15)$ \\
\hline $\mathrm{O}(2)-\mathrm{C}(12)$ & $1.391(13)$ \\
\hline $\mathrm{O}(2)-\mathrm{C}(13)$ & $1.400(12)$ \\
\hline $\mathrm{O}(3)-\mathrm{C}(33)$ & $1.174(15)$ \\
\hline $\mathrm{O}(4)-\mathrm{C}(38)$ & $1.358(12)$ \\
\hline $\mathrm{O}(4)-\mathrm{C}(37)$ & $1.395(12)$ \\
\hline $\mathrm{O}(5)-\mathrm{C}(58)$ & $1.194(12)$ \\
\hline $\mathrm{O}(6)-\mathrm{C}(62)$ & $1.329(11)$ \\
\hline $\mathrm{O}(6)-\mathrm{C}(63)$ & $1.364(11)$ \\
\hline $\mathrm{O}(7)-\mathrm{C}(83)$ & $1.234(12)$ \\
\hline $\mathrm{O}(8)-\mathrm{C}(87)$ & $1.356(11)$ \\
\hline $\mathrm{O}(8)-\mathrm{C}(88)$ & $1.397(12)$ \\
\hline $\mathrm{N}(1)-\mathrm{C}(8)$ & $1.380(15)$ \\
\hline $\mathrm{N}(1)-\mathrm{C}(7)$ & $1.421(15)$ \\
\hline $\mathrm{N}(1)-\mathrm{C}(9)$ & $1.459(17)$ \\
\hline $\mathrm{N}(2)-\mathrm{C}(12)$ & $1.238(15)$ \\
\hline $\mathrm{N}(2)-\mathrm{N}(3)$ & $1.464(13)$ \\
\hline $\mathrm{N}(3)-\mathrm{C}(13)$ & $1.266(15)$ \\
\hline $\mathrm{N}(4)-\mathrm{C}(33)$ & $1.388(16)$ \\
\hline $\mathrm{N}(4)-\mathrm{C}(32)$ & $1.418(14)$ \\
\hline $\mathrm{N}(4)-\mathrm{C}(34)$ & $1.461(17)$ \\
\hline $\mathrm{N}(5)-\mathrm{C}(37)$ & $1.257(15)$ \\
\hline $\mathrm{N}(5)-\mathrm{N}(6)$ & $1.379(15)$ \\
\hline $\mathrm{N}(6)-\mathrm{C}(38)$ & $1.228(16)$ \\
\hline$N(7)-C(57)$ & $1.379(12)$ \\
\hline $\mathrm{N}(7)-\mathrm{C}(58)$ & $1.384(12)$ \\
\hline$N(7)-C(59)$ & $1.475(14)$ \\
\hline $\mathrm{N}(8)-\mathrm{C}(62)$ & $1.337(12)$ \\
\hline $\mathrm{N}(8)-\mathrm{N}(9)$ & $1.414(11)$ \\
\hline $\mathrm{N}(9)-\mathrm{C}(63)$ & $1.297(12)$ \\
\hline $\mathrm{N}(10)-\mathrm{C}(83)$ & $1.357(13)$ \\
\hline $\mathrm{N}(10)-\mathrm{C}(82)$ & $1.411(12)$ \\
\hline $\mathrm{N}(10)-\mathrm{C}(84)$ & $1.452(14)$ \\
\hline
\end{tabular}




\begin{tabular}{|c|c|}
\hline $\mathrm{N}(11)-\mathrm{C}(87)$ & $1.307(14)$ \\
\hline $\mathrm{N}(11)-\mathrm{N}(12)$ & $1.417(13)$ \\
\hline $\mathrm{N}(12)-\mathrm{C}(88)$ & $1.332(14)$ \\
\hline$C(1)-C(2)$ & $1.484(13)$ \\
\hline$C(1)-C(11)$ & $1.507(15)$ \\
\hline$C(1)-C(8)$ & $1.545(14)$ \\
\hline $\mathrm{C}(1)-\mathrm{C}(10)$ & $1.548(14)$ \\
\hline$C(2)-C(7)$ & $1.388(14)$ \\
\hline$C(2)-C(3)$ & $1.435(13)$ \\
\hline$C(3)-C(4)$ & $1.437(16)$ \\
\hline $\mathrm{C}(3)-\mathrm{H}(3)$ & 0.9500 \\
\hline $\mathrm{C}(4)-\mathrm{C}(5)$ & $1.278(16)$ \\
\hline$C(5)-C(6)$ & $1.493(16)$ \\
\hline $\mathrm{C}(5)-\mathrm{H}(5)$ & 0.9500 \\
\hline$C(6)-C(7)$ & $1.372(16)$ \\
\hline $\mathrm{C}(6)-\mathrm{H}(6)$ & 0.9500 \\
\hline $\mathrm{C}(9)-\mathrm{H}(9 \mathrm{~A})$ & 0.9800 \\
\hline C(9)-H(9B) & 0.9800 \\
\hline C(9)-H(9C) & 0.9800 \\
\hline $\mathrm{C}(10)-\mathrm{H}(10 \mathrm{~A})$ & 0.9800 \\
\hline $\mathrm{C}(10)-\mathrm{H}(10 \mathrm{~B})$ & 0.9800 \\
\hline $\mathrm{C}(10)-\mathrm{H}(10 \mathrm{C})$ & 0.9800 \\
\hline $\mathrm{C}(11)-\mathrm{C}(12)$ & $1.451(16)$ \\
\hline $\mathrm{C}(11)-\mathrm{H}(11 \mathrm{~A})$ & 0.9900 \\
\hline $\mathrm{C}(11)-\mathrm{H}(11 \mathrm{~B})$ & 0.9900 \\
\hline$C(13)-C(14)$ & $1.500(16)$ \\
\hline$C(14)-C(15)$ & $1.330(17)$ \\
\hline$C(14)-C(19)$ & $1.430(15)$ \\
\hline$C(15)-C(16)$ & $1.350(16)$ \\
\hline $\mathrm{C}(15)-\mathrm{H}(15)$ & 0.9500 \\
\hline$C(16)-C(17)$ & $1.418(15)$ \\
\hline $\mathrm{C}(16)-\mathrm{H}(16)$ & 0.9500 \\
\hline$C(17)-C(18)$ & $1.441(14)$ \\
\hline$C(17)-C(20)$ & $1.482(14)$ \\
\hline$C(18)-C(19)$ & $1.407(13)$ \\
\hline $\mathrm{C}(18)-\mathrm{H}(18)$ & 0.9500 \\
\hline C(19)-H(19) & 0.9500 \\
\hline$C(20)-C(25)$ & $1.357(18)$ \\
\hline$C(20)-C(21)$ & $1.371(16)$ \\
\hline
\end{tabular}




\begin{tabular}{|c|c|}
\hline$C(21)-C(22)$ & $1.432(17)$ \\
\hline $\mathrm{C}(21)-\mathrm{H}(21)$ & 0.9500 \\
\hline$C(22)-C(23)$ & $1.317(16)$ \\
\hline $\mathrm{C}(22)-\mathrm{H}(22)$ & 0.9500 \\
\hline$C(23)-C(24)$ & $1.521(16)$ \\
\hline $\mathrm{C}(23)-\mathrm{H}(23)$ & 0.9500 \\
\hline$C(24)-C(25)$ & $1.354(19)$ \\
\hline $\mathrm{C}(24)-\mathrm{H}(24)$ & 0.9500 \\
\hline $\mathrm{C}(25)-\mathrm{H}(25)$ & 0.9500 \\
\hline$C(26)-C(27)$ & $1.487(15)$ \\
\hline$C(26)-C(35)$ & $1.530(14)$ \\
\hline$C(26)-C(36)$ & $1.550(16)$ \\
\hline$C(26)-C(33)$ & $1.584(16)$ \\
\hline $\mathrm{C}(27)-\mathrm{C}(28)$ & $1.403(14)$ \\
\hline$C(27)-C(32)$ & $1.423(15)$ \\
\hline$C(28)-C(29)$ & $1.383(15)$ \\
\hline $\mathrm{C}(28)-\mathrm{H}(28)$ & 0.9500 \\
\hline$C(29)-C(30)$ & $1.408(18)$ \\
\hline$C(30)-C(31)$ & $1.426(18)$ \\
\hline $\mathrm{C}(30)-\mathrm{H}(30)$ & 0.9500 \\
\hline$C(31)-C(32)$ & $1.340(17)$ \\
\hline $\mathrm{C}(31)-\mathrm{H}(31)$ & 0.9500 \\
\hline $\mathrm{C}(34)-\mathrm{H}(34 \mathrm{~A})$ & 0.9800 \\
\hline $\mathrm{C}(34)-\mathrm{H}(34 \mathrm{~B})$ & 0.9800 \\
\hline $\mathrm{C}(34)-\mathrm{H}(34 \mathrm{C})$ & 0.9800 \\
\hline $\mathrm{C}(35)-\mathrm{H}(35 \mathrm{~A})$ & 0.9800 \\
\hline $\mathrm{C}(35)-\mathrm{H}(35 \mathrm{~B})$ & 0.9800 \\
\hline $\mathrm{C}(35)-\mathrm{H}(35 \mathrm{C})$ & 0.9800 \\
\hline$C(36)-C(37)$ & $1.499(15)$ \\
\hline $\mathrm{C}(36)-\mathrm{H}(36 \mathrm{~A})$ & 0.9900 \\
\hline $\mathrm{C}(36)-\mathrm{H}(36 \mathrm{~B})$ & 0.9900 \\
\hline C(38)-C(39) & $1.480(13)$ \\
\hline$C(39)-C(44)$ & $1.356(14)$ \\
\hline$C(39)-C(40)$ & $1.390(15)$ \\
\hline$C(40)-C(41)$ & $1.370(15)$ \\
\hline $\mathrm{C}(40)-\mathrm{H}(40)$ & 0.9500 \\
\hline$C(41)-C(42)$ & $1.473(15)$ \\
\hline $\mathrm{C}(41)-\mathrm{H}(41)$ & 0.9500 \\
\hline $\mathrm{C}(42)-\mathrm{C}(43)$ & $1.449(14)$ \\
\hline
\end{tabular}




\begin{tabular}{|c|c|}
\hline$C(42)-C(45)$ & $1.463(15)$ \\
\hline C(43)-C(44) & $1.388(13)$ \\
\hline $\mathrm{C}(43)-\mathrm{H}(43)$ & 0.9500 \\
\hline C(44)-H(44) & 0.9500 \\
\hline$C(45)-C(46)$ & $1.327(15)$ \\
\hline$C(45)-C(50)$ & $1.389(16)$ \\
\hline $\mathrm{C}(46)-\mathrm{C}(47)$ & $1.377(15)$ \\
\hline C(46)-H(46) & 0.9500 \\
\hline $\mathrm{C}(47)-\mathrm{C}(48)$ & $1.498(15)$ \\
\hline $\mathrm{C}(47)-\mathrm{H}(47)$ & 0.9500 \\
\hline C(48)-C(49) & $1.395(13)$ \\
\hline $\mathrm{C}(48)-\mathrm{H}(48)$ & 0.9500 \\
\hline$C(49)-C(50)$ & $1.418(13)$ \\
\hline $\mathrm{C}(49)-\mathrm{H}(49)$ & 0.9500 \\
\hline $\mathrm{C}(50)-\mathrm{H}(50)$ & 0.9500 \\
\hline$C(51)-C(58)$ & $1.514(12)$ \\
\hline$C(51)-C(52)$ & $1.516(12)$ \\
\hline$C(51)-C(60)$ & $1.538(12)$ \\
\hline$C(51)-C(61)$ & $1.557(12)$ \\
\hline$C(52)-C(53)$ & $1.378(11)$ \\
\hline$C(52)-C(57)$ & $1.380(13)$ \\
\hline$C(53)-C(54)$ & $1.362(16)$ \\
\hline $\mathrm{C}(53)-\mathrm{H}(53)$ & 0.9500 \\
\hline $\mathrm{C}(54)-\mathrm{C}(55)$ & $1.415(16)$ \\
\hline$C(55)-C(56)$ & $1.309(15)$ \\
\hline $\mathrm{C}(55)-\mathrm{H}(55)$ & 0.9500 \\
\hline$C(56)-C(57)$ & $1.421(14)$ \\
\hline $\mathrm{C}(56)-\mathrm{H}(56)$ & 0.9500 \\
\hline $\mathrm{C}(59)-\mathrm{H}(59 \mathrm{~A})$ & 0.9800 \\
\hline $\mathrm{C}(59)-\mathrm{H}(59 \mathrm{~B})$ & 0.9800 \\
\hline $\mathrm{C}(59)-\mathrm{H}(59 \mathrm{C})$ & 0.9800 \\
\hline $\mathrm{C}(60)-\mathrm{H}(60 \mathrm{~A})$ & 0.9800 \\
\hline $\mathrm{C}(60)-\mathrm{H}(60 \mathrm{~B})$ & 0.9800 \\
\hline $\mathrm{C}(60)-\mathrm{H}(60 \mathrm{C})$ & 0.9800 \\
\hline$C(61)-C(62)$ & $1.497(11)$ \\
\hline $\mathrm{C}(61)-\mathrm{H}(61 \mathrm{~A})$ & 0.9900 \\
\hline $\mathrm{C}(61)-\mathrm{H}(61 \mathrm{~B})$ & 0.9900 \\
\hline$C(63)-C(64)$ & $1.434(11)$ \\
\hline$C(64)-C(69)$ & $1.385(13)$ \\
\hline
\end{tabular}




\begin{tabular}{|c|c|}
\hline$C(64)-C(65)$ & $1.411(13)$ \\
\hline$C(65)-C(66)$ & $1.410(14)$ \\
\hline $\mathrm{C}(65)-\mathrm{H}(65)$ & 0.9500 \\
\hline$C(66)-C(67)$ & $1.343(14)$ \\
\hline $\mathrm{C}(66)-\mathrm{H}(66)$ & 0.9500 \\
\hline $\mathrm{C}(67)-\mathrm{C}(68)$ & $1.392(15)$ \\
\hline $\mathrm{C}(67)-\mathrm{C}(70)$ & $1.500(13)$ \\
\hline$C(68)-C(69)$ & $1.368(14)$ \\
\hline $\mathrm{C}(68)-\mathrm{H}(68)$ & 0.9500 \\
\hline $\mathrm{C}(69)-\mathrm{H}(69)$ & 0.9500 \\
\hline$C(70)-C(71)$ & $1.365(15)$ \\
\hline$C(70)-C(75)$ & $1.432(13)$ \\
\hline$C(71)-C(72)$ & $1.380(13)$ \\
\hline $\mathrm{C}(71)-\mathrm{H}(71)$ & 0.9500 \\
\hline$C(72)-C(73)$ & $1.426(14)$ \\
\hline $\mathrm{C}(72)-\mathrm{H}(72)$ & 0.9500 \\
\hline$C(73)-C(74)$ & $1.275(14)$ \\
\hline $\mathrm{C}(73)-\mathrm{H}(73)$ & 0.9500 \\
\hline$C(74)-C(75)$ & $1.411(12)$ \\
\hline $\mathrm{C}(74)-\mathrm{H}(74)$ & 0.9500 \\
\hline $\mathrm{C}(75)-\mathrm{H}(75)$ & 0.9500 \\
\hline$C(76)-C(77)$ & $1.503(12)$ \\
\hline$C(76)-C(83)$ & $1.541(12)$ \\
\hline$C(76)-C(85)$ & $1.546(13)$ \\
\hline$C(76)-C(86)$ & $1.556(12)$ \\
\hline $\mathrm{C}(77)-\mathrm{C}(78)$ & $1.377(13)$ \\
\hline $\mathrm{C}(77)-\mathrm{C}(82)$ & $1.424(14)$ \\
\hline C(78)-C(79) & $1.395(14)$ \\
\hline $\mathrm{C}(78)-\mathrm{H}(78)$ & 0.9500 \\
\hline$C(79)-C(80)$ & $1.403(15)$ \\
\hline $\mathrm{C}(80)-\mathrm{C}(81)$ & $1.385(15)$ \\
\hline $\mathrm{C}(80)-\mathrm{H}(80)$ & 0.9500 \\
\hline $\mathrm{C}(81)-\mathrm{C}(82)$ & $1.375(16)$ \\
\hline $\mathrm{C}(81)-\mathrm{H}(81)$ & 0.9500 \\
\hline $\mathrm{C}(84)-\mathrm{H}(84 \mathrm{~A})$ & 0.9800 \\
\hline $\mathrm{C}(84)-\mathrm{H}(84 \mathrm{~B})$ & 0.9800 \\
\hline $\mathrm{C}(84)-\mathrm{H}(84 \mathrm{C})$ & 0.9800 \\
\hline $\mathrm{C}(85)-\mathrm{H}(85 \mathrm{~A})$ & 0.9800 \\
\hline $\mathrm{C}(85)-\mathrm{H}(85 \mathrm{~B})$ & 0.9800 \\
\hline
\end{tabular}




\begin{tabular}{|c|c|}
\hline $\mathrm{C}(85)-\mathrm{H}(85 \mathrm{C})$ & 0.9800 \\
\hline C(86)-C(87) & $1.461(13)$ \\
\hline $\mathrm{C}(86)-\mathrm{H}(86 \mathrm{~A})$ & 0.9900 \\
\hline $\mathrm{C}(86)-\mathrm{H}(86 \mathrm{~B})$ & 0.9900 \\
\hline C(88)-C(89) & $1.455(13)$ \\
\hline C(89)-C(90) & $1.360(15)$ \\
\hline C(89)-C(94) & $1.477(13)$ \\
\hline C(90)-C(91) & $1.374(15)$ \\
\hline C(90)-H(90) & 0.9500 \\
\hline $\mathrm{C}(91)-\mathrm{C}(92)$ & $1.353(14)$ \\
\hline C(91)-H(91) & 0.9500 \\
\hline C(92)-C(93) & $1.390(13)$ \\
\hline $\mathrm{C}(92)-\mathrm{C}(95)$ & $1.502(12)$ \\
\hline C(93)-C(94) & $1.418(13)$ \\
\hline C(93)-H(93) & 0.9500 \\
\hline C(94)-H(94) & 0.9500 \\
\hline$C(95)-C(100)$ & $1.388(15)$ \\
\hline C(95)-C(96) & $1.446(13)$ \\
\hline C(96)-C(97) & $1.414(13)$ \\
\hline C(96)-H(96) & 0.9500 \\
\hline C(97)-C(98) & $1.289(15)$ \\
\hline C(97)-H(97) & 0.9500 \\
\hline C(98)-C(99) & $1.414(16)$ \\
\hline C(98)-H(98) & 0.9500 \\
\hline C(99)-C(100) & $1.374(16)$ \\
\hline C(99)-H(99) & 0.9500 \\
\hline $\mathrm{C}(100)-\mathrm{H}(100)$ & 0.9500 \\
\hline $\mathrm{C}(12)-\mathrm{O}(2)-\mathrm{C}(13)$ & $99.3(8)$ \\
\hline $\mathrm{C}(38)-\mathrm{O}(4)-\mathrm{C}(37)$ & $102.8(7)$ \\
\hline $\mathrm{C}(62)-\mathrm{O}(6)-\mathrm{C}(63)$ & $106.5(7)$ \\
\hline $\mathrm{C}(87)-\mathrm{O}(8)-\mathrm{C}(88)$ & $102.7(7)$ \\
\hline $\mathrm{C}(8)-\mathrm{N}(1)-\mathrm{C}(7)$ & $108.9(10)$ \\
\hline $\mathrm{C}(8)-\mathrm{N}(1)-\mathrm{C}(9)$ & $124.4(11)$ \\
\hline $\mathrm{C}(7)-\mathrm{N}(1)-\mathrm{C}(9)$ & $126.7(10)$ \\
\hline $\mathrm{C}(12)-\mathrm{N}(2)-\mathrm{N}(3)$ & $106.5(9)$ \\
\hline $\mathrm{C}(13)-\mathrm{N}(3)-\mathrm{N}(2)$ & 104.2(9) \\
\hline $\mathrm{C}(33)-\mathrm{N}(4)-\mathrm{C}(32)$ & $111.0(10)$ \\
\hline $\mathrm{C}(33)-\mathrm{N}(4)-\mathrm{C}(34)$ & $118.5(10)$ \\
\hline
\end{tabular}




\begin{tabular}{|c|c|}
\hline $\mathrm{C}(32)-\mathrm{N}(4)-\mathrm{C}(34)$ & $130.4(11)$ \\
\hline $\mathrm{C}(37)-\mathrm{N}(5)-\mathrm{N}(6)$ & $108.5(11)$ \\
\hline $\mathrm{C}(38)-\mathrm{N}(6)-\mathrm{N}(5)$ & $107.7(11)$ \\
\hline $\mathrm{C}(57)-\mathrm{N}(7)-\mathrm{C}(58)$ & $111.9(8)$ \\
\hline $\mathrm{C}(57)-\mathrm{N}(7)-\mathrm{C}(59)$ & $123.6(9)$ \\
\hline $\mathrm{C}(58)-\mathrm{N}(7)-\mathrm{C}(59)$ & $124.5(9)$ \\
\hline $\mathrm{C}(62)-\mathrm{N}(8)-\mathrm{N}(9)$ & $104.4(7)$ \\
\hline $\mathrm{C}(63)-\mathrm{N}(9)-\mathrm{N}(8)$ & $108.4(7)$ \\
\hline $\mathrm{C}(83)-\mathrm{N}(10)-\mathrm{C}(82)$ & $112.0(8)$ \\
\hline $\mathrm{C}(83)-\mathrm{N}(10)-\mathrm{C}(84)$ & $126.5(8)$ \\
\hline $\mathrm{C}(82)-\mathrm{N}(10)-\mathrm{C}(84)$ & $121.5(9)$ \\
\hline $\mathrm{C}(87)-\mathrm{N}(11)-\mathrm{N}(12)$ & $106.7(9)$ \\
\hline $\mathrm{C}(88)-\mathrm{N}(12)-\mathrm{N}(11)$ & $105.5(8)$ \\
\hline $\mathrm{C}(2)-\mathrm{C}(1)-\mathrm{C}(11)$ & $115.9(8)$ \\
\hline $\mathrm{C}(2)-\mathrm{C}(1)-\mathrm{C}(8)$ & $99.8(8)$ \\
\hline $\mathrm{C}(11)-\mathrm{C}(1)-\mathrm{C}(8)$ & 111.3(9) \\
\hline$C(2)-C(1)-C(10)$ & $110.5(8)$ \\
\hline$C(11)-C(1)-C(10)$ & $111.0(9)$ \\
\hline$C(8)-C(1)-C(10)$ & 107.6(8) \\
\hline$C(7)-C(2)-C(3)$ & 118.9(9) \\
\hline$C(7)-C(2)-C(1)$ & $111.7(8)$ \\
\hline$C(3)-C(2)-C(1)$ & $129.3(9)$ \\
\hline$C(2)-C(3)-C(4)$ & $113.8(9)$ \\
\hline $\mathrm{C}(2)-\mathrm{C}(3)-\mathrm{H}(3)$ & 123.1 \\
\hline $\mathrm{C}(4)-\mathrm{C}(3)-\mathrm{H}(3)$ & 123.1 \\
\hline$C(5)-C(4)-C(3)$ & $126.3(11)$ \\
\hline$C(5)-C(4)-B r(1)$ & $120.4(9)$ \\
\hline$C(3)-C(4)-\operatorname{Br}(1)$ & $113.1(8)$ \\
\hline$C(4)-C(5)-C(6)$ & $121.4(11)$ \\
\hline $\mathrm{C}(4)-\mathrm{C}(5)-\mathrm{H}(5)$ & 119.3 \\
\hline $\mathrm{C}(6)-\mathrm{C}(5)-\mathrm{H}(5)$ & 119.3 \\
\hline$C(7)-C(6)-C(5)$ & $112.3(11)$ \\
\hline $\mathrm{C}(7)-\mathrm{C}(6)-\mathrm{H}(6)$ & 123.8 \\
\hline $\mathrm{C}(5)-\mathrm{C}(6)-\mathrm{H}(6)$ & 123.8 \\
\hline$C(6)-C(7)-C(2)$ & $127.0(11)$ \\
\hline$C(6)-C(7)-N(1)$ & $124.0(10)$ \\
\hline $\mathrm{C}(2)-\mathrm{C}(7)-\mathrm{N}(1)$ & 108.9(9) \\
\hline $\mathrm{O}(1)-\mathrm{C}(8)-\mathrm{N}(1)$ & $122.2(11)$ \\
\hline $\mathrm{O}(1)-\mathrm{C}(8)-\mathrm{C}(1)$ & $127.7(11)$ \\
\hline
\end{tabular}




\begin{tabular}{|c|c|}
\hline $\mathrm{N}(1)-\mathrm{C}(8)-\mathrm{C}(1)$ & $110.1(10)$ \\
\hline $\mathrm{N}(1)-\mathrm{C}(9)-\mathrm{H}(9 \mathrm{~A})$ & 109.5 \\
\hline N(1)-C(9)-H(9B) & 109.5 \\
\hline $\mathrm{H}(9 \mathrm{~A})-\mathrm{C}(9)-\mathrm{H}(9 \mathrm{~B})$ & 109.5 \\
\hline N(1)-C(9)-H(9C) & 109.5 \\
\hline $\mathrm{H}(9 \mathrm{~A})-\mathrm{C}(9)-\mathrm{H}(9 \mathrm{C})$ & 109.5 \\
\hline H(9B)-C(9)-H(9C) & 109.5 \\
\hline $\mathrm{C}(1)-\mathrm{C}(10)-\mathrm{H}(10 \mathrm{~A})$ & 109.5 \\
\hline $\mathrm{C}(1)-\mathrm{C}(10)-\mathrm{H}(10 \mathrm{~B})$ & 109.5 \\
\hline $\mathrm{H}(10 \mathrm{~A})-\mathrm{C}(10)-\mathrm{H}(10 \mathrm{~B})$ & 109.5 \\
\hline $\mathrm{C}(1)-\mathrm{C}(10)-\mathrm{H}(10 \mathrm{C})$ & 109.5 \\
\hline $\mathrm{H}(10 \mathrm{~A})-\mathrm{C}(10)-\mathrm{H}(10 \mathrm{C})$ & 109.5 \\
\hline $\mathrm{H}(10 \mathrm{~B})-\mathrm{C}(10)-\mathrm{H}(10 \mathrm{C})$ & 109.5 \\
\hline $\mathrm{C}(12)-\mathrm{C}(11)-\mathrm{C}(1)$ & $116.5(10)$ \\
\hline $\mathrm{C}(12)-\mathrm{C}(11)-\mathrm{H}(11 \mathrm{~A})$ & 108.2 \\
\hline $\mathrm{C}(1)-\mathrm{C}(11)-\mathrm{H}(11 \mathrm{~A})$ & 108.2 \\
\hline $\mathrm{C}(12)-\mathrm{C}(11)-\mathrm{H}(11 \mathrm{~B})$ & 108.2 \\
\hline $\mathrm{C}(1)-\mathrm{C}(11)-\mathrm{H}(11 \mathrm{~B})$ & 108.2 \\
\hline $\mathrm{H}(11 \mathrm{~A})-\mathrm{C}(11)-\mathrm{H}(11 \mathrm{~B})$ & 107.3 \\
\hline $\mathrm{N}(2)-\mathrm{C}(12)-\mathrm{O}(2)$ & $114.8(9)$ \\
\hline $\mathrm{N}(2)-\mathrm{C}(12)-\mathrm{C}(11)$ & $130.4(11)$ \\
\hline $\mathrm{O}(2)-\mathrm{C}(12)-\mathrm{C}(11)$ & $114.5(10)$ \\
\hline $\mathrm{N}(3)-\mathrm{C}(13)-\mathrm{O}(2)$ & $114.5(9)$ \\
\hline N(3)-C(13)-C(14) & $132.4(11)$ \\
\hline $\mathrm{O}(2)-\mathrm{C}(13)-\mathrm{C}(14)$ & $112.1(9)$ \\
\hline C(15)-C(14)-C(19) & $122.1(11)$ \\
\hline $\mathrm{C}(15)-\mathrm{C}(14)-\mathrm{C}(13)$ & $124.8(11)$ \\
\hline$C(19)-C(14)-C(13)$ & $113.2(10)$ \\
\hline$C(14)-C(15)-C(16)$ & $120.0(11)$ \\
\hline $\mathrm{C}(14)-\mathrm{C}(15)-\mathrm{H}(15)$ & 120.0 \\
\hline $\mathrm{C}(16)-\mathrm{C}(15)-\mathrm{H}(15)$ & 120.0 \\
\hline$C(15)-C(16)-C(17)$ & $124.8(12)$ \\
\hline $\mathrm{C}(15)-\mathrm{C}(16)-\mathrm{H}(16)$ & 117.6 \\
\hline $\mathrm{C}(17)-\mathrm{C}(16)-\mathrm{H}(16)$ & 117.6 \\
\hline$C(16)-C(17)-C(18)$ & $113.3(9)$ \\
\hline$C(16)-C(17)-C(20)$ & $124.6(10)$ \\
\hline C(18)-C(17)-C(20) & $121.9(9)$ \\
\hline $\mathrm{C}(19)-\mathrm{C}(18)-\mathrm{C}(17)$ & $122.3(9)$ \\
\hline $\mathrm{C}(19)-\mathrm{C}(18)-\mathrm{H}(18)$ & 118.8 \\
\hline
\end{tabular}




\begin{tabular}{|c|c|}
\hline $\mathrm{C}(17)-\mathrm{C}(18)-\mathrm{H}(18)$ & 118.8 \\
\hline$C(18)-C(19)-C(14)$ & $116.8(9)$ \\
\hline $\mathrm{C}(18)-\mathrm{C}(19)-\mathrm{H}(19)$ & 121.6 \\
\hline $\mathrm{C}(14)-\mathrm{C}(19)-\mathrm{H}(19)$ & 121.6 \\
\hline$C(25)-C(20)-C(21)$ & $120.0(11)$ \\
\hline$C(25)-C(20)-C(17)$ & $121.5(11)$ \\
\hline$C(21)-C(20)-C(17)$ & $118.5(9)$ \\
\hline$C(20)-C(21)-C(22)$ & $120.5(11)$ \\
\hline $\mathrm{C}(20)-\mathrm{C}(21)-\mathrm{H}(21)$ & 119.8 \\
\hline $\mathrm{C}(22)-\mathrm{C}(21)-\mathrm{H}(21)$ & 119.8 \\
\hline$C(23)-C(22)-C(21)$ & $121.3(12)$ \\
\hline $\mathrm{C}(23)-\mathrm{C}(22)-\mathrm{H}(22)$ & 119.3 \\
\hline $\mathrm{C}(21)-\mathrm{C}(22)-\mathrm{H}(22)$ & 119.3 \\
\hline$C(22)-C(23)-C(24)$ & $117.0(10)$ \\
\hline $\mathrm{C}(22)-\mathrm{C}(23)-\mathrm{H}(23)$ & 121.5 \\
\hline $\mathrm{C}(24)-\mathrm{C}(23)-\mathrm{H}(23)$ & 121.5 \\
\hline$C(25)-C(24)-C(23)$ & $119.0(11)$ \\
\hline $\mathrm{C}(25)-\mathrm{C}(24)-\mathrm{H}(24)$ & 120.5 \\
\hline $\mathrm{C}(23)-\mathrm{C}(24)-\mathrm{H}(24)$ & 120.5 \\
\hline$C(24)-C(25)-C(20)$ & $121.8(14)$ \\
\hline $\mathrm{C}(24)-\mathrm{C}(25)-\mathrm{H}(25)$ & 119.1 \\
\hline $\mathrm{C}(20)-\mathrm{C}(25)-\mathrm{H}(25)$ & 119.1 \\
\hline$C(27)-C(26)-C(35)$ & $112.2(9)$ \\
\hline$C(27)-C(26)-C(36)$ & $117.4(9)$ \\
\hline$C(35)-C(26)-C(36)$ & $106.7(9)$ \\
\hline$C(27)-C(26)-C(33)$ & $104.0(9)$ \\
\hline$C(35)-C(26)-C(33)$ & $105.9(9)$ \\
\hline$C(36)-C(26)-C(33)$ & $110.1(9)$ \\
\hline$C(28)-C(27)-C(32)$ & $121.1(10)$ \\
\hline$C(28)-C(27)-C(26)$ & $131.2(10)$ \\
\hline$C(32)-C(27)-C(26)$ & $107.6(9)$ \\
\hline$C(29)-C(28)-C(27)$ & $116.4(10)$ \\
\hline $\mathrm{C}(29)-\mathrm{C}(28)-\mathrm{H}(28)$ & 121.8 \\
\hline $\mathrm{C}(27)-\mathrm{C}(28)-\mathrm{H}(28)$ & 121.8 \\
\hline$C(28)-C(29)-C(30)$ & $123.4(11)$ \\
\hline$C(28)-C(29)-B r(2)$ & $118.5(8)$ \\
\hline$C(30)-C(29)-B r(2)$ & 118.1(9) \\
\hline$C(29)-C(30)-C(31)$ & $118.1(12)$ \\
\hline $\mathrm{C}(29)-\mathrm{C}(30)-\mathrm{H}(30)$ & 120.9 \\
\hline
\end{tabular}




\begin{tabular}{|c|c|}
\hline $\mathrm{C}(31)-\mathrm{C}(30)-\mathrm{H}(30)$ & 120.9 \\
\hline$C(32)-C(31)-C(30)$ & $119.6(12)$ \\
\hline $\mathrm{C}(32)-\mathrm{C}(31)-\mathrm{H}(31)$ & 120.2 \\
\hline $\mathrm{C}(30)-\mathrm{C}(31)-\mathrm{H}(31)$ & 120.2 \\
\hline $\mathrm{C}(31)-\mathrm{C}(32)-\mathrm{N}(4)$ & $128.0(11)$ \\
\hline $\mathrm{C}(31)-\mathrm{C}(32)-\mathrm{C}(27)$ & $121.3(10)$ \\
\hline $\mathrm{N}(4)-\mathrm{C}(32)-\mathrm{C}(27)$ & $110.7(10)$ \\
\hline $\mathrm{O}(3)-\mathrm{C}(33)-\mathrm{N}(4)$ & $126.3(11)$ \\
\hline $\mathrm{O}(3)-\mathrm{C}(33)-\mathrm{C}(26)$ & $127.3(11)$ \\
\hline $\mathrm{N}(4)-\mathrm{C}(33)-\mathrm{C}(26)$ & $106.2(9)$ \\
\hline $\mathrm{N}(4)-\mathrm{C}(34)-\mathrm{H}(34 \mathrm{~A})$ & 109.5 \\
\hline N(4)-C(34)-H(34B) & 109.5 \\
\hline $\mathrm{H}(34 \mathrm{~A})-\mathrm{C}(34)-\mathrm{H}(34 \mathrm{~B})$ & 109.5 \\
\hline $\mathrm{N}(4)-\mathrm{C}(34)-\mathrm{H}(34 \mathrm{C})$ & 109.5 \\
\hline $\mathrm{H}(34 \mathrm{~A})-\mathrm{C}(34)-\mathrm{H}(34 \mathrm{C})$ & 109.5 \\
\hline $\mathrm{H}(34 \mathrm{~B})-\mathrm{C}(34)-\mathrm{H}(34 \mathrm{C})$ & 109.5 \\
\hline $\mathrm{C}(26)-\mathrm{C}(35)-\mathrm{H}(35 \mathrm{~A})$ & 109.5 \\
\hline $\mathrm{C}(26)-\mathrm{C}(35)-\mathrm{H}(35 \mathrm{~B})$ & 109.5 \\
\hline $\mathrm{H}(35 \mathrm{~A})-\mathrm{C}(35)-\mathrm{H}(35 \mathrm{~B})$ & 109.5 \\
\hline $\mathrm{C}(26)-\mathrm{C}(35)-\mathrm{H}(35 \mathrm{C})$ & 109.5 \\
\hline $\mathrm{H}(35 \mathrm{~A})-\mathrm{C}(35)-\mathrm{H}(35 \mathrm{C})$ & 109.5 \\
\hline $\mathrm{H}(35 \mathrm{~B})-\mathrm{C}(35)-\mathrm{H}(35 \mathrm{C})$ & 109.5 \\
\hline$C(37)-C(36)-C(26)$ & $112.2(10)$ \\
\hline $\mathrm{C}(37)-\mathrm{C}(36)-\mathrm{H}(36 \mathrm{~A})$ & 109.2 \\
\hline $\mathrm{C}(26)-\mathrm{C}(36)-\mathrm{H}(36 \mathrm{~A})$ & 109.2 \\
\hline $\mathrm{C}(37)-\mathrm{C}(36)-\mathrm{H}(36 \mathrm{~B})$ & 109.2 \\
\hline $\mathrm{C}(26)-\mathrm{C}(36)-\mathrm{H}(36 \mathrm{~B})$ & 109.2 \\
\hline $\mathrm{H}(36 \mathrm{~A})-\mathrm{C}(36)-\mathrm{H}(36 \mathrm{~B})$ & 107.9 \\
\hline $\mathrm{N}(5)-\mathrm{C}(37)-\mathrm{O}(4)$ & $108.9(10)$ \\
\hline N(5)-C(37)-C(36) & $131.0(11)$ \\
\hline $\mathrm{O}(4)-\mathrm{C}(37)-\mathrm{C}(36)$ & $120.1(9)$ \\
\hline $\mathrm{N}(6)-\mathrm{C}(38)-\mathrm{O}(4)$ & $112.0(9)$ \\
\hline N(6)-C(38)-C(39) & $132.2(10)$ \\
\hline $\mathrm{O}(4)-\mathrm{C}(38)-\mathrm{C}(39)$ & $115.7(8)$ \\
\hline$C(44)-C(39)-C(40)$ & 118.2(9) \\
\hline C(44)-C(39)-C(38) & $122.0(9)$ \\
\hline C(40)-C(39)-C(38) & 119.7(9) \\
\hline C(41)-C(40)-C(39) & $123.8(11)$ \\
\hline $\mathrm{C}(41)-\mathrm{C}(40)-\mathrm{H}(40)$ & 118.1 \\
\hline
\end{tabular}




\begin{tabular}{|c|c|}
\hline $\mathrm{C}(39)-\mathrm{C}(40)-\mathrm{H}(40)$ & 118.1 \\
\hline$C(40)-C(41)-C(42)$ & $119.3(10)$ \\
\hline $\mathrm{C}(40)-\mathrm{C}(41)-\mathrm{H}(41)$ & 120.4 \\
\hline $\mathrm{C}(42)-\mathrm{C}(41)-\mathrm{H}(41)$ & 120.4 \\
\hline$C(43)-C(42)-C(45)$ & $122.9(10)$ \\
\hline$C(43)-C(42)-C(41)$ & $114.7(9)$ \\
\hline$C(45)-C(42)-C(41)$ & $122.4(9)$ \\
\hline$C(44)-C(43)-C(42)$ & $121.6(9)$ \\
\hline $\mathrm{C}(44)-\mathrm{C}(43)-\mathrm{H}(43)$ & 119.2 \\
\hline $\mathrm{C}(42)-\mathrm{C}(43)-\mathrm{H}(43)$ & 119.2 \\
\hline$C(39)-C(44)-C(43)$ & $122.1(9)$ \\
\hline $\mathrm{C}(39)-\mathrm{C}(44)-\mathrm{H}(44)$ & 118.9 \\
\hline $\mathrm{C}(43)-\mathrm{C}(44)-\mathrm{H}(44)$ & 118.9 \\
\hline$C(46)-C(45)-C(50)$ & $121.5(10)$ \\
\hline$C(46)-C(45)-C(42)$ & $120.9(11)$ \\
\hline$C(50)-C(45)-C(42)$ & $117.3(10)$ \\
\hline$C(45)-C(46)-C(47)$ & $121.8(11)$ \\
\hline $\mathrm{C}(45)-\mathrm{C}(46)-\mathrm{H}(46)$ & 119.1 \\
\hline $\mathrm{C}(47)-\mathrm{C}(46)-\mathrm{H}(46)$ & 119.1 \\
\hline$C(46)-C(47)-C(48)$ & $120.2(10)$ \\
\hline $\mathrm{C}(46)-\mathrm{C}(47)-\mathrm{H}(47)$ & 119.9 \\
\hline $\mathrm{C}(48)-\mathrm{C}(47)-\mathrm{H}(47)$ & 119.9 \\
\hline$C(49)-C(48)-C(47)$ & 114.6(9) \\
\hline $\mathrm{C}(49)-\mathrm{C}(48)-\mathrm{H}(48)$ & 122.7 \\
\hline $\mathrm{C}(47)-\mathrm{C}(48)-\mathrm{H}(48)$ & 122.7 \\
\hline$C(48)-C(49)-C(50)$ & $122.2(10)$ \\
\hline $\mathrm{C}(48)-\mathrm{C}(49)-\mathrm{H}(49)$ & 118.9 \\
\hline $\mathrm{C}(50)-\mathrm{C}(49)-\mathrm{H}(49)$ & 118.9 \\
\hline$C(45)-C(50)-C(49)$ & 119.2(9) \\
\hline $\mathrm{C}(45)-\mathrm{C}(50)-\mathrm{H}(50)$ & 120.4 \\
\hline $\mathrm{C}(49)-\mathrm{C}(50)-\mathrm{H}(50)$ & 120.4 \\
\hline $\mathrm{C}(58)-\mathrm{C}(51)-\mathrm{C}(52)$ & $102.2(7)$ \\
\hline $\mathrm{C}(58)-\mathrm{C}(51)-\mathrm{C}(60)$ & $107.5(7)$ \\
\hline$C(52)-C(51)-C(60)$ & $110.3(7)$ \\
\hline$C(58)-C(51)-C(61)$ & $112.7(7)$ \\
\hline$C(52)-C(51)-C(61)$ & $116.6(7)$ \\
\hline$C(60)-C(51)-C(61)$ & $107.2(7)$ \\
\hline$C(53)-C(52)-C(57)$ & $120.2(8)$ \\
\hline$C(53)-C(52)-C(51)$ & $131.3(8)$ \\
\hline
\end{tabular}




\begin{tabular}{|c|c|}
\hline $\mathrm{C}(57)-\mathrm{C}(52)-\mathrm{C}(51)$ & $108.4(7)$ \\
\hline $\mathrm{C}(54)-\mathrm{C}(53)-\mathrm{C}(52)$ & $118.3(9)$ \\
\hline $\mathrm{C}(54)-\mathrm{C}(53)-\mathrm{H}(53)$ & 120.8 \\
\hline $\mathrm{C}(52)-\mathrm{C}(53)-\mathrm{H}(53)$ & 120.8 \\
\hline $\mathrm{C}(53)-\mathrm{C}(54)-\mathrm{C}(55)$ & $121.5(11)$ \\
\hline $\mathrm{C}(53)-\mathrm{C}(54)-\mathrm{Br}(3)$ & $119.5(9)$ \\
\hline $\mathrm{C}(55)-\mathrm{C}(54)-\mathrm{Br}(3)$ & $119.0(9)$ \\
\hline $\mathrm{C}(56)-\mathrm{C}(55)-\mathrm{C}(54)$ & $120.2(11)$ \\
\hline $\mathrm{C}(56)-\mathrm{C}(55)-\mathrm{H}(55)$ & 119.9 \\
\hline $\mathrm{C}(54)-\mathrm{C}(55)-\mathrm{H}(55)$ & 119.9 \\
\hline $\mathrm{C}(55)-\mathrm{C}(56)-\mathrm{C}(57)$ & $119.4(10)$ \\
\hline $\mathrm{C}(55)-\mathrm{C}(56)-\mathrm{H}(56)$ & 120.3 \\
\hline $\mathrm{C}(57)-\mathrm{C}(56)-\mathrm{H}(56)$ & 120.3 \\
\hline $\mathrm{N}(7)-\mathrm{C}(57)-\mathrm{C}(52)$ & $109.6(8)$ \\
\hline $\mathrm{N}(7)-\mathrm{C}(57)-\mathrm{C}(56)$ & $130.3(9)$ \\
\hline$C(52)-C(57)-C(56)$ & $120.1(9)$ \\
\hline $\mathrm{O}(5)-\mathrm{C}(58)-\mathrm{N}(7)$ & $125.8(9)$ \\
\hline $\mathrm{O}(5)-\mathrm{C}(58)-\mathrm{C}(51)$ & $126.9(9)$ \\
\hline $\mathrm{N}(7)-\mathrm{C}(58)-\mathrm{C}(51)$ & $106.8(8)$ \\
\hline N(7)-C(59)-H(59A) & 109.5 \\
\hline N(7)-C(59)-H(59B) & 109.5 \\
\hline $\mathrm{H}(59 \mathrm{~A})-\mathrm{C}(59)-\mathrm{H}(59 \mathrm{~B})$ & 109.5 \\
\hline $\mathrm{N}(7)-\mathrm{C}(59)-\mathrm{H}(59 \mathrm{C})$ & 109.5 \\
\hline $\mathrm{H}(59 \mathrm{~A})-\mathrm{C}(59)-\mathrm{H}(59 \mathrm{C})$ & 109.5 \\
\hline H(59B)-C(59)-H(59C) & 109.5 \\
\hline $\mathrm{C}(51)-\mathrm{C}(60)-\mathrm{H}(60 \mathrm{~A})$ & 109.5 \\
\hline $\mathrm{C}(51)-\mathrm{C}(60)-\mathrm{H}(60 \mathrm{~B})$ & 109.5 \\
\hline $\mathrm{H}(60 \mathrm{~A})-\mathrm{C}(60)-\mathrm{H}(60 \mathrm{~B})$ & 109.5 \\
\hline $\mathrm{C}(51)-\mathrm{C}(60)-\mathrm{H}(60 \mathrm{C})$ & 109.5 \\
\hline $\mathrm{H}(60 \mathrm{~A})-\mathrm{C}(60)-\mathrm{H}(60 \mathrm{C})$ & 109.5 \\
\hline $\mathrm{H}(60 \mathrm{~B})-\mathrm{C}(60)-\mathrm{H}(60 \mathrm{C})$ & 109.5 \\
\hline $\mathrm{C}(62)-\mathrm{C}(61)-\mathrm{C}(51)$ & $112.5(7)$ \\
\hline$C(62)-C(61)-H(61 A)$ & 109.1 \\
\hline $\mathrm{C}(51)-\mathrm{C}(61)-\mathrm{H}(61 \mathrm{~A})$ & 109.1 \\
\hline $\mathrm{C}(62)-\mathrm{C}(61)-\mathrm{H}(61 \mathrm{~B})$ & 109.1 \\
\hline $\mathrm{C}(51)-\mathrm{C}(61)-\mathrm{H}(61 \mathrm{~B})$ & 109.1 \\
\hline $\mathrm{H}(61 \mathrm{~A})-\mathrm{C}(61)-\mathrm{H}(61 \mathrm{~B})$ & 107.8 \\
\hline $\mathrm{O}(6)-\mathrm{C}(62)-\mathrm{N}(8)$ & 111.1(8) \\
\hline $\mathrm{O}(6)-\mathrm{C}(62)-\mathrm{C}(61)$ & $119.7(8)$ \\
\hline
\end{tabular}




\begin{tabular}{ll}
$\mathrm{N}(8)-\mathrm{C}(62)-\mathrm{C}(61)$ & $129.1(8)$ \\
$\mathrm{N}(9)-\mathrm{C}(63)-\mathrm{O}(6)$ & $109.5(7)$ \\
$\mathrm{N}(9)-\mathrm{C}(63)-\mathrm{C}(64)$ & $130.8(8)$ \\
$\mathrm{O}(6)-\mathrm{C}(63)-\mathrm{C}(64)$ & $119.6(7)$ \\
$\mathrm{C}(69)-\mathrm{C}(64)-\mathrm{C}(65)$ & $118.5(8)$ \\
$\mathrm{C}(69)-\mathrm{C}(64)-\mathrm{C}(63)$ & $122.6(8)$ \\
$\mathrm{C}(65)-\mathrm{C}(64)-\mathrm{C}(63)$ & $118.8(8)$ \\
$\mathrm{C}(66)-\mathrm{C}(65)-\mathrm{C}(64)$ & $119.1(9)$ \\
$\mathrm{C}(66)-\mathrm{C}(65)-\mathrm{H}(65)$ & 120.5 \\
$\mathrm{C}(64)-\mathrm{C}(65)-\mathrm{H}(65)$ & 120.5 \\
$\mathrm{C}(67)-\mathrm{C}(66)-\mathrm{C}(65)$ & $119.8(10)$ \\
$\mathrm{C}(67)-\mathrm{C}(66)-\mathrm{H}(66)$ & 120.1 \\
$\mathrm{C}(65)-\mathrm{C}(66)-\mathrm{H}(66)$ & 120.1 \\
$\mathrm{C}(66)-\mathrm{C}(67)-\mathrm{C}(68)$ & $121.3(10)$ \\
$\mathrm{C}(66)-\mathrm{C}(67)-\mathrm{C}(70)$ & $118.6(9)$ \\
$\mathrm{C}(68)-\mathrm{C}(67)-\mathrm{C}(70)$ & $120.1(9)$ \\
$\mathrm{C}(69)-\mathrm{C}(68)-\mathrm{C}(67)$ & $119.7(10)$ \\
$\mathrm{C}(69)-\mathrm{C}(68)-\mathrm{H}(68)$ & 120.2 \\
$\mathrm{C}(67)-\mathrm{C}(68)-\mathrm{H}(68)$ & 120.2 \\
$\mathrm{C}(68)-\mathrm{C}(69)-\mathrm{C}(64)$ & $120.8(10)$ \\
$\mathrm{C}(68)-\mathrm{C}(69)-\mathrm{H}(69)$ & 119.6 \\
$\mathrm{C}(64)-\mathrm{C}(69)-\mathrm{H}(69)$ & 119.6 \\
$\mathrm{C}(71)-\mathrm{C}(70)-\mathrm{C}(75)$ & $115.5(8)$ \\
$\mathrm{C}(71)-\mathrm{C}(70)-\mathrm{C}(67)$ & $123.9(9)$ \\
$\mathrm{C}(75)-\mathrm{C}(70)-\mathrm{C}(67)$ & $120.6(8)$ \\
$\mathrm{C}(70)-\mathrm{C}(71)-\mathrm{C}(72)$ & $123.2(10)$ \\
$\mathrm{C}(70)-\mathrm{C}(71)-\mathrm{H}(71)$ & 118.4 \\
$\mathrm{C}(74)-\mathrm{C}(75)-\mathrm{C}(75)-\mathrm{C}(75)-\mathrm{H}(75)-\mathrm{H}(70)-\mathrm{H}(71)$ & 118.4 \\
$\mathrm{C}(71)-\mathrm{C}(72)-\mathrm{C}(73)$ & $116.7(9)$ \\
$\mathrm{C}(71)-\mathrm{C}(72)-\mathrm{H}(72)$ & 121.7 \\
$\mathrm{C}(73)-\mathrm{C}(72)-\mathrm{H}(72)$ & 121.7 \\
$\mathrm{C}(74)-\mathrm{C}(73)-\mathrm{C}(72)$ & $124.0(10)$ \\
$\mathrm{C}(74)-\mathrm{C}(73)-\mathrm{H}(73)$ & 118.0 \\
$\mathrm{C}(72)-\mathrm{C}(73)-\mathrm{H}(73)$ & 118.0 \\
$\mathrm{C}(73) \mathrm{C}(75)$ & $117.9(9)$ \\
\hline
\end{tabular}




\begin{tabular}{|c|c|}
\hline $\mathrm{C}(70)-\mathrm{C}(75)-\mathrm{H}(75)$ & 118.9 \\
\hline$C(77)-C(76)-C(83)$ & $102.0(7)$ \\
\hline$C(77)-C(76)-C(85)$ & $110.6(7)$ \\
\hline$C(83)-C(76)-C(85)$ & $108.3(7)$ \\
\hline$C(77)-C(76)-C(86)$ & 113.2(7) \\
\hline$C(83)-C(76)-C(86)$ & $111.1(7)$ \\
\hline$C(85)-C(76)-C(86)$ & $111.3(7)$ \\
\hline$C(78)-C(77)-C(82)$ & $119.5(9)$ \\
\hline $\mathrm{C}(78)-\mathrm{C}(77)-\mathrm{C}(76)$ & $131.4(9)$ \\
\hline$C(82)-C(77)-C(76)$ & $108.9(8)$ \\
\hline$C(77)-C(78)-C(79)$ & $118.5(9)$ \\
\hline $\mathrm{C}(77)-\mathrm{C}(78)-\mathrm{H}(78)$ & 120.7 \\
\hline $\mathrm{C}(79)-\mathrm{C}(78)-\mathrm{H}(78)$ & 120.7 \\
\hline$C(78)-C(79)-C(80)$ & $121.2(9)$ \\
\hline $\mathrm{C}(78)-\mathrm{C}(79)-\mathrm{Br}(4)$ & $120.5(8)$ \\
\hline $\mathrm{C}(80)-\mathrm{C}(79)-\mathrm{Br}(4)$ & $118.4(8)$ \\
\hline$C(81)-C(80)-C(79)$ & $120.8(10)$ \\
\hline $\mathrm{C}(81)-\mathrm{C}(80)-\mathrm{H}(80)$ & 119.6 \\
\hline $\mathrm{C}(79)-\mathrm{C}(80)-\mathrm{H}(80)$ & 119.6 \\
\hline $\mathrm{C}(82)-\mathrm{C}(81)-\mathrm{C}(80)$ & $117.8(10)$ \\
\hline $\mathrm{C}(82)-\mathrm{C}(81)-\mathrm{H}(81)$ & 121.1 \\
\hline $\mathrm{C}(80)-\mathrm{C}(81)-\mathrm{H}(81)$ & 121.1 \\
\hline $\mathrm{C}(81)-\mathrm{C}(82)-\mathrm{N}(10)$ & $129.7(10)$ \\
\hline $\mathrm{C}(81)-\mathrm{C}(82)-\mathrm{C}(77)$ & $122.2(9)$ \\
\hline $\mathrm{N}(10)-\mathrm{C}(82)-\mathrm{C}(77)$ & $108.2(8)$ \\
\hline $\mathrm{O}(7)-\mathrm{C}(83)-\mathrm{N}(10)$ & $126.7(9)$ \\
\hline $\mathrm{O}(7)-\mathrm{C}(83)-\mathrm{C}(76)$ & $124.6(9)$ \\
\hline $\mathrm{N}(10)-\mathrm{C}(83)-\mathrm{C}(76)$ & $108.5(7)$ \\
\hline $\mathrm{N}(10)-\mathrm{C}(84)-\mathrm{H}(84 \mathrm{~A})$ & 109.5 \\
\hline $\mathrm{N}(10)-\mathrm{C}(84)-\mathrm{H}(84 \mathrm{~B})$ & 109.5 \\
\hline $\mathrm{H}(84 \mathrm{~A})-\mathrm{C}(84)-\mathrm{H}(84 \mathrm{~B})$ & 109.5 \\
\hline $\mathrm{N}(10)-\mathrm{C}(84)-\mathrm{H}(84 \mathrm{C})$ & 109.5 \\
\hline $\mathrm{H}(84 \mathrm{~A})-\mathrm{C}(84)-\mathrm{H}(84 \mathrm{C})$ & 109.5 \\
\hline $\mathrm{H}(84 \mathrm{~B})-\mathrm{C}(84)-\mathrm{H}(84 \mathrm{C})$ & 109.5 \\
\hline $\mathrm{C}(76)-\mathrm{C}(85)-\mathrm{H}(85 \mathrm{~A})$ & 109.5 \\
\hline $\mathrm{C}(76)-\mathrm{C}(85)-\mathrm{H}(85 \mathrm{~B})$ & 109.5 \\
\hline $\mathrm{H}(85 \mathrm{~A})-\mathrm{C}(85)-\mathrm{H}(85 \mathrm{~B})$ & 109.5 \\
\hline $\mathrm{C}(76)-\mathrm{C}(85)-\mathrm{H}(85 \mathrm{C})$ & 109.5 \\
\hline $\mathrm{H}(85 \mathrm{~A})-\mathrm{C}(85)-\mathrm{H}(85 \mathrm{C})$ & 109.5 \\
\hline
\end{tabular}




\begin{tabular}{|c|c|}
\hline $\mathrm{H}(85 \mathrm{~B})-\mathrm{C}(85)-\mathrm{H}(85 \mathrm{C})$ & 109.5 \\
\hline$C(87)-C(86)-C(76)$ & $117.1(8)$ \\
\hline $\mathrm{C}(87)-\mathrm{C}(86)-\mathrm{H}(86 \mathrm{~A})$ & 108.0 \\
\hline $\mathrm{C}(76)-\mathrm{C}(86)-\mathrm{H}(86 \mathrm{~A})$ & 108.0 \\
\hline $\mathrm{C}(87)-\mathrm{C}(86)-\mathrm{H}(86 \mathrm{~B})$ & 108.0 \\
\hline $\mathrm{C}(76)-\mathrm{C}(86)-\mathrm{H}(86 \mathrm{~B})$ & 108.0 \\
\hline $\mathrm{H}(86 \mathrm{~A})-\mathrm{C}(86)-\mathrm{H}(86 \mathrm{~B})$ & 107.3 \\
\hline $\mathrm{N}(11)-\mathrm{C}(87)-\mathrm{O}(8)$ & 113.3(9) \\
\hline $\mathrm{N}(11)-\mathrm{C}(87)-\mathrm{C}(86)$ & $128.3(9)$ \\
\hline $\mathrm{O}(8)-\mathrm{C}(87)-\mathrm{C}(86)$ & 118.1(8) \\
\hline $\mathrm{N}(12)-\mathrm{C}(88)-\mathrm{O}(8)$ & $111.5(8)$ \\
\hline $\mathrm{N}(12)-\mathrm{C}(88)-\mathrm{C}(89)$ & $125.7(9)$ \\
\hline $\mathrm{O}(8)-\mathrm{C}(88)-\mathrm{C}(89)$ & $122.8(8)$ \\
\hline $\mathrm{C}(90)-\mathrm{C}(89)-\mathrm{C}(88)$ & 124.7(9) \\
\hline C(90)-C(89)-C(94) & $121.4(9)$ \\
\hline C(88)-C(89)-C(94) & $113.9(8)$ \\
\hline C(89)-C(90)-C(91) & $118.1(10)$ \\
\hline C(89)-C(90)-H(90) & 121.0 \\
\hline $\mathrm{C}(91)-\mathrm{C}(90)-\mathrm{H}(90)$ & 121.0 \\
\hline $\mathrm{C}(92)-\mathrm{C}(91)-\mathrm{C}(90)$ & $124.1(10)$ \\
\hline $\mathrm{C}(92)-\mathrm{C}(91)-\mathrm{H}(91)$ & 118.0 \\
\hline $\mathrm{C}(90)-\mathrm{C}(91)-\mathrm{H}(91)$ & 118.0 \\
\hline C(91)-C(92)-C(93) & $120.1(9)$ \\
\hline $\mathrm{C}(91)-\mathrm{C}(92)-\mathrm{C}(95)$ & $123.0(8)$ \\
\hline C(93)-C(92)-C(95) & $116.8(8)$ \\
\hline $\mathrm{C}(92)-\mathrm{C}(93)-\mathrm{C}(94)$ & $119.9(9)$ \\
\hline $\mathrm{C}(92)-\mathrm{C}(93)-\mathrm{H}(93)$ & 120.0 \\
\hline $\mathrm{C}(94)-\mathrm{C}(93)-\mathrm{H}(93)$ & 120.0 \\
\hline C(93)-C(94)-C(89) & $116.4(9)$ \\
\hline $\mathrm{C}(93)-\mathrm{C}(94)-\mathrm{H}(94)$ & 121.8 \\
\hline C(89)-C(94)-H(94) & 121.8 \\
\hline$C(100)-C(95)-C(96)$ & $115.1(8)$ \\
\hline $\mathrm{C}(100)-\mathrm{C}(95)-\mathrm{C}(92)$ & $123.4(9)$ \\
\hline $\mathrm{C}(96)-\mathrm{C}(95)-\mathrm{C}(92)$ & $121.4(8)$ \\
\hline $\mathrm{C}(97)-\mathrm{C}(96)-\mathrm{C}(95)$ & $120.6(9)$ \\
\hline $\mathrm{C}(97)-\mathrm{C}(96)-\mathrm{H}(96)$ & 119.7 \\
\hline $\mathrm{C}(95)-\mathrm{C}(96)-\mathrm{H}(96)$ & 119.7 \\
\hline $\mathrm{C}(98)-\mathrm{C}(97)-\mathrm{C}(96)$ & $118.7(10)$ \\
\hline C(98)-C(97)-H(97) & 120.6 \\
\hline
\end{tabular}




$\begin{array}{ll}\mathrm{C}(96)-\mathrm{C}(97)-\mathrm{H}(97) & 120.6 \\ \mathrm{C}(97)-\mathrm{C}(98)-\mathrm{C}(99) & 125.2(10) \\ \mathrm{C}(97)-\mathrm{C}(98)-\mathrm{H}(98) & 117.4 \\ \mathrm{C}(99)-\mathrm{C}(98)-\mathrm{H}(98) & 117.4 \\ \mathrm{C}(100)-\mathrm{C}(99)-\mathrm{C}(98) & 115.4(11) \\ \mathrm{C}(100)-\mathrm{C}(99)-\mathrm{H}(99) & 122.3 \\ \mathrm{C}(98)-\mathrm{C}(99)-\mathrm{H}(99) & 122.3 \\ \mathrm{C}(99)-\mathrm{C}(100)-\mathrm{C}(95) & 124.6(11) \\ \mathrm{C}(99)-\mathrm{C}(100)-\mathrm{H}(100) & 117.7 \\ \mathrm{C}(95)-\mathrm{C}(100)-\mathrm{H}(100) & 117.7\end{array}$

Symmetry transformations used to generate equivalent atoms: 
Table 4. Anisotropic displacement parameters $\left(\AA^{2} \times 10^{3}\right)$ for kwq-c-48b. The anisotropic displacement factor exponent takes the form: $-2 \pi^{2}\left[h^{2} a^{* 2} U^{11}+\ldots+2 h k a^{*} b^{*} U^{12}\right]$

\begin{tabular}{|c|c|c|c|c|c|c|}
\hline & $\mathrm{U}^{11}$ & $\mathrm{U}^{22}$ & $\mathrm{U}^{33}$ & $\mathrm{U}^{23}$ & $\mathrm{U}^{13}$ & $\mathrm{U}^{12}$ \\
\hline $\operatorname{Br}(1)$ & $71(1)$ & $50(1)$ & $21(1)$ & $-15(1)$ & $12(1)$ & $-40(1)$ \\
\hline $\operatorname{Br}(2)$ & $43(1)$ & $34(1)$ & $31(1)$ & $7(1)$ & $0(1)$ & $9(1)$ \\
\hline $\operatorname{Br}(3)$ & $43(1)$ & $44(1)$ & $18(1)$ & $-14(1)$ & $1(1)$ & $25(1)$ \\
\hline $\operatorname{Br}(4)$ & $30(1)$ & $28(1)$ & $27(1)$ & $7(1)$ & $13(1)$ & $-7(1)$ \\
\hline $\mathrm{O}(1)$ & $39(2)$ & $39(2)$ & $38(2)$ & $1(1)$ & $3(1)$ & $-1(1)$ \\
\hline $\mathrm{O}(2)$ & $17(2)$ & $14(2)$ & $14(2)$ & 1(1) & $2(1)$ & $-1(1)$ \\
\hline $\mathrm{O}(3)$ & $35(2)$ & $35(2)$ & $34(2)$ & $0(1)$ & $5(1)$ & $-1(1)$ \\
\hline $\mathrm{O}(4)$ & $14(2)$ & $14(2)$ & $14(2)$ & $-1(1)$ & $0(1)$ & $0(1)$ \\
\hline $\mathrm{O}(5)$ & $25(2)$ & $26(2)$ & $23(2)$ & $0(1)$ & $4(1)$ & $2(1)$ \\
\hline $\mathrm{O}(6)$ & $14(2)$ & $12(2)$ & $12(2)$ & $1(1)$ & $1(1)$ & $0(1)$ \\
\hline $\mathrm{O}(7)$ & $24(2)$ & $24(2)$ & $24(2)$ & $0(1)$ & $4(1)$ & $1(1)$ \\
\hline $\mathrm{O}(8)$ & $16(2)$ & $15(2)$ & $15(2)$ & $0(1)$ & $3(1)$ & $0(1)$ \\
\hline $\mathrm{N}(1)$ & $28(2)$ & $28(2)$ & $27(2)$ & $1(1)$ & $3(1)$ & $0(1)$ \\
\hline $\mathrm{N}(2)$ & $23(2)$ & $23(2)$ & $22(2)$ & $1(1)$ & $2(1)$ & $0(1)$ \\
\hline $\mathrm{N}(3)$ & $30(2)$ & $29(2)$ & $29(2)$ & $1(1)$ & $4(1)$ & $0(1)$ \\
\hline $\mathrm{N}(4)$ & $28(2)$ & $27(2)$ & $27(2)$ & $-1(1)$ & $2(1)$ & $0(1)$ \\
\hline $\mathrm{N}(5)$ & $34(2)$ & $35(3)$ & $33(2)$ & $0(1)$ & $4(1)$ & $-1(1)$ \\
\hline $\mathrm{N}(6)$ & $38(3)$ & $39(3)$ & $38(3)$ & $0(1)$ & $5(1)$ & $-2(1)$ \\
\hline $\mathrm{N}(7)$ & $14(2)$ & $15(2)$ & $13(2)$ & $1(1)$ & $2(1)$ & $0(1)$ \\
\hline $\mathrm{N}(8)$ & $17(2)$ & $16(2)$ & $16(2)$ & $1(1)$ & $2(1)$ & $-1(1)$ \\
\hline $\mathrm{N}(9)$ & $12(2)$ & $11(2)$ & $11(2)$ & $2(1)$ & $2(1)$ & $0(1)$ \\
\hline $\mathrm{N}(10)$ & $20(2)$ & $18(2)$ & $18(2)$ & $-1(1)$ & $2(1)$ & $1(1)$ \\
\hline $\mathrm{N}(11)$ & $29(2)$ & $29(2)$ & $28(2)$ & $-1(1)$ & $4(1)$ & $1(1)$ \\
\hline $\mathrm{N}(12)$ & $23(2)$ & $23(2)$ & $22(2)$ & $-2(1)$ & $3(1)$ & $1(1)$ \\
\hline$C(1)$ & $17(2)$ & $17(2)$ & $17(2)$ & $0(1)$ & $2(1)$ & $0(1)$ \\
\hline$C(2)$ & $16(2)$ & $15(2)$ & $16(2)$ & $1(1)$ & $2(1)$ & $0(1)$ \\
\hline$C(3)$ & $21(2)$ & $20(2)$ & $19(2)$ & $0(1)$ & $3(1)$ & $-1(1)$ \\
\hline C(4) & $20(2)$ & $20(2)$ & $20(2)$ & $0(1)$ & $3(1)$ & $-1(1)$ \\
\hline$C(5)$ & $24(2)$ & $23(2)$ & $23(2)$ & $0(1)$ & $4(1)$ & $-1(1)$ \\
\hline$C(6)$ & $30(3)$ & $30(3)$ & $30(3)$ & 1(1) & $5(1)$ & $1(1)$ \\
\hline$C(7)$ & $20(2)$ & $20(2)$ & $20(2)$ & $1(1)$ & $3(1)$ & $1(1)$ \\
\hline$C(8)$ & $23(2)$ & $24(2)$ & $23(2)$ & $1(1)$ & $2(1)$ & $-1(1)$ \\
\hline$C(9)$ & $40(3)$ & $41(3)$ & $40(3)$ & $1(1)$ & $5(1)$ & $1(1)$ \\
\hline$C(10)$ & $25(2)$ & $23(2)$ & $23(2)$ & $0(1)$ & $3(1)$ & $0(1)$ \\
\hline
\end{tabular}




\begin{tabular}{|c|c|c|c|c|c|c|}
\hline $\mathrm{C}(11)$ & $28(3)$ & $28(3)$ & $28(3)$ & $0(1)$ & $3(1)$ & $0(1)$ \\
\hline $\mathrm{C}(12)$ & $22(2)$ & $20(2)$ & $20(2)$ & $0(1)$ & 2(1) & $-1(1)$ \\
\hline $\mathrm{C}(13)$ & $22(2)$ & $21(2)$ & $21(2)$ & $0(1)$ & 2(1) & $-1(1)$ \\
\hline $\mathrm{C}(14)$ & $28(3)$ & $27(3)$ & $27(3)$ & $0(1)$ & $4(1)$ & $0(1)$ \\
\hline$C(15)$ & $28(2)$ & $27(2)$ & $27(2)$ & $0(1)$ & $4(1)$ & $1(1)$ \\
\hline$C(16)$ & $33(3)$ & $32(3)$ & $32(3)$ & $0(1)$ & 4(1) & 1(1) \\
\hline $\mathrm{C}(17)$ & $17(2)$ & $16(2)$ & $15(2)$ & $0(1)$ & 2(1) & $-1(1)$ \\
\hline $\mathrm{C}(18)$ & $14(2)$ & $14(2)$ & $14(2)$ & $0(1)$ & 1(1) & $0(1)$ \\
\hline $\mathrm{C}(19)$ & $18(2)$ & $18(2)$ & $17(2)$ & $1(1)$ & 2(1) & $0(1)$ \\
\hline$C(20)$ & $17(2)$ & $16(2)$ & $16(2)$ & $0(1)$ & $2(1)$ & $-1(1)$ \\
\hline $\mathrm{C}(21)$ & $25(3)$ & $25(3)$ & $25(2)$ & $1(1)$ & $3(1)$ & $0(1)$ \\
\hline $\mathrm{C}(22)$ & $34(3)$ & $34(3)$ & $34(3)$ & $1(1)$ & $4(1)$ & $0(1)$ \\
\hline$C(23)$ & $13(2)$ & $13(2)$ & $11(2)$ & $1(1)$ & $1(1)$ & $-1(1)$ \\
\hline$C(24)$ & $32(3)$ & $32(3)$ & $31(3)$ & $0(1)$ & $4(1)$ & 1(1) \\
\hline$C(25)$ & $40(3)$ & $40(3)$ & $39(3)$ & $0(1)$ & $5(1)$ & $1(1)$ \\
\hline$C(26)$ & $21(2)$ & $21(2)$ & $20(2)$ & $0(1)$ & $3(1)$ & $0(1)$ \\
\hline$C(27)$ & $21(2)$ & $21(2)$ & $21(2)$ & $0(1)$ & $4(1)$ & $0(1)$ \\
\hline$C(28)$ & $20(2)$ & $20(2)$ & $19(2)$ & $0(1)$ & $3(1)$ & $0(1)$ \\
\hline $\mathrm{C}(29)$ & $23(2)$ & $22(2)$ & $22(2)$ & $0(1)$ & 2(1) & $0(1)$ \\
\hline $\mathrm{C}(30)$ & $38(3)$ & $37(3)$ & $38(3)$ & $0(1)$ & $5(1)$ & $0(1)$ \\
\hline $\mathrm{C}(31)$ & $29(2)$ & $28(2)$ & $29(2)$ & $0(1)$ & $4(1)$ & $0(1)$ \\
\hline $\mathrm{C}(32)$ & $24(2)$ & $23(2)$ & $23(2)$ & $0(1)$ & $3(1)$ & $-1(1)$ \\
\hline $\mathrm{C}(33)$ & $29(3)$ & $28(3)$ & $28(2)$ & $0(1)$ & 4(1) & $-1(1)$ \\
\hline$C(34)$ & $40(3)$ & $40(3)$ & $39(3)$ & $-1(1)$ & $6(1)$ & $0(1)$ \\
\hline$C(35)$ & $22(2)$ & $22(2)$ & $22(2)$ & $0(1)$ & $3(1)$ & $0(1)$ \\
\hline$C(36)$ & $28(2)$ & $27(2)$ & $27(2)$ & $0(1)$ & $4(1)$ & $-1(1)$ \\
\hline$C(37)$ & $21(2)$ & $20(2)$ & $20(2)$ & $1(1)$ & 2(1) & $-1(1)$ \\
\hline$C(38)$ & $17(2)$ & $17(2)$ & $17(2)$ & $1(1)$ & 2(1) & $0(1)$ \\
\hline $\mathrm{C}(39)$ & $16(2)$ & $16(2)$ & $16(2)$ & $1(1)$ & $2(1)$ & $0(1)$ \\
\hline $\mathrm{C}(40)$ & $23(2)$ & $23(2)$ & $24(2)$ & $0(1)$ & $2(1)$ & $-1(1)$ \\
\hline $\mathrm{C}(41)$ & $21(2)$ & $22(2)$ & $22(2)$ & $0(1)$ & $2(1)$ & $0(1)$ \\
\hline $\mathrm{C}(42)$ & $19(2)$ & $19(2)$ & $19(2)$ & $1(1)$ & $2(1)$ & $0(1)$ \\
\hline $\mathrm{C}(43)$ & $20(2)$ & $19(2)$ & $20(2)$ & $0(1)$ & $3(1)$ & $0(1)$ \\
\hline $\mathrm{C}(44)$ & $18(2)$ & $18(2)$ & $18(2)$ & $1(1)$ & $3(1)$ & $0(1)$ \\
\hline $\mathrm{C}(45)$ & $20(2)$ & $20(2)$ & $21(2)$ & $0(1)$ & $2(1)$ & $0(1)$ \\
\hline$C(46)$ & $24(2)$ & $24(2)$ & $24(2)$ & $1(1)$ & $3(1)$ & $-1(1)$ \\
\hline $\mathrm{C}(47)$ & $27(2)$ & $26(2)$ & $27(2)$ & $1(1)$ & $3(1)$ & $0(1)$ \\
\hline$C(48)$ & $14(2)$ & $14(2)$ & $14(2)$ & $0(1)$ & 1(1) & $0(1)$ \\
\hline$C(49)$ & $20(2)$ & $20(2)$ & $20(2)$ & $0(1)$ & $3(1)$ & $0(1)$ \\
\hline
\end{tabular}




\begin{tabular}{|c|c|c|c|c|c|c|}
\hline $\mathrm{C}(50)$ & $17(2)$ & $17(2)$ & $18(2)$ & $0(1)$ & $3(1)$ & $0(1)$ \\
\hline $\mathrm{C}(51)$ & $12(2)$ & 11(2) & $12(2)$ & $0(1)$ & 1(1) & 1(1) \\
\hline $\mathrm{C}(52)$ & $10(2)$ & $9(2)$ & $9(2)$ & $0(1)$ & $0(1)$ & $-1(1)$ \\
\hline $\mathrm{C}(53)$ & $10(2)$ & $10(2)$ & $9(2)$ & $-1(1)$ & 1(1) & 1(1) \\
\hline$C(54)$ & $28(2)$ & $27(3)$ & $27(2)$ & $0(1)$ & 2(1) & $0(1)$ \\
\hline $\mathrm{C}(55)$ & $20(2)$ & $18(2)$ & $19(2)$ & $-1(1)$ & 1(1) & 1(1) \\
\hline $\mathrm{C}(56)$ & $22(2)$ & $21(2)$ & $22(2)$ & $0(1)$ & 1(1) & $-1(1)$ \\
\hline $\mathrm{C}(57)$ & $15(2)$ & $15(2)$ & $15(2)$ & $1(1)$ & 2(1) & $0(1)$ \\
\hline C(58) & $15(2)$ & $15(2)$ & $14(2)$ & $1(1)$ & 2(1) & 1(1) \\
\hline $\mathrm{C}(59)$ & $31(3)$ & $31(3)$ & $30(3)$ & $1(1)$ & $4(1)$ & $-1(1)$ \\
\hline $\mathrm{C}(60)$ & $18(2)$ & $17(2)$ & $17(2)$ & $-1(1)$ & $1(1)$ & $0(1)$ \\
\hline $\mathrm{C}(61)$ & $8(2)$ & $8(2)$ & $7(2)$ & $0(1)$ & $0(1)$ & $1(1)$ \\
\hline $\mathrm{C}(62)$ & $9(2)$ & $8(2)$ & $9(2)$ & $0(1)$ & $2(1)$ & 1(1) \\
\hline $\mathrm{C}(63)$ & $11(2)$ & $10(2)$ & $9(2)$ & $0(1)$ & $0(1)$ & 1(1) \\
\hline $\mathrm{C}(64)$ & $7(2)$ & $6(2)$ & $5(2)$ & $0(1)$ & $0(1)$ & $0(1)$ \\
\hline $\mathrm{C}(65)$ & $22(2)$ & $22(2)$ & $23(2)$ & $0(1)$ & 2(1) & $0(1)$ \\
\hline C(66) & $26(2)$ & $25(2)$ & $25(2)$ & $1(1)$ & 2(1) & $-1(1)$ \\
\hline $\mathrm{C}(67)$ & $13(2)$ & $12(2)$ & $11(2)$ & $0(1)$ & 2(1) & $0(1)$ \\
\hline $\mathrm{C}(68)$ & $21(2)$ & $21(2)$ & $21(2)$ & $0(1)$ & 2(1) & $0(1)$ \\
\hline $\mathrm{C}(69)$ & $17(2)$ & $18(2)$ & $18(2)$ & $0(1)$ & $3(1)$ & $-1(1)$ \\
\hline $\mathrm{C}(70)$ & $14(2)$ & $13(2)$ & $13(2)$ & $0(1)$ & 2(1) & $0(1)$ \\
\hline $\mathrm{C}(71)$ & $20(2)$ & 21(2) & $21(2)$ & $1(1)$ & $2(1)$ & $0(1)$ \\
\hline$C(72)$ & $17(2)$ & $17(2)$ & $16(2)$ & $1(1)$ & $2(1)$ & $0(1)$ \\
\hline$C(73)$ & $19(2)$ & $18(2)$ & $18(2)$ & $0(1)$ & $3(1)$ & $0(1)$ \\
\hline $\mathrm{C}(74)$ & $13(2)$ & $13(2)$ & $12(2)$ & $0(1)$ & $0(1)$ & $1(1)$ \\
\hline$C(75)$ & $13(2)$ & $12(2)$ & $12(2)$ & $0(1)$ & $1(1)$ & $-1(1)$ \\
\hline$C(76)$ & $12(2)$ & $12(2)$ & $11(2)$ & $0(1)$ & $1(1)$ & $0(1)$ \\
\hline $\mathrm{C}(77)$ & $13(2)$ & $13(2)$ & $13(2)$ & $0(1)$ & $1(1)$ & $0(1)$ \\
\hline $\mathrm{C}(78)$ & $17(2)$ & $17(2)$ & $17(2)$ & $0(1)$ & $2(1)$ & $0(1)$ \\
\hline$C(79)$ & $23(2)$ & $22(2)$ & $23(2)$ & $0(1)$ & $2(1)$ & $0(1)$ \\
\hline $\mathrm{C}(80)$ & $19(2)$ & $18(2)$ & $18(2)$ & $0(1)$ & 2(1) & $-1(1)$ \\
\hline $\mathrm{C}(81)$ & $27(2)$ & $26(2)$ & $27(2)$ & $0(1)$ & $2(1)$ & $-1(1)$ \\
\hline $\mathrm{C}(82)$ & $18(2)$ & $17(2)$ & $17(2)$ & $0(1)$ & $1(1)$ & $0(1)$ \\
\hline $\mathrm{C}(83)$ & $13(2)$ & $12(2)$ & $13(2)$ & $0(1)$ & 1(1) & 1(1) \\
\hline $\mathrm{C}(84)$ & $28(2)$ & $28(2)$ & $27(2)$ & $-1(1)$ & $3(1)$ & $0(1)$ \\
\hline$C(85)$ & $20(2)$ & $20(2)$ & $20(2)$ & $0(1)$ & $2(1)$ & $-1(1)$ \\
\hline$C(86)$ & $10(2)$ & $9(2)$ & $10(2)$ & $0(1)$ & $2(1)$ & $0(1)$ \\
\hline C(87) & $15(2)$ & $14(2)$ & $14(2)$ & $0(1)$ & $3(1)$ & $0(1)$ \\
\hline $\mathrm{C}(88)$ & $16(2)$ & $16(2)$ & $15(2)$ & 1(1) & $3(1)$ & $0(1)$ \\
\hline
\end{tabular}




\begin{tabular}{lcccccc}
$\mathrm{C}(89)$ & $14(2)$ & $13(2)$ & $12(2)$ & $1(1)$ & $2(1)$ & $-1(1)$ \\
$\mathrm{C}(90)$ & $22(2)$ & $22(2)$ & $23(2)$ & $0(1)$ & $3(1)$ & $1(1)$ \\
$\mathrm{C}(91)$ & $22(2)$ & $23(2)$ & $23(2)$ & $0(1)$ & $2(1)$ & $1(1)$ \\
$\mathrm{C}(92)$ & $9(2)$ & $9(2)$ & $9(2)$ & $1(1)$ & $2(1)$ & $-1(1)$ \\
$\mathrm{C}(93)$ & $15(2)$ & $16(2)$ & $15(2)$ & $0(1)$ & $2(1)$ & $1(1)$ \\
$\mathrm{C}(94)$ & $16(2)$ & $16(2)$ & $16(2)$ & $1(1)$ & $2(1)$ & $0(1)$ \\
$\mathrm{C}(95)$ & $8(2)$ & $7(2)$ & $8(2)$ & $1(1)$ & $1(1)$ & $0(1)$ \\
$\mathrm{C}(96)$ & $14(2)$ & $14(2)$ & $13(2)$ & $0(1)$ & $2(1)$ & $2(1)$ \\
$\mathrm{C}(97)$ & $18(2)$ & $18(2)$ & $17(2)$ & $0(1)$ & $2(1)$ & $0(1)$ \\
$\mathrm{C}(98)$ & $19(2)$ & $18(2)$ & $18(2)$ & $0(1)$ & $3(1)$ & $-1(1)$ \\
$\mathrm{C}(99)$ & $28(3)$ & $29(3)$ & $28(3)$ & $-1(1)$ & $3(1)$ & $0(1)$ \\
$\mathrm{C}(100)$ & $26(3)$ & $26(3)$ & $26(2)$ & $0(1)$ & $2(1)$ & $1(1)$ \\
& & & & & & \\
\hline
\end{tabular}


Table 5. Hydrogen coordinates ( x 10 $)$ and isotropic displacement parameters $\left(\AA^{2} \times 10^{3}\right)$ for kwq-c-48b.

\begin{tabular}{|c|c|c|c|c|}
\hline & $\mathrm{x}$ & $\mathrm{y}$ & $\mathrm{z}$ & $\mathrm{U}(\mathrm{eq})$ \\
\hline $\mathrm{H}(3)$ & 4449 & -2018 & 1066 & 24 \\
\hline $\mathrm{H}(5)$ & 3123 & 2188 & 1089 & 28 \\
\hline $\mathrm{H}(6)$ & 1266 & 1551 & 730 & 36 \\
\hline $\mathrm{H}(9 \mathrm{~A})$ & -645 & -1154 & 210 & 61 \\
\hline H(9B) & 189 & 297 & 176 & 61 \\
\hline $\mathrm{H}(9 \mathrm{C})$ & -518 & 98 & 467 & 61 \\
\hline $\mathrm{H}(10 \mathrm{~A})$ & 4285 & -3486 & 476 & 35 \\
\hline $\mathrm{H}(10 \mathrm{~B})$ & 3458 & -2414 & 246 & 35 \\
\hline $\mathrm{H}(10 \mathrm{C})$ & 3199 & -4176 & 244 & 35 \\
\hline $\mathrm{H}(11 \mathrm{~A})$ & 2161 & -5319 & 657 & 34 \\
\hline $\mathrm{H}(11 \mathrm{~B})$ & 3293 & -4737 & 887 & 34 \\
\hline $\mathrm{H}(15)$ & 2368 & -7446 & 1642 & 33 \\
\hline $\mathrm{H}(16)$ & 2284 & -8801 & 2067 & 39 \\
\hline $\mathrm{H}(18)$ & -300 & -6002 & 2335 & 17 \\
\hline H(19) & -241 & -4604 & 1889 & 21 \\
\hline $\mathrm{H}(21)$ & 2794 & -9308 & 2530 & 30 \\
\hline $\mathrm{H}(22)$ & 2953 & -10580 & 2997 & 41 \\
\hline $\mathrm{H}(23)$ & 1569 & -10120 & 3331 & 15 \\
\hline $\mathrm{H}(24)$ & -351 & -8701 & 3141 & 38 \\
\hline $\mathrm{H}(25)$ & -431 & -7490 & 2686 & 48 \\
\hline $\mathrm{H}(28)$ & 4460 & -878 & -249 & 24 \\
\hline $\mathrm{H}(30)$ & 5755 & -5084 & -398 & 45 \\
\hline $\mathrm{H}(31)$ & 3867 & -5645 & -710 & 34 \\
\hline $\mathrm{H}(34 \mathrm{~A})$ & 684 & -4560 & -1171 & 60 \\
\hline $\mathrm{H}(34 \mathrm{~B})$ & 1245 & -5797 & -934 & 60 \\
\hline $\mathrm{H}(34 \mathrm{C})$ & 2054 & -5193 & -1185 & 60 \\
\hline $\mathrm{H}(35 \mathrm{~A})$ & 1591 & -289 & -223 & 33 \\
\hline $\mathrm{H}(35 \mathrm{~B})$ & 1501 & -2071 & -199 & 33 \\
\hline $\mathrm{H}(35 \mathrm{C})$ & 450 & -1160 & -404 & 33 \\
\hline $\mathrm{H}(36 \mathrm{~A})$ & 1336 & 547 & -825 & 33 \\
\hline $\mathrm{H}(36 \mathrm{~B})$ & 2477 & 921 & -575 & 33 \\
\hline $\mathrm{H}(40)$ & 5302 & -131 & -1789 & 28 \\
\hline $\mathrm{H}(41)$ & 5448 & -1351 & -2236 & 26 \\
\hline
\end{tabular}




\begin{tabular}{|c|c|c|c|c|}
\hline $\mathrm{H}(43)$ & 1988 & -3160 & -2158 & 24 \\
\hline $\mathrm{H}(44)$ & 2100 & -2133 & -1681 & 22 \\
\hline $\mathrm{H}(46)$ & 4818 & -1778 & -2725 & 29 \\
\hline $\mathrm{H}(47)$ & 4907 & -3028 & -3175 & 32 \\
\hline $\mathrm{H}(48)$ & 3425 & -5102 & -3316 & 16 \\
\hline $\mathrm{H}(49)$ & 2374 & -6049 & -2922 & 24 \\
\hline $\mathrm{H}(50)$ & 2543 & -4864 & -2448 & 21 \\
\hline $\mathrm{H}(53)$ & -1770 & -19109 & -6082 & 12 \\
\hline $\mathrm{H}(55)$ & -3101 & -23356 & -6107 & 23 \\
\hline $\mathrm{H}(56)$ & -4267 & -22846 & -5733 & 26 \\
\hline $\mathrm{H}(59 \mathrm{~A})$ & -4990 & -21798 & -5247 & 46 \\
\hline $\mathrm{H}(59 \mathrm{~B})$ & -6092 & -21088 & -5472 & 46 \\
\hline $\mathrm{H}(59 \mathrm{C})$ & -5627 & -20268 & -5161 & 46 \\
\hline $\mathrm{H}(60 \mathrm{~A})$ & -1889 & -18807 & -5238 & 26 \\
\hline $\mathrm{H}(60 \mathrm{~B})$ & -2085 & -17033 & -5248 & 26 \\
\hline $\mathrm{H}(60 \mathrm{C})$ & -1244 & -17816 & -5474 & 26 \\
\hline $\mathrm{H}(61 \mathrm{~A})$ & -3464 & -15814 & -5641 & 9 \\
\hline $\mathrm{H}(61 \mathrm{~B})$ & -2551 & -16395 & -5871 & 9 \\
\hline $\mathrm{H}(65)$ & -4622 & -13467 & -6590 & 27 \\
\hline $\mathrm{H}(66)$ & -4810 & -12368 & -7076 & 31 \\
\hline $\mathrm{H}(68)$ & -7530 & -15275 & -7356 & 26 \\
\hline $\mathrm{H}(69)$ & -7268 & -16471 & -6894 & 21 \\
\hline $\mathrm{H}(71)$ & -4648 & -12020 & -7538 & 25 \\
\hline $\mathrm{H}(72)$ & -4911 & -10907 & -8012 & 20 \\
\hline $\mathrm{H}(73)$ & -6932 & -10922 & -8281 & 22 \\
\hline $\mathrm{H}(74)$ & -8399 & -12449 & -8161 & 16 \\
\hline $\mathrm{H}(75)$ & -8155 & -13553 & -7678 & 15 \\
\hline $\mathrm{H}(78)$ & -343 & -20283 & -4770 & 20 \\
\hline $\mathrm{H}(80)$ & 1205 & -16161 & -4616 & 22 \\
\hline $\mathrm{H}(81)$ & -307 & -15526 & -4305 & 32 \\
\hline $\mathrm{H}(84 \mathrm{~A})$ & -3015 & -16351 & -3863 & 42 \\
\hline $\mathrm{H}(84 \mathrm{~B})$ & -2499 & -15314 & -4112 & 42 \\
\hline $\mathrm{H}(84 \mathrm{C})$ & -1566 & -15976 & -3840 & 42 \\
\hline $\mathrm{H}(85 \mathrm{~A})$ & -3244 & -19108 & -4804 & 30 \\
\hline $\mathrm{H}(85 \mathrm{~B})$ & -4109 & -20041 & -4606 & 30 \\
\hline $\mathrm{H}(85 \mathrm{C})$ & -3131 & -20891 & -4786 & 30 \\
\hline $\mathrm{H}(86 \mathrm{~A})$ & -2702 & -21791 & -4173 & 12 \\
\hline $\mathrm{H}(86 \mathrm{~B})$ & -1767 & -22081 & -4416 & 12 \\
\hline $\mathrm{H}(90)$ & 2264 & -20940 & -3207 & 27 \\
\hline
\end{tabular}




\begin{tabular}{lrrrr}
$\mathrm{H}(91)$ & 2634 & -19701 & -2753 & 27 \\
$\mathrm{H}(93)$ & -748 & -17853 & -2842 & 18 \\
$\mathrm{H}(94)$ & -1279 & -19181 & -3304 & 19 \\
$\mathrm{H}(96)$ & 2917 & -18972 & -2304 & 17 \\
$\mathrm{H}(97)$ & 3210 & -17821 & -1824 & 21 \\
$\mathrm{H}(98)$ & 1977 & -15963 & -1725 & 22 \\
$\mathrm{H}(99)$ & 140 & -15337 & -2036 & 34 \\
$\mathrm{H}(100)$ & -213 & -16518 & -2501 & 31 \\
\hline
\end{tabular}


Table 6. Torsion angles $\left[{ }^{\circ}\right]$ for kwq-c-48b.

\begin{tabular}{|c|c|}
\hline $\mathrm{C}(12)-\mathrm{N}(2)-\mathrm{N}(3)-\mathrm{C}(13)$ & $-0.9(12)$ \\
\hline $\mathrm{C}(37)-\mathrm{N}(5)-\mathrm{N}(6)-\mathrm{C}(38)$ & $-3.1(14)$ \\
\hline $\mathrm{C}(62)-\mathrm{N}(8)-\mathrm{N}(9)-\mathrm{C}(63)$ & $-2.0(9)$ \\
\hline $\mathrm{C}(87)-\mathrm{N}(11)-\mathrm{N}(12)-\mathrm{C}(88)$ & $-4.2(12)$ \\
\hline $\mathrm{C}(11)-\mathrm{C}(1)-\mathrm{C}(2)-\mathrm{C}(7)$ & $-126.9(10)$ \\
\hline $\mathrm{C}(8)-\mathrm{C}(1)-\mathrm{C}(2)-\mathrm{C}(7)$ & $-7.3(11)$ \\
\hline $\mathrm{C}(10)-\mathrm{C}(1)-\mathrm{C}(2)-\mathrm{C}(7)$ & $105.8(10)$ \\
\hline $\mathrm{C}(11)-\mathrm{C}(1)-\mathrm{C}(2)-\mathrm{C}(3)$ & $56.6(14)$ \\
\hline $\mathrm{C}(8)-\mathrm{C}(1)-\mathrm{C}(2)-\mathrm{C}(3)$ & 176.1(9) \\
\hline $\mathrm{C}(10)-\mathrm{C}(1)-\mathrm{C}(2)-\mathrm{C}(3)$ & $-70.8(13)$ \\
\hline $\mathrm{C}(7)-\mathrm{C}(2)-\mathrm{C}(3)-\mathrm{C}(4)$ & $0.9(13)$ \\
\hline$C(1)-C(2)-C(3)-C(4)$ & $177.3(9)$ \\
\hline $\mathrm{C}(2)-\mathrm{C}(3)-\mathrm{C}(4)-\mathrm{C}(5)$ & $-2.8(15)$ \\
\hline$C(2)-C(3)-C(4)-B r(1)$ & $-177.3(7)$ \\
\hline$C(3)-C(4)-C(5)-C(6)$ & $5.0(17)$ \\
\hline $\mathrm{Br}(1)-\mathrm{C}(4)-\mathrm{C}(5)-\mathrm{C}(6)$ & $179.2(8)$ \\
\hline$C(4)-C(5)-C(6)-C(7)$ & $-5.1(15)$ \\
\hline$C(5)-C(6)-C(7)-C(2)$ & $3.6(16)$ \\
\hline$C(5)-C(6)-C(7)-N(1)$ & $180.0(9)$ \\
\hline$C(3)-C(2)-C(7)-C(6)$ & $-1.9(16)$ \\
\hline$C(1)-C(2)-C(7)-C(6)$ & $-178.8(10)$ \\
\hline $\mathrm{C}(3)-\mathrm{C}(2)-\mathrm{C}(7)-\mathrm{N}(1)$ & $-178.7(9)$ \\
\hline $\mathrm{C}(1)-\mathrm{C}(2)-\mathrm{C}(7)-\mathrm{N}(1)$ & $4.4(12)$ \\
\hline $\mathrm{C}(8)-\mathrm{N}(1)-\mathrm{C}(7)-\mathrm{C}(6)$ & $-175.8(11)$ \\
\hline $\mathrm{C}(9)-\mathrm{N}(1)-\mathrm{C}(7)-\mathrm{C}(6)$ & $3.7(17)$ \\
\hline $\mathrm{C}(8)-\mathrm{N}(1)-\mathrm{C}(7)-\mathrm{C}(2)$ & $1.2(12)$ \\
\hline $\mathrm{C}(9)-\mathrm{N}(1)-\mathrm{C}(7)-\mathrm{C}(2)$ & $-179.3(10)$ \\
\hline $\mathrm{C}(7)-\mathrm{N}(1)-\mathrm{C}(8)-\mathrm{O}(1)$ & $176.9(10)$ \\
\hline $\mathrm{C}(9)-\mathrm{N}(1)-\mathrm{C}(8)-\mathrm{O}(1)$ & $-2.6(17)$ \\
\hline $\mathrm{C}(7)-\mathrm{N}(1)-\mathrm{C}(8)-\mathrm{C}(1)$ & $-6.0(11)$ \\
\hline $\mathrm{C}(9)-\mathrm{N}(1)-\mathrm{C}(8)-\mathrm{C}(1)$ & $174.5(10)$ \\
\hline $\mathrm{C}(2)-\mathrm{C}(1)-\mathrm{C}(8)-\mathrm{O}(1)$ & $-175.1(11)$ \\
\hline $\mathrm{C}(11)-\mathrm{C}(1)-\mathrm{C}(8)-\mathrm{O}(1)$ & $-52.3(14)$ \\
\hline $\mathrm{C}(10)-\mathrm{C}(1)-\mathrm{C}(8)-\mathrm{O}(1)$ & $69.6(14)$ \\
\hline $\mathrm{C}(2)-\mathrm{C}(1)-\mathrm{C}(8)-\mathrm{N}(1)$ & $8.0(10)$ \\
\hline $\mathrm{C}(11)-\mathrm{C}(1)-\mathrm{C}(8)-\mathrm{N}(1)$ & $130.8(10)$ \\
\hline $\mathrm{C}(10)-\mathrm{C}(1)-\mathrm{C}(8)-\mathrm{N}(1)$ & $-107.3(10)$ \\
\hline
\end{tabular}




\begin{tabular}{|c|c|}
\hline$C(2)-C(1)-C(11)-C(12)$ & $50.8(14)$ \\
\hline $\mathrm{C}(8)-\mathrm{C}(1)-\mathrm{C}(11)-\mathrm{C}(12)$ & $-62.2(13)$ \\
\hline $\mathrm{C}(10)-\mathrm{C}(1)-\mathrm{C}(11)-\mathrm{C}(12)$ & 177.9(9) \\
\hline $\mathrm{N}(3)-\mathrm{N}(2)-\mathrm{C}(12)-\mathrm{O}(2)$ & $-4.9(12)$ \\
\hline $\mathrm{N}(3)-\mathrm{N}(2)-\mathrm{C}(12)-\mathrm{C}(11)$ & $-178.0(12)$ \\
\hline $\mathrm{C}(13)-\mathrm{O}(2)-\mathrm{C}(12)-\mathrm{N}(2)$ & $8.0(12)$ \\
\hline $\mathrm{C}(13)-\mathrm{O}(2)-\mathrm{C}(12)-\mathrm{C}(11)$ & $-177.8(9)$ \\
\hline $\mathrm{C}(1)-\mathrm{C}(11)-\mathrm{C}(12)-\mathrm{N}(2)$ & $17.8(19)$ \\
\hline $\mathrm{C}(1)-\mathrm{C}(11)-\mathrm{C}(12)-\mathrm{O}(2)$ & $-155.3(9)$ \\
\hline $\mathrm{N}(2)-\mathrm{N}(3)-\mathrm{C}(13)-\mathrm{O}(2)$ & $6.2(12)$ \\
\hline $\mathrm{N}(2)-\mathrm{N}(3)-\mathrm{C}(13)-\mathrm{C}(14)$ & $173.6(12)$ \\
\hline $\mathrm{C}(12)-\mathrm{O}(2)-\mathrm{C}(13)-\mathrm{N}(3)$ & $-8.6(11)$ \\
\hline $\mathrm{C}(12)-\mathrm{O}(2)-\mathrm{C}(13)-\mathrm{C}(14)$ & $-178.5(9)$ \\
\hline $\mathrm{N}(3)-\mathrm{C}(13)-\mathrm{C}(14)-\mathrm{C}(15)$ & $179.8(13)$ \\
\hline $\mathrm{O}(2)-\mathrm{C}(13)-\mathrm{C}(14)-\mathrm{C}(15)$ & $-12.6(16)$ \\
\hline $\mathrm{N}(3)-\mathrm{C}(13)-\mathrm{C}(14)-\mathrm{C}(19)$ & $0.4(18)$ \\
\hline $\mathrm{O}(2)-\mathrm{C}(13)-\mathrm{C}(14)-\mathrm{C}(19)$ & $168.0(9)$ \\
\hline$C(19)-C(14)-C(15)-C(16)$ & $1.8(19)$ \\
\hline $\mathrm{C}(13)-\mathrm{C}(14)-\mathrm{C}(15)-\mathrm{C}(16)$ & $-177.5(12)$ \\
\hline $\mathrm{C}(14)-\mathrm{C}(15)-\mathrm{C}(16)-\mathrm{C}(17)$ & $-6(2)$ \\
\hline$C(15)-C(16)-C(17)-C(18)$ & $9.5(18)$ \\
\hline$C(15)-C(16)-C(17)-C(20)$ & $-175.6(11)$ \\
\hline $\mathrm{C}(16)-\mathrm{C}(17)-\mathrm{C}(18)-\mathrm{C}(19)$ & $-8.8(15)$ \\
\hline $\mathrm{C}(20)-\mathrm{C}(17)-\mathrm{C}(18)-\mathrm{C}(19)$ & 176.1(10) \\
\hline$C(17)-C(18)-C(19)-C(14)$ & $5.3(15)$ \\
\hline $\mathrm{C}(15)-\mathrm{C}(14)-\mathrm{C}(19)-\mathrm{C}(18)$ & $-1.4(17)$ \\
\hline $\mathrm{C}(13)-\mathrm{C}(14)-\mathrm{C}(19)-\mathrm{C}(18)$ & $178.0(9)$ \\
\hline$C(16)-C(17)-C(20)-C(25)$ & $-160.3(13)$ \\
\hline $\mathrm{C}(18)-\mathrm{C}(17)-\mathrm{C}(20)-\mathrm{C}(25)$ & $14.2(16)$ \\
\hline$C(16)-C(17)-C(20)-C(21)$ & $19.8(17)$ \\
\hline$C(18)-C(17)-C(20)-C(21)$ & $-165.7(10)$ \\
\hline$C(25)-C(20)-C(21)-C(22)$ & $-1.7(18)$ \\
\hline$C(17)-C(20)-C(21)-C(22)$ & $178.2(11)$ \\
\hline$C(20)-C(21)-C(22)-C(23)$ & $-3(2)$ \\
\hline $\mathrm{C}(21)-\mathrm{C}(22)-\mathrm{C}(23)-\mathrm{C}(24)$ & $6.9(18)$ \\
\hline $\mathrm{C}(22)-\mathrm{C}(23)-\mathrm{C}(24)-\mathrm{C}(25)$ & $-7.1(19)$ \\
\hline$C(23)-C(24)-C(25)-C(20)$ & $3(2)$ \\
\hline $\mathrm{C}(21)-\mathrm{C}(20)-\mathrm{C}(25)-\mathrm{C}(24)$ & $1(2)$ \\
\hline$C(17)-C(20)-C(25)-C(24)$ & $-178.5(13)$ \\
\hline
\end{tabular}




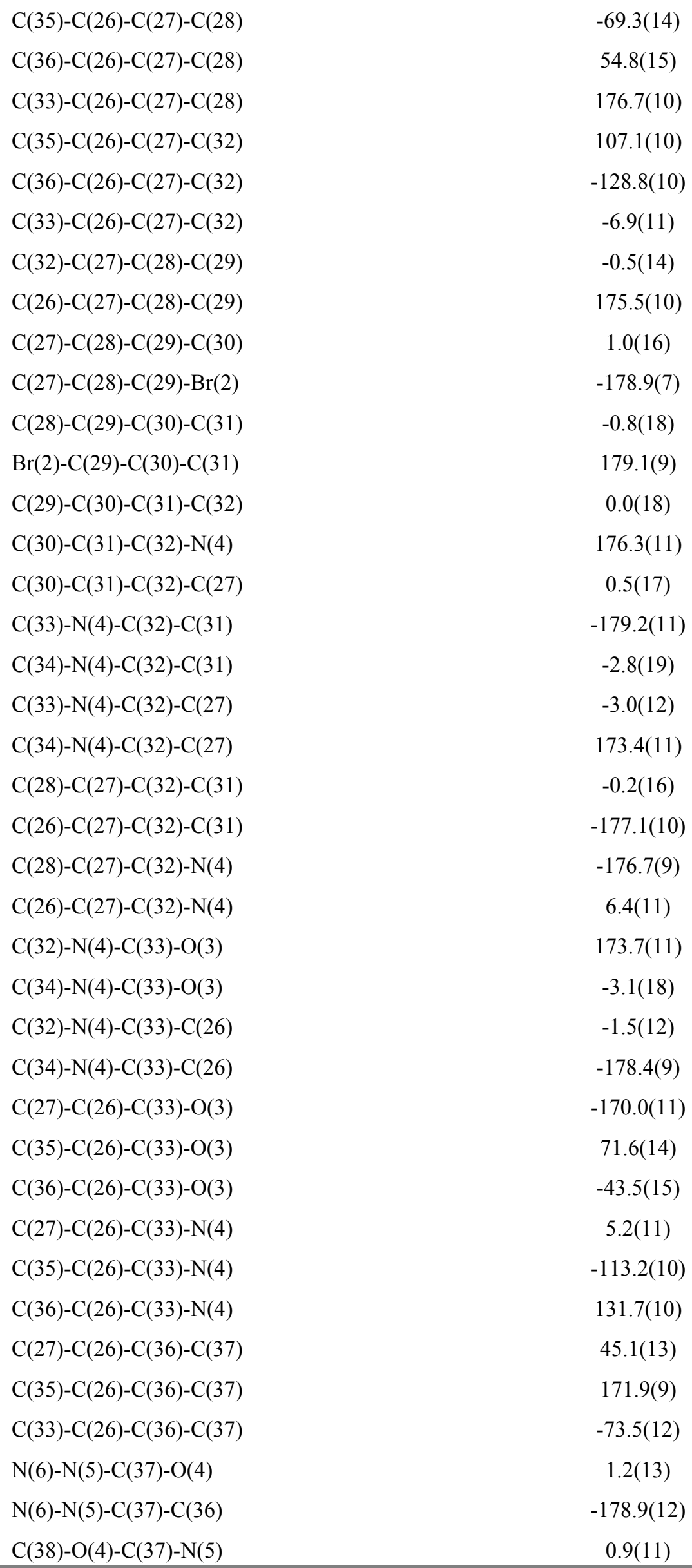




\begin{tabular}{|c|c|}
\hline $\mathrm{C}(38)-\mathrm{O}(4)-\mathrm{C}(37)-\mathrm{C}(36)$ & $-179.1(10)$ \\
\hline $\mathrm{C}(26)-\mathrm{C}(36)-\mathrm{C}(37)-\mathrm{N}(5)$ & $-117.9(14)$ \\
\hline $\mathrm{C}(26)-\mathrm{C}(36)-\mathrm{C}(37)-\mathrm{O}(4)$ & $62.1(13)$ \\
\hline $\mathrm{N}(5)-\mathrm{N}(6)-\mathrm{C}(38)-\mathrm{O}(4)$ & $3.7(13)$ \\
\hline $\mathrm{N}(5)-\mathrm{N}(6)-\mathrm{C}(38)-\mathrm{C}(39)$ & $-179.7(11)$ \\
\hline $\mathrm{C}(37)-\mathrm{O}(4)-\mathrm{C}(38)-\mathrm{N}(6)$ & $-3.0(12)$ \\
\hline $\mathrm{C}(37)-\mathrm{O}(4)-\mathrm{C}(38)-\mathrm{C}(39)$ & $179.9(9)$ \\
\hline $\mathrm{N}(6)-\mathrm{C}(38)-\mathrm{C}(39)-\mathrm{C}(44)$ & $-173.8(12)$ \\
\hline $\mathrm{O}(4)-\mathrm{C}(38)-\mathrm{C}(39)-\mathrm{C}(44)$ & $2.6(14)$ \\
\hline $\mathrm{N}(6)-\mathrm{C}(38)-\mathrm{C}(39)-\mathrm{C}(40)$ & $3.4(18)$ \\
\hline $\mathrm{O}(4)-\mathrm{C}(38)-\mathrm{C}(39)-\mathrm{C}(40)$ & $179.9(9)$ \\
\hline$C(44)-C(39)-C(40)-C(41)$ & $-4.3(16)$ \\
\hline $\mathrm{C}(38)-\mathrm{C}(39)-\mathrm{C}(40)-\mathrm{C}(41)$ & $178.3(10)$ \\
\hline $\mathrm{C}(39)-\mathrm{C}(40)-\mathrm{C}(41)-\mathrm{C}(42)$ & $5.4(17)$ \\
\hline $\mathrm{C}(40)-\mathrm{C}(41)-\mathrm{C}(42)-\mathrm{C}(43)$ & $-1.5(15)$ \\
\hline$C(40)-C(41)-C(42)-C(45)$ & $-179.2(11)$ \\
\hline $\mathrm{C}(45)-\mathrm{C}(42)-\mathrm{C}(43)-\mathrm{C}(44)$ & $174.5(10)$ \\
\hline$C(41)-C(42)-C(43)-C(44)$ & $-3.2(15)$ \\
\hline$C(40)-C(39)-C(44)-C(43)$ & $-0.7(16)$ \\
\hline $\mathrm{C}(38)-\mathrm{C}(39)-\mathrm{C}(44)-\mathrm{C}(43)$ & $176.6(9)$ \\
\hline $\mathrm{C}(42)-\mathrm{C}(43)-\mathrm{C}(44)-\mathrm{C}(39)$ & $4.4(16)$ \\
\hline$C(43)-C(42)-C(45)-C(46)$ & $153.7(11)$ \\
\hline$C(41)-C(42)-C(45)-C(46)$ & $-28.7(17)$ \\
\hline$C(43)-C(42)-C(45)-C(50)$ & $-20.6(16)$ \\
\hline$C(41)-C(42)-C(45)-C(50)$ & $157.0(11)$ \\
\hline $\mathrm{C}(50)-\mathrm{C}(45)-\mathrm{C}(46)-\mathrm{C}(47)$ & $-4.2(18)$ \\
\hline $\mathrm{C}(42)-\mathrm{C}(45)-\mathrm{C}(46)-\mathrm{C}(47)$ & $-178.3(11)$ \\
\hline$C(45)-C(46)-C(47)-C(48)$ & $8.2(17)$ \\
\hline $\mathrm{C}(46)-\mathrm{C}(47)-\mathrm{C}(48)-\mathrm{C}(49)$ & $-7.7(15)$ \\
\hline $\mathrm{C}(47)-\mathrm{C}(48)-\mathrm{C}(49)-\mathrm{C}(50)$ & $3.7(15)$ \\
\hline$C(46)-C(45)-C(50)-C(49)$ & $-0.1(17)$ \\
\hline$C(42)-C(45)-C(50)-C(49)$ & $174.2(10)$ \\
\hline$C(48)-C(49)-C(50)-C(45)$ & $0.1(16)$ \\
\hline $\mathrm{C}(58)-\mathrm{C}(51)-\mathrm{C}(52)-\mathrm{C}(53)$ & $173.5(9)$ \\
\hline$C(60)-C(51)-C(52)-C(53)$ & $-72.5(12)$ \\
\hline$C(61)-C(51)-C(52)-C(53)$ & $50.1(13)$ \\
\hline$C(58)-C(51)-C(52)-C(57)$ & $-9.2(9)$ \\
\hline$C(60)-C(51)-C(52)-C(57)$ & $104.8(8)$ \\
\hline $\mathrm{C}(61)-\mathrm{C}(51)-\mathrm{C}(52)-\mathrm{C}(57)$ & $-132.6(8)$ \\
\hline
\end{tabular}




\begin{tabular}{|c|c|}
\hline $\mathrm{C}(57)-\mathrm{C}(52)-\mathrm{C}(53)-\mathrm{C}(54)$ & $-3.1(13)$ \\
\hline $\mathrm{C}(51)-\mathrm{C}(52)-\mathrm{C}(53)-\mathrm{C}(54)$ & $173.9(9)$ \\
\hline $\mathrm{C}(52)-\mathrm{C}(53)-\mathrm{C}(54)-\mathrm{C}(55)$ & $4.0(15)$ \\
\hline $\mathrm{C}(52)-\mathrm{C}(53)-\mathrm{C}(54)-\mathrm{Br}(3)$ & $-176.3(7)$ \\
\hline $\mathrm{C}(53)-\mathrm{C}(54)-\mathrm{C}(55)-\mathrm{C}(56)$ & $-4.6(17)$ \\
\hline $\operatorname{Br}(3)-C(54)-C(55)-C(56)$ & $175.7(8)$ \\
\hline$C(54)-C(55)-C(56)-C(57)$ & $3.9(15)$ \\
\hline $\mathrm{C}(58)-\mathrm{N}(7)-\mathrm{C}(57)-\mathrm{C}(52)$ & $2.4(11)$ \\
\hline $\mathrm{C}(59)-\mathrm{N}(7)-\mathrm{C}(57)-\mathrm{C}(52)$ & $-180.0(9)$ \\
\hline $\mathrm{C}(58)-\mathrm{N}(7)-\mathrm{C}(57)-\mathrm{C}(56)$ & $-177.8(10)$ \\
\hline $\mathrm{C}(59)-\mathrm{N}(7)-\mathrm{C}(57)-\mathrm{C}(56)$ & $-0.2(16)$ \\
\hline $\mathrm{C}(53)-\mathrm{C}(52)-\mathrm{C}(57)-\mathrm{N}(7)$ & $-177.7(8)$ \\
\hline $\mathrm{C}(51)-\mathrm{C}(52)-\mathrm{C}(57)-\mathrm{N}(7)$ & $4.7(10)$ \\
\hline $\mathrm{C}(53)-\mathrm{C}(52)-\mathrm{C}(57)-\mathrm{C}(56)$ & $2.6(14)$ \\
\hline $\mathrm{C}(51)-\mathrm{C}(52)-\mathrm{C}(57)-\mathrm{C}(56)$ & $-175.0(8)$ \\
\hline $\mathrm{C}(55)-\mathrm{C}(56)-\mathrm{C}(57)-\mathrm{N}(7)$ & $177.2(10)$ \\
\hline $\mathrm{C}(55)-\mathrm{C}(56)-\mathrm{C}(57)-\mathrm{C}(52)$ & $-3.0(15)$ \\
\hline $\mathrm{C}(57)-\mathrm{N}(7)-\mathrm{C}(58)-\mathrm{O}(5)$ & $178.8(9)$ \\
\hline $\mathrm{C}(59)-\mathrm{N}(7)-\mathrm{C}(58)-\mathrm{O}(5)$ & $1.2(15)$ \\
\hline $\mathrm{C}(57)-\mathrm{N}(7)-\mathrm{C}(58)-\mathrm{C}(51)$ & $-8.5(10)$ \\
\hline $\mathrm{C}(59)-\mathrm{N}(7)-\mathrm{C}(58)-\mathrm{C}(51)$ & $173.9(8)$ \\
\hline $\mathrm{C}(52)-\mathrm{C}(51)-\mathrm{C}(58)-\mathrm{O}(5)$ & $-176.9(9)$ \\
\hline $\mathrm{C}(60)-\mathrm{C}(51)-\mathrm{C}(58)-\mathrm{O}(5)$ & $67.0(12)$ \\
\hline $\mathrm{C}(61)-\mathrm{C}(51)-\mathrm{C}(58)-\mathrm{O}(5)$ & $-51.0(12)$ \\
\hline $\mathrm{C}(52)-\mathrm{C}(51)-\mathrm{C}(58)-\mathrm{N}(7)$ & $10.5(9)$ \\
\hline $\mathrm{C}(60)-\mathrm{C}(51)-\mathrm{C}(58)-\mathrm{N}(7)$ & $-105.6(8)$ \\
\hline $\mathrm{C}(61)-\mathrm{C}(51)-\mathrm{C}(58)-\mathrm{N}(7)$ & $136.4(8)$ \\
\hline $\mathrm{C}(58)-\mathrm{C}(51)-\mathrm{C}(61)-\mathrm{C}(62)$ & $-64.3(9)$ \\
\hline $\mathrm{C}(52)-\mathrm{C}(51)-\mathrm{C}(61)-\mathrm{C}(62)$ & $53.5(10)$ \\
\hline $\mathrm{C}(60)-\mathrm{C}(51)-\mathrm{C}(61)-\mathrm{C}(62)$ & $177.6(7)$ \\
\hline $\mathrm{C}(63)-\mathrm{O}(6)-\mathrm{C}(62)-\mathrm{N}(8)$ & $-2.8(9)$ \\
\hline $\mathrm{C}(63)-\mathrm{O}(6)-\mathrm{C}(62)-\mathrm{C}(61)$ & $-179.4(7)$ \\
\hline $\mathrm{N}(9)-\mathrm{N}(8)-\mathrm{C}(62)-\mathrm{O}(6)$ & $2.9(10)$ \\
\hline $\mathrm{N}(9)-\mathrm{N}(8)-\mathrm{C}(62)-\mathrm{C}(61)$ & 179.2(8) \\
\hline $\mathrm{C}(51)-\mathrm{C}(61)-\mathrm{C}(62)-\mathrm{O}(6)$ & $-161.0(7)$ \\
\hline $\mathrm{C}(51)-\mathrm{C}(61)-\mathrm{C}(62)-\mathrm{N}(8)$ & $23.1(12)$ \\
\hline $\mathrm{N}(8)-\mathrm{N}(9)-\mathrm{C}(63)-\mathrm{O}(6)$ & $0.4(9)$ \\
\hline N(8)-N(9)-C(63)-C(64) & $176.8(9)$ \\
\hline $\mathrm{C}(62)-\mathrm{O}(6)-\mathrm{C}(63)-\mathrm{N}(9)$ & $1.4(9)$ \\
\hline
\end{tabular}




\begin{tabular}{|c|c|}
\hline $\mathrm{C}(62)-\mathrm{O}(6)-\mathrm{C}(63)-\mathrm{C}(64)$ & $-175.5(7)$ \\
\hline N(9)-C(63)-C(64)-C(69) & $-7.5(15)$ \\
\hline $\mathrm{O}(6)-\mathrm{C}(63)-\mathrm{C}(64)-\mathrm{C}(69)$ & $168.6(8)$ \\
\hline $\mathrm{N}(9)-\mathrm{C}(63)-\mathrm{C}(64)-\mathrm{C}(65)$ & $169.2(9)$ \\
\hline $\mathrm{O}(6)-\mathrm{C}(63)-\mathrm{C}(64)-\mathrm{C}(65)$ & $-14.7(12)$ \\
\hline$C(69)-C(64)-C(65)-C(66)$ & $-11.5(14)$ \\
\hline$C(63)-C(64)-C(65)-C(66)$ & $171.6(9)$ \\
\hline $\mathrm{C}(64)-\mathrm{C}(65)-\mathrm{C}(66)-\mathrm{C}(67)$ & $7.9(16)$ \\
\hline $\mathrm{C}(65)-\mathrm{C}(66)-\mathrm{C}(67)-\mathrm{C}(68)$ & $-1.9(16)$ \\
\hline$C(65)-C(66)-C(67)-C(70)$ & $179.9(10)$ \\
\hline $\mathrm{C}(66)-\mathrm{C}(67)-\mathrm{C}(68)-\mathrm{C}(69)$ & $-0.6(16)$ \\
\hline $\mathrm{C}(70)-\mathrm{C}(67)-\mathrm{C}(68)-\mathrm{C}(69)$ & $177.6(10)$ \\
\hline $\mathrm{C}(67)-\mathrm{C}(68)-\mathrm{C}(69)-\mathrm{C}(64)$ & $-3.2(16)$ \\
\hline $\mathrm{C}(65)-\mathrm{C}(64)-\mathrm{C}(69)-\mathrm{C}(68)$ & $9.3(14)$ \\
\hline $\mathrm{C}(63)-\mathrm{C}(64)-\mathrm{C}(69)-\mathrm{C}(68)$ & $-174.0(9)$ \\
\hline$C(66)-C(67)-C(70)-C(71)$ & $22.8(15)$ \\
\hline $\mathrm{C}(68)-\mathrm{C}(67)-\mathrm{C}(70)-\mathrm{C}(71)$ & $-155.4(10)$ \\
\hline$C(66)-C(67)-C(70)-C(75)$ & $-156.1(10)$ \\
\hline $\mathrm{C}(68)-\mathrm{C}(67)-\mathrm{C}(70)-\mathrm{C}(75)$ & $25.7(14)$ \\
\hline $\mathrm{C}(75)-\mathrm{C}(70)-\mathrm{C}(71)-\mathrm{C}(72)$ & $-1.1(15)$ \\
\hline $\mathrm{C}(67)-\mathrm{C}(70)-\mathrm{C}(71)-\mathrm{C}(72)$ & $-180.0(10)$ \\
\hline $\mathrm{C}(70)-\mathrm{C}(71)-\mathrm{C}(72)-\mathrm{C}(73)$ & $4.1(16)$ \\
\hline $\mathrm{C}(71)-\mathrm{C}(72)-\mathrm{C}(73)-\mathrm{C}(74)$ & $-8.4(16)$ \\
\hline $\mathrm{C}(72)-\mathrm{C}(73)-\mathrm{C}(74)-\mathrm{C}(75)$ & $8.9(16)$ \\
\hline$C(73)-C(74)-C(75)-C(70)$ & $-5.3(15)$ \\
\hline $\mathrm{C}(71)-\mathrm{C}(70)-\mathrm{C}(75)-\mathrm{C}(74)$ & $1.4(14)$ \\
\hline $\mathrm{C}(67)-\mathrm{C}(70)-\mathrm{C}(75)-\mathrm{C}(74)$ & $-179.6(9)$ \\
\hline $\mathrm{C}(83)-\mathrm{C}(76)-\mathrm{C}(77)-\mathrm{C}(78)$ & $179.2(10)$ \\
\hline $\mathrm{C}(85)-\mathrm{C}(76)-\mathrm{C}(77)-\mathrm{C}(78)$ & $-65.7(13)$ \\
\hline $\mathrm{C}(86)-\mathrm{C}(76)-\mathrm{C}(77)-\mathrm{C}(78)$ & $59.9(13)$ \\
\hline $\mathrm{C}(83)-\mathrm{C}(76)-\mathrm{C}(77)-\mathrm{C}(82)$ & $-6.4(9)$ \\
\hline $\mathrm{C}(85)-\mathrm{C}(76)-\mathrm{C}(77)-\mathrm{C}(82)$ & $108.7(9)$ \\
\hline $\mathrm{C}(86)-\mathrm{C}(76)-\mathrm{C}(77)-\mathrm{C}(82)$ & $-125.7(8)$ \\
\hline $\mathrm{C}(82)-\mathrm{C}(77)-\mathrm{C}(78)-\mathrm{C}(79)$ & $2.3(14)$ \\
\hline $\mathrm{C}(76)-\mathrm{C}(77)-\mathrm{C}(78)-\mathrm{C}(79)$ & $176.3(9)$ \\
\hline $\mathrm{C}(77)-\mathrm{C}(78)-\mathrm{C}(79)-\mathrm{C}(80)$ & $-3.1(15)$ \\
\hline $\mathrm{C}(77)-\mathrm{C}(78)-\mathrm{C}(79)-\mathrm{Br}(4)$ & 177.1(7) \\
\hline $\mathrm{C}(78)-\mathrm{C}(79)-\mathrm{C}(80)-\mathrm{C}(81)$ & $1.2(15)$ \\
\hline $\mathrm{Br}(4)-\mathrm{C}(79)-\mathrm{C}(80)-\mathrm{C}(81)$ & $-179.0(8)$ \\
\hline
\end{tabular}




\begin{tabular}{|c|c|}
\hline $\mathrm{C}(79)-\mathrm{C}(80)-\mathrm{C}(81)-\mathrm{C}(82)$ & $1.5(15)$ \\
\hline $\mathrm{C}(80)-\mathrm{C}(81)-\mathrm{C}(82)-\mathrm{N}(10)$ & $178.5(9)$ \\
\hline $\mathrm{C}(80)-\mathrm{C}(81)-\mathrm{C}(82)-\mathrm{C}(77)$ & $-2.2(15)$ \\
\hline $\mathrm{C}(83)-\mathrm{N}(10)-\mathrm{C}(82)-\mathrm{C}(81)$ & $178.9(10)$ \\
\hline $\mathrm{C}(84)-\mathrm{N}(10)-\mathrm{C}(82)-\mathrm{C}(81)$ & $-2.6(16)$ \\
\hline $\mathrm{C}(83)-\mathrm{N}(10)-\mathrm{C}(82)-\mathrm{C}(77)$ & $-0.4(11)$ \\
\hline C(84)-N(10)-C(82)-C(77) & $178.0(9)$ \\
\hline $\mathrm{C}(78)-\mathrm{C}(77)-\mathrm{C}(82)-\mathrm{C}(81)$ & $0.3(14)$ \\
\hline $\mathrm{C}(76)-\mathrm{C}(77)-\mathrm{C}(82)-\mathrm{C}(81)$ & $-174.9(9)$ \\
\hline $\mathrm{C}(78)-\mathrm{C}(77)-\mathrm{C}(82)-\mathrm{N}(10)$ & $179.7(8)$ \\
\hline $\mathrm{C}(76)-\mathrm{C}(77)-\mathrm{C}(82)-\mathrm{N}(10)$ & $4.6(10)$ \\
\hline $\mathrm{C}(82)-\mathrm{N}(10)-\mathrm{C}(83)-\mathrm{O}(7)$ & $-179.3(9)$ \\
\hline $\mathrm{C}(84)-\mathrm{N}(10)-\mathrm{C}(83)-\mathrm{O}(7)$ & $2.4(16)$ \\
\hline $\mathrm{C}(82)-\mathrm{N}(10)-\mathrm{C}(83)-\mathrm{C}(76)$ & $-3.8(10)$ \\
\hline $\mathrm{C}(84)-\mathrm{N}(10)-\mathrm{C}(83)-\mathrm{C}(76)$ & $177.9(9)$ \\
\hline $\mathrm{C}(77)-\mathrm{C}(76)-\mathrm{C}(83)-\mathrm{O}(7)$ & $-178.2(8)$ \\
\hline $\mathrm{C}(85)-\mathrm{C}(76)-\mathrm{C}(83)-\mathrm{O}(7)$ & $65.1(11)$ \\
\hline $\mathrm{C}(86)-\mathrm{C}(76)-\mathrm{C}(83)-\mathrm{O}(7)$ & $-57.4(11)$ \\
\hline $\mathrm{C}(77)-\mathrm{C}(76)-\mathrm{C}(83)-\mathrm{N}(10)$ & $6.1(9)$ \\
\hline $\mathrm{C}(85)-\mathrm{C}(76)-\mathrm{C}(83)-\mathrm{N}(10)$ & $-110.5(8)$ \\
\hline $\mathrm{C}(86)-\mathrm{C}(76)-\mathrm{C}(83)-\mathrm{N}(10)$ & $127.0(8)$ \\
\hline $\mathrm{C}(77)-\mathrm{C}(76)-\mathrm{C}(86)-\mathrm{C}(87)$ & $47.5(10)$ \\
\hline $\mathrm{C}(83)-\mathrm{C}(76)-\mathrm{C}(86)-\mathrm{C}(87)$ & $-66.5(10)$ \\
\hline C(85)-C(76)-C(86)-C(87) & $172.7(8)$ \\
\hline $\mathrm{N}(12)-\mathrm{N}(11)-\mathrm{C}(87)-\mathrm{O}(8)$ & $5.7(12)$ \\
\hline $\mathrm{N}(12)-\mathrm{N}(11)-\mathrm{C}(87)-\mathrm{C}(86)$ & $179.2(10)$ \\
\hline $\mathrm{C}(88)-\mathrm{O}(8)-\mathrm{C}(87)-\mathrm{N}(11)$ & $-4.8(11)$ \\
\hline $\mathrm{C}(88)-\mathrm{O}(8)-\mathrm{C}(87)-\mathrm{C}(86)$ & $-178.9(8)$ \\
\hline $\mathrm{C}(76)-\mathrm{C}(86)-\mathrm{C}(87)-\mathrm{N}(11)$ & $-117.4(12)$ \\
\hline $\mathrm{C}(76)-\mathrm{C}(86)-\mathrm{C}(87)-\mathrm{O}(8)$ & $55.7(11)$ \\
\hline $\mathrm{N}(11)-\mathrm{N}(12)-\mathrm{C}(88)-\mathrm{O}(8)$ & $1.4(11)$ \\
\hline $\mathrm{N}(11)-\mathrm{N}(12)-\mathrm{C}(88)-\mathrm{C}(89)$ & $-178.6(10)$ \\
\hline $\mathrm{C}(87)-\mathrm{O}(8)-\mathrm{C}(88)-\mathrm{N}(12)$ & $1.8(10)$ \\
\hline $\mathrm{C}(87)-\mathrm{O}(8)-\mathrm{C}(88)-\mathrm{C}(89)$ & $-178.2(9)$ \\
\hline $\mathrm{N}(12)-\mathrm{C}(88)-\mathrm{C}(89)-\mathrm{C}(90)$ & $7.6(17)$ \\
\hline $\mathrm{O}(8)-\mathrm{C}(88)-\mathrm{C}(89)-\mathrm{C}(90)$ & $-172.4(10)$ \\
\hline $\mathrm{N}(12)-\mathrm{C}(88)-\mathrm{C}(89)-\mathrm{C}(94)$ & $-173.9(9)$ \\
\hline $\mathrm{O}(8)-\mathrm{C}(88)-\mathrm{C}(89)-\mathrm{C}(94)$ & $6.1(13)$ \\
\hline C(88)-C(89)-C(90)-C(91) & $178.0(10)$ \\
\hline
\end{tabular}




$\begin{array}{lc}C(94)-C(89)-C(90)-C(91) & -0.4(16) \\ C(89)-C(90)-C(91)-C(92) & -0.8(17) \\ C(90)-C(91)-C(92)-C(93) & 0.1(17) \\ C(90)-C(91)-C(92)-C(95) & 177.7(10) \\ C(91)-C(92)-C(93)-C(94) & 1.7(14) \\ C(95)-C(92)-C(93)-C(94) & -176.0(9) \\ C(92)-C(93)-C(94)-C(89) & -2.7(14) \\ C(90)-C(89)-C(94)-C(93) & 2.1(14) \\ C(88)-C(89)-C(94)-C(93) & -176.5(9) \\ C(91)-C(92)-C(95)-C(100) & 167.1(11) \\ C(93)-C(92)-C(95)-C(100) & -15.3(14) \\ C(91)-C(92)-C(95)-C(96) & -8.1(14) \\ C(93)-C(92)-C(95)-C(96) & 169.5(8) \\ C(100)-C(95)-C(96)-C(97) & 4.8(13) \\ C(92)-C(95)-C(96)-C(97) & -179.7(9) \\ C(95)-C(96)-C(97)-C(98) & -5.8(15) \\ C(96)-C(97)-C(98)-C(99) & 5.5(17) \\ C(97)-C(98)-C(99)-C(100) & -4.0(17) \\ C(98)-C(99)-C(100)-C(95) & 3.0(18) \\ C(96)-C(95)-C(100)-C(99) & -3.5(16) \\ C(92)-C(95)-C(100)-C(99) & -179.0(10) \\ & \end{array}$

Symmetry transformations used to generate equivalent atoms: 
(2) (R)-9aa

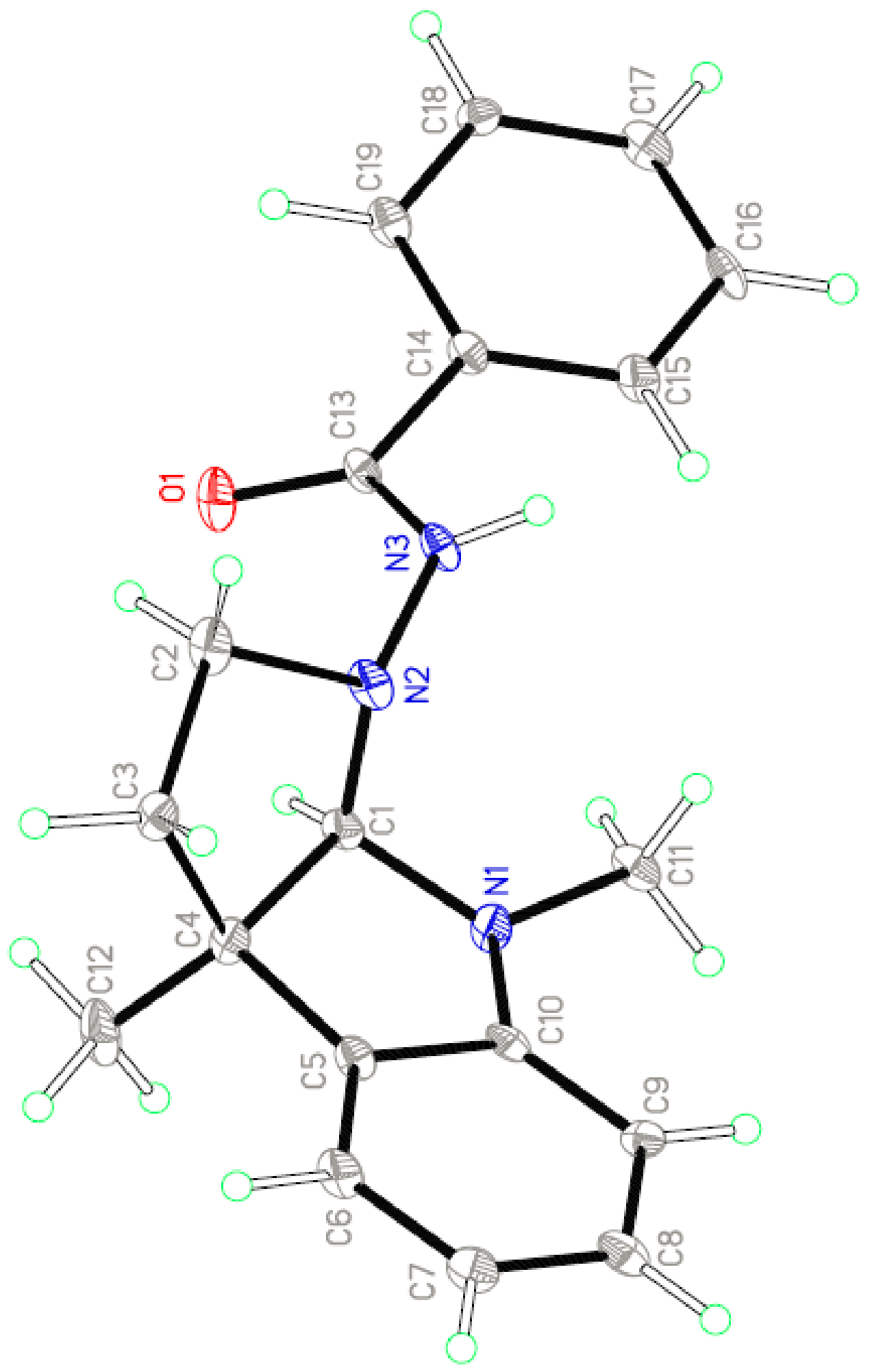

S118 
Table 1. Crystal data and structure refinement for kwq-c-63.

Identification code

Empirical formula

Formula weight

Temperature

Wavelength

Crystal system

Space group

Unit cell dimensions

Volume

Z

Density (calculated)

Absorption coefficient

$\mathrm{F}(000)$

Crystal size

Theta range for data collection

Index ranges

Reflections collected

Independent reflections

Completeness to theta $=27.51^{\circ}$

Absorption correction

Max. and min. transmission

Refinement method

Data / restraints / parameters

Goodness-of-fit on $\mathrm{F}^{2}$

Final $\mathrm{R}$ indices $[\mathrm{I}>2 \operatorname{sigma}(\mathrm{I})]$

$\mathrm{R}$ indices (all data)

Absolute structure parameter

Extinction coefficient

Largest diff. peak and hole kwq-c-63

$\mathrm{C}_{19} \mathrm{H}_{21} \mathrm{~N}_{3} \mathrm{O}$

307.39

100(2) K

$0.71073 \AA$

Orthorhombic

$P 2{ }_{1} 22$

$\mathrm{a}=10.259(2) \AA$

$\alpha=90^{\circ}$

$\mathrm{b}=22.885(5) \AA$

$\beta=90^{\circ}$.

$\mathrm{c}=6.9775(14) \AA$

$\gamma=90^{\circ}$

1638.2(6) $\AA^{3}$

4

$1.246 \mathrm{Mg} / \mathrm{m}^{3}$

$0.079 \mathrm{~mm}^{-1}$

656

$0.46 \times 0.21 \times 0.11 \mathrm{~mm}^{3}$

2.18 to $27.51^{\circ}$.

$-13 \leq \mathrm{h} \leq 13,-29 \leq \mathrm{k} \leq 29,-8 \leq 1 \leq 9$

19543

$3726[\mathrm{R}($ int $)=0.0984]$

$99.6 \%$

Semi-empirical from equivalents

0.7456 and 0.4307

Full-matrix least-squares on $\mathrm{F}^{2}$

$3726 / 0 / 211$

1.225

$\mathrm{R} 1=0.1174, \mathrm{wR} 2=0.2653$

$\mathrm{R} 1=0.1488, \mathrm{wR} 2=0.2818$

$-1(6)$

$0.031(4)$

0.445 and -0.462 e. $\AA^{-3}$ 
Table 2. Atomic coordinates $\left(\mathrm{x} 10^{4}\right)$ and equivalent isotropic displacement parameters $\left(\AA^{2} \times 10^{3}\right)$ for kwq-c-63. $U(\mathrm{eq})$ is defined as one third of the trace of the orthogonalized $\mathrm{U}^{\mathrm{ij}}$ tensor.

\begin{tabular}{|c|c|c|c|c|}
\hline & $\mathrm{x}$ & $\mathrm{y}$ & $\mathrm{z}$ & $\mathrm{U}(\mathrm{eq})$ \\
\hline $\mathrm{O}(1)$ & $10316(4)$ & $7418(2)$ & $5013(8)$ & $37(1)$ \\
\hline $\mathrm{N}(1)$ & $7999(5)$ & $6987(2)$ & $9820(9)$ & $31(1)$ \\
\hline $\mathrm{N}(2)$ & $8231(5)$ & $6734(2)$ & $6469(8)$ & $30(1)$ \\
\hline $\mathrm{N}(3)$ & $8130(5)$ & $7279(2)$ & $5469(8)$ & $27(1)$ \\
\hline $\mathrm{C}(1)$ & $8862(6)$ & $6782(2)$ & $8313(9)$ & $26(1)$ \\
\hline$C(2)$ & $8931(7)$ & $6269(2)$ & $5369(11)$ & $34(2)$ \\
\hline$C(3)$ & $9068(7)$ & $5801(2)$ & $6867(10)$ & $31(1)$ \\
\hline$C(4)$ & $9157(7)$ & $6133(2)$ & $8851(10)$ & $31(2)$ \\
\hline$C(5)$ & $8014(6)$ & $5989(2)$ & $10140(9)$ & $26(1)$ \\
\hline$C(6)$ & $7593(6)$ & $5461(2)$ & $10895(10)$ & $31(2)$ \\
\hline$C(7)$ & $6526(7)$ & $5439(3)$ & $12084(10)$ & $34(2)$ \\
\hline $\mathrm{C}(8)$ & $5862(7)$ & $5947(3)$ & $12552(10)$ & $35(2)$ \\
\hline $\mathrm{C}(9)$ & $6277(6)$ & $6493(3)$ & $11835(9)$ & $26(1)$ \\
\hline$C(10)$ & $7340(6)$ & $6501(2)$ & $10625(9)$ & $27(1)$ \\
\hline$C(11)$ & $7409(6)$ & $7565(2)$ & $9837(10)$ & $30(1)$ \\
\hline$C(12)$ & 10441(6) & $6064(3)$ & $9873(14)$ & $47(2)$ \\
\hline$C(13)$ & $9176(6)$ & $7595(2)$ & 4912(9) & $24(1)$ \\
\hline$C(14)$ & $8885(6)$ & $8212(2)$ & $4253(9)$ & $25(1)$ \\
\hline$C(15)$ & $7853(6)$ & $8535(2)$ & $5015(11)$ & $30(1)$ \\
\hline$C(16)$ & $7707(6)$ & $9125(2)$ & $4469(10)$ & $34(2)$ \\
\hline$C(17)$ & $8568(7)$ & $9373(3)$ & $3201(11)$ & $32(2)$ \\
\hline$C(18)$ & $9566(6)$ & $9049(3)$ & 2397(9) & $27(1)$ \\
\hline$C(19)$ & $9730(7)$ & $8464(3)$ & $2946(10)$ & $32(2)$ \\
\hline
\end{tabular}


Table 3. Bond lengths $[\AA]$ and angles $\left[{ }^{\circ}\right]$ for kwq-c- 63 .

\begin{tabular}{|c|c|}
\hline $\mathrm{O}(1)-\mathrm{C}(13)$ & $1.240(7)$ \\
\hline $\mathrm{N}(1)-\mathrm{C}(10)$ & $1.418(7)$ \\
\hline $\mathrm{N}(1)-\mathrm{C}(1)$ & $1.453(8)$ \\
\hline $\mathrm{N}(1)-\mathrm{C}(11)$ & $1.454(7)$ \\
\hline $\mathrm{N}(2)-\mathrm{N}(3)$ & $1.433(7)$ \\
\hline $\mathrm{N}(2)-\mathrm{C}(1)$ & $1.444(8)$ \\
\hline $\mathrm{N}(2)-\mathrm{C}(2)$ & $1.495(8)$ \\
\hline $\mathrm{N}(3)-\mathrm{C}(13)$ & $1.351(8)$ \\
\hline $\mathrm{N}(3)-\mathrm{H}(3)$ & 0.8800 \\
\hline$C(1)-C(4)$ & $1.561(8)$ \\
\hline $\mathrm{C}(1)-\mathrm{H}(1)$ & 1.0000 \\
\hline$C(2)-C(3)$ & $1.503(9)$ \\
\hline $\mathrm{C}(2)-\mathrm{H}(2 \mathrm{~A})$ & 0.9900 \\
\hline $\mathrm{C}(2)-\mathrm{H}(2 \mathrm{~B})$ & 0.9900 \\
\hline$C(3)-C(4)$ & $1.582(9)$ \\
\hline $\mathrm{C}(3)-\mathrm{H}(3 \mathrm{~A})$ & 0.9900 \\
\hline $\mathrm{C}(3)-\mathrm{H}(3 \mathrm{~B})$ & 0.9900 \\
\hline$C(4)-C(12)$ & $1.506(10)$ \\
\hline$C(4)-C(5)$ & $1.514(9)$ \\
\hline$C(5)-C(6)$ & $1.387(8)$ \\
\hline$C(5)-C(10)$ & $1.402(8)$ \\
\hline$C(6)-C(7)$ & $1.375(10)$ \\
\hline $\mathrm{C}(6)-\mathrm{H}(6)$ & 0.9500 \\
\hline$C(7)-C(8)$ & $1.385(10)$ \\
\hline $\mathrm{C}(7)-\mathrm{H}(7)$ & 0.9500 \\
\hline$C(8)-C(9)$ & $1.411(8)$ \\
\hline $\mathrm{C}(8)-\mathrm{H}(8)$ & 0.9500 \\
\hline $\mathrm{C}(9)-\mathrm{C}(10)$ & $1.379(9)$ \\
\hline $\mathrm{C}(9)-\mathrm{H}(9)$ & 0.9500 \\
\hline $\mathrm{C}(11)-\mathrm{H}(11 \mathrm{~A})$ & 0.9800 \\
\hline $\mathrm{C}(11)-\mathrm{H}(11 \mathrm{~B})$ & 0.9800 \\
\hline $\mathrm{C}(11)-\mathrm{H}(11 \mathrm{C})$ & 0.9800 \\
\hline $\mathrm{C}(12)-\mathrm{H}(12 \mathrm{~A})$ & 0.9800 \\
\hline $\mathrm{C}(12)-\mathrm{H}(12 \mathrm{~B})$ & 0.9800 \\
\hline $\mathrm{C}(12)-\mathrm{H}(12 \mathrm{C})$ & 0.9800 \\
\hline$C(13)-C(14)$ & $1.514(7)$ \\
\hline C(14)-C(19) & $1.384(9)$ \\
\hline
\end{tabular}




\begin{tabular}{|c|c|}
\hline$C(14)-C(15)$ & $1.397(8)$ \\
\hline$C(15)-C(16)$ & $1.411(8)$ \\
\hline $\mathrm{C}(15)-\mathrm{H}(15)$ & 0.9500 \\
\hline$C(16)-C(17)$ & $1.372(10)$ \\
\hline C(16)-H(16) & 0.9500 \\
\hline $\mathrm{C}(17)-\mathrm{C}(18)$ & $1.382(9)$ \\
\hline $\mathrm{C}(17)-\mathrm{H}(17)$ & 0.9500 \\
\hline C(18)-C(19) & $1.401(8)$ \\
\hline $\mathrm{C}(18)-\mathrm{H}(18)$ & 0.9500 \\
\hline C(19)-H(19) & 0.9500 \\
\hline $\mathrm{C}(10)-\mathrm{N}(1)-\mathrm{C}(1)$ & $108.9(4)$ \\
\hline $\mathrm{C}(10)-\mathrm{N}(1)-\mathrm{C}(11)$ & $120.8(5)$ \\
\hline $\mathrm{C}(1)-\mathrm{N}(1)-\mathrm{C}(11)$ & $123.6(5)$ \\
\hline $\mathrm{N}(3)-\mathrm{N}(2)-\mathrm{C}(1)$ & $113.6(4)$ \\
\hline $\mathrm{N}(3)-\mathrm{N}(2)-\mathrm{C}(2)$ & $113.8(5)$ \\
\hline $\mathrm{C}(1)-\mathrm{N}(2)-\mathrm{C}(2)$ & $107.2(5)$ \\
\hline $\mathrm{C}(13)-\mathrm{N}(3)-\mathrm{N}(2)$ & $123.3(5)$ \\
\hline $\mathrm{C}(13)-\mathrm{N}(3)-\mathrm{H}(3)$ & 118.4 \\
\hline $\mathrm{N}(2)-\mathrm{N}(3)-\mathrm{H}(3)$ & 118.4 \\
\hline $\mathrm{N}(2)-\mathrm{C}(1)-\mathrm{N}(1)$ & $113.4(5)$ \\
\hline $\mathrm{N}(2)-\mathrm{C}(1)-\mathrm{C}(4)$ & $103.2(5)$ \\
\hline $\mathrm{N}(1)-\mathrm{C}(1)-\mathrm{C}(4)$ & $104.6(5)$ \\
\hline $\mathrm{N}(2)-\mathrm{C}(1)-\mathrm{H}(1)$ & 111.7 \\
\hline $\mathrm{N}(1)-\mathrm{C}(1)-\mathrm{H}(1)$ & 111.7 \\
\hline $\mathrm{C}(4)-\mathrm{C}(1)-\mathrm{H}(1)$ & 111.7 \\
\hline $\mathrm{N}(2)-\mathrm{C}(2)-\mathrm{C}(3)$ & $101.2(5)$ \\
\hline $\mathrm{N}(2)-\mathrm{C}(2)-\mathrm{H}(2 \mathrm{~A})$ & 111.5 \\
\hline $\mathrm{C}(3)-\mathrm{C}(2)-\mathrm{H}(2 \mathrm{~A})$ & 111.5 \\
\hline $\mathrm{N}(2)-\mathrm{C}(2)-\mathrm{H}(2 \mathrm{~B})$ & 111.5 \\
\hline $\mathrm{C}(3)-\mathrm{C}(2)-\mathrm{H}(2 \mathrm{~B})$ & 111.5 \\
\hline $\mathrm{H}(2 \mathrm{~A})-\mathrm{C}(2)-\mathrm{H}(2 \mathrm{~B})$ & 109.3 \\
\hline $\mathrm{C}(2)-\mathrm{C}(3)-\mathrm{C}(4)$ & $105.8(5)$ \\
\hline $\mathrm{C}(2)-\mathrm{C}(3)-\mathrm{H}(3 \mathrm{~A})$ & 110.6 \\
\hline $\mathrm{C}(4)-\mathrm{C}(3)-\mathrm{H}(3 \mathrm{~A})$ & 110.6 \\
\hline $\mathrm{C}(2)-\mathrm{C}(3)-\mathrm{H}(3 \mathrm{~B})$ & 110.6 \\
\hline $\mathrm{C}(4)-\mathrm{C}(3)-\mathrm{H}(3 \mathrm{~B})$ & 110.6 \\
\hline $\mathrm{H}(3 \mathrm{~A})-\mathrm{C}(3)-\mathrm{H}(3 \mathrm{~B})$ & 108.7 \\
\hline$C(12)-C(4)-C(5)$ & $111.9(6)$ \\
\hline
\end{tabular}




\begin{tabular}{|c|c|}
\hline$C(12)-C(4)-C(1)$ & $112.6(5)$ \\
\hline$C(5)-C(4)-C(1)$ & $101.5(5)$ \\
\hline$C(12)-C(4)-C(3)$ & $114.5(6)$ \\
\hline$C(5)-C(4)-C(3)$ & $111.7(5)$ \\
\hline$C(1)-C(4)-C(3)$ & $103.6(5)$ \\
\hline$C(6)-C(5)-C(10)$ & $118.9(6)$ \\
\hline$C(6)-C(5)-C(4)$ & $131.0(5)$ \\
\hline$C(10)-C(5)-C(4)$ & $110.1(5)$ \\
\hline$C(7)-C(6)-C(5)$ & $120.6(6)$ \\
\hline $\mathrm{C}(7)-\mathrm{C}(6)-\mathrm{H}(6)$ & 119.7 \\
\hline $\mathrm{C}(5)-\mathrm{C}(6)-\mathrm{H}(6)$ & 119.7 \\
\hline$C(6)-C(7)-C(8)$ & $120.2(6)$ \\
\hline $\mathrm{C}(6)-\mathrm{C}(7)-\mathrm{H}(7)$ & 119.9 \\
\hline $\mathrm{C}(8)-\mathrm{C}(7)-\mathrm{H}(7)$ & 119.9 \\
\hline$C(7)-C(8)-C(9)$ & $120.7(7)$ \\
\hline $\mathrm{C}(7)-\mathrm{C}(8)-\mathrm{H}(8)$ & 119.7 \\
\hline $\mathrm{C}(9)-\mathrm{C}(8)-\mathrm{H}(8)$ & 119.7 \\
\hline$C(10)-C(9)-C(8)$ & $117.9(6)$ \\
\hline $\mathrm{C}(10)-\mathrm{C}(9)-\mathrm{H}(9)$ & 121.1 \\
\hline $\mathrm{C}(8)-\mathrm{C}(9)-\mathrm{H}(9)$ & 121.1 \\
\hline$C(9)-C(10)-C(5)$ & $121.7(5)$ \\
\hline $\mathrm{C}(9)-\mathrm{C}(10)-\mathrm{N}(1)$ & $129.1(5)$ \\
\hline $\mathrm{C}(5)-\mathrm{C}(10)-\mathrm{N}(1)$ & $109.0(5)$ \\
\hline $\mathrm{N}(1)-\mathrm{C}(11)-\mathrm{H}(11 \mathrm{~A})$ & 109.5 \\
\hline $\mathrm{N}(1)-\mathrm{C}(11)-\mathrm{H}(11 \mathrm{~B})$ & 109.5 \\
\hline $\mathrm{H}(11 \mathrm{~A})-\mathrm{C}(11)-\mathrm{H}(11 \mathrm{~B})$ & 109.5 \\
\hline $\mathrm{N}(1)-\mathrm{C}(11)-\mathrm{H}(11 \mathrm{C})$ & 109.5 \\
\hline $\mathrm{H}(11 \mathrm{~A})-\mathrm{C}(11)-\mathrm{H}(11 \mathrm{C})$ & 109.5 \\
\hline $\mathrm{H}(11 \mathrm{~B})-\mathrm{C}(11)-\mathrm{H}(11 \mathrm{C})$ & 109.5 \\
\hline $\mathrm{C}(4)-\mathrm{C}(12)-\mathrm{H}(12 \mathrm{~A})$ & 109.5 \\
\hline $\mathrm{C}(4)-\mathrm{C}(12)-\mathrm{H}(12 \mathrm{~B})$ & 109.5 \\
\hline $\mathrm{H}(12 \mathrm{~A})-\mathrm{C}(12)-\mathrm{H}(12 \mathrm{~B})$ & 109.5 \\
\hline $\mathrm{C}(4)-\mathrm{C}(12)-\mathrm{H}(12 \mathrm{C})$ & 109.5 \\
\hline $\mathrm{H}(12 \mathrm{~A})-\mathrm{C}(12)-\mathrm{H}(12 \mathrm{C})$ & 109.5 \\
\hline $\mathrm{H}(12 \mathrm{~B})-\mathrm{C}(12)-\mathrm{H}(12 \mathrm{C})$ & 109.5 \\
\hline $\mathrm{O}(1)-\mathrm{C}(13)-\mathrm{N}(3)$ & $123.9(5)$ \\
\hline $\mathrm{O}(1)-\mathrm{C}(13)-\mathrm{C}(14)$ & $120.5(5)$ \\
\hline $\mathrm{N}(3)-\mathrm{C}(13)-\mathrm{C}(14)$ & $115.5(5)$ \\
\hline $\mathrm{C}(19)-\mathrm{C}(14)-\mathrm{C}(15)$ & $120.3(5)$ \\
\hline
\end{tabular}




$\begin{array}{ll}\mathrm{C}(19)-\mathrm{C}(14)-\mathrm{C}(13) & 117.8(5) \\ \mathrm{C}(15)-\mathrm{C}(14)-\mathrm{C}(13) & 121.8(5) \\ \mathrm{C}(14)-\mathrm{C}(15)-\mathrm{C}(16) & 118.9(6) \\ \mathrm{C}(14)-\mathrm{C}(15)-\mathrm{H}(15) & 120.5 \\ \mathrm{C}(16)-\mathrm{C}(15)-\mathrm{H}(15) & 120.5 \\ \mathrm{C}(17)-\mathrm{C}(16)-\mathrm{C}(15) & 120.1(6) \\ \mathrm{C}(17)-\mathrm{C}(16)-\mathrm{H}(16) & 120.0 \\ \mathrm{C}(15)-\mathrm{C}(16)-\mathrm{H}(16) & 120.0 \\ \mathrm{C}(16)-\mathrm{C}(17)-\mathrm{C}(18) & 121.1(6) \\ \mathrm{C}(16)-\mathrm{C}(17)-\mathrm{H}(17) & 119.4 \\ \mathrm{C}(18)-\mathrm{C}(17)-\mathrm{H}(17) & 119.4 \\ \mathrm{C}(17)-\mathrm{C}(18)-\mathrm{C}(19) & 119.3(6) \\ \mathrm{C}(17)-\mathrm{C}(18)-\mathrm{H}(18) & 120.3 \\ \mathrm{C}(19)-\mathrm{C}(18)-\mathrm{H}(18) & 120.3 \\ \mathrm{C}(14)-\mathrm{C}(19)-\mathrm{C}(18) & 120.2(6) \\ \mathrm{C}(14)-\mathrm{C}(19)-\mathrm{H}(19) & 119.9 \\ \mathrm{C}(18)-\mathrm{C}(19)-\mathrm{H}(19) & 119.9\end{array}$

Symmetry transformations used to generate equivalent atoms: 
Table 4. Anisotropic displacement parameters $\left(\AA^{2} \times 10^{3}\right)$ for kwq-c-63. The anisotropic displacement factor exponent takes the form: $-2 \pi^{2}\left[h^{2} a^{* 2} U^{11}+\ldots+2 h k a^{*} b^{*} U^{12}\right]$

\begin{tabular}{|c|c|c|c|c|c|c|}
\hline & $\mathrm{U}^{11}$ & $\mathrm{U}^{22}$ & $\mathrm{U}^{33}$ & $\mathrm{U}^{23}$ & $\mathrm{U}^{13}$ & $\mathrm{U}^{12}$ \\
\hline $\mathrm{O}(1)$ & $31(2)$ & $13(2)$ & $67(3)$ & $-7(2)$ & $6(2)$ & $2(2)$ \\
\hline $\mathrm{N}(1)$ & $34(3)$ & $16(2)$ & $42(3)$ & $3(2)$ & $10(3)$ & $1(2)$ \\
\hline $\mathrm{N}(2)$ & $36(3)$ & $7(2)$ & $46(3)$ & $2(2)$ & $-2(3)$ & $-2(2)$ \\
\hline $\mathrm{N}(3)$ & $27(3)$ & $10(2)$ & $42(3)$ & $3(2)$ & $-6(2)$ & $1(2)$ \\
\hline$C(1)$ & $31(3)$ & $13(3)$ & $34(3)$ & $7(2)$ & $1(3)$ & $-1(2)$ \\
\hline$C(2)$ & $35(4)$ & $12(3)$ & $56(5)$ & $-6(3)$ & $6(3)$ & $-3(2)$ \\
\hline$C(3)$ & $41(4)$ & $12(3)$ & $40(4)$ & $-3(3)$ & $8(3)$ & $3(2)$ \\
\hline$C(4)$ & $38(4)$ & $11(3)$ & $43(4)$ & $5(3)$ & $15(3)$ & $3(2)$ \\
\hline$C(5)$ & $29(3)$ & $14(2)$ & $35(3)$ & $2(3)$ & $-2(3)$ & $-1(2)$ \\
\hline$C(6)$ & $36(4)$ & $10(2)$ & $47(4)$ & $4(3)$ & $0(3)$ & $-3(2)$ \\
\hline$C(7)$ & $43(4)$ & $22(3)$ & $39(4)$ & $4(3)$ & $-3(3)$ & $-6(3)$ \\
\hline $\mathrm{C}(8)$ & $36(4)$ & $28(3)$ & $42(4)$ & $11(3)$ & $-10(3)$ & $-4(3)$ \\
\hline$C(9)$ & $39(3)$ & $20(3)$ & $20(3)$ & $-2(2)$ & $-1(3)$ & $0(3)$ \\
\hline$C(10)$ & $31(3)$ & $17(3)$ & $32(3)$ & $9(2)$ & $-7(3)$ & $0(2)$ \\
\hline$C(11)$ & $43(4)$ & $10(2)$ & $37(3)$ & $-2(2)$ & $-11(3)$ & $0(2)$ \\
\hline$C(12)$ & $34(4)$ & $18(3)$ & $89(6)$ & $10(4)$ & $10(4)$ & $6(3)$ \\
\hline$C(13)$ & $30(3)$ & $12(2)$ & $31(3)$ & $-5(2)$ & $-5(3)$ & $5(2)$ \\
\hline$C(14)$ & $30(3)$ & $12(3)$ & $32(3)$ & $-1(2)$ & $-3(3)$ & $0(2)$ \\
\hline$C(15)$ & $31(3)$ & $16(3)$ & $43(4)$ & $5(3)$ & $2(3)$ & $-3(2)$ \\
\hline$C(16)$ & $34(3)$ & $15(3)$ & $52(4)$ & $3(3)$ & $-10(3)$ & $9(2)$ \\
\hline$C(17)$ & $44(4)$ & $13(3)$ & $40(4)$ & $-4(3)$ & $-11(3)$ & $-2(3)$ \\
\hline$C(18)$ & $39(4)$ & $20(3)$ & $24(3)$ & $0(2)$ & $3(3)$ & $0(3)$ \\
\hline$C(19)$ & $39(4)$ & $13(3)$ & $44(4)$ & $0(3)$ & $-1(3)$ & $3(2)$ \\
\hline
\end{tabular}


Table 5. Hydrogen coordinates ( x 10 $)$ and isotropic displacement parameters $\left(\AA^{2} \times 10^{3}\right)$ for kwq-c-63.

\begin{tabular}{|c|c|c|c|c|}
\hline & $\mathrm{x}$ & $\mathrm{y}$ & $\mathrm{z}$ & $\mathrm{U}(\mathrm{eq})$ \\
\hline $\mathrm{H}(3)$ & 7348 & 7414 & 5204 & 32 \\
\hline $\mathrm{H}(1)$ & 9677 & 7021 & 8239 & 32 \\
\hline $\mathrm{H}(2 \mathrm{~A})$ & 9791 & 6407 & 4913 & 41 \\
\hline $\mathrm{H}(2 \mathrm{~B})$ & 8410 & 6132 & 4263 & 41 \\
\hline $\mathrm{H}(3 \mathrm{~A})$ & 9864 & 5567 & 6641 & 37 \\
\hline $\mathrm{H}(3 \mathrm{~B})$ & 8304 & 5537 & 6848 & 37 \\
\hline $\mathrm{H}(6)$ & 8046 & 5111 & 10587 & 37 \\
\hline $\mathrm{H}(7)$ & 6242 & 5075 & 12587 & 41 \\
\hline $\mathrm{H}(8)$ & 5120 & 5927 & 13364 & 42 \\
\hline $\mathrm{H}(9)$ & 5838 & 6843 & 12175 & 31 \\
\hline $\mathrm{H}(11 \mathrm{~A})$ & 7053 & 7646 & 11113 & 45 \\
\hline $\mathrm{H}(11 \mathrm{~B})$ & 6706 & 7580 & 8887 & 45 \\
\hline $\mathrm{H}(11 \mathrm{C})$ & 8071 & 7859 & 9521 & 45 \\
\hline $\mathrm{H}(12 \mathrm{~A})$ & 10552 & 5655 & 10261 & 70 \\
\hline $\mathrm{H}(12 \mathrm{~B})$ & 10453 & 6315 & 11012 & 70 \\
\hline $\mathrm{H}(12 \mathrm{C})$ & 11153 & 6176 & 9012 & 70 \\
\hline $\mathrm{H}(15)$ & 7258 & 8360 & 5887 & 36 \\
\hline $\mathrm{H}(16)$ & 7013 & 9351 & 4979 & 41 \\
\hline $\mathrm{H}(17)$ & 8477 & 9773 & 2870 & 39 \\
\hline $\mathrm{H}(18)$ & 10135 & 9221 & 1483 & 33 \\
\hline H(19) & 10423 & 8241 & 2419 & 39 \\
\hline
\end{tabular}


Table 6. Torsion angles $\left[{ }^{\circ}\right]$ for kwq-c-63.

\begin{tabular}{|c|c|}
\hline $\mathrm{C}(1)-\mathrm{N}(2)-\mathrm{N}(3)-\mathrm{C}(13)$ & $62.0(8)$ \\
\hline $\mathrm{C}(2)-\mathrm{N}(2)-\mathrm{N}(3)-\mathrm{C}(13)$ & $-61.1(7)$ \\
\hline $\mathrm{N}(3)-\mathrm{N}(2)-\mathrm{C}(1)-\mathrm{N}(1)$ & $81.0(6)$ \\
\hline $\mathrm{C}(2)-\mathrm{N}(2)-\mathrm{C}(1)-\mathrm{N}(1)$ & $-152.4(5)$ \\
\hline $\mathrm{N}(3)-\mathrm{N}(2)-\mathrm{C}(1)-\mathrm{C}(4)$ & $-166.5(5)$ \\
\hline $\mathrm{C}(2)-\mathrm{N}(2)-\mathrm{C}(1)-\mathrm{C}(4)$ & $-39.8(6)$ \\
\hline $\mathrm{C}(10)-\mathrm{N}(1)-\mathrm{C}(1)-\mathrm{N}(2)$ & $86.8(6)$ \\
\hline $\mathrm{C}(11)-\mathrm{N}(1)-\mathrm{C}(1)-\mathrm{N}(2)$ & $-64.5(7)$ \\
\hline $\mathrm{C}(10)-\mathrm{N}(1)-\mathrm{C}(1)-\mathrm{C}(4)$ & $-24.9(7)$ \\
\hline $\mathrm{C}(11)-\mathrm{N}(1)-\mathrm{C}(1)-\mathrm{C}(4)$ & $-176.2(5)$ \\
\hline $\mathrm{N}(3)-\mathrm{N}(2)-\mathrm{C}(2)-\mathrm{C}(3)$ & $171.3(5)$ \\
\hline $\mathrm{C}(1)-\mathrm{N}(2)-\mathrm{C}(2)-\mathrm{C}(3)$ & $44.8(6)$ \\
\hline $\mathrm{N}(2)-\mathrm{C}(2)-\mathrm{C}(3)-\mathrm{C}(4)$ & $-30.2(6)$ \\
\hline $\mathrm{N}(2)-\mathrm{C}(1)-\mathrm{C}(4)-\mathrm{C}(12)$ & $143.0(6)$ \\
\hline $\mathrm{N}(1)-\mathrm{C}(1)-\mathrm{C}(4)-\mathrm{C}(12)$ & $-98.2(7)$ \\
\hline $\mathrm{N}(2)-\mathrm{C}(1)-\mathrm{C}(4)-\mathrm{C}(5)$ & $-97.2(6)$ \\
\hline $\mathrm{N}(1)-\mathrm{C}(1)-\mathrm{C}(4)-\mathrm{C}(5)$ & $21.6(7)$ \\
\hline $\mathrm{N}(2)-\mathrm{C}(1)-\mathrm{C}(4)-\mathrm{C}(3)$ & $18.8(6)$ \\
\hline $\mathrm{N}(1)-\mathrm{C}(1)-\mathrm{C}(4)-\mathrm{C}(3)$ & $137.6(5)$ \\
\hline $\mathrm{C}(2)-\mathrm{C}(3)-\mathrm{C}(4)-\mathrm{C}(12)$ & $-115.2(6)$ \\
\hline$C(2)-C(3)-C(4)-C(5)$ & $116.2(6)$ \\
\hline $\mathrm{C}(2)-\mathrm{C}(3)-\mathrm{C}(4)-\mathrm{C}(1)$ & $7.7(7)$ \\
\hline$C(12)-C(4)-C(5)-C(6)$ & $-69.6(9)$ \\
\hline$C(1)-C(4)-C(5)-C(6)$ & $170.1(7)$ \\
\hline$C(3)-C(4)-C(5)-C(6)$ & $60.3(9)$ \\
\hline$C(12)-C(4)-C(5)-C(10)$ & $108.5(6)$ \\
\hline $\mathrm{C}(1)-\mathrm{C}(4)-\mathrm{C}(5)-\mathrm{C}(10)$ & $-11.8(7)$ \\
\hline $\mathrm{C}(3)-\mathrm{C}(4)-\mathrm{C}(5)-\mathrm{C}(10)$ & $-121.6(6)$ \\
\hline$C(10)-C(5)-C(6)-C(7)$ & $0.6(10)$ \\
\hline$C(4)-C(5)-C(6)-C(7)$ & $178.6(7)$ \\
\hline $\mathrm{C}(5)-\mathrm{C}(6)-\mathrm{C}(7)-\mathrm{C}(8)$ & $-0.4(10)$ \\
\hline $\mathrm{C}(6)-\mathrm{C}(7)-\mathrm{C}(8)-\mathrm{C}(9)$ & $-0.7(11)$ \\
\hline $\mathrm{C}(7)-\mathrm{C}(8)-\mathrm{C}(9)-\mathrm{C}(10)$ & $1.5(10)$ \\
\hline $\mathrm{C}(8)-\mathrm{C}(9)-\mathrm{C}(10)-\mathrm{C}(5)$ & $-1.3(9)$ \\
\hline $\mathrm{C}(8)-\mathrm{C}(9)-\mathrm{C}(10)-\mathrm{N}(1)$ & $-175.4(6)$ \\
\hline$C(6)-C(5)-C(10)-C(9)$ & $0.3(10)$ \\
\hline $\mathrm{C}(4)-\mathrm{C}(5)-\mathrm{C}(10)-\mathrm{C}(9)$ & $-178.1(6)$ \\
\hline
\end{tabular}




$\begin{array}{lc}\mathrm{C}(6)-\mathrm{C}(5)-\mathrm{C}(10)-\mathrm{N}(1) & 175.4(6) \\ \mathrm{C}(4)-\mathrm{C}(5)-\mathrm{C}(10)-\mathrm{N}(1) & -3.0(7) \\ \mathrm{C}(1)-\mathrm{N}(1)-\mathrm{C}(10)-\mathrm{C}(9) & -167.3(6) \\ \mathrm{C}(11)-\mathrm{N}(1)-\mathrm{C}(10)-\mathrm{C}(9) & -15.1(10) \\ \mathrm{C}(1)-\mathrm{N}(1)-\mathrm{C}(10)-\mathrm{C}(5) & 18.1(7) \\ \mathrm{C}(11)-\mathrm{N}(1)-\mathrm{C}(10)-\mathrm{C}(5) & 170.3(6) \\ \mathrm{N}(2)-\mathrm{N}(3)-\mathrm{C}(13)-\mathrm{O}(1) & 8.4(10) \\ \mathrm{N}(2)-\mathrm{N}(3)-\mathrm{C}(13)-\mathrm{C}(14) & -167.7(5) \\ \mathrm{O}(1)-\mathrm{C}(13)-\mathrm{C}(14)-\mathrm{C}(19) & 31.0(9) \\ \mathrm{N}(3)-\mathrm{C}(13)-\mathrm{C}(14)-\mathrm{C}(19) & -152.7(6) \\ \mathrm{O}(1)-\mathrm{C}(13)-\mathrm{C}(14)-\mathrm{C}(15) & -144.9(7) \\ \mathrm{N}(3)-\mathrm{C}(13)-\mathrm{C}(14)-\mathrm{C}(15) & 31.4(8) \\ \mathrm{C}(19)-\mathrm{C}(14)-\mathrm{C}(15)-\mathrm{C}(16) & -1.6(10) \\ \mathrm{C}(13)-\mathrm{C}(14)-\mathrm{C}(15)-\mathrm{C}(16) & 174.2(6) \\ \mathrm{C}(14)-\mathrm{C}(15)-\mathrm{C}(16)-\mathrm{C}(17) & 0.3(10) \\ \mathrm{C}(15)-\mathrm{C}(16)-\mathrm{C}(17)-\mathrm{C}(18) & 1.8(10) \\ \mathrm{C}(16)-\mathrm{C}(17)-\mathrm{C}(18)-\mathrm{C}(19) & -2.5(10) \\ \mathrm{C}(15)-\mathrm{C}(14)-\mathrm{C}(19)-\mathrm{C}(18) & 0.9(10) \\ \mathrm{C}(13)-\mathrm{C}(14)-\mathrm{C}(19)-\mathrm{C}(18) & -175.1(6) \\ \mathrm{C}(17)-\mathrm{C}(18)-\mathrm{C}(19)-\mathrm{C}(14) & 1.2(10) \\ & \end{array}$

Symmetry transformations used to generate equivalent atoms: 
Table 7. Hydrogen bonds for kwq-c-63 [ $\AA$ and $\left.{ }^{\circ}\right]$.

\begin{tabular}{lcccc}
\hline D-H...A & $d(D-H)$ & $d(H . . . A)$ & $d(D \ldots A)$ & $<($ DHA $)$ \\
\hline $\mathrm{N}(3)-H(3) \ldots \mathrm{O}(1) \# 1$ & 0.88 & 2.13 & $2.988(7)$ & 166.7 \\
\hline
\end{tabular}

Symmetry transformations used to generate equivalent atoms:

$\# 1 \mathrm{x}-1 / 2,-\mathrm{y}+3 / 2,-\mathrm{z}+1$ 


\section{References:}

1. (a) Zhang, J.; Gu, Q.; Shi, X.; You, S. Chem. Commun. 2013, 49, 7750. (b) Neuville, L.; Bigot, A.; Tran Huu Dau, M. E.; Zhu, J. J. Org. Chem. 1999, 64, 7638.

2. (a) Uray, G.; Celotto. C. J. Label Compd. Radiopharm. 1987, 24, 1455. (b) Dannenberg, C. A.; Bizet, V.; Zou, L. -H; Bolm, C. Eur. J. Org. Chem. 2015, 77.

3. (a) He, B.; Song, J.; Wang, H.-W.; Qing, Y. Faming Zhuanli Shenqing Gongkai Shuomingshu CN101121717A (2007). (b) Kulkarni, M. G.; Dhondge, A. P.; Borhade, A. S.; Gaikwad, D. D.; Chavhan, S. W.; Shaikh, Y. B.; Ningdale, V. B.; Desai, M. P.; Birhade, D. R.; Shinde, M. P. Tetrahedron Lett. 2009, 50, 2411. 
9. The copies of ${ }^{1} \mathrm{H}$ and ${ }^{13} \mathrm{C}$ NMR spectra

1b

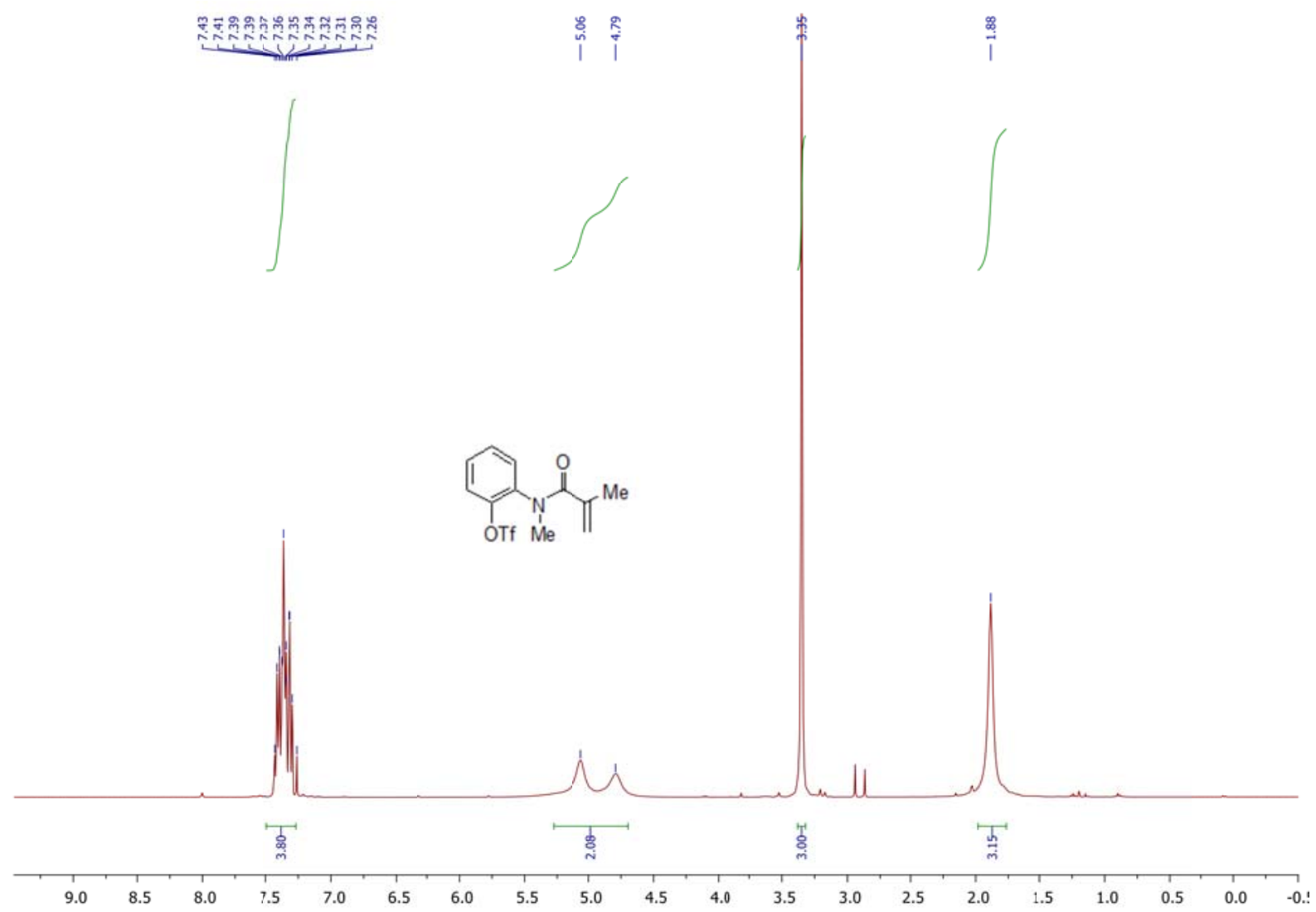

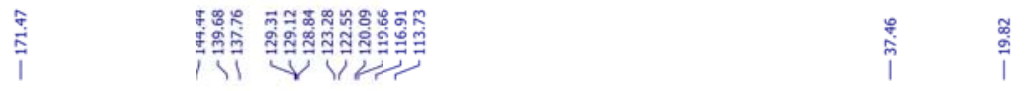
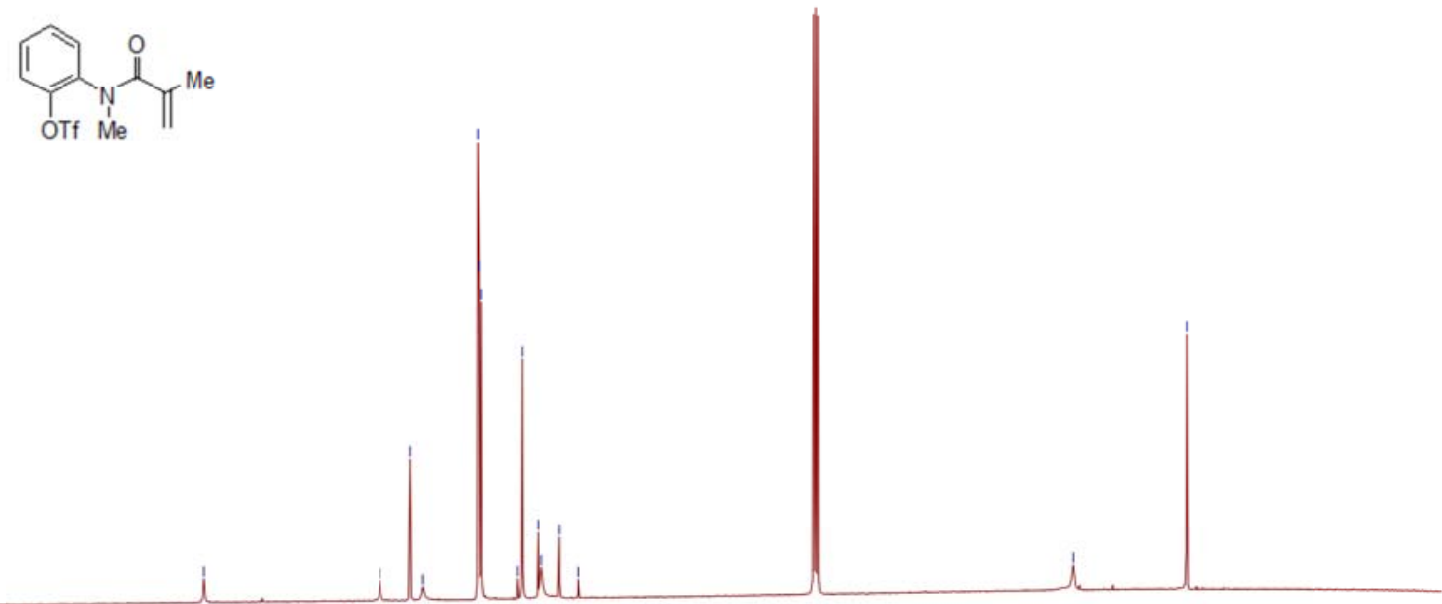

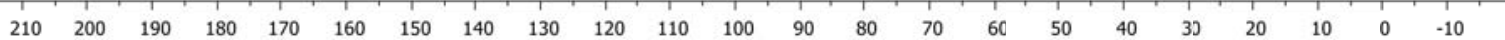



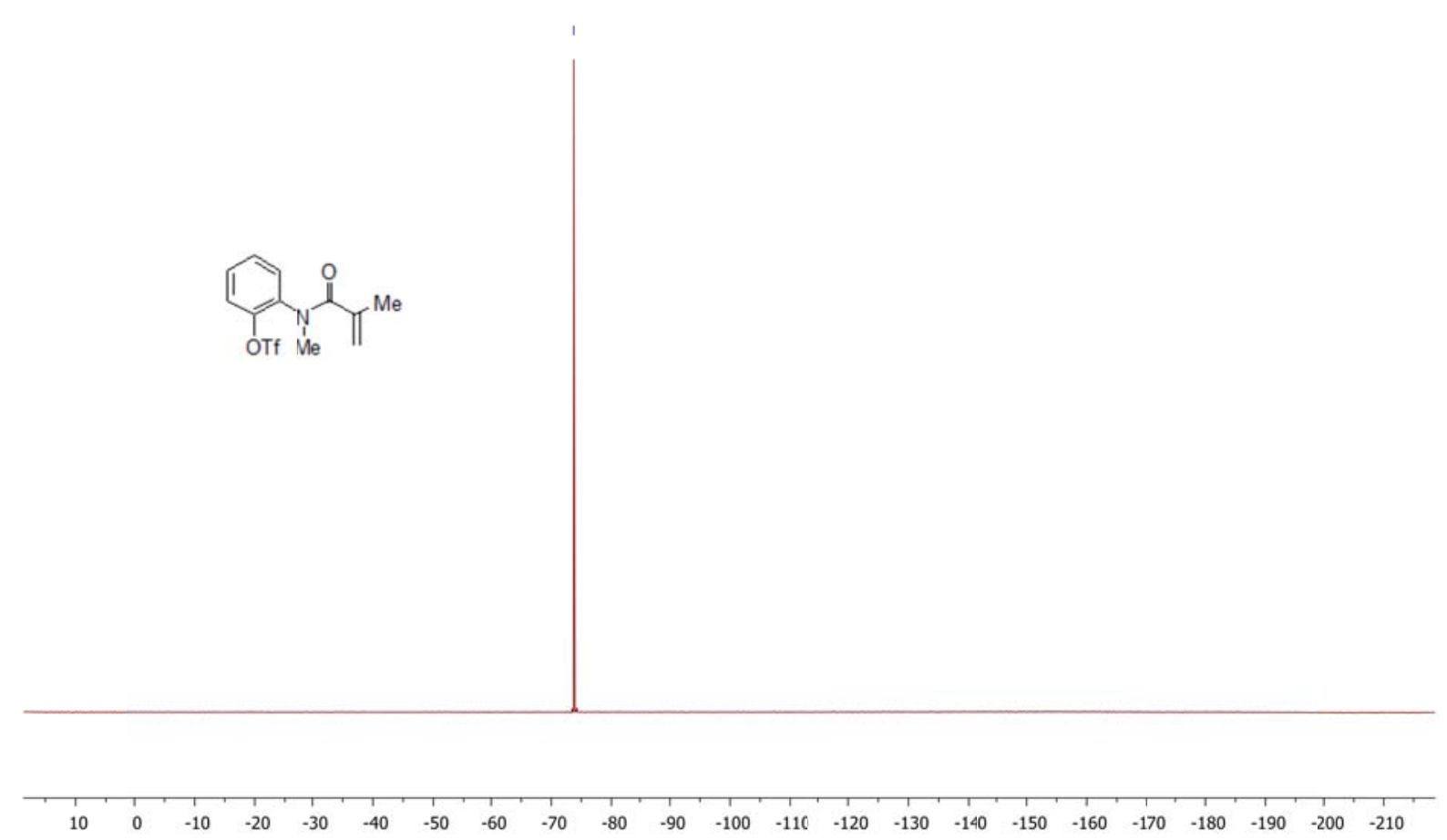

1d
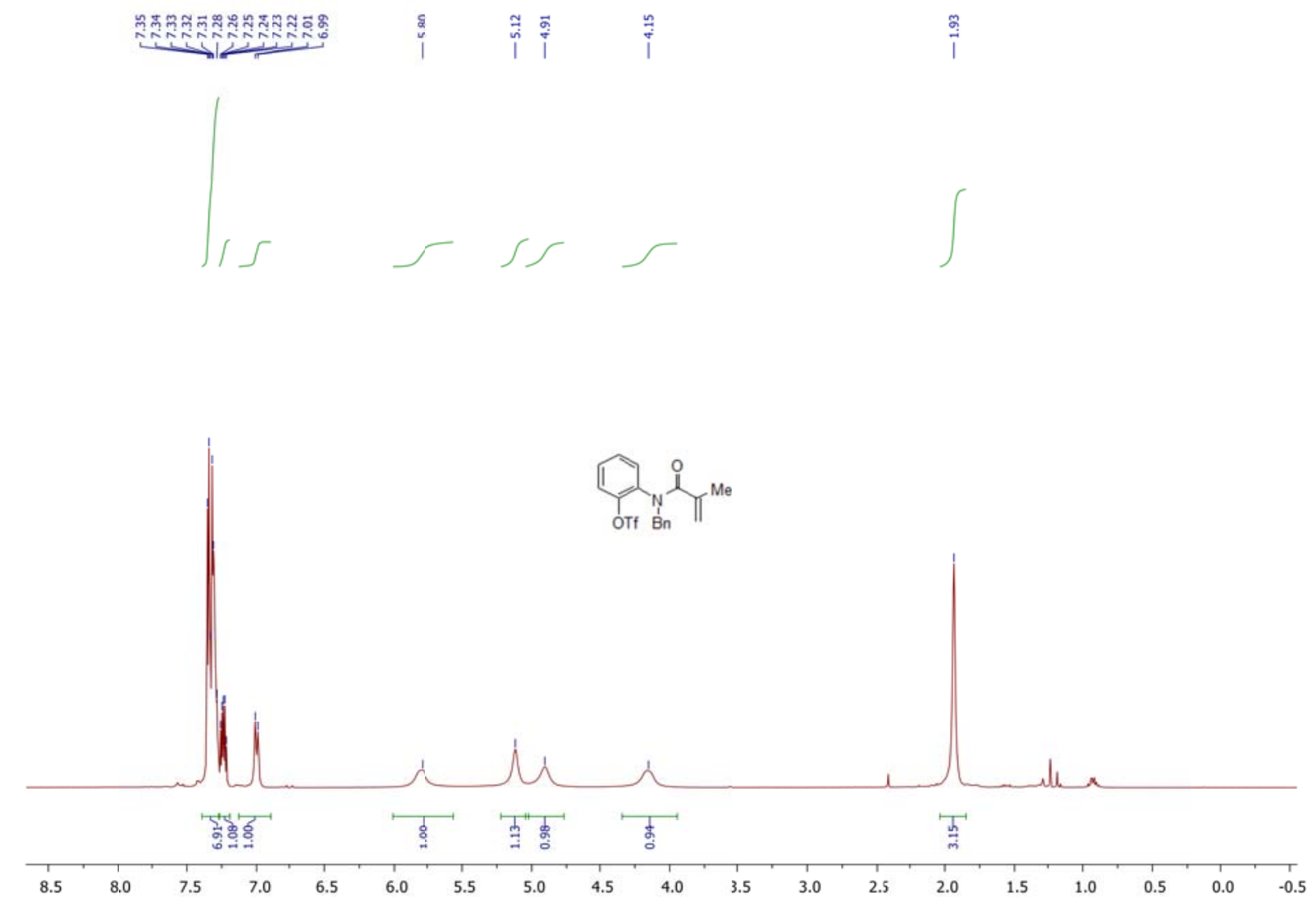

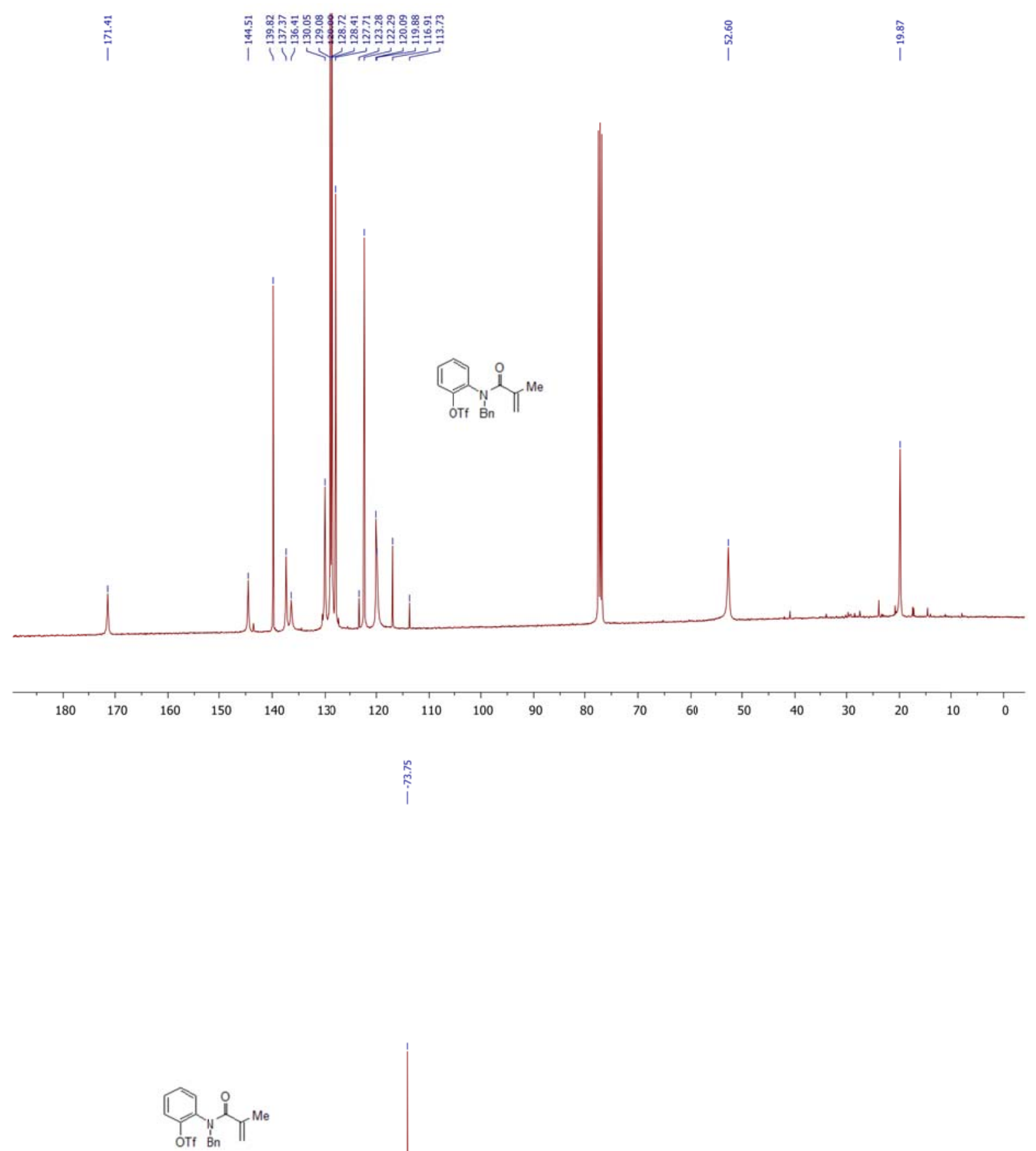

$\begin{array}{lllllllllllllllllllllllllll}1 & 0 & 0 & -10 & -20 & -30 & -40 & -50 & -60 & -70 & -80 & -90 & -100 & -110 & -120 & -130 & -140 & -150 & -160 & -170 & -180 & -190 & -200 & -210\end{array}$ 
$1 g$

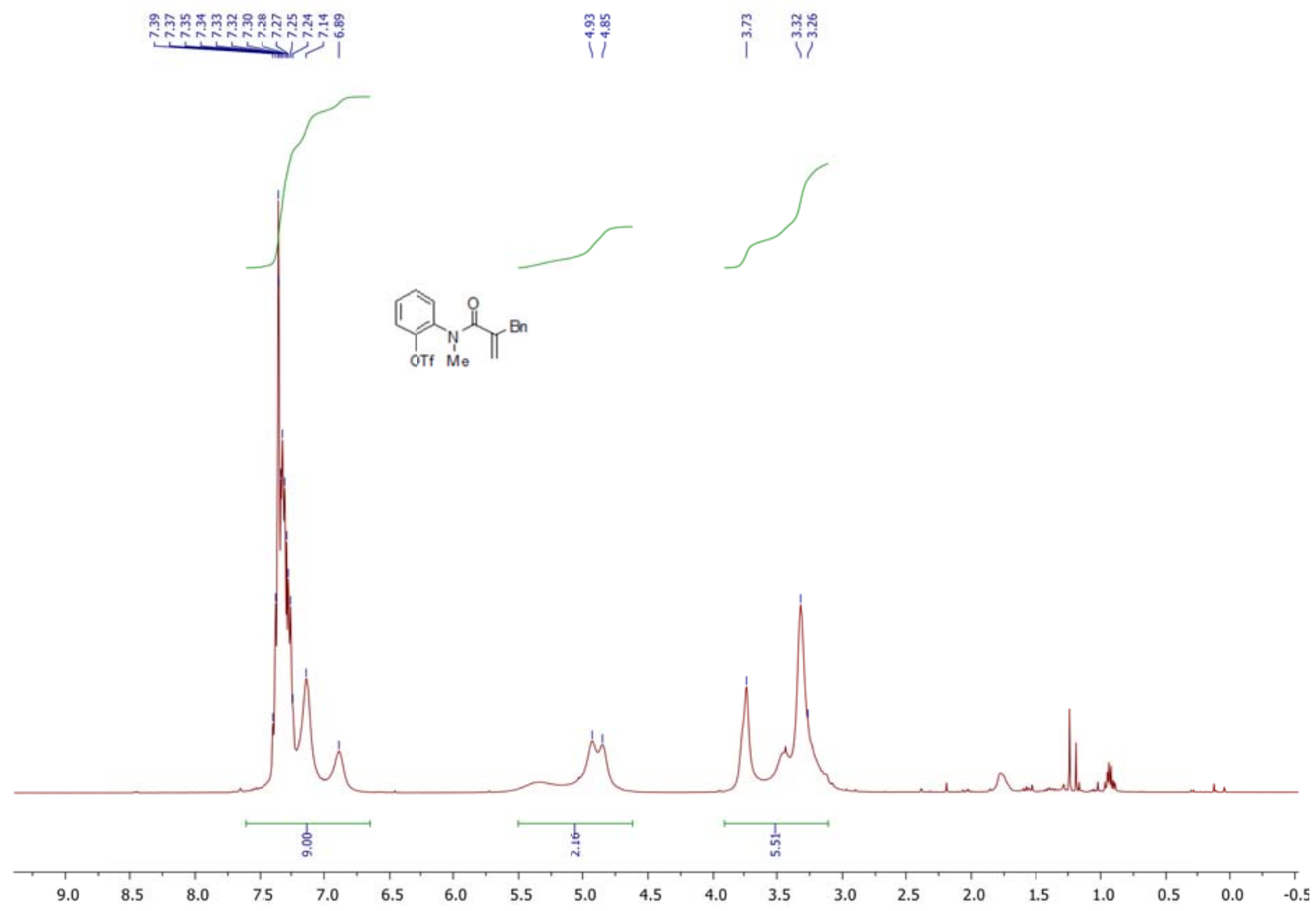

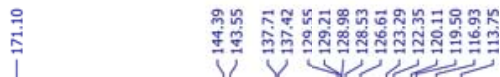

雚

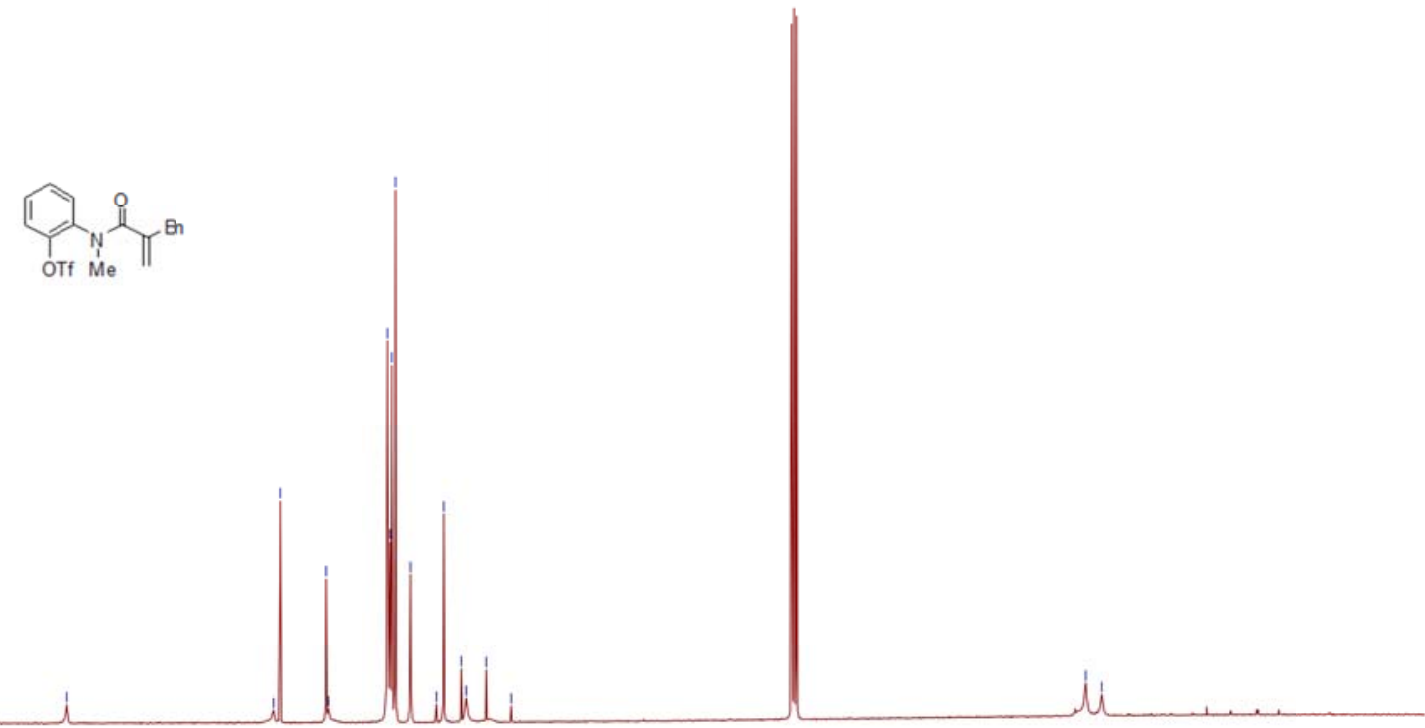

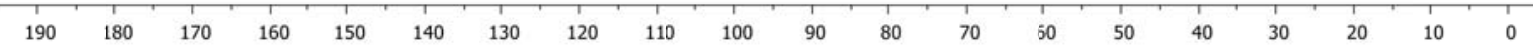


$\underbrace{\infty}_{\substack{N \\ M e}} \pi^{B n}$

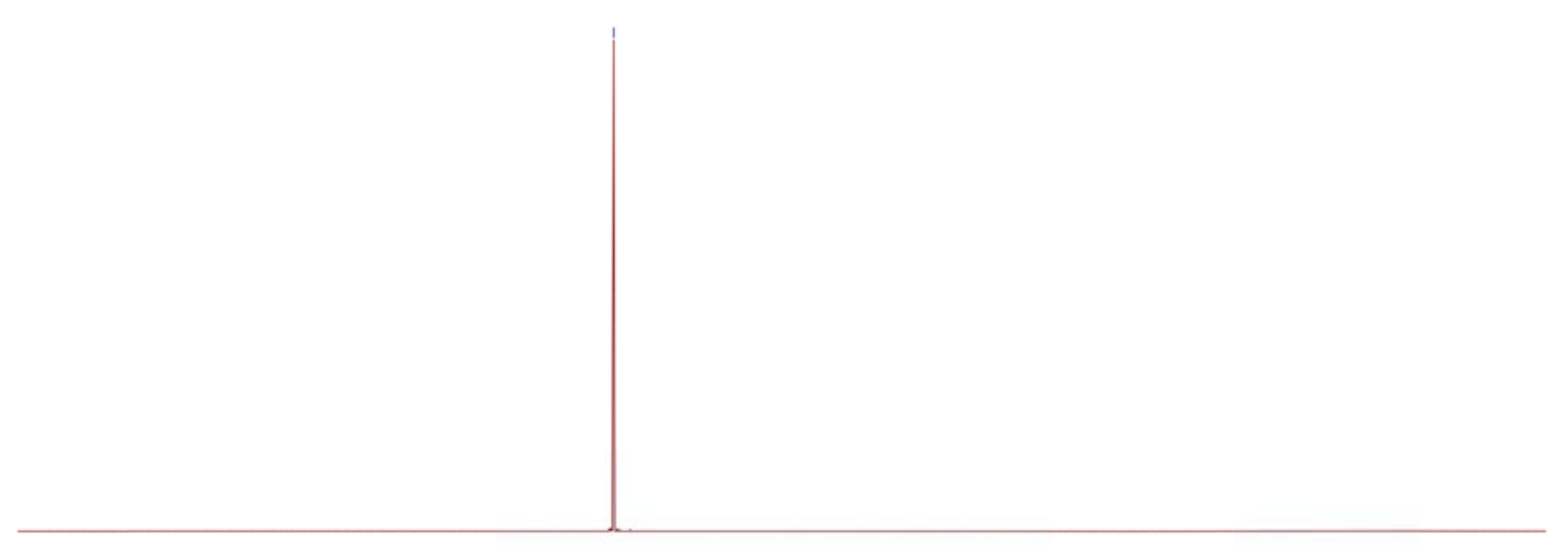

$\begin{array}{lllllllllllllllllllllllll}10 & 0 & -10 & -20 & -30 & -40 & -50 & -60 & -70 & -80 & -90 & -100 & -110 & -120 & -130 & -140 & -150 & -160 & -170 & -180 & -190 & -200 & -210\end{array}$

1h
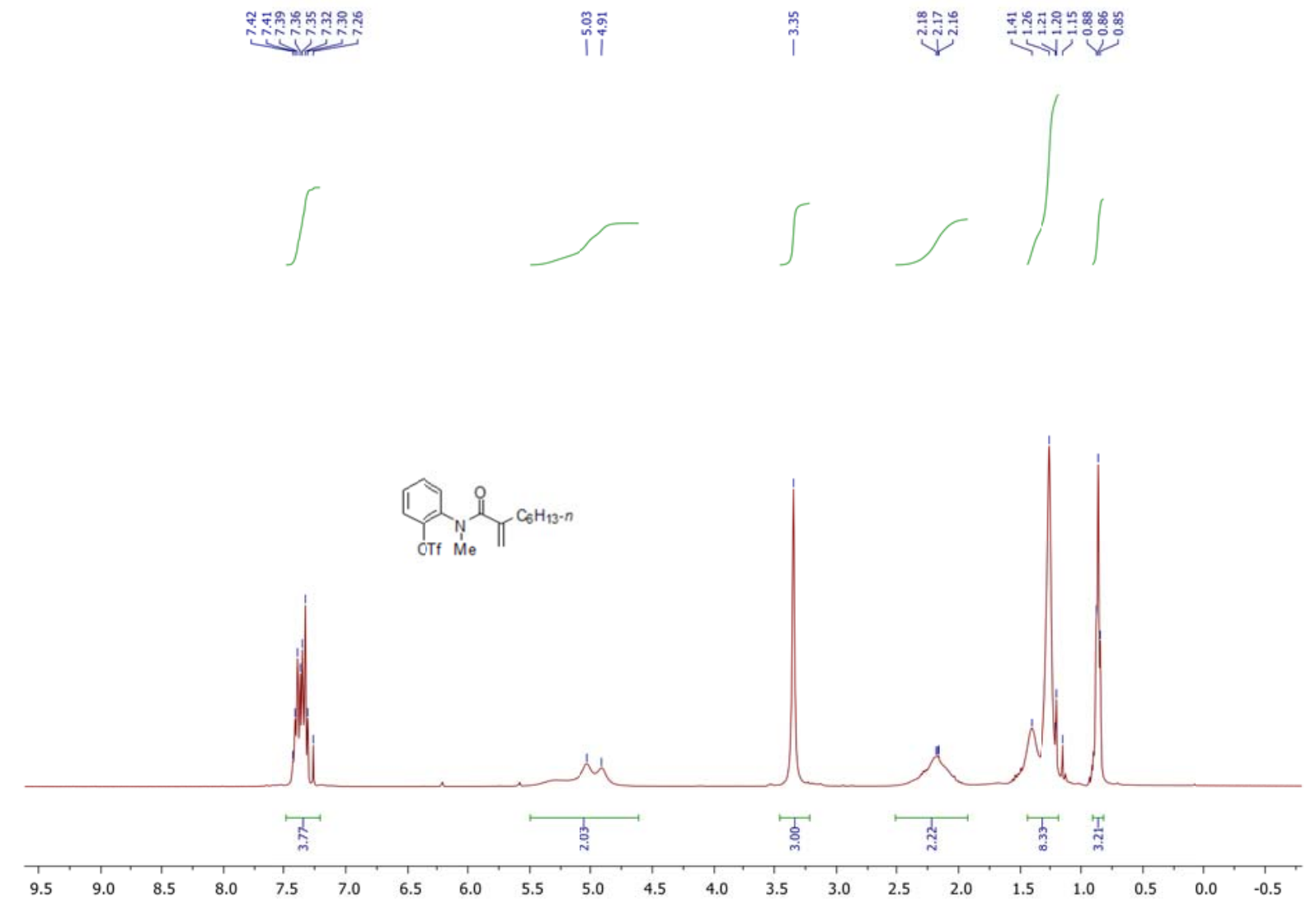

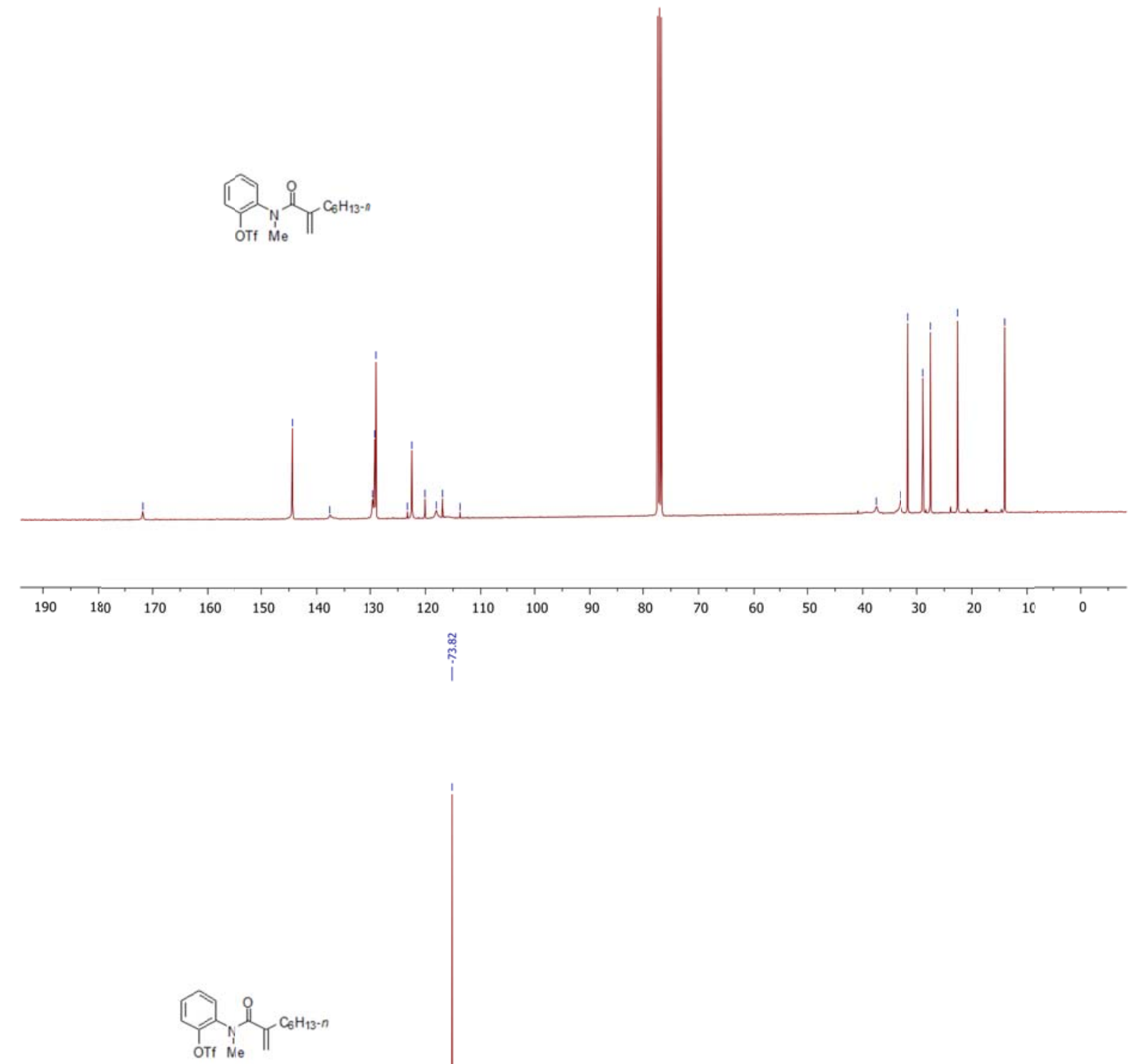

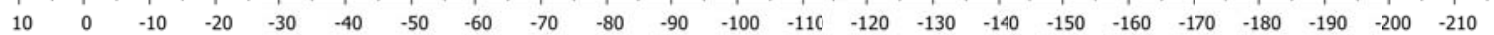


$1 \mathbf{i}$

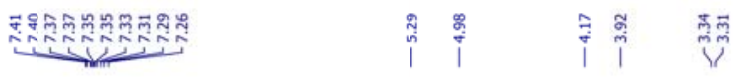
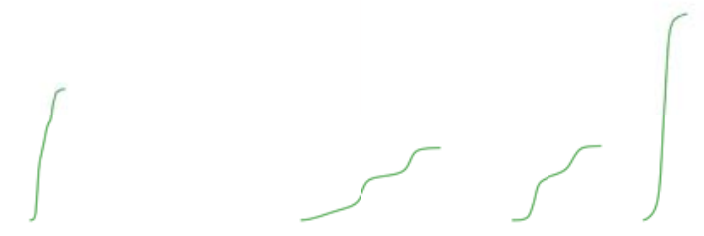

OTf Me $_{\substack{N \\ \text { OMe }}}$

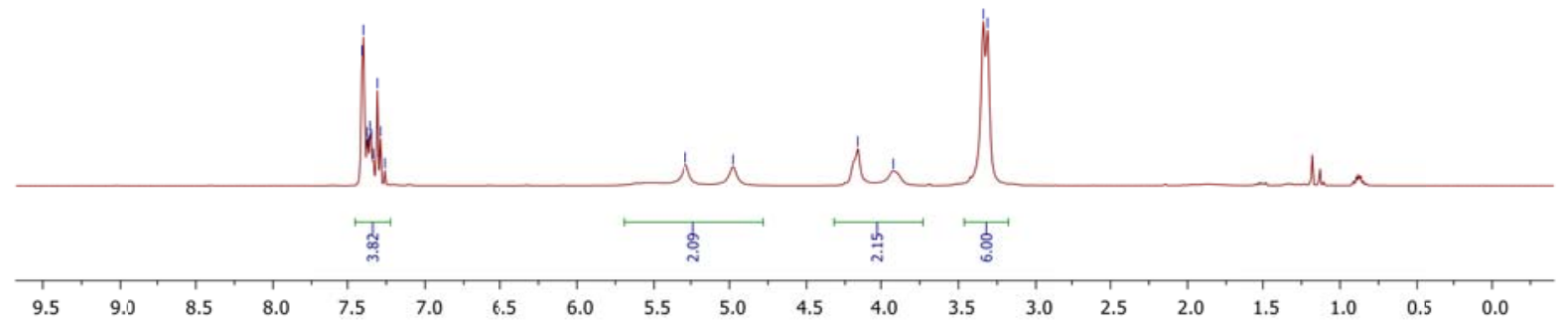

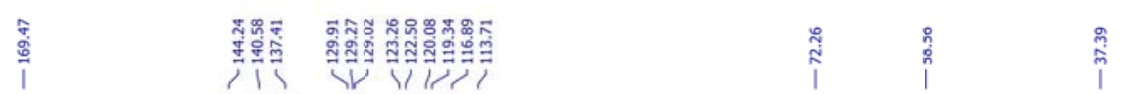

OTf Me

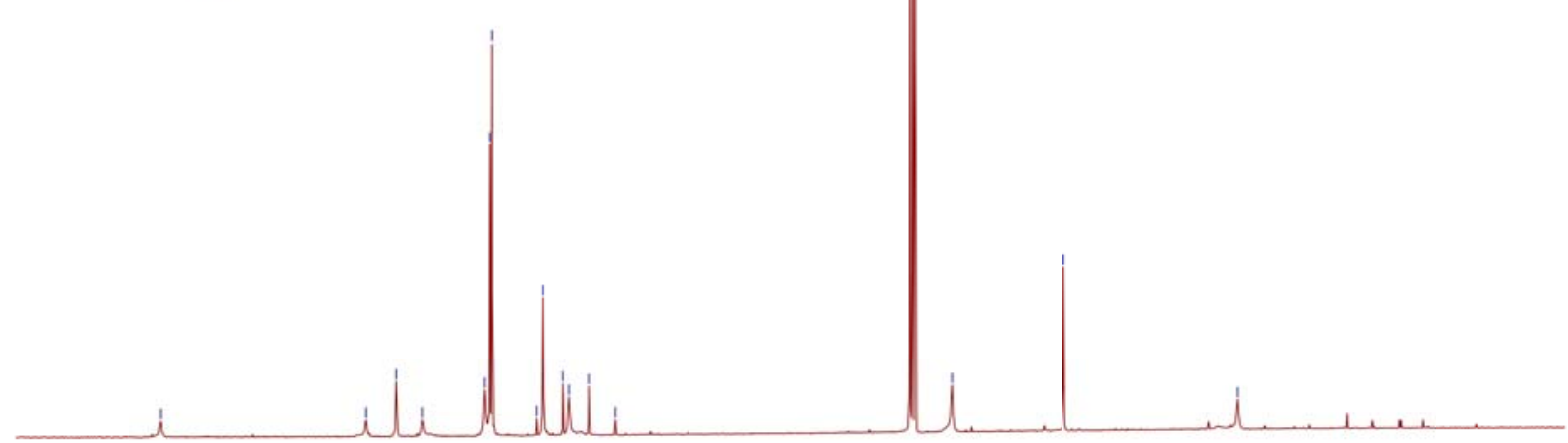

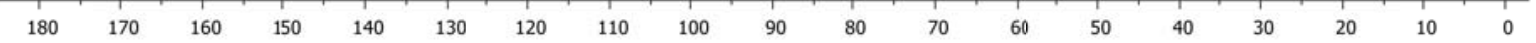




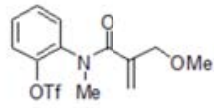

$\begin{array}{lllllllllllllllllllllll}10 & 0 & -10 & -20 & -30 & -40 & -50 & -60 & -70 & -80 & -90 & -100 & -110 & -120 & -130 & -140 & -150 & -160 & -170 & -180 & -190 & -200 & -210\end{array}$

1j

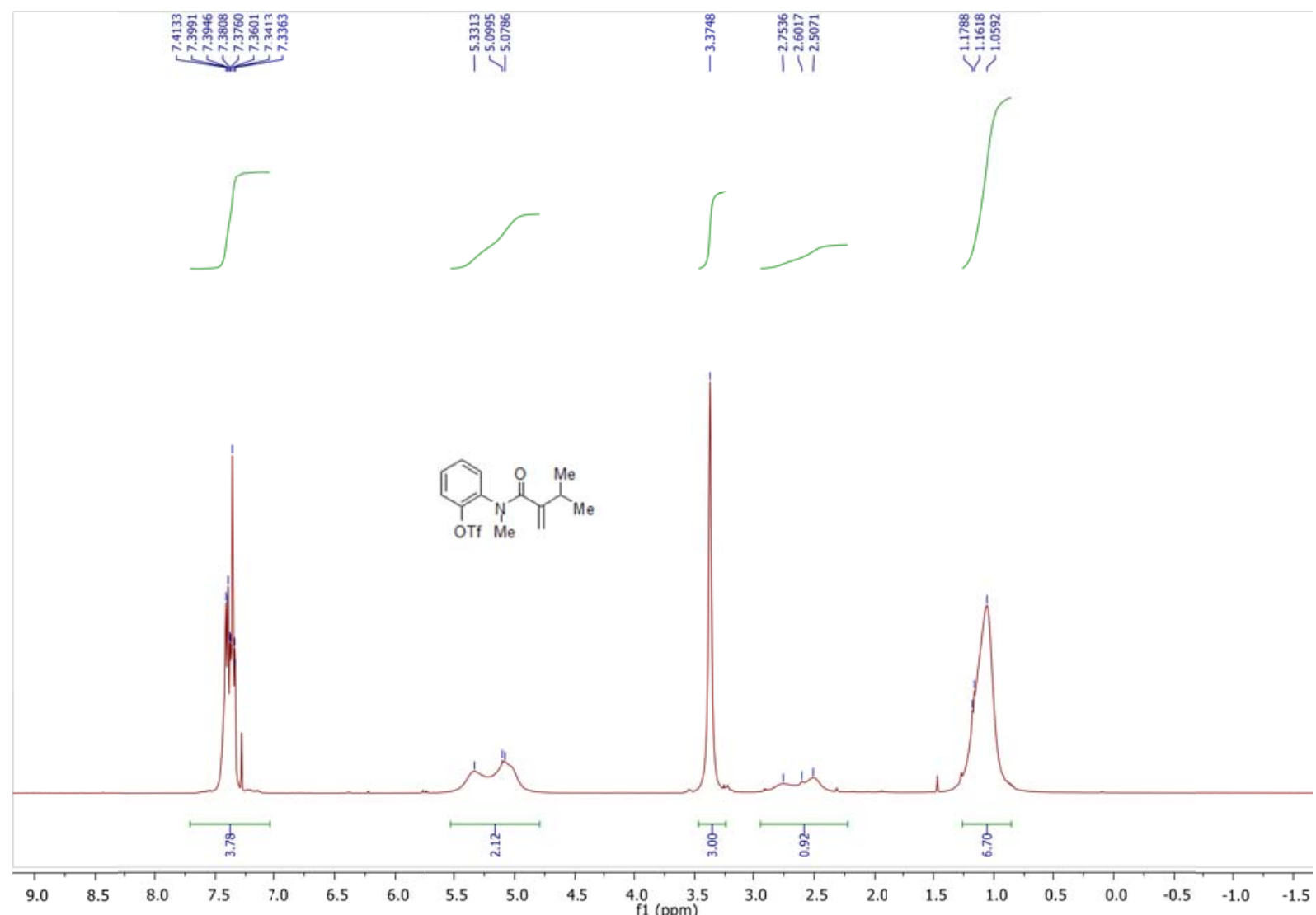



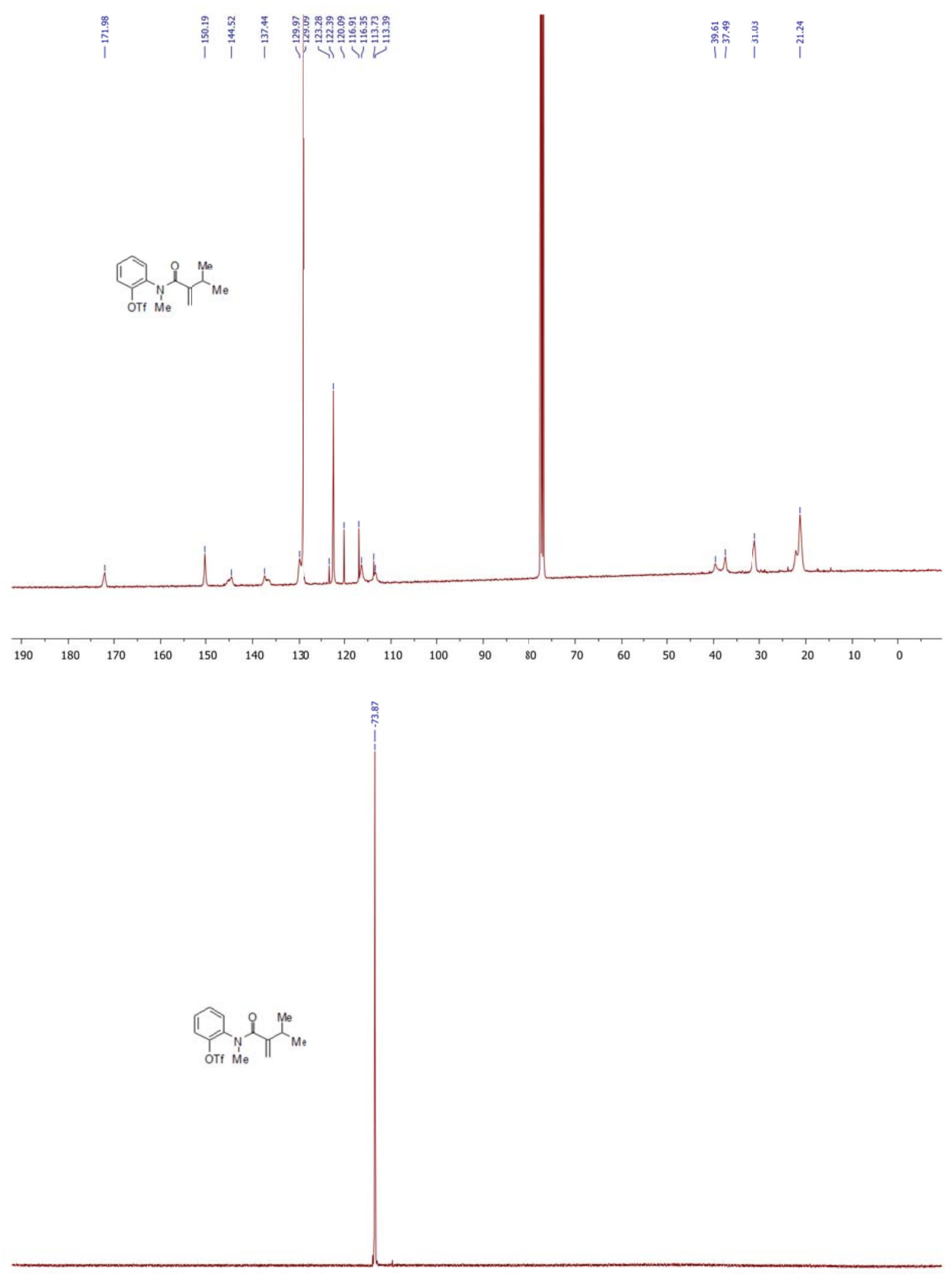

$\begin{array}{lllllllllllllllllllllllll}1 & 10 & 0 & -10 & -20 & -30 & -40 & -50 & -60 & -70 & -80 & -90 & -100 & -110 & -120 & -130 & -140 & -150 & -160 & -170 & -180 & -190 & -200 & -210 & 1\end{array}$ 


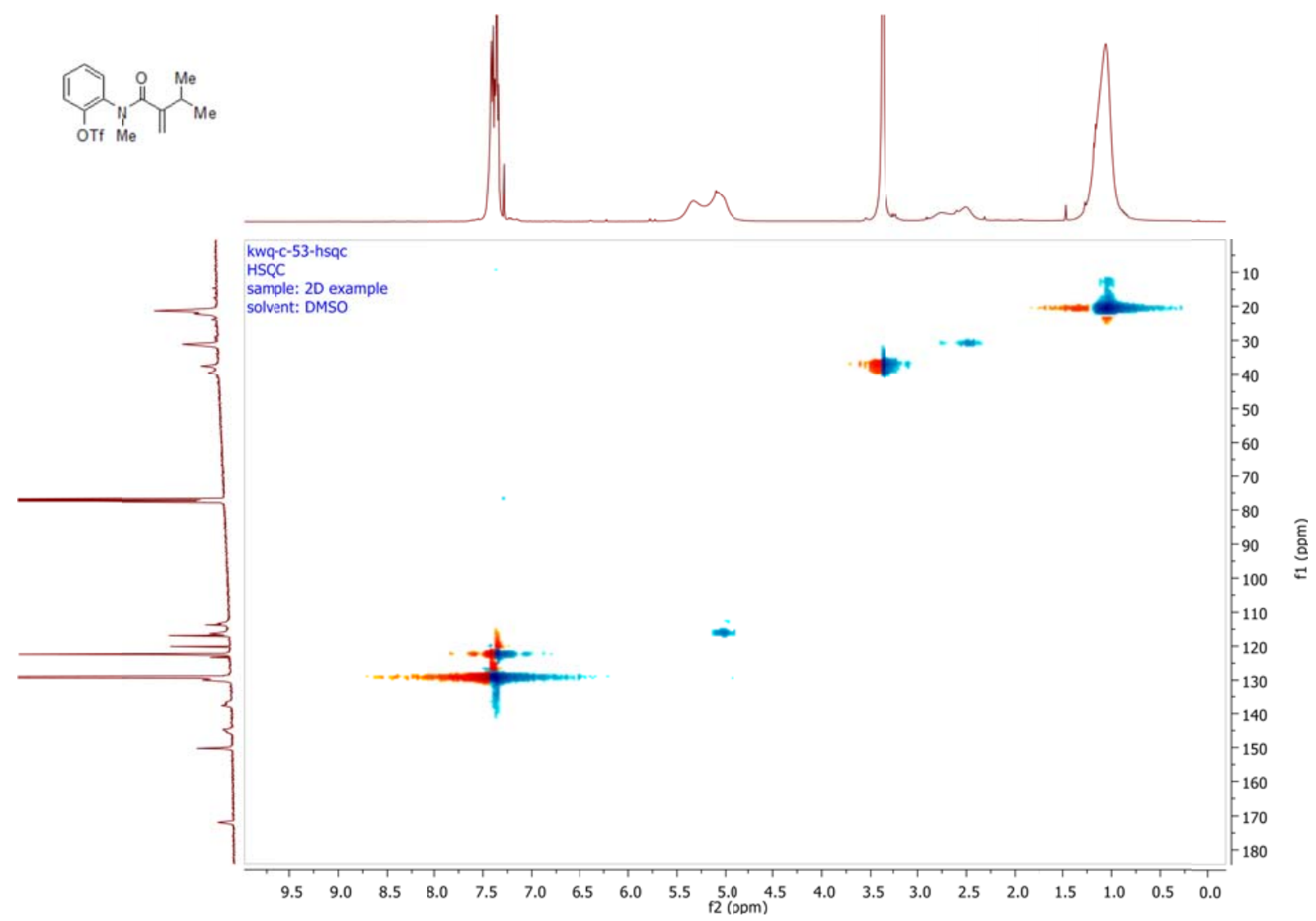

$1 \mathrm{k}$

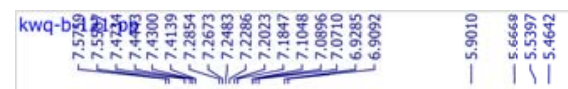
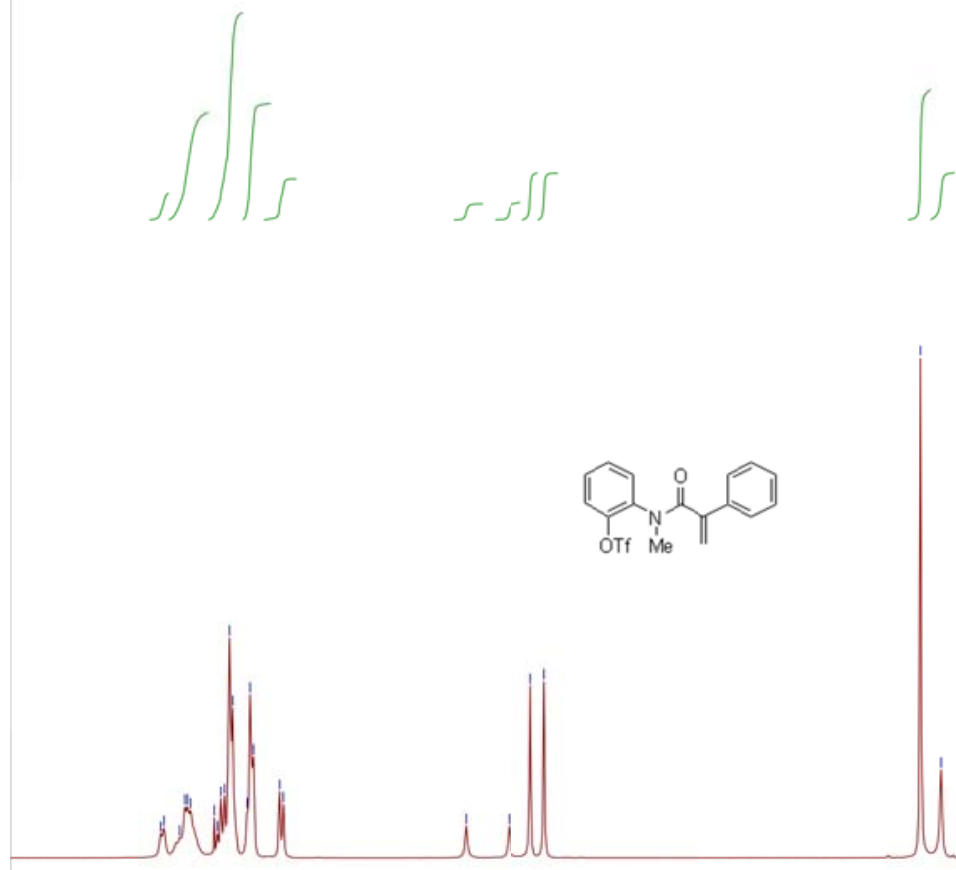

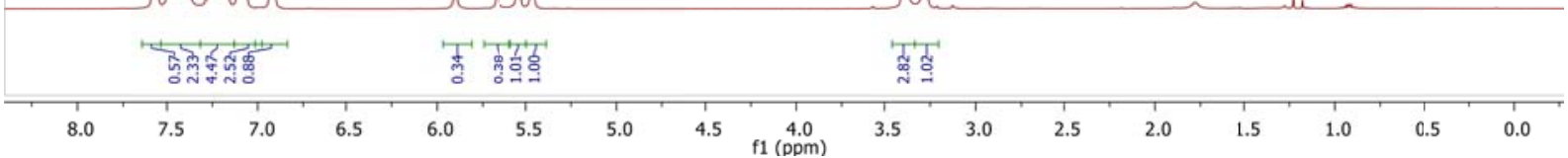



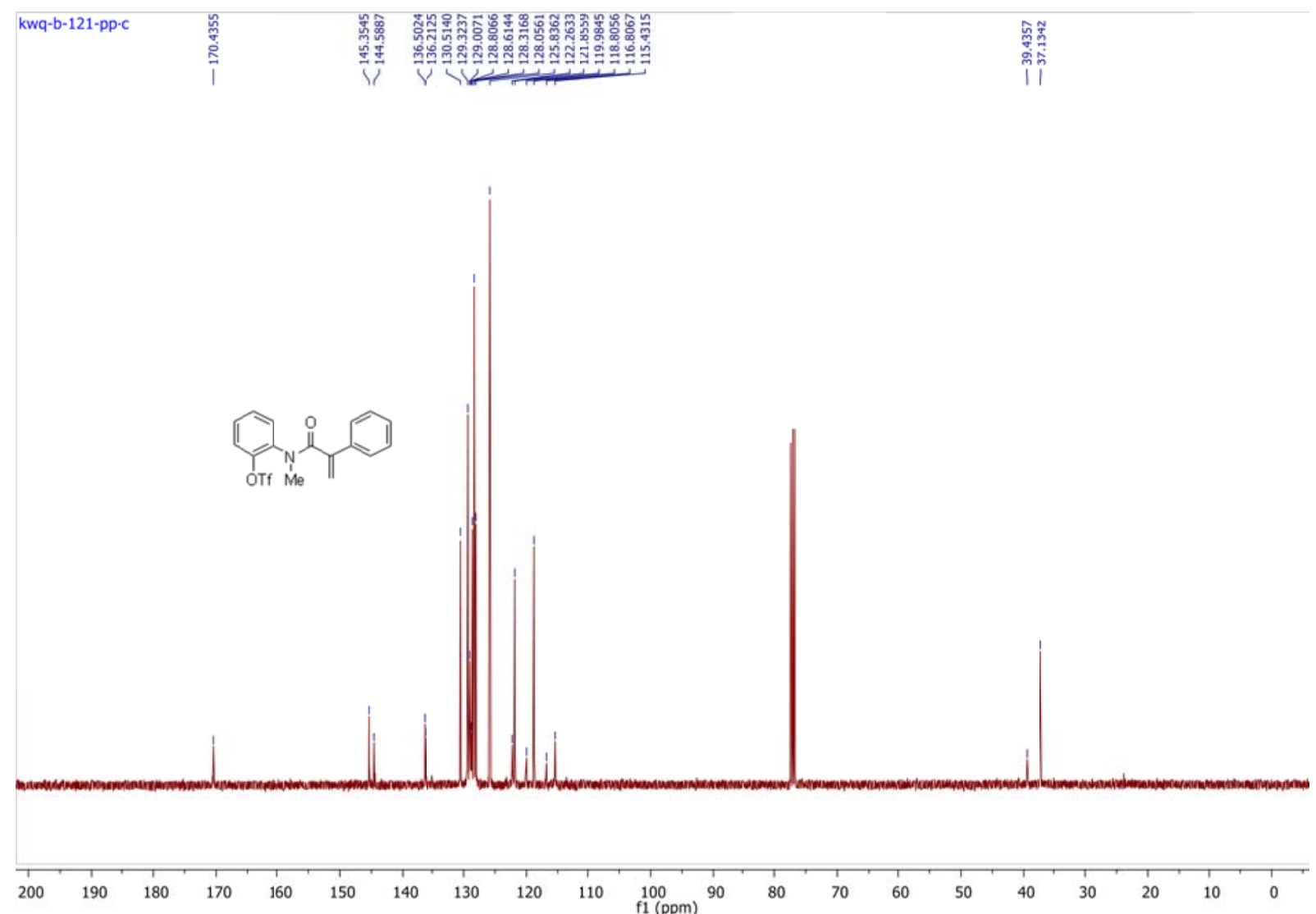

kwq-b-121-pp-f
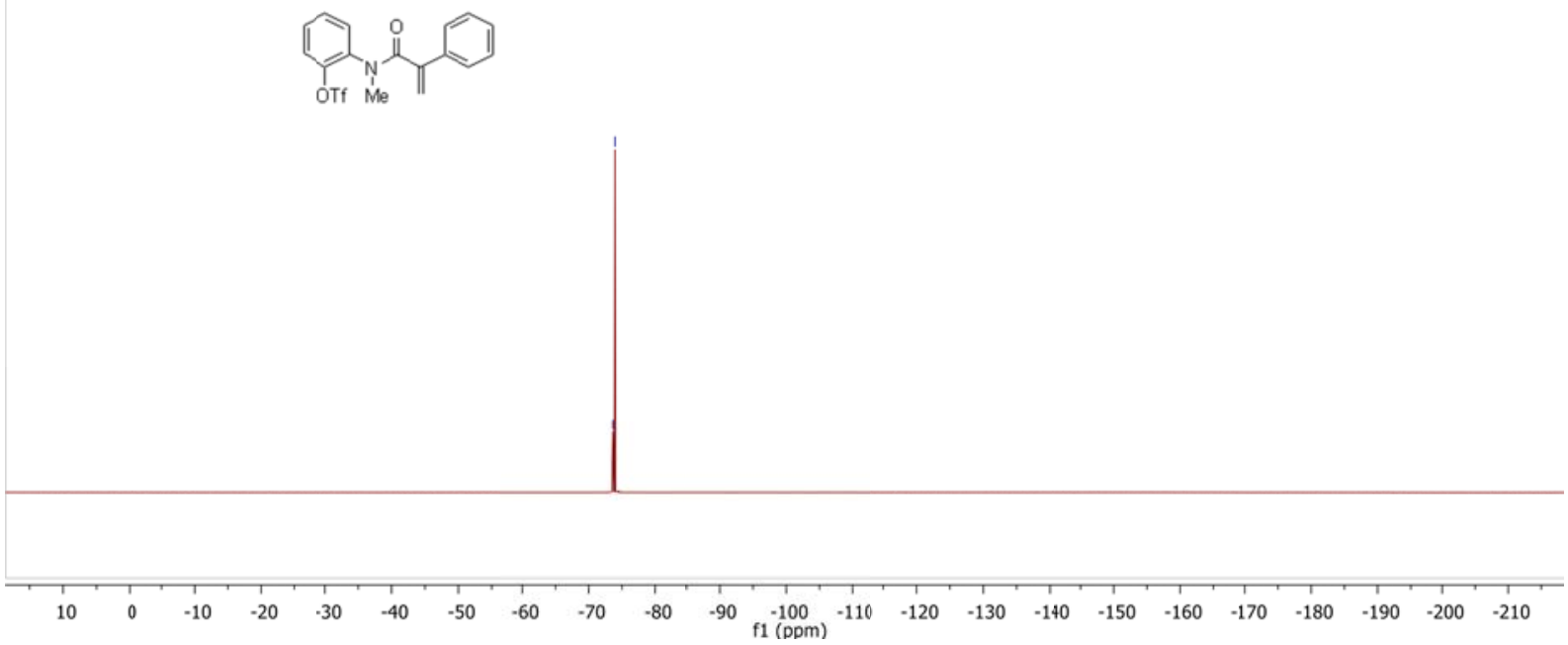
11

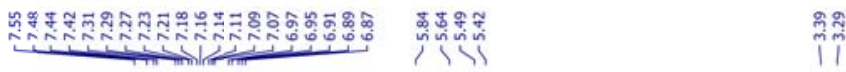

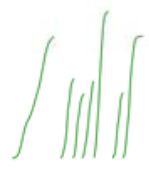

$1 / \int$

OTf $\mathrm{Me}$

whin

U

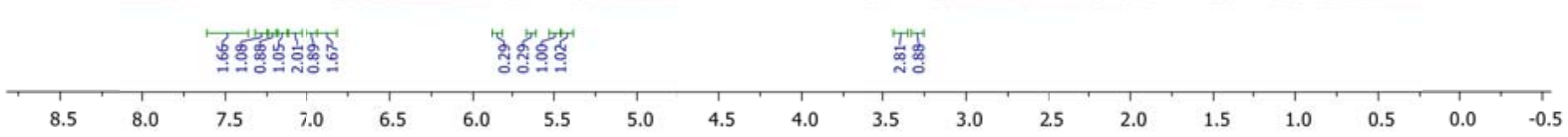

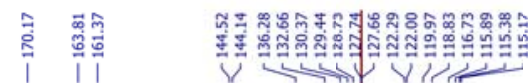

$\underbrace{}_{\text {OTf }}$

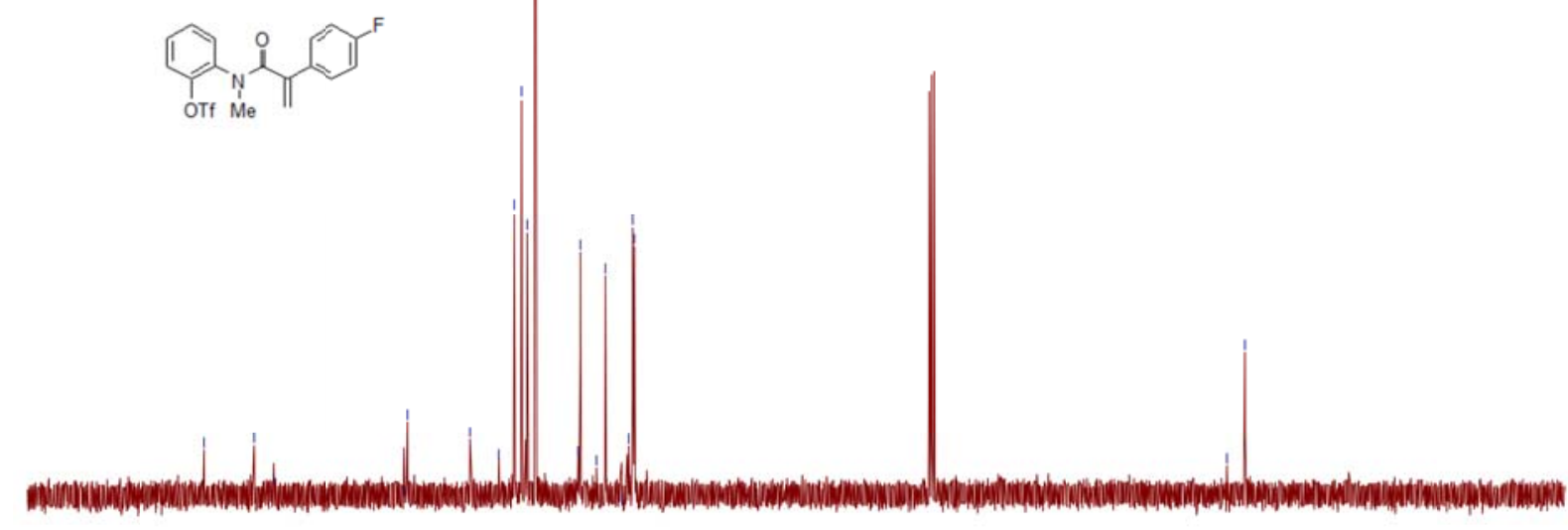

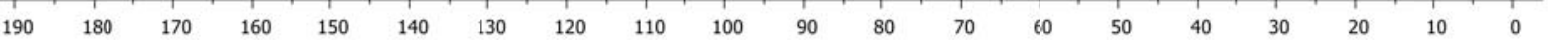


กับ
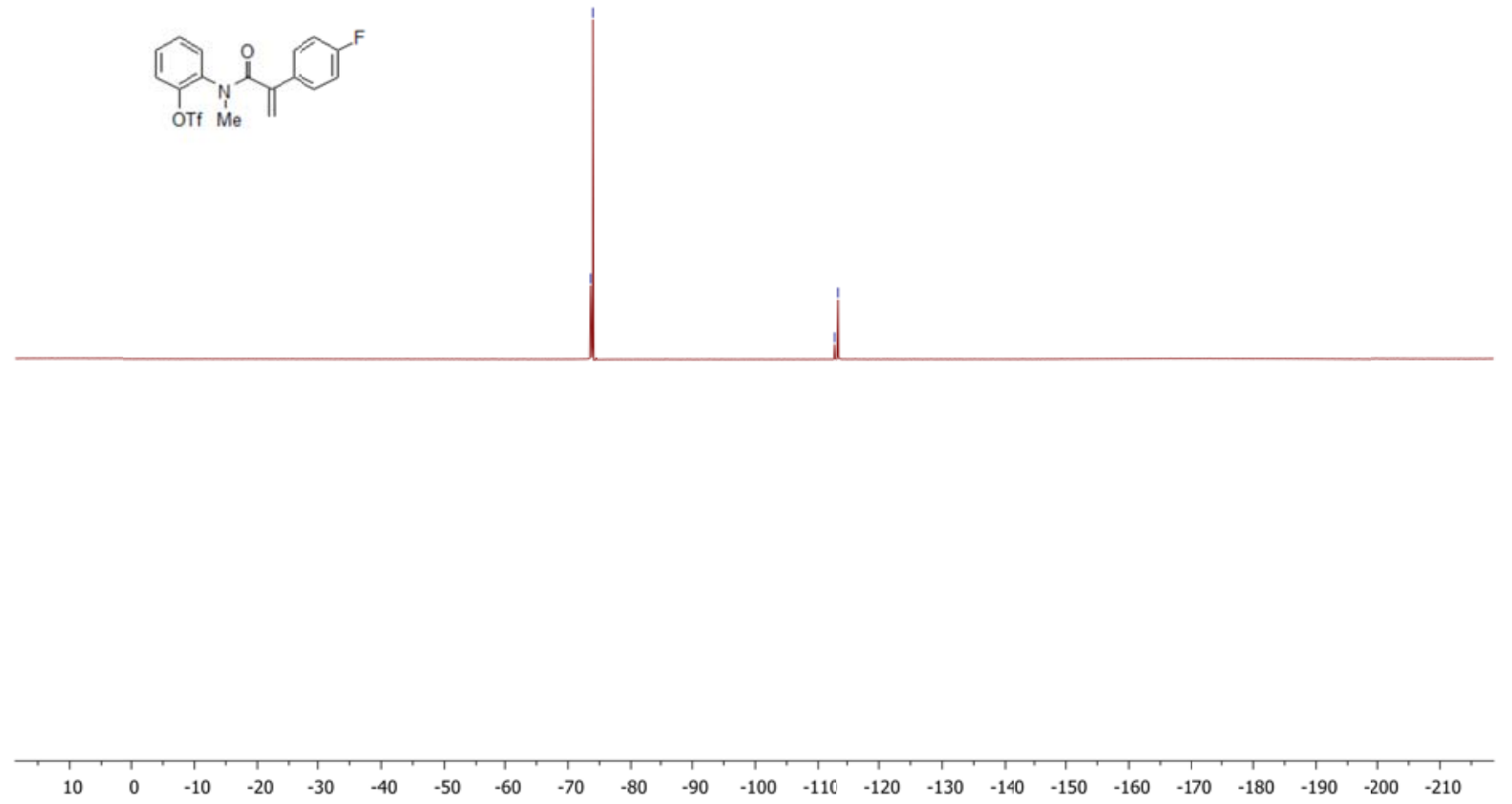

\section{$1 \mathrm{~m}$}

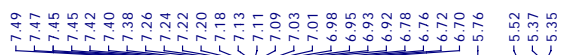

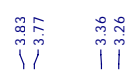

$\int\|\| \int$

$\iint$

$\mid 1$

OTf

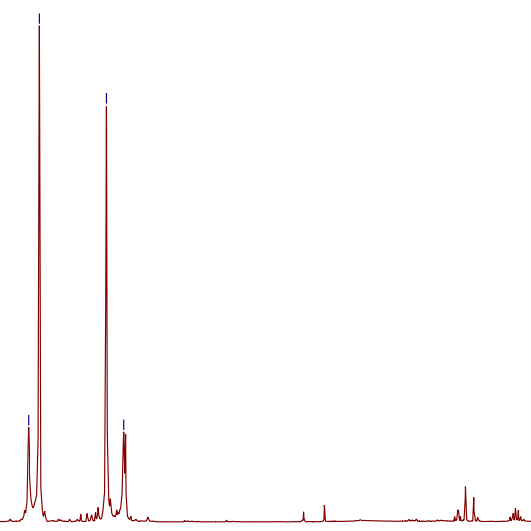

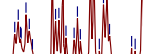
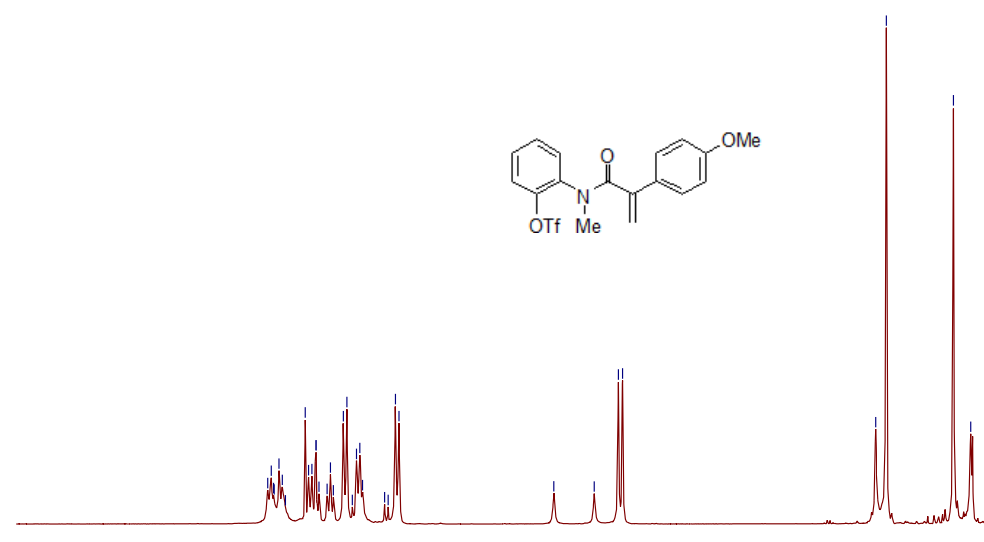

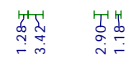

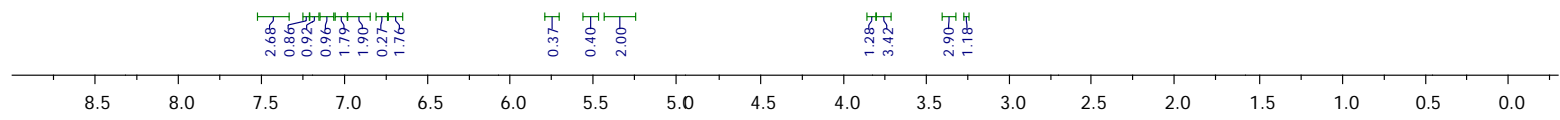




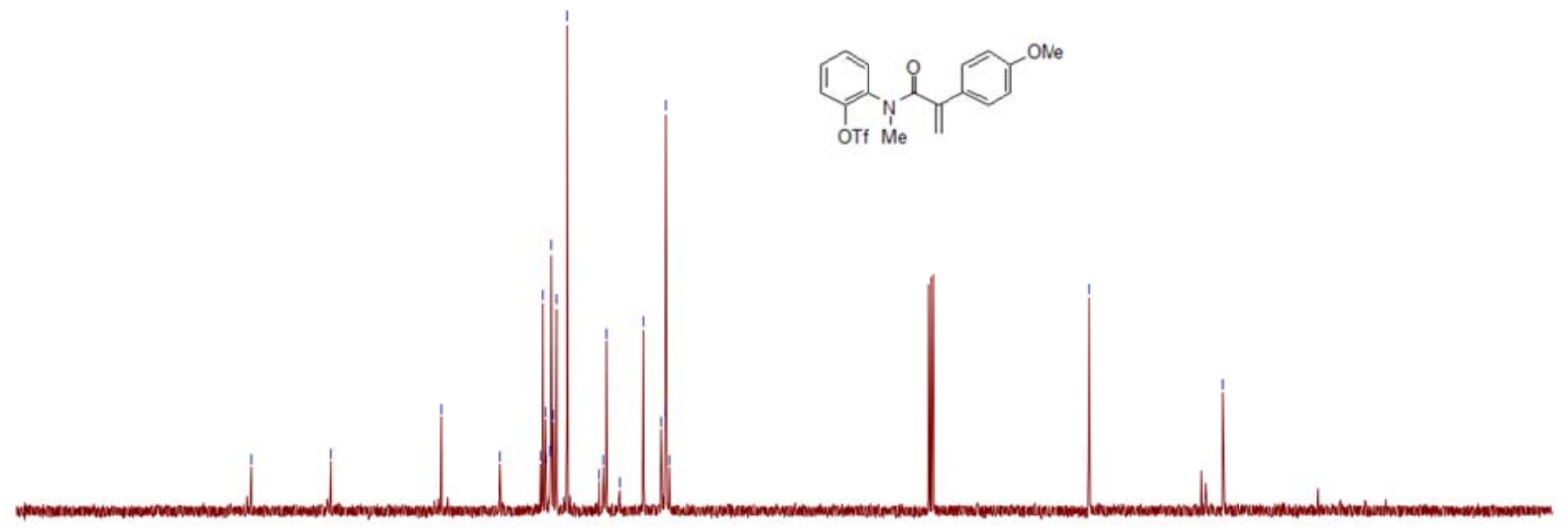

ชั

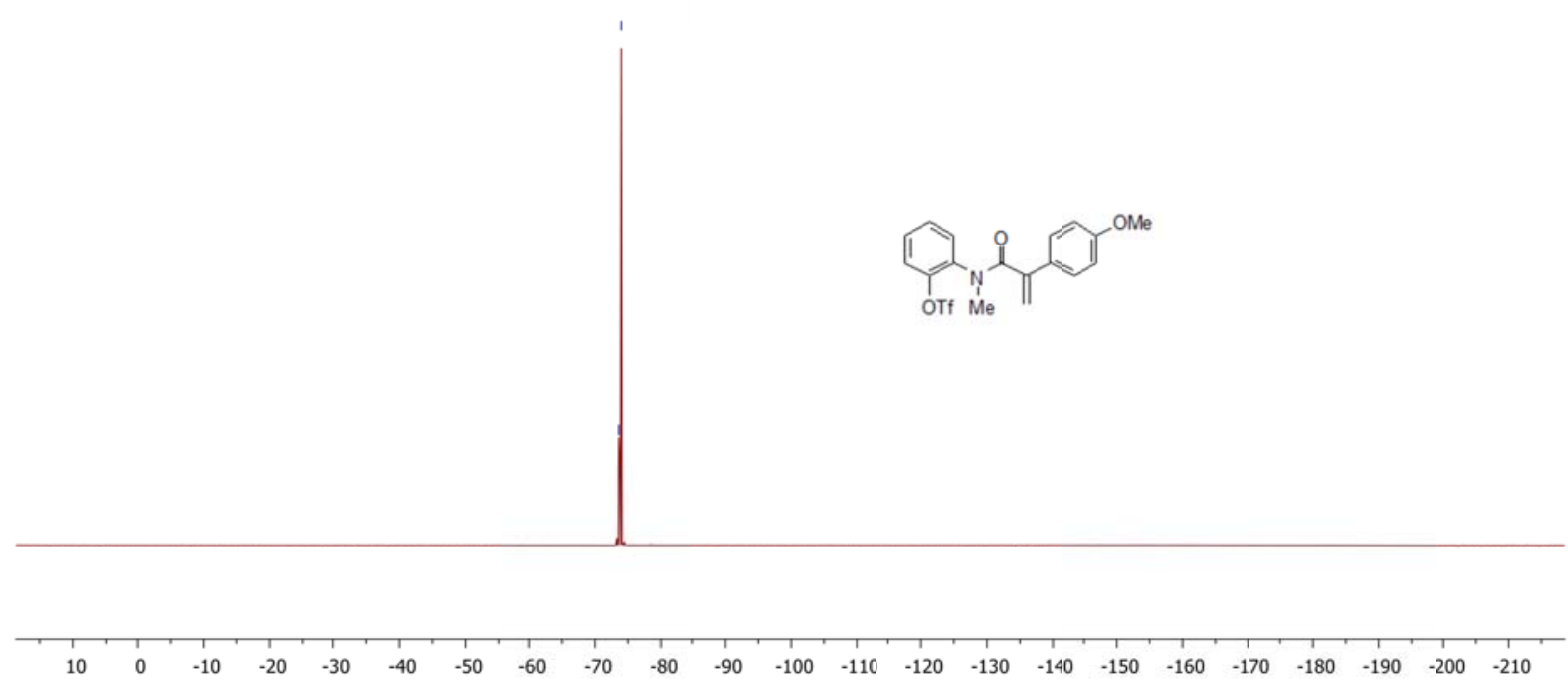


$1 n$

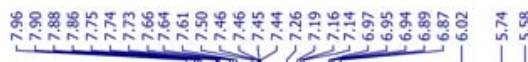

$\|_{1} \int_{\|}$<smiles>CC=C[Te]</smiles>

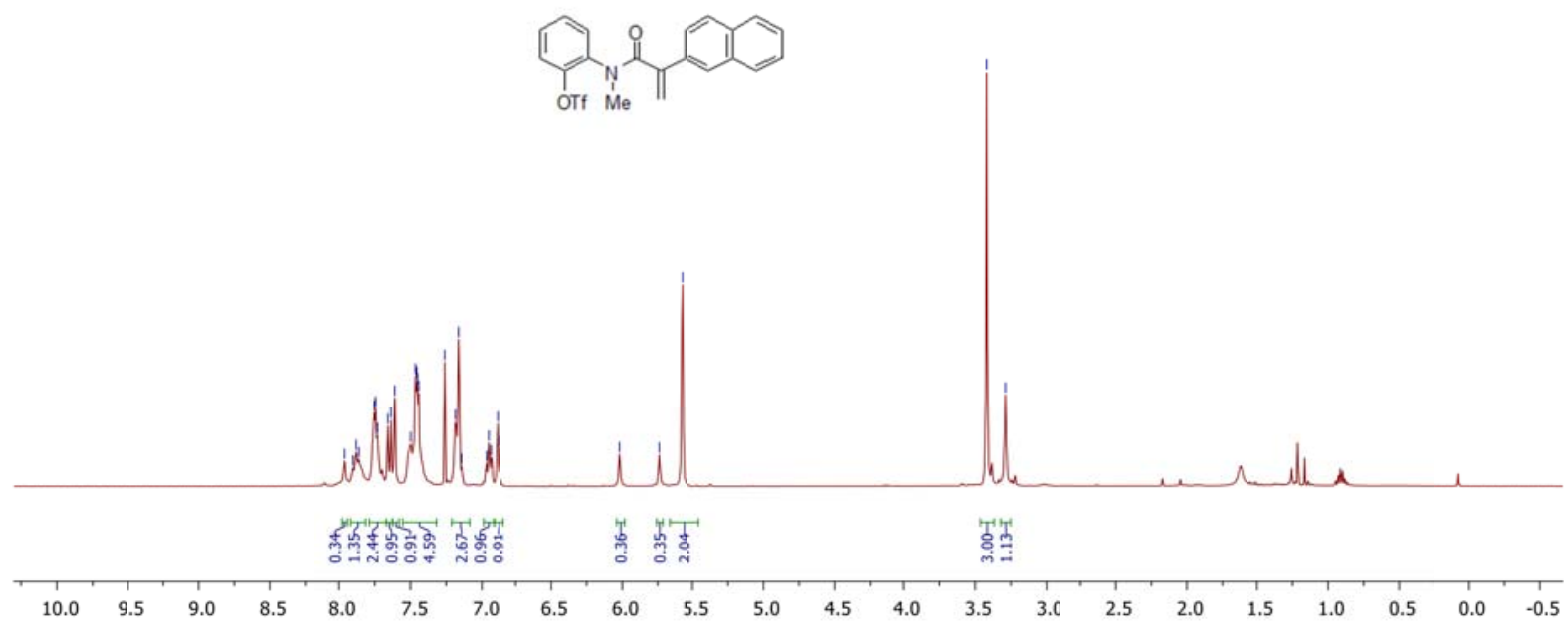

OTf Me

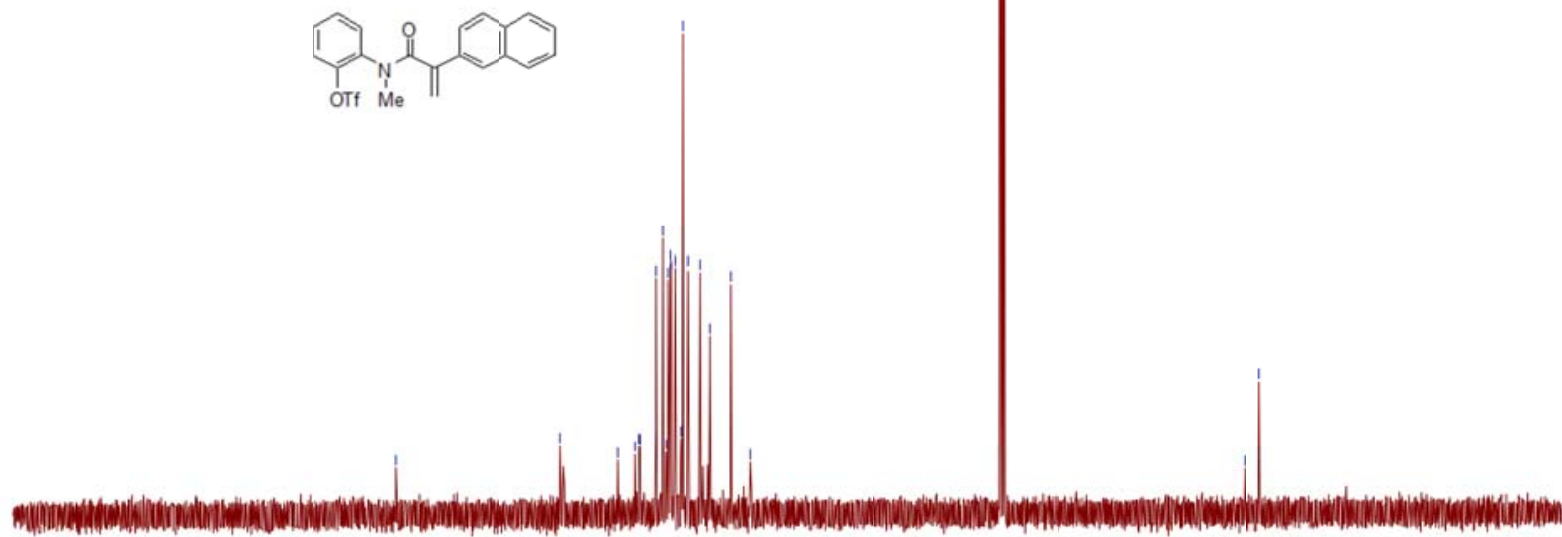

$\begin{array}{lllllllllllllllllllllll}220 & 210 & 200 & 190 & 180 & 170 & 160 & 150 & 140 & 130 & 120 & 110 & 100 & 90 & 80 & 70 & 60 & 50 & 40 & 30 & 20 & 10 & 0\end{array}$ 


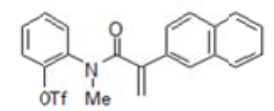

$\begin{array}{lllllllllllllllllllllllllll}10 & 0 & -10 & -20 & -30 & -40 & -50 & -60 & -70 & -80 & -90 & -100 & -110 & -120 & -130 & -140 & -150 & -160 & -170 & -180 & -190 & -200 & -210\end{array}$

10

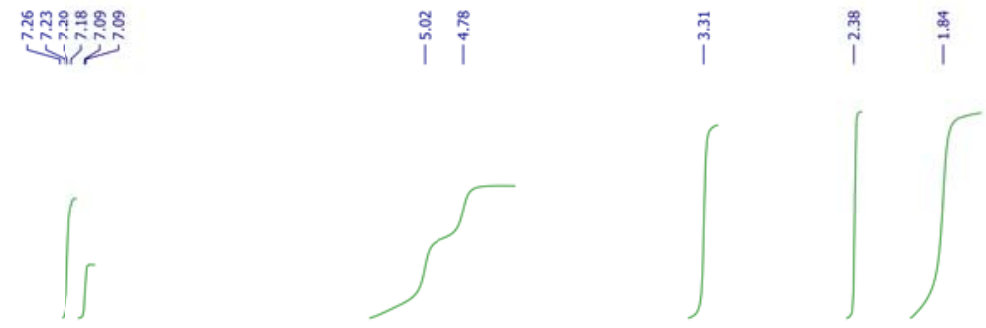

Me

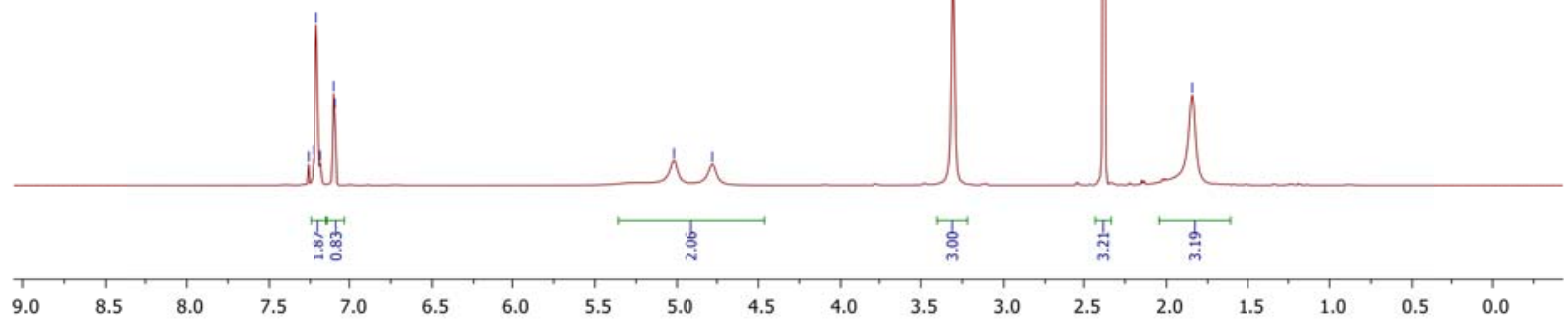



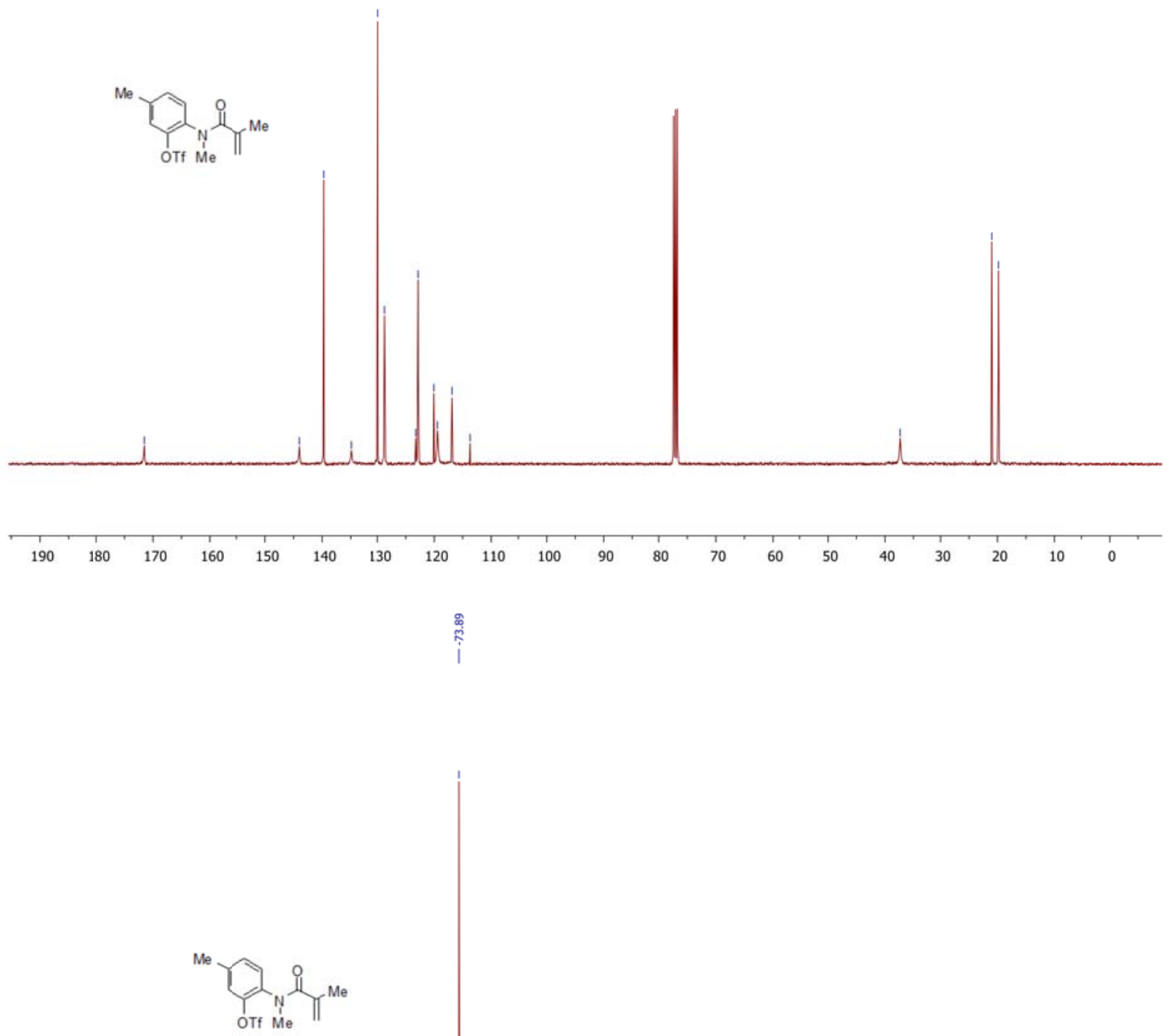

$\begin{array}{llllllllllllllllllllllllll}10 & 0 & -10 & -20 & -30 & -40 & -50 & -60 & -70 & -80 & -90 & -100 & -110 & -120 & -130 & -140 & -150 & -160 & -170 & -180 & -190 & -200 & -210\end{array}$ 
$1 p$

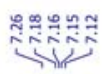

$\stackrel{8}{i}$

$\stackrel{\substack{0 \\ i}}{i} \stackrel{\infty}{i}$
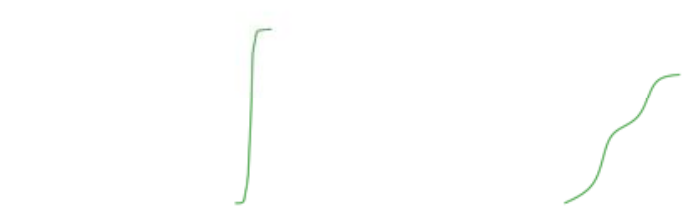

$\overbrace{\text { OTf Me }}^{\text {Me }}$
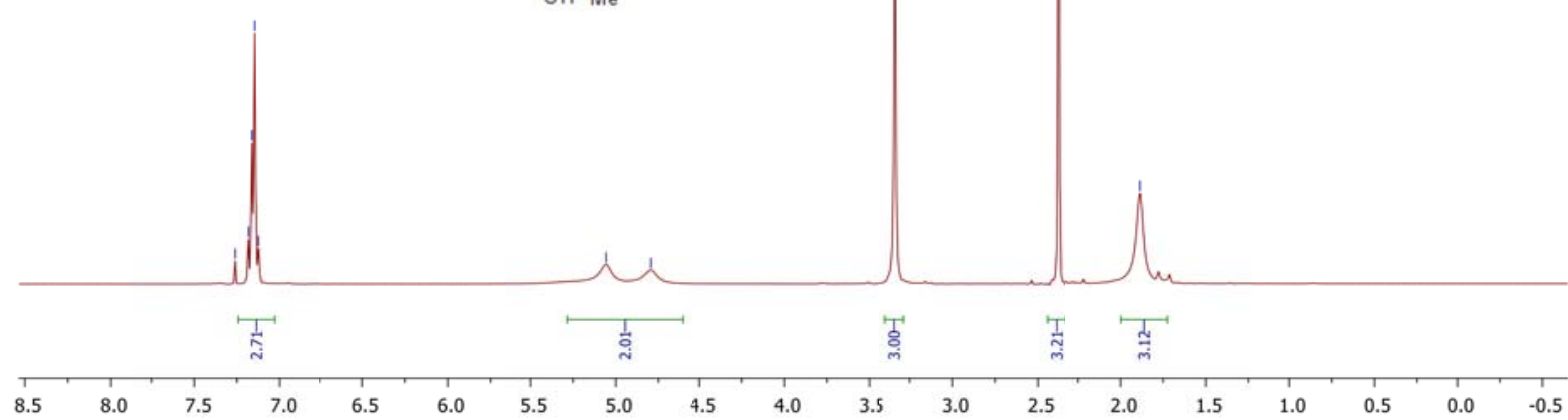

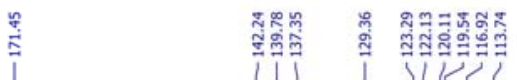

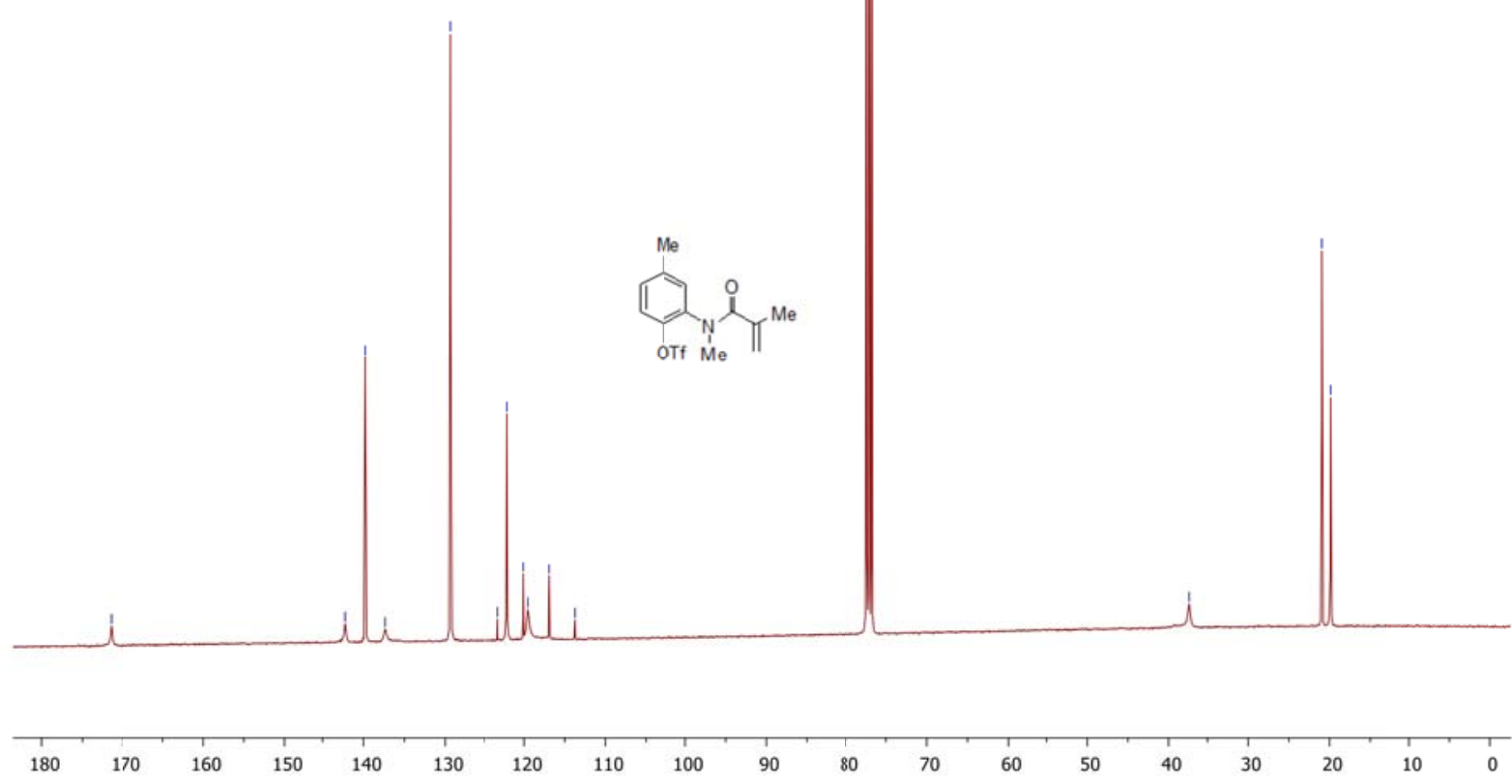



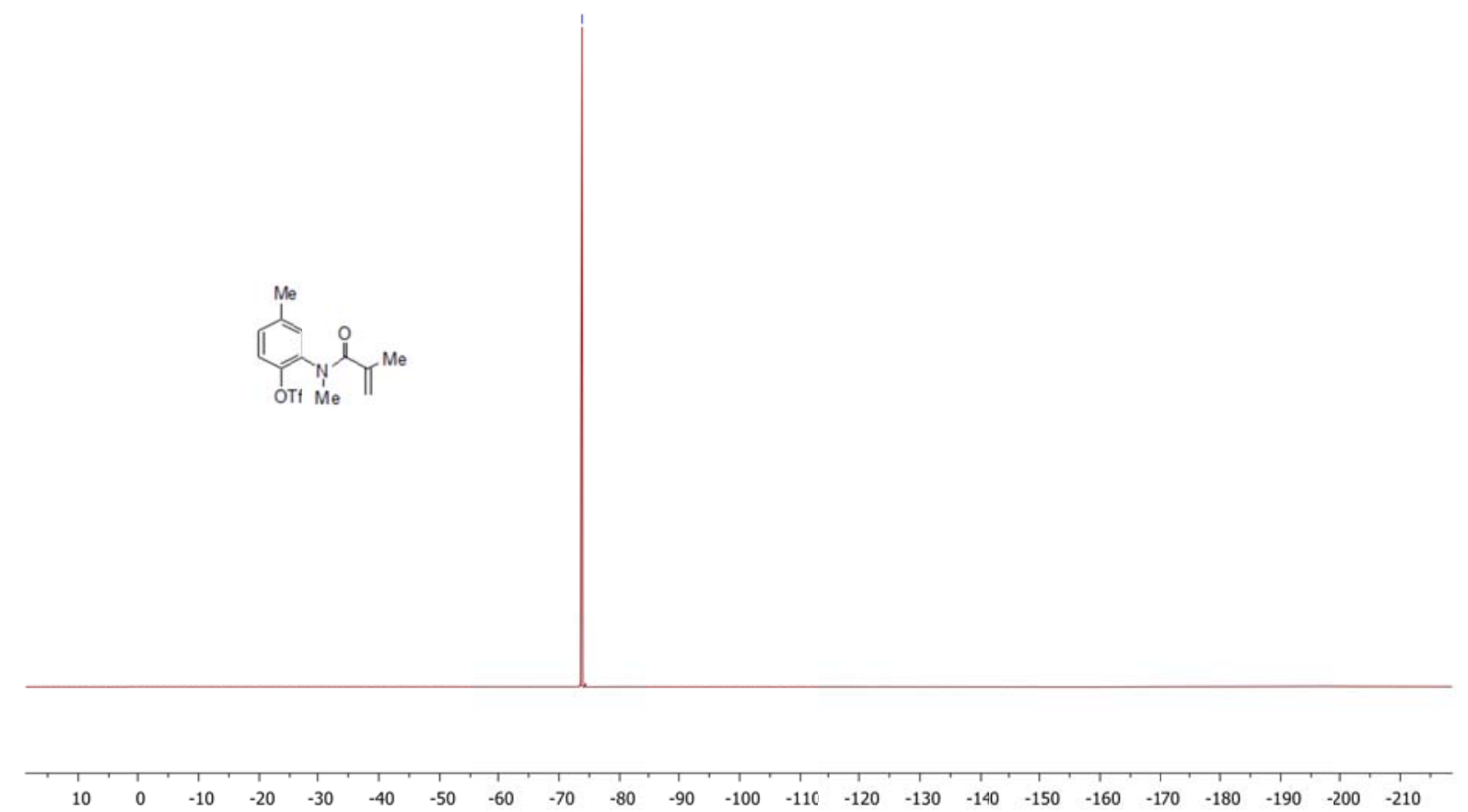

$1 q$

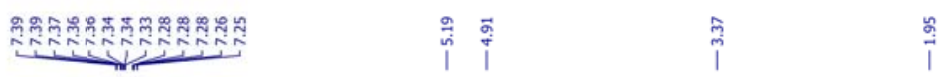
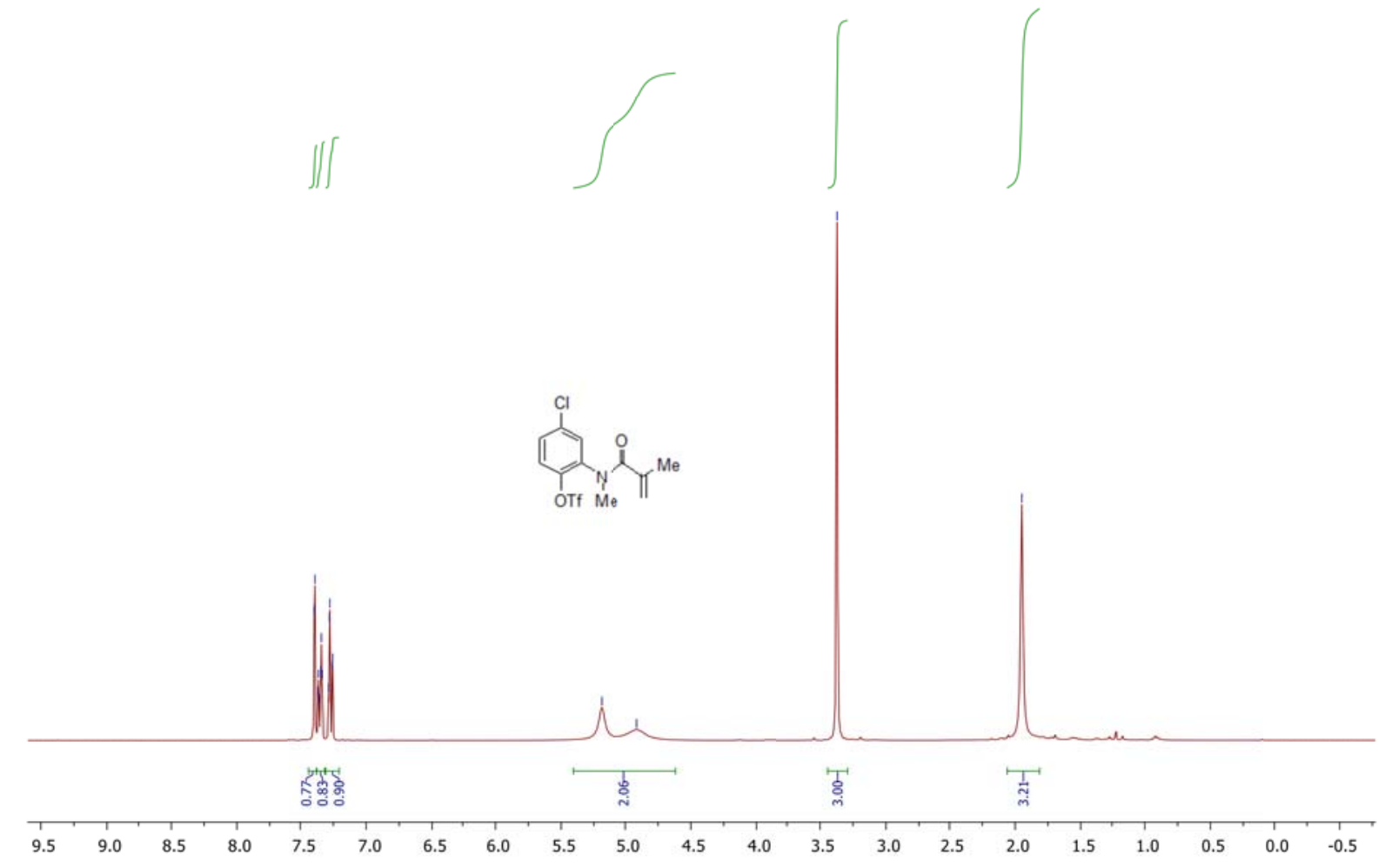

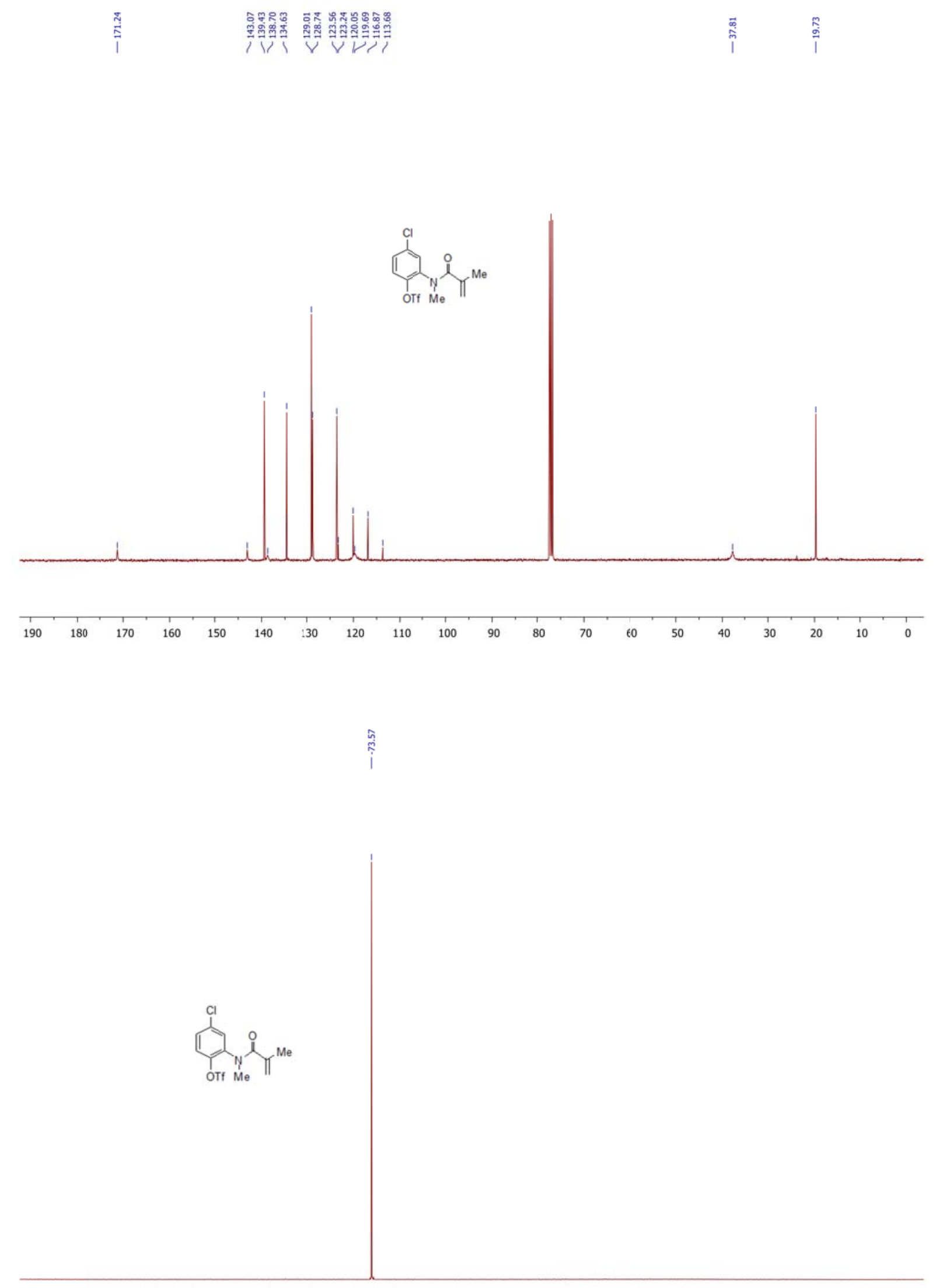

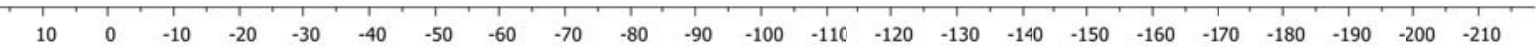




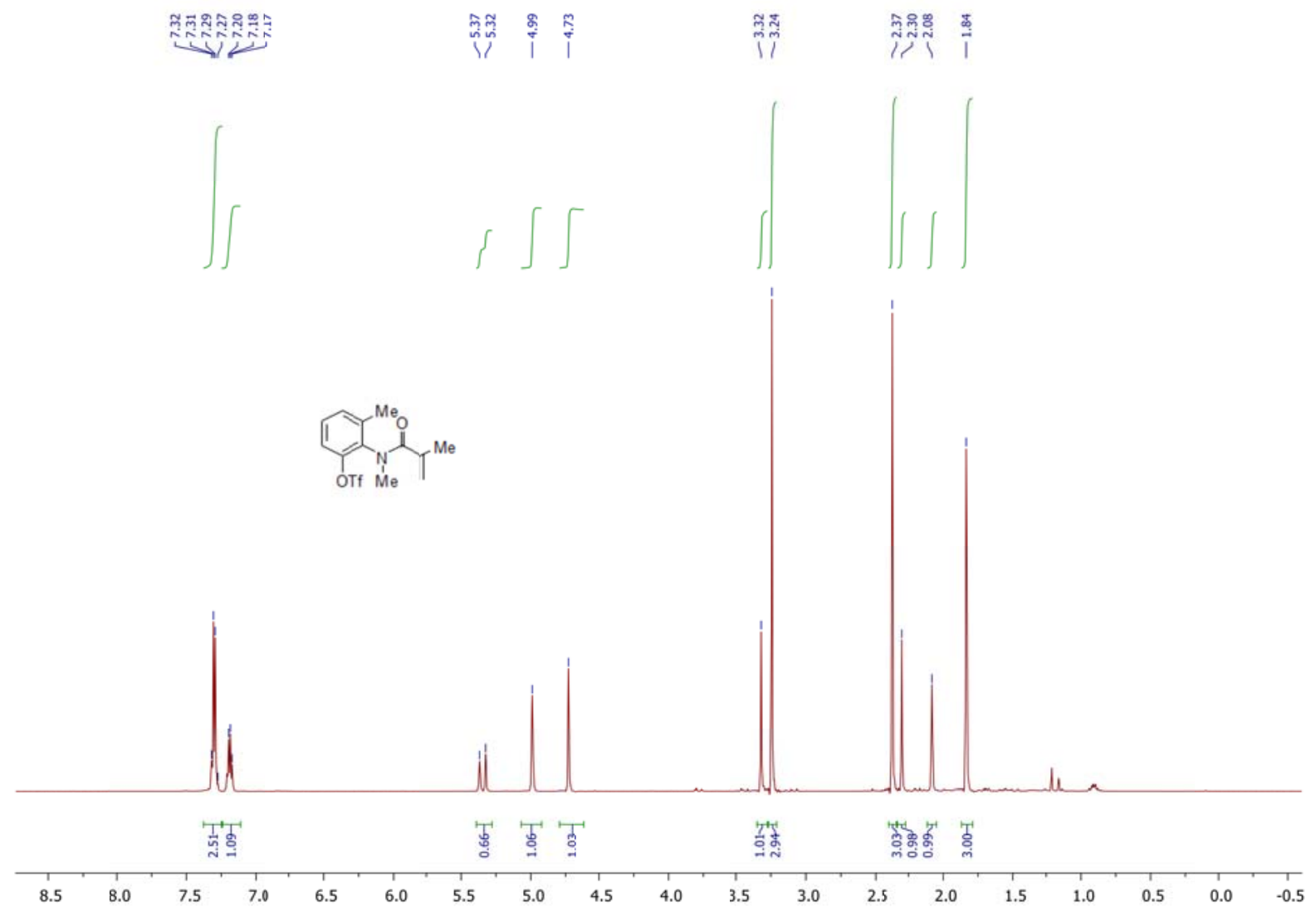

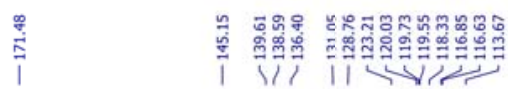

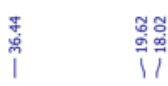

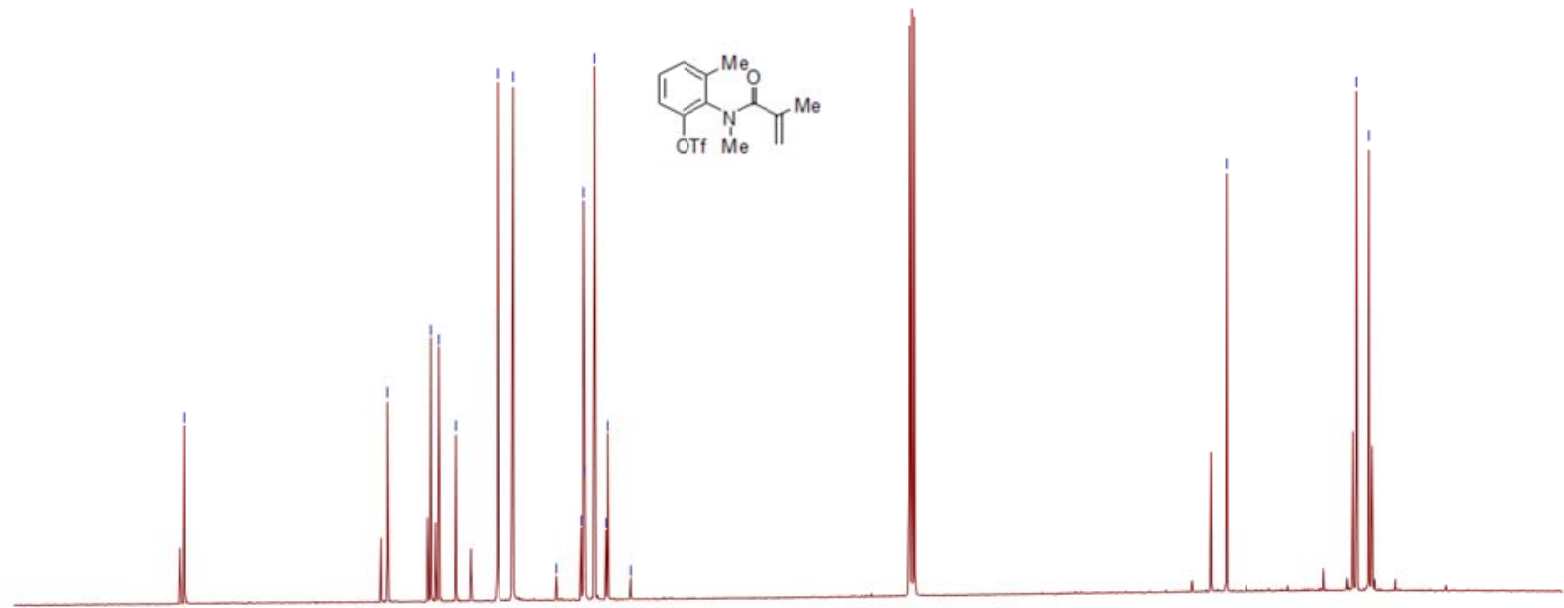

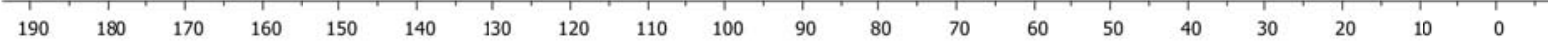



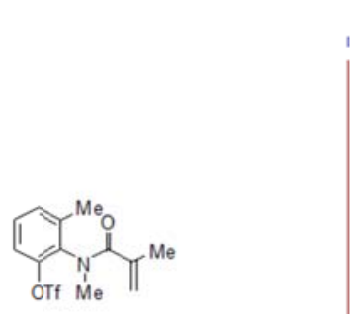

$\begin{array}{llllllllllllllllllllllllllll}1 & 0 & 0 & -10 & -20 & -30 & -40 & -50 & -60 & -70 & -80 & -90 & -100 & -110 & -120 & -130 & -140 & -150 & -160 & -170 & -180 & -190 & -200 & -210 & \end{array}$

$1 s$

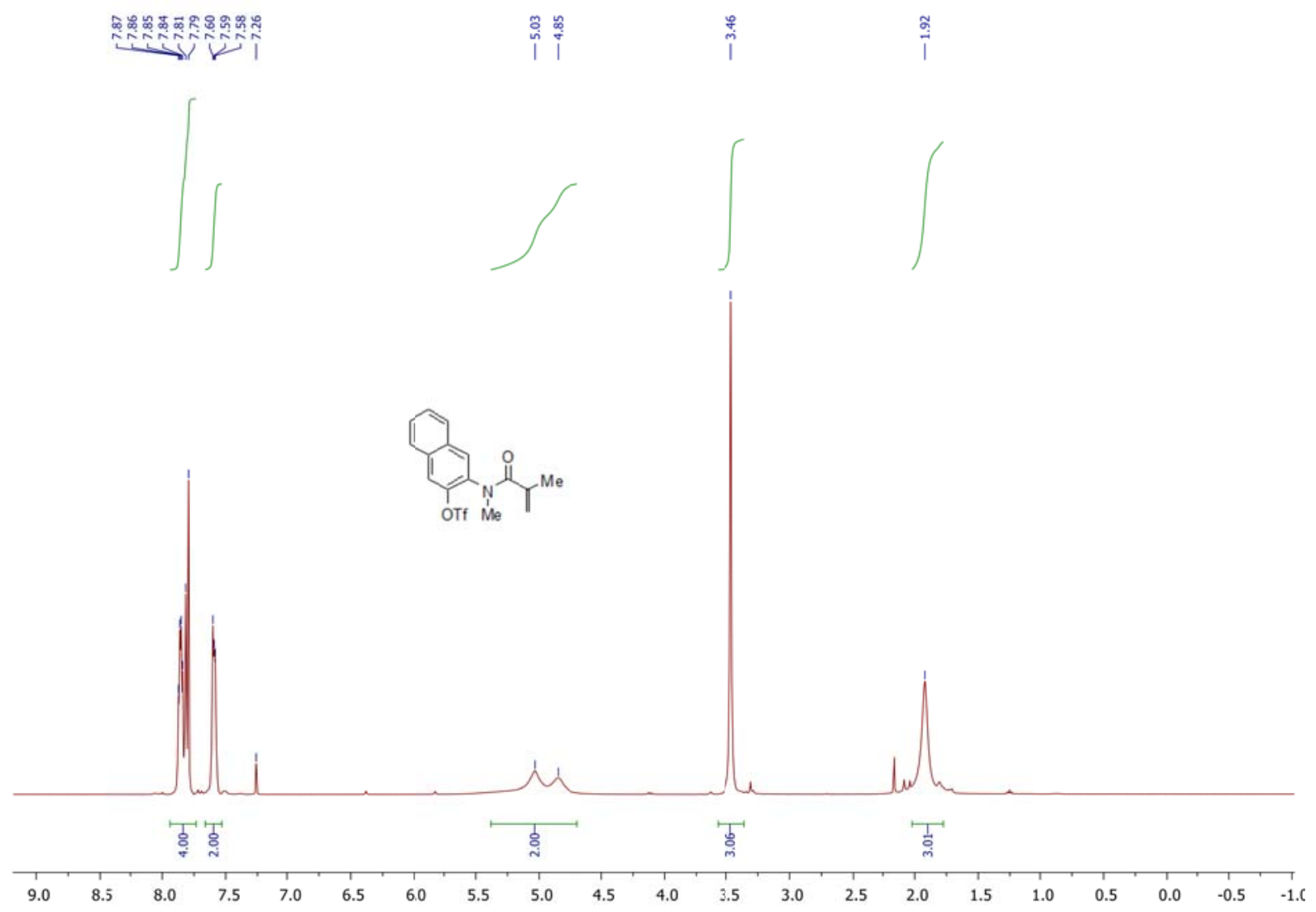



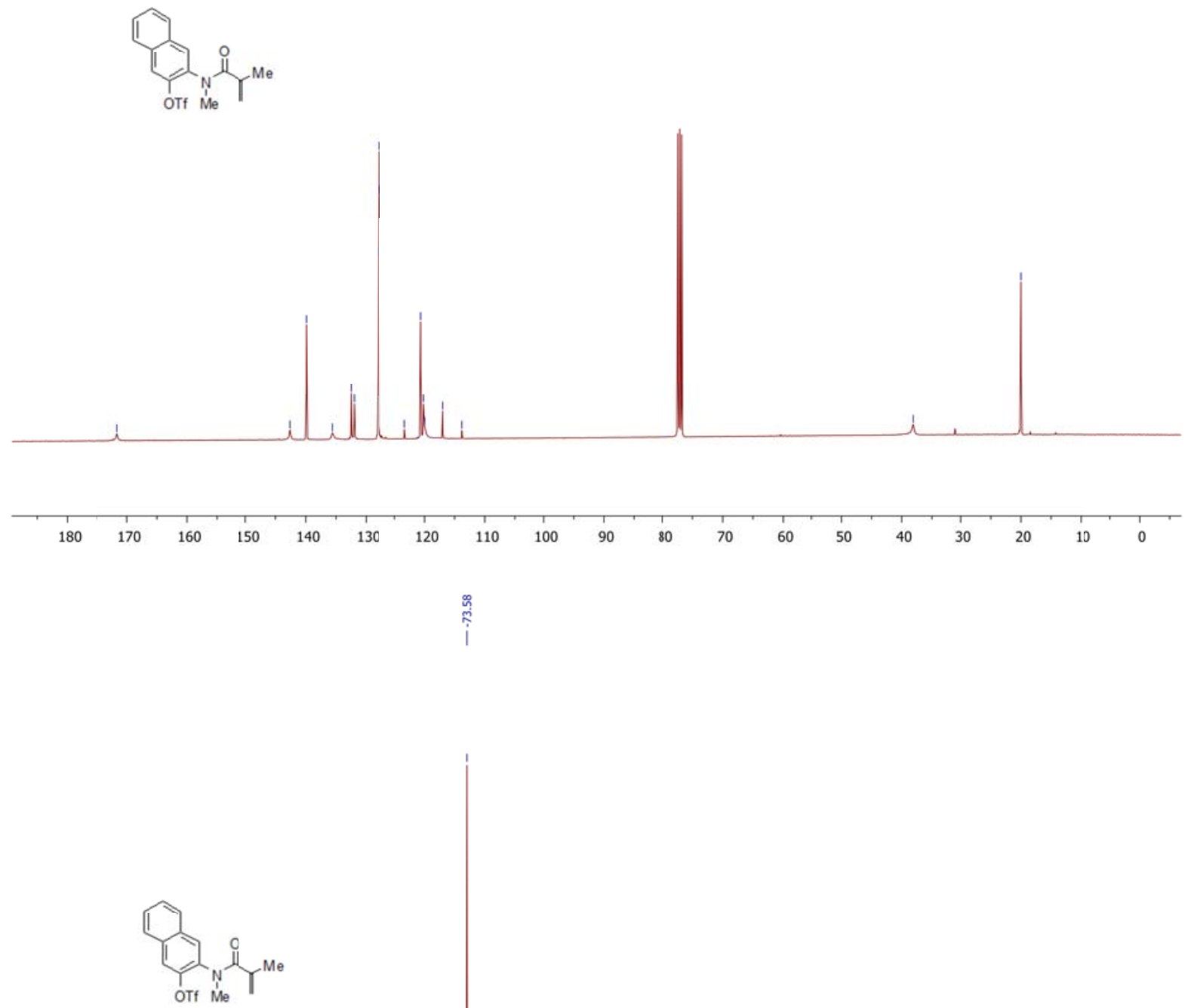

$\begin{array}{llllllllllllllllllllllllllllll}10 & 0 & -10 & -20 & -30 & -40 & -50 & -60 & -70 & -80 & -90 & -100 & -110 & -120 & -130 & -140 & -150 & -160 & -170 & -180 & -190 & -200 & -210\end{array}$ 
$3 a a$

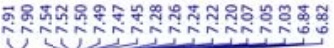

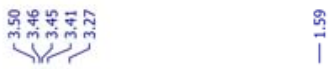

/ / I
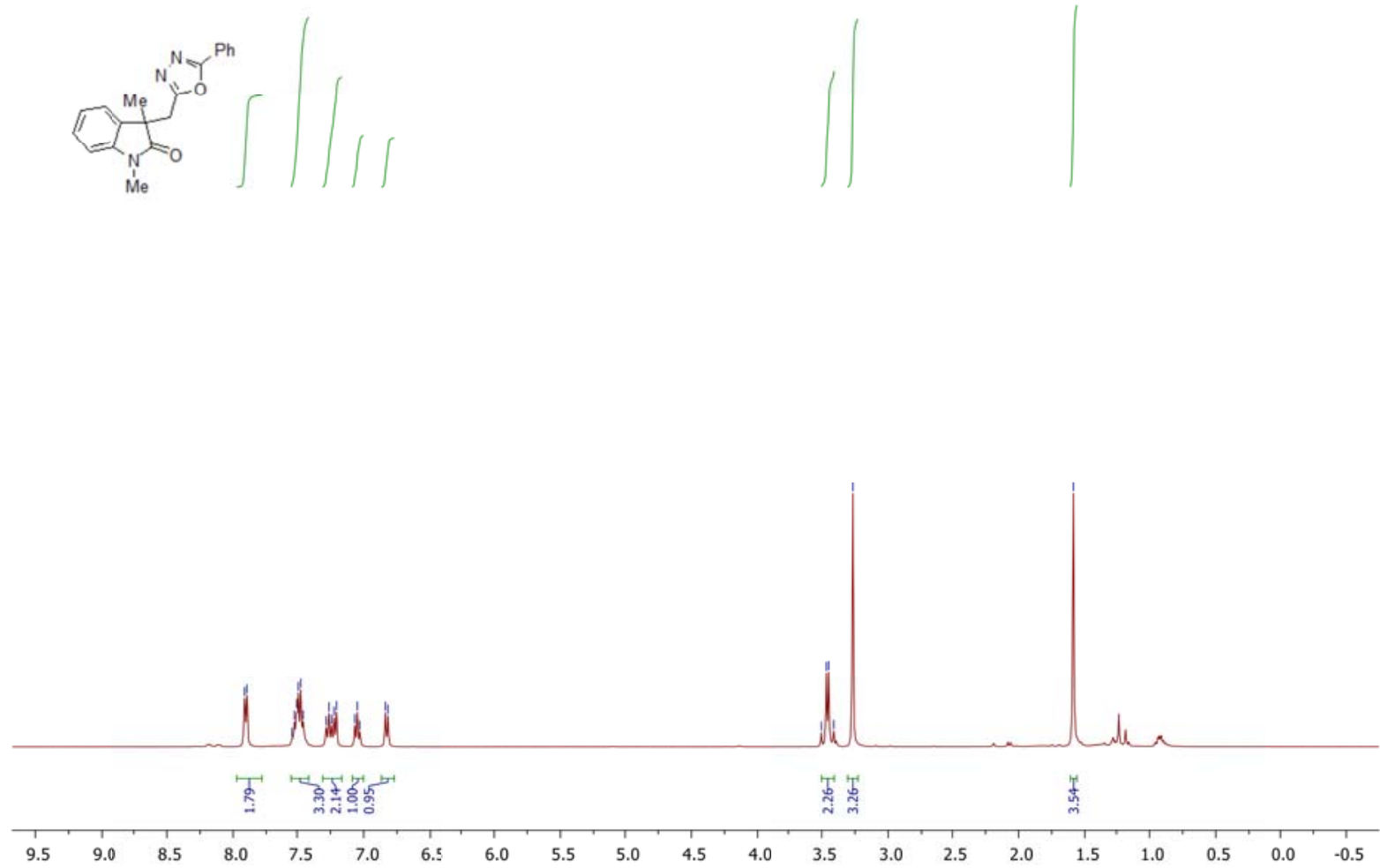

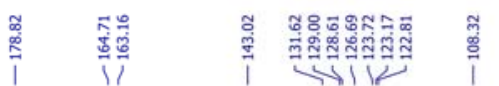

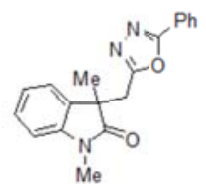

$220 \quad 210 \quad 200$

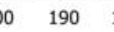

$\begin{array}{llll}170 & 160 & 150 & 140\end{array}$

$130 \quad 120$

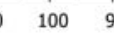

80

60

50

$30 \quad 20$

100 
$3 a b$

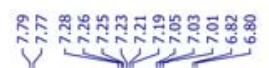

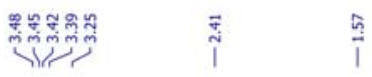
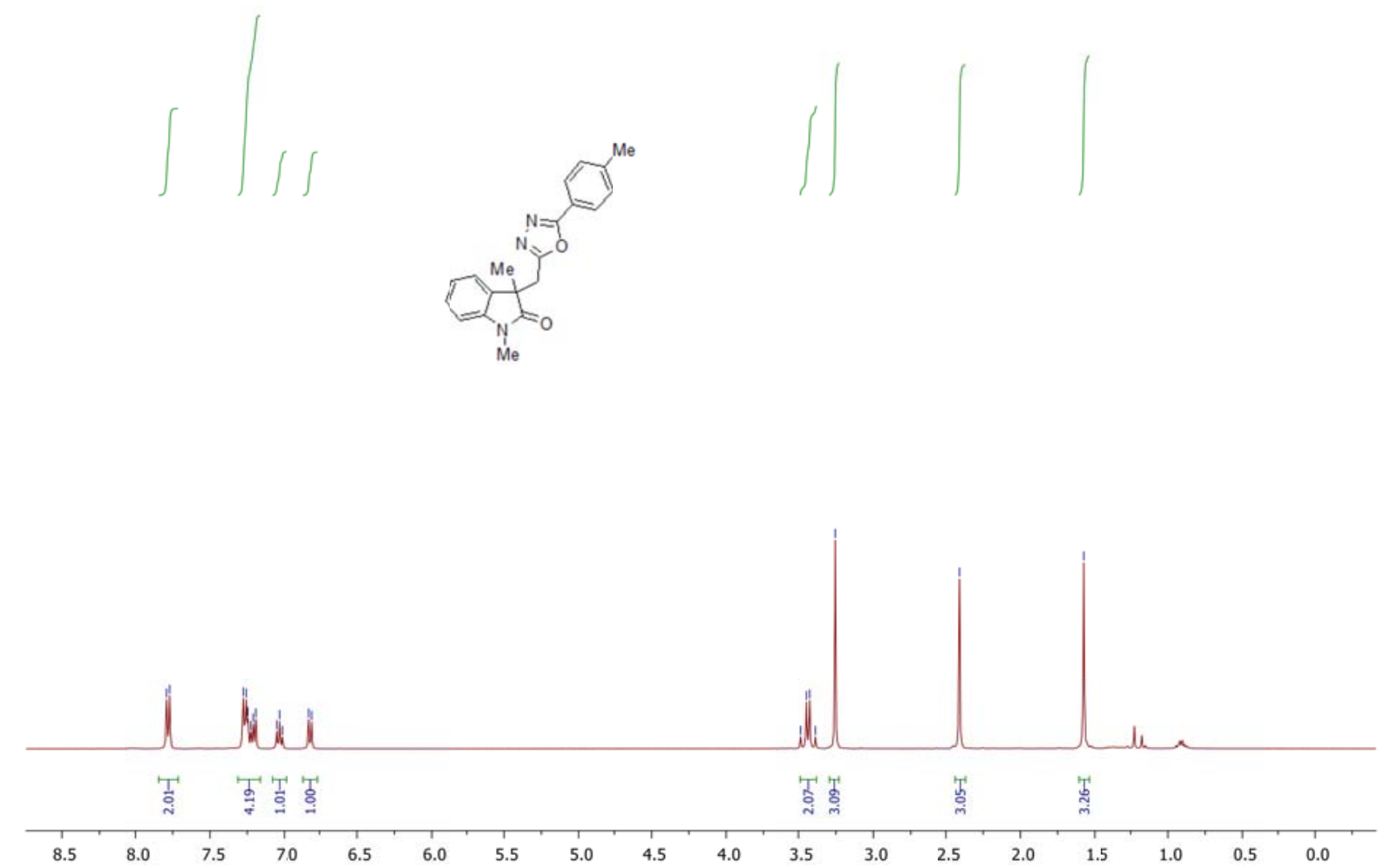

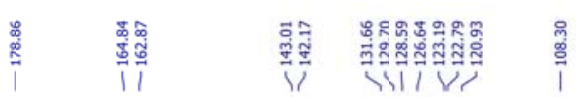

ईิ

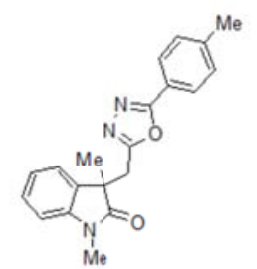

190

180

17

160

$50 \quad 140$

1301
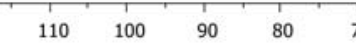

$60 \quad 50$

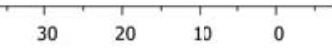


$3 a c$

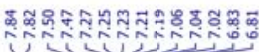

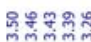

i

$\iiint S$

lilit

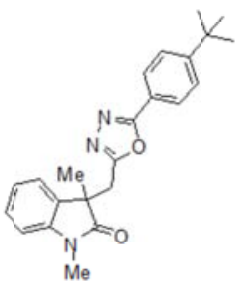

Hex.

it.
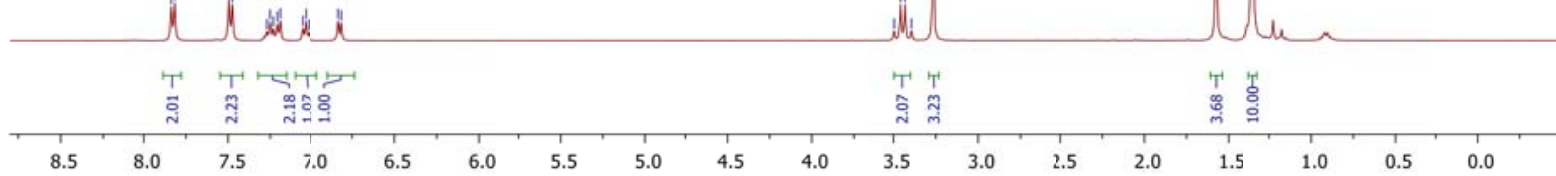

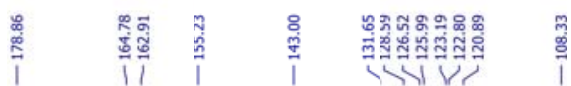

|
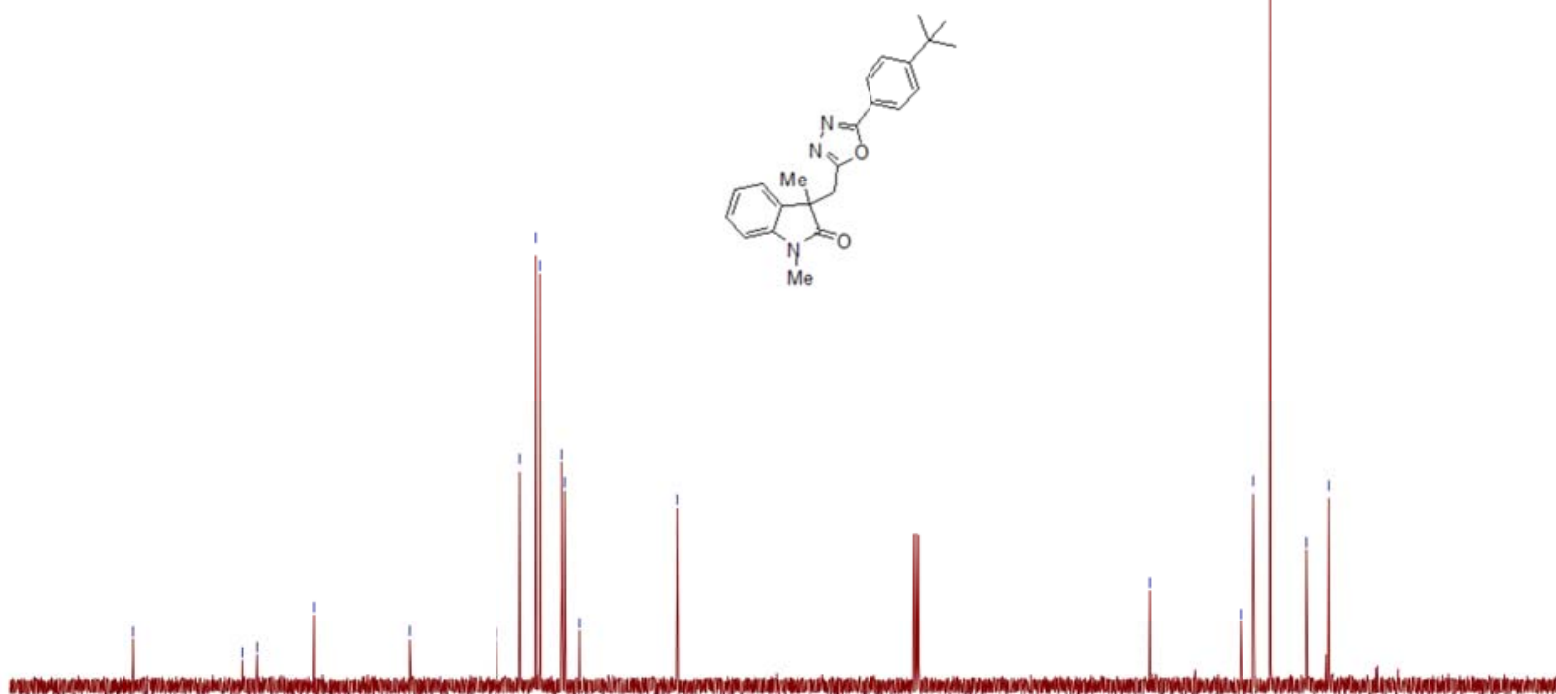

$\begin{array}{lllllllllllllllllllll}190 & 180 & 170 & 160 & 150 & 140 & 130 & 120 & 110 & 100 & 90 & 80 & 70 & 60 & 50 & 40 & 30 & 20 & 10 & 0\end{array}$ 

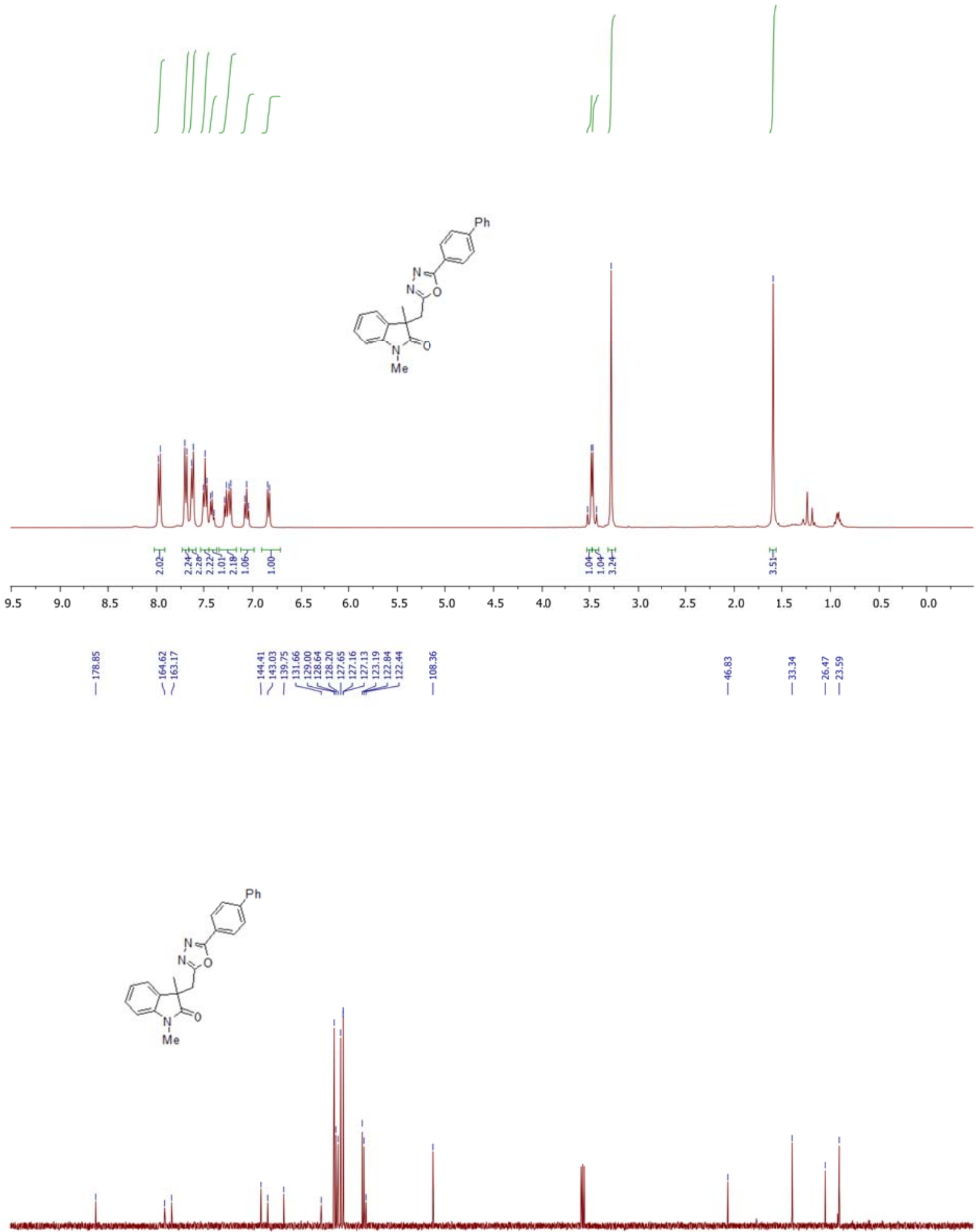

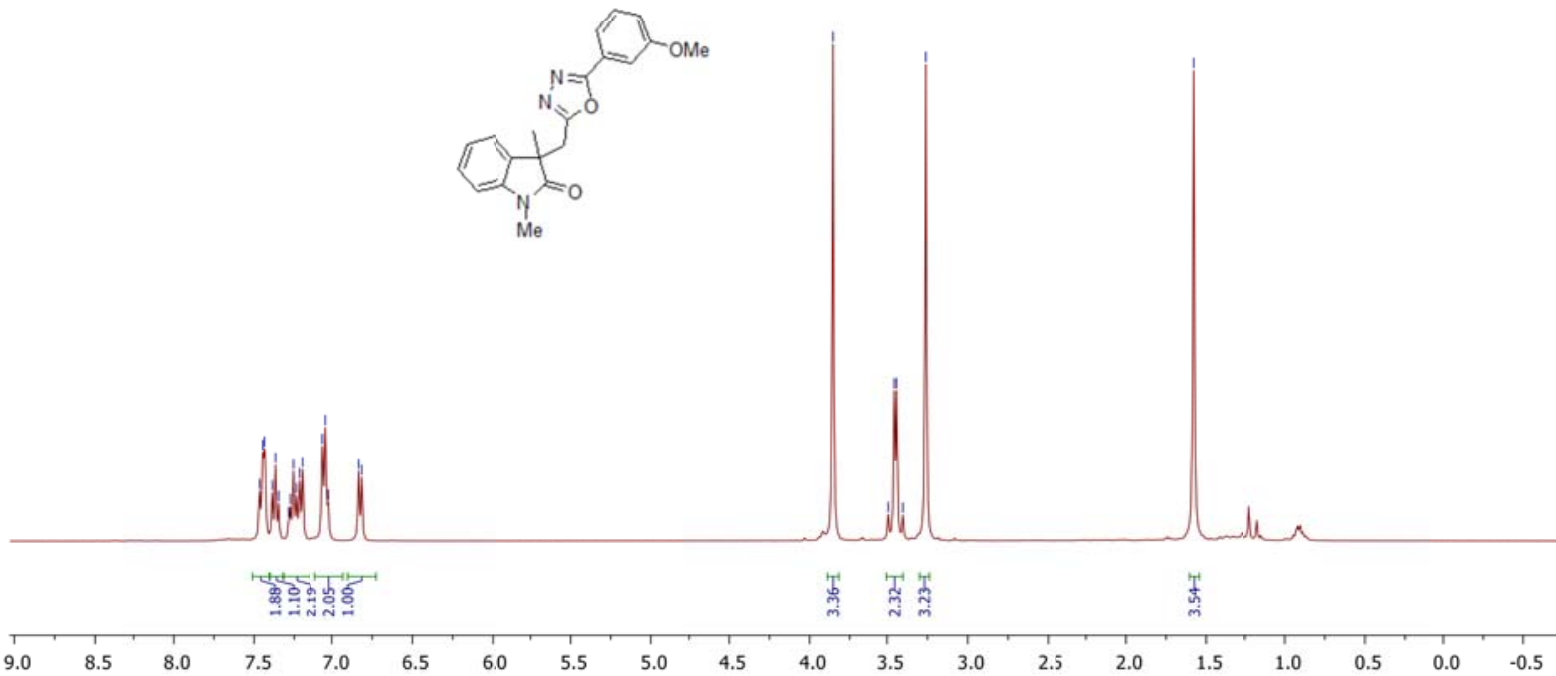

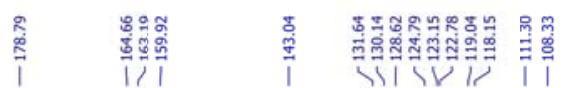
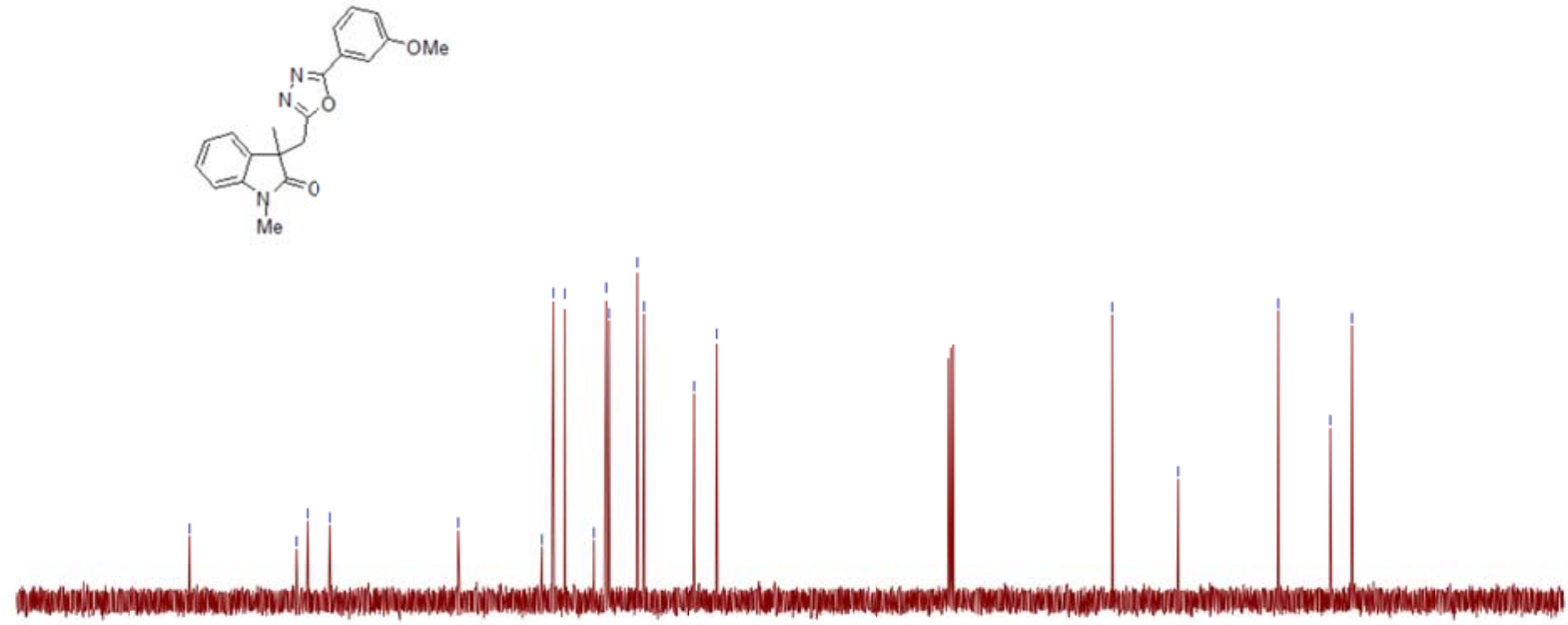

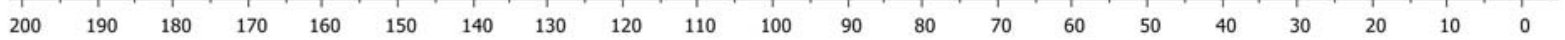


3af

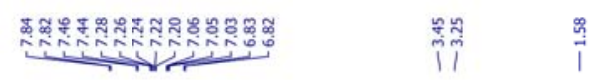
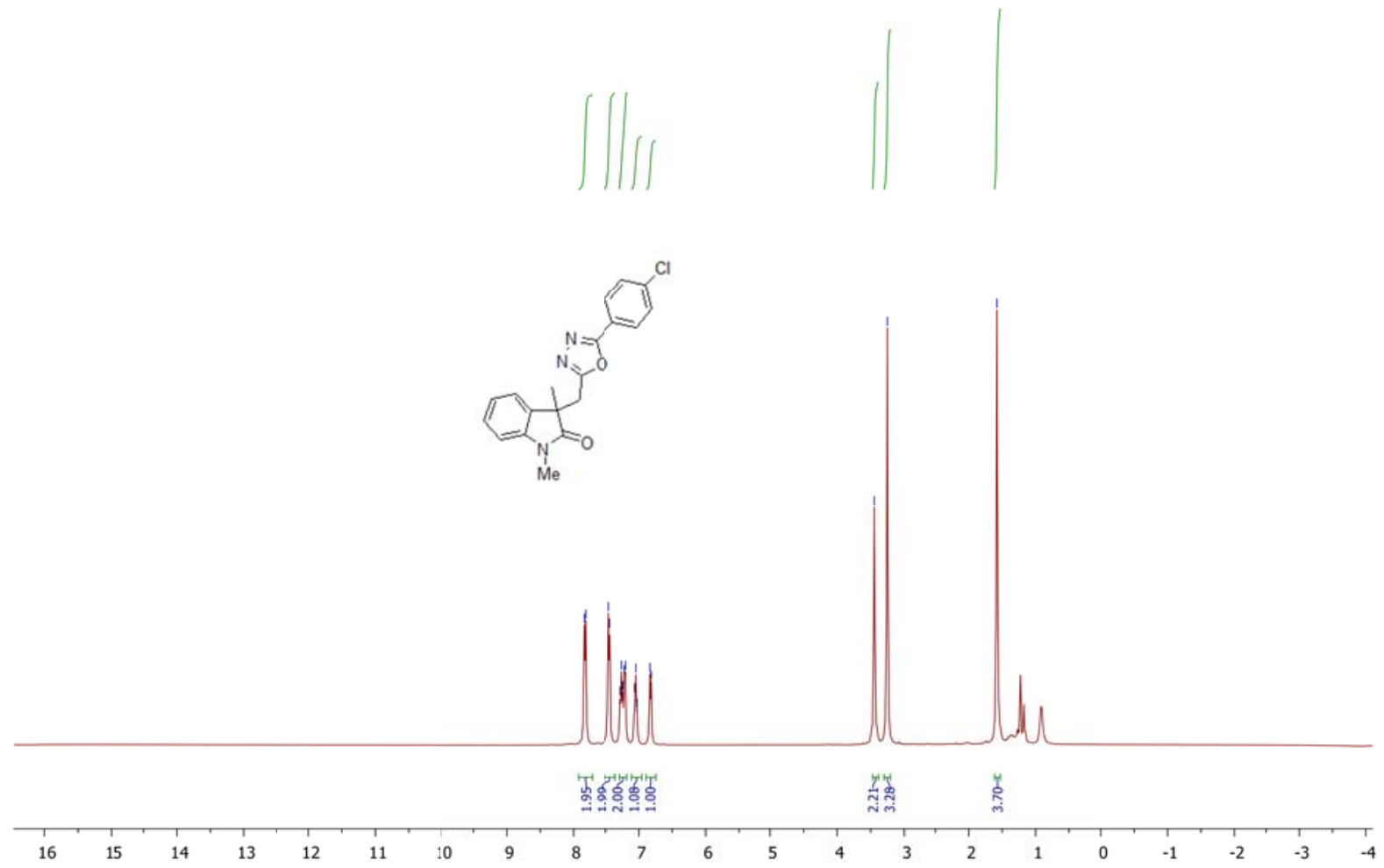

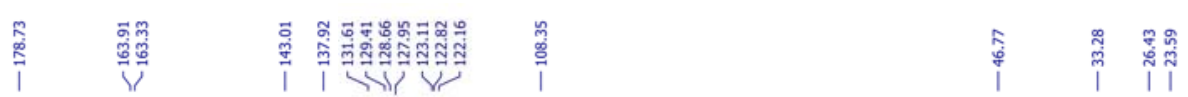
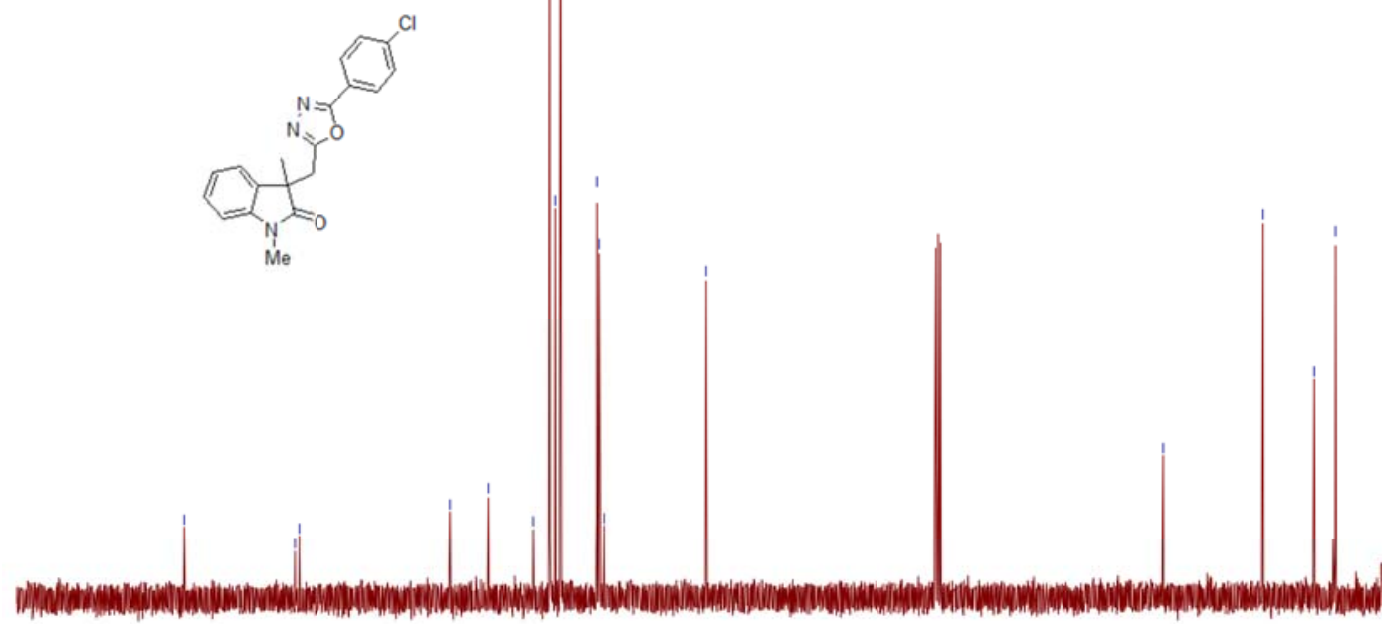

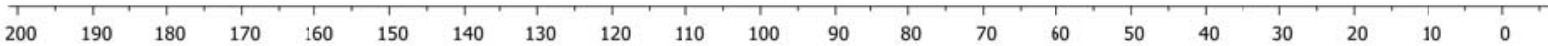


$3 a g$

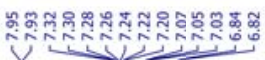

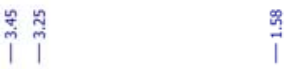

$\int \mid \| \iint$
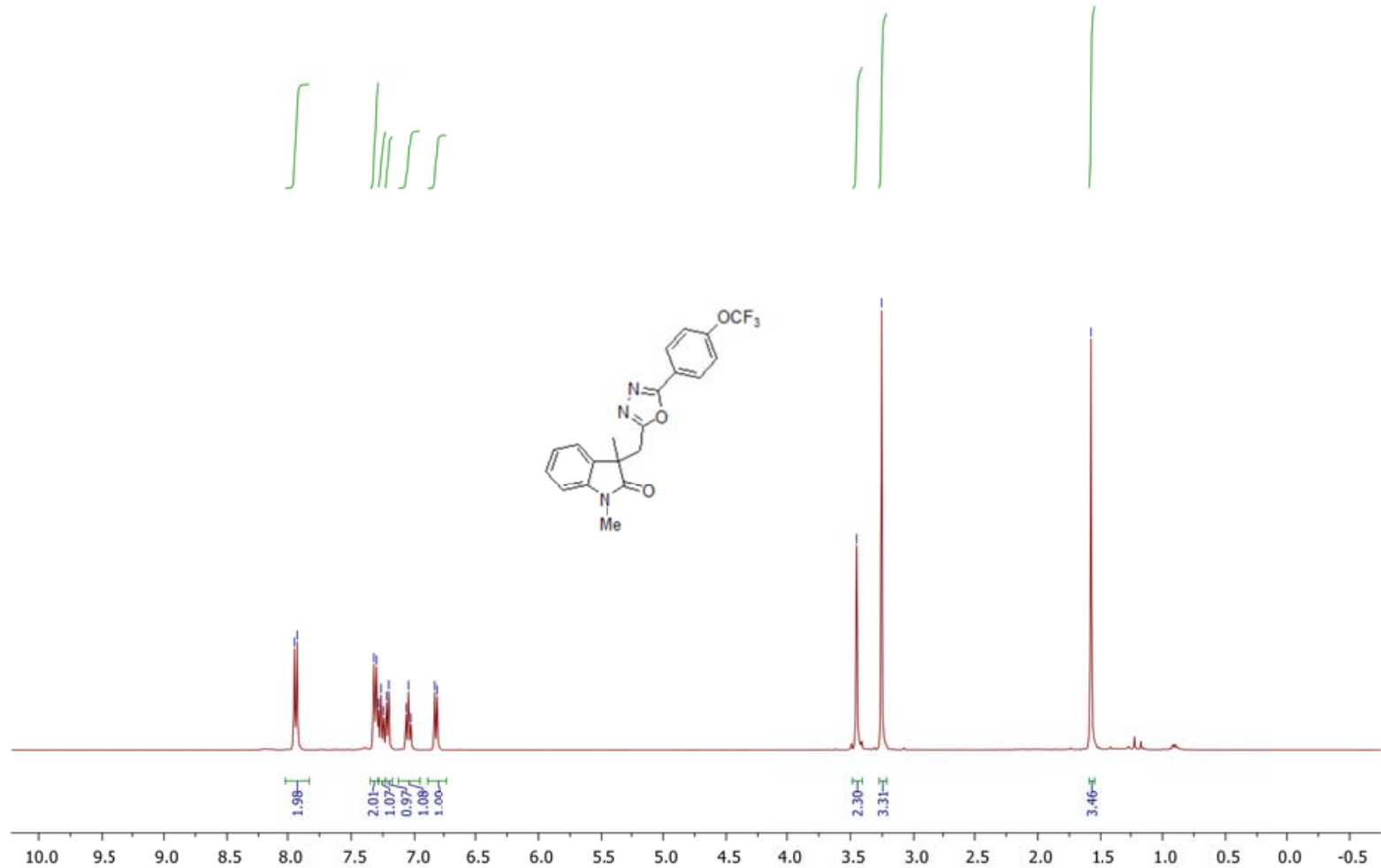

I.

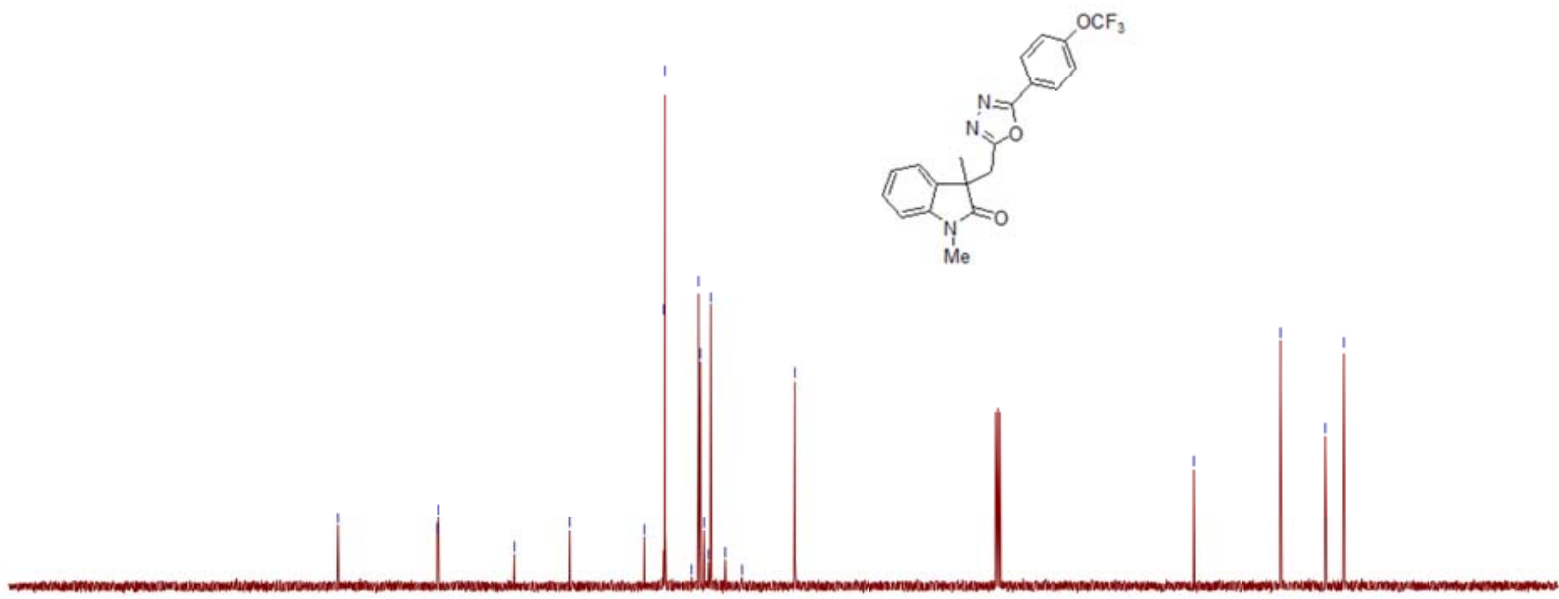

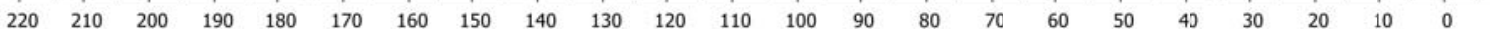


3ah

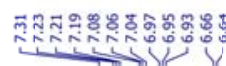

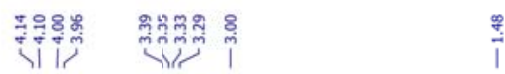
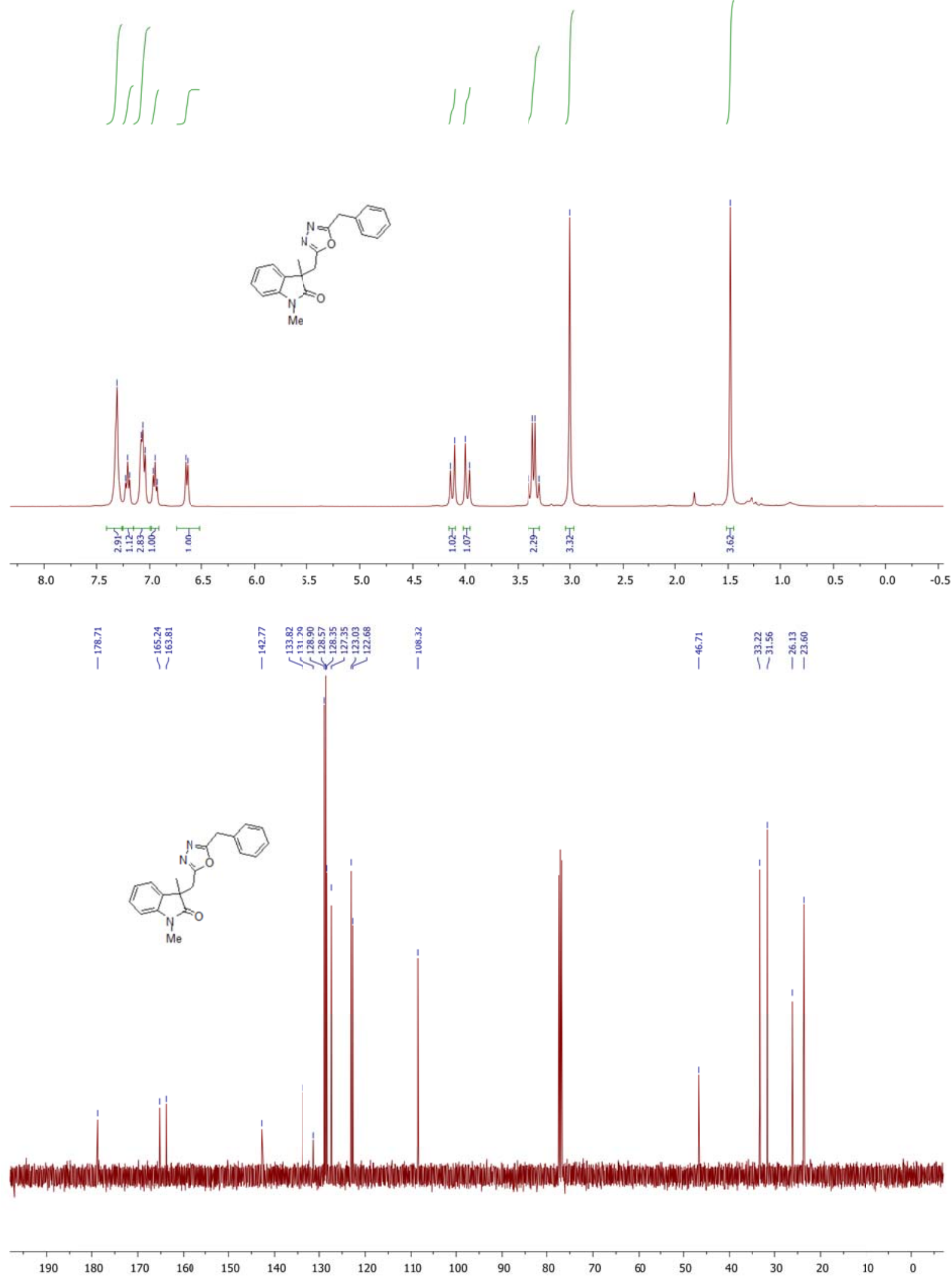

S161 
$3 a \mathbf{i}$

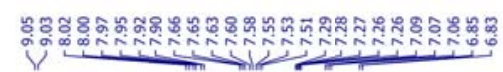

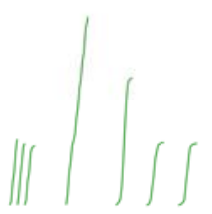

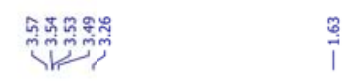

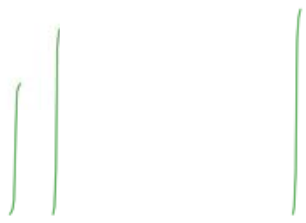

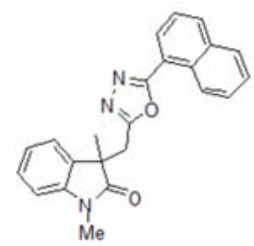

ind

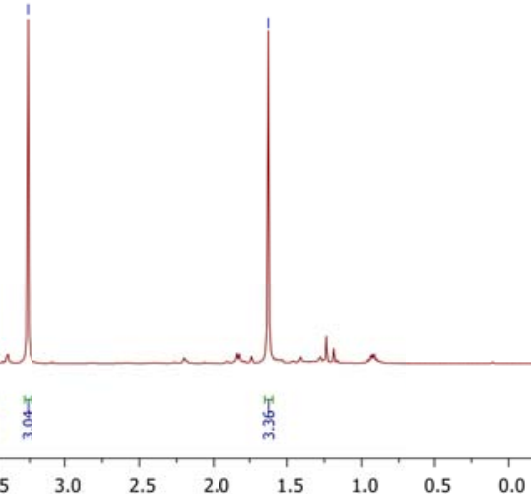

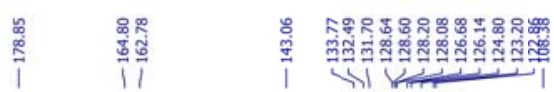

离

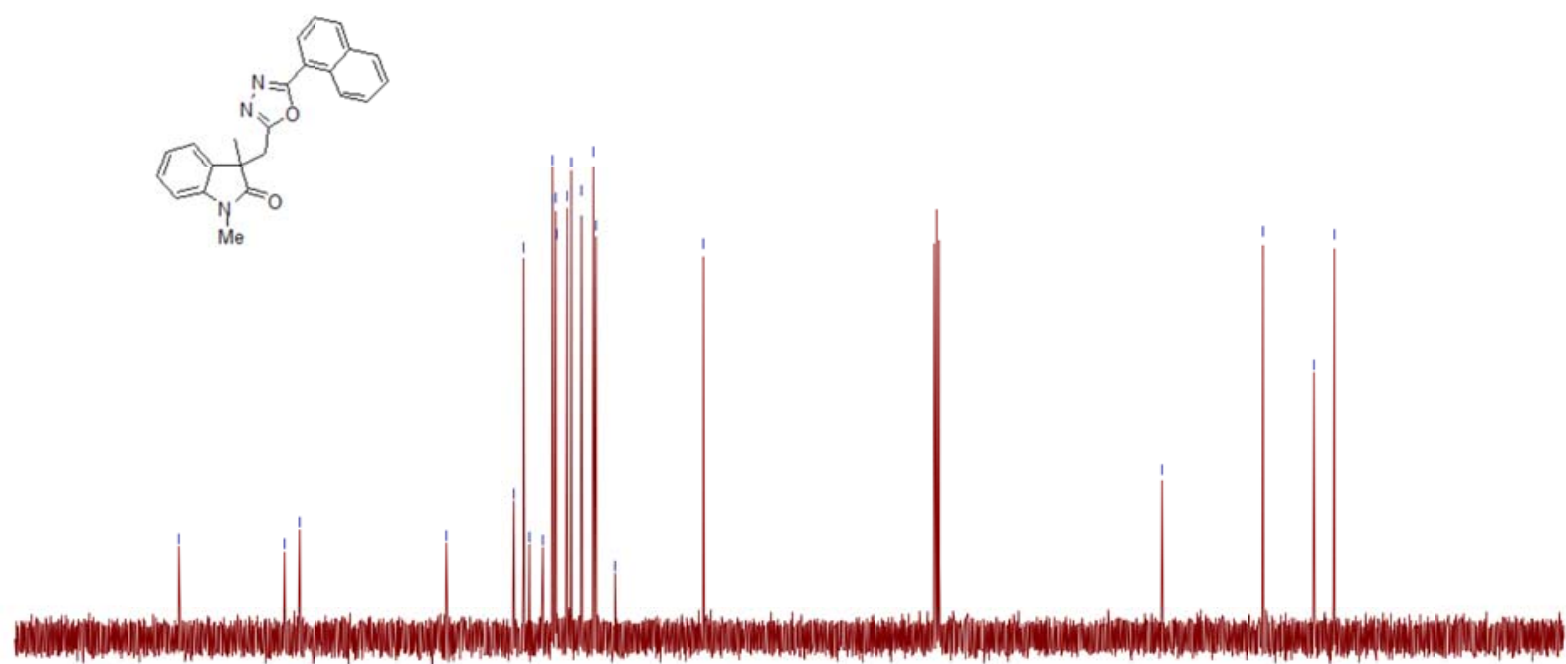

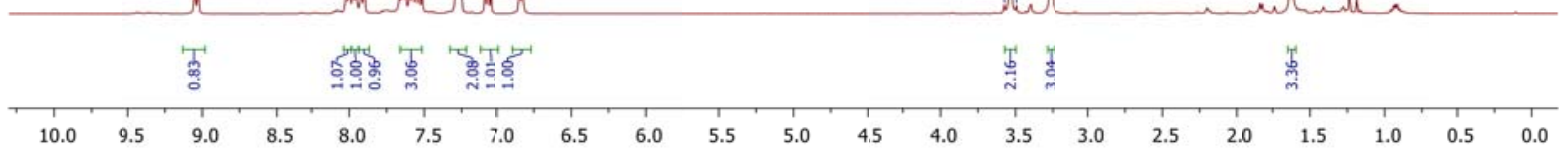

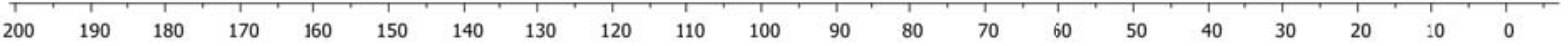


3aj

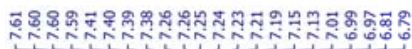

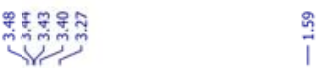
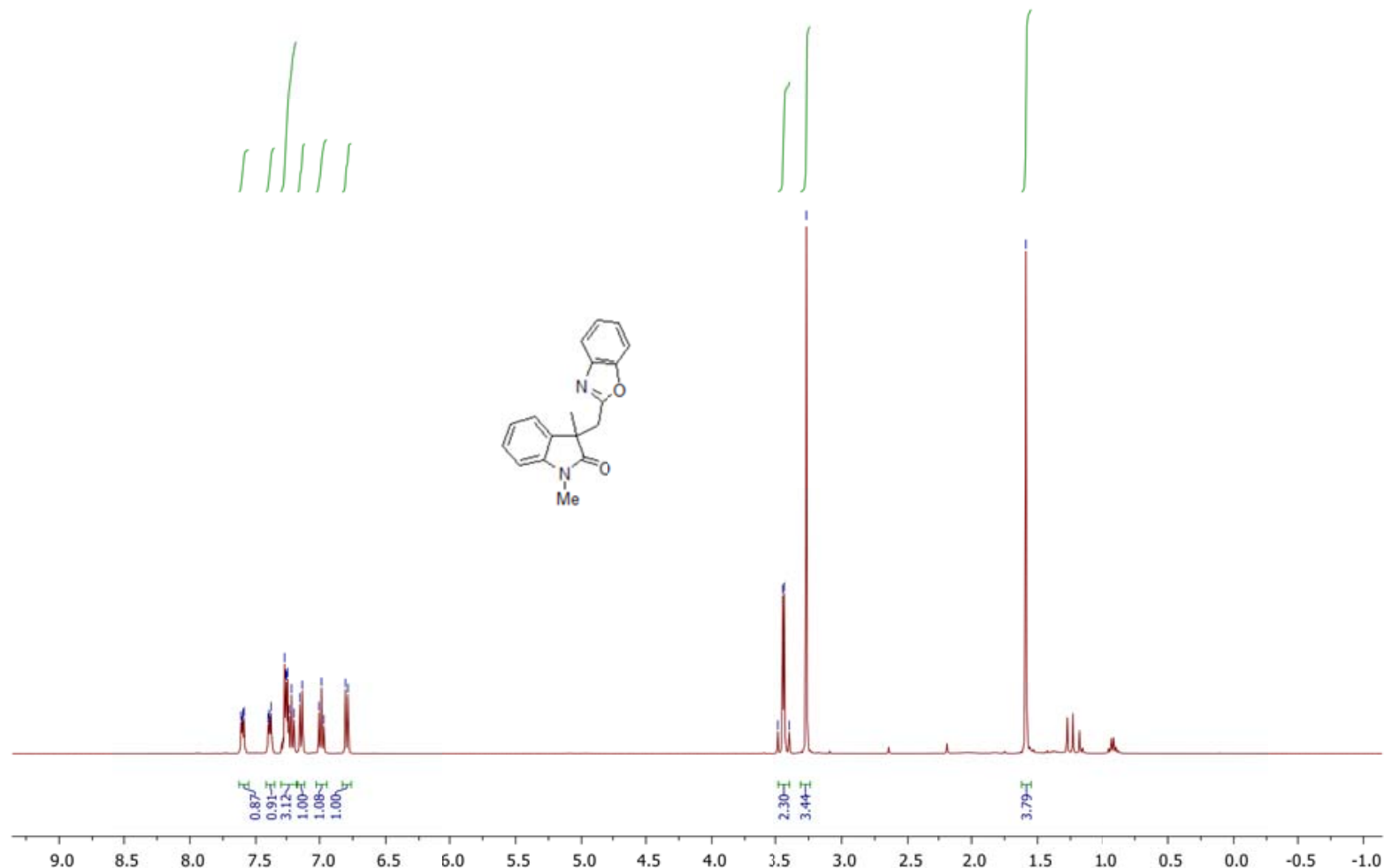

兹
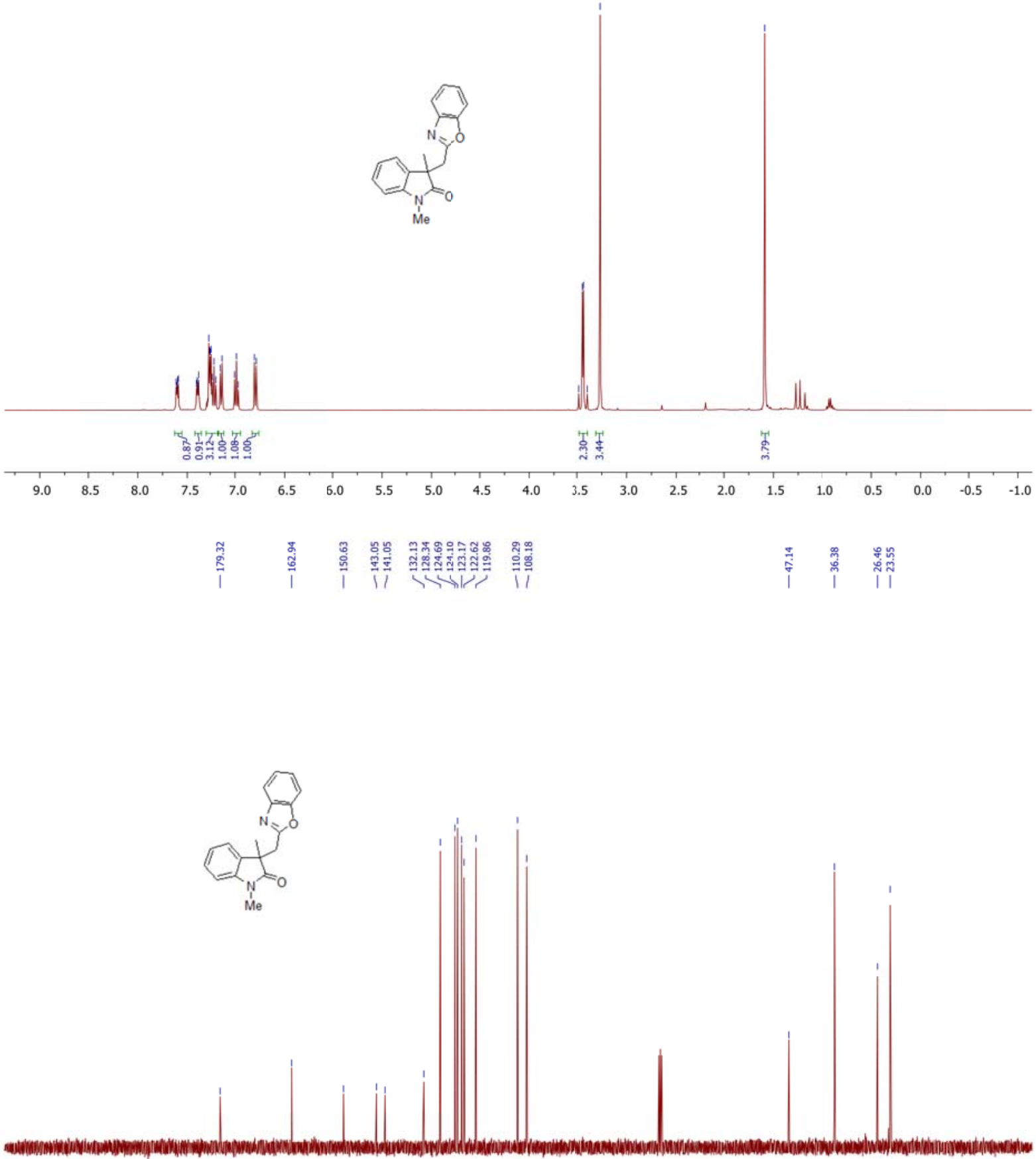

$\begin{array}{lllllllllllllllllllllll}220 & 210 & 200 & 190 & 180 & 170 & 160 & 150 & 140 & 130 & 120 & 110 & 100 & 90 & 80 & 70 & 60 & 50 & 40 & 30 & 20 & 10 & 0\end{array}$ 


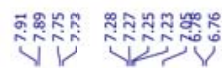

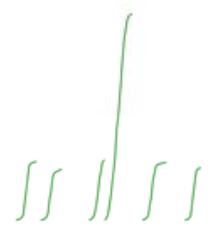

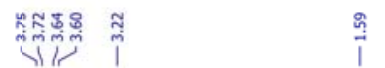
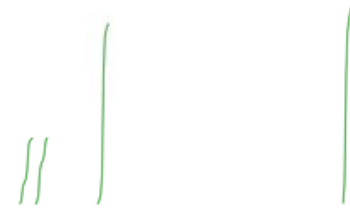

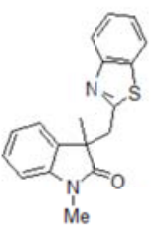

is

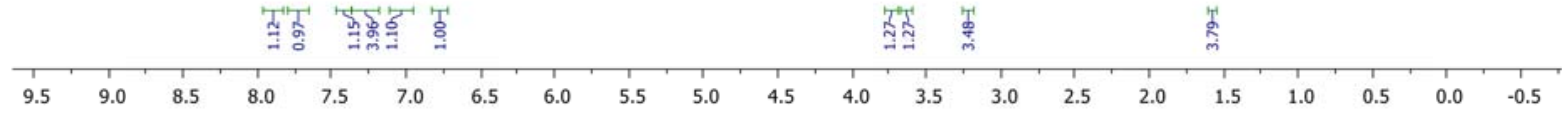

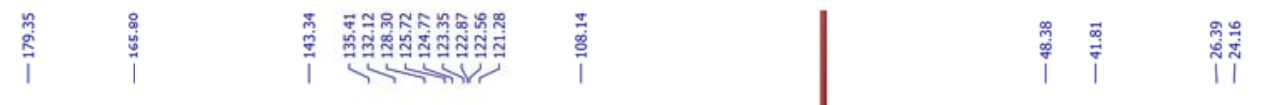
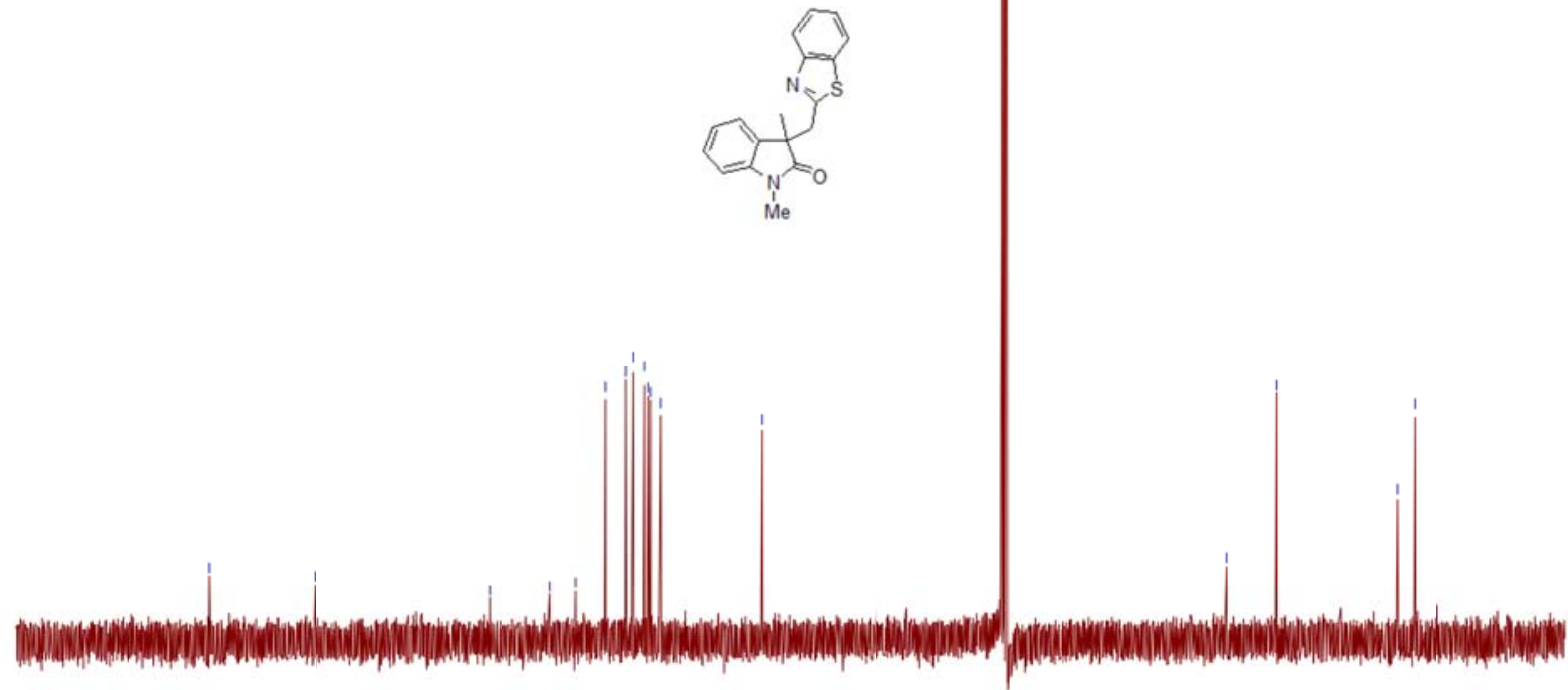

$\begin{array}{llllllllllllllllllll}200 & 190 & 180 & 170 & 160 & 150 & 140 & 130 & 120 & 110 & 100 & 90 & 80 & 70 & 60 & 50 & 40 & 30 & 20 & 10\end{array}$ 
3da
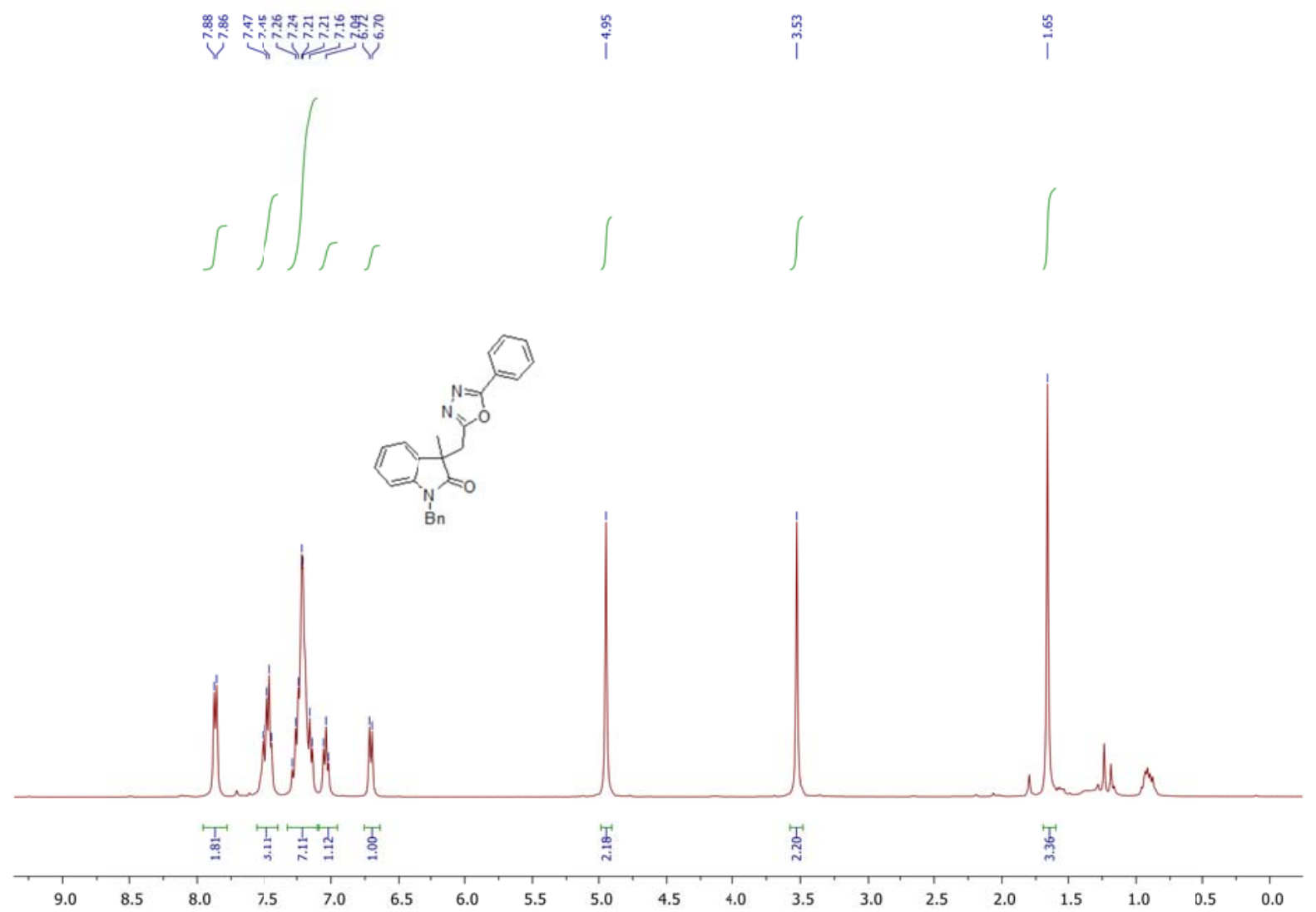

(1)

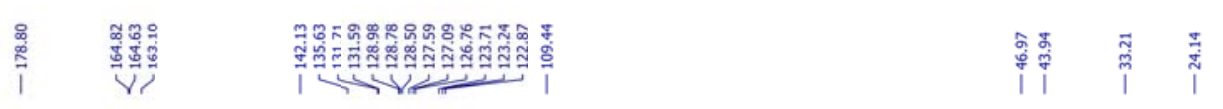<smiles>O=C(Cc1nnc(-c2ccccc2)o1)c1ccccc1</smiles>

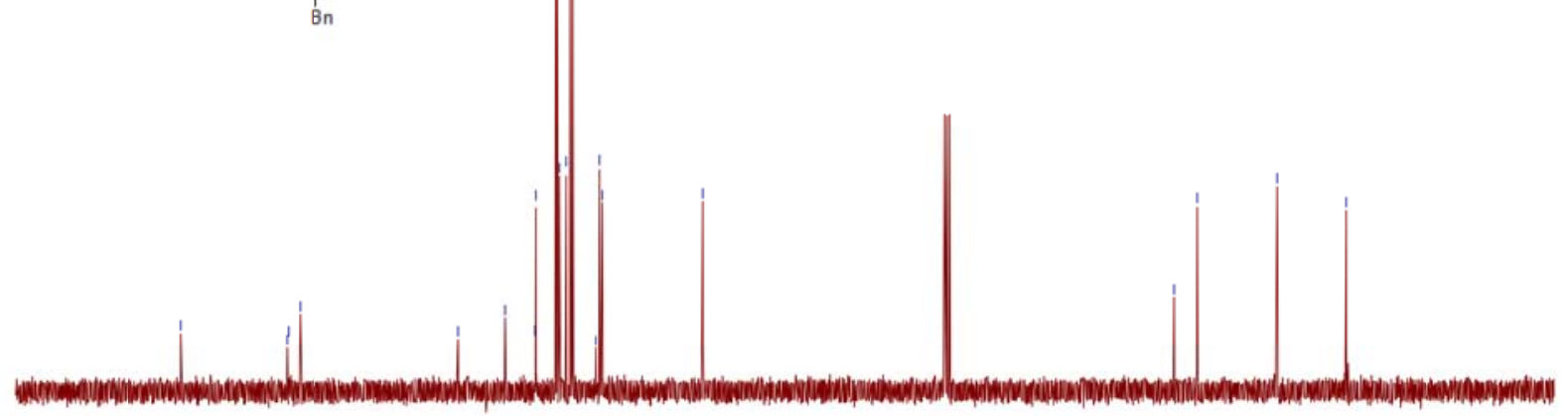

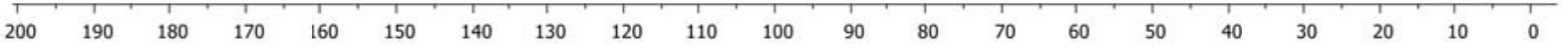


3gd

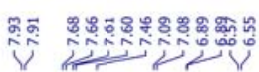

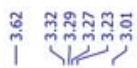

\|\| $1 / 1 \int_{1}$
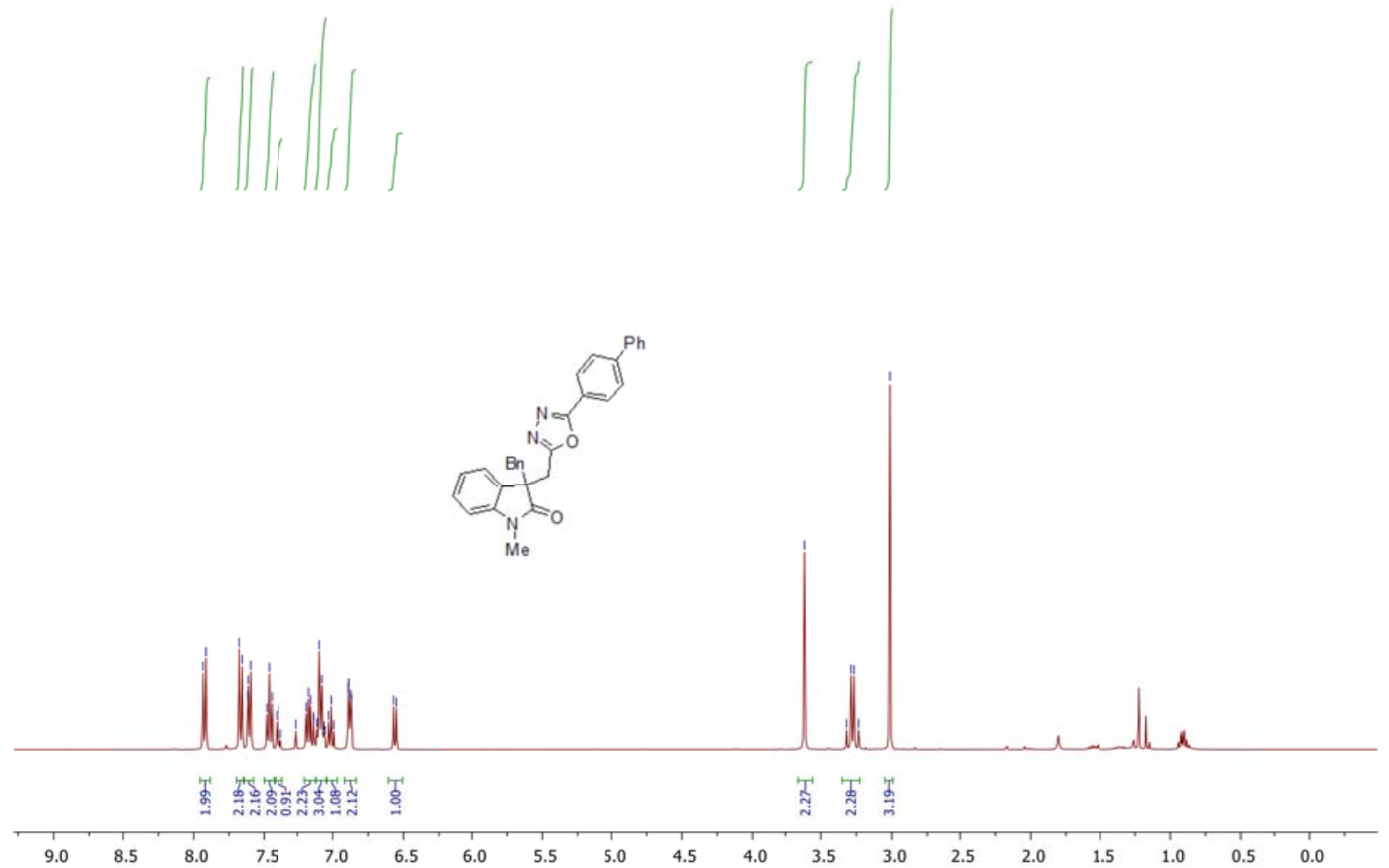

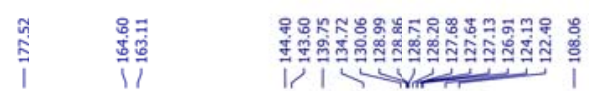

芯

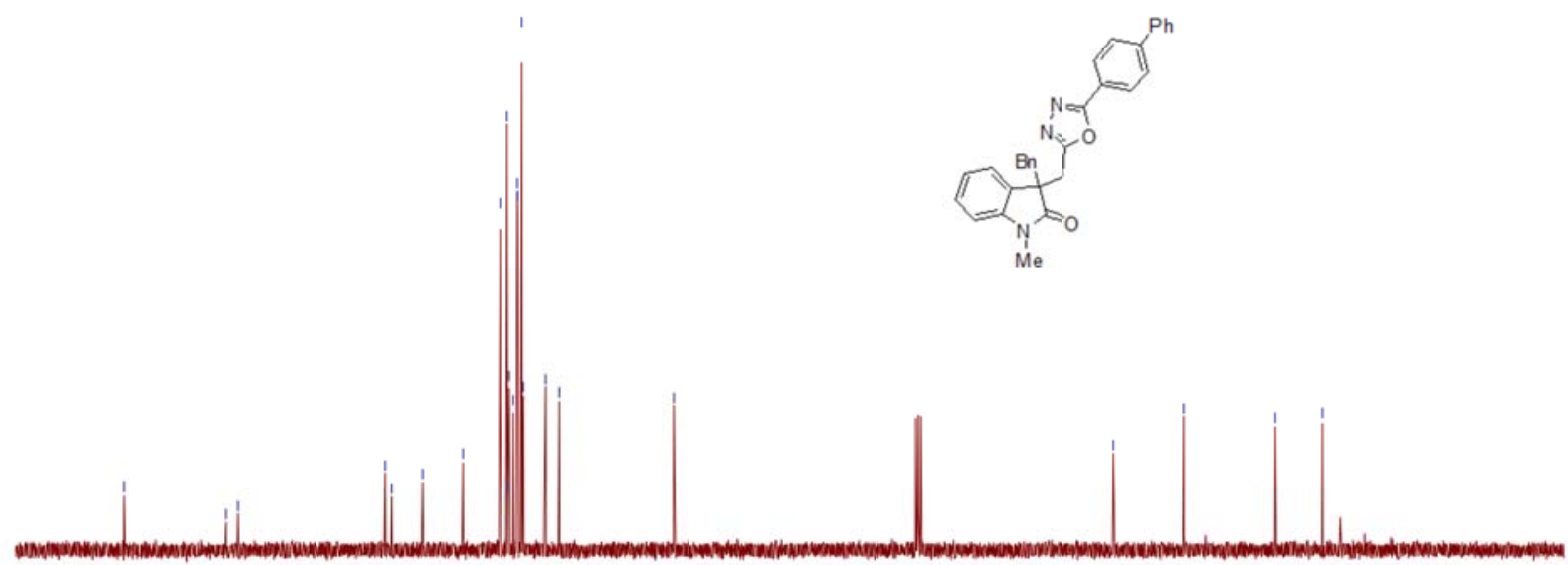

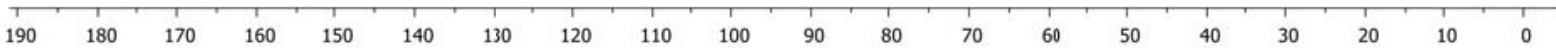



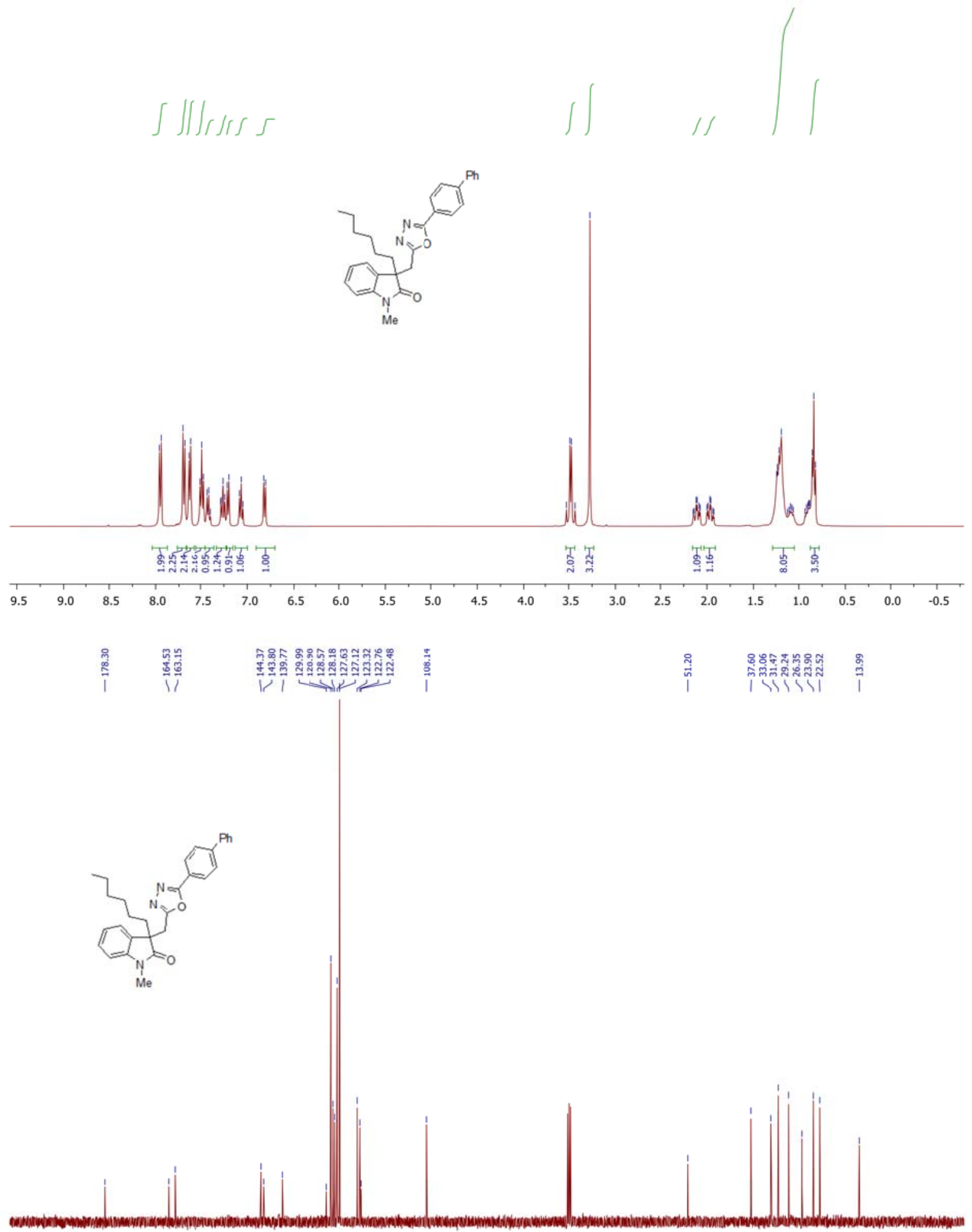

if

|रा।डर

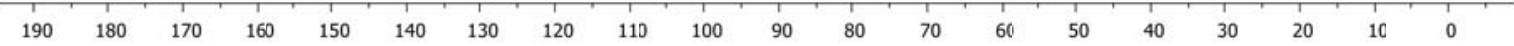


3jd

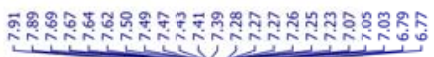

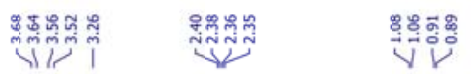

$\int \| \int / \iint$
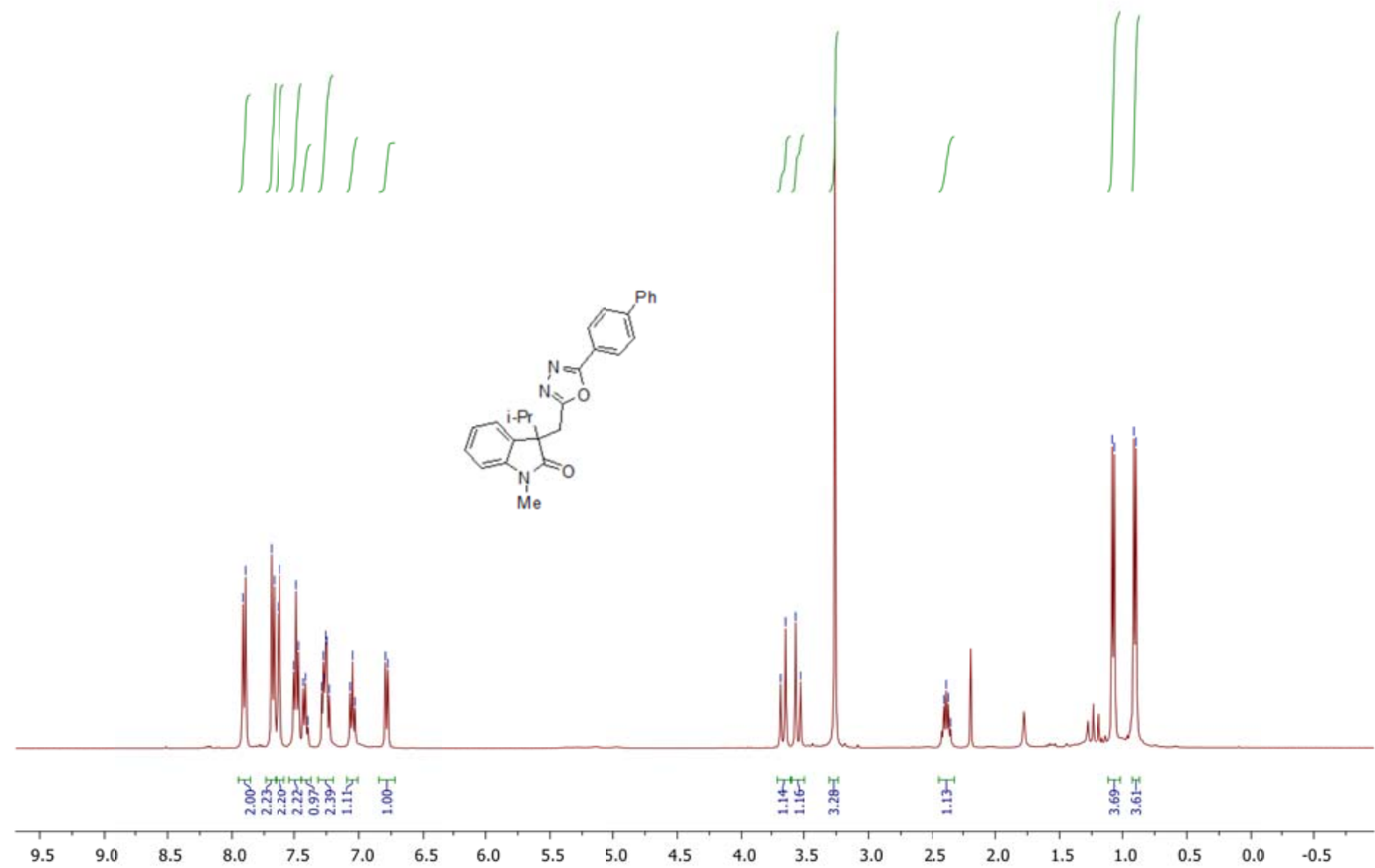

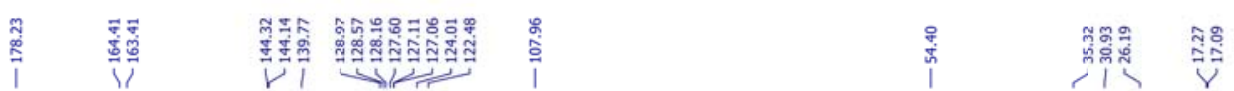

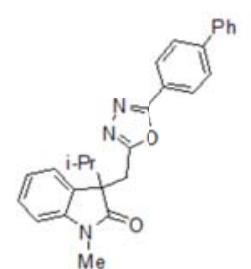

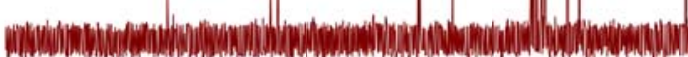

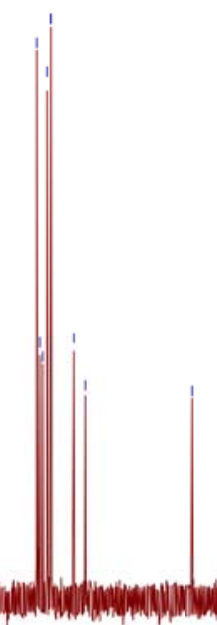

$190 \quad 180$

$170 \quad 160$

$\begin{array}{llll}130 & 120 & 110 & 100\end{array}$

$90 \quad 80$

$\begin{array}{lll}70 \quad 60 \quad 50 & 0\end{array}$

30 


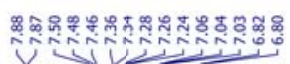

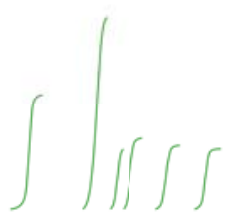

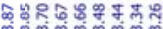

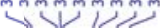
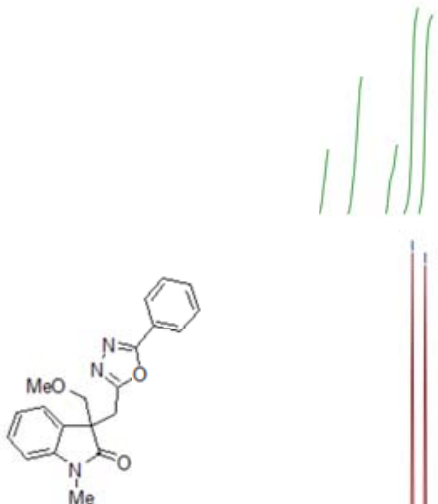

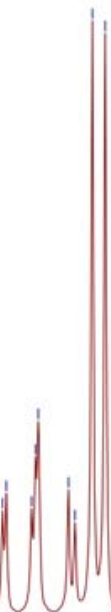

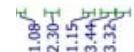

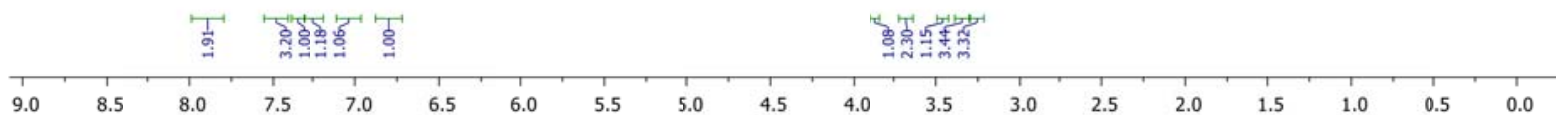

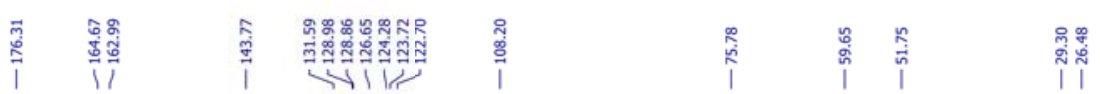<smiles></smiles>

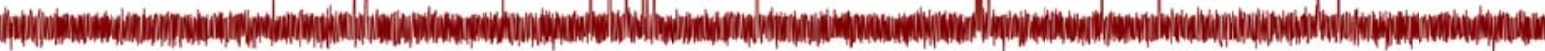

$\begin{array}{llllllllllllllllllllll}210 & 200 & 190 & 180 & 170 & 160 & 150 & 140 & 130 & 120 & 110 & 100 & 90 & 80 & 70 & 60 & 50 & 40 & 30 & 20 & 10 & 0\end{array}$


$3 k a$

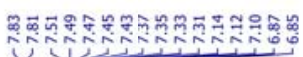

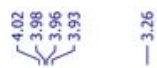
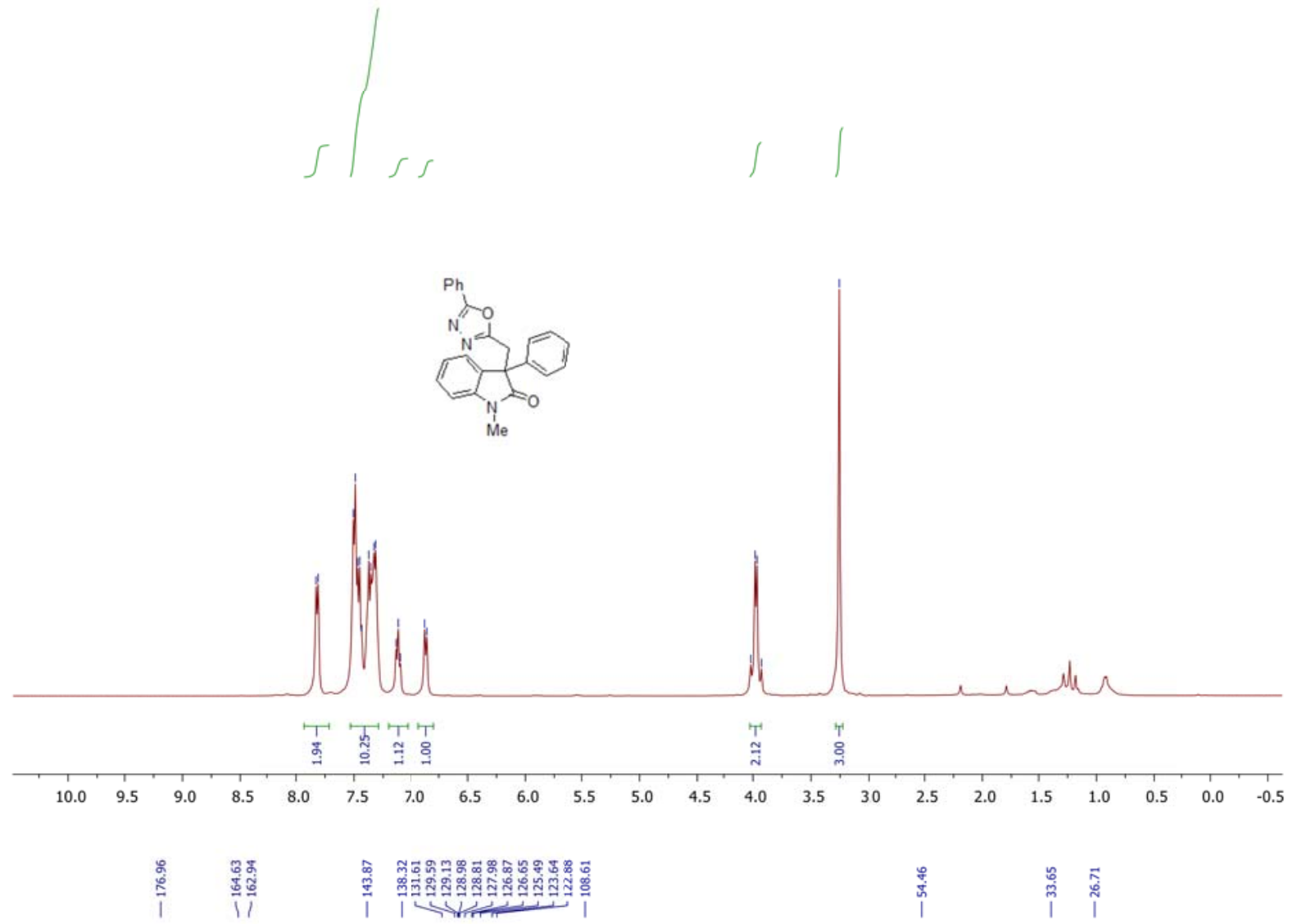

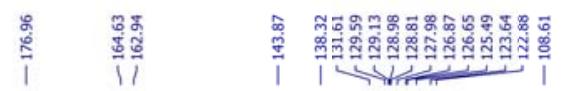

।
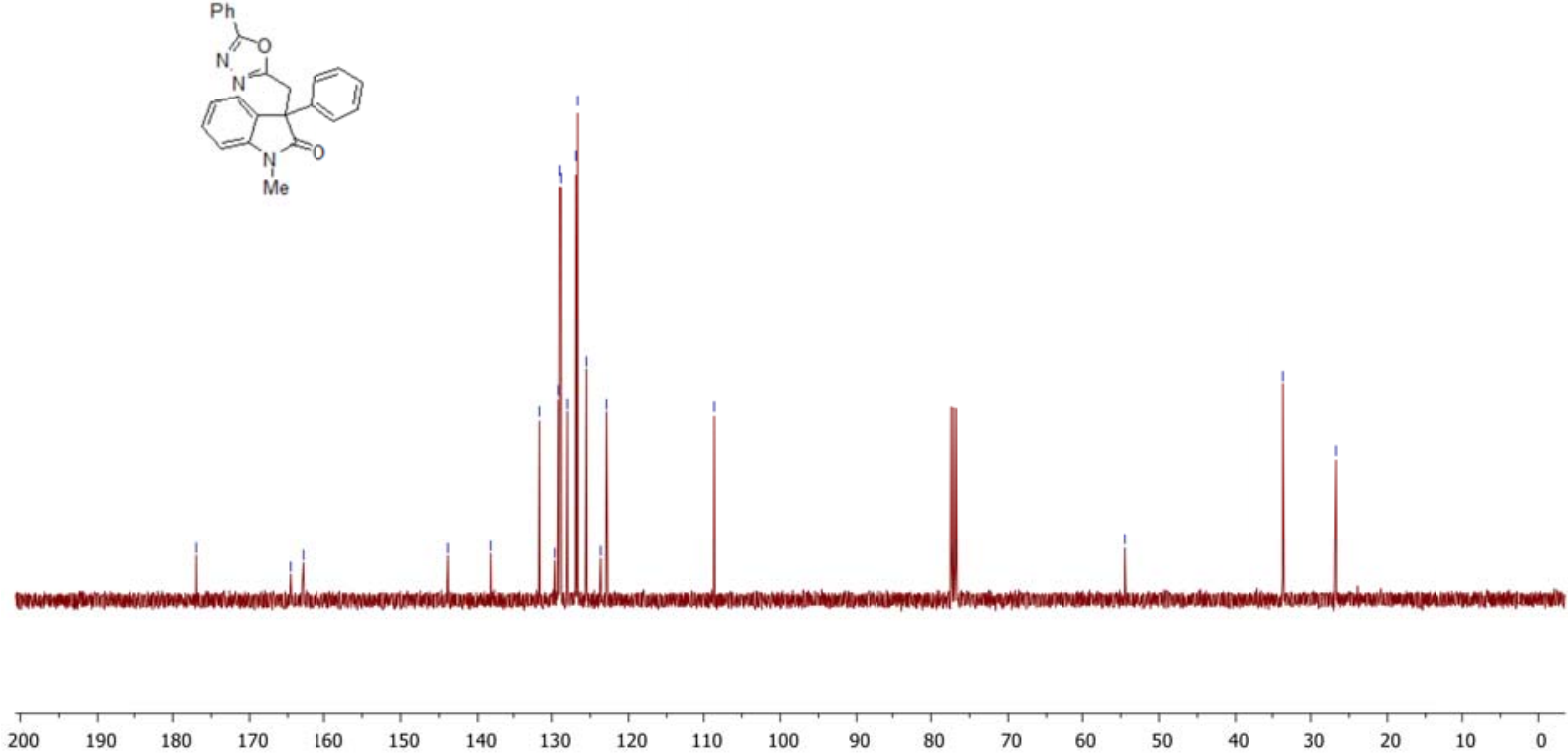

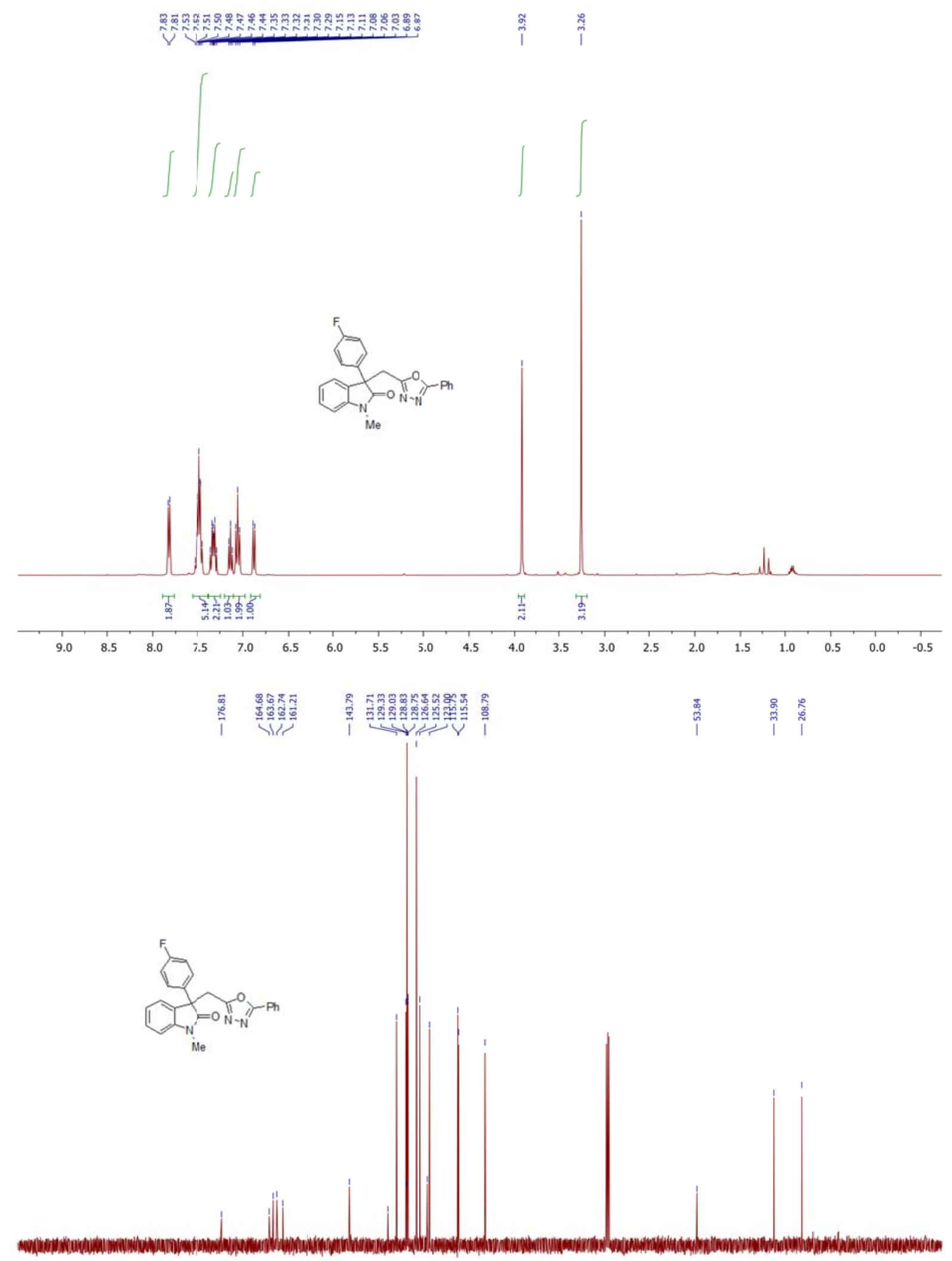

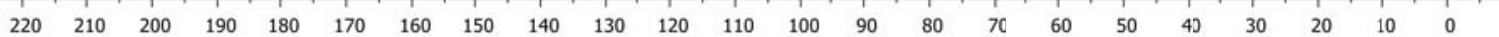




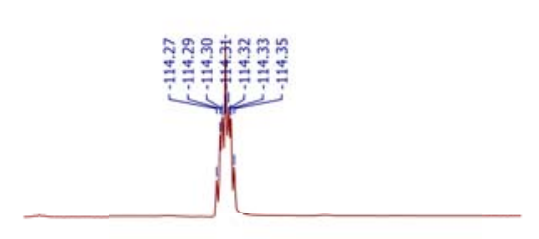

고ำำㄱำำำ

过计

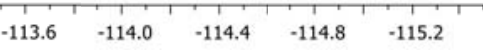

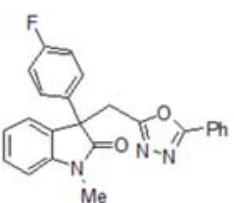

Me

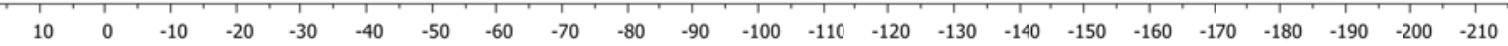

\section{3ma}

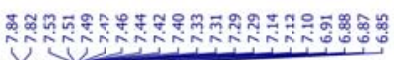

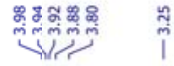
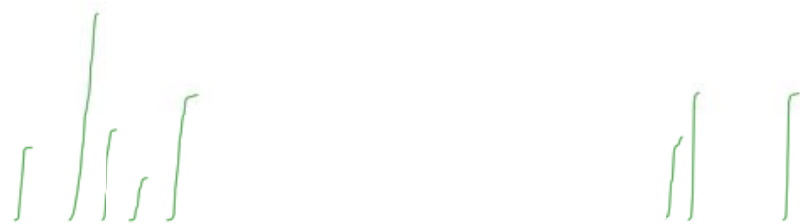

$\mathrm{MeO}$
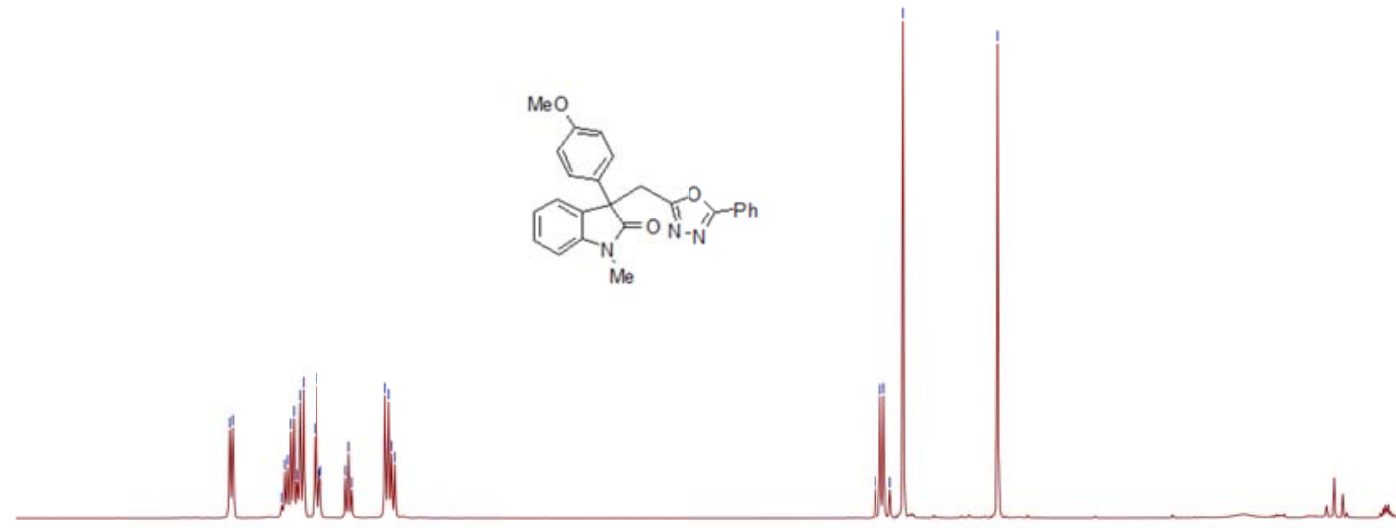

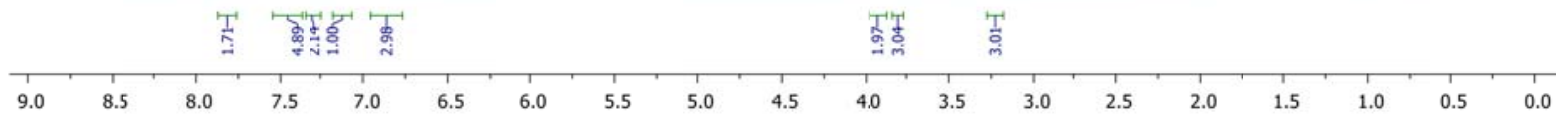




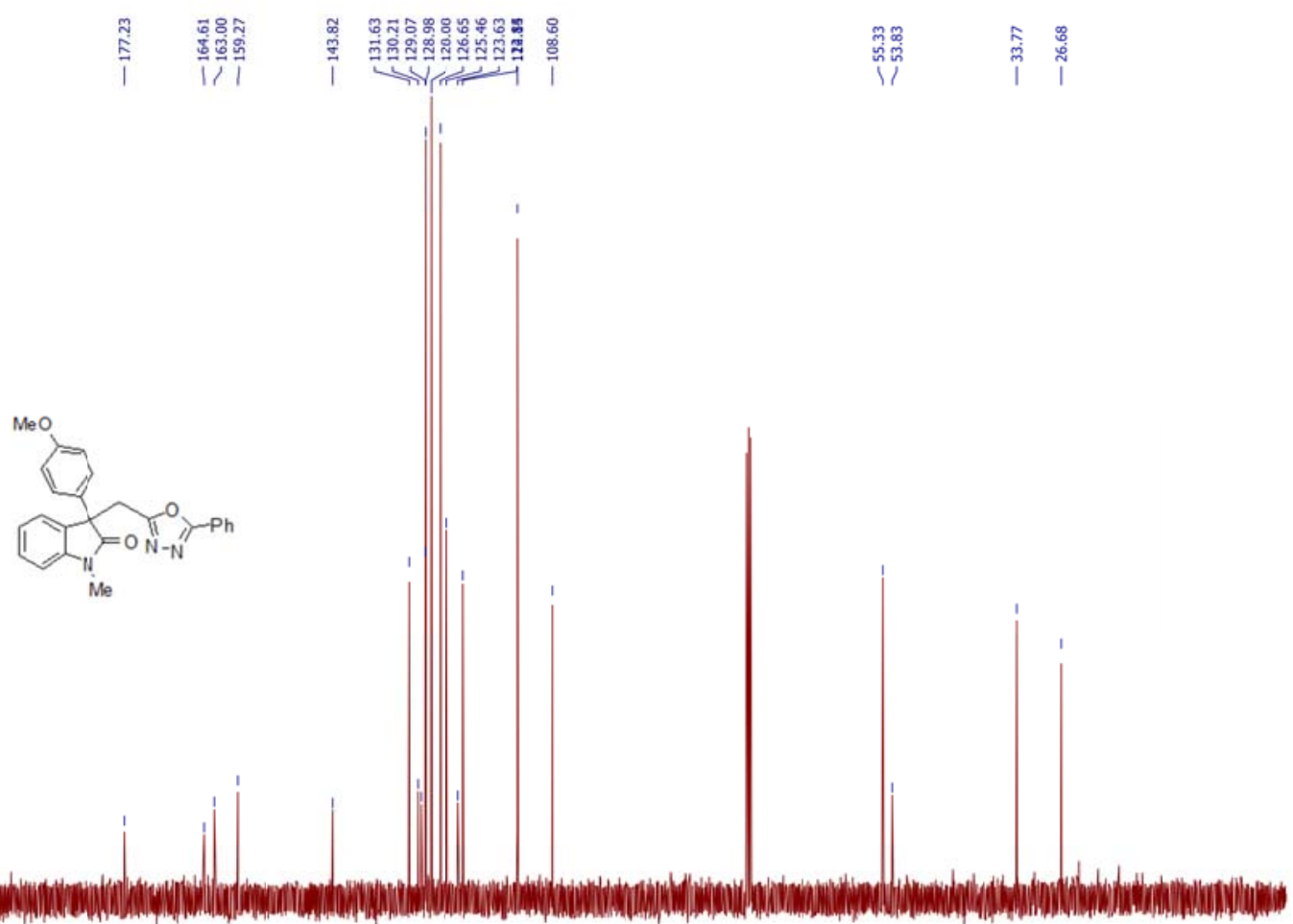

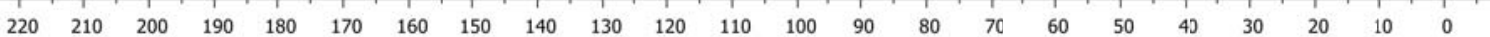

3nd

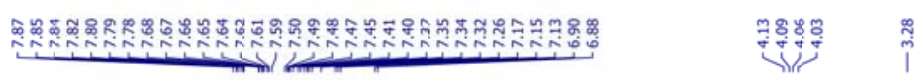
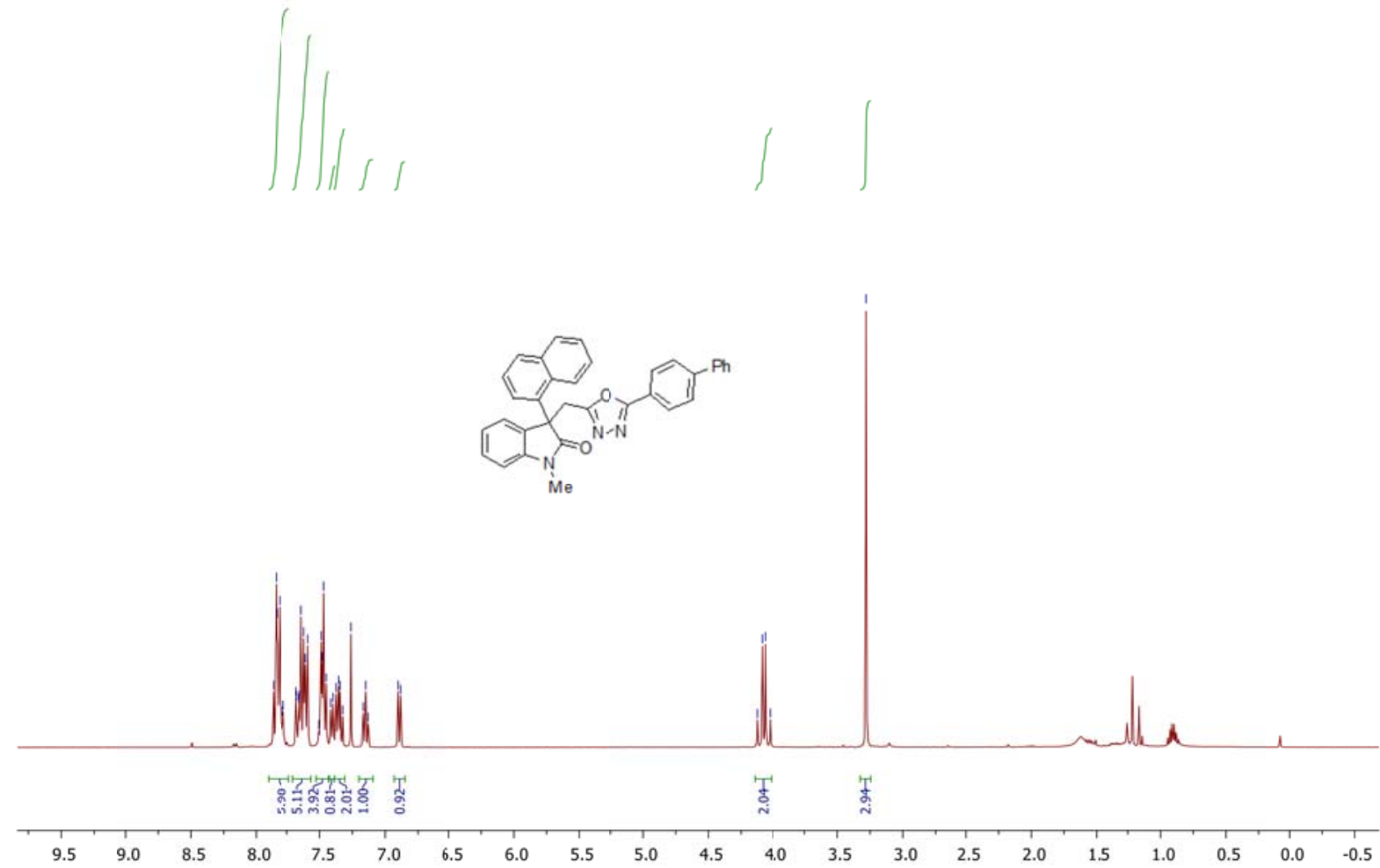

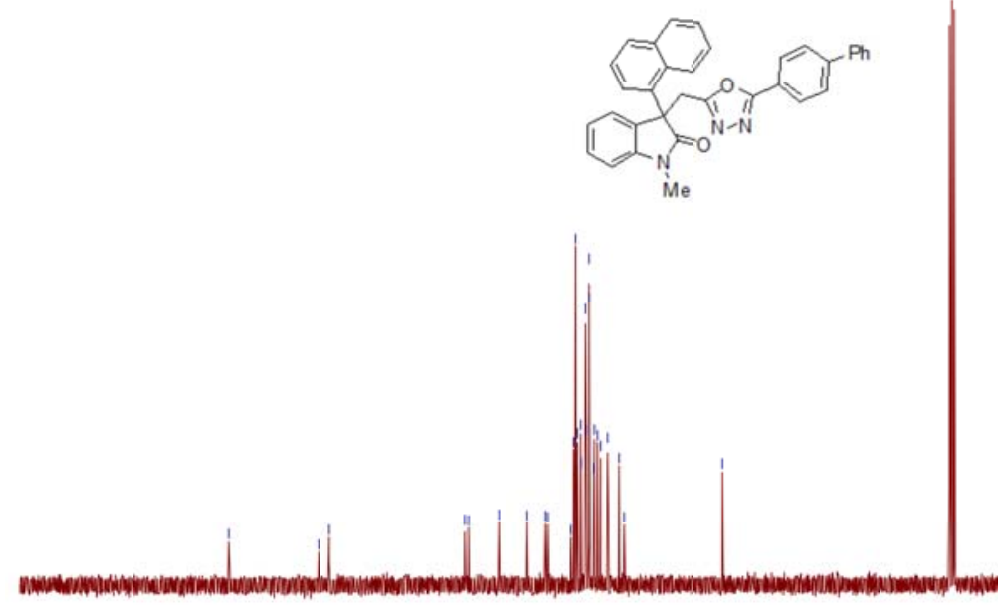

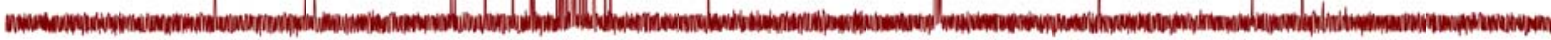

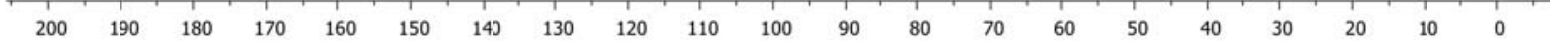

3od

ฟ

$\iiint \iint$

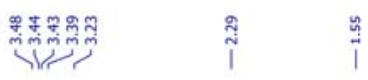
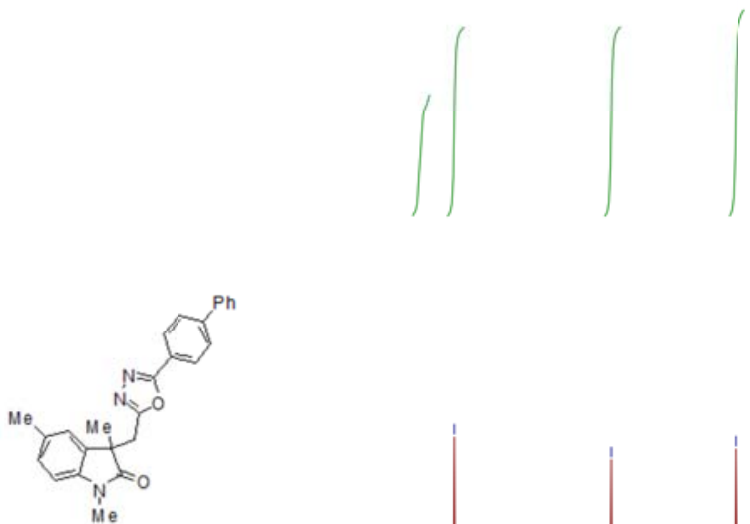
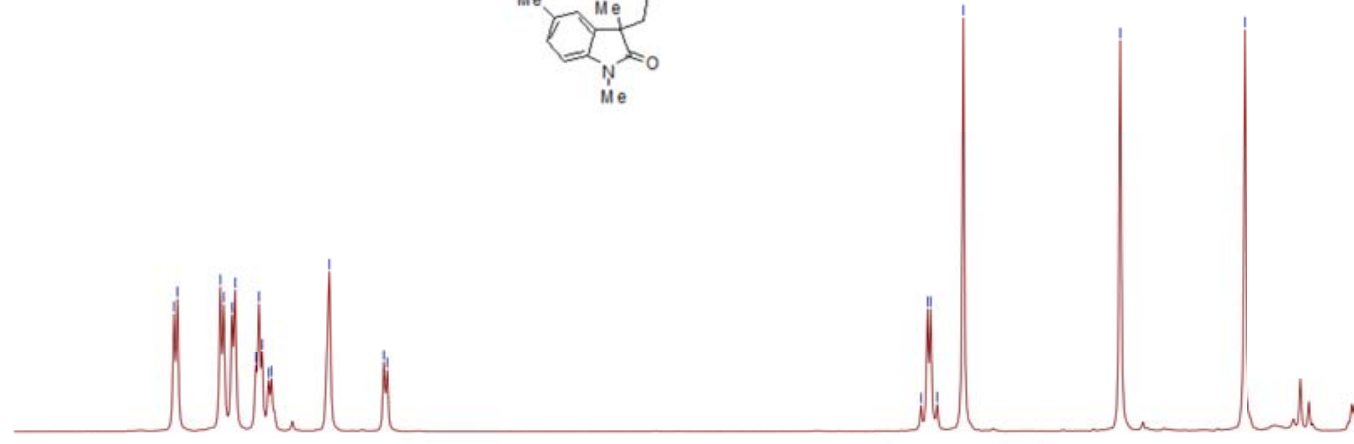

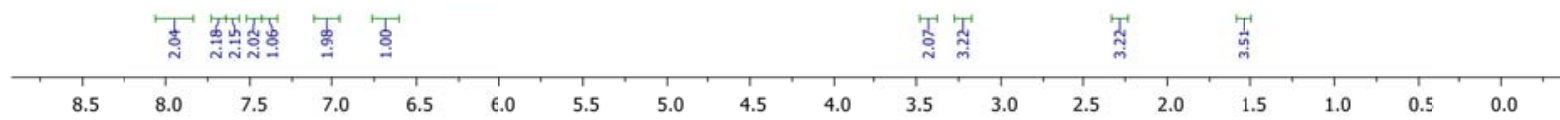




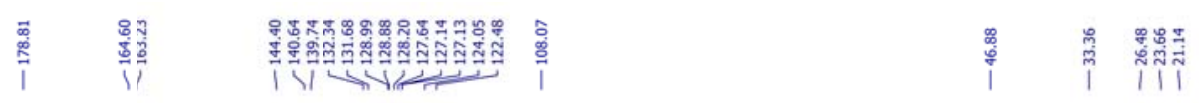
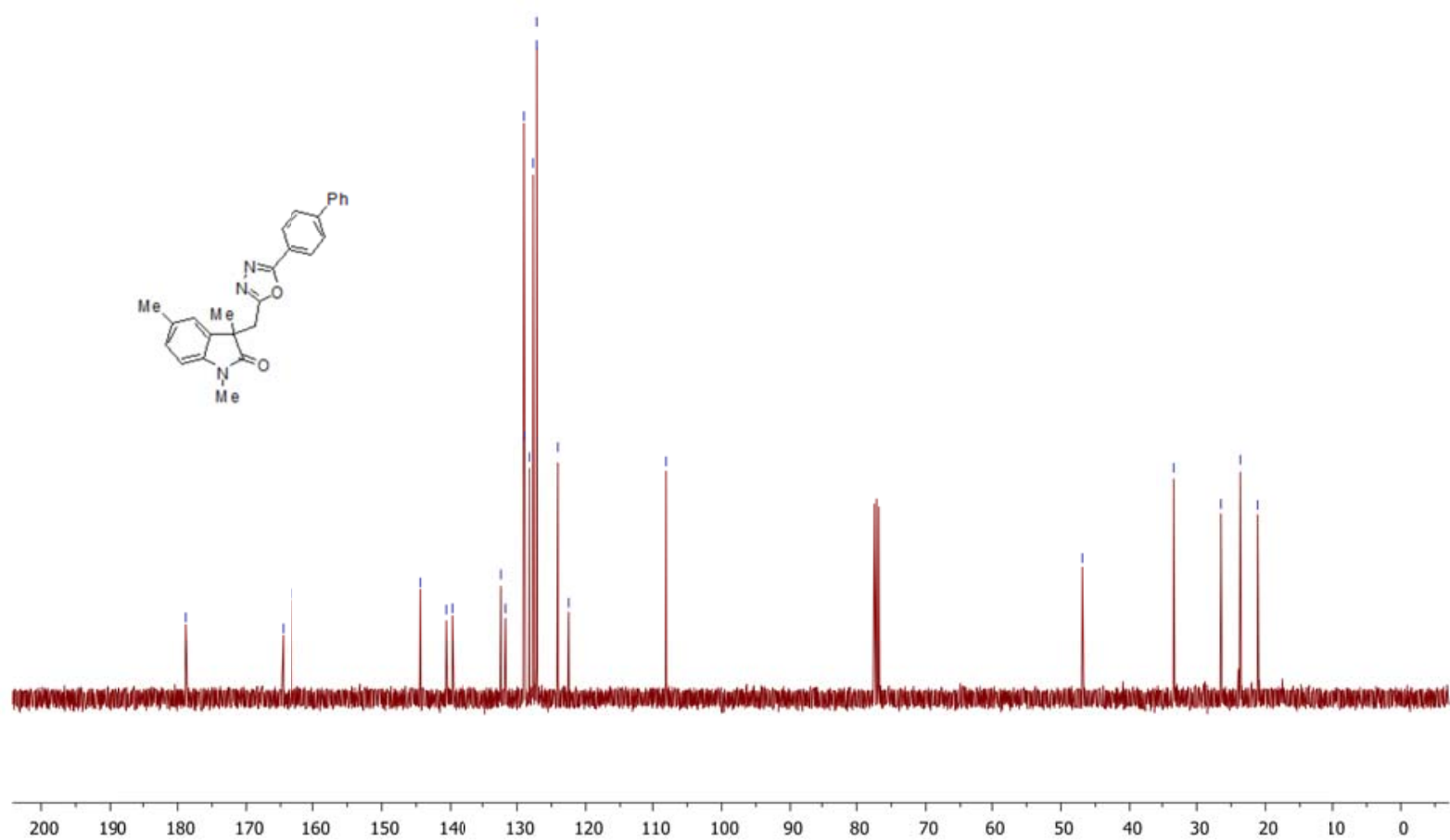

3pd

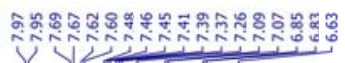

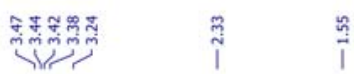

$\int\|\| \iiint$
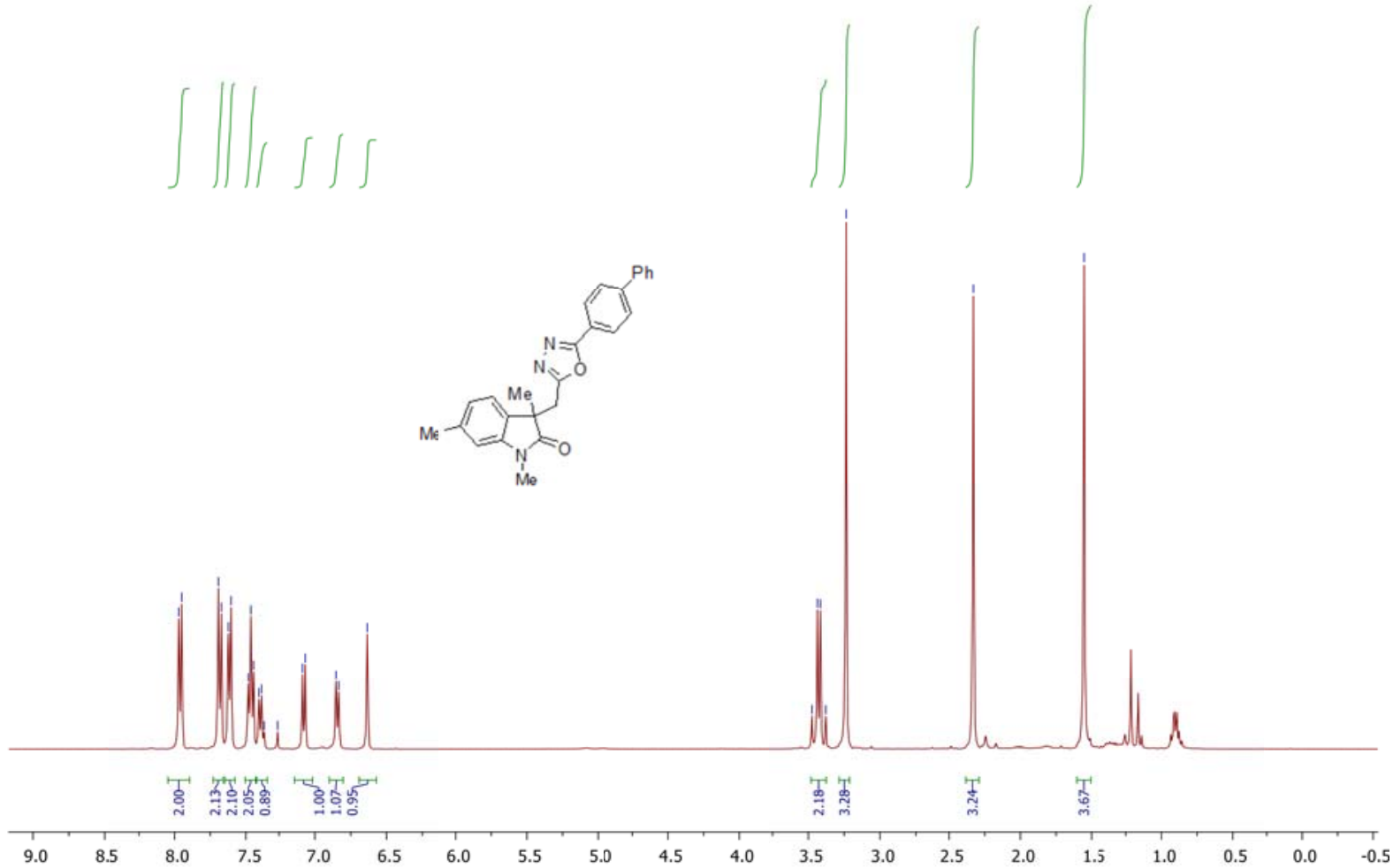


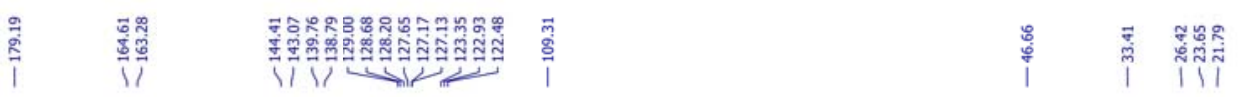

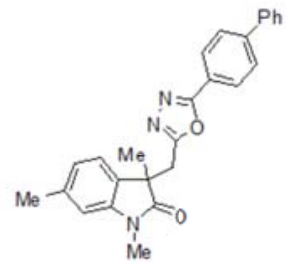

\begin{tabular}{llllllllllllllllllllllll}
\hline 190 & 180 & 170 & 160 & 150 & 140 & 130 & 120 & 110 & 100 & 90 & 80 & 70 & 60 & 50 & 40 & 30 & 20 & 10 & 0
\end{tabular}

3qd

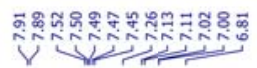

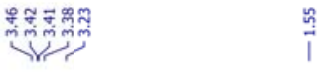
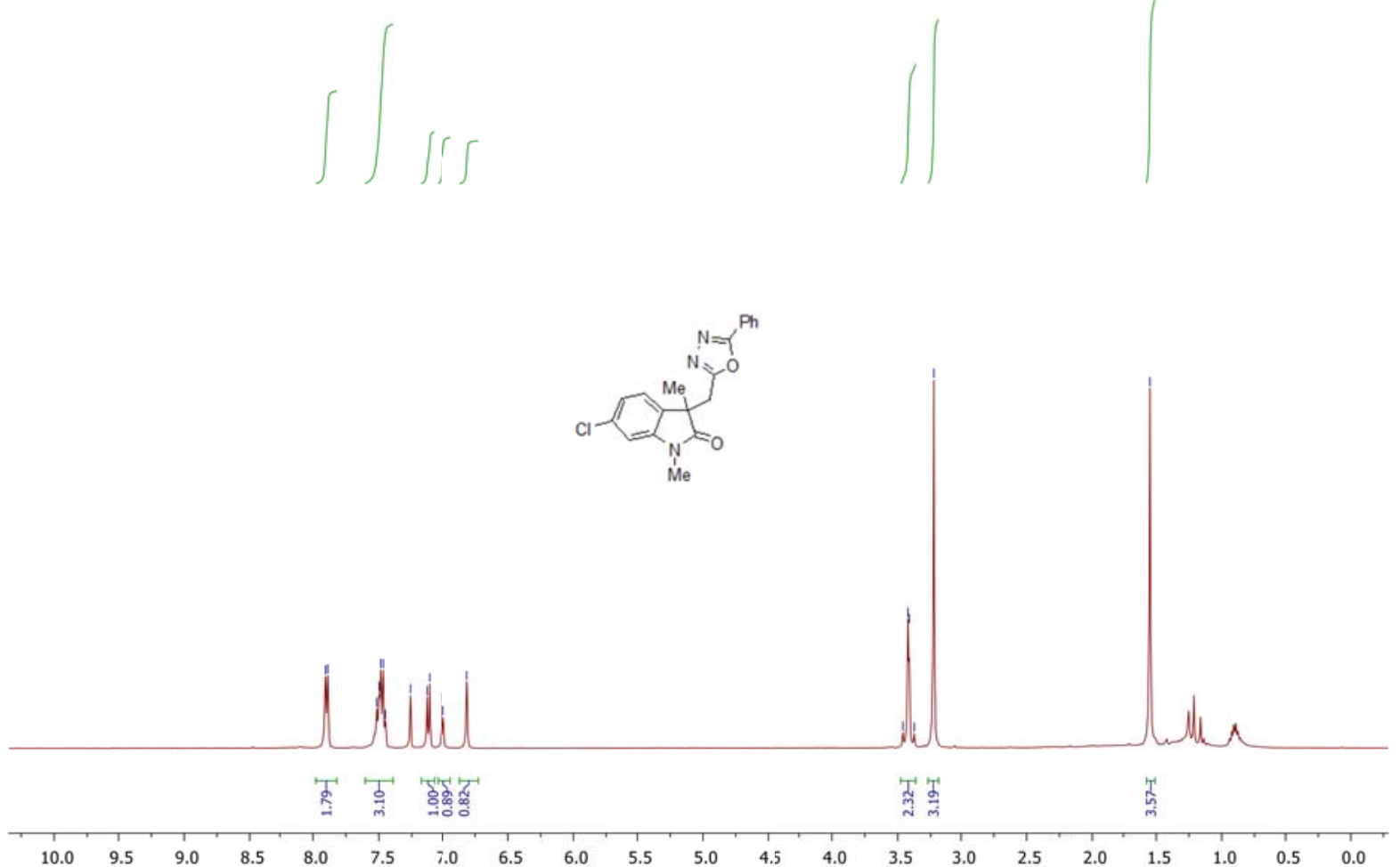


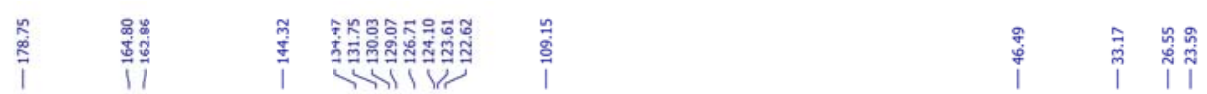
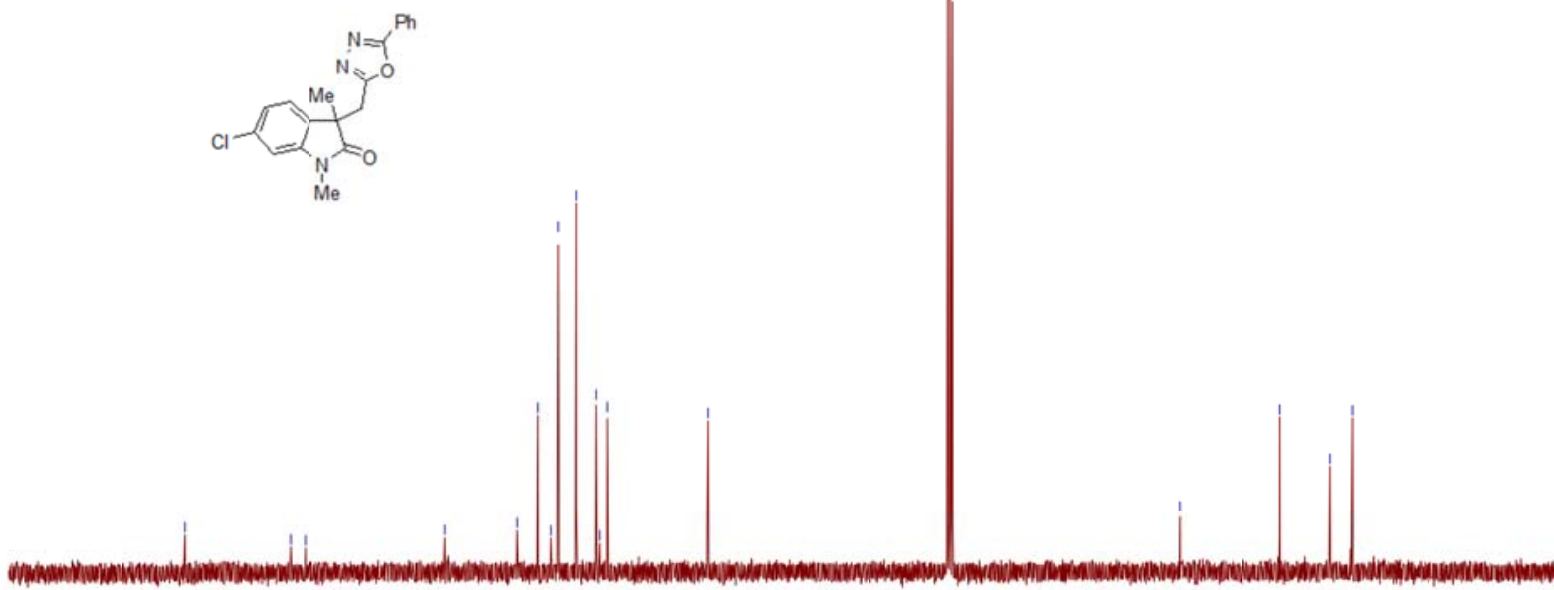

$\begin{array}{llllllllllllllllllllllllll} & 200 & 190 & 180 & 170 & 160 & 150 & 140 & 130 & 120 & 110 & 100 & 90 & 80 & 70 & 60 & 50 & 40 & 30 & 20 & 10 & 0\end{array}$

3rd

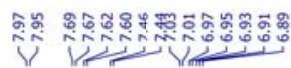

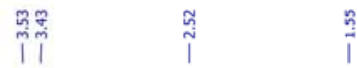

$\int\|\| \|$
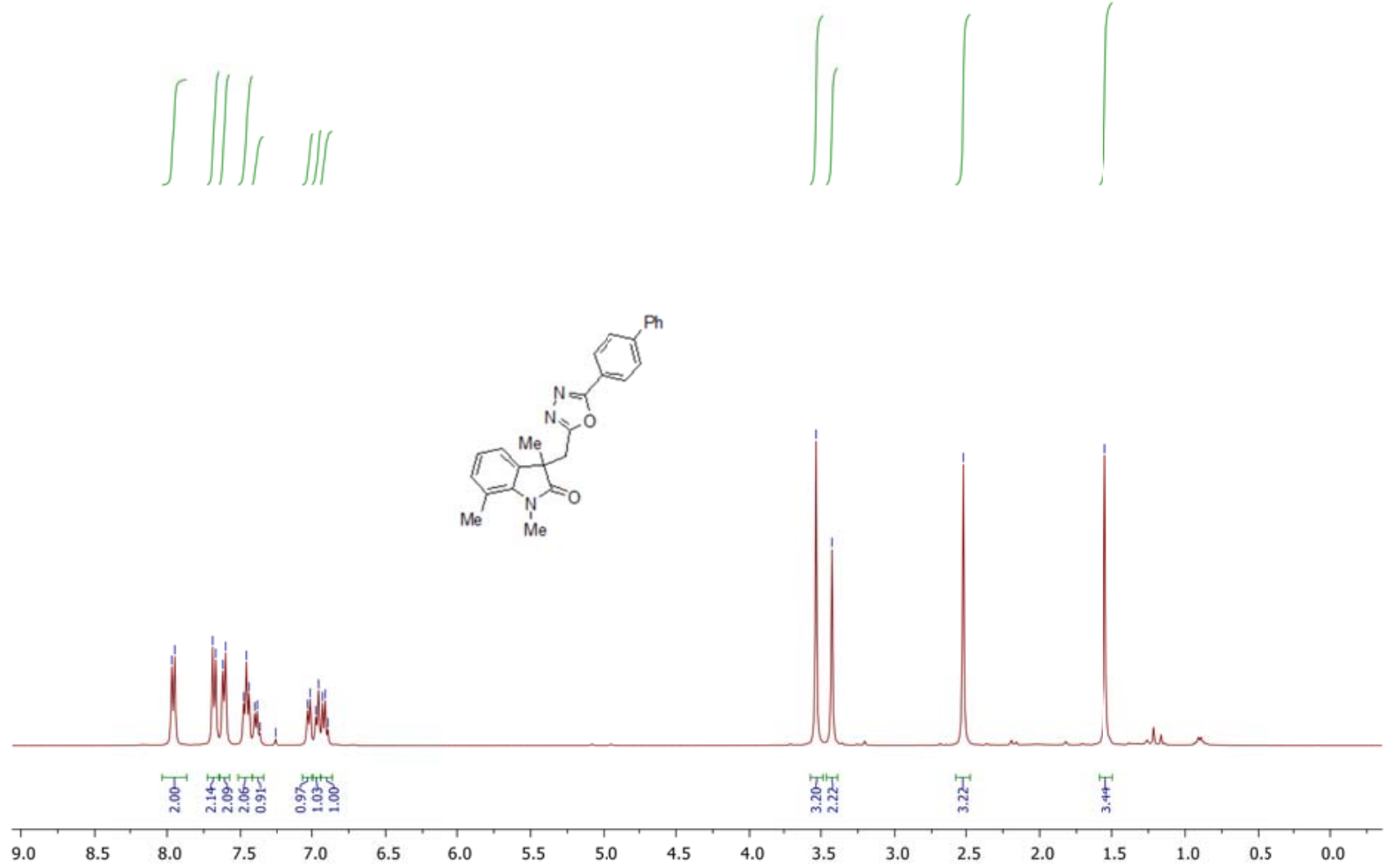

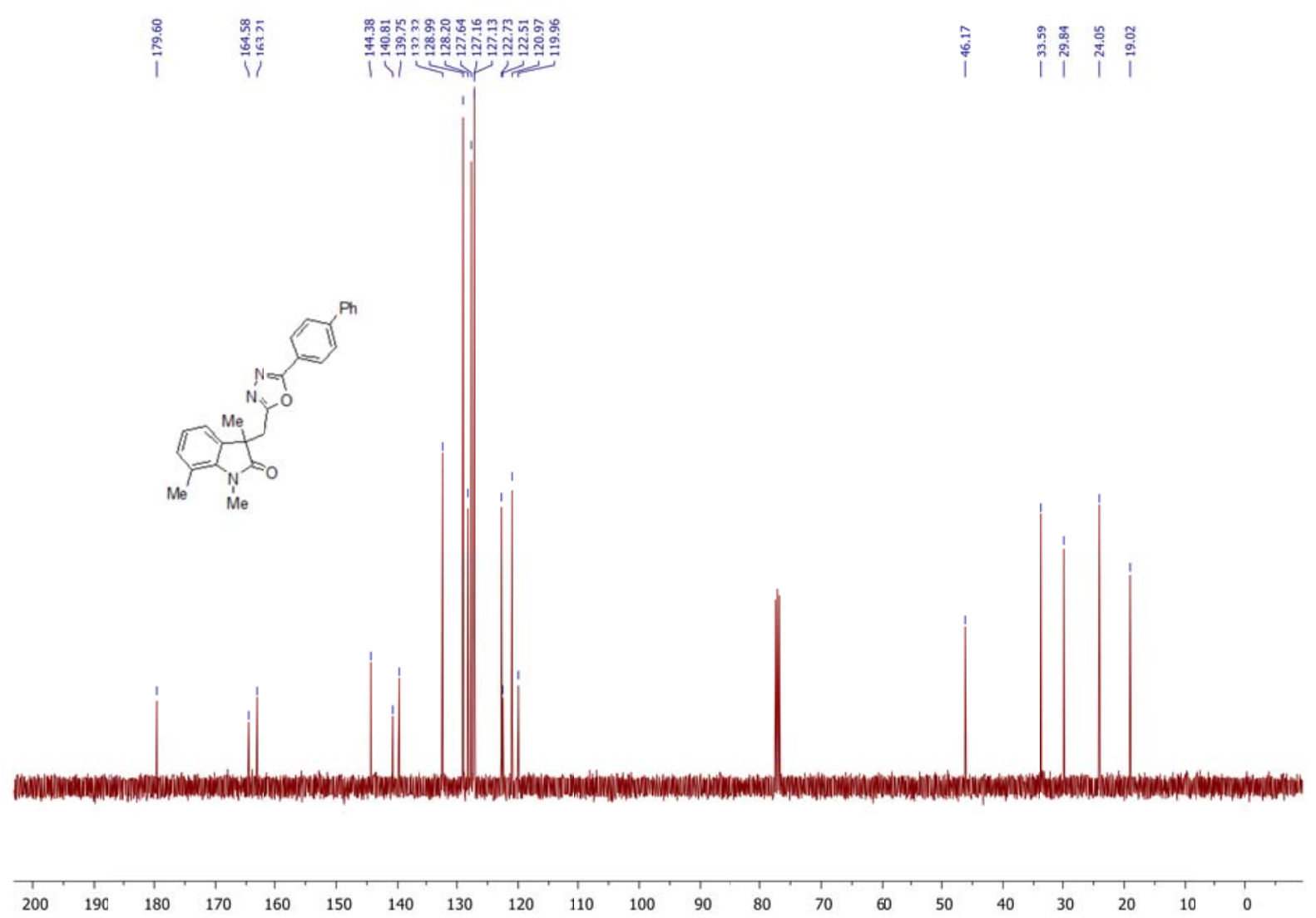

3sa

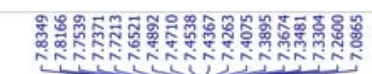

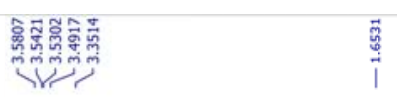

$\iiint$
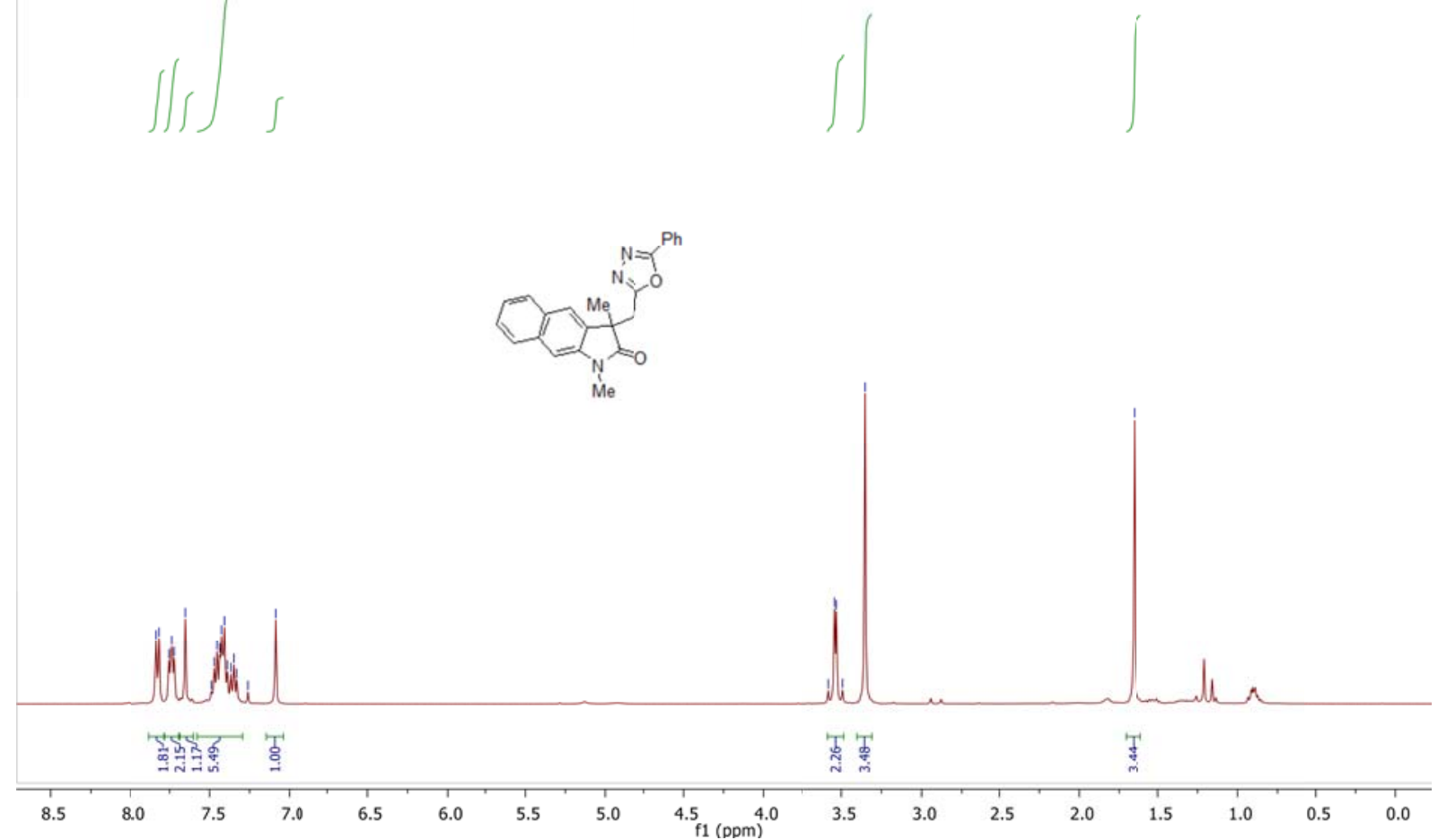


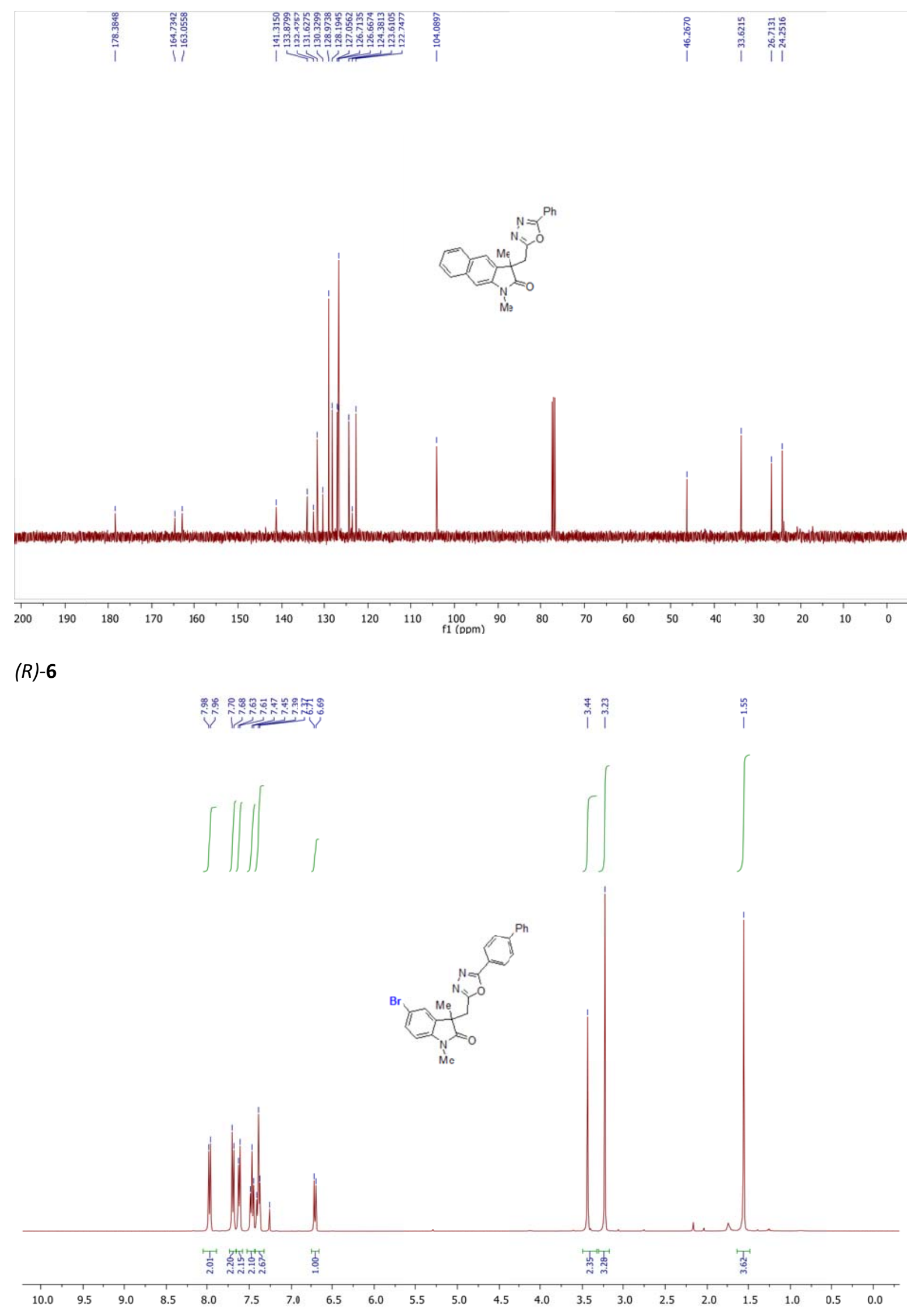



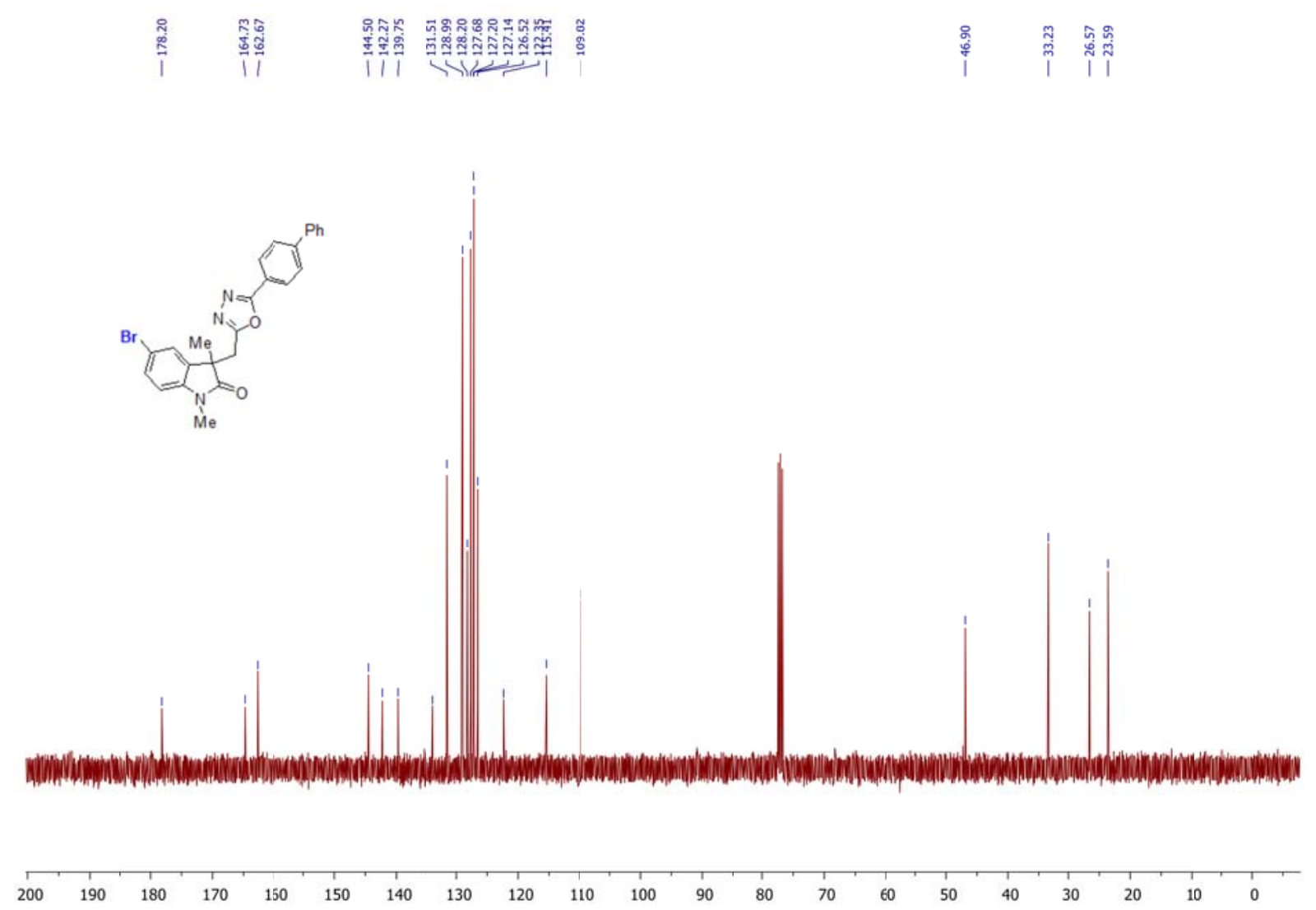

$9 a a$

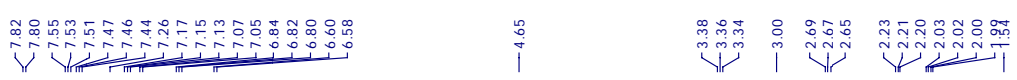
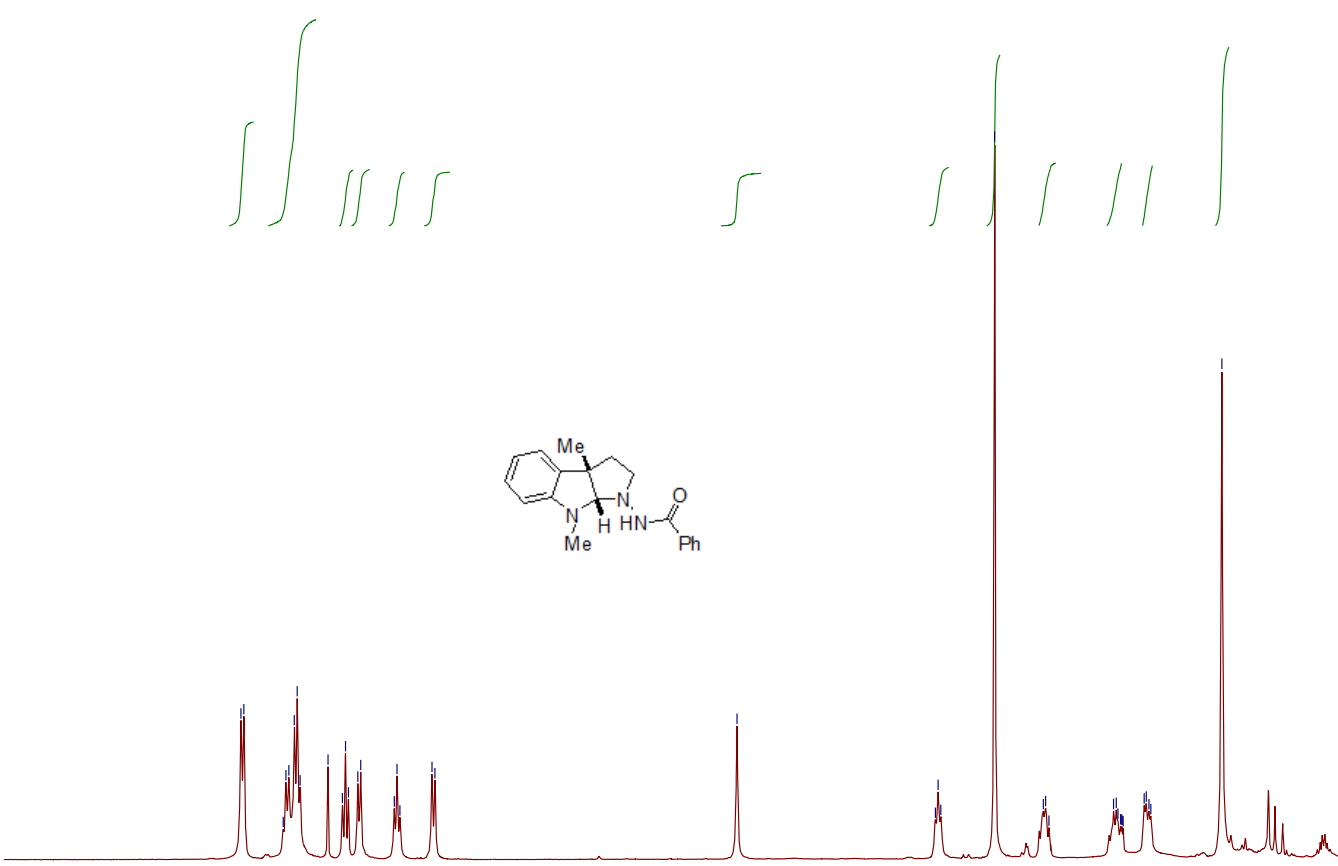

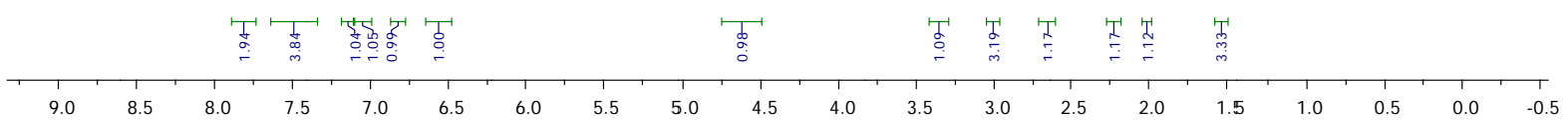




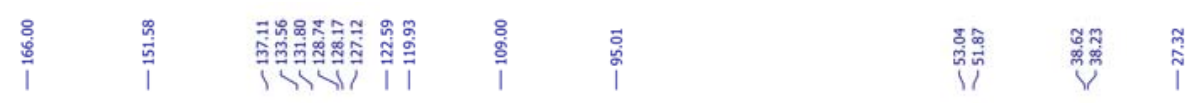

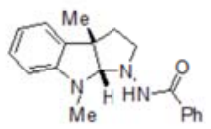

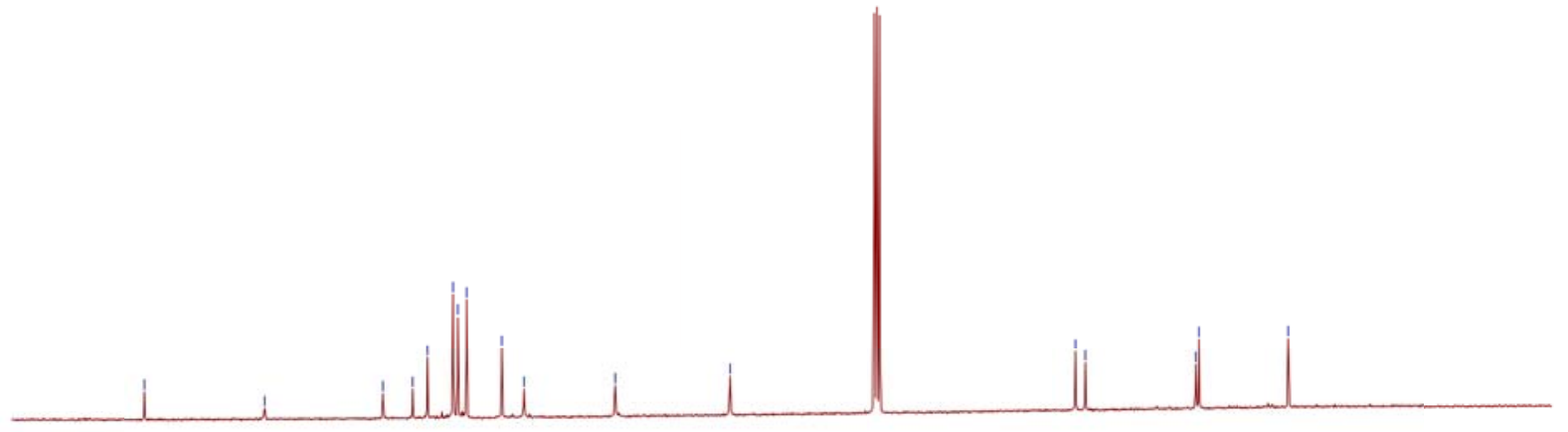

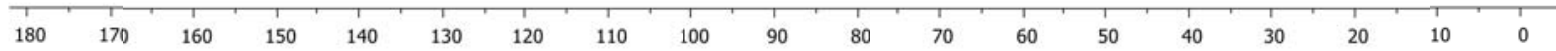
舟

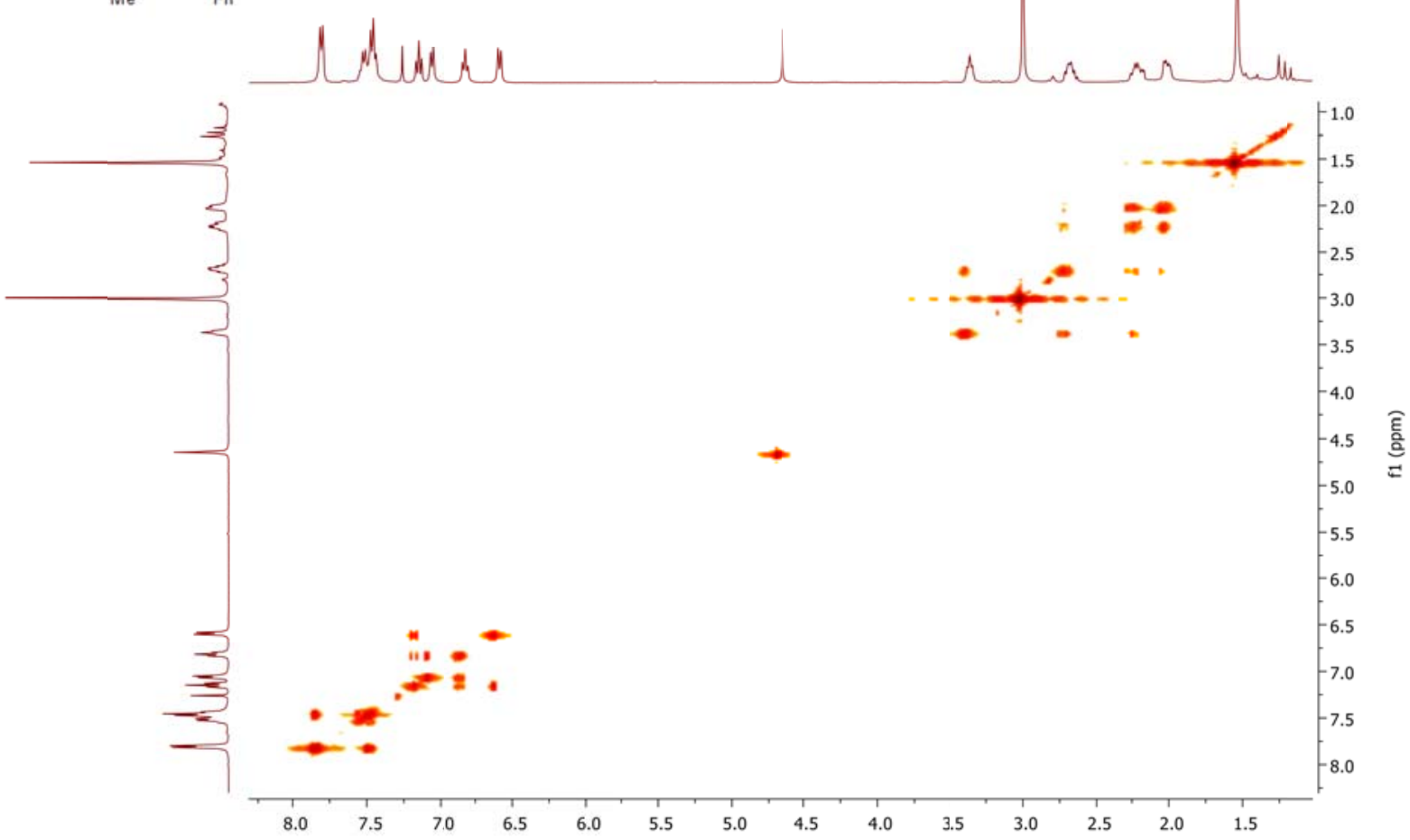




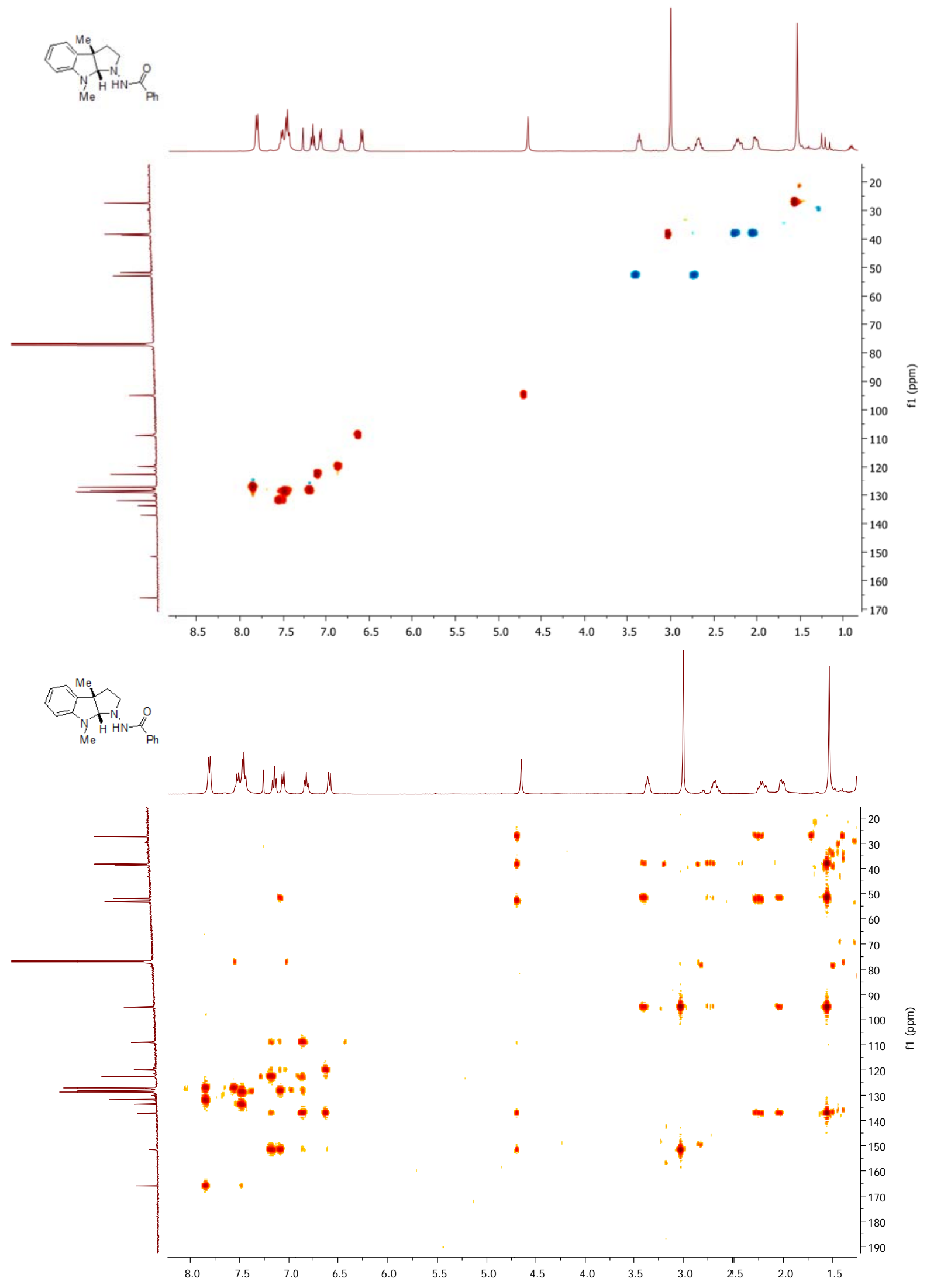


9aa-D3
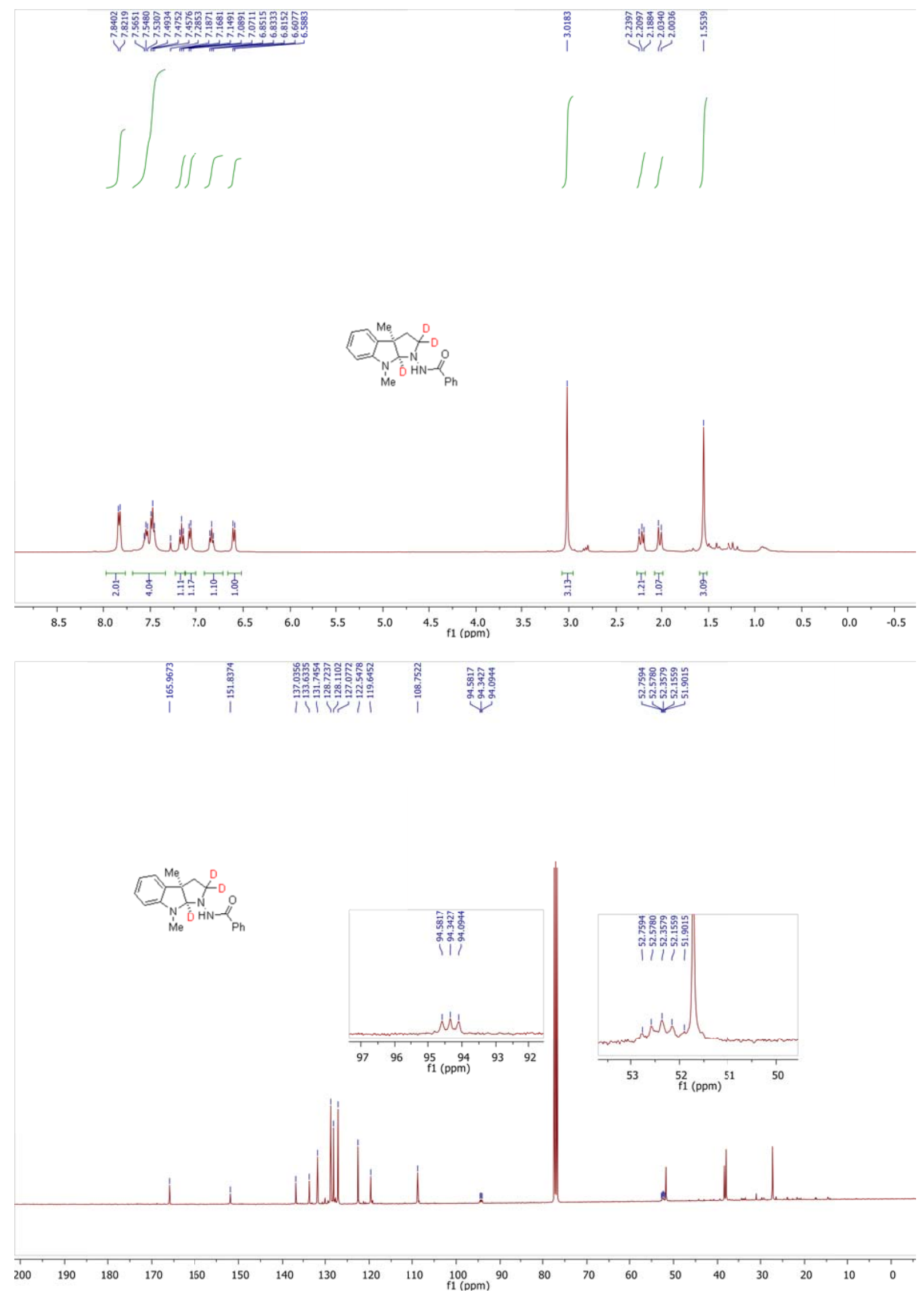

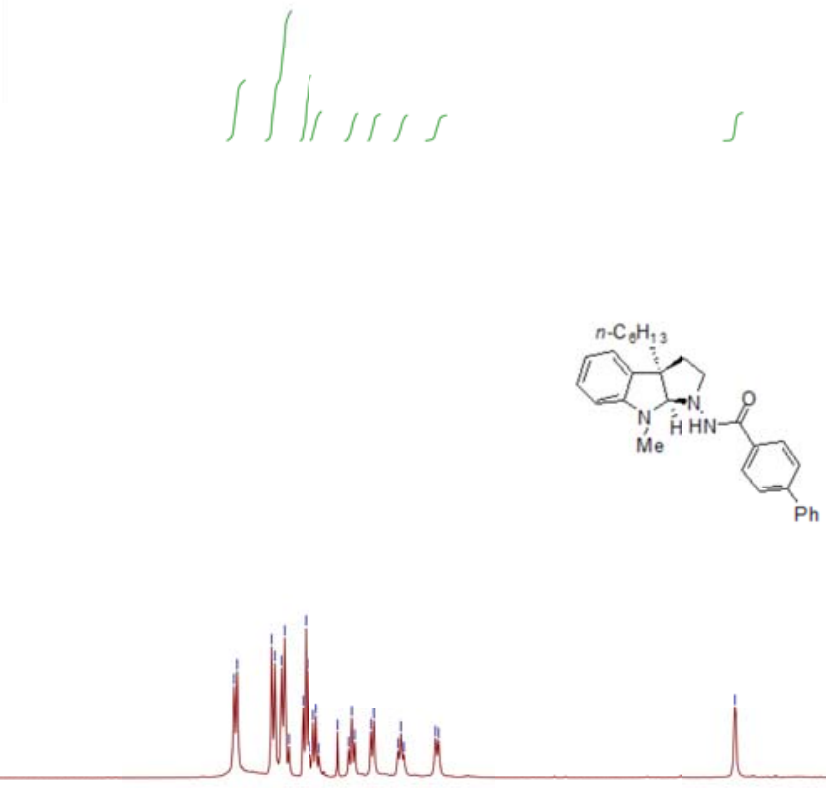

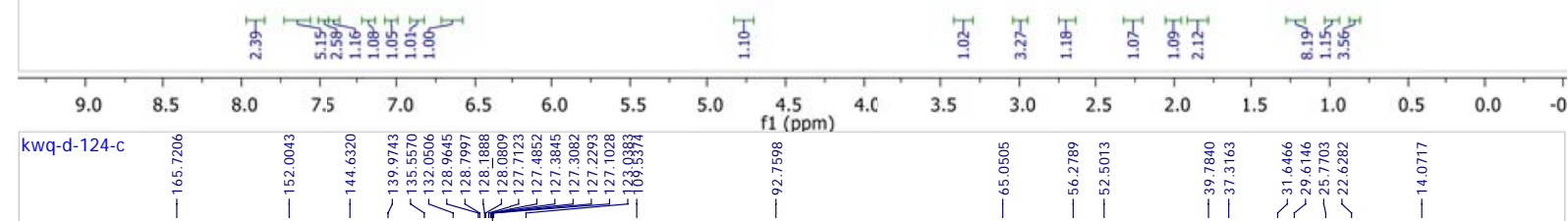

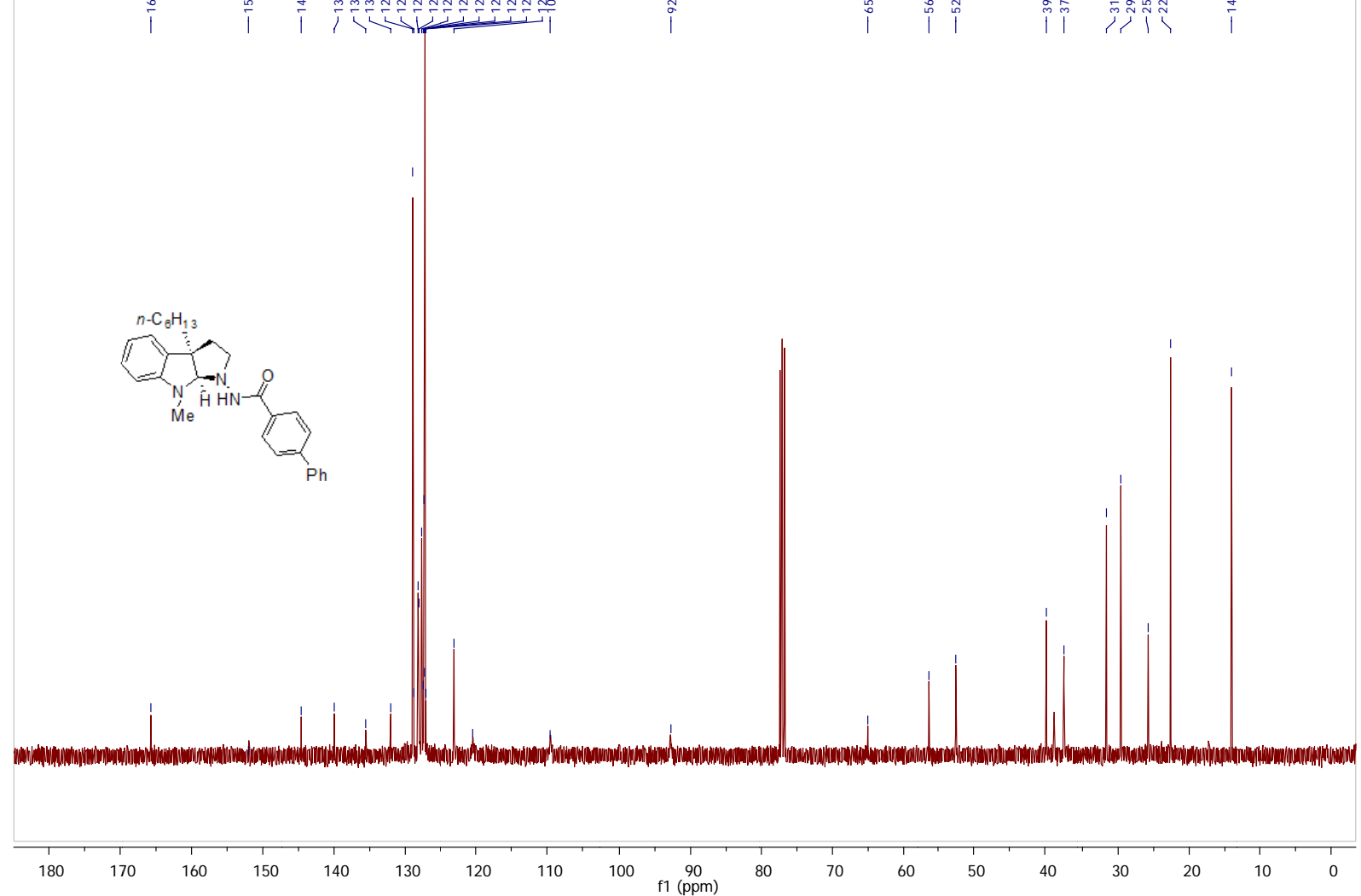


9kd

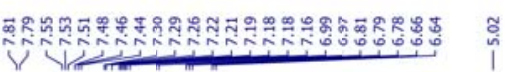

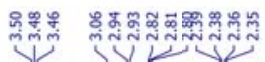
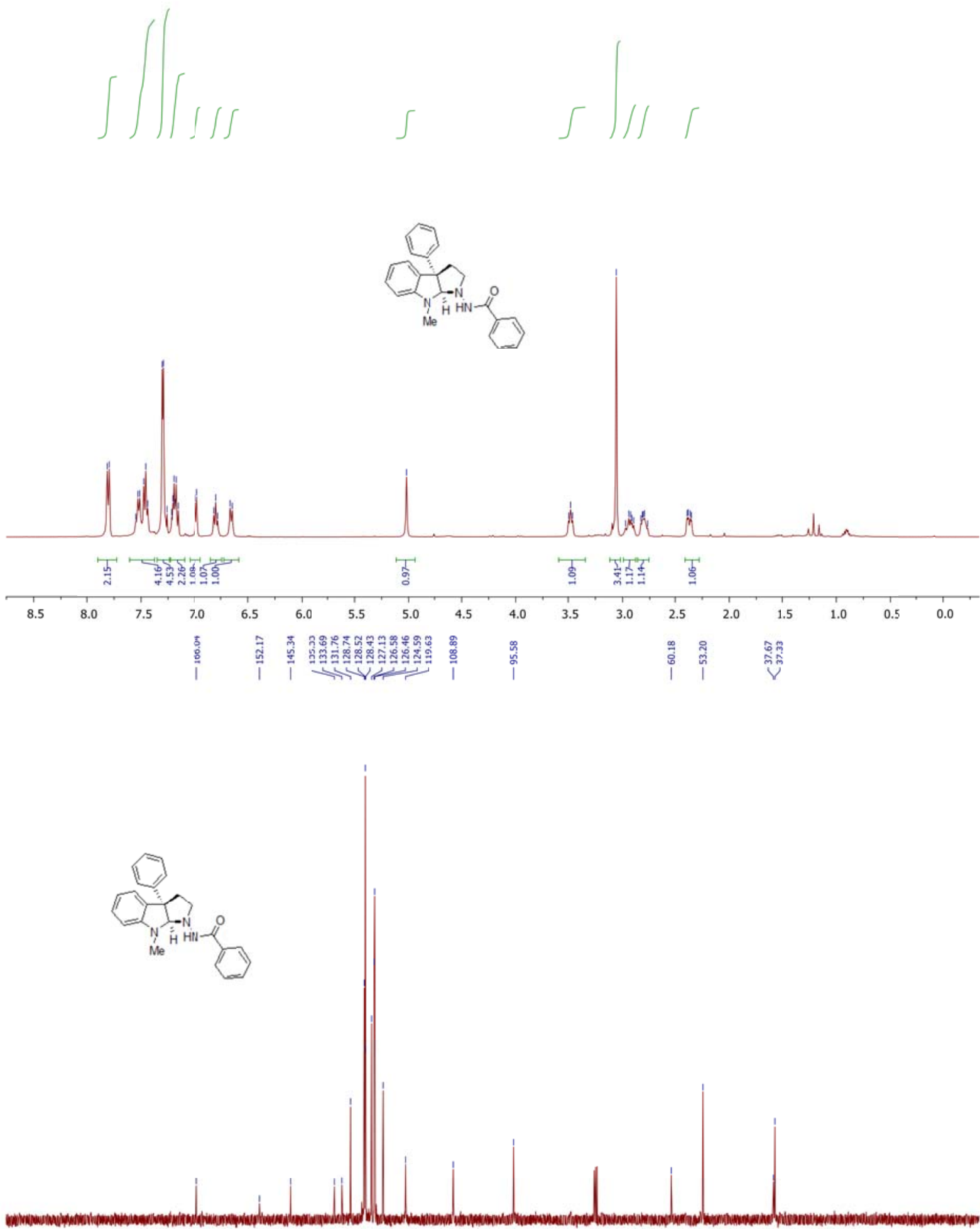

$\begin{array}{lllllllllll}200 & 190 & 180 & 170 & 160 & 150 & 140 & 130 & 120 & 110 & 100\end{array}$ 

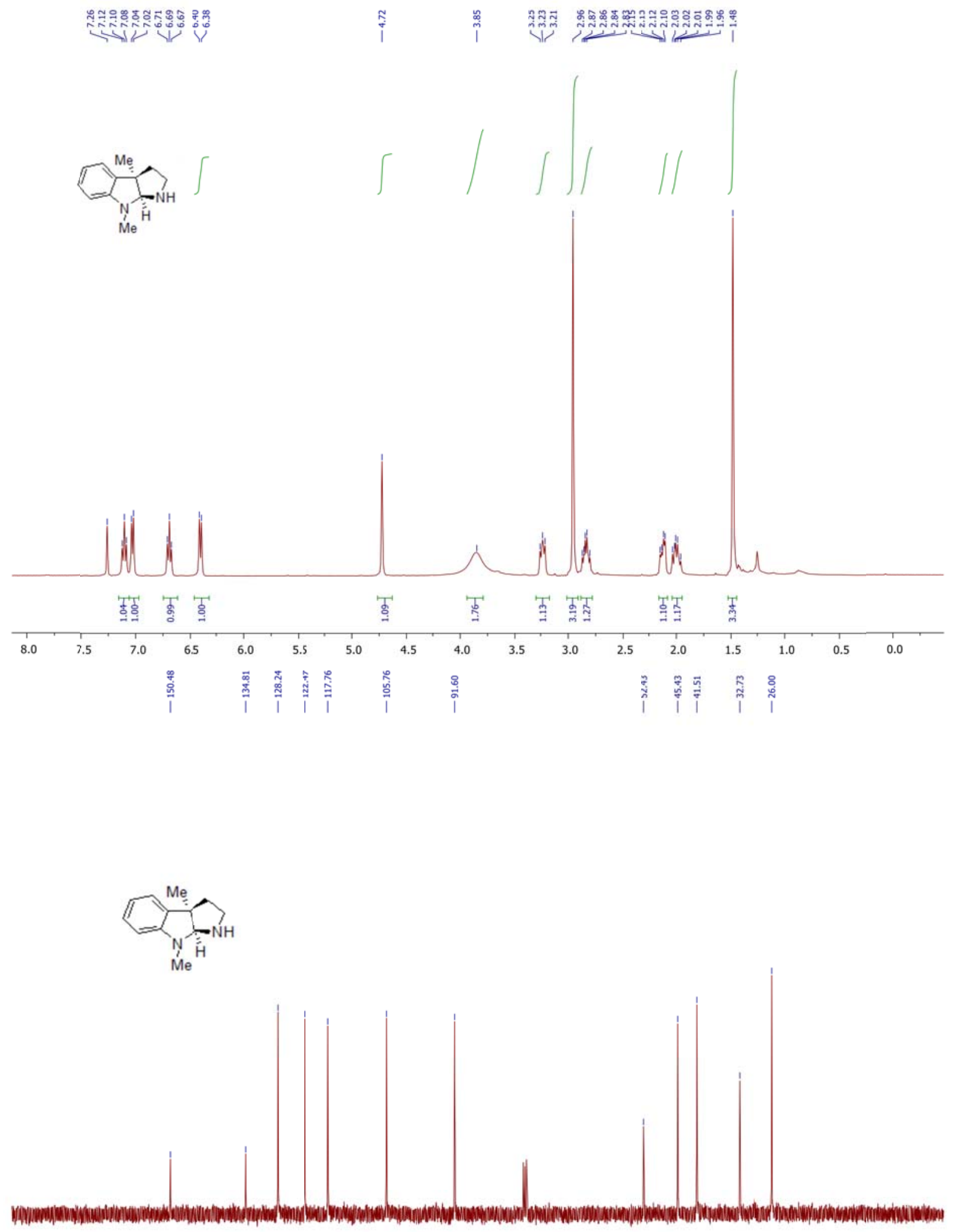

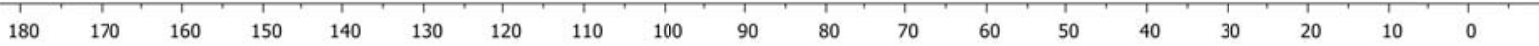



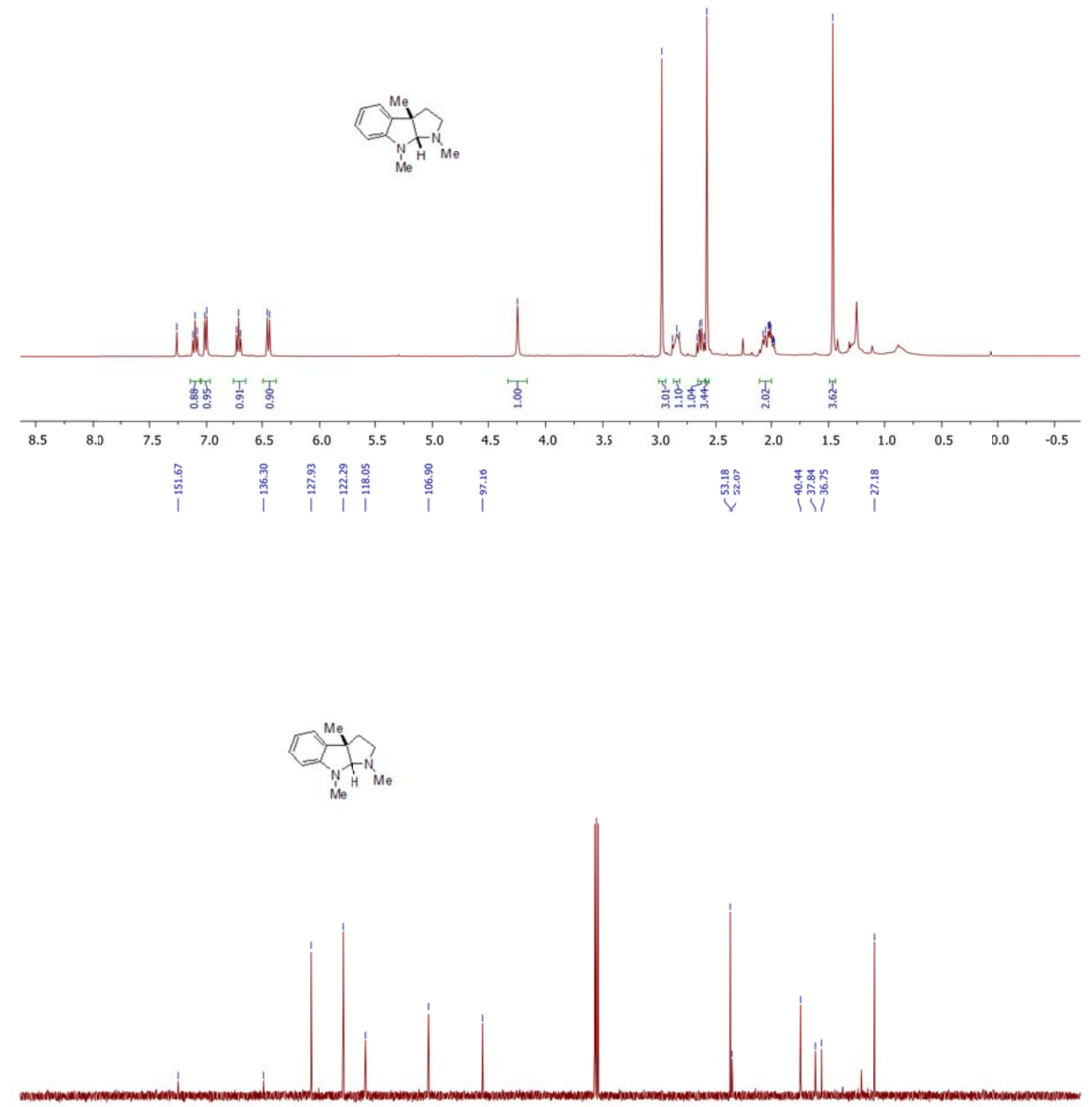

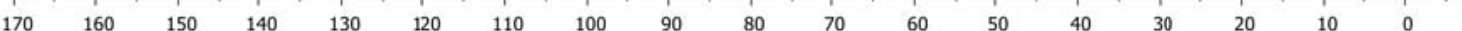


11

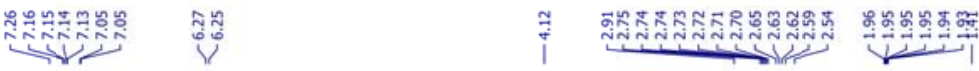
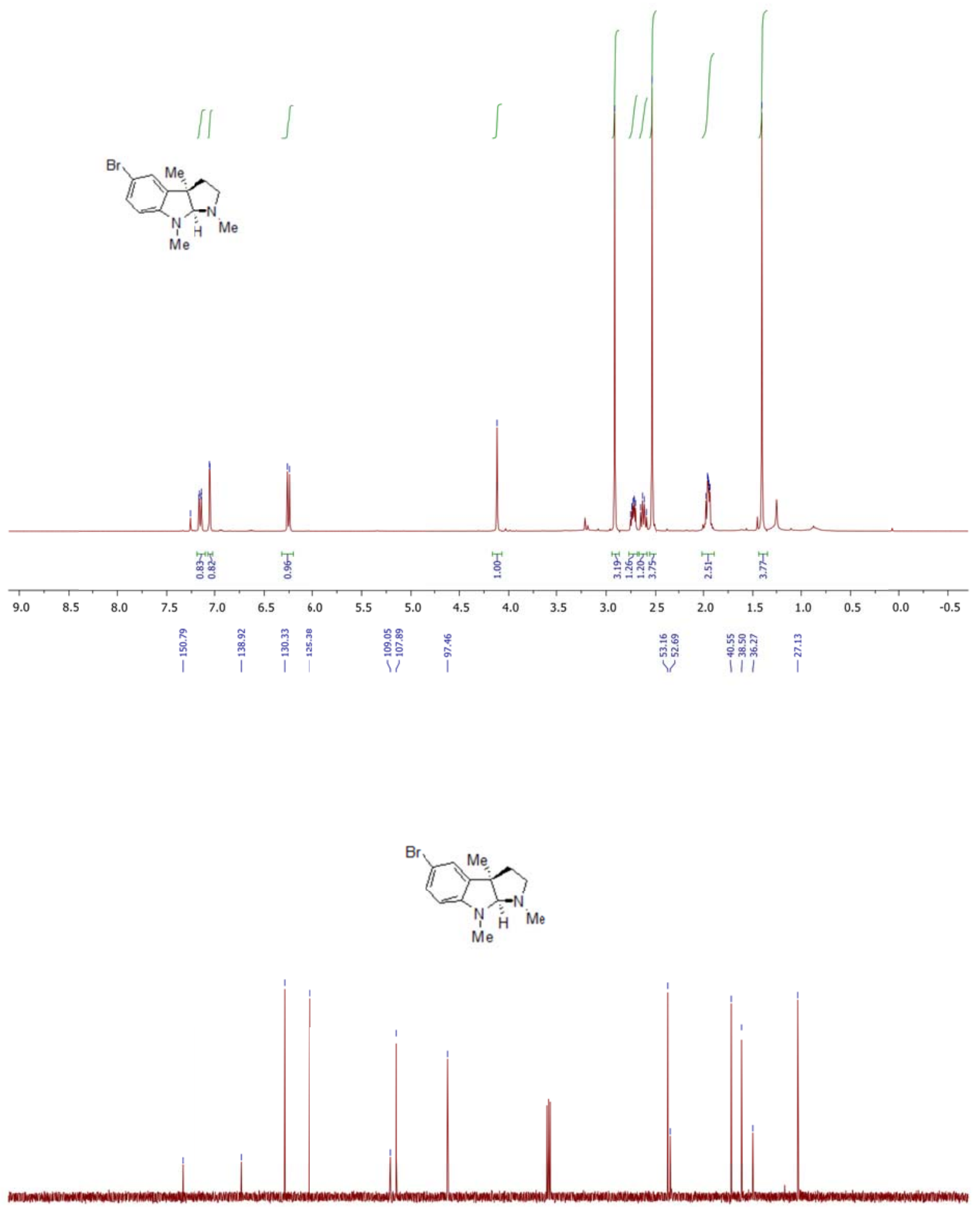

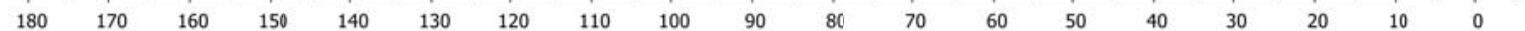


4b

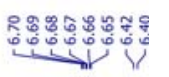

1

$\iint$
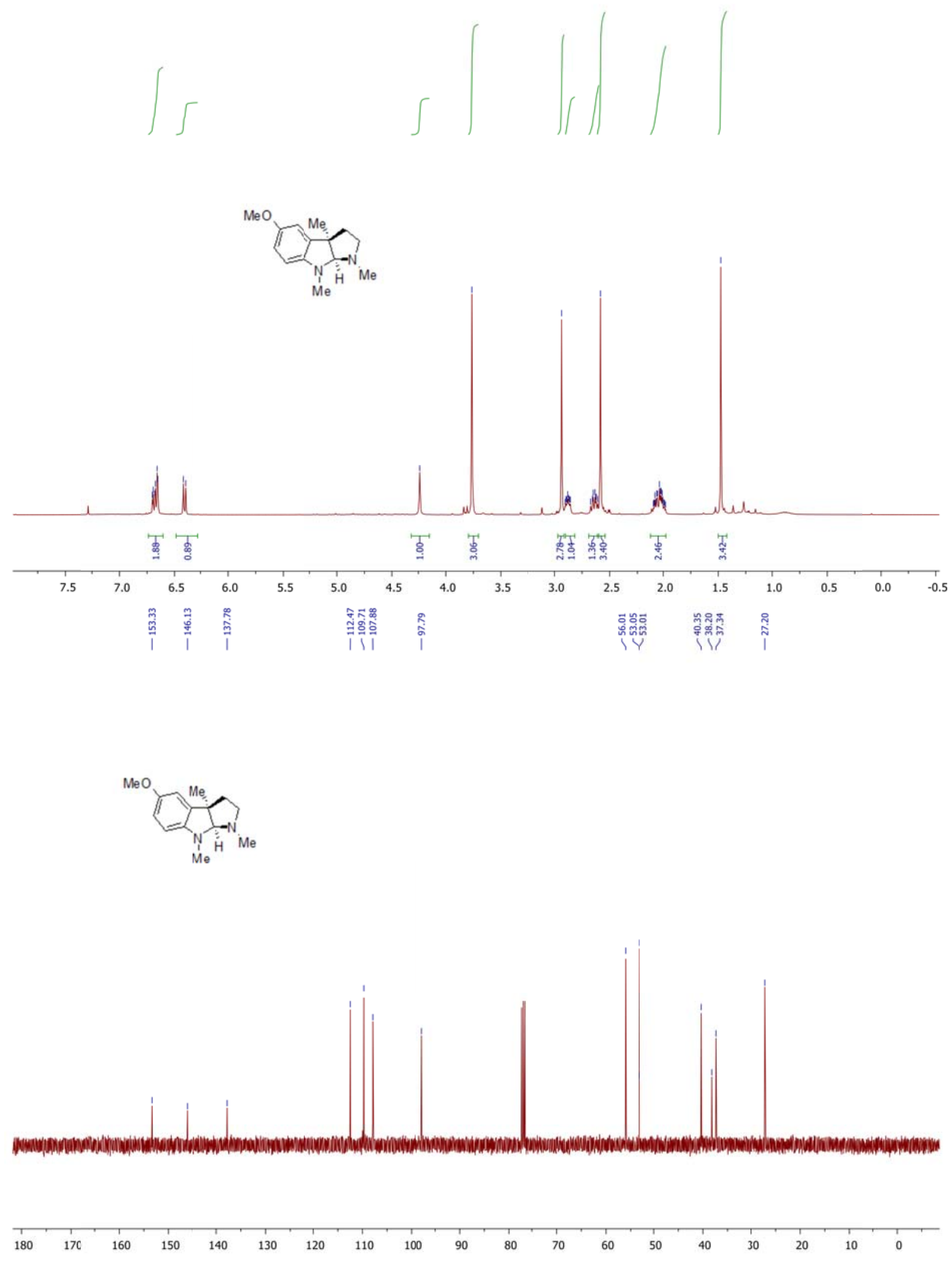

S189 